Baltic Astronomy, vol. 10, 1-318, 2001.

\title{
GENERAL CATALOG OF GALACTIC CARBON STARS BY C. B.STEPHENSON. THIRD EDITION
}

\author{
A. Alksnis, A. Balklavs, U. Dzervitis, I. Eglitis, \\ O. Paupers and I. Pundure \\ Institute of Astronomy, University of Latvia, \\ Raina bulv. 19, Riga, LV-1586, Latvia
}

Received October 1, 2000.

\begin{abstract}
The catalog is an updated and revised version of Stephenson's Catalogue of Galactic Cool Carbon Stars (2nd edition). It includes 6891 entries. For each star the following information is given: equatorial (2000.0) and galactic coordinates, blue, visual and infrared magnitudes, spectral classification, references, designations in the most significant catalogs and coordinate precision classes. The main catalog is supplemented by remarks containing information for which there was no place in entries of the main part, as well as some occasional notes about the peculiarities of specific stars.
\end{abstract}

Key words: catalogs - stars: carbon, fundamental parameters

\section{INTRODUCTION}

Most types of carbon stars are easily recognizable on low resolution blue-green and photographic infrared spectra. Spectral surveys carried out with Schmidt telescopes were the main sources for the search and discovery of galactic carbon stars. The total number of known carbon stars is sufficiently small to keep all of them in a single catalog. B. C. Stephenson of the Warner and Swasey Observatory has maintained a General Catalogue of Cool Carbon Stars for many years. He has published its two paper editions, CCCS and CCGCS (Stephenson 1973, 1989).

At a business meeting of the Working Group on Peculiar Red Giants during IAU Symposium 177 on "The Carbon Star Phenomenon" (May 27-31, 1996) in Antalya, Turkey, it was reported that 
Dr. Stephenson will no longer continue work on the catalog, although there is a need for its updating. A. Alksnis of the Radioastrophysical Observatory, Latvian Academy of Sciences, agreed to consider the possibility of assuming responsibility for updating the catalog (Wing 1997). As a result, a group of astronomers at the Radioastrophysical Observatory of the Latvian Academy of Sciences, now the Astrophysical Observatory of the Institute of Astronomy, University of Latvia, agreed to undertake supplementing and updating of the General Catalogue of Cool Galactic Carbon Stars.

This new printed version of the Catalogue is based on the machine-readable version of the 2 nd edition assembled by Charles Knox at the Department of Astronomy, Case Western Reserve University.

The sources of data of new carbon stars and of new data of known objects of this class mainly were the papers published in journals or other astronomical publications.

In comparison with the previous edition of the catalog, the identification criteria of carbon stars were widened. Carbon stars are recognized in the optical spectral region from the Swan bands of the $\mathrm{C}_{2}$ molecule, from the $\mathrm{CN}$ bands in the near-infrared and from the $11.2 \mu \mathrm{m}$ band of $\mathrm{SiC}$. We have included also stars classified from the $3.1 \mu \mathrm{m}$ band, and we have added carbon star candidates identified by the N. Epchtein group from the $[12-25]$ vs. $K-L$ two-color diagram (Guglielmo et al. 1998 and references therein).

Since the appearance of the 2nd edition of the catalog, the results of the largest surveys of the galactic carbon stars have been based on objective-prism infrared spectra.

Infrared objective-prism surveys of seven low-latitude southern fields between galactic longitudes $245^{\circ}$ and $311^{\circ}$ were completed using the Curtis Schmidt telescope at Cerro Tololo and of nine lowlatitude northern fields between $57^{\circ}$ and $136^{\circ}$ using the Burrell Schmidt telescope of the Warner and Swasey Observatory at Kitt Peak. These studies have led to 209 new discoveries of carbon stars, many of which were confirmed by slit spectra (Aaronson et al. 1989, 1990).

Four-degree prism infrared spectra of the Kiso $105 \mathrm{~cm}$ Schmidt telescope were used to search for cool carbon stars in several areas: in a 240 square-degree area in Cygnus (near $\ell=85^{\circ}$ ), in a 240 squaredegree area in the Serpens-Aquila-Scutum region (near $\ell=32^{\circ}$ ), in a 235 square-degree area in the Perseus-Camelopardalis region (near 
$\ell=145^{\circ}$ ), in a 230 square-degree area in the Monoceros region (near $\ell=206^{\circ}$ ), and in a 230 square-degree area in the Vulpecula region (near $\ell=60^{\circ}$ ) (Maehara \& Soyano 1988, 1990; Soyano \& Maehara $1991,1993,1999)$. As a result, 145 new carbon stars were discovered and many known carbon stars were confirmed.

An unpublished list of southern carbon stars, identified by MacConnell (2000) on infrared plates taken with the Curtis Schmidt telescope of the University of Michigan at Cerro Tololo, added 169 new entries to the catalog and improved positions for 257 of the known carbon stars.

Since 1989 red sensitized Kodak F-type plates of the First Byurakan Spectral Sky Survey $\left(|\mathrm{b}|>30^{\circ}, \delta>-15^{\circ}\right)$ obtained with the 1 meter Schmidt telescope of the Byurakan Observatory, Armenia, have been used to search for and classify faint late-type stars (Gigoyan et al. 1998 and references therein). Thus 39 new carbon stars were added to the CCGCS, mostly at high galactic latitudes.

The discovery of dwarf carbon stars, seven of them newly added to the Catalogue, has stimulated further search for and study of faint carbon stars at high galactic latitudes.

A color-magnitude diagram based on direct blue and red UKST and POSS survey plates, used by Totten \& Irwin (1998) to select faint candidate carbon stars at high galactic latitudes, and followup spectrophotometry on the 2.5 meter Isaac Newton Telescope, La Palma, resulted in 28 newly discovered faint high latitude carbon stars.

Medium resolution slit spectra in the wavelength range 450-700 $\mathrm{nm}$ obtained with the ESO 3.6 meter telescope confirmed carbon star candidates discovered earlier in eight galactic bulge fields with the Canada-France-Hawaii 3.6 meter telescope (Azzopardi et al. 1991). This resulted in 31 new entries in the Catalogue.

We had to delete the entries Nos. 273, 293, 391, 651, 675, 1077, $1111,1224,1260,5832$ and 5920 from CCGCS (each of them actually doubles another entry) and to correct the positions of Nos. 434, 1191,1259 and 5878, because RA(1950) and DEC(1950) for the stars BC 186 - BC 200 (Platais 1981) were included in CCGCS without reduction to the equinox of 1900 .

We have included in the catalog more than 200 infrared carbon star candidates identified through their location in the IRAS color [12-25] vs. near-IR color $K-L$ diagram (Guglielmo et al. 1998 and 
references therein). Their spectra are noted by lower case c, as it is used by these authors.

Altogether almost a thousand new carbon stars have been added to the Catalogue. Their surface distribution is shown in Fig. 1 in equatorial coordinates and in Fig. 2 in galactic coordinates. A similar distribution for all known galactic carbon stars is shown in Figs. 3 and 4.

The carbon stars represented in this catalog include mainly optically identified stars corresponding to the $\mathrm{R}, \mathrm{N}$ or MK classification. The R0-R3 types sometimes are called "warm carbon stars". In the infrared wavelength region we meet "infrared carbon stars", "extreme-" or "dusty carbon stars". A dozen "dwarf carbon stars" or "carbon dwarfs" are known. Evidently, there is no need to call all these types as "cool carbon stars"; a shorter term "carbon stars" is good enough. Thus we can shorten the title of this catalog used in the second edition to the "General Catalogue of Galactic Carbon Stars" (CGCS).

The present catalog includes many Population II carbon stars belonging to the $\mathrm{CH}$ type. More complete catalogs of $\mathrm{CH}$ and related stars were published by Bartkevičius \& Šleivytè (1983), Šleivytė \& Bartkevičius (1990) and Bartkevičius (1996).

The positions of all carbon stars were compared with those in the electronic version of the General Catalogue of Variable Stars available at the Sternberg Astronomical Institute home page (http://www.sai.msu.ru) to identify carbon variable stars. A difference up to 2.5 arcmin in both coordinates was used for the selection of possible variable carbon stars, which were then inspected closer individually to confirm or reject the identity. More than a hundred new names of variable stars were included in the column "Designations".

For most of the stars, which are included in the 2nd edition of the Catalog, no new data have been added, and the respective entries in the new edition of the Catalog (Table 1) remain unchanged. The only change is in the equinox of the equatorial coordinates: they are now reduced to the equinox 2000.0. For the new entries we tried to maintain the style of the 2 nd edition. Therefore we had to repeat the description of the catalog columns from the 2nd edition, modifying them accordingly. Quotations from the 2 nd edition are given in the quotation marks $(\ll \ldots . . \gg)$. 


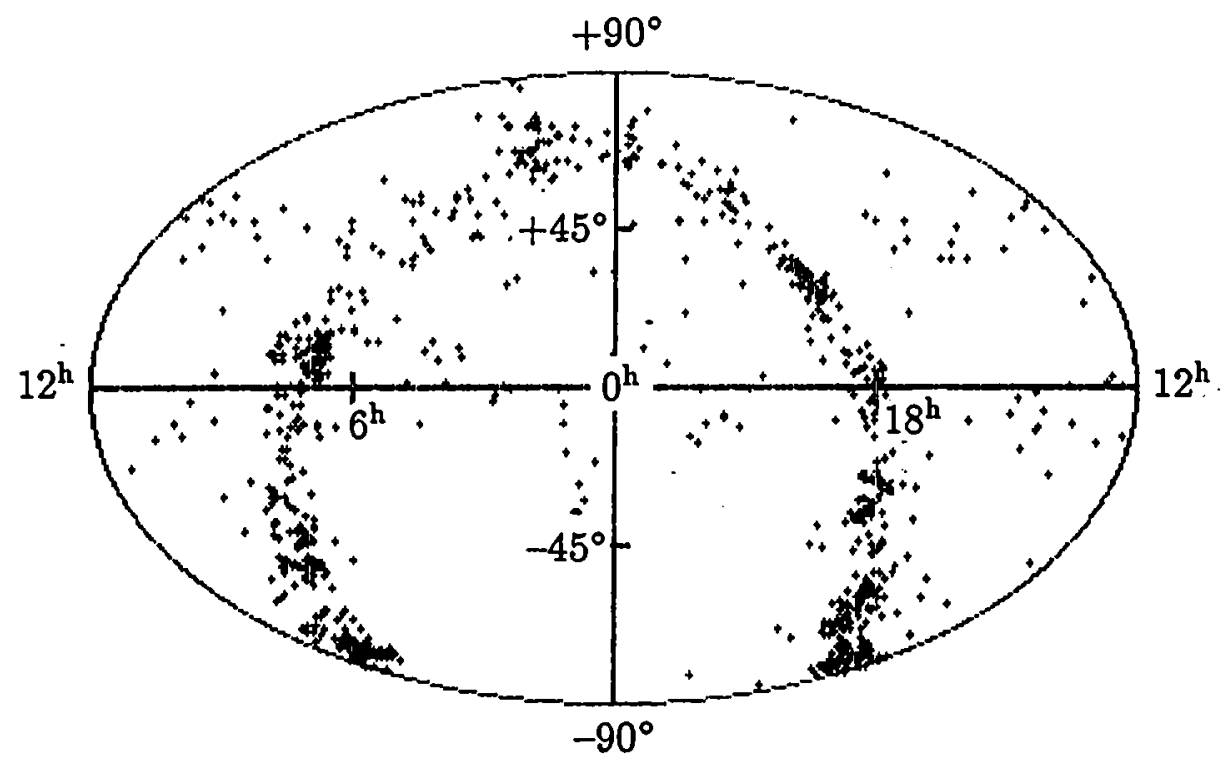

Fig. 1. Surface distribution of new carbon stars in equatorial coordinates.

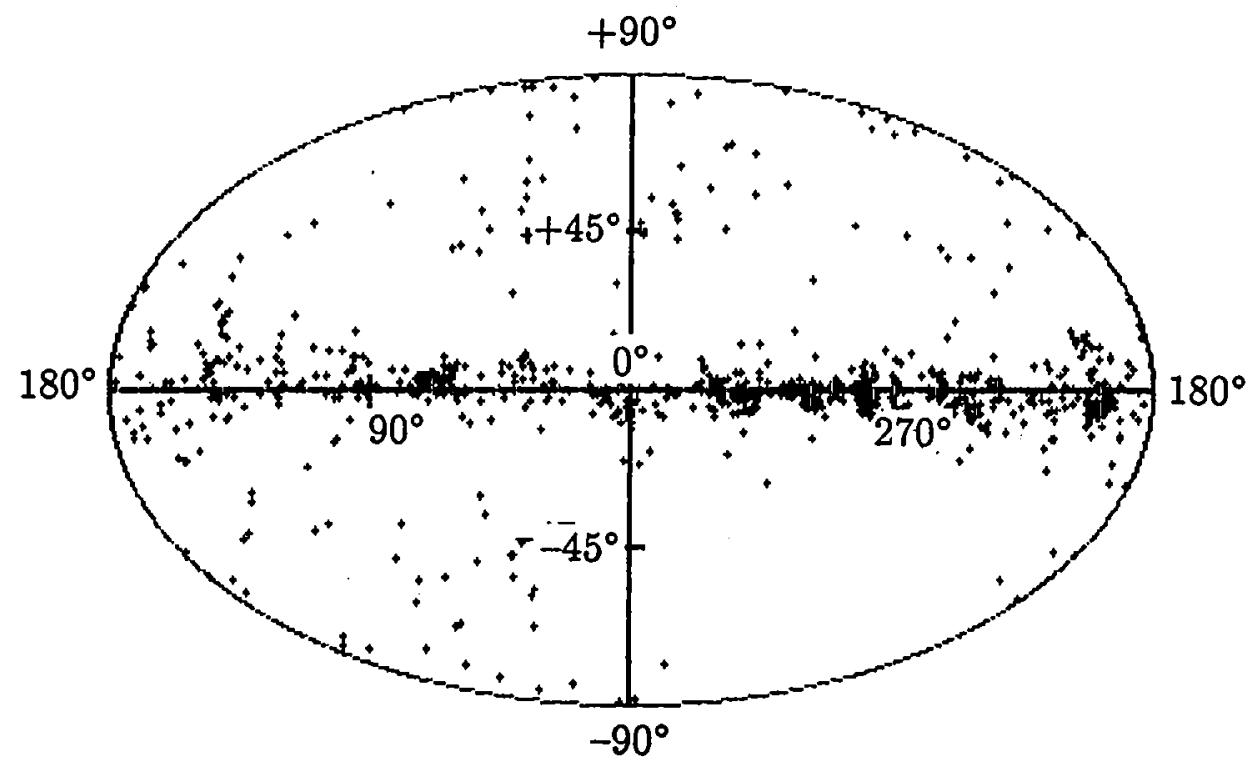

Fig. 2. Surface distribution of new carbon stars in galactic coordinates. 


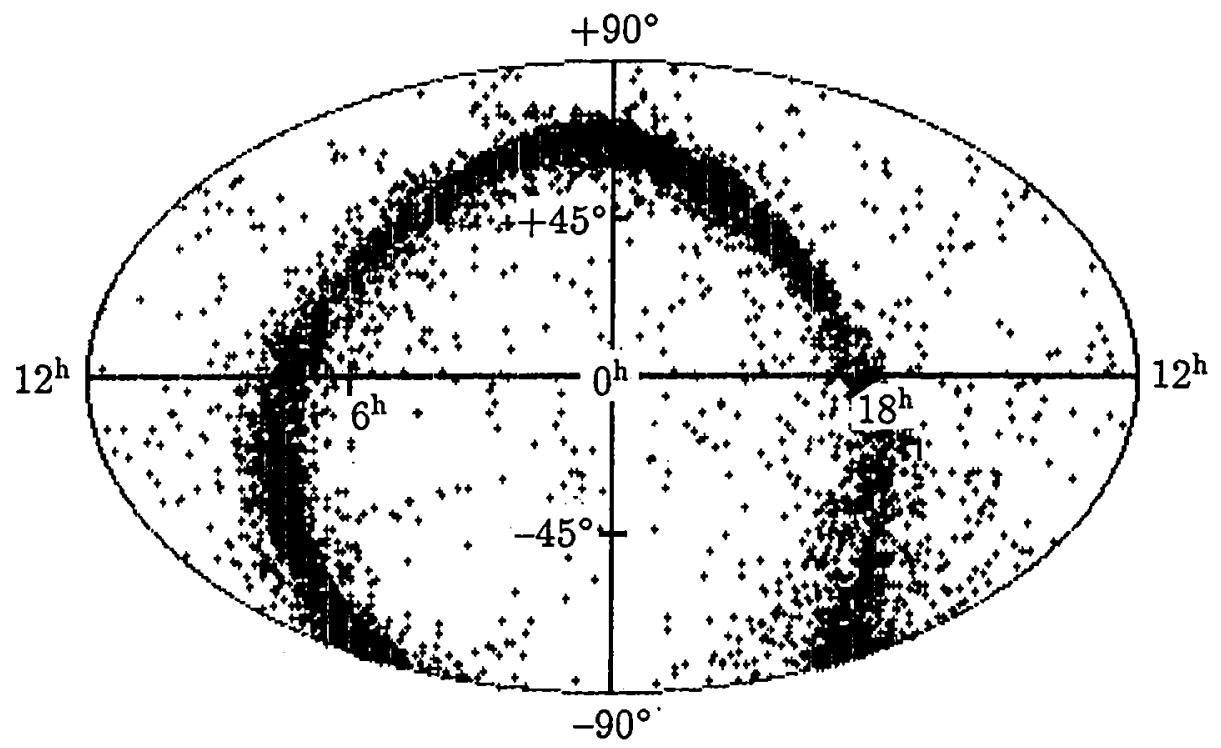

Fig. 3. Surface distribution of all known carbon stars in equatorial coordinates.

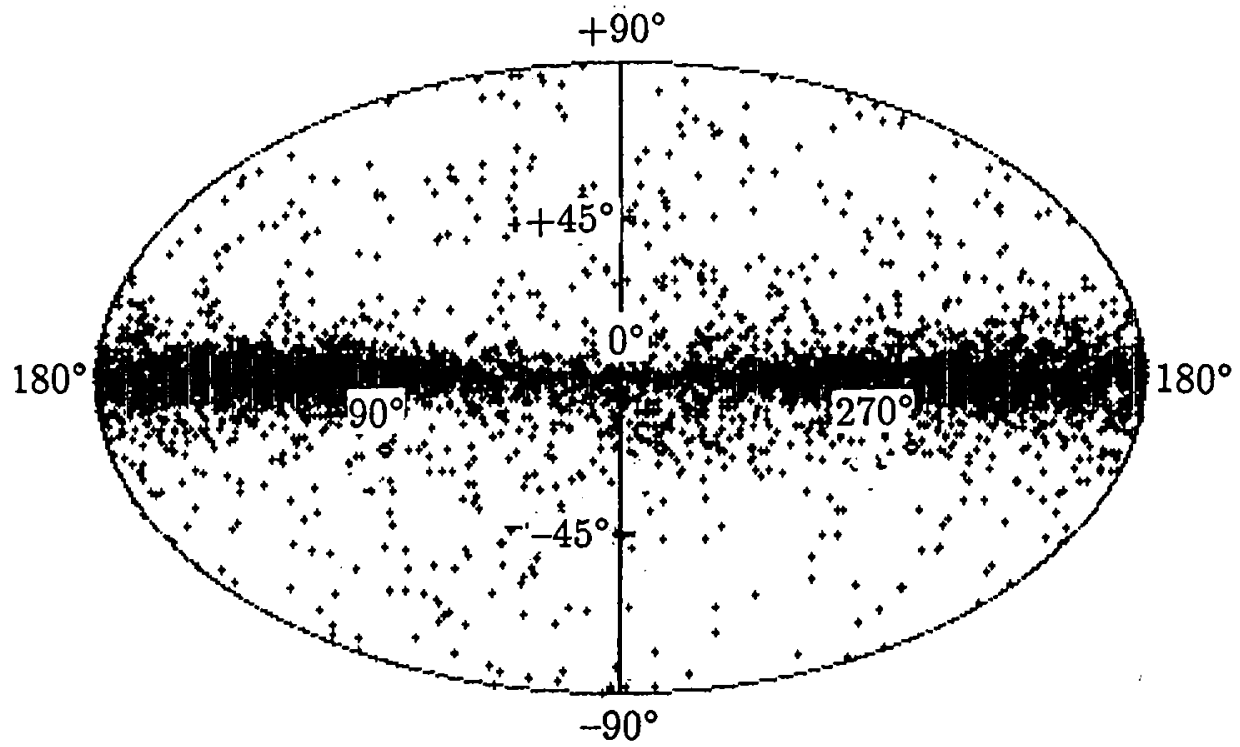

Fig. 4. Surface distribution of all known carbon stars in galactic coordinates. 


\section{DESCRIPTION OF THE CATALOG (TABLE 1): COLUMN HEADINGS}

RA(2000), DEC(2000). These are the equatorial coordinates, referred to the equinox and equator 2000 .

$\ll$ In case of multiple finding lists, the coordinates are averages from multiple sources, heavily weighted in favor of the most accurate, if any. Where Ste is included among the designations, the star has almost invariably been measured for position at the Warner and Swasey Obs. on one or even more objective-prism plates, with a resulting precision of 1-2 arcseconds. The astrometric reduction procedure used by Stephenson is explained in Publs. Warner and Swasey Obs. Vol. 2, No. 4, Sec. II. More than 2000 stars of Table 1 have post-CCCS position determinations done at the Warner and Swasey Observatory. $\gg$

As in the machine-readable version of the CGCCS, the coordinates are given to $0.1 \mathrm{sec}$ in RA and 1 arcsec in DEC, notwithstanding the precision of the position of the object. The estimated precision of the coordinates is indicated in the last column. For many stars of CGCCS the equatorial coordinates have been improved on the basis of new determinations, e.g., by Aaronson et al. (1989, 1990), MacConnell (2000), Maehara \& Soyano (1988, 1990), Soyano \& Maehara (1991, 1993, 1999).

For more than 300 carbon stars equatorial coordinates were taken from the Hipparcos Catalogue after approximation to the format of this catalog. These stars can be recognized as bright stars with the highest precision class 0 in the last column of the Table.

CGCS. This is the number from the first column (No.) of Table 1 from the 2nd edition (CGCCS). We have not introduced a new running number to avoid overcrowding of identifiers for an object. For new entries the running numbers begin with No. 6001 and are given in the sequence of R.A.(2000).

b, v, i. $\ll$ These are, as available, blue, visual and infrared magnitudes. The infrared magnitudes are normally the sort defined by a Kodak N-type photographic plate exposed through a filter similar to the Schott RG8 or 695 filter. Such infrared magnitudes are close to the system of Kron \& Smith (1951), and have now been published for several thousand carbon stars. For $b, v$ and $i$ alike, the 
magnitudes essentially all come from photographic plates; thousands of them are based only upon an average plate calibration used for an entire survey. Thus the magnitudes are almost never accurate to 0.1 mag, to which they are commonly given, and errors of over a whole magnitude should be common, even discounting variability. $\gg$

For some stars $v$ magnitudes from Paupers et al. (1993) are used. For the newly added stars, especially for the infrared (or dusty) carbon stars, which are observed only in the infrared, column $i$ gives the magnitude in the shortest wavelength band with corresponding notation.

long, lat. $\ll$ This is galactic longitude and latitude, in the system currently in use. $\gg$ They are computed from RA(2000) and $\operatorname{Dec}(2000)$.

Spectrum. «This column displays the earliest more reliable determinations of spectral type in the $\mathrm{R} / \mathrm{N}$ notation. Here also are classifications in the scheme of Morgan \& Keenan (1941); this scheme is still a good compact description of the spectrum. Sources of quoted classifications are coded here in parentheses; most of these are listed in the explanation of the "Designations" column, which is devoted to finding lists. Below we list abbreviations for papers consisting mainly of re-observation of stars selected from finding lists. We also include here the abbreviations HD and HDE which are occasionally cited under the "Spectrum" column. $\gg$ However, these abbreviations are omitted in the "Designations" column where a pure HD or HDE number is given. These abbreviations are as follows.

Bidun Bidelman, unpublished.

De Dean (1976).

HD, HDE Henry Draper Catalogue and the first extension, Harvard Annals $91-100$. The second extension, HA 112, is cited as CannMay49.

Her52 Herbig (1952).

IRAS C Little-Marenin et al. (1987).

K-M Keenan \& Morgan (1941).

Lund21 Lundmark (1921).

San41 Sanford (1941).

San Sanford (1944). 
Sh Shane (1928).

War Warner (1963).

WPB Bidelman (1954).

Yam Yamashita $(1972,1975)$.

Designations. «This gives the star's occurence in various finding lists, including unpublished ones. Some CCCS stars repeated here are still called unpublished, which means only that I had no reference to a separate publication when the CCCS went to press. For some of those stars, the CCCS is the only place they have been published; for others, they have since appeared in separate lists by their discoverers. In the latter case I have sometimes changed the discoverer designation to reflect separate subsequent publication, and sometimes not; in the great majority of cases, CCCS stars there called unpublished have since been confirmed in other surveys.

There are finding lists with no special concentration on carbon stars. These are the Bright Star Catalog number, cited, as in all editions of the Bright Star Catalog, as an HR number (and usually put at the head of the list); HD or HDE numbers (listed as a number without other characters); BD or CD numbers in a notation close to the usual one; and the GCVS designation. The intent was to give CD numbers only if the CD magnitude is 9.5 or brighter. Any CPD (Cape Photographic Durchmusterung) nos. in Table 1 are inadvertent unless spelled out as such, a fact due to the circumstance that carbon stars are red stars and the $C D$ is red-biased, not only relative to the CPD but even, though this does not appear to be widely known, relative to the BD.

Other finding lists; an explanation for the coding of these follows. The largest unpublished ones are by Stephenson (Ste), a combination of stars now published in the CCCS and infrared survey products appearing in the present catalog; Sanduleak (Sndlk), whose entries are almost exclusively confined to CCCS stars; Victor Blanco (VB); and MacConnell (MacCon). Although there are several published MacConnell lists cited here, some of the "MacCons" may have since become separately published; but see also the special note under MacCon in the table to follow.

Coding: Whenever a number appears separated from the initial part of the designation by a blank space, the number is a published one assigned by the corresponding discoverer, hyphens if any separating table numbers from numbers within tables. Numbers following 
alphabetic abbreviations without a blank space represent the year of publication where this was for some reason helpful to me (generally, where the same author has produced more than one paper). So W58 1 means star No. 1 in a table of carbon stars in Westerlund (1958). $\gg$

The abbreviations mean the following:

Aar89

Aar90

AbrGig89a

AbrGig89b

AbrGig90

AbrGig93

AbrGig94

Ack

$\mathrm{AFGL}=\mathrm{CRL}$

AGHA97

AGSh95

Akyol

Alb

Alks72

All77

ALR

ALRW91

Balz56

Barb70

$\mathrm{BC}$
Aaronson, Blanco, Cook and Schechter (1989).

Aaronson, Blanco, Cook, Olszewski, Schechter (1990).

Abramyan and Gigoyan (1989a).

Abramyan and Gigoyan (1989b).

Abramyan and Gigoyan (1990).

Abramyan and Gigoyan (1993).

Abramyan and Gigoyan (1994).

Ackermann (1970).

Infrared sky survey, U.S. Air Force, Hanscom Field, MA. Abramyan, Gigoyan, Hambaryan and Azzopardi (1997).

Abramyan, Gigoyan and Shakhbazyan (1995).

Akyol et al. (1974).

Albers (1967).

Alksne and Ozolina (1972).

Allen, Hyland, Longmore, Caswell, Goss and Haynes (1977).

Azzopardi et al. (1985).

Azzopardi, Lequeur, Rebeirot and Westerlund (1991).

Balz (1956).

Barbier (1970).

Baldone carbon star number, assigned at Baldone, Latvia:

BC 10 - BC 33 Alksne and Ozolina (1974).

BC 34 - BC 39 Daube and Ozolina (1974).

BC 40 - BC 49 Alksne and Ozolina (1975).

BC 50 - BC 62 Alksne and Ozolina (1976).

BC 63 - BC 77 Alksnis, Alksne, Ozolina and Eglitis (1976).

BC 78 - BC 109 Alksnis, Alksne and Ozolina (1977). 
BC 110 - BC 184 Alksnis, Alksne, Ozolina and Platais (1978).

BC 185

Platais (1979).

BC 186 - BC 200 Platais (1981).

BC 201 - BC 217 Alksne and Alksnis (1981).

BC 218 - BC 234 Alksne, Alksnis and Eglitis (1981).

BC 235 - BC 245 Alksnis, Alksne and Eglitis (1983).

BC 246 - BC 255 Alksnis and Ozolina (1983).

BC 256 - BC 272 Alksnis, Alksne, Platais and Ozolina (1987).

BC 273 - BC 310 Alksnis, Alksne, Ozolina and Platais (1988).

BC 311 - BC 318 Alksnis, Alksne, Ozolina and Platais (1988).

BC 1 - BC 318 Alksne, Alksnis, Ozolina and Platais (1989).

Bid80

BidMac

Blanc55

B-M

Bon75

Boes

Bot91

BSD

C

Cann21

Cann22

Cann26

CannMay38

CannMay 49

Catchp68

CCCS

CCGCS
Bidelman (1980).

Bidelman and MacConnell (1973).

Blanco, Nassau et al. (1955).

Blanco and Munch (1955).

Bond (1975).

Boeshaar, unpublished.

Bothun, Elias, Macalpine, Matthews, Mould,

Neugebauer, Reid (1991).

The Bergedorfer Spektral-Durchmusterung

(Schwassmann and Van Rhijn 1935).

The Case infrared surveys at low galactic latitudes

(Nassau and Blanco 1954, 1957; Blanco 1958).

The carbon star numbers are continuous.

Cannon (1921).

Cannon (1922).

Cannon (1926).

Cannon and Mayall (1938).

Cannon and Mayall (1949). The Annie J. Cannon Memorial.

Catchpole (1968).

Catalogue of Cool Carbon Stars, 1st edition (Stephenson 1973).

A General Catalogue of Cool Galactic Carbon Stars, 2nd edition (Stephenson 1989) 
CH96 Cohen and Hitchon (1996).

CK77 Cohen and Kuhi (1977).

CohKuh Cohen and Kuhi (1976).

Crg61 Cregg (1961).

Cut89 Cutri, Low, Kleinmann, Olszewski, Willner, Campbell and Gillett (1989).

CWWVS Cohen, Wainscoat, Walker, Volk and Schwartz (1989).

CWWV96 Cohen, Wainscoat, Walker and Volk (1996).

D The Dearborn Observatory objective-prism surveys for red stars (Lee et al. 1940-44). The carbon stars thus found were abstracted as lists of exclusively carbon stars, in the papers just cited, and were duplicated in longer lists containing all red stars found; the $\mathrm{D}$ numbers given in Table 1 refer to the short lists, with one or two exceptions spelled out in the notes to the table. The notes also refer in other contexts to the "longer" Dearborn lists, with the meaning just explained. The "short" list numbers for carbon stars are continuous from paper to paper.

Daub71 Daube (1971).

DLKSH77 Dahn, Liebert, Kron, Spinrad and Hintzen (1977).

Dol62 Dolidze (1962).

Dol68 Dolidze (1968).

Dol71a Dolidze (1971a).

Dol71b Dolidze (1971b).

Dril72 Drilling (1972).

Epc87 Epchtein, Le Bertre, Lepine, Margues dos Santos, Matsuura and Picazzio (1987).

Epc90 Epchtein, Le Bertre and Lepine (1990).

Esp Espin (1924).

Fea97 Feast (1997).

Feast77 Feast et al. (1977).

Flem12 Fleming (1912).

Fou92 Fouque, Le Bertre, Epchtein, Guglielmo and Kerchbaum (1992).

Fr75 Frogel, Dickinson and Hyland (1975).

Fuen Fuenmayor (1981). 
FueSto95 Fuenmayor and Stock (1995).

GAA Gigoyan, Hambaryan and Azzopardi (1998).

GAM Gigoyan, Azzopardi and Muratorio (1999).

GBE98 Guglielmo, Le Betre and Epchtein (1998).

GEAS97 Guglielmo, Epchtein, Arditti and Sevre (1997).

GCVS General Catalogue of Variable Stars Volumes I-III, 4th edition (1999).

GGHCG Grasdalen, Gehrz, Hackwell, Castelaz and Gullison (1983).

GH76 Gehrz and Hackwell (1976).

GigAmb Gigoyan and Hambaryan (1996).

GJ93 Groenewegen and de Jong (1993).

GJB93 Groenewegen, de Jong and Baas (1993).

GJBSW Groenewegen, de Jong, van der Bliek, Slijkhuis and Willems (1992).

GJG94 Groenewegen, de Jong and Geballe (1994).

GMAC Green, Margon, Anderson and Cook (1994).

GMAM92 Green, Margon, Anderson and MacConnell (1992).

GMM91 Green, Margon and MacConnell (1991).

GOL97 Groenewegen, Oudmaijer and Ludwig (1997).

Gor68 Gordon (1968).

GreMar90 Green and Margon (1990).

GreMar94 Green and Margon (1994).

Gug93 Guglielmo, Epchtein, Le Betre, Fouque, Hron, Kerschbaum and Lepine (1993).

GWSK Groenewegen, Whitelock, Smith, Kerschbaum (1998).

Ha85 Hartwick and Cowley (1985).

$\mathrm{HanBl}$ Hansen and Blanco (1975).

Har Hardorp, Lubeck and Stephenson (1972). Spectral type $\mathrm{ER}=$ early $\mathrm{R}, \mathrm{LN}=$ Late $\mathrm{N}$, etc.

Harw Harwood (1962).

Hen Rybski (1972). Most of the carbon stars discussed by Rybski were found on objective-prism plates by Henize, and by him assigned the numbers which Rybski refers to as Hen numbers and Stephenson quotes in Table 1. There is no connection between these Hen numbers and others 
occurring in the literature - even in the case of the occasional Hen numbers for carbon stars cited by Wray (1966), who however also assigns his own numbers and which are quoted in Table 1 as Wray.

IDS Index Catalogue of Double Stars. Not mentioned if ADS number given.

JBGHJKL Jones, Bryja, Gehrz, Harrison, Johnson, Klebe and Lawrence (1990).

Jon78 Jones, Merrill, Puetter and Willner (1978).

Joy98 Joyce (1998).

Krum L. E. Krumenaker, unpublished. At Warner and Swasey we had by 1972 taken duplicate objective-prism plates of the northern Milky Way for about half of the fields surveyed in the Hamburg-Cleveland survey for luminous stars in the Northern Milky Way. Although carbon stars were identified and measured for position by the Hamburg workers, and eventually published (Hardorp et al. 1972), the Hamburg limiting magnitude for carbon stars was not very faint (cf. Hardorp et al. 1972). Hence Krumenaker searched for carbon stars on the new Case material already mentioned. On the other hand, the magnitude limit for $\mathrm{R} / \mathrm{N}$ classification is already fairly uniform between Hamburg and Cleveland (Stephenson 1989).

Krum75 Krumenaker (1975).

Kub W. R. Kubinec, unpublished. These carbon stars are discussed by Kubinec, without position data, in Publs. Warner and Swasey Obs. 1, No. 3. He measured the approximate positions specially for Table 1.

Kur96 Kurtanidze, unpublished (1996).

KurNik88 Kurtanidze and Nikolashvili (1988).

KurNik88b Kurtanidze and Nikolashvili (1988b).

KurNik89 Kurtanidze and Nikolashvili (1989).

KurNik90 Kurtanidze and Nikolashvili (1990).

KurNik2000 Kurtanidze and Nikolashvili (2000).

Kurt78 Kurtanidze (1978, 80, 80a, 81, 88).

Kurt80

Kurt80a

Kurt81

Kurt88 
Kurtun Kurtanidze, unpublished.

KurtWest Kurtanidze and Westerlund (1980).

LE83 Lloyd Evans (1983).

LE90 Lloyd Evans (1990).

LE91 Lloyd Evans (1991).

LeB92 Le Bertre (1992).

LFOP93 Loup, Forveile, Omont and Paul (1993).

Lit Little-Marenin et al. (1987). These are carbon star classi-

fications from IRAS spectra (11.2 $\mu \mathrm{m} \mathrm{SiC} \mathrm{emission).}$

LKVR76 Low, Kurtz, Vrba and Rieke (1976).

Lo74 Lockwood (1974).

LR77 Lebofsky and Rieke (1977).

LSKR78 Lebofsky, Sargent, Kleinmann and Rieke (1978).

LSLSLCFB Liebert, Schmidt, Lesser, Stepanian, Lipowetsky, Chaffee,

Foltz and Bergeron (1994).

MacCon With running nos. 1-466, MacConnell (1988).

MacCon MacConnell, unpublished, at least originally.

MacCon67 MacConnell (1967, 79, 81, 82, 97)

MacCon79

MacCon81

MacCon82

MacCon97

MacCon2000 MacConnell, unpublished (2000).

MaeSoy Maehara and Soyano (1986a).

MaeSoy2 Maehara and Soyano (1986b).

MaeSoy88 Maehara and Soyano (1988).

MaeSoy90 Maehara and Soyano (1990).

Marg . Margon et al. (1984).

May51 Mayall (1951).

MayCann40 Mayall and Cannon (1940).

Mayun N. U. Mayall, unpublished, communicated to C. B.

Stephenson by W. P. Bidelman.

$\mathrm{McC} \quad$ McCarthy (1960).

Merr50 Merrill and Burwell (1950).

MerSt76 Merrill and Stein (1976). 
MGSJ97 Moody, Gregory, Soukup and Jaderlund (1997).

Mich W.P. Bidelman and D. J. MacConnell, unpublished. The observations were made on $108 \AA / \mathrm{mm}$ objectiveprism plates taken with the Schmidt telescope of the University of Michigan at Cerro Tololo.

Mik78 Mikami (1978).

Mic97 Mickaelian (1997).

MSB Merrill, Sanford and Burwell (1933, 1942).

MSGAL85 Mould, Schneider, Gordon, Aaronson and Liebert (1985).

Nass64 Nassau, Stephenson, and Caprioli (1964).

NassCam Nassau and Cameron (1956). Cited only if the star did not appear later with a $\mathrm{C}$ number.

NassCol Nassau and Colacevich (1950). The numerical C types quoted from this paper are defined in the paper; they essentially describe the $\mathrm{CN}$ strength and the redness of the spectrum in the infrared.

NassSte61 Nassau and Stephenson (1961).

NassvanAl Nassau and van Albada (1949).

NETC Nguyen-Q-Rieu, Epchtein, Truong-Bach and Cohen (1987).

Nik Nikolashvili (1987a).

Nik2 Nikolashvili (1987b).

Nik3 Nikolashvili (1987c).

NSV New Catalogue of Suspected Variable Stars (Kukarkin et al. 1982).

OLFHHS Omont, Loup, Forveille, Hekkert, Habing and Sivagnanam (1993).

OrlRod74 Orlov and Rodriges (1974).

Perr59 Perraud and Pelletier (1959).

Perr61 Perraud (1961).

Pes Pesch, unpublished, Case objective-prism plate.

Pes67 Pesch (1967).

Phil67 Philip (1967).

Pick11 Pickering (1911).

Po78 Poulakos (1978). 
Pots The Potsdam Spektral-Durchmusterung (Becker 1931, Becker and Bruck 1935-38).

Poul Poulakos (1972).

Richt Richter, Schaifers and Wentzel (1961).

Ros51 Rosino (1951).

San49 Sanford (1949).

SandPhil Sanduleak and Philip (1968).

Schai59 Schaifers (1959).

Schai60 Schaifers (1960).

SHLE96 Sarre, Hurst and Lloyd Evans (1996).

Slet Slettebak, Keenan and Brundage (1969).

Smith37 Smith (1937).

Sndlk N. Sanduleak, unpublished, various objective-prism plates taken at Cerro Tololo.

SndPes Sanduleak and Pesch (1982).

SndPes88 Sanduleak and Pesch (1988).

Sop85 Sopka, Hildebrand, Jaffe, Gatley, Roellig and Werner (1985).

SoyMae91 Soyano and Maehara (1991).

SoyMae93 Soyano and Maehara (1993).

Soymae99 Soyano and Maehara (1999).

Ste Stephenson, unpublished, various objective-prism plates (Case, Hamburg, Bosscha, Cerro Tololo) unless slit spectrograms mentioned in the notes.

Ste65 Stephenson (1965).

Ste76 Stephenson (1976).

Ste85 Stephenson (1985).

Sto Stock and Wroblewski (1972).

Thack49 Thackeray (1949).

The68 The (1968).

Tot98 Totten and Irwin (1998).

Upgr60 Upgren (1960).

Upgr68 Upgren and Grossenbacher (1968).

Van Vandervoort (1958).

VB V. Blanco, unpublished. 


$\begin{array}{ll}\text { Vet } & \text { Vetesnik (1979). } \\ \text { VKL92 } & \text { Volk, Kwok and Langill (1992). } \\ \text { Vogt } & \text { Vogt (1973). } \\ \text { VyssBalz58 } & \text { Vyssotsky and Balz (1958). } \\ \text { W } & \text { Westerlund (1971). } \\ \text { W58 } & \text { Westerlund (1958). } \\ \text { W65 } & \text { Westerlund (1965). } \\ \text { War65 } & \text { Warner (1965). } \\ \text { War93 } & \text { Warren, Irwin, Evans, Liebert, Osmer, Hewett (1993). } \\ \text { Wil73 } & \text { Wilson, Schwartz and Epstein (1973). } \\ \text { WMFCMC } & \text { Whitelock, Menzies, Feast, Catchpole, Marang, Carter } \\ & \text { (1995). } \\ \text { Wray } & \text { Wray (1966). CE = H } \alpha \text { emission. } \\ \text { WY77 } & \text { Wing and Yorka (1977). }\end{array}$

$\ll$ Except for the occurrence of the CCCS numbers, the ordering of the carbon star-finding lists under "designations" in Table 1 is generally the order in which I have become aware of them. The major exception is myself (Ste), whom I have usually listed last regardless of history. The overwhelming majority of these separately-found identifications for the same carbon star are, to the best of my knowledge - and certainly for my own surveys - completely independent rediscoveries. $\gg$

n. $\ll$ Four symbols were introduced in the CCCS and are retained here. (1) An asterisk indicates that Table 1 equates two or more data in literal contradiction to one or more papers cited; for example, a much later paper may be reporting a new carbon star already so identified in a much earlier paper, which implies that perhaps there are two carbon stars in the area. This was a natural flag to use in the CCCS, where carbon stars were mainly reported in a few large and well-known surveys. It is no longer natural now, where the main pre-publication comparison has been with the CCCS, and there have meanwhile been many relatively short lists. Thus, I have added few if any new asterisks. Finally, a single asterisk meant that my fusing of two or more reports was based on coordinates alone, without any plate re-examination. In fact, many or most of these cases have now been routinely re-examined through later, systematic, 
surveys; but I have retained the asterisk to show the historical order. (2) A double asterisk means the same as a single one, except that I have specially re-examined plates with the identification problem in mind. As in (1), many of these have been further checked routinely by later, systematic surveys, where the finding of only one star allows the strong presumption that only one carbon star is involved. There are few or no new double asterisks in the present edition. (3) A plus sign signals a GCVS identification made by me by comparing an objective prism plate with a published identification chart. These, too, are mostly holdovers from the CCCS, because the host of more accurate coordinates for carbon stars in the present edition usually restricts any challenge to identifying a carbon star with a GCVS cool-giant variability type to the question (at least on deep plates): is there any other banded spectrum near the carbon star? (4) An R signals further remarks at the end of Table 1.»

The object of the remarks is twofold. First, remarks are intended as an extension to the entries "Designations" and "Spectrum" of the catalog comprising matter for which there was no place in the Table. Second, remarks contain information about considerable disagreement in the position of a star determined by different authors or cases when there is some doubt if the data refer to the same star. We repeat Stephenson's remarks, although in a shortened form, in which he confirms discoveries or points to certain spectral and photometric peculiarities (outstanding spectral lines and bands, very red color etc.). Also we indicate the cases when a carbon star lies in the sky within boundaries of some star clusters and note conclusions about the membership, if possible. Also the stars with such distinguishing features as circumstellar silicate envelopes, detached gas and dust shells, maser emission, binarity and bipolar molecular outflows are marked.

P. Estimated precision class of the equatorial coordinates of the star: $0-1$ for \pm 0.1 arcsec, 2 for \pm 1 arcsec and 3 for \pm 0.1 arcmin. Values of the precision class have been estimated by comparing coordinates of the stars determined by different authors. The highest precision class 0 was assigned only to the positions determined by Hipparcos, by MacConnell (2000) and for dwarf carbon stars. 


\section{REJECTED STARS}

Table 2 contains 21 stars that have been declared as carbon or possible carbon stars but this classification was rejected in other publications. This table is a supplement to Table 2 in the 2nd edition of the Catalogue. The first five columns coincide with those in the Catalogue (Table 1), the 6th column gives the reference to the publication (with abbreviations of Section 2), the designation and the carbon spectral type, the 7th column gives the same for the publications in which this classification has been rejected.

\section{CROSS-CORRELATION CGCS - R.A. (2000)}

After reduction of equatorial coordinates to the equinox 2000, the sequence of CCGCS=CGCS numbers in the catalog is newly arranged, and some stars are difficult to find according to their CGCS number, especially circumpolar stars. The same refers to several stars for which equatorial coordinates in the 2nd edition are in error. To help find these stars identified with their CCGCS number, crosscorrelation between this number and their coordinates is given in Table 3.

ACKNOWLEDGMENTS. We are grateful to Robert F. Wing, Chairman of the IAU Working Group on Peculiar Red Giant Stars for encouragement to start this work. We thank the authors who sent us reprints of their papers containing lists of carbon stars, especially, Hideo Maehara and Takao Soyano from the National Astronomical Observatory of Japan, Omar M. Kurtanidze and Maria G. Nikolashvili from Abastumani Astrophysical Observatory. We are thankful to D. Jack MacConnell from Space Telescope Science Institute (U.S.A.) who put at our disposal his unpublished list of new southern carbon stars and also pointed to the corrections needed for coordinates of several stars in the 2 nd edition of the Catalogue. We are also thankful to William Buscombe from Northwestern University (U.S.A.) for supplying us with his latest catalogs of MK Spectral Classification. Our work has been supported by the grant No. 96.0225 from the Latvian Council of Science. 
Table 2. Stars that have been published as carbon but probably or definitely are not.

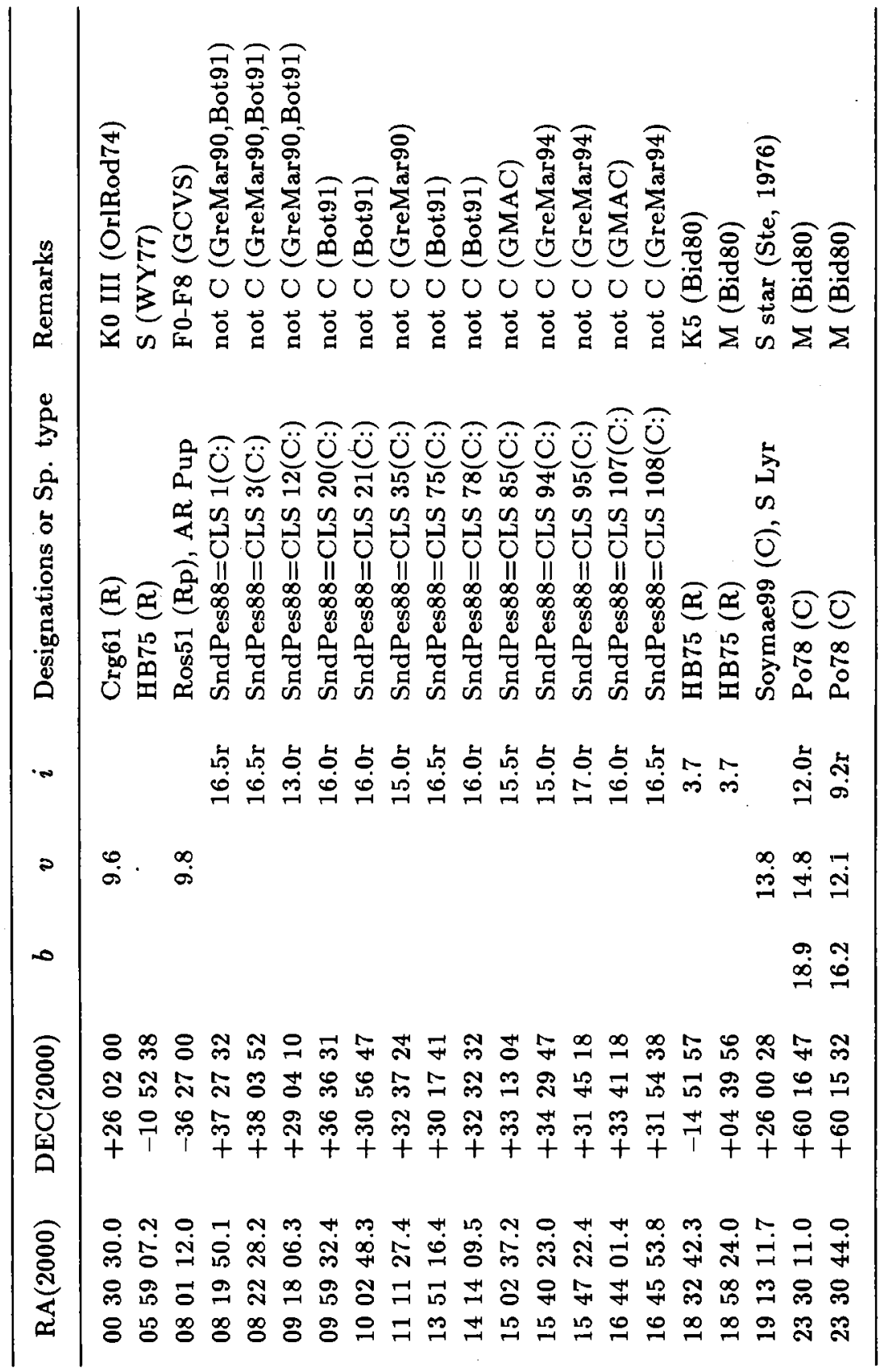


Table 3. Stars with the largest differences RA(2000) - RA(1900).

\begin{tabular}{|c|c|c|c|c|c|}
\hline CGCS & $\alpha, \delta(2000)$ & CGCS & $\alpha, \delta(2000)$ & CGCS & $\alpha, \delta(2000)$ \\
\hline 5 & $0006+7004$ & 2947 & $1103-7145$ & 3761 & $1709-7507$ \\
\hline 81 & $0035+7044$ & 2967 & $1107-8147$ & 3765 & $1718-8132$ \\
\hline 83 & $0035+7031$ & 2970 & $1110-7415$ & 3806 & $1726-7429$ \\
\hline 122 & $0050+7139$ & 2985 & $1115-7042$ & 3871 & $1752-7809$ \\
\hline 133 & $0054+7205$ & 2992 & $1118-7451$ & 3910 & $1811-8037$ \\
\hline 163 & $0102+7150$ & 2994 & $1118-7506$ & 4302 & $1921+7633$ \\
\hline 172 & $0108+7015$ & 3011 & $1122-7045$ & 4485 & $1924+8521$ \\
\hline 208 & $0120+7134$ & 3012 & $1123-7248$ & 5322 & $2126+7013$ \\
\hline 261 & $0145+7119$ & 3049 & $1132-7238$ & 5406 & $2135+7837$ \\
\hline 333 & $0222+7340$ & 3062 & $1133-7312$ & 5452 & $2144+7338$ \\
\hline 506 & $0334+7238$ & 3083 & $1139-7233$ & 5476 & $2143+8258$ \\
\hline 526 & $0339+7053$ & 3137 & $1157-7826$ & 5483 & $2148+7751$ \\
\hline 931 & $0517-7110$ & 3156 & $1203+7025$ & 5529 & $2156+7124$ \\
\hline 984 & $0526-7004$ & 3195 & $1213-7335$ & 5725 & $2243+7447$ \\
\hline 1138 & $0551-7544$ & 3218 & $1221-7602$ & 5874 & $2324+7221$ \\
\hline 1212 & $0554-8403$ & 3222 & $1223-7530$ & 5910 & $2340+7019$ \\
\hline 1380 & 0638-7005 & 3368 & $1321-7426$ & 5973 & $0000+5953$ \\
\hline 1425 & $0644-7239$ & 3425 & $1344-7036$ & 5974 & $0000+5658$ \\
\hline 1599 & $0705-7300$ & 3448 & $1359-7921$ & 5975 & $0000+6134$ \\
\hline 1876 & $0754+7213$ & 3529 & $1449-7431$ & 5976 & $0001+6021$ \\
\hline 1901 & $0745-7119$ & 3562 & $1509-7203$ & 5977 & $0001+6455$ \\
\hline 2282 & $0846+7432$ & 3569 & $1514-7004$ & 5978 & $0001+6357$ \\
\hline 2438 & $0906-7046$ & 3582 & $1501+8303$ & 5979 & $0002+6259$ \\
\hline 2531 & $0924-7943$ & 3591 & $1506+8245$ & 5980 & $0002-0249$ \\
\hline 2643 & $0948-7234$ & 3594 & $1528-7555$ & 5981 & $0002+5958$ \\
\hline 2664 & $0952-7522$ & 3615 & $1556-8546$ & 5982 & $0002+5602$ \\
\hline 2688 & $0959-7212$ & 3620 & $1525+7959$ & 5983 & $0002+6337$ \\
\hline 2715 & $1017+7706$ & 3657 & $1606-7659$ & 5984 & $0003+5944$ \\
\hline 2719 & $1017+7325$ & 3689 & $1629-7552$ & 5985 & $0004+6418$ \\
\hline 2744 & $1017-7030$ & 3722 & $1647-7333$ & 5986 & $0004+6055$ \\
\hline 2918 & $1057-7506$ & 3724 & $1649-7245$ & 5987 & $0004+4333$ \\
\hline
\end{tabular}




\section{REFERENCES}

Aaronson M., Blanco V.M., Cook K.H., Schechter P.L. 1989, ApJS, 70, 637

Aaronson M., Blanco V.M., Cook K.H., Olszewski E.W., Schechter P.L. 1990, ApJS, 73, 841

Abramyan G.V., Gigoyan K.S. 1989a, Astrofizika, 31, 223

Abramyan G.V., Gigoyan K.S. 1989b, Astrofizika, 31, 601

Abramyan G.V., Gigoyan K.S. 1990, Astrofizika, 32, 501

Abramyan G.V., Gigoyan K.S. 1993, Astrofizika, 36, 181

Abramyan G.V., Gigoyan K.S. 1994, Astrofizika, 37, 585

Abramyan G.V., Gigoyan K.S., Shahbazian G.M. 1995, Astrofizika, 38, 351

Abramyan G.V., Gigoyan K.S., Hambaryan V.V., Azzopardi M. 1997, Astrofizika, 40, 131

Ackermann G. 1970, A\&A, 8, 315

Akyol M.U., Hidajat B., Balazs L.G., Voelcker K. 1974, A\&AS, 18, 427

Albers H. 1967, PASP, 79, 259

Alksne Z., Alksnis A. 1981, Issled. Solntsa Krasnykh Zvezd, No. 12, 24

Alksne Z., Ozolina V. 1972, Astron. Circular, Moscow, No. 738, 7

Alksne Z., Ozolina V. 1974, Issled. Solntsa Krasnykh Zvezd, No. 1, 5

Alksne Z., Ozolina V. 1975, Issled. Solntsa Krasnykh Zvezd, No. 3, 29

Alksne Z., Ozolina V. 1976, Issled. Solntsa Krasnykh Zvezd, No. 4, 5

Alksne Z., Alksnis A., Eglitis I. 1981, Issled. Solntsa Krasnykh Zvezd, No. 13,5

Alksne Z., Alksnis A., Ozolina V., Platais I. 1989, Issled. Solntsa Krasnykh Zvezd, No. 30,40

Alksnis A., Ozolina V. 1983, Issled. Solntsa Krasnykh Zvezd, No. 19, 40

Alksnis A., Alksne Z., Eglitis I. 1983, Nauchnye Informatsii, Moscow, No. 52,138

Alksnis A., Alksne Z., Ozolina V. 1977, Issled. Solntsa Krasnykh Zvezd, No. 6,55

Alksnis A., Alksne Z., Ozolina V., Eglitis I. 1976, Issled. Solntsa Krasnykh Zvezd, No. 5, 15

Alksnis A., Alksne Z., Ozolina V., Platais I. 1978, Issled. Solntsa Krasnykh Zvezd, No. 8, 5 
Alksnis A., Alksne Z., Ozolina V., Platais I. 1988b, Issled. Solntsa Krasnykh Zvezd, No. 29, 16

Alksnis A., Alksne Z., Ozolina V., Platais I. 1988a, Nauchnye Informatsii, Moscow, No. 65, 162

Alksnis A., Alksne Z., Platais I., Ozolina V. 1987, Issled. Solntsa Krasnykh Zvezd, No. 25, 5

Allen D.A. 1980, MNRAS, 192, 521

Allen D.A., Hyland A.R., Longmore A.J., Caswell J.L., Goss W.M., Haynes R.F. 1977, ApJ, 217, 108

Azzopardi M., Lequeux J., Rebeirot E. 1985, A\&A, 145, No. 2, L4

Azzopardi M., Lequeux J., Rebeirot E., Westerlund B.E. 1991, A\&AS, 88, 265

Bartkevičius A. 1996, Baltic Astronomy, 5, 217

Bartkevičius A., Śleivytè J. 1983, Bull. Vilnius Obs., No. 64, 24

Balz A.G.A. 1956, Publ. Leander McCormick Obs., 13, pt. 1

Barbier M. 1970, A\&AS, 2, 201

Becker F. 1931, Publ. Astrophys. Obs. Potsdam, 27

Becker F., Bruck H. 1935-8, Publ. Obs. Potsdam, 28

Bidelman W.P. 1954, ApJS, 1, 175

Bidelman W.P. 1956, The Carbon Stars - an Astrophysical Enigma, in Vistas in Astronomy, Vol. 2, ed. A. Beer, Pergamon Press, N. Y.

Bidelman W.P. 1965, PASP, 77, 392

Bidelman W.P. 1971, ApJ, 165, L7

Bidelman W.P. 1980, Publ. Warner and Swasey Obs., 6, 183

Bidelman W.P., MacConnell D.J. 1973, AJ, 78, 730

Blanco B.M., Blanco V.M., McCarthy M.F. 1978, Nature, 271, 638

Blanco V.M. 1958, ApJ, 127, 191

Blanco V.M. 1965, in Galactic Structure, Stars and Stellar Systems, Vol. 5, eds. A. Blaauw \& M. Schmidt, Univ. Chicago Press, p. 241

Blanco V.M., Munch L. 1955, Bol. Obs. Tonantzintla y Tacubaya, No. 12 Blanco V.M., Nassau J.J., Stock J., Wehlau W. 1955, ApJ, 121, 637

Bond H.E. 1975, ApJ, 202, L47

Bothun G., Elias J.H., Macalpine G., Matthews K., Mould J.R., Neugebauer G., Reid I.N. 1991, AJ, 101, 2220

Cannon A.J. 1921, Circ. Harvard Obs., No. 224

Cannon A.J. 1922, Circ. Harvard Obs., No. 231 
Cannon A.J. 1926, Bull. Harvard Obs., No. 837

Cannon A.J., Mayall M.W. 1938, Bull. Harvard Obs., No. 908

Cannon A.J., Mayall M.W. 1949, Ann. Harvard Obs., 112

Catchpole R.M. 1968, PASP, 80, 744

Cohen M., Hitchon K. 1996, AJ, 111, 962

Cohen M., Kuhi L.V. 1976, PASP, 88, 535

Cohen M., Kuhi L.V. 1977, PASP, 89, 829

Cohen M., Wainscoat R.J., Walker H.J., Volk K. 1996, AJ, 111, 1333

Cohen M., Wainscoat R.J., Walker H.J., Volk K., Schwartz D.E. 1989, AJ, 97,1759

Cregg T. 1961, PASP, 73, 453

Cutri R.M., Low F.G., Kleinmann S.G., Olszewski E.W., Willner S.P., Campbell B., Gillett F.C. 1989, AJ, 97, 866

Dahn C.C., Liebert J., Kron R.G., Spinrad H., Hintzen P.M. 1977, ApJ, 216, 757

Daube I. 1971, Astron. Circular, Moscow, No. 661, 6

Daube I., Ozolina V. 1974, Issled. Solntsa Krasnykh Zvezd, No. 1, 14

Dean C.A. 1976, AJ, 81, 364

Dolidze A.C. 1962, Astron. Circular, Moscow, No. 228, 13

Dolidze A.C. 1968, Astron. Circular, Moscow, No. 457, 2

Dolidze A.C. 1971a, Astron. Circular, Moscow, No. 616, 7

Dolidze A.C. 1971b, Astron. Circular, Moscow, No. 629, 8

Drilling J.S. 1972, PASP, 84, 35

Engels D. 1994, A\&A,285,497.

Epchtein N., Le Bertre T., Lepine J.R.D. 1990, A\&A, 227, 82

Epchtein N., Le Bertre T., Lepine J.R.D., Marques dos Santos P.,

Matsuura O.T., Picazzio E. 1987, A\&AS, 71, 39

ESA, The Hipparcos and Tycho Catalogues, 1997, SP-1200

Espin T.E. 1924, MNRAS, 85, 93

Feast M. 1997, Observatory, 117, 300

Feast M.W., Catchpole R.M., Warren P.R. 1977, Observatory, 97, 140

Fitzgerald M.P. 1968, AJ, 73, 983

Fleming W.S. 1912, Ann. Harvard Obs., 56, 165

Fouque P., Le Bertre T., Epchtein N., Guglielmo F., Kerschbaum F. 1992, A\&AS, 93, 151 
Frogel J.A., Dickinson D.F., Hyland A.R. 1975, ApJ, 201, 392

Fuenmayor F.J. 1981, Rev. Mex. A\&A, 6, 83

Fuenmayor F.J., Stock J. 1995, in The Future Utilization of Schmidt Telescopes (IAU Colloq. 148), eds. J. Chapman et al., ASP Conference Series, 84, 311

General Catalogue of Variable Stars. 1999, Volumes I-III, 4th ed., Moscow Gehrz R.D., Hackwell J.A. 1976, ApJ, 206, L161

Gigoyan K.S., Hambaryan V.V. 1996, Astrofizika, 39, 523

Gigoyan K.S., Hambaryan V.V., Azzopardi M. 1998, Astrofizika, 41, 545

Gigoyan K.S., Azzopardi M., Muratorio G. 1999, Astrofizika, 42, 155

Gonzales G., Gonzales G. 1956, Bol. Obs. Tonantzintla y Tacubaya, No. 14,19

Gordon C.P. 1968, PASP, 80, 597

Grasdalen G.L., Gehrz R.D., Hackwell J.A., Catelaz M., Gullison D. 1983, ApJS, 53, 413

Green P.J., Margon B. 1990, PASP, 102, 1372

Green P.J., Margon B. 1994, ApJ, 423, 733

Green P.J., Margon B., MacConnell D.J. 1991, ApJ, 380, L31

Green P.J., Margon B., Anderson S.F., Cook K.H. 1994, ApJ, 434, 319

Green P.J., Margon B., Anderson S.F., MacConnell D.J. 1992, ApJ, 400, 659

Greene A.E., Wing R.F. 1971, ApJ, 163, 309

Groenewegen M.T.A., de Jong T. 1993, A\&AS, 101, 267

Groenewegen M.T.A., de Jong T., Baas F. 1993, A\&AS, 101, 513

Groenewegen M.T.A., de Jong T., Geballe T.R. 1994, A\&A, 287, 163

Groenewegen M.T.A., Oudmaijer R.D., Ludwig H.-G. 1997, MNRAS, 292, 686

Groenewegen M.T.A., Whitelock P.A., Smith C.H., Kerschbaum F. 1998, MNRAS, 293, 18

Groenewegen M.T.A., de Jong T., van der Bliek N.S., Slijkhuis S., Willems F.J. 1992, A\&A, 253, 150

Guglielmo F., Le Betre T., Epchtein N. 1998, A\&A, 334, 609

Guglielmo F., Epchtein N., Arditti F., Sevre F. 1997, A\&AS, 122, 489

Guglielmo F., Epchtein N., Le Betre T., Fouque P., Hron J., Kerschbaum F., Lepine J.R.D. 1993, A\&AS, 99, 31

Hansen O.L., Blanco V.M. 1975, AJ, 80, 1011 
Hardorp J., Lubeck K., Stephenson C.B. 1973, A\&A, 22, 129

Hartwick F.D.A., Cowley A.P. 1985, AJ, 90, 2244

Harwood M. 1962, Ann. Leiden Obs., 21, 387

Henize K.G. 1976, ApJS, 30, 491

Herbig G.H. 1952, Trans. I.A.U., Vol. 8, 807

Hess S., Hoffleit D., Osborn W. 1969, AJ, 74, 718

IRAS Science Team. 1986, A\&AS, 65, 607

Jones B., Merrill K.M., Puetter R.C., Willner S.P. 1978, AJ, 83, 1437

Jones T.J., Bryja C.O., Gehrz R.D., Harrison T.C., Johnson J.I., Klebe

D.I., Lawrence G. 1990, ApJS, 74, 785

Joyce R.R. 1998, AJ, 115, 2059

Keenan P.C. 1950, AJ, 55, 172

Keenan P.C., Morgan W.W. 1941, ApJ, 94, 501

Kron G.E., Smith J.L. 1951, ApJ, 113, 324

Krumenaker L. E. 1975, PASP, 87, 185

Kukarkin B.V., Kholopov P.N., Artiukhina N.M., Fedorovich V.P., Frolov M.S., Goranskij V.P., Gorynya N.A., Karitskaya E.A., Kireeva N.N., Kukarkina N.P., Kurochkin N.E., Medvedeva G.I., Perova N.B., Ponomareva G.A., Samus' N.N., Shugarov S.Yu. 1982, New Catalogue of Suspected Variable Stars, Nauka Publishing House, Moscow Kurtanidze O.M. 1973, Astron. Circular, Moscow, No. 1036, 8 Kurtanidze O.M. 1980a, Astrofizika, 16, 190

Kurtanidze O.M. 1980, Astron. Circular, Moscow, No. 1109, 3 Kurtanidze O.M. 1996, unpublished

Kurtanidze O.M., Nikolashvili M.G. 1981, Astrofizika, 17, 576

Kurtanidze O.M., Nikolashvili M.G. 1988, Astrofizika, 29, 405

Kurtanidze O.M., Nikolashvili M.G. 1988b, Astrofizika, 29, 470

Kurtanidze O.M., Nikolashvili M.G. 1988, Georg. Inst. Sci. Tech. Inf. No. 430 - G88.

Kurtanidze O.M., Nikolashvili M.G. 1989, Astrofizika, 31, 507

Kurtanidze O.M., Nikolashvili M.G. 1990, Astrofizika, 32, 173

Kurtanidze O.M., Nikolashvili M.G. 2000, in The Carbon Star Phenomenon, (IAU Symp. 177), ed. R. F. Wing, Kluwer Academic Publishers, p. 554

Kurtanidze O.M., West R.M. 1980, A\&AS, 39, 35

Le Bertre T. 1992, A\&AS, 94, 377 
Lebofsky M.J., Rieke G.H. 1977, AJ, 82, 646

Lebofsky M.J., Sargent D.G., Kleinmann S.G., Rieke G.H. 1978, ApJ, 219, 487

Lee O.J., Bartlett T.J. 1942, Ann. Dearborn Obs., 5, pt. 3

Lee O.J., Gore G.D., Bartlett T.J. 1944, Ann. Dearborn Obs., 5, pt. 7

Lee O.J., Baldwin R.B., Hamlin D.W., Kinnaird R.F. 1940, Ann. Dearborn Obs., 4 , pt. 16

Liebert J., Schmidt G.D., Lesser M., Stepanian J.A., Lipovetsky V.A., Chaffee F.H., Foltz C.B., Bergeron P. 1994, ApJ, 421, 733

Little-Marenin I., Ramsay M.E., Stephenson C.B., Little S.J., Price S.D. 1987, AJ, 93, 663

Lloyd Evans T. 1983, Observatory, 103, 276

Lloyd Evans T. 1990, MNRAS, 243, 336

Lloyd Evans T. 1991, MNRAS, 249, 409

Lockwood G.W. 1974, ApJ, 192, 113

Loup C., Forveille T., Omont A., Paul J.F. 1993, A\&A, 99, 231

Low F.G., Kurtz R.F., Vrba F.J., Rieke G.H. 1976, ApJ, 206, L153

Lundmark K. 1921, PASP, 33, 314

Luyten W.J. 1956, AJ, 61, 261

Luyten W.J., Merrill P.W. 1954, PASP, 66, 207

McCarthy M.F. 1960, Ric. Astron., 6, 301

MacConnell D.J. 1967, PASP, 79, 266

MacConnell D.J. 1979, A\&AS, 38, 335

MacConnell D.J. 1981, A\&AS, 44, 387

MacConnell D.J. 1982, A\&AS, 48, 355

MacConnell D.J. 1988, AJ, 96, 354

MacConnell D.J. 1997, BA, 6, 105

MacConnell D.J. 2000, personal communication

MacConnell D.J., Frye R.L., Upgren A.R. 1972, AJ, 77, 384

Maehara H., Soyano T. 1986a, Ann. Tokyo Obs., 21, 293

Maehara H., Soyano T. 1986b, Ann. Tokyo Obs., 21, 423

Maehara H., Soyano T. 1988, Ann. Tokyo Obs., 22, 59

Maehara H., Sayano T. 1990, Publ. Natl. Astron. Obs. Japan, 1, 207

Margon B., Aaronson M., Liebert J., Monet D. 1984, AJ, 89, 274

Mayall M.W. 1951, Bull. Harvard Obs., No. 920, 32 
Mayall M.W., Cannon A.J. 1940, Bull. Harvard Obs., No. 913

Merrill K.M., Stein W.A. 1976, PASP, 88, 874

Merrill P.W. 1926, PASP, 38, 175

Merrill P.W., Burwell C.G. 1950, ApJ, 112, 72

Merrill P.W., Sanford R.F., Burwell C.G. 1933, PASP, 45, 306

Merrill P.W., Sanford R.F., Burwell C.G. 1942, PASP, 54, 107

Mickaelian A.M. 1997, Astrofizika, 40, 5

Mikami T. 1978, Ann. Tokyo Obs., 17, 1

Moody J.W., Gregory S.A., Soukup M.S., Jaderlund E.C. 1997, AJ, 113, 1022

Mould J.R., Schneider D.P., Gordon G.A., Aaronson M., Liebert J.W. 1985, PASP, 97, 130

Nassau J.J., Blanco V.M. 1954, ApJ, 120, 129

Nassau J.J., Blanco V.M. 1957, ApJ, 125, 195

Nassau J.J., Cameron D.M. 1956, ApJ, 124, 346

Nassau J.J., Colacevech A. 1950, ApJ, 111, 199

Nassau J.J., Stephenson C.B. 1961, ApJ, 133, 920

Nassau J.J., Stephenson C.B., Caprioli G. 1964, ApJ, 139, 864

Nassau J.J., van Albada G.B. 1949, ApJ, 109, 391

Neugebauer G., Leighton R.B. 1969, NASA Special Publ., No. 3047

Nguyen-Q-Rieu, Epchtein N., Truong-Bach, Cohen M. 1987, A\&A, 180, 117

Nikolashvili M.G. 1987a, Astrofizika, 26, 209

Nikolashvili M.G. 1987b, Astrofizika, 26, 559

Nikolashvili M.G. 1987c, Astrofizika, 27, 197

Omont A., Loup C., Forveille T., te Lintel Hekkert P., Habing H., Sivagnanam P. 1993, A\&A, 267, 515

Orlov M.Ya., Rodriges M.G. 1974, Astron. Circular, Moscow, No. 813

Paupers O., Žaime D., Eglītis I. 1993, Baltic Astromomy, 2, 268

Perraud H. 1961, J. des Observateurs, 44, 247

Perraud H., Pelletier, B. 1959, J. des Observateurs, 42, 33

Pesch P. 1967, ApJ, 149, L65

Philip A.G.D. 1967, PASP, 79, 366

Philip A.G.D. 1968, AJ, 73, S113

Pickering E.C. 1911, Circ. Harvard Obs., No. 167 
Platais I. 1979, Issled. Solntsa Krasnykh Zvezd, No. 10, 23

Platais I. 1981, Issled. Solntsa Krasnykh Zvezd, No. 12, 19

Poulakos C. 1972, Mem. Soc. Astron. Ital., 42, 419

Poulakos C. 1978, A\&AS, 1978, 32, 395

Richer H. 1972, ApJ, 172, L63

Richter G., Schaifers K., Wenzel W. 1961, Mitt. Veränd. Sterne, No. 526

Rosino L. 1951, ApJ, 113, 60

Rybski P.M. 1972, unpublished Ph.D. Thesis, Northwestern University

Sanduleak N., Pesch P. 1982, PASP, 94, 690

Sanduleak N., Pesch P. 1988, ApJS, 66, 387

Sanduleak N., Philip A.G.D. 1968, PASP, 80, 437

Sanduleak N., Stephenson C.B. 1973, ApJ, 185, 899

Sanford R.F. 1926, PASP, 38, 177

Sanford R.F. 1941, PASP, 53, 290

Sanford R.F. 1944, ApJ, 99, 145

Sanford R.F. 1949, PASP, 61, 43

Sanford R.F. 1950a, ApJ, 111, 270

Sanford R.F. 1950b, ApJ, 111, 262

Sarre P.J., Hurst M.E., Lloyd Evans T. 1996, ApJ, 471, L107

Schaifers K. 1959, Mitt Veränd. Sterne, No. 385

Schaifers K. 1960, Mitt Veränd. Sterne, No. 432

Schwassmann A., van Rhijn P.J. 1935-53, Bergedorfer Spektral-Durchmusterung, Hamburger Sternwarte

Shane C.D. 1928, Bull. Lick Obs., 13, 123

Šleivytė J., Bartkevičius A. 1990, Bull. Vilnius Obs., No. 85, 3

Slettebak A., Keenan P.C., Brundage R.K. 1969, AJ, 74, 373

Smith C.E. 1937, Bull. Lick Obs., 18, 39

Sopka R.J., Hildebrand R., Jaffe D.T., Gatley I., Roellig T., Werner M. 1985, ApJ, 294, 242

Soyano T., Maehara H. 1991, Publ. Nat. Astron. Obs. Japan, 2, 203

Soyano T., Maehara H. 1993, Publ. Nat. Astron. Obs. Japan, 3, 259

Soyano T., Maehara H. 1999, Publ. Nat. Astron. Obs. Japan, 5, 149

Stephenson C.B. 1965, ApJ, 142, 712

Stephenson C.B. 1973, ApJ, 186, 589

Stephenson C.B. 1973, Publ. Warner and Swasey Obs., 1, No. 4 
Stephenson C.B. 1976, Publ. Warner and Swasey Obs., 2, No. 2

Stephenson C.B. 1985, AJ, 90, 784

Stephenson C.B. 1989, Publ. Warner and Swasey Obs., 3, No. 2

Stock J., Wroblewski H. 1972, Publ. Dept. Astron. Univ. Chile, 2, No. 3

Swope H. 1943, Ann. Harvard Obs., 109, No. 9

Thackeray A.D. 1949, Mon. Notes Astron. Soc. Southern Africa, 8, 15

The P.S. 1968, PASP, 80, 104

Totten E.J., Irwin M.J. 1998, MNRAS, 294, 1

Ulrich B.T., Neugebauer G., McCammon D., Leighton R.B., Hughes E.E., Becklin E. 1966, ApJ, 146, 288

Upgren A.R. 1960, AJ, 65, 644

Upgren A.R., Grossenbacher R. 1968, PASP, 80, 342

Van den Bergh S. 1971, PASP, 83, 819

Van der Hucht K.A., Conti P.S., Lundstrom I., Stenholm B. 1981, Space Sci. Rev., 28, 227

Vandervoort G.L. 1958, AJ, 63, 477

Vetesnik M. 1979, Bull. Astron. Inst. Czech., 30, 1

Vogt S.S. 1973, AJ, 78, 389

Volk K., Kwok S., Langill P.P. 1992, ApJ, 391, 285

Vyssotsky A.N., Balz A.G.A. 1958, Publ. Leander McCormick Obs., 13, pt. 2

Warner B. 1963, MNRAS, 126, 61

Warner B. 1965, MNRAS, 129, 263

Warren S.J, Irwin M.J., Evans D.W., Liebert J., Osmer P.S., Hewett P.C. 1993, MNRAS, 261, 185

Weaver W.B. 1972, PASP, 84, 854

Westerlund B. 1958, ApJS, 4, 73

Westerlund B. 1965, MNRAS, 130, 45

Westerlund B. 1971, A\&AS, 4, 51

Whitelock P., Menzies J., Feast M., Catchpole R., Marang F., Carter B. 1995, MNRAS, 276, 219

Wilson W.J., Schwartz P.R., Epstein E.E. 1973, ApJ, 183, 871

Wing R.F. 1997, Newsletter of Chemically Peculiar Red Giant Stars, No. 20,1

Wing R.F., Yorka S.B. 1977, MNRAS, 178, 383 
Wisniewski W.Z., Wing R.F., Spinrad H., Johnson H.L. 1967, ApJ, 148, L29

Wray J.D. 1966, unpublished Ph.D. Thesis, Northwestern University

Wyckoff S. 1970, ApJ, 162, 203

Yamashita Y. 1972, Ann. Tokyo Obs., 13, 169

Yamashita Y. 1975, Ann. Tokyo Obs., 15, 47 


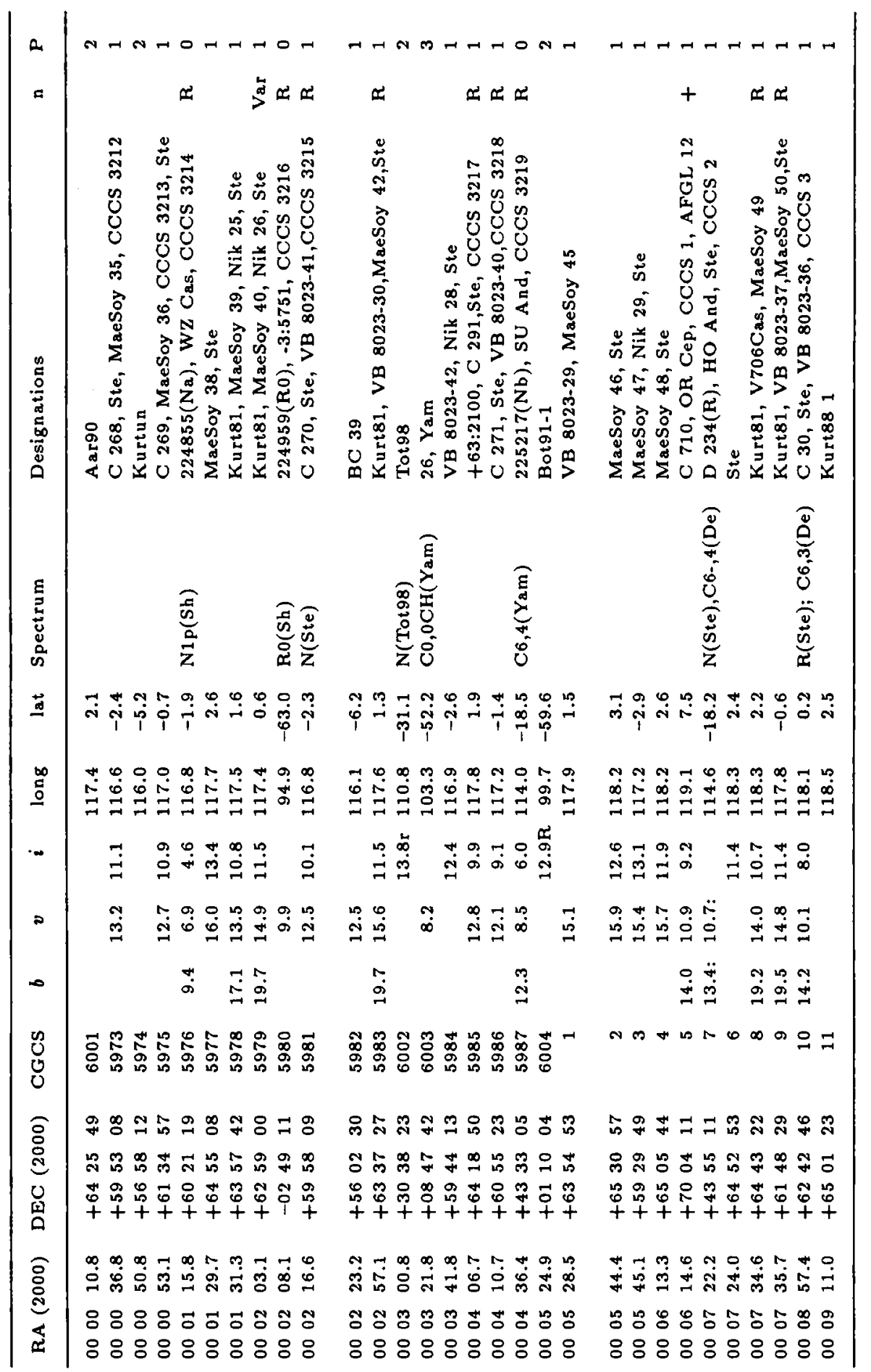


A.Alksnis, A. Balklavs, U. Dzervitis, I. Eglitis et al.

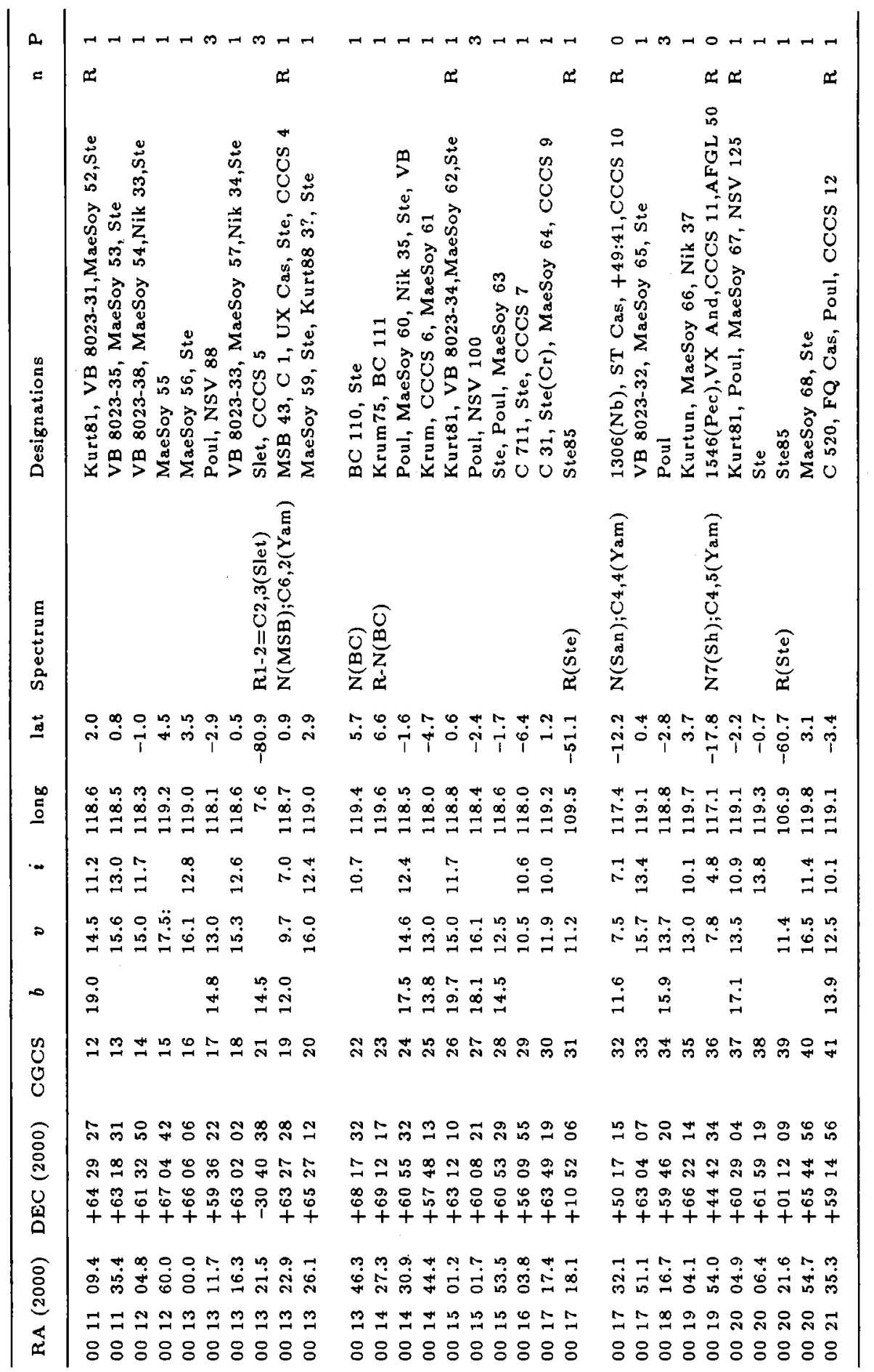




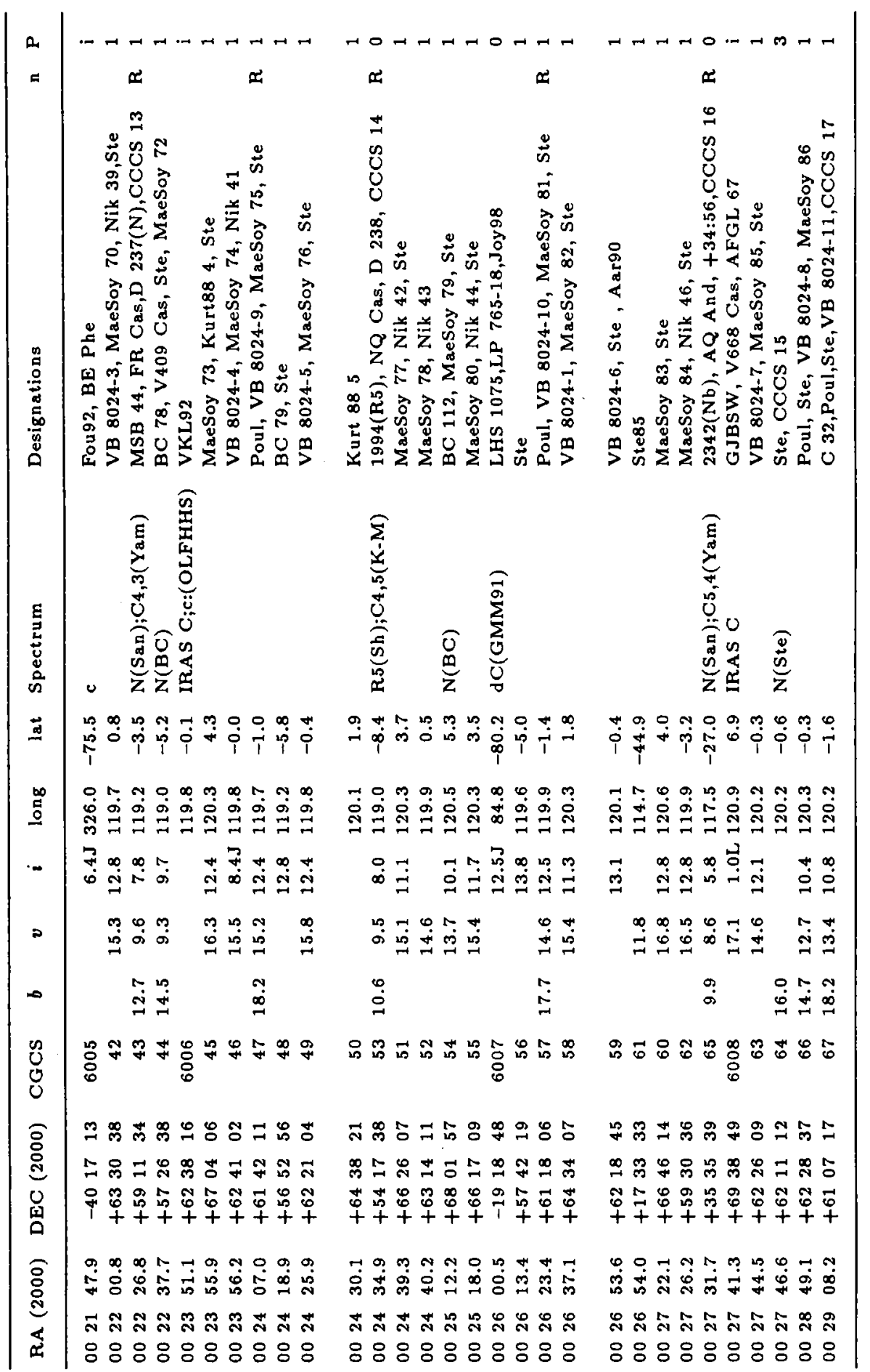




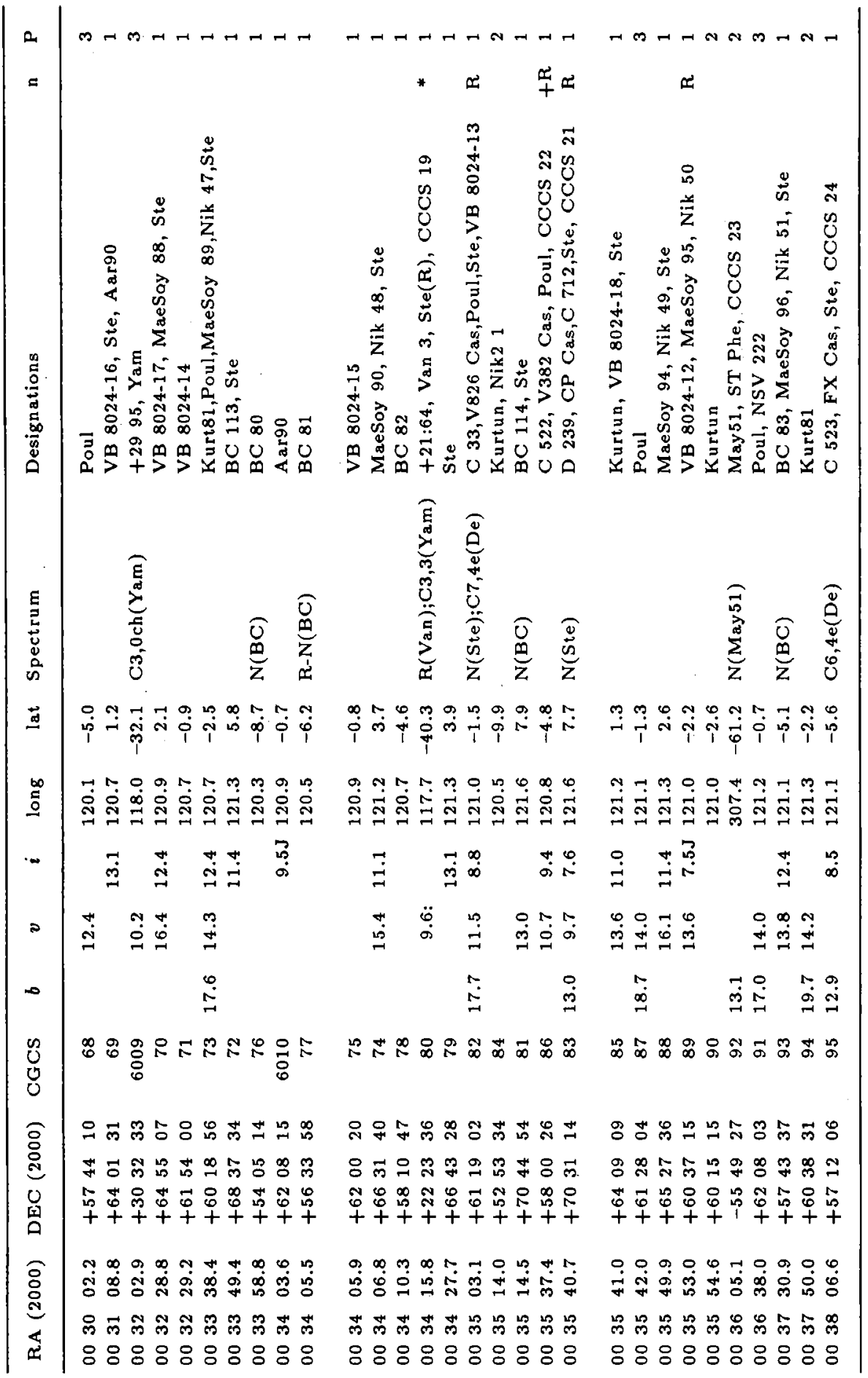




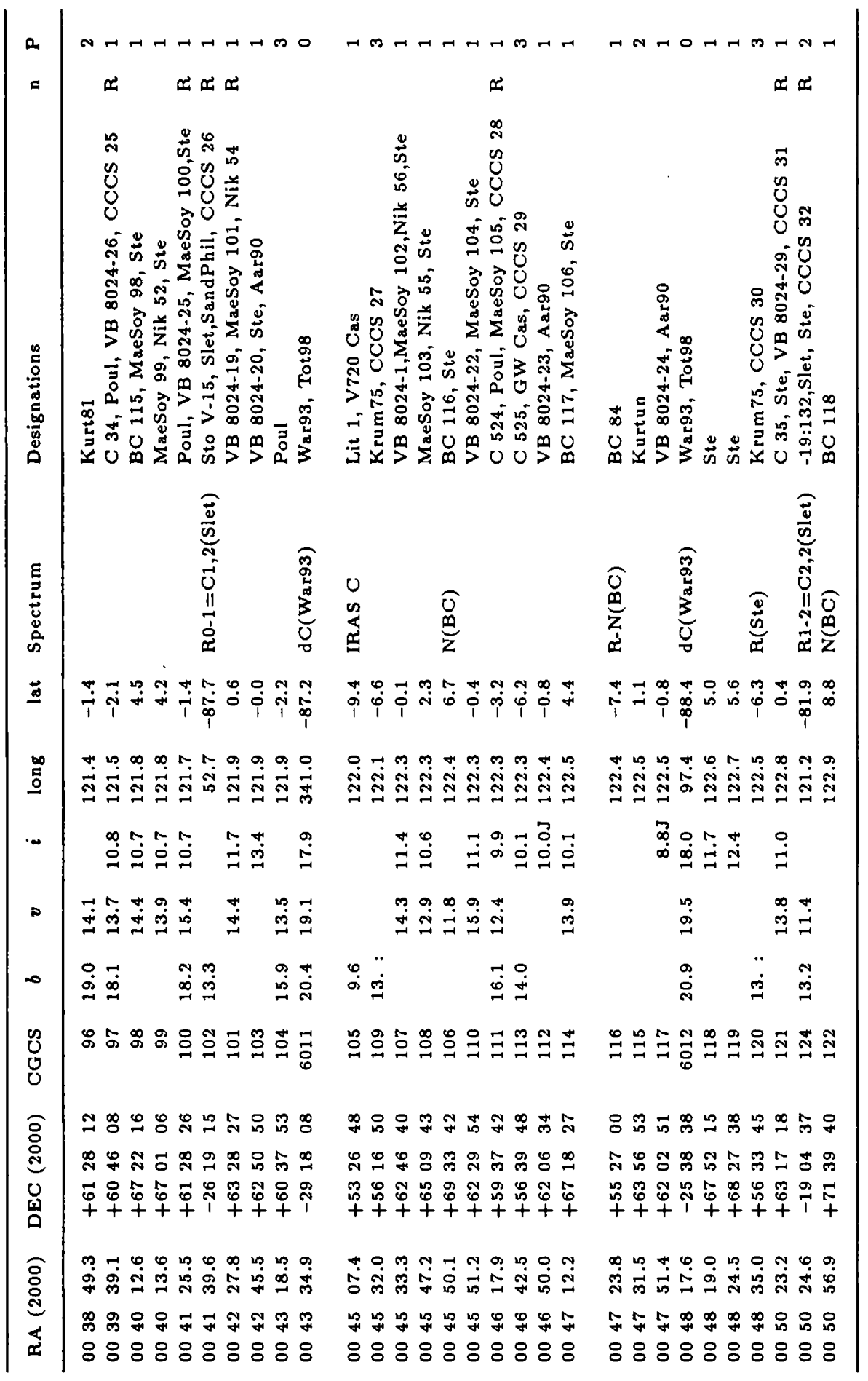




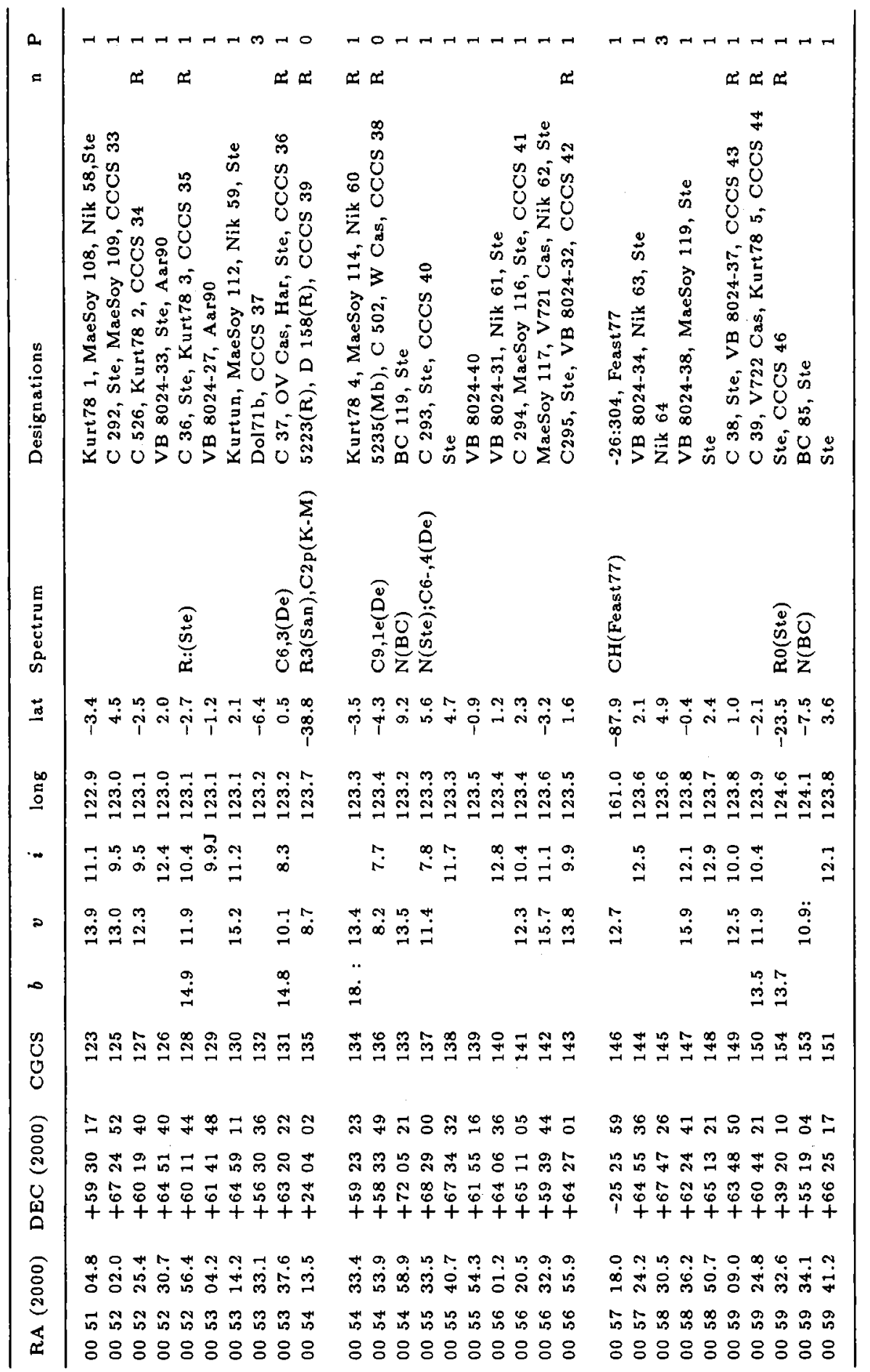




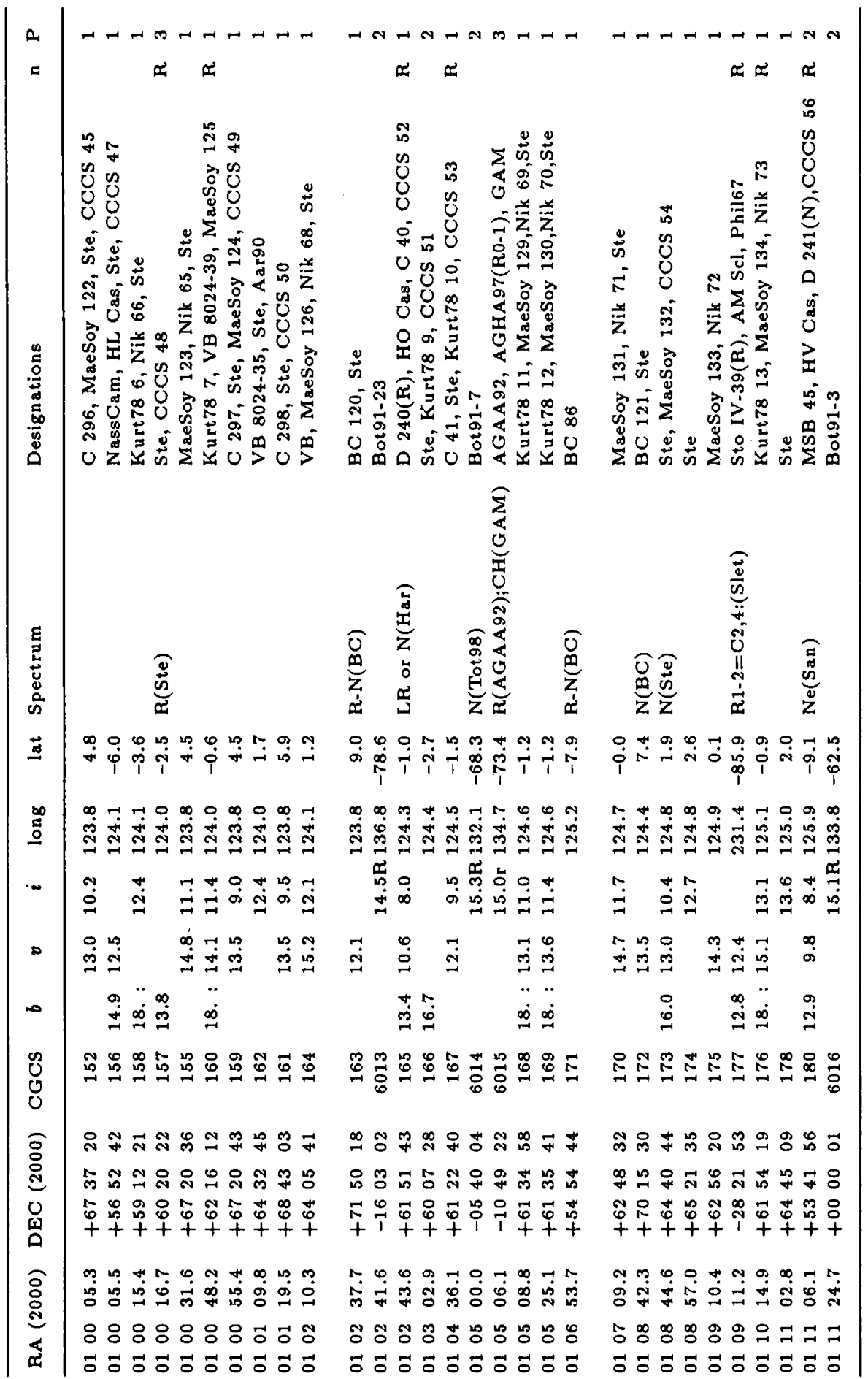




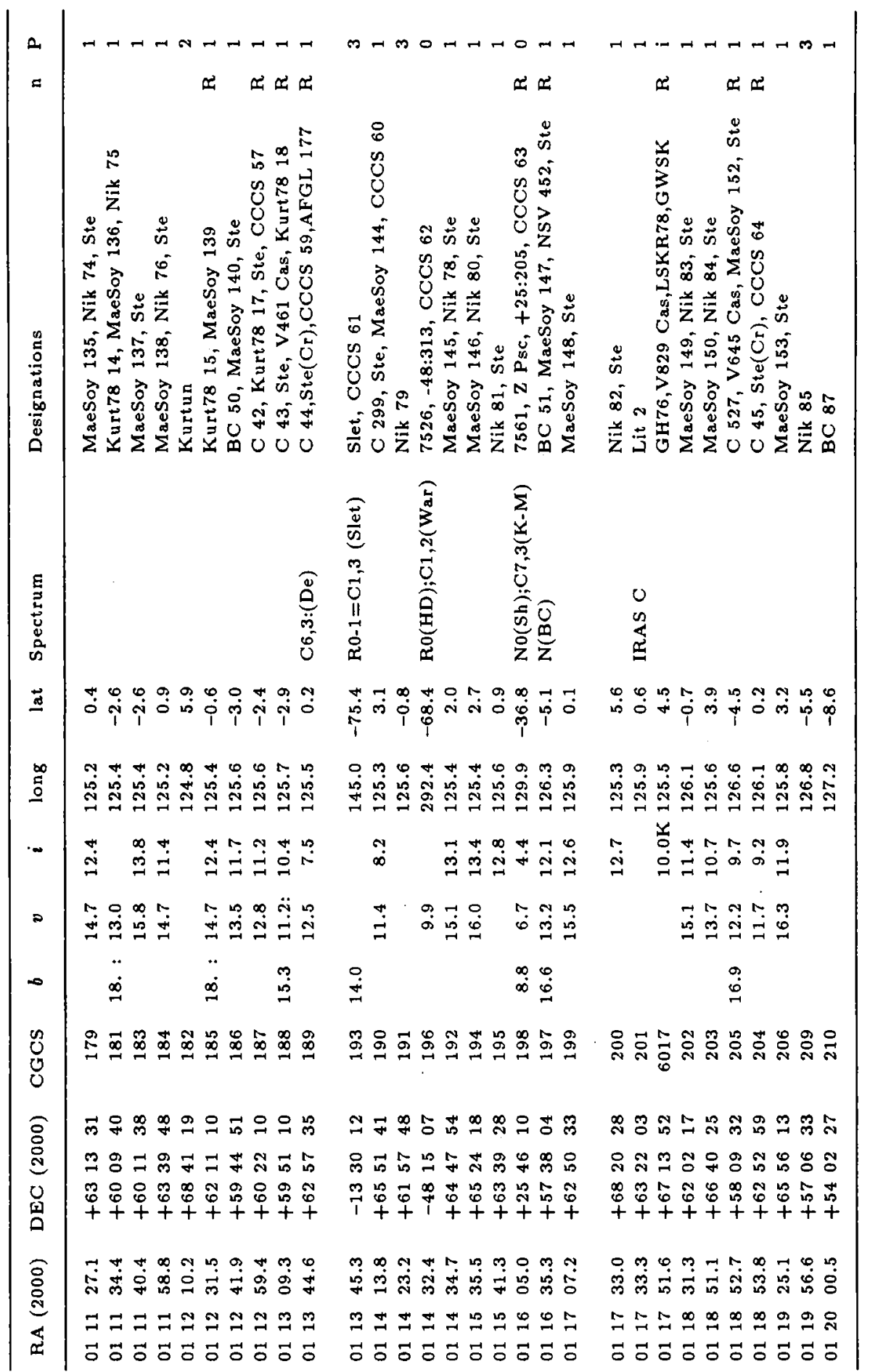




\begin{tabular}{|c|c|c|c|}
\hline Q & 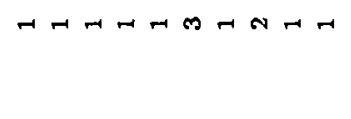 & 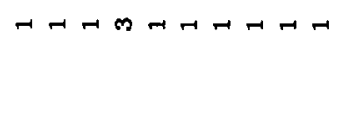 & 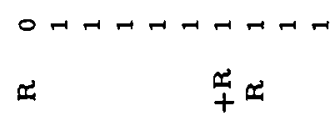 \\
\hline 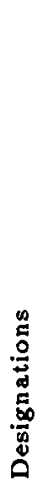 & 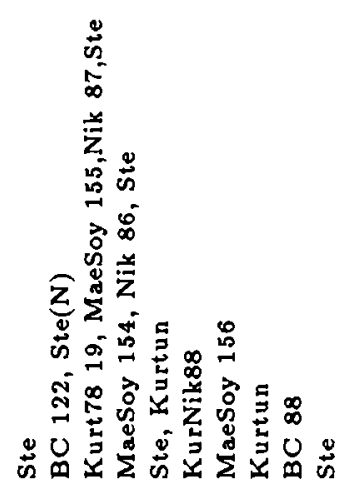 & 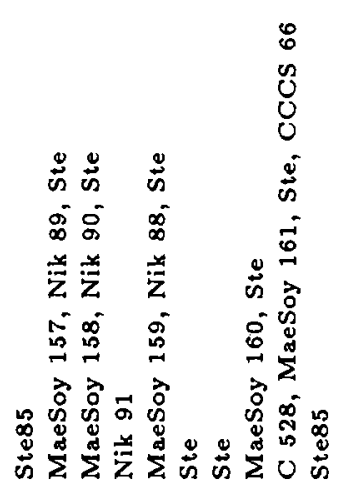 & 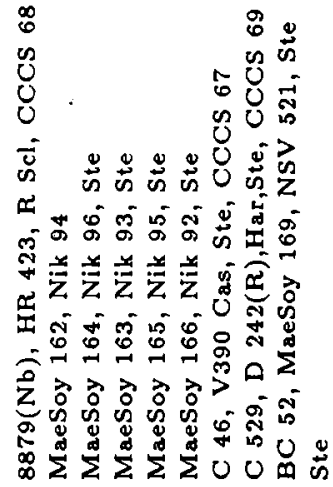 \\
\hline 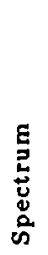 & 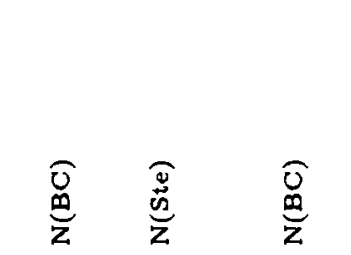 & 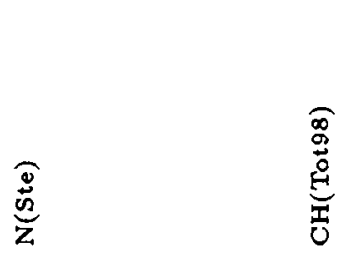 & 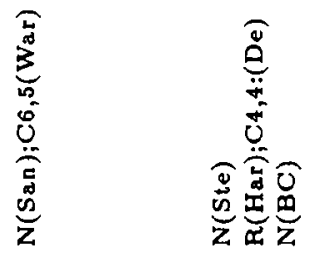 \\
\hline 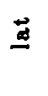 & 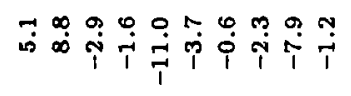 & 分 & 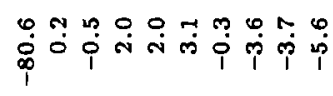 \\
\hline 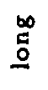 & 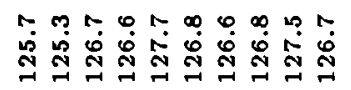 & 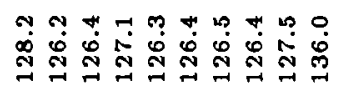 & 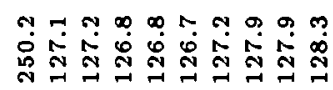 \\
\hline - & 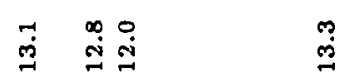 & 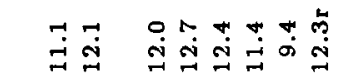 & 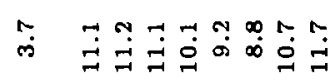 \\
\hline$\Rightarrow$ & 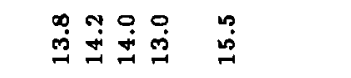 & 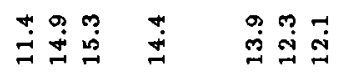 & 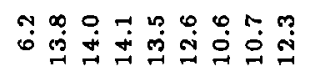 \\
\hline$\infty$ & $\ddot{\infty}$ & & 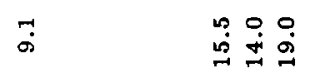 \\
\hline Uू & 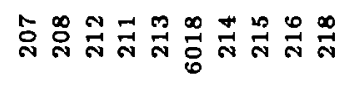 & 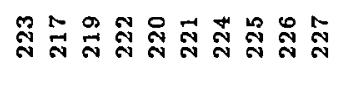 & 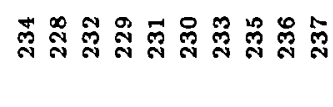 \\
\hline 용 & ロ & 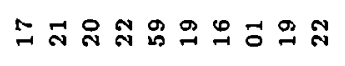 & 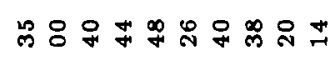 \\
\hline 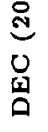 & 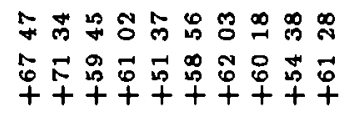 & 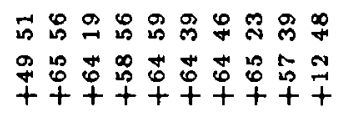 & 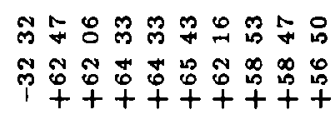 \\
\hline ठิ & 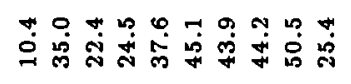 & 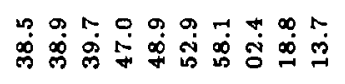 & 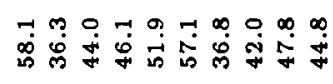 \\
\hline 药 & 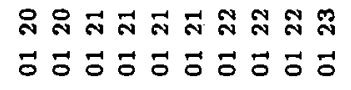 & 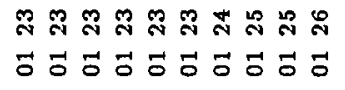 & 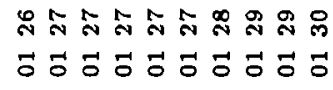 \\
\hline
\end{tabular}




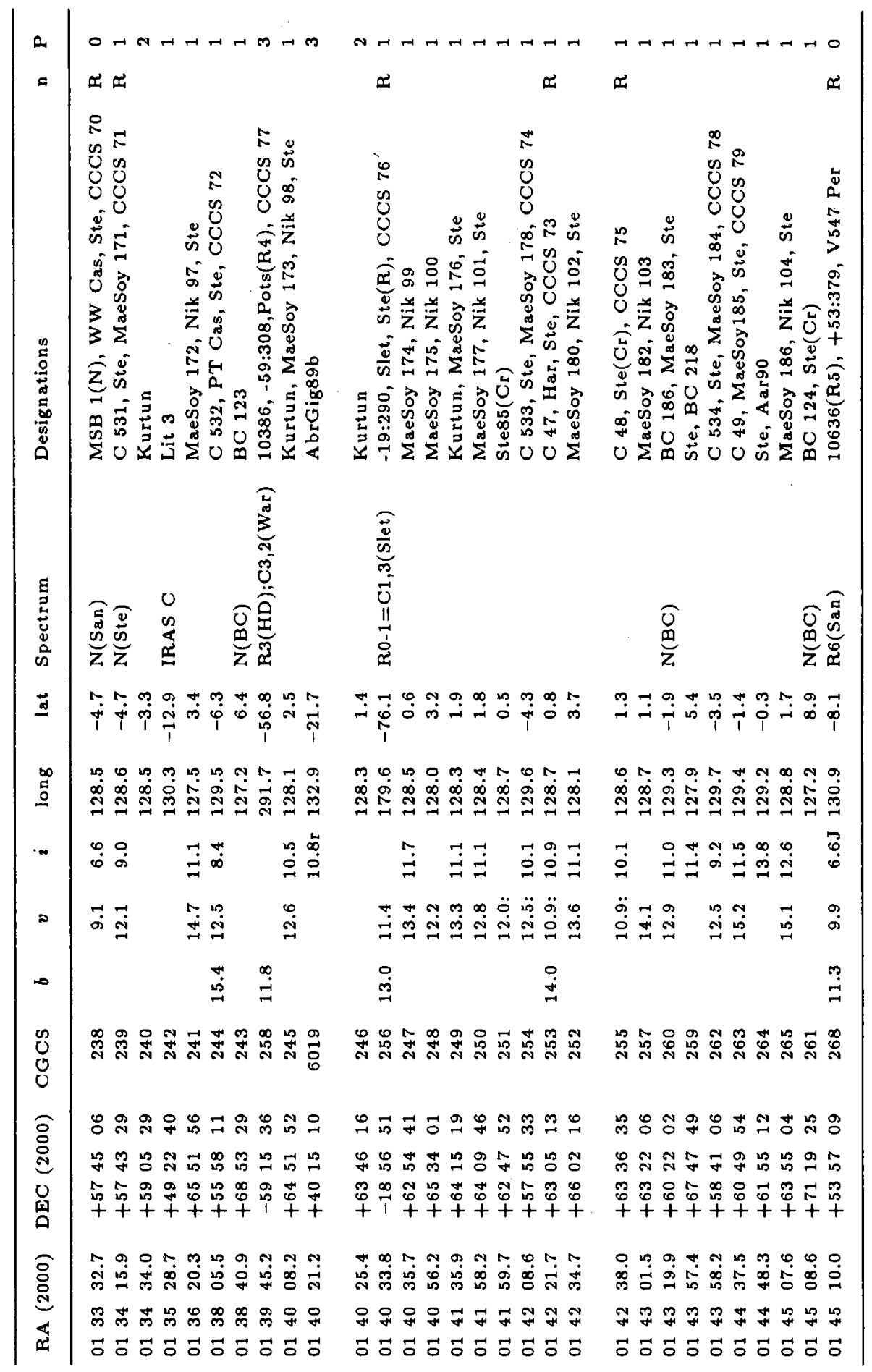




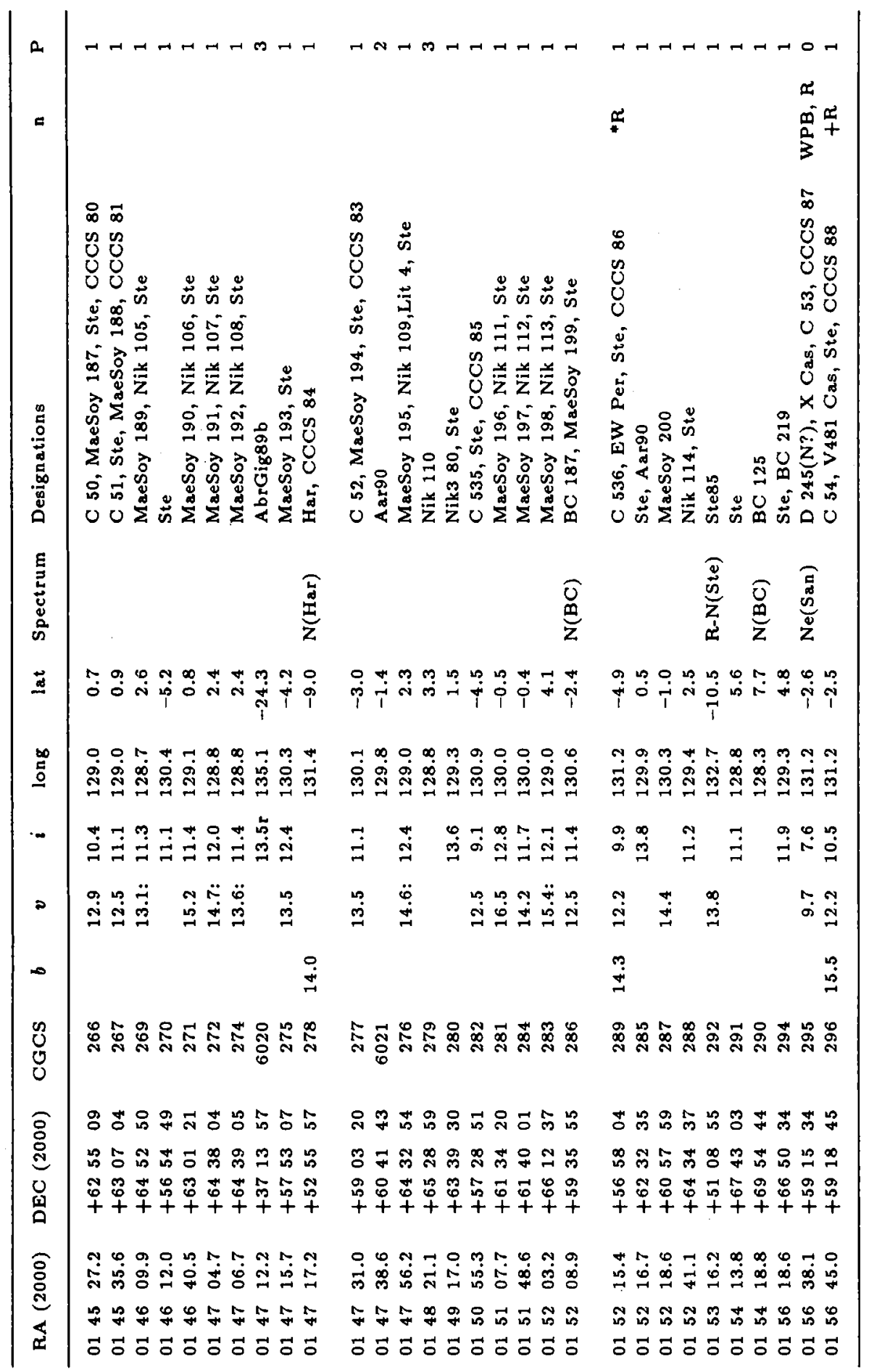




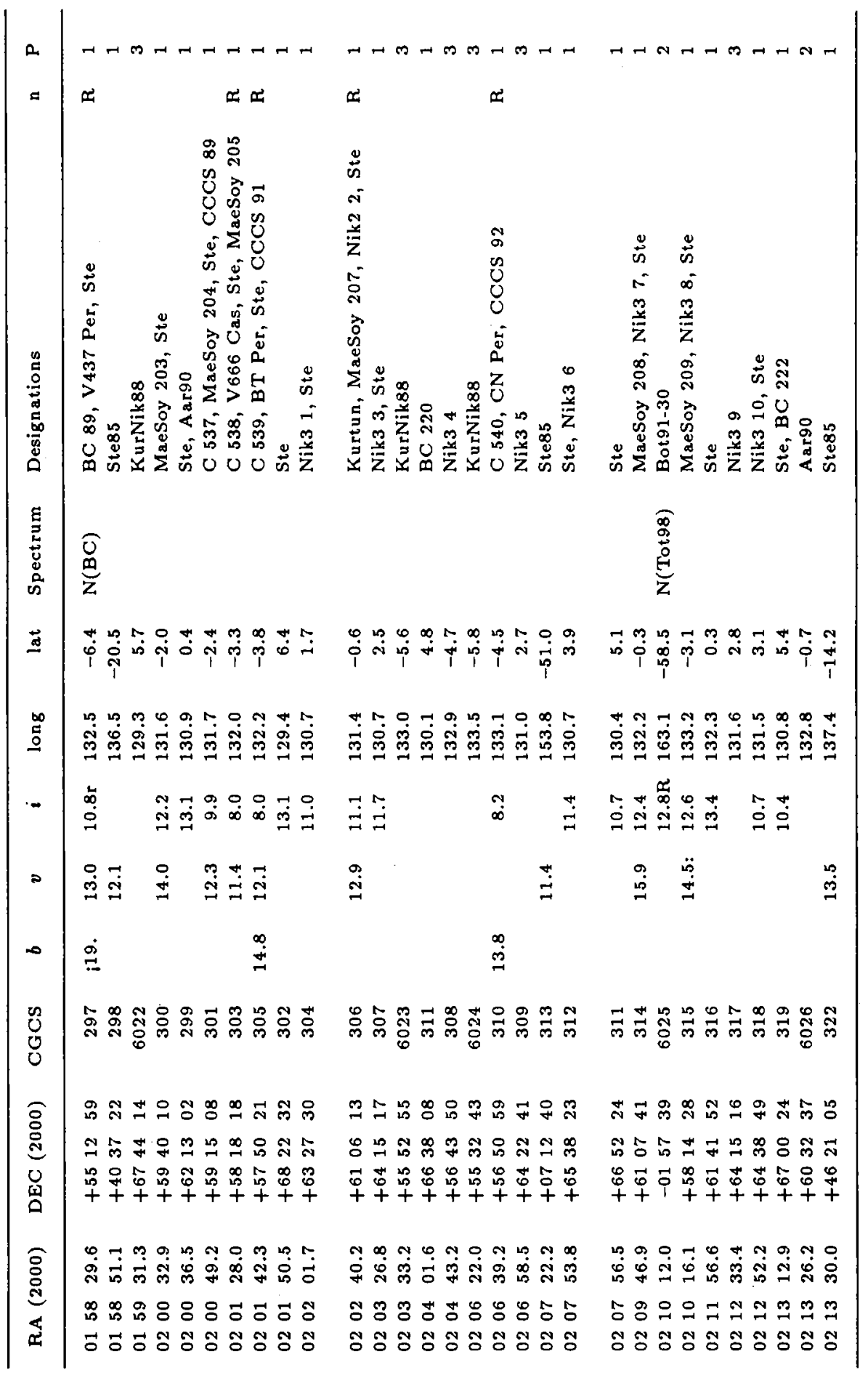




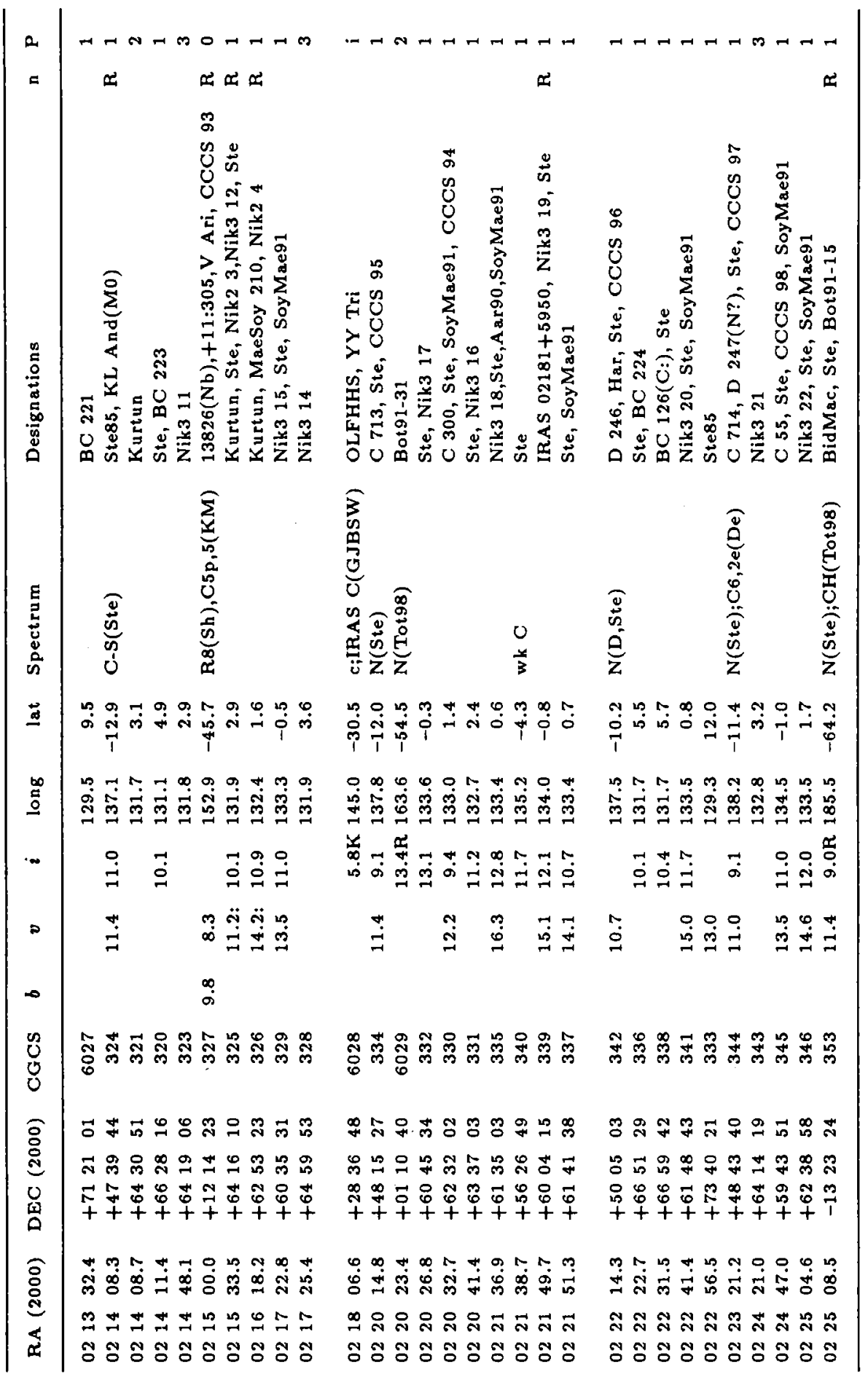




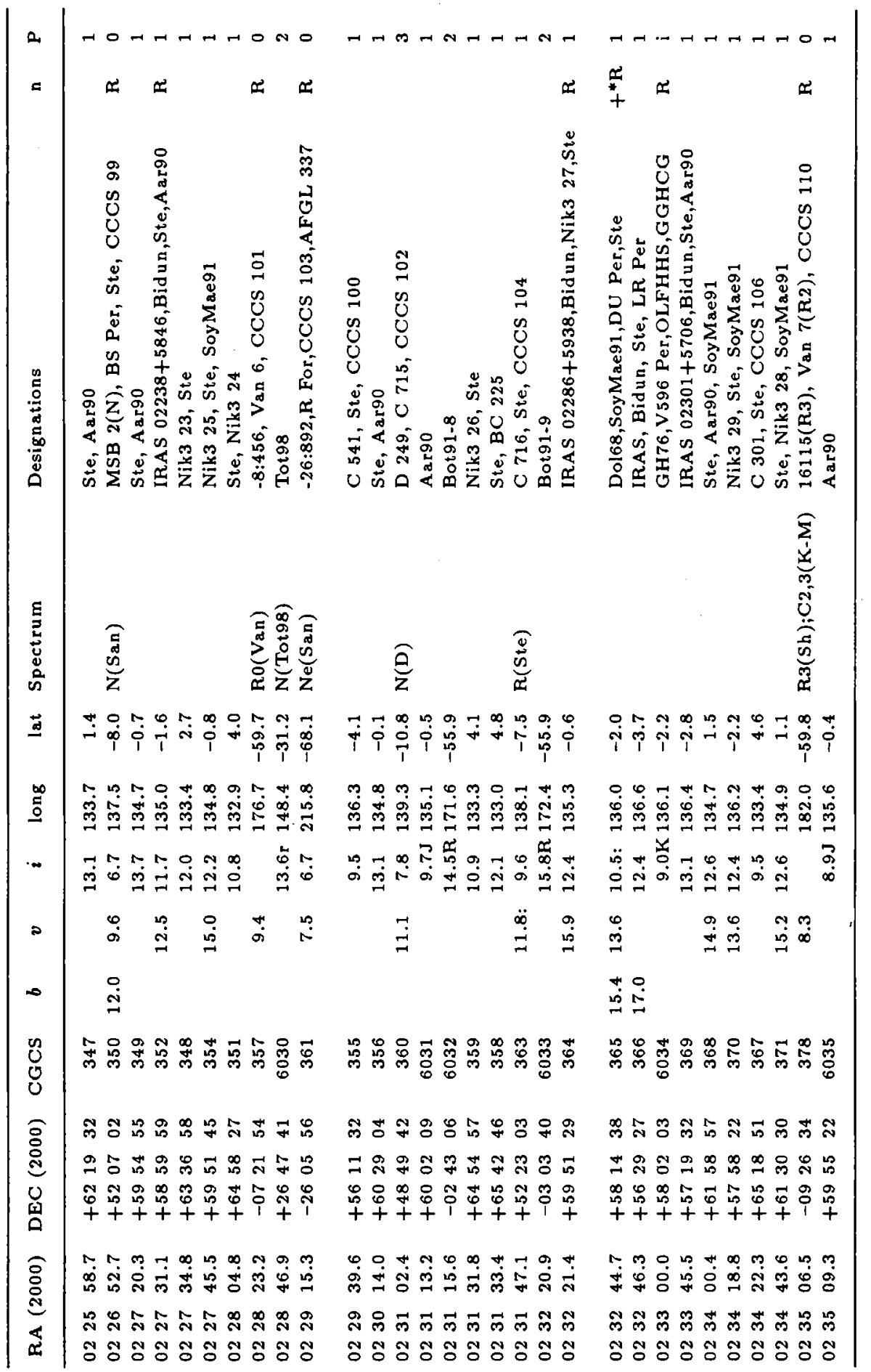




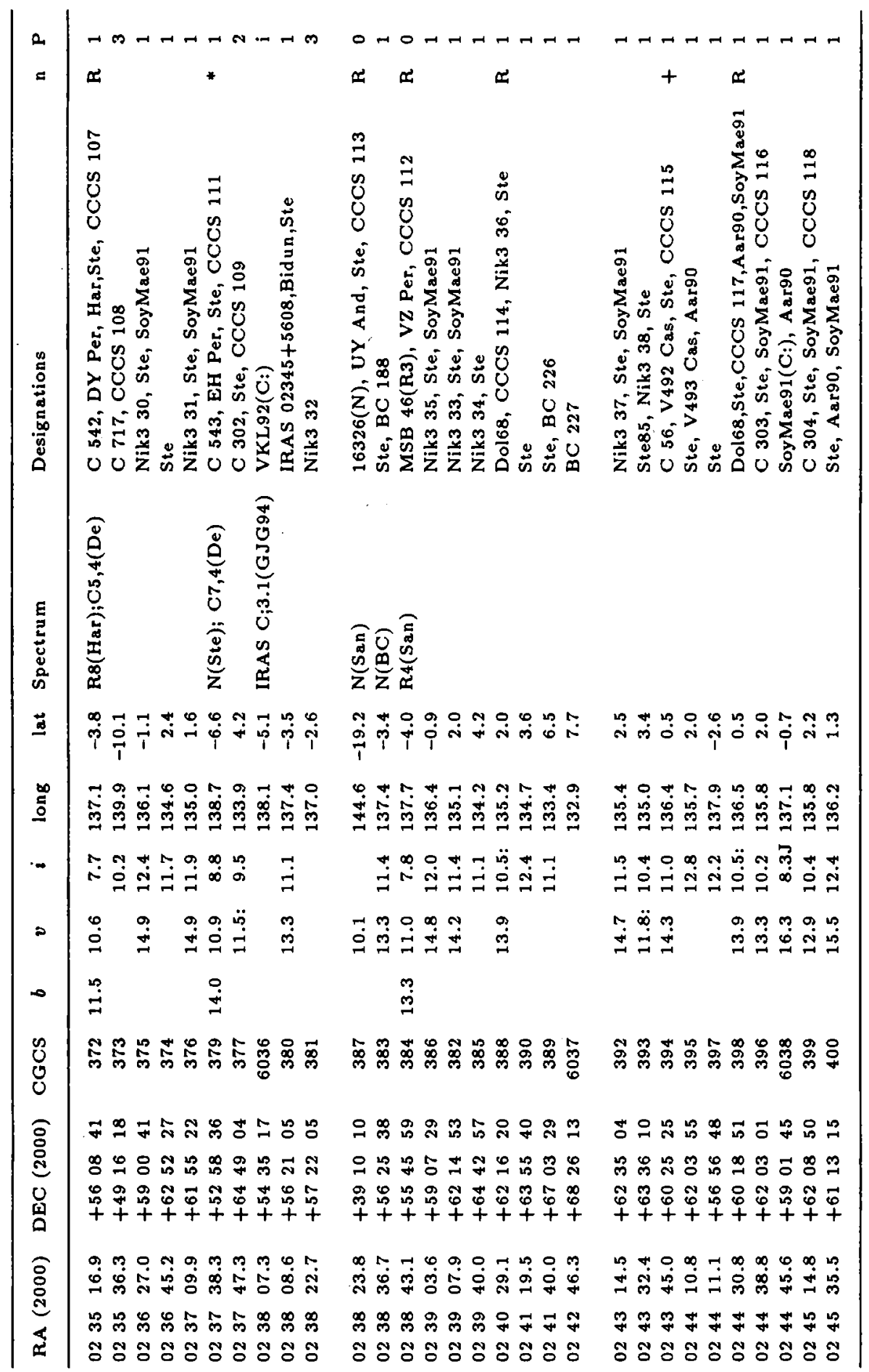




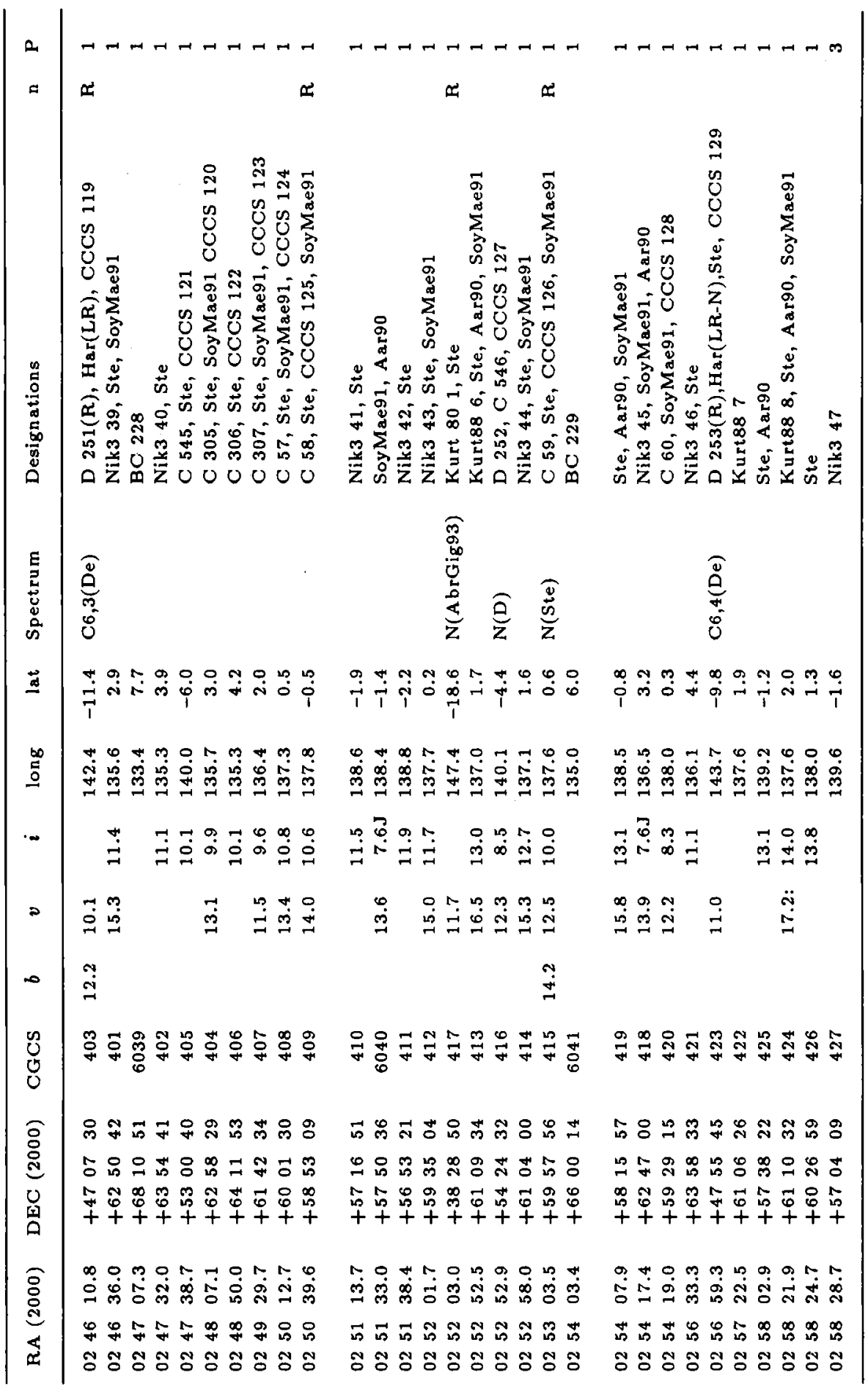




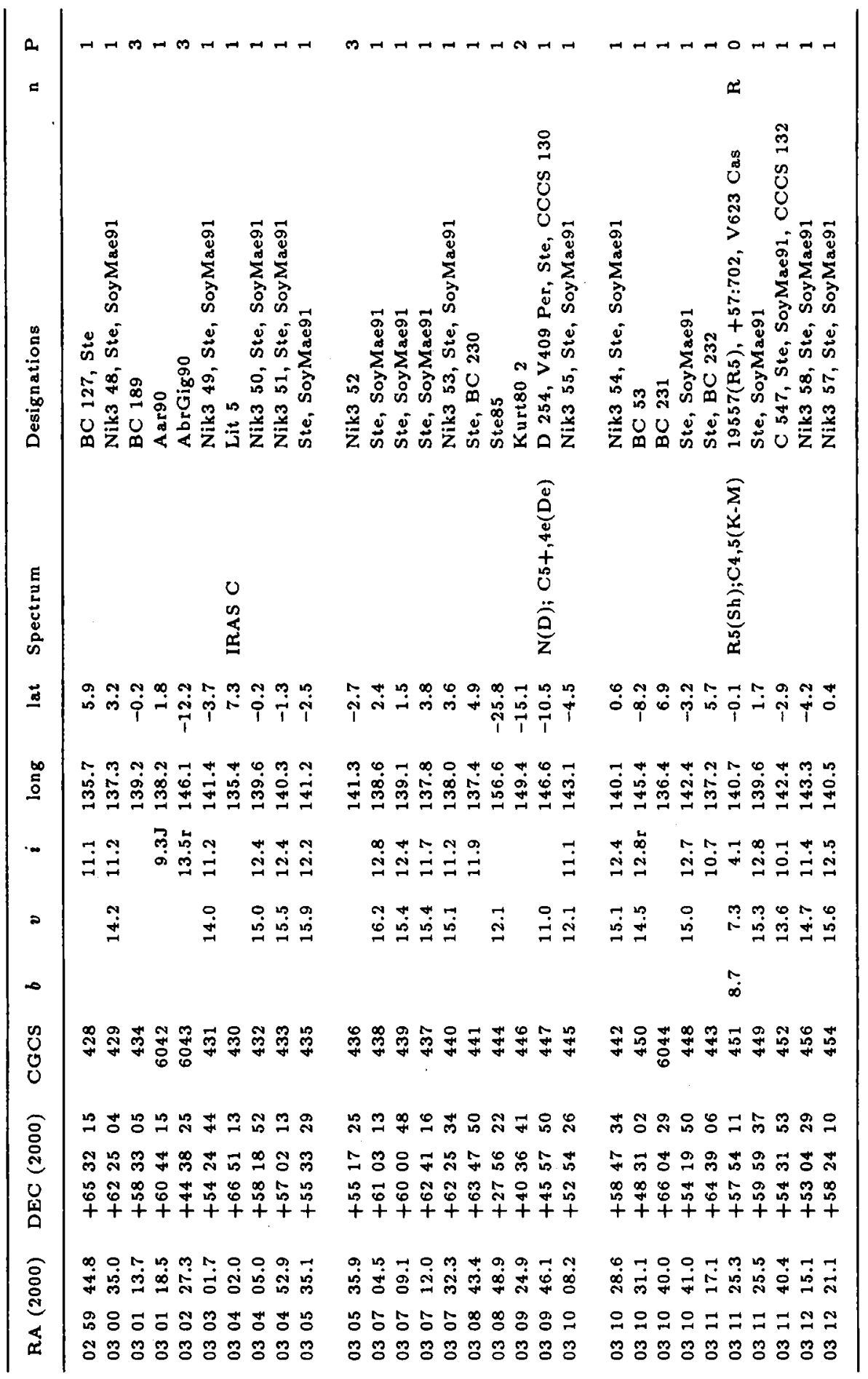




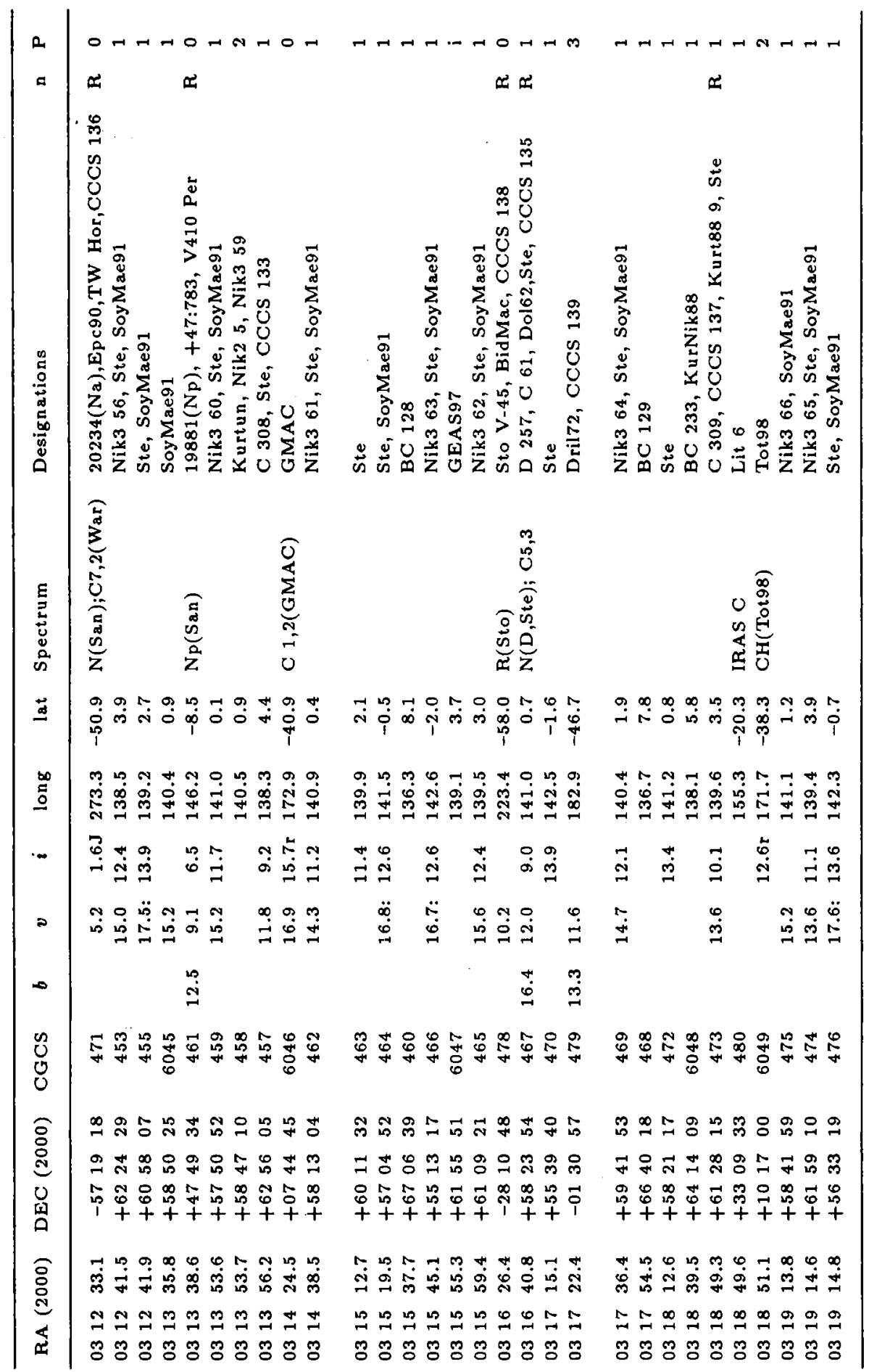




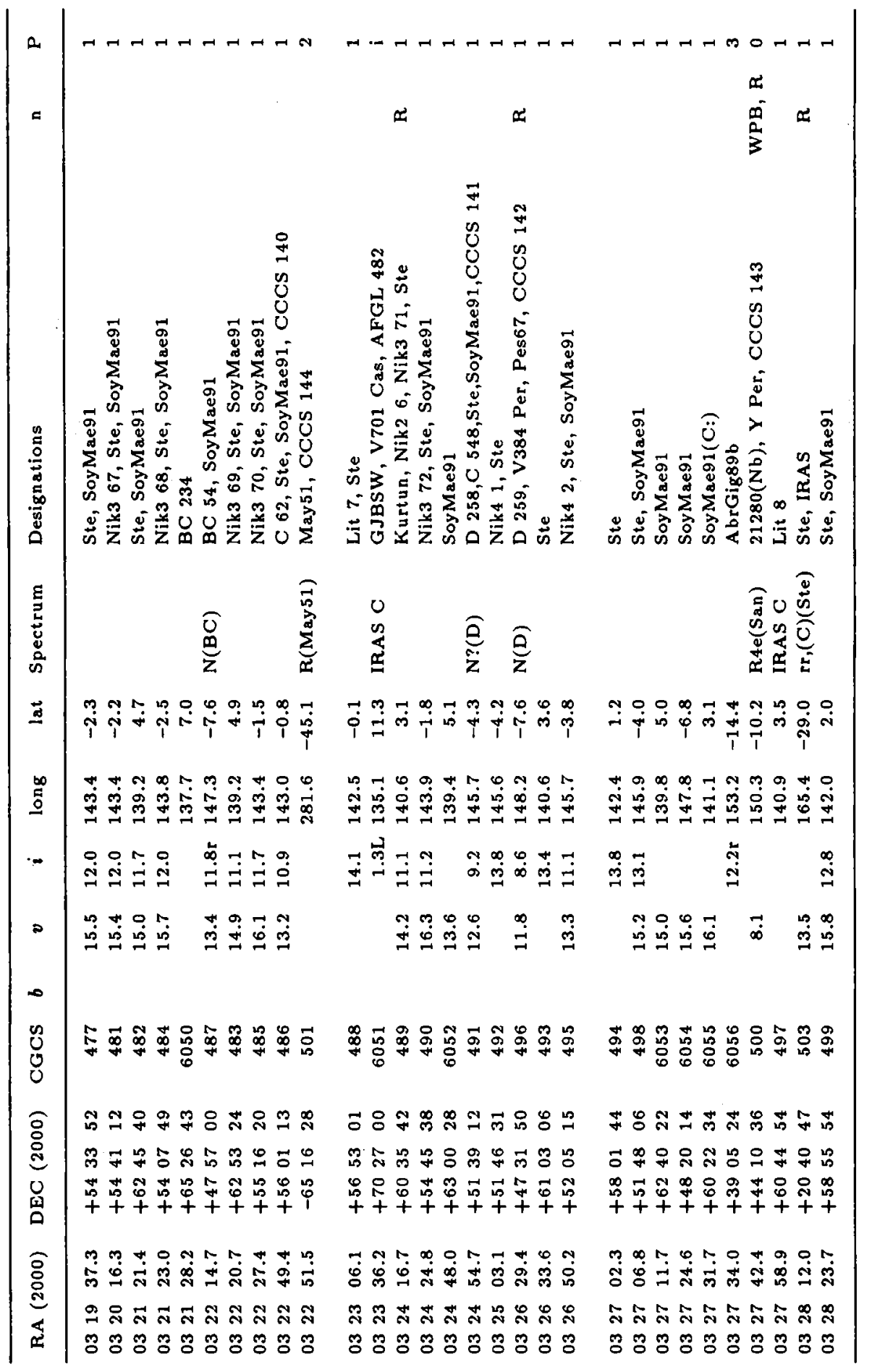




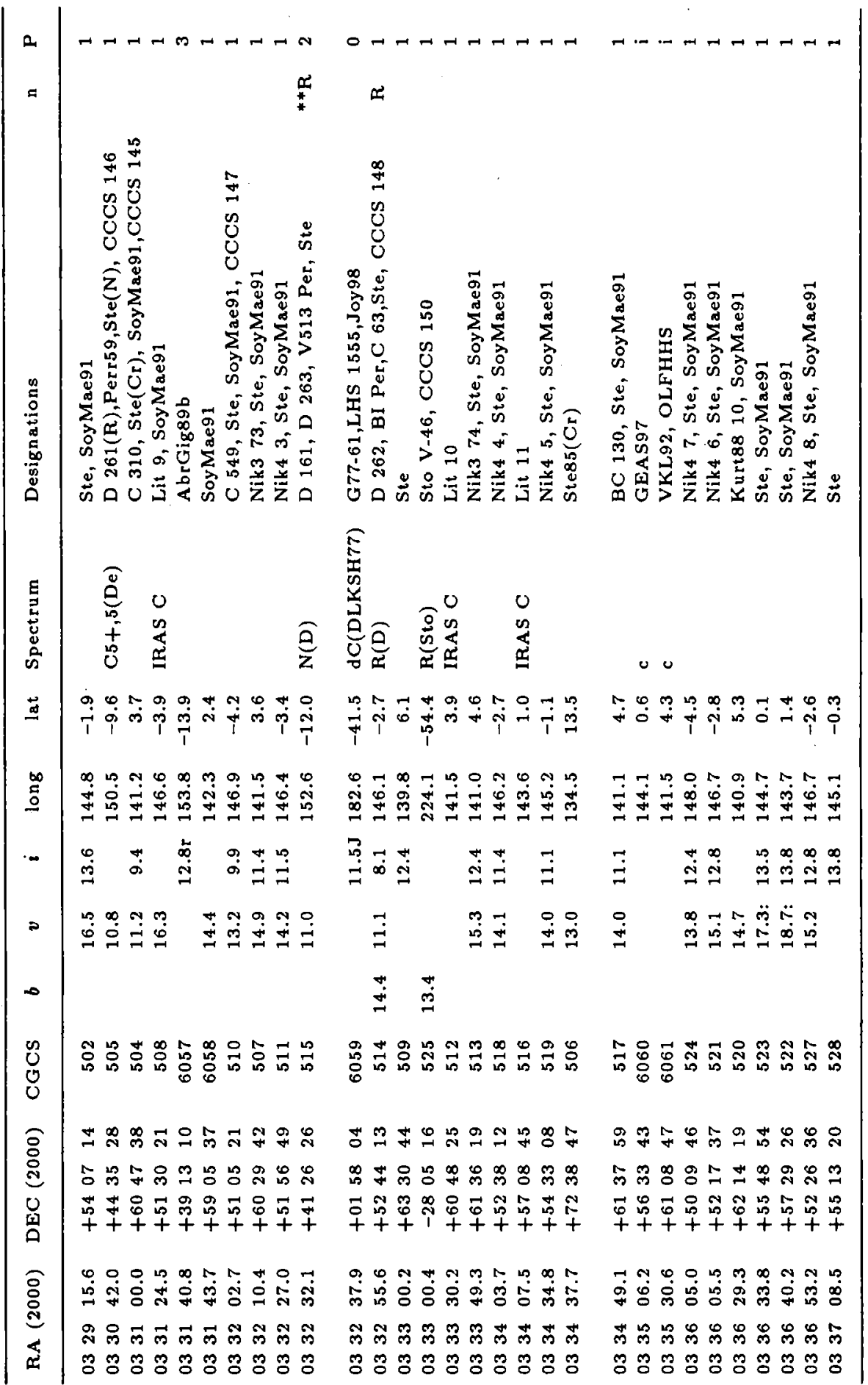




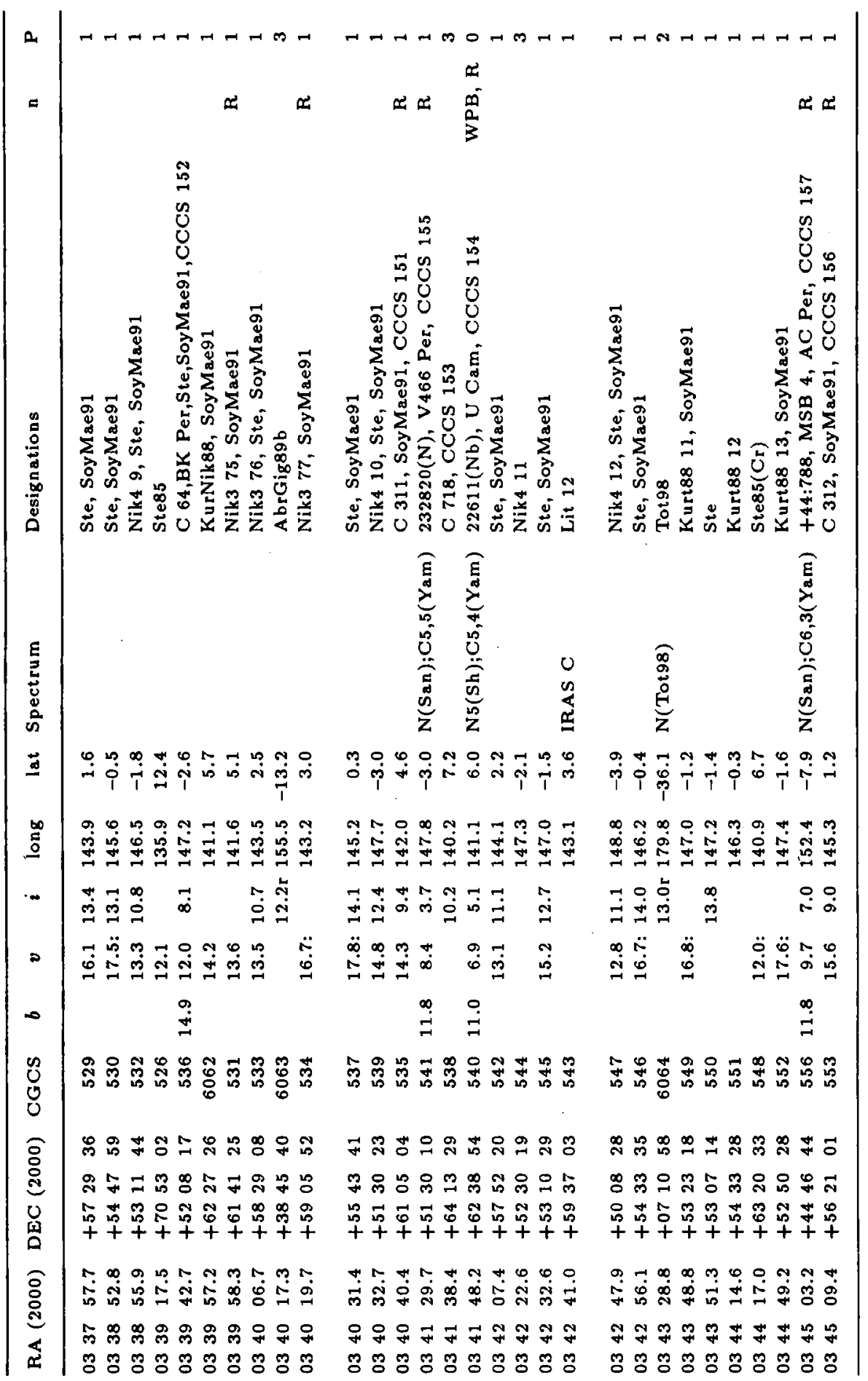




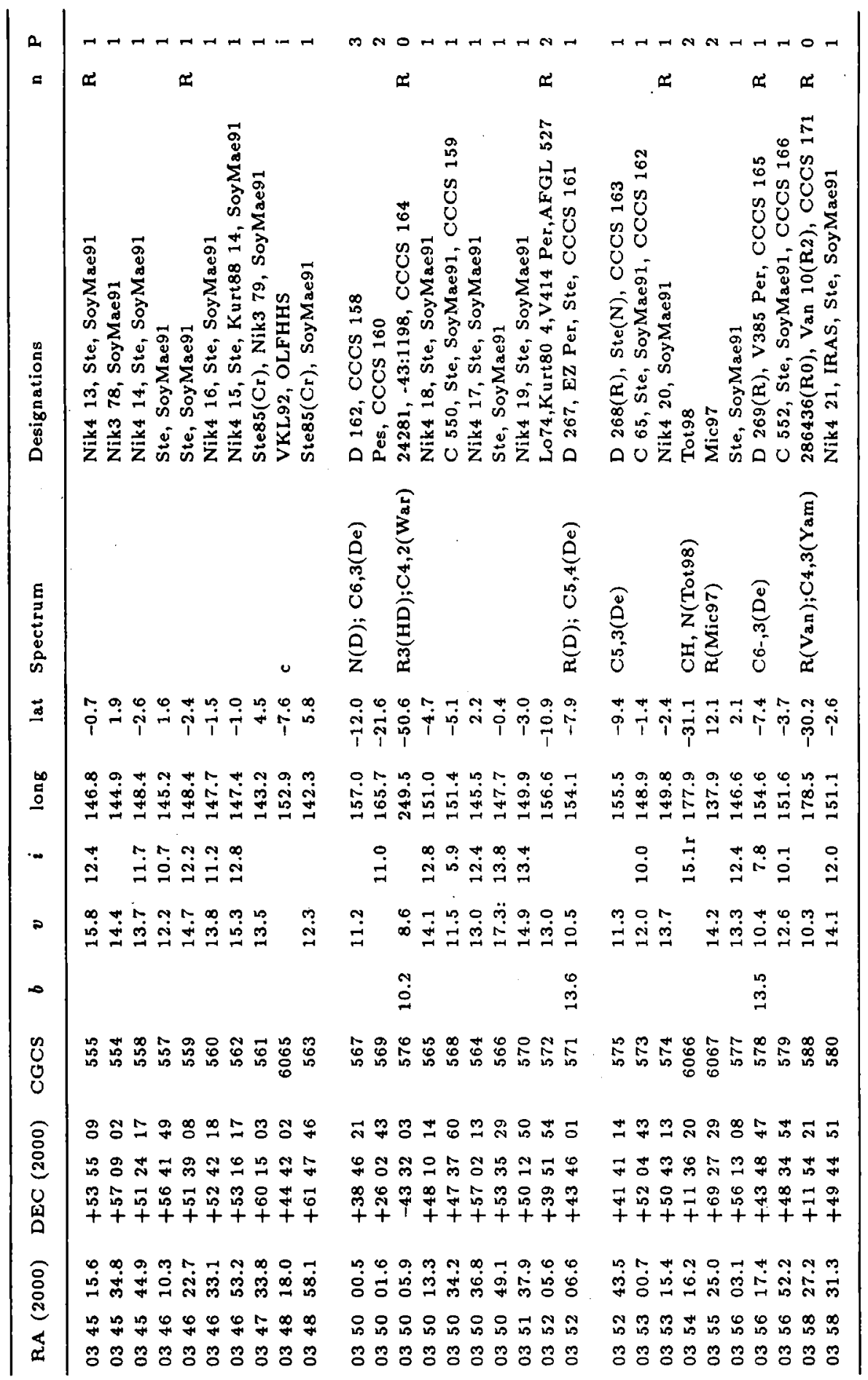




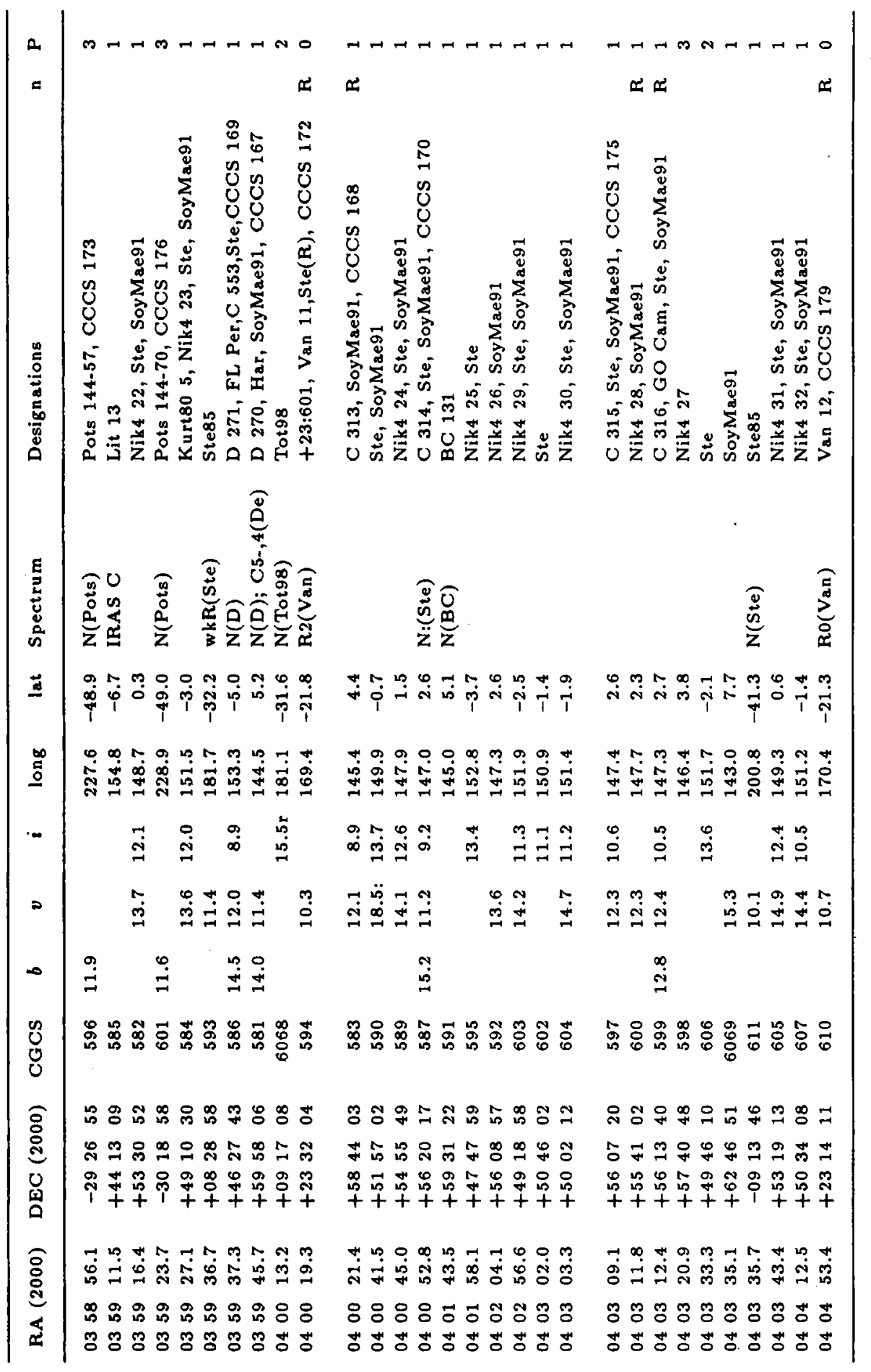




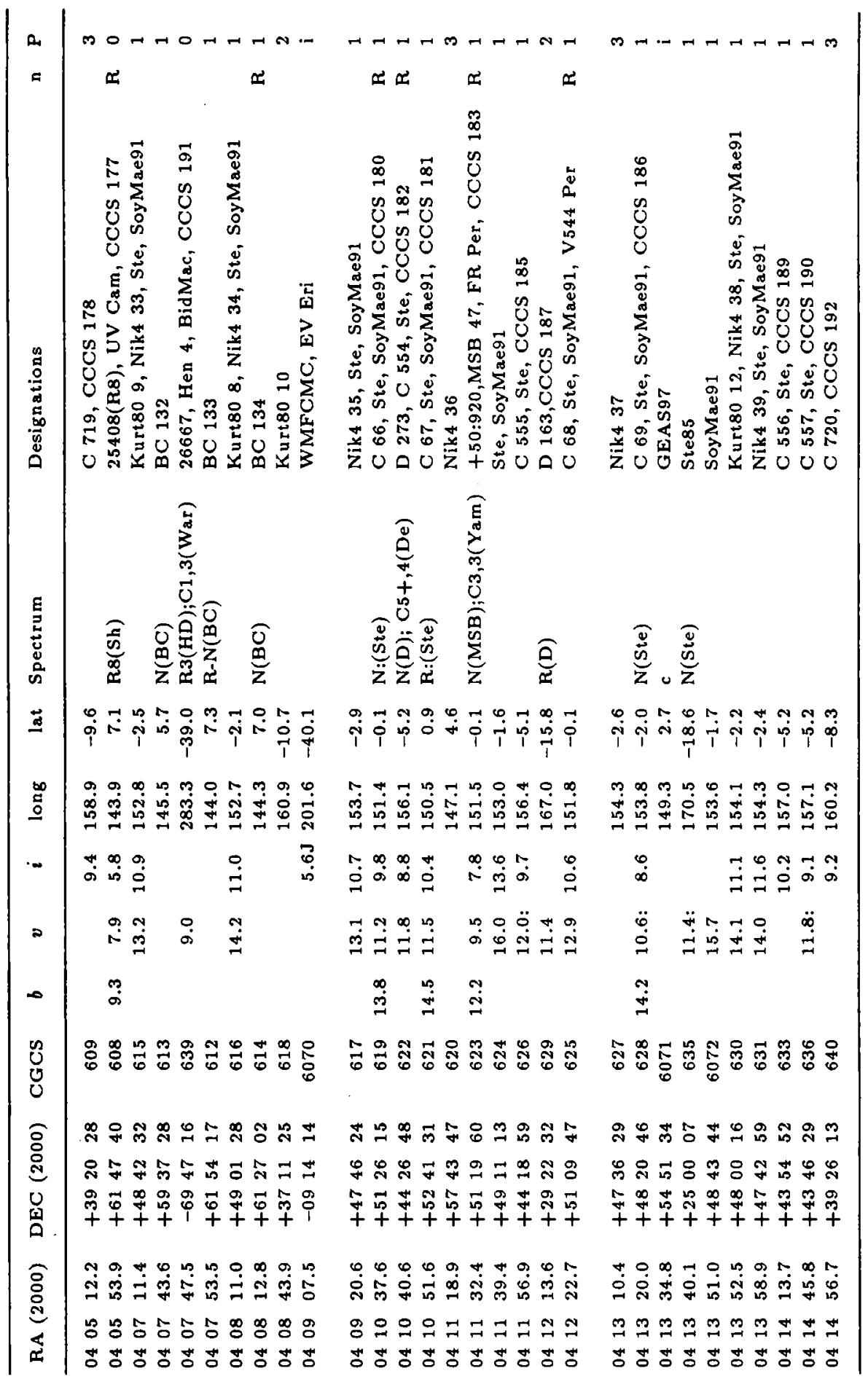




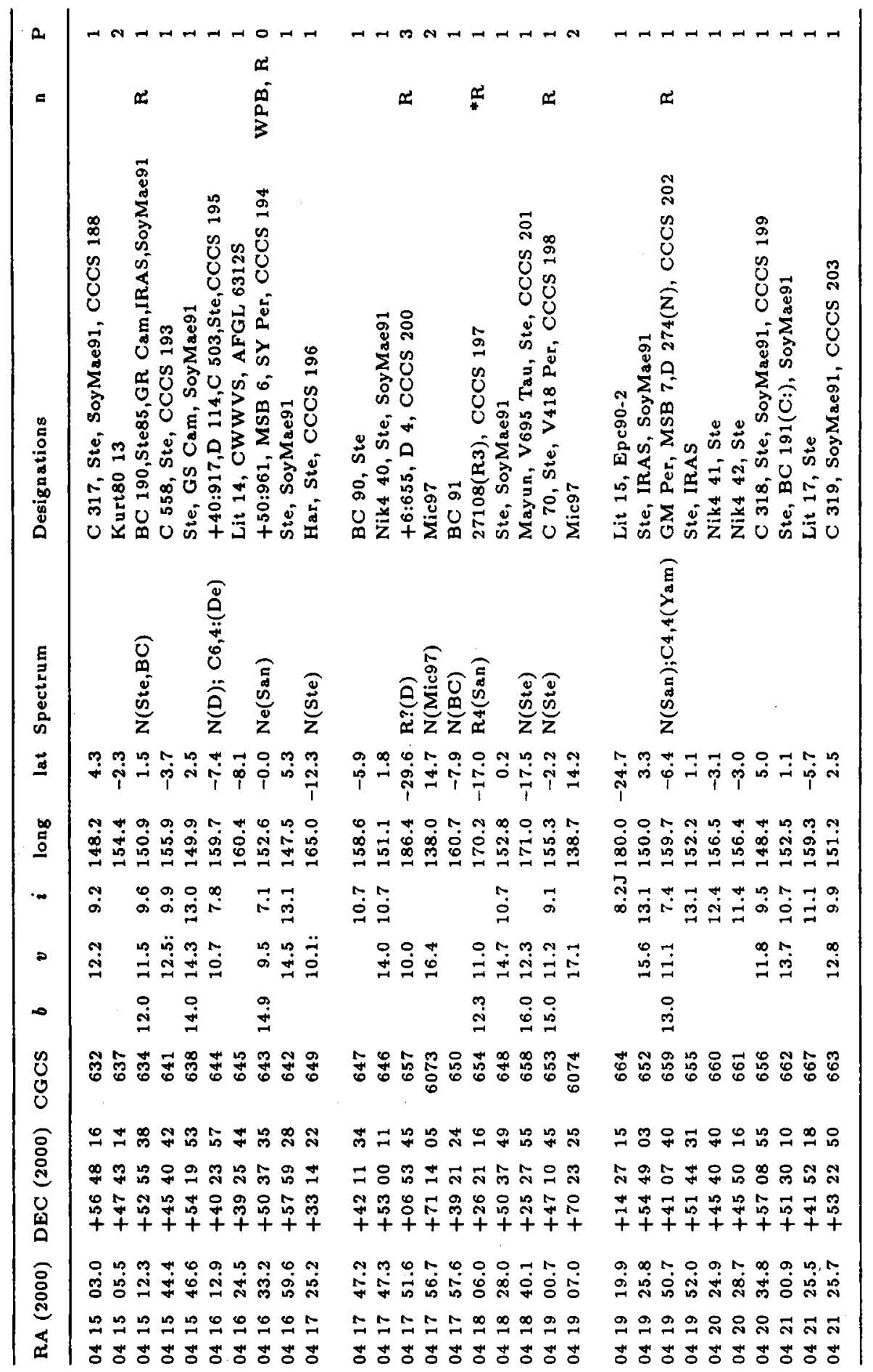




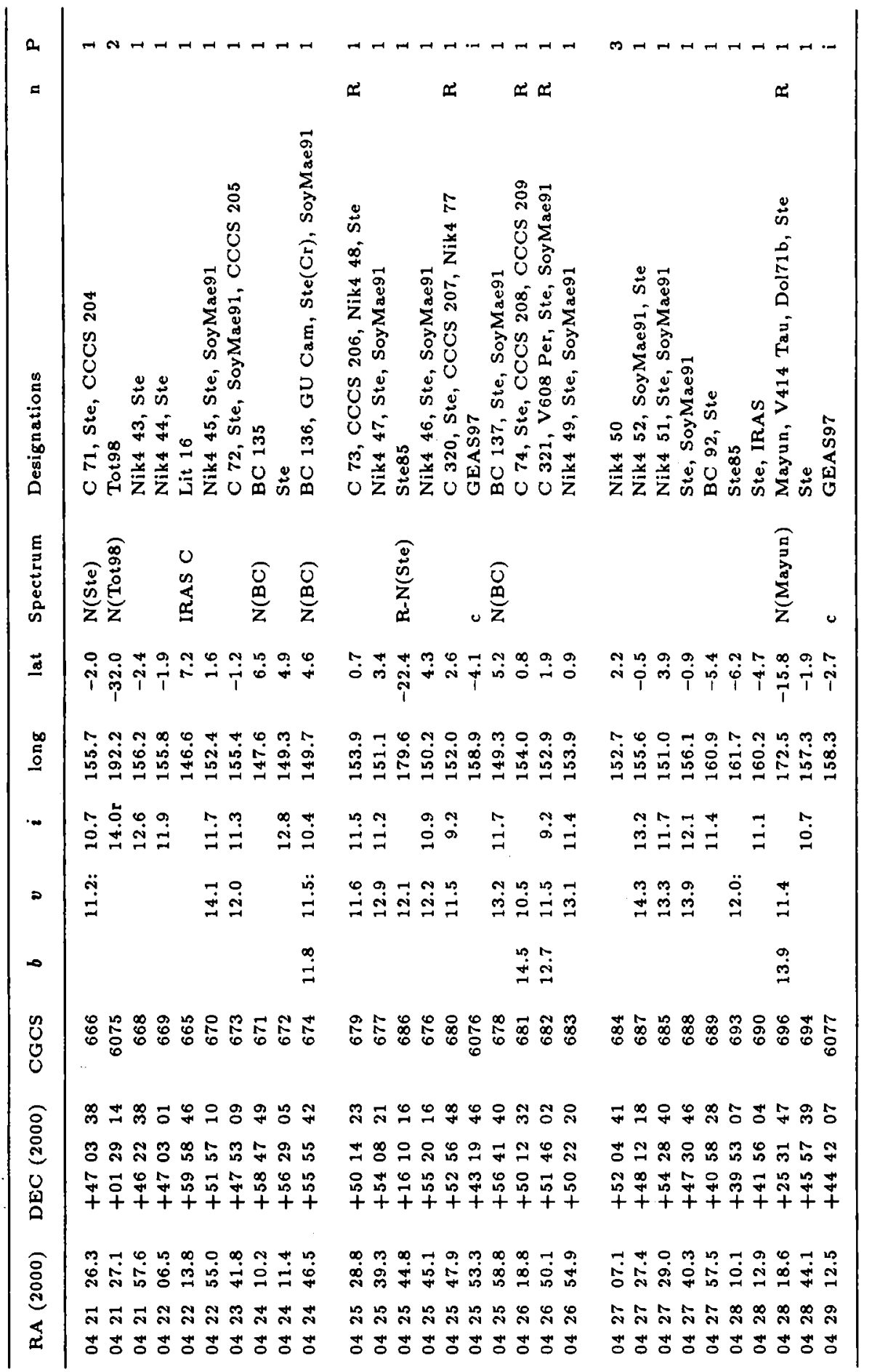




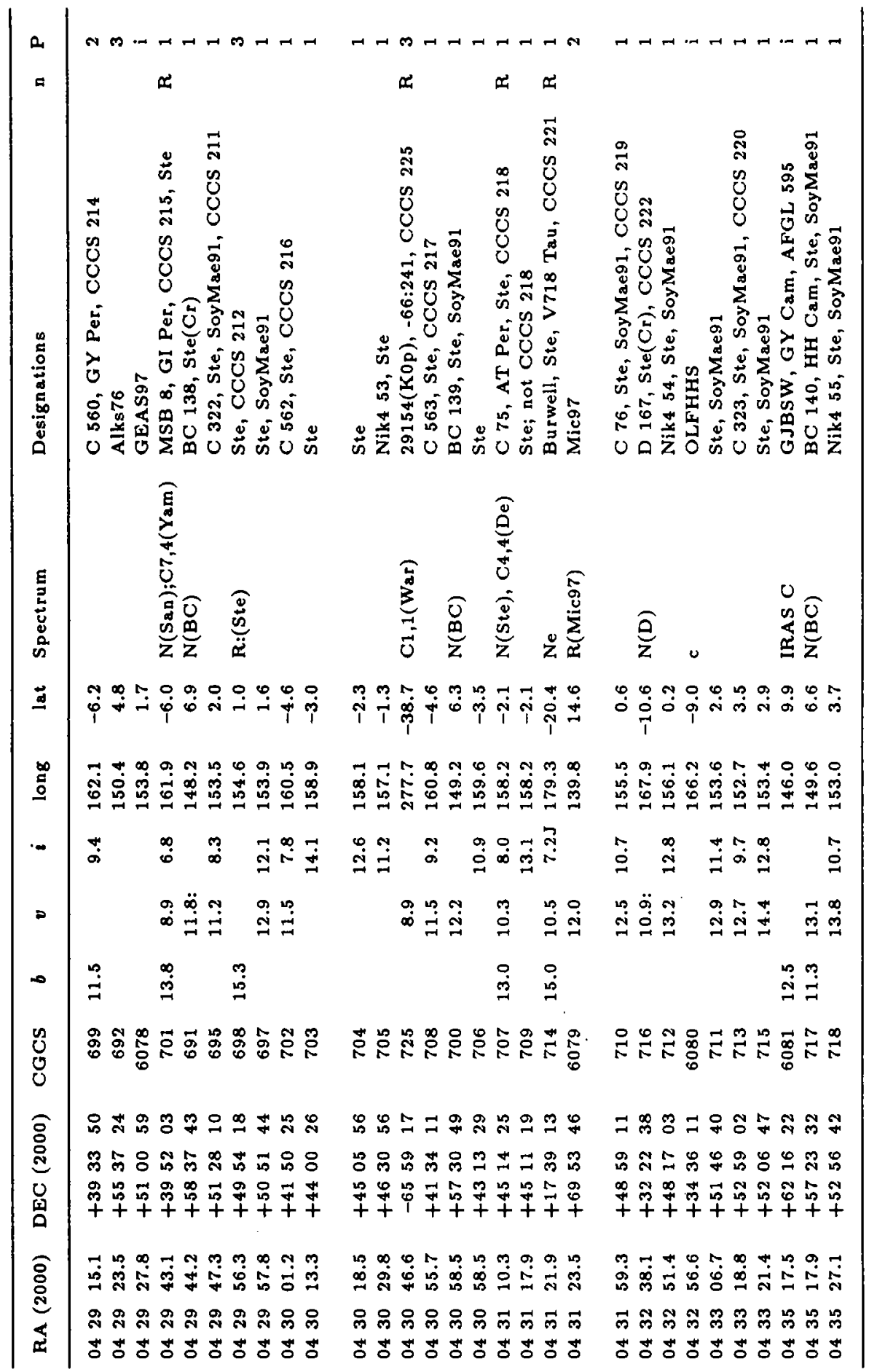




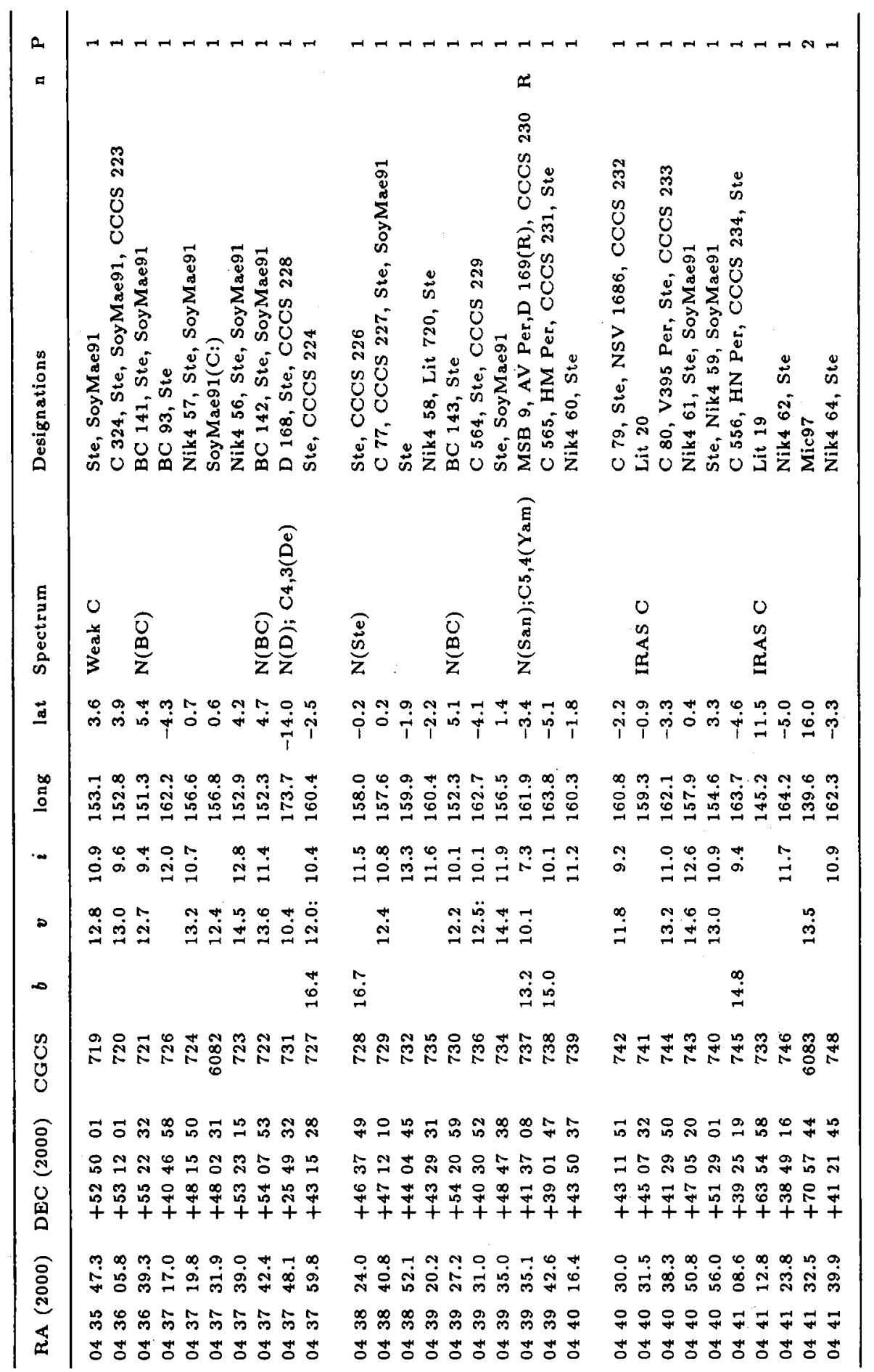




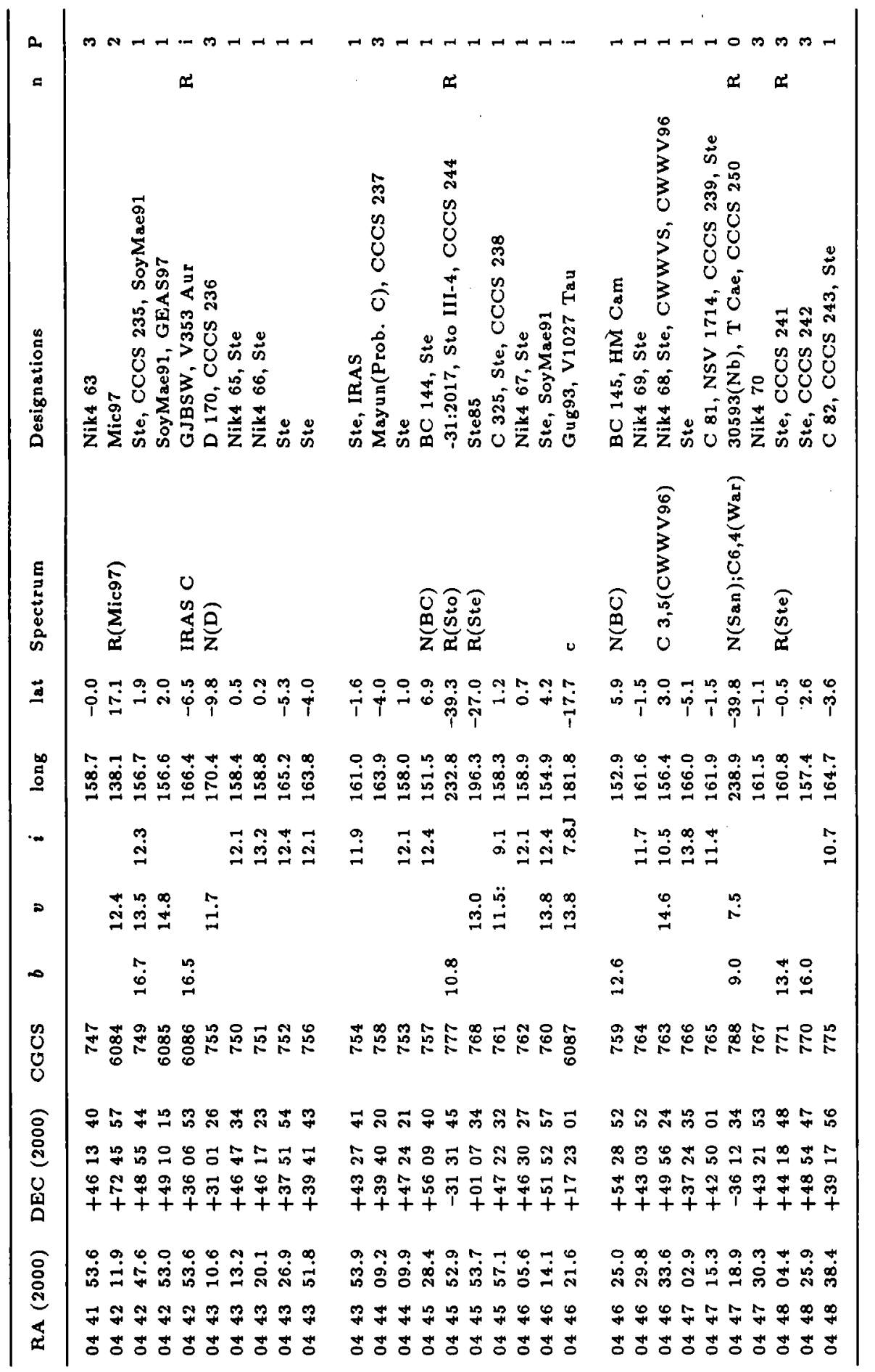




\begin{tabular}{|c|c|c|c|}
\hline a & 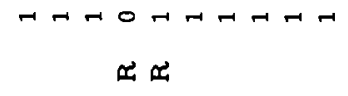 & 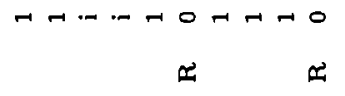 & 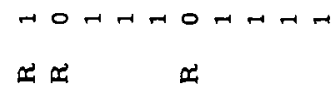 \\
\hline 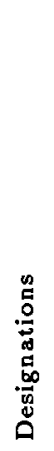 & 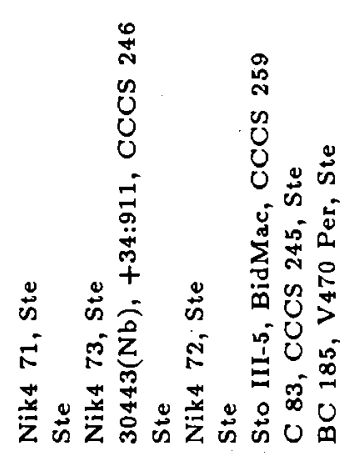 & 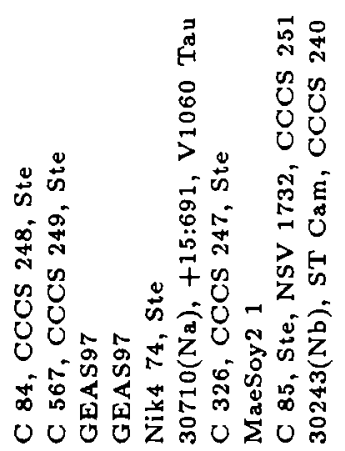 & 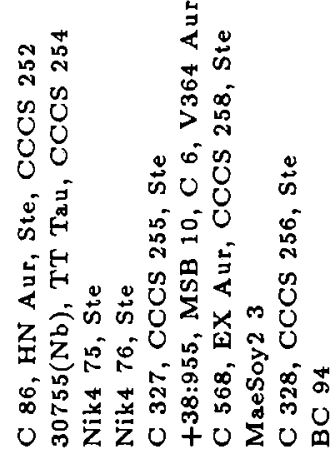 \\
\hline 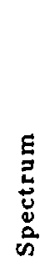 & 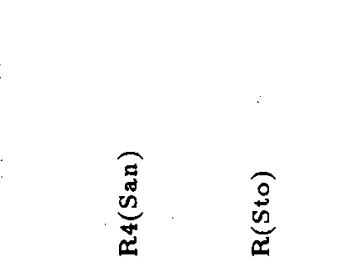 & 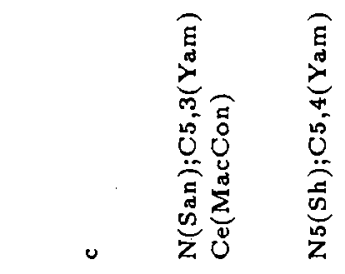 & 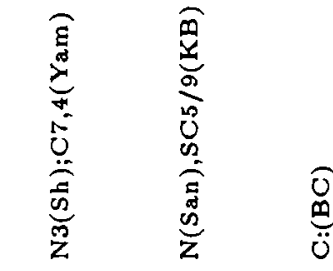 \\
\hline$\underline{\underline{a}}$ & 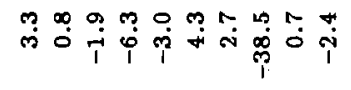 & 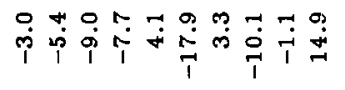 & 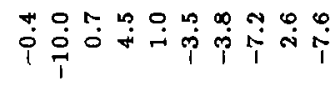 \\
\hline$\stackrel{\infty}{0}$ & 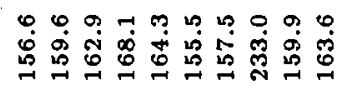 & 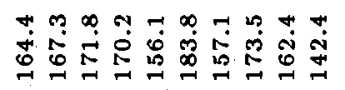 & 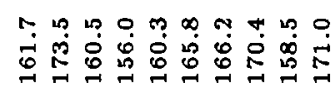 \\
\hline هـ' & 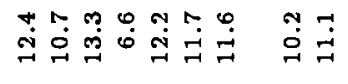 & 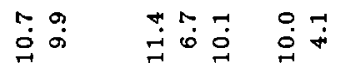 & 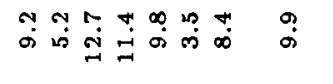 \\
\hline D & $\stackrel{+}{\ddot{m}}$ & $\begin{array}{lll}\overrightarrow{0} & \stackrel{0}{\infty} \stackrel{\infty}{0} & 0 \\
0 & =\end{array}$ & $\begin{array}{lll}\infty & 0 & 0 \\
\infty & \dot{m} & \dot{n}\end{array}$ \\
\hline$\infty$ & 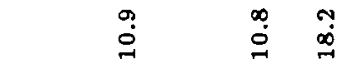 & $\stackrel{\sim}{\rightrightarrows}$ & ฯุ \\
\hline$\bigcup_{\circlearrowright}^{\infty}$ & 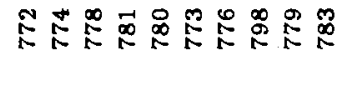 & 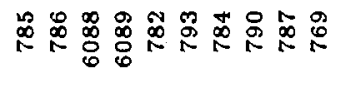 & 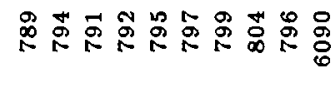 \\
\hline \&ิ & 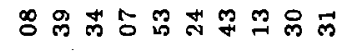 & 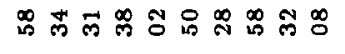 & 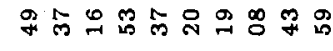 \\
\hline 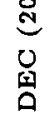 & 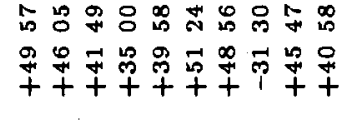 & 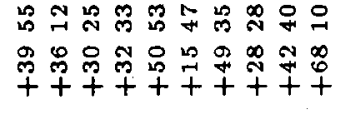 & 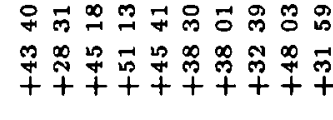 \\
\hline ठิ & 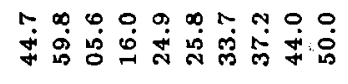 & 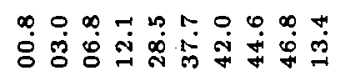 & 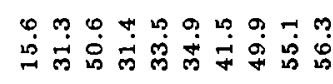 \\
\hline జ & 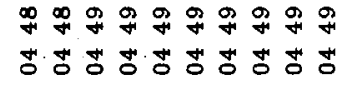 & 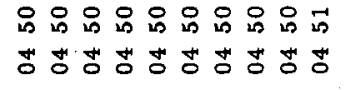 & 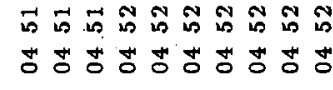 \\
\hline
\end{tabular}




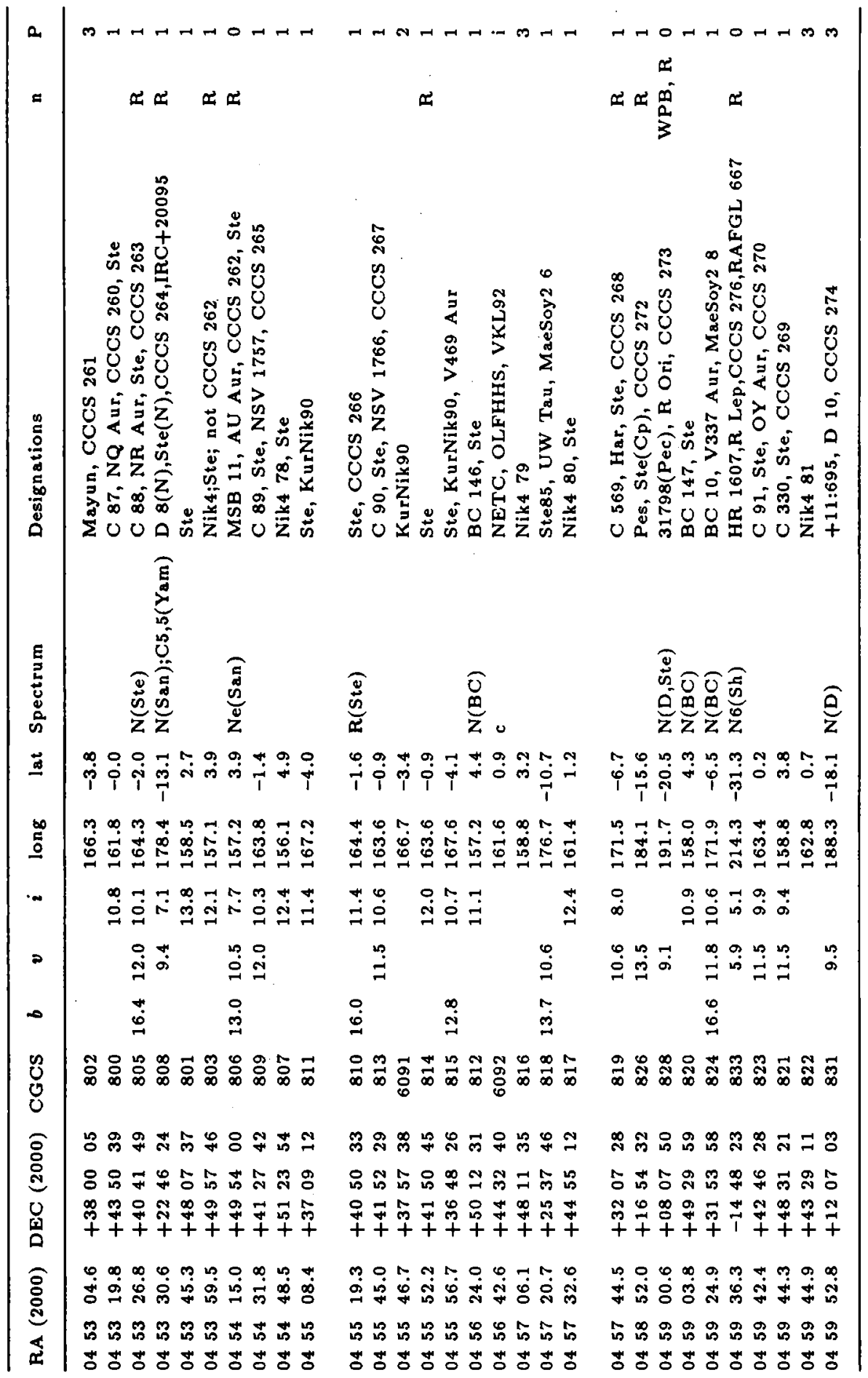




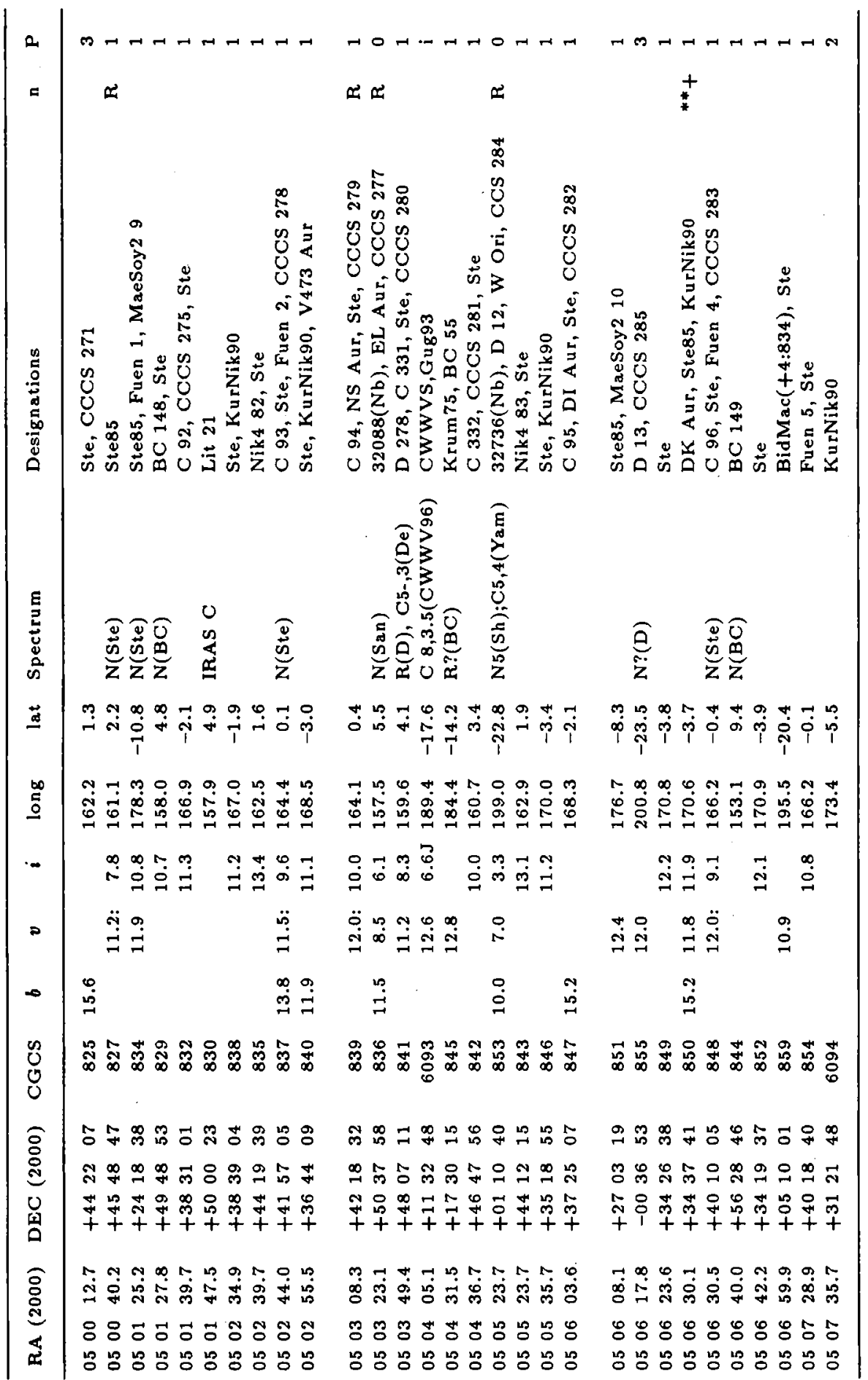




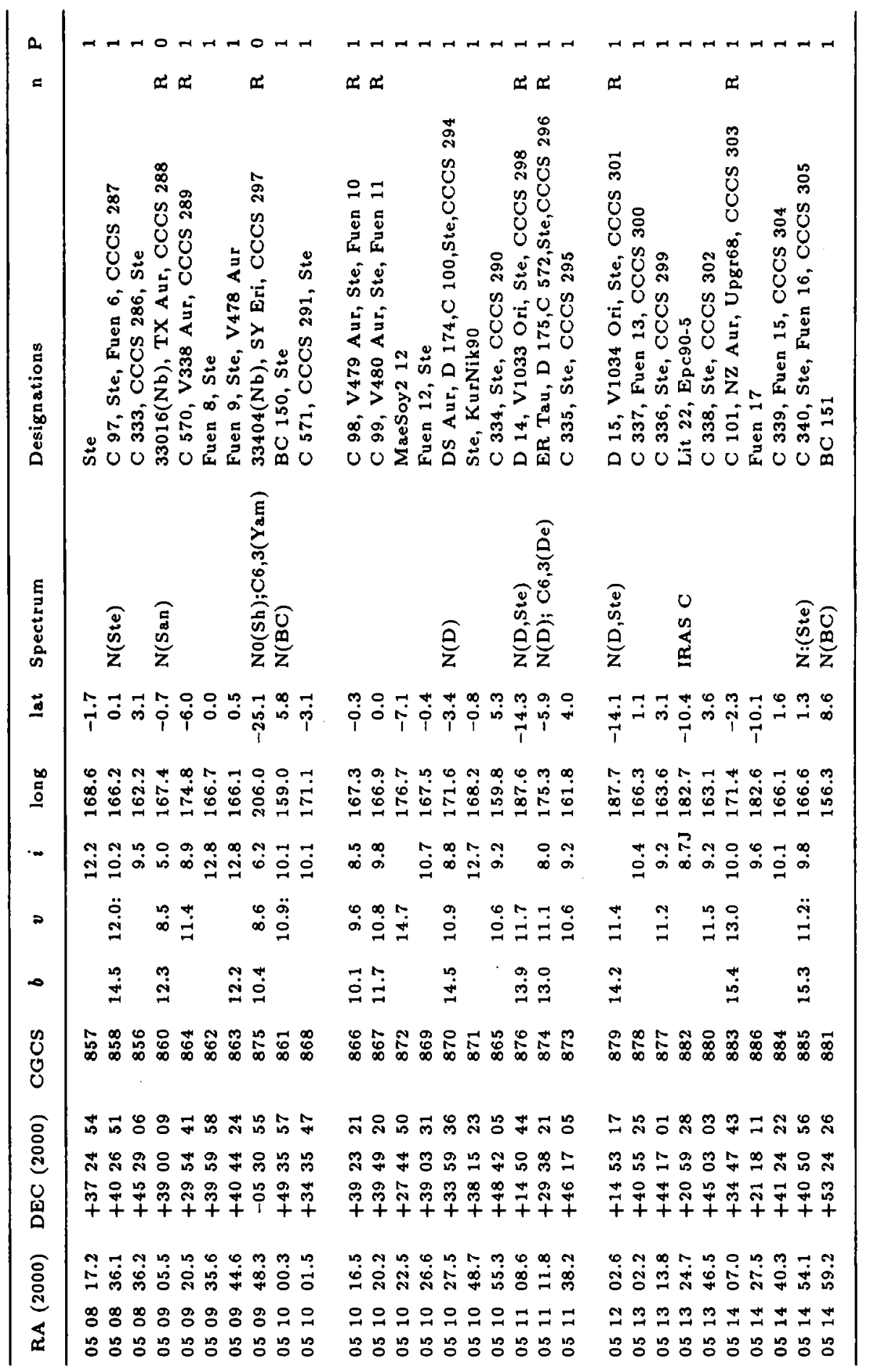




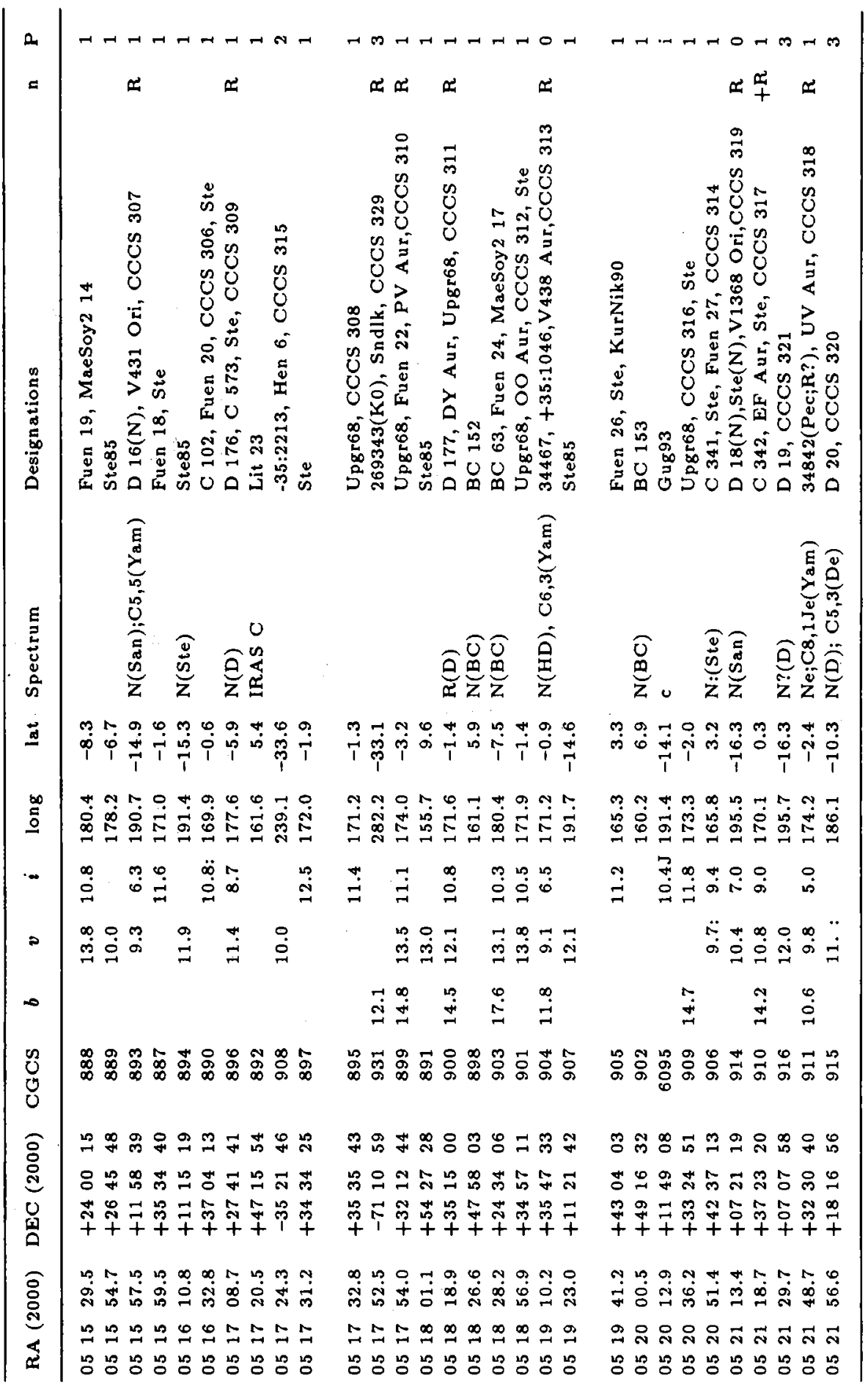




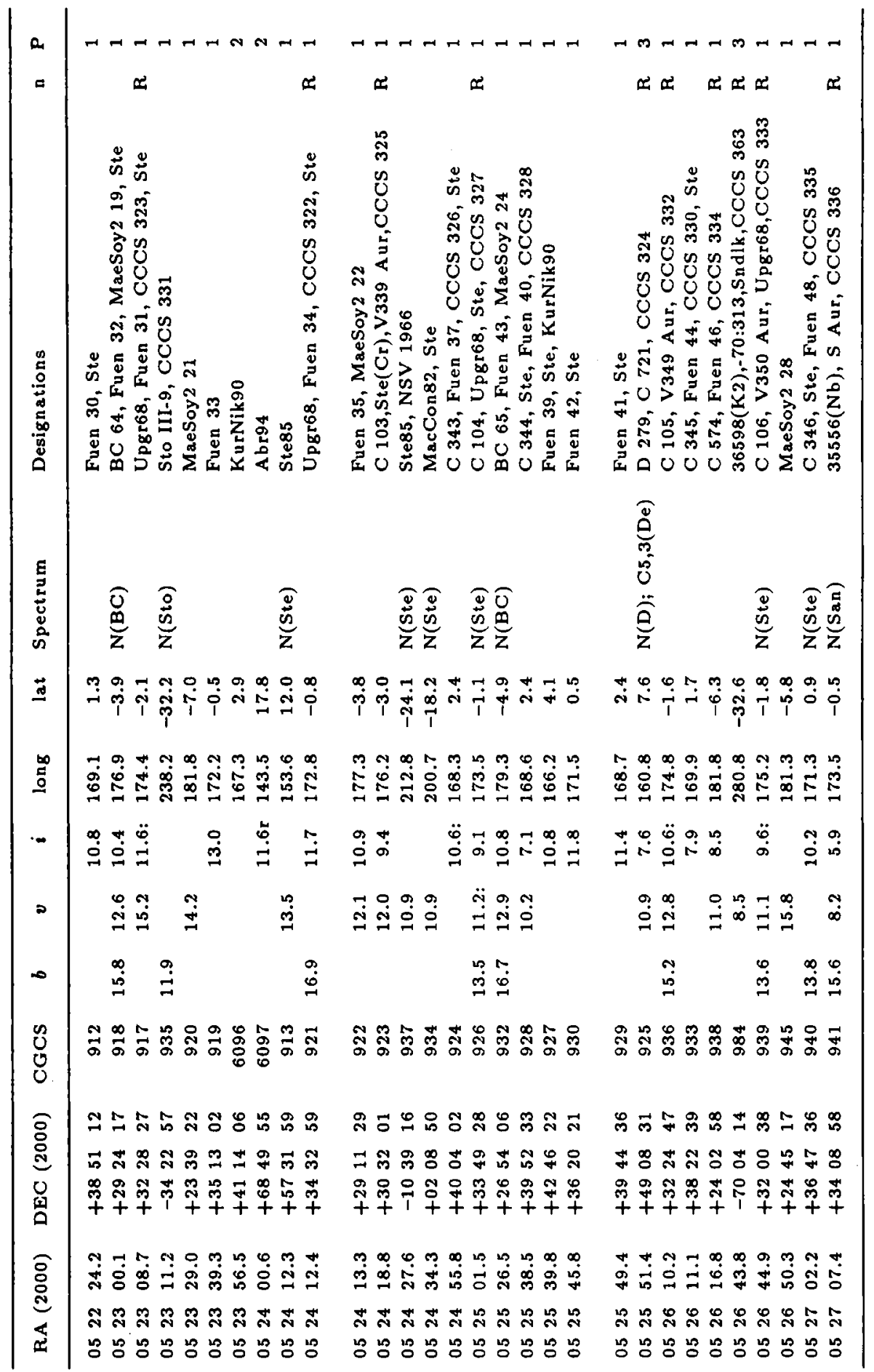




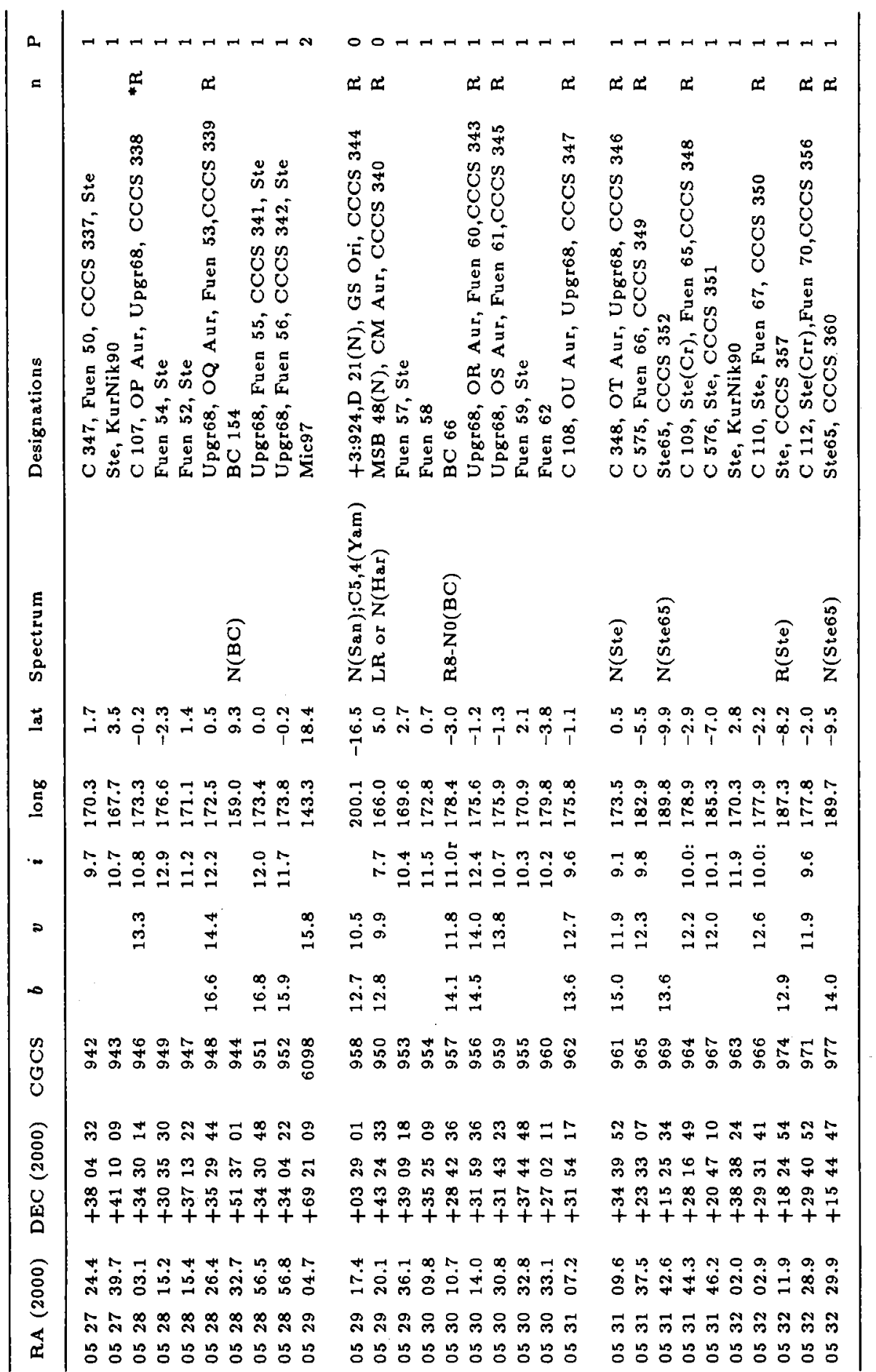




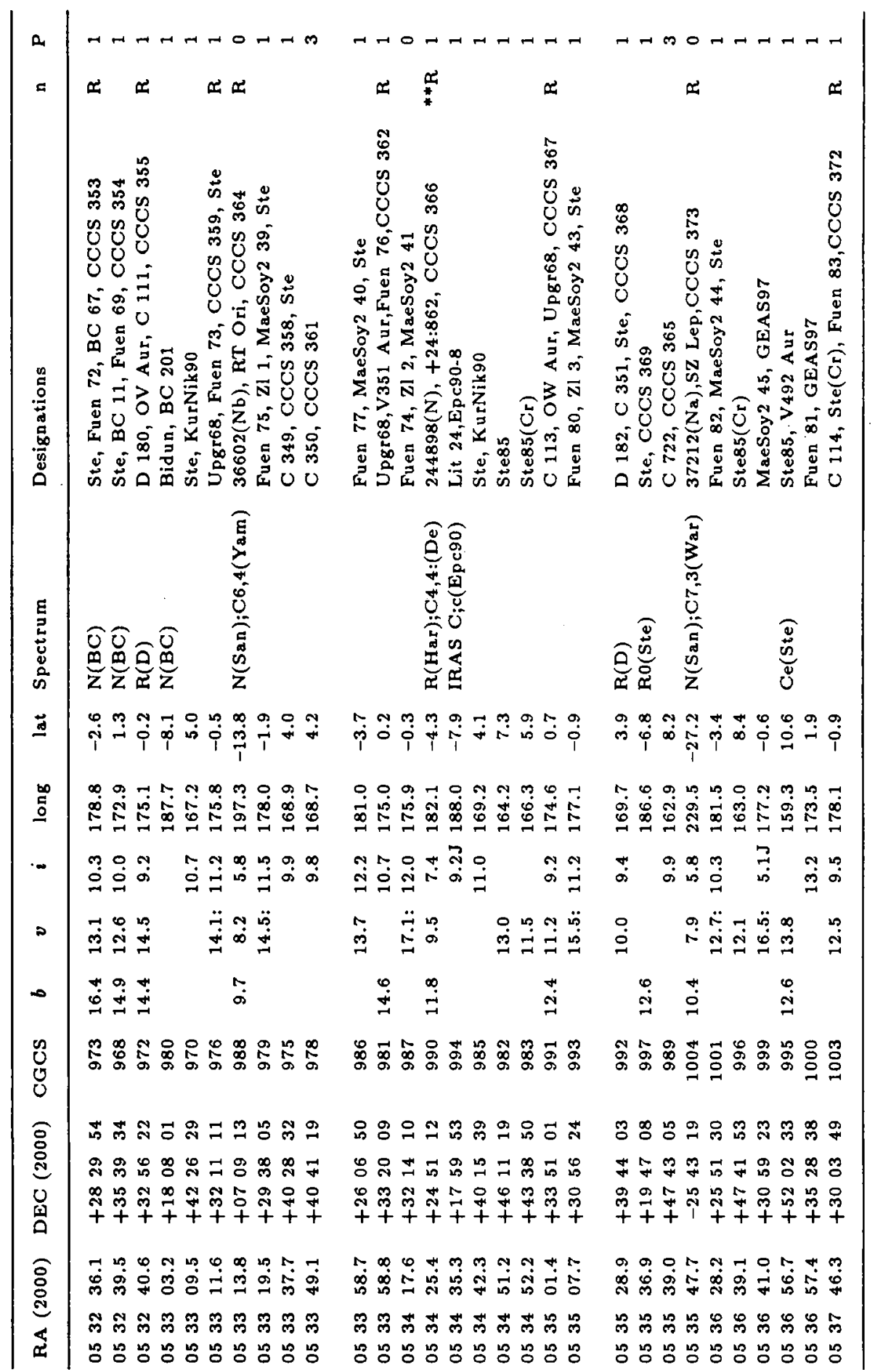




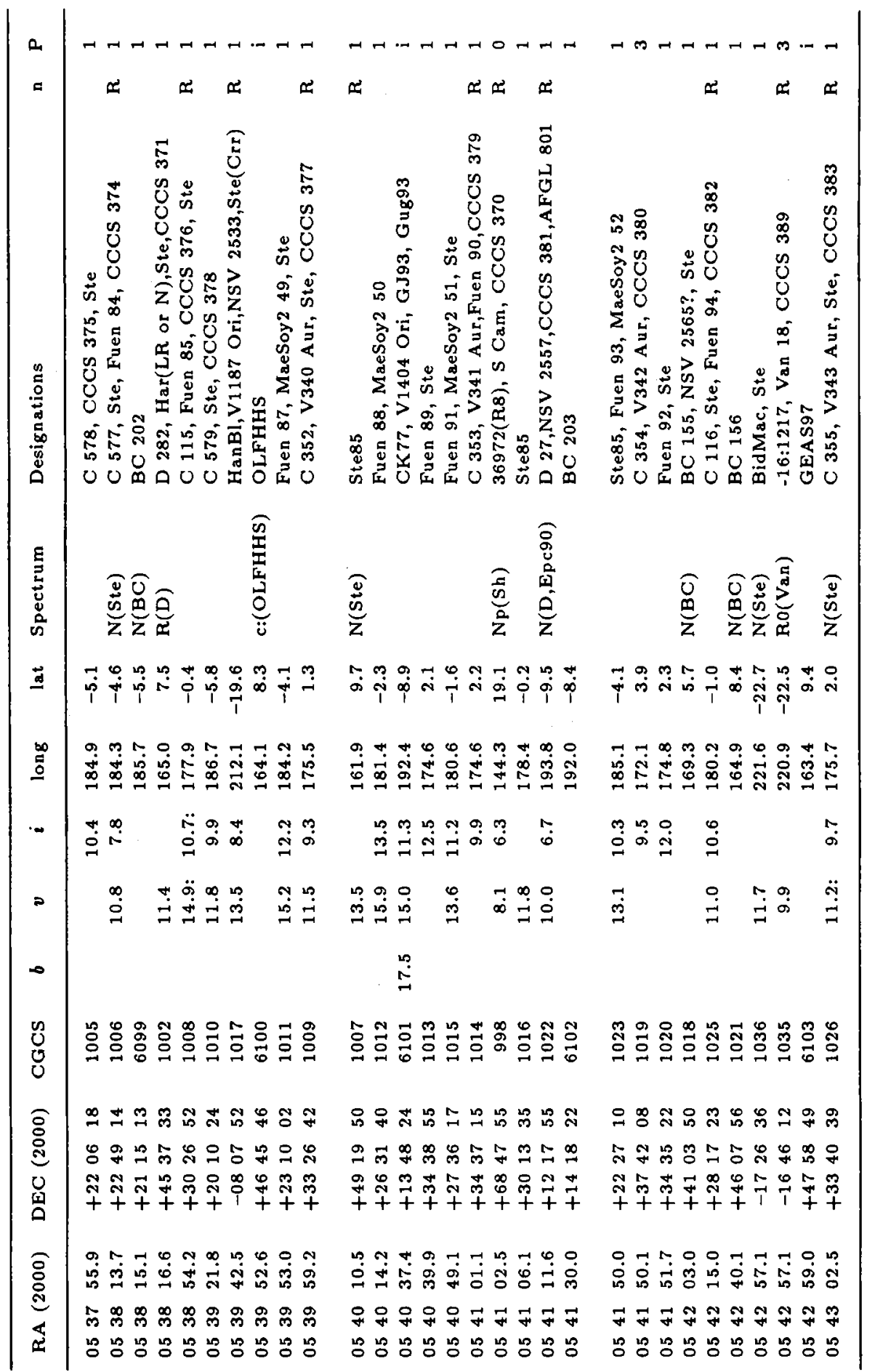




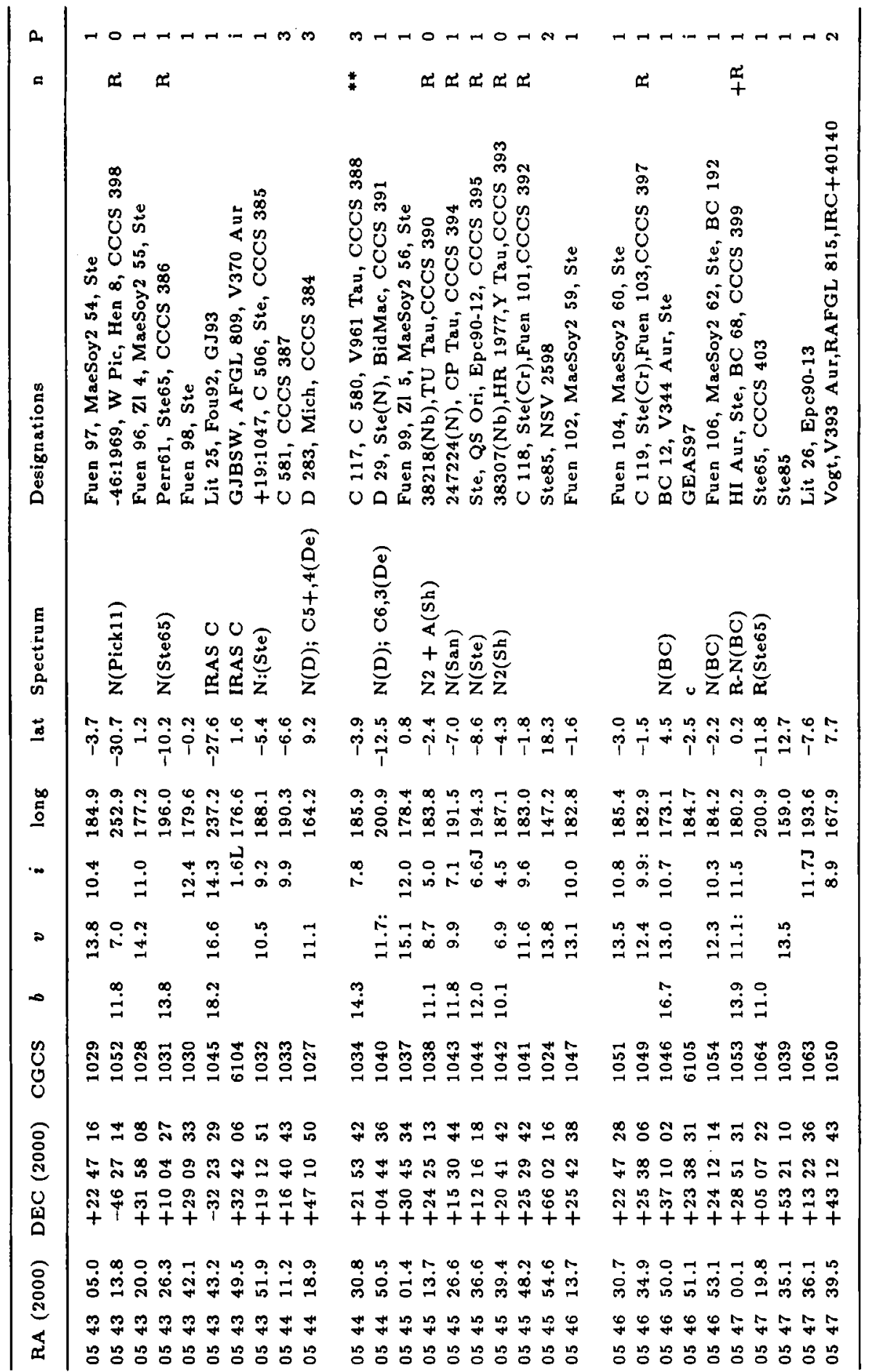




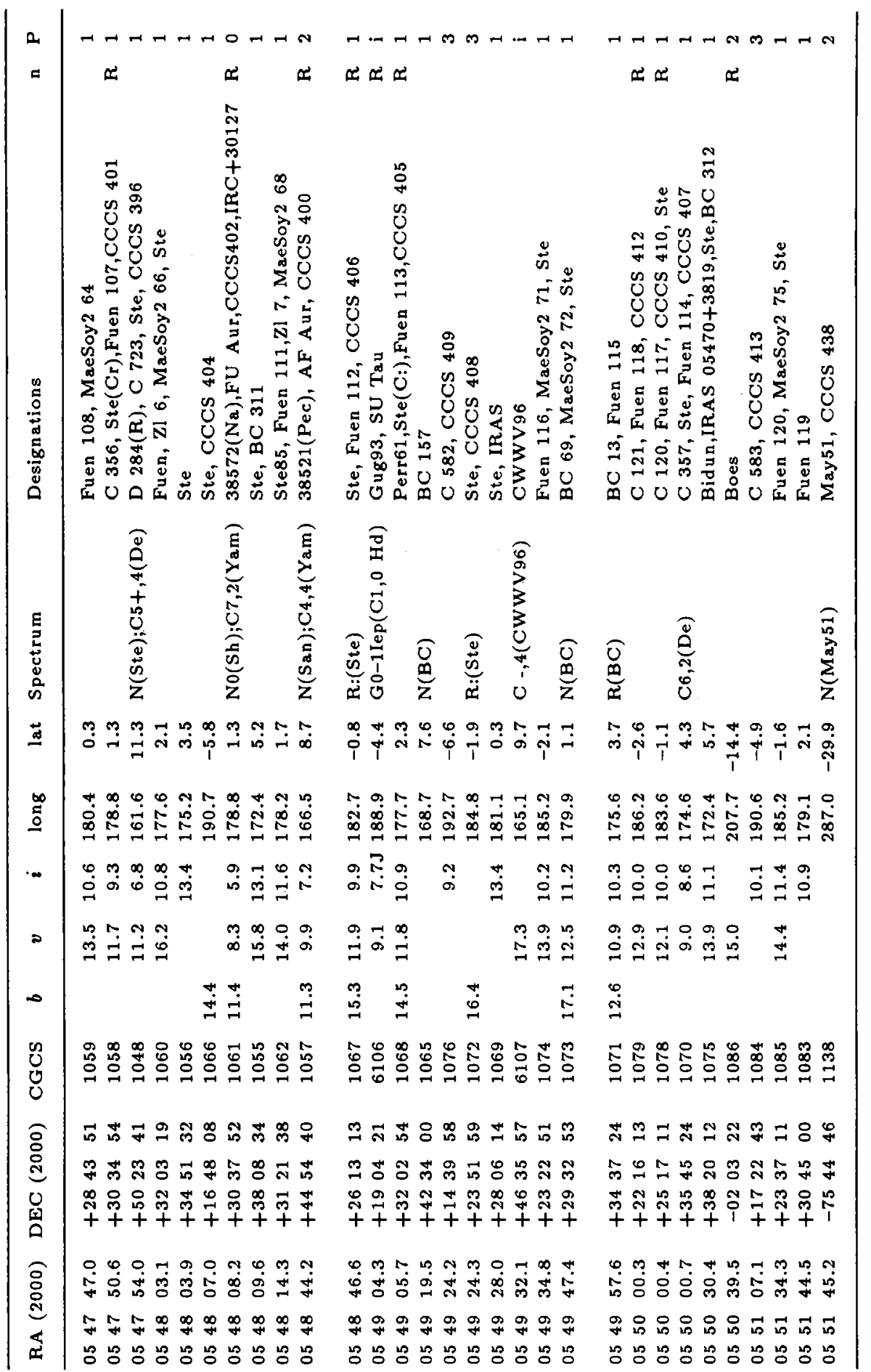




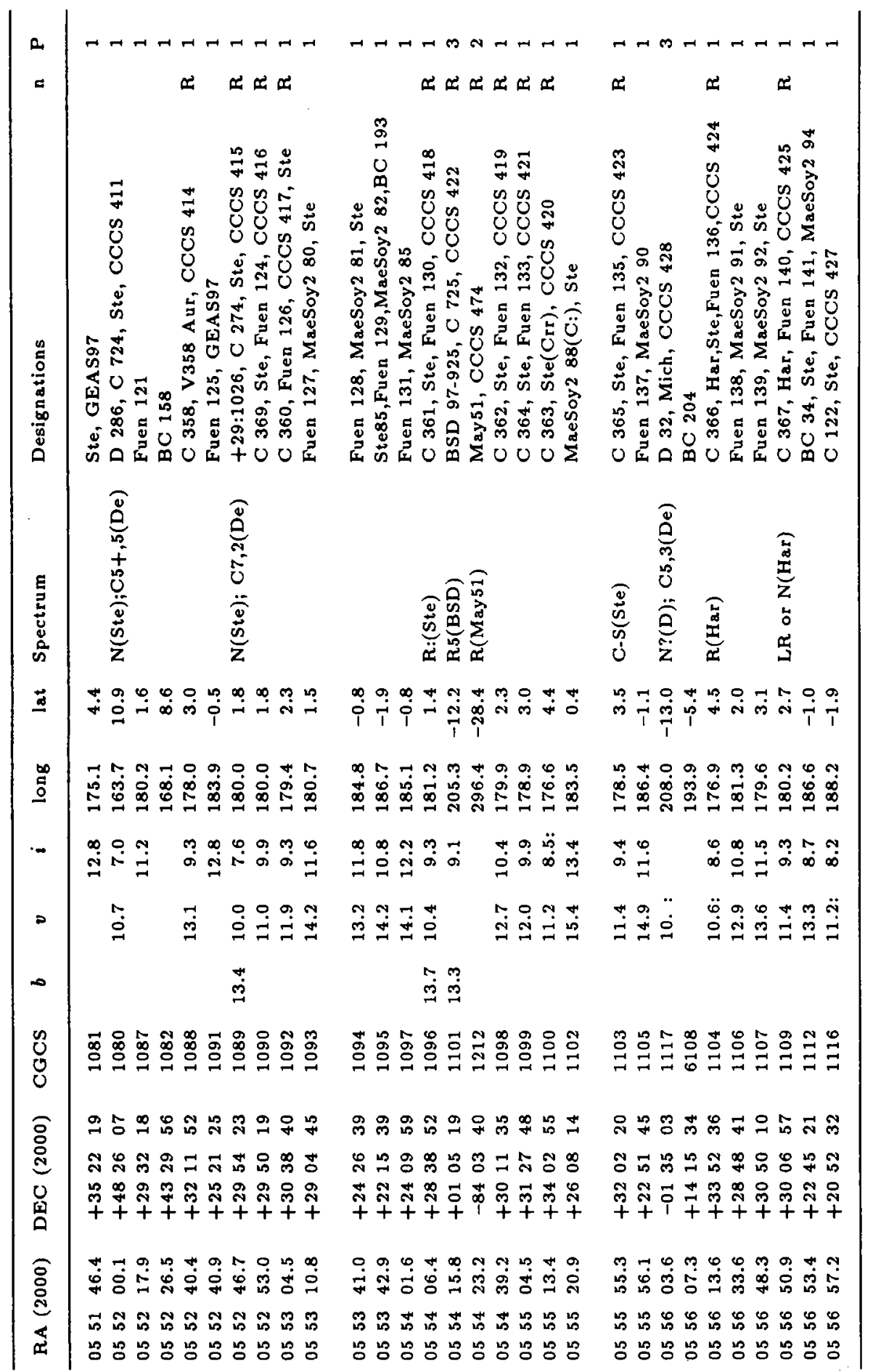




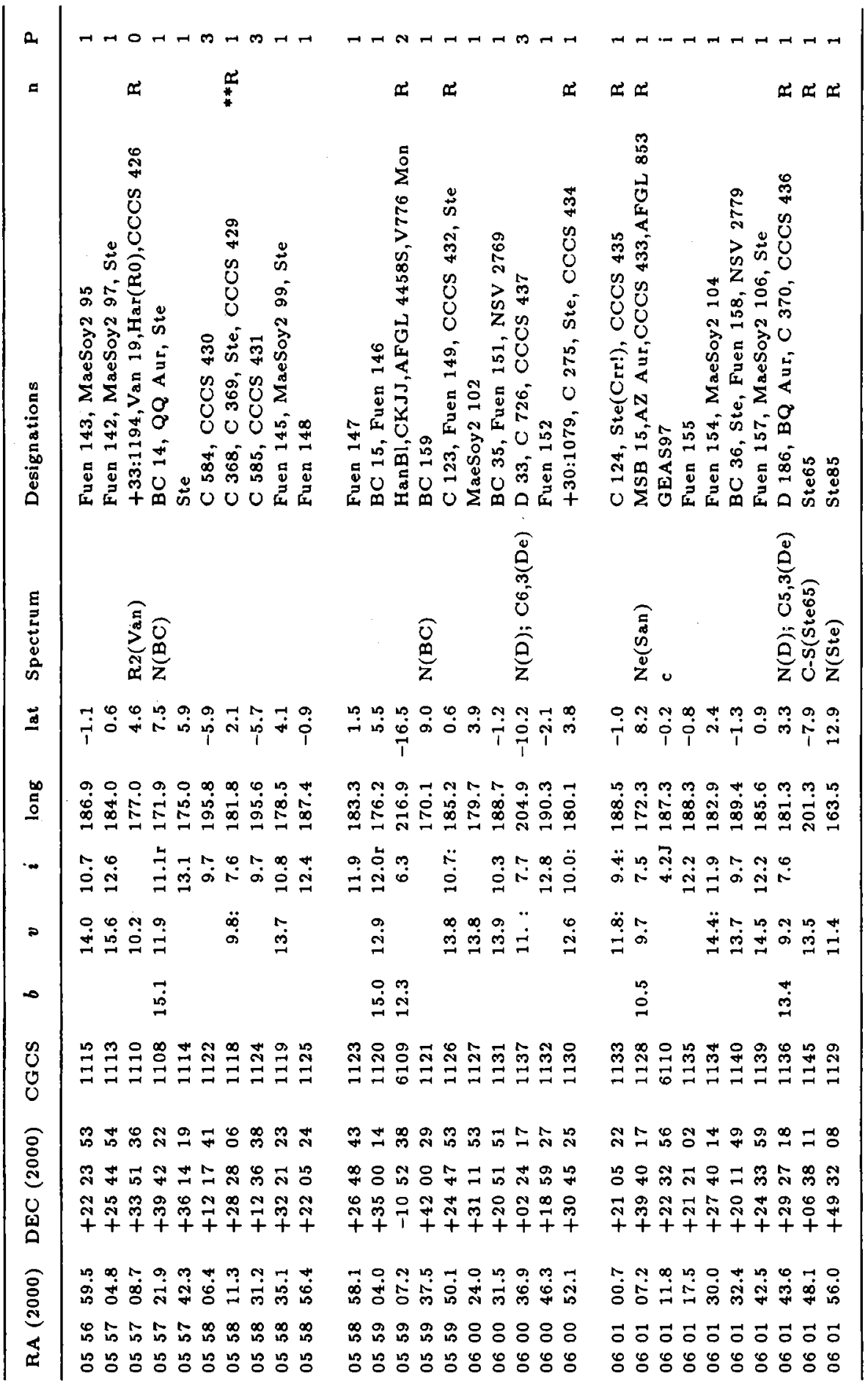




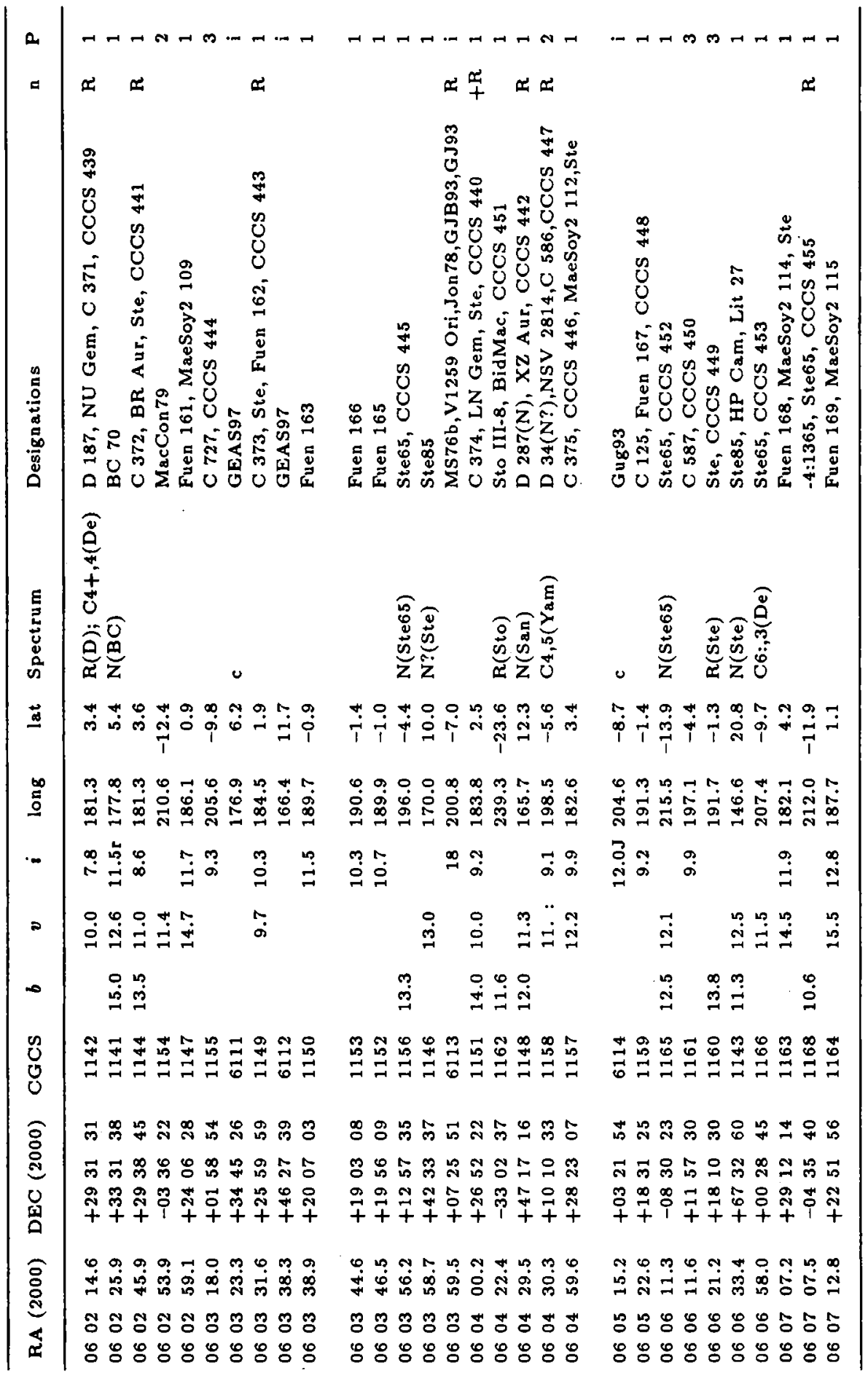




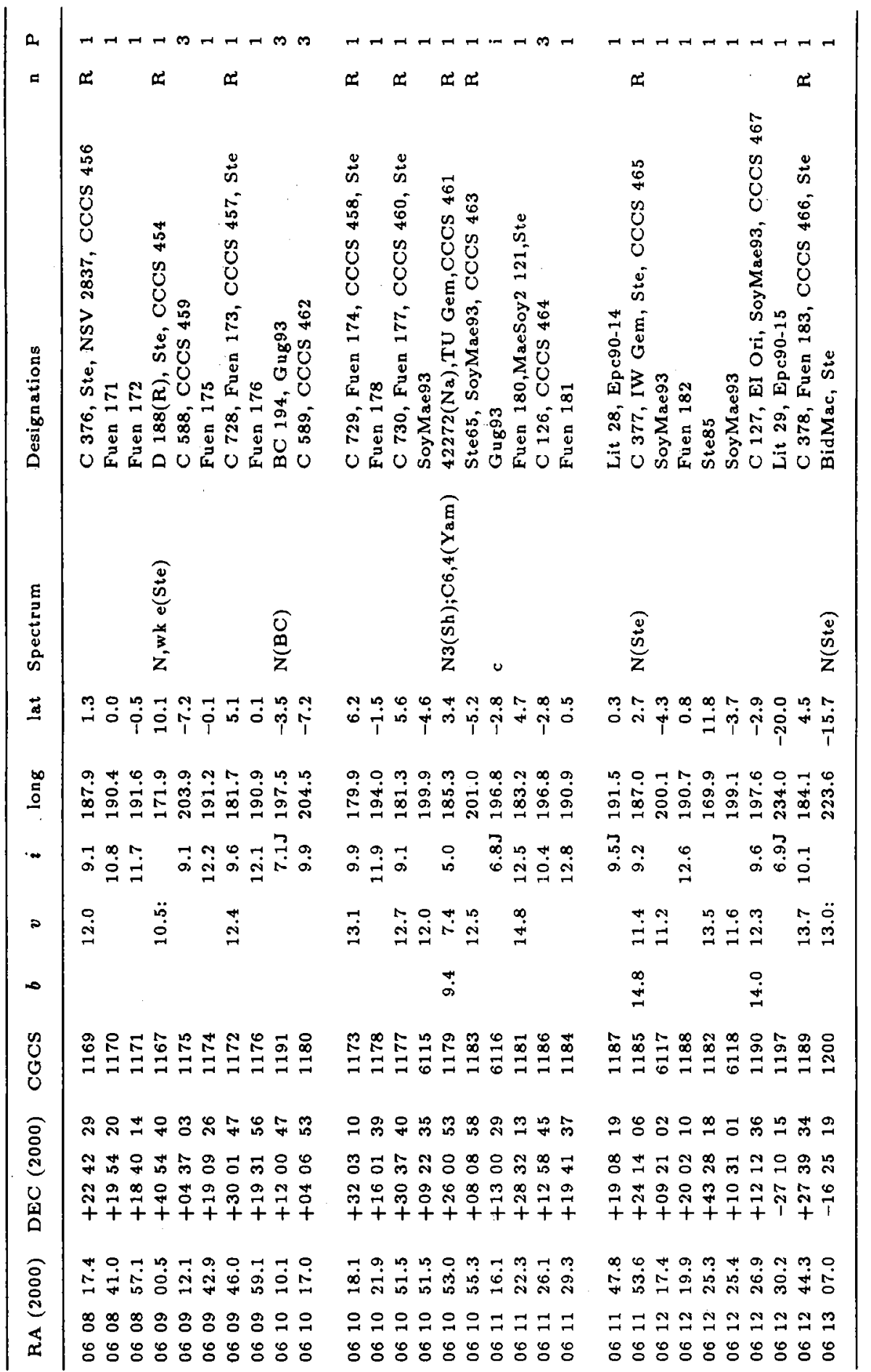




\begin{tabular}{|c|c|c|c|}
\hline a & 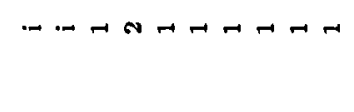 & 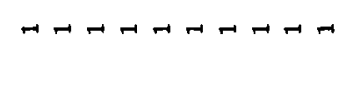 & 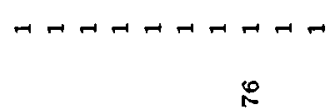 \\
\hline هُ & 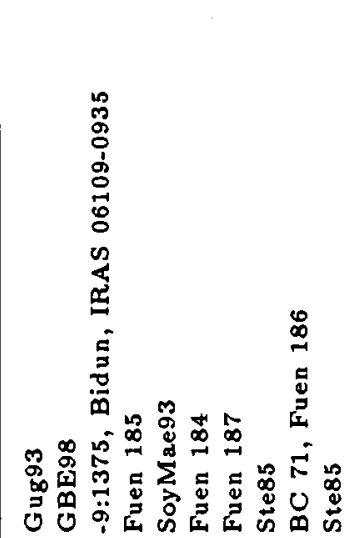 & 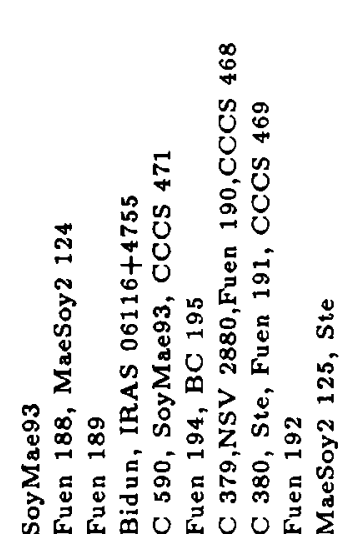 & 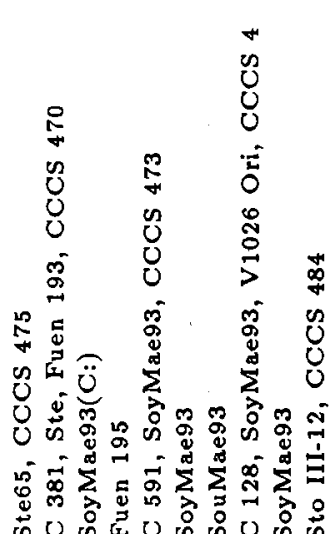 \\
\hline & 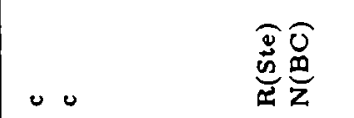 & $\begin{array}{l}\hat{0} \\
\stackrel{m}{z}\end{array}$ & 高 \\
\hline$\underline{\mathbf{d}}$ & 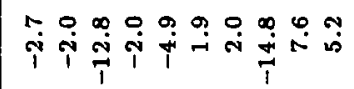 & $\vec{T}$ & 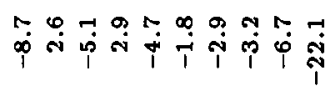 \\
\hline 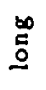 & 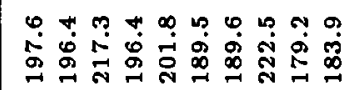 & 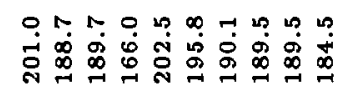 & 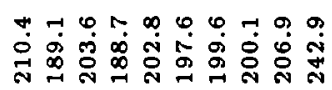 \\
\hline • & 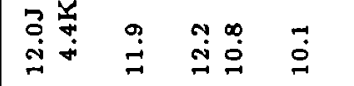 & 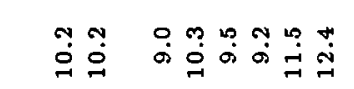 & $\stackrel{\infty}{\infty} \stackrel{\substack{0 \\
\infty}}{=} \underset{0}{\infty}$ \\
\hline a & 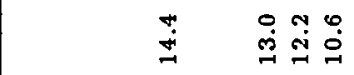 & 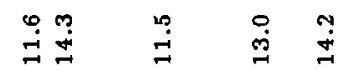 & 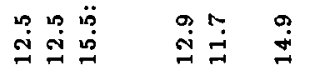 \\
\hline$\infty$ & $\stackrel{\ddot{g}}{\leftrightarrow}$ & & $\stackrel{\infty}{\stackrel{\infty}{\oplus}}$ \\
\hline O্ & 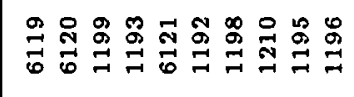 & 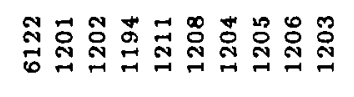 & 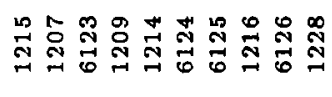 \\
\hline ๕ & 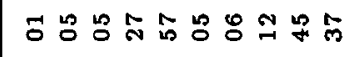 & 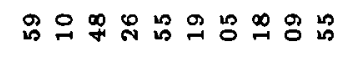 & 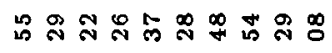 \\
\hline 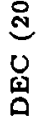 & 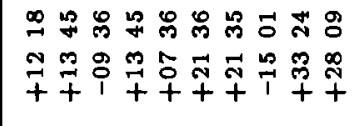 & 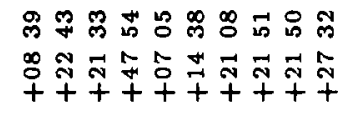 & 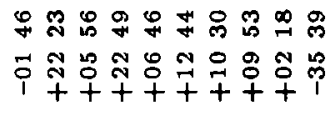 \\
\hline ڤे & 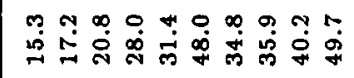 & 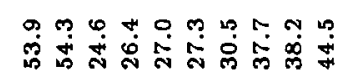 & 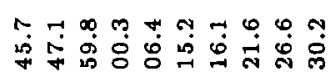 \\
\hline 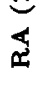 & 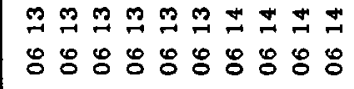 & 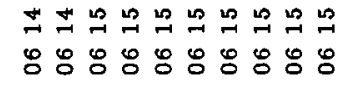 & 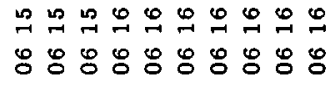 \\
\hline
\end{tabular}




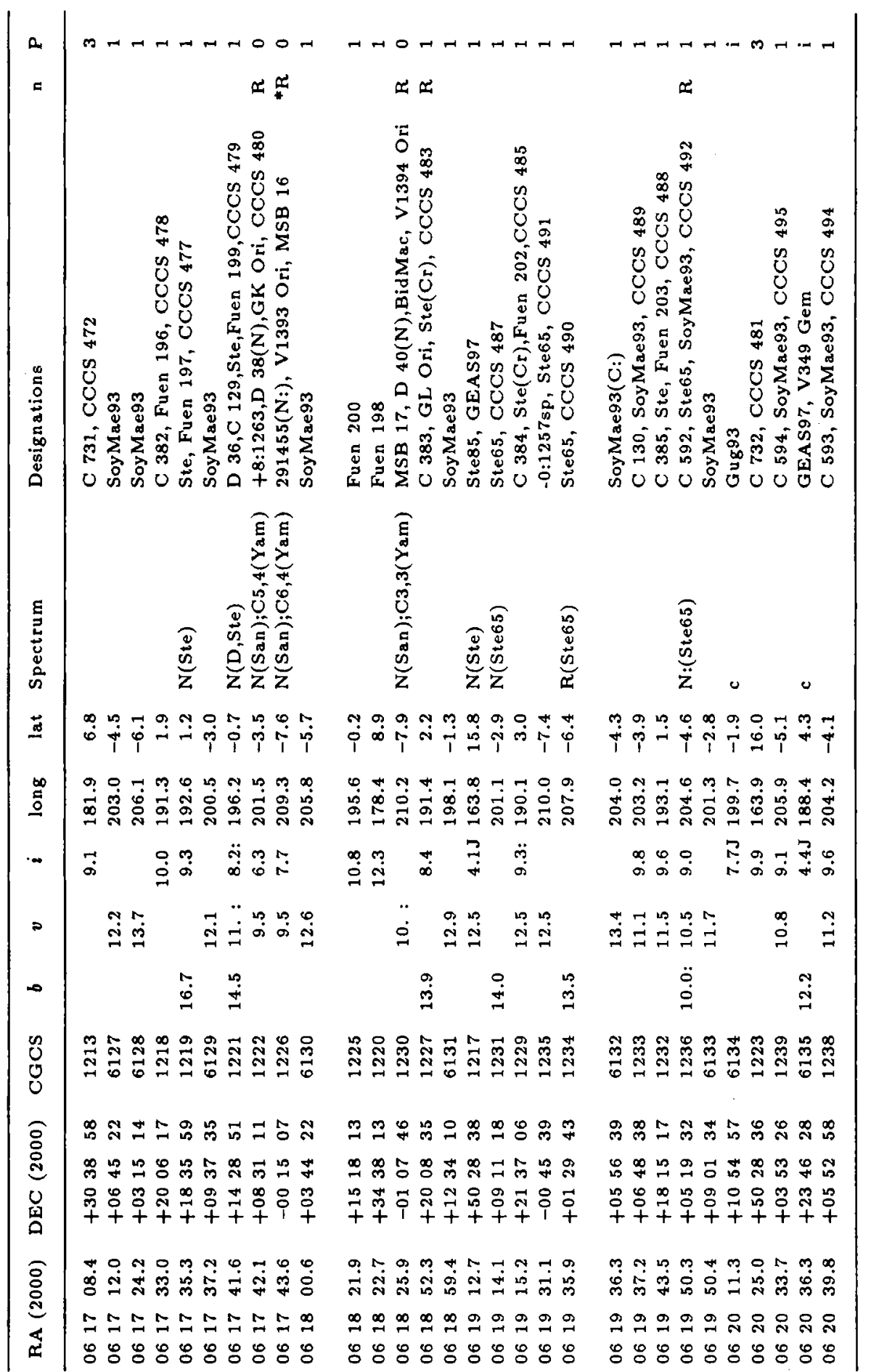




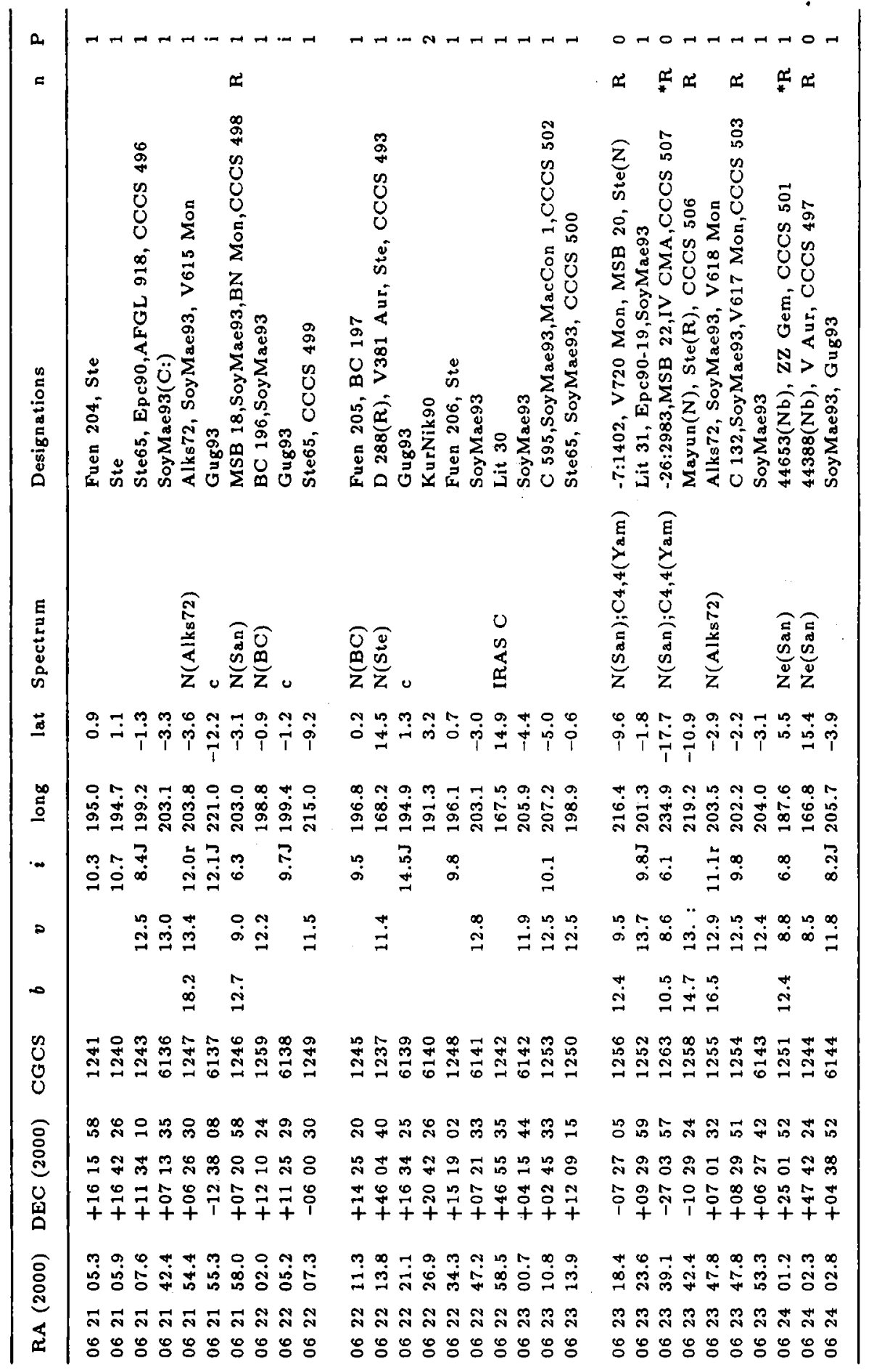




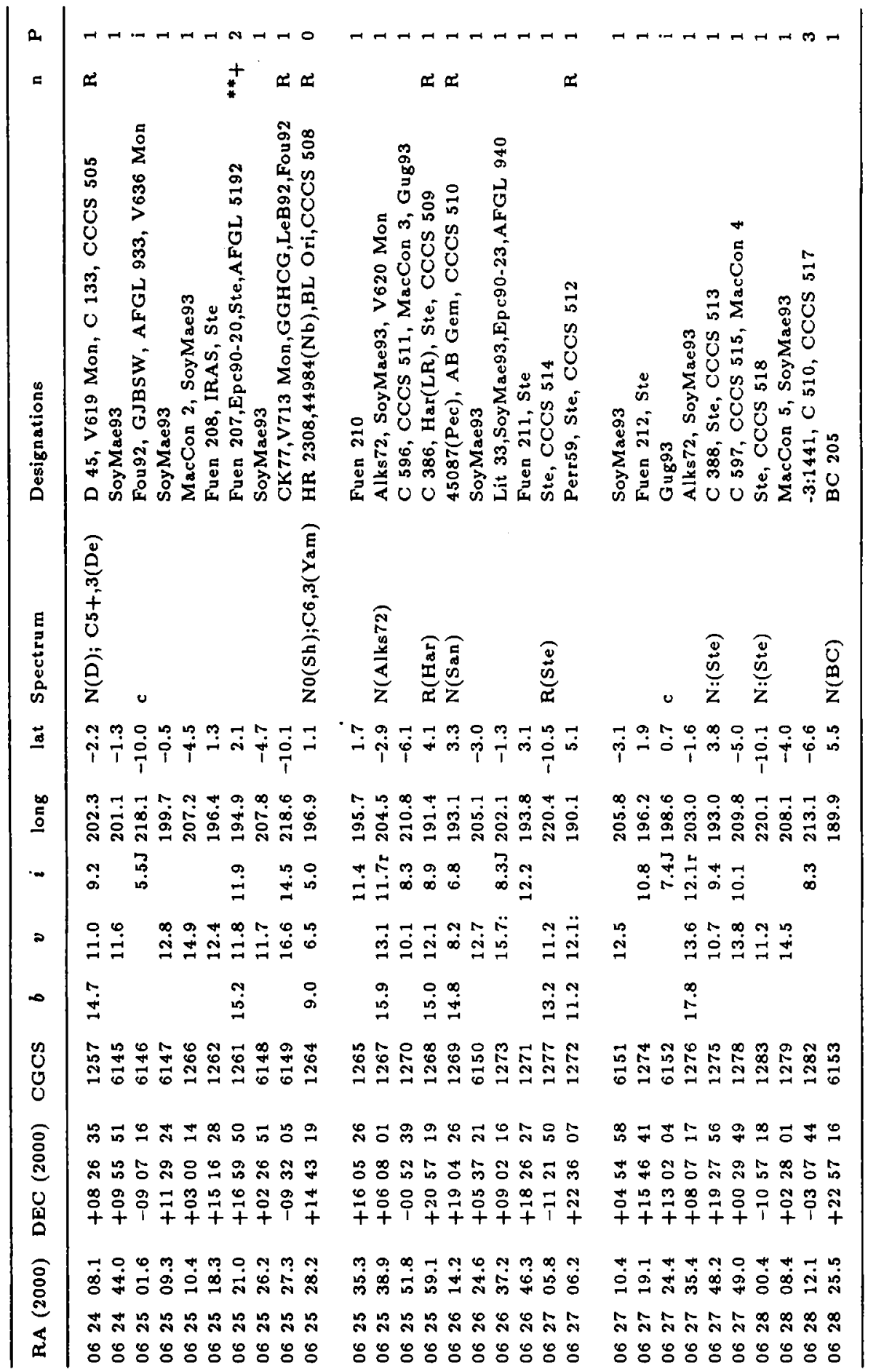




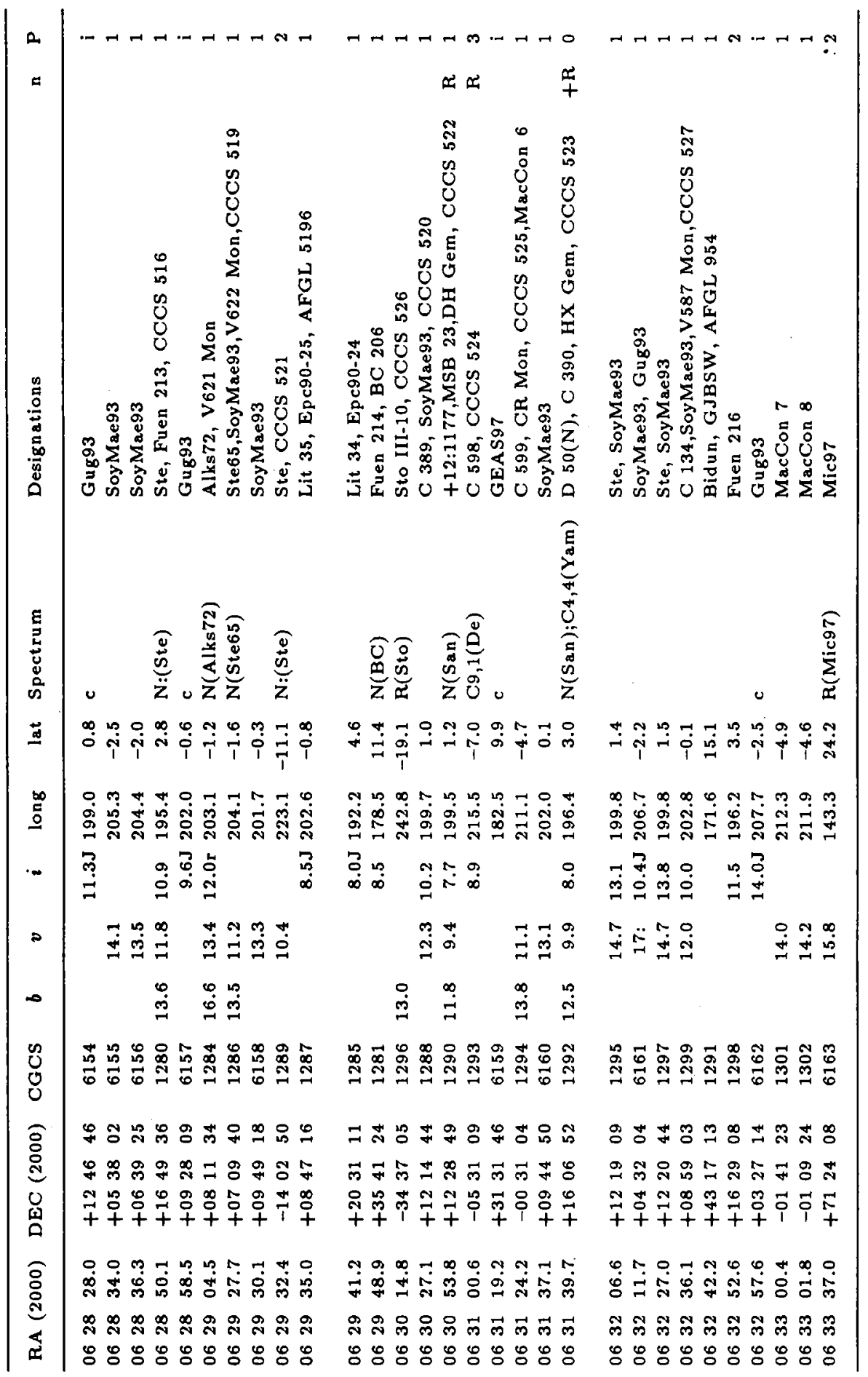




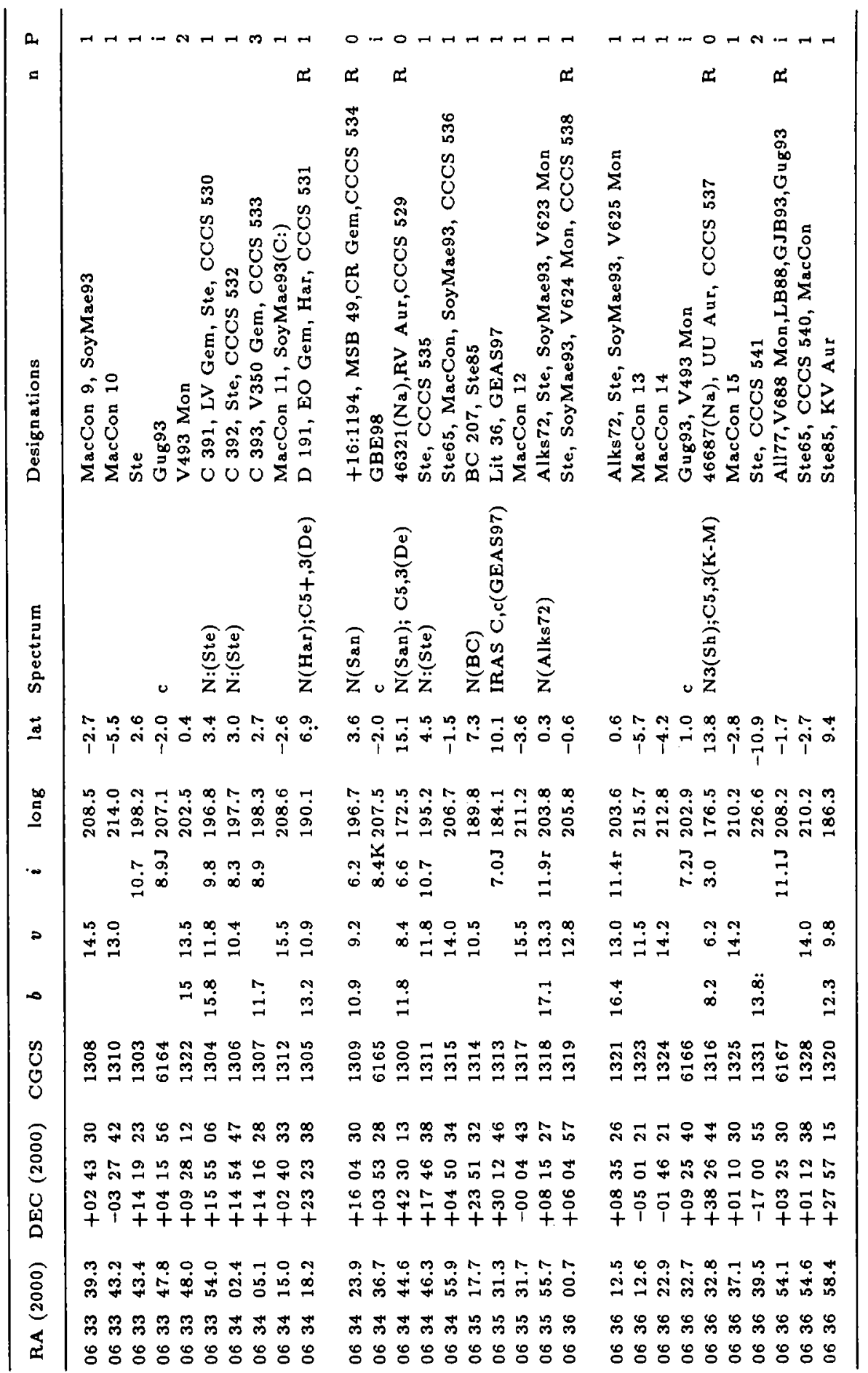




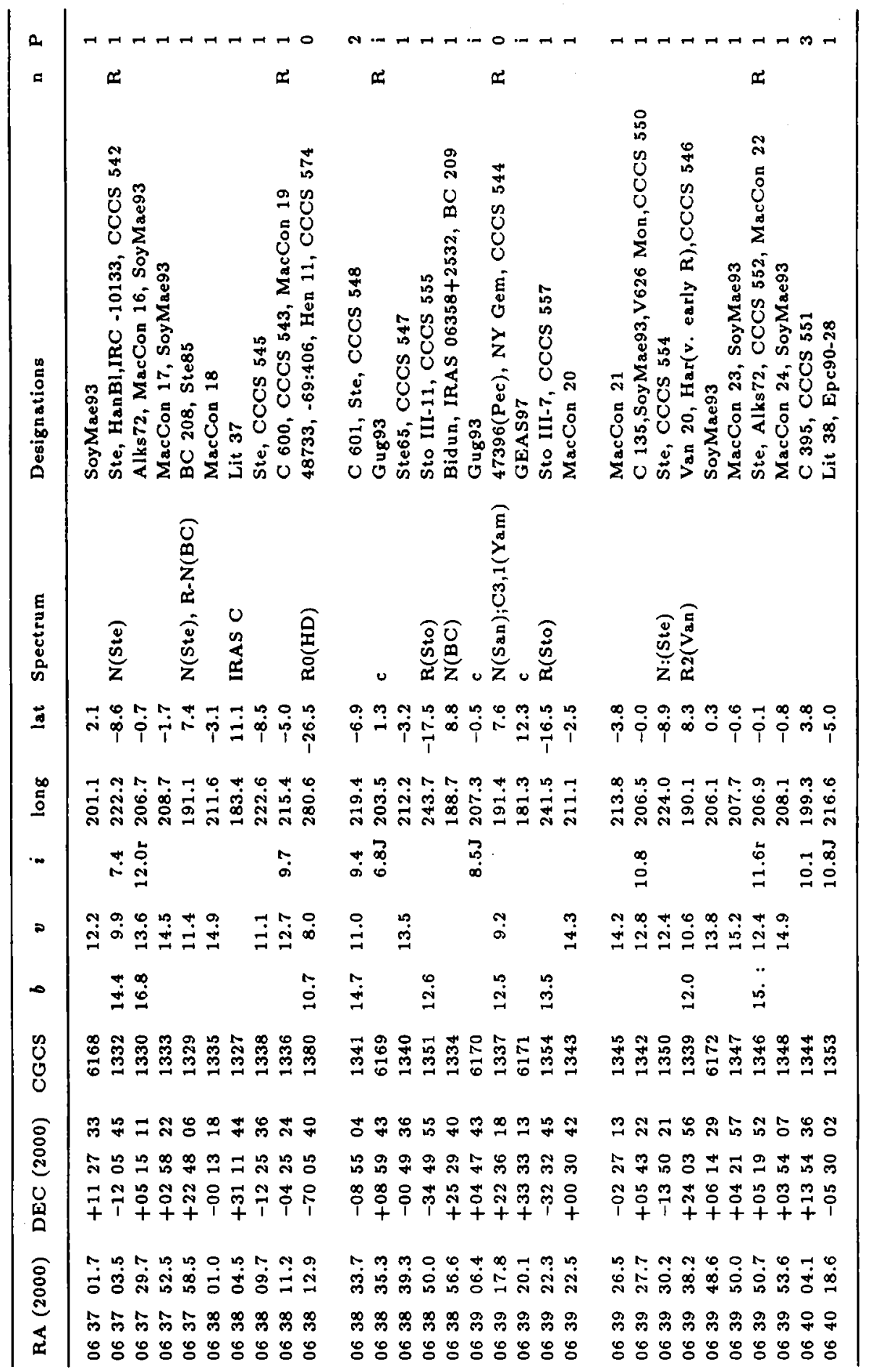




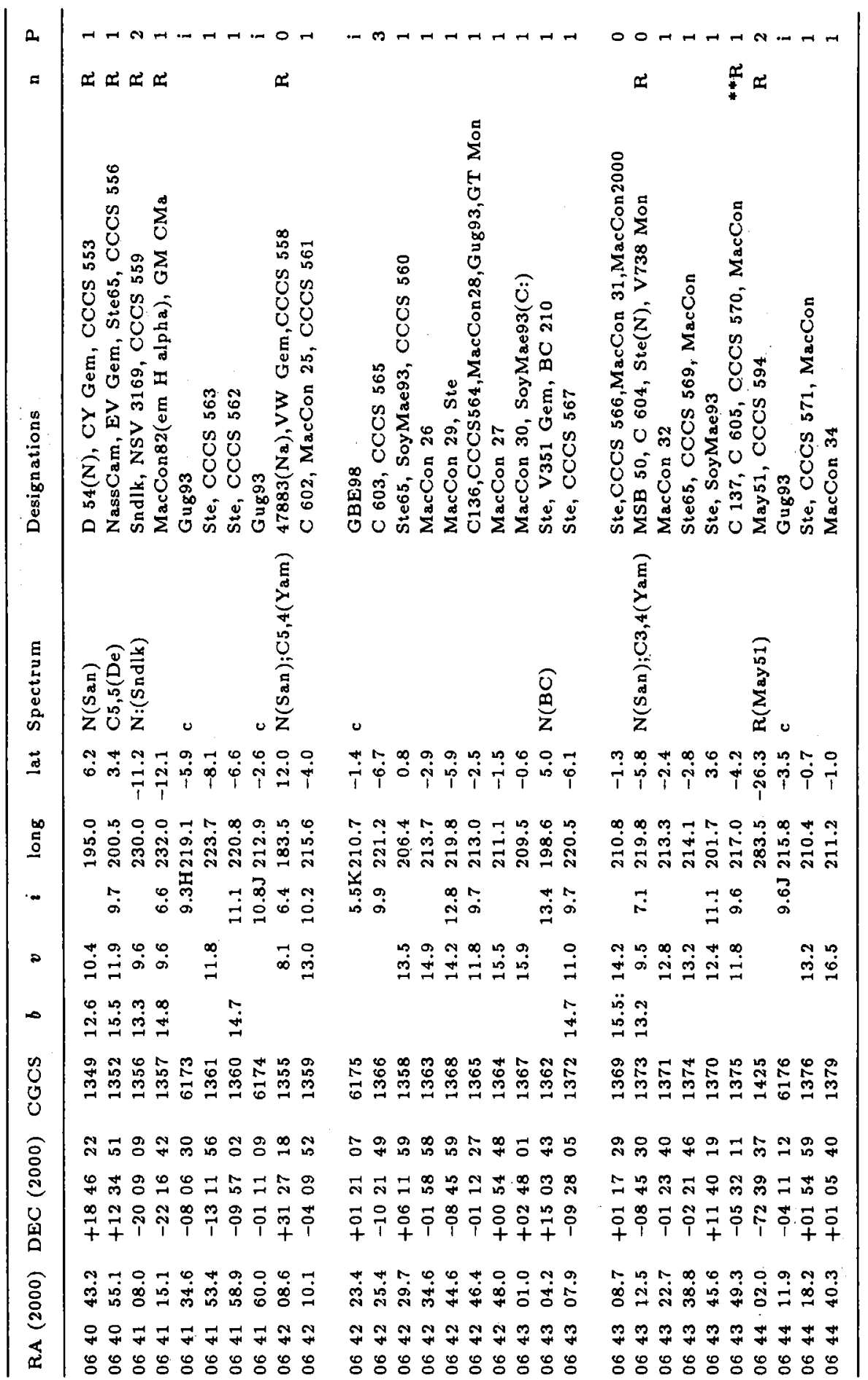




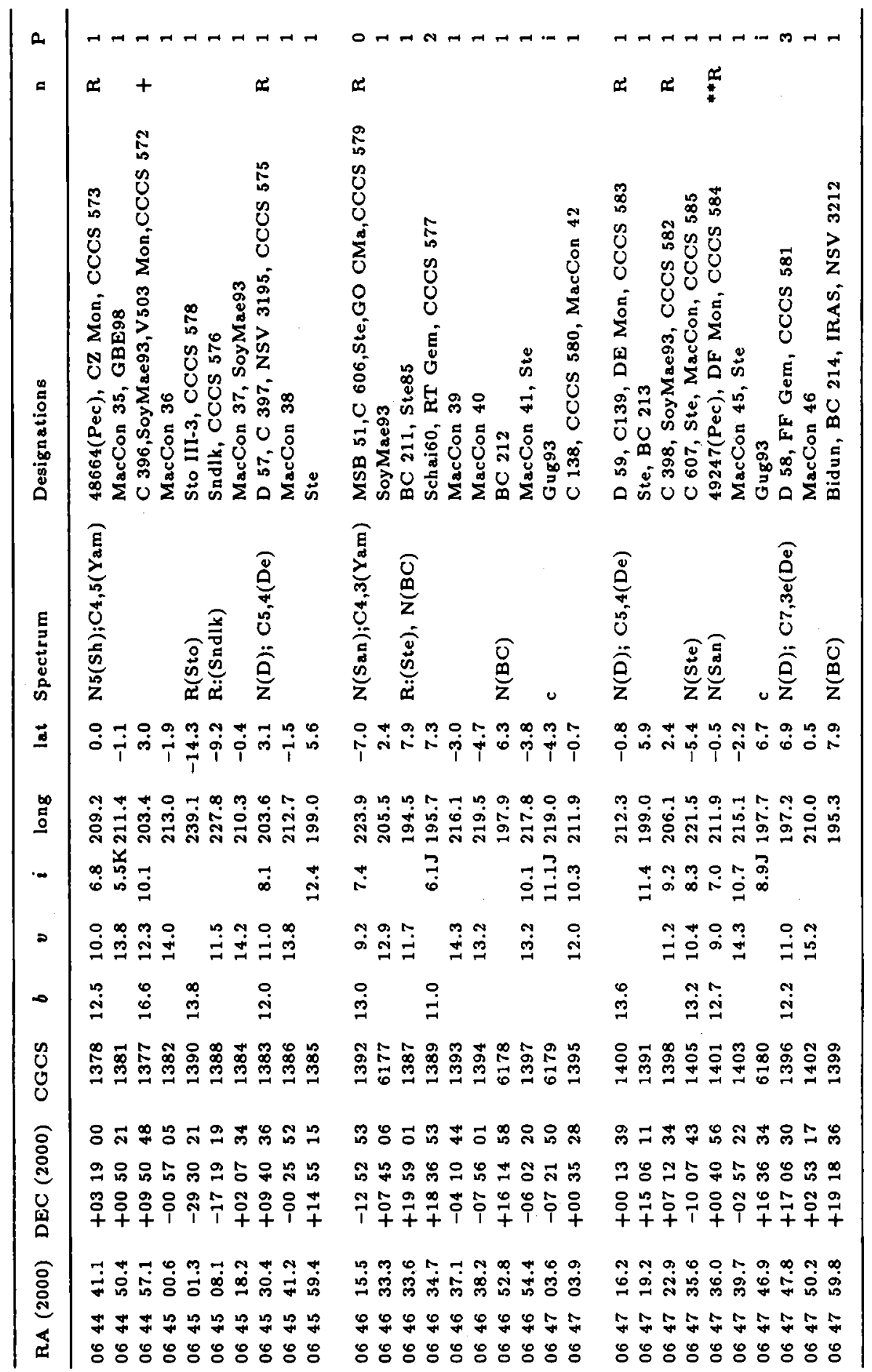




\begin{tabular}{|c|c|c|c|}
\hline D & 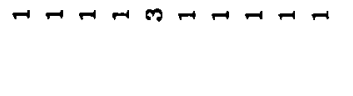 & 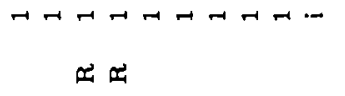 & 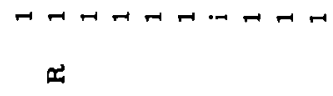 \\
\hline 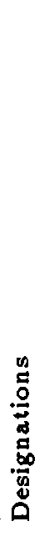 & 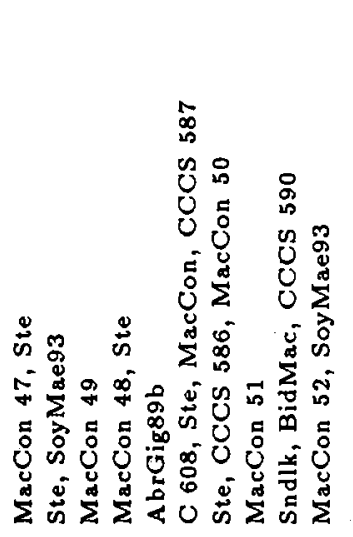 & 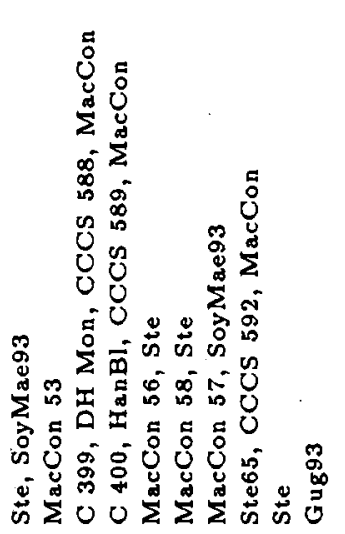 & 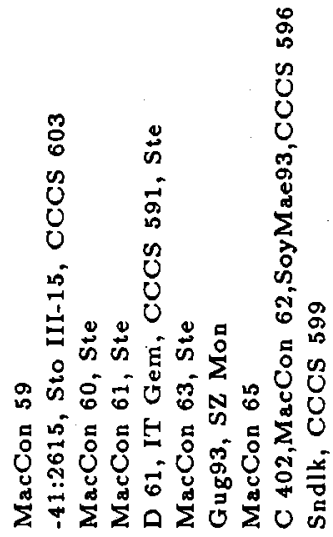 \\
\hline 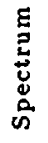 & 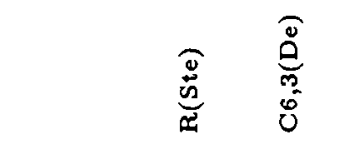 & 0 & 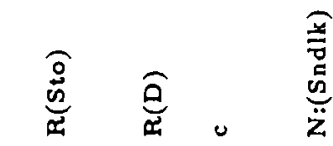 \\
\hline $\overrightarrow{\mathrm{z}}$ & 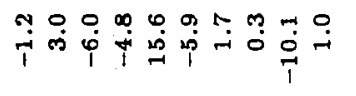 & 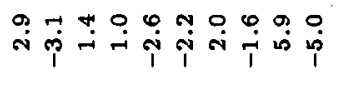 & 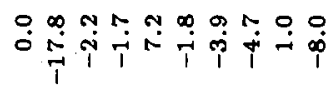 \\
\hline$\stackrel{\infty}{0}$ & 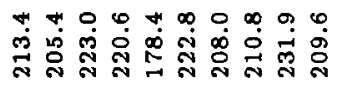 & 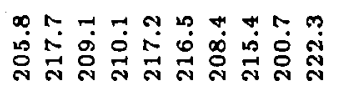 & 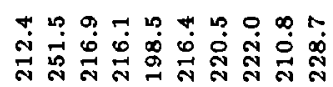 \\
\hline$\omega$ & 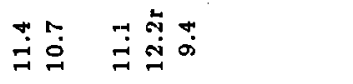 & 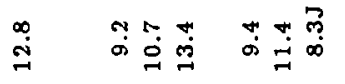 & 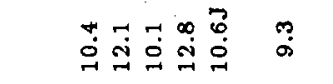 \\
\hline D & 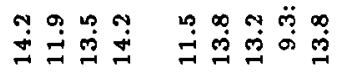 & 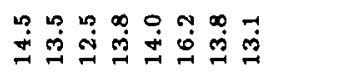 & 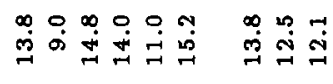 \\
\hline$\infty$ & $\stackrel{\ddot{m}}{\ddot{n}}$ & $\overrightarrow{\dot{n}}$ & $\stackrel{\sim}{\underset{H}{*}} \quad \stackrel{n}{\leftrightarrows}$ \\
\hline 仓ু & 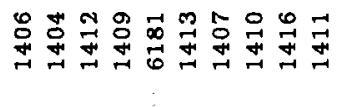 & 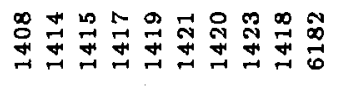 & 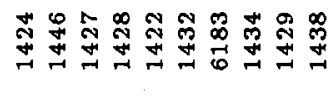 \\
\hline \&ิ & 呚祍 & 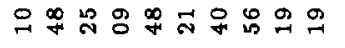 & 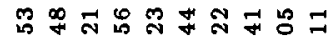 \\
\hline 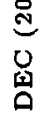 & 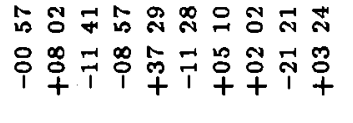 & 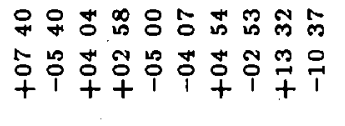 & 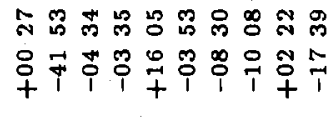 \\
\hline 용 & 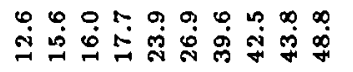 & تُن & 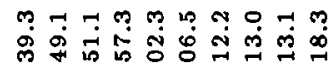 \\
\hline 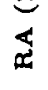 & 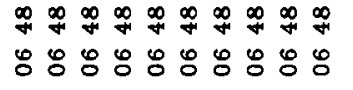 & 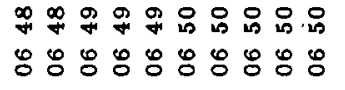 & 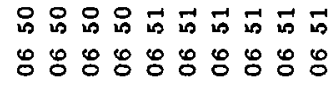 \\
\hline
\end{tabular}




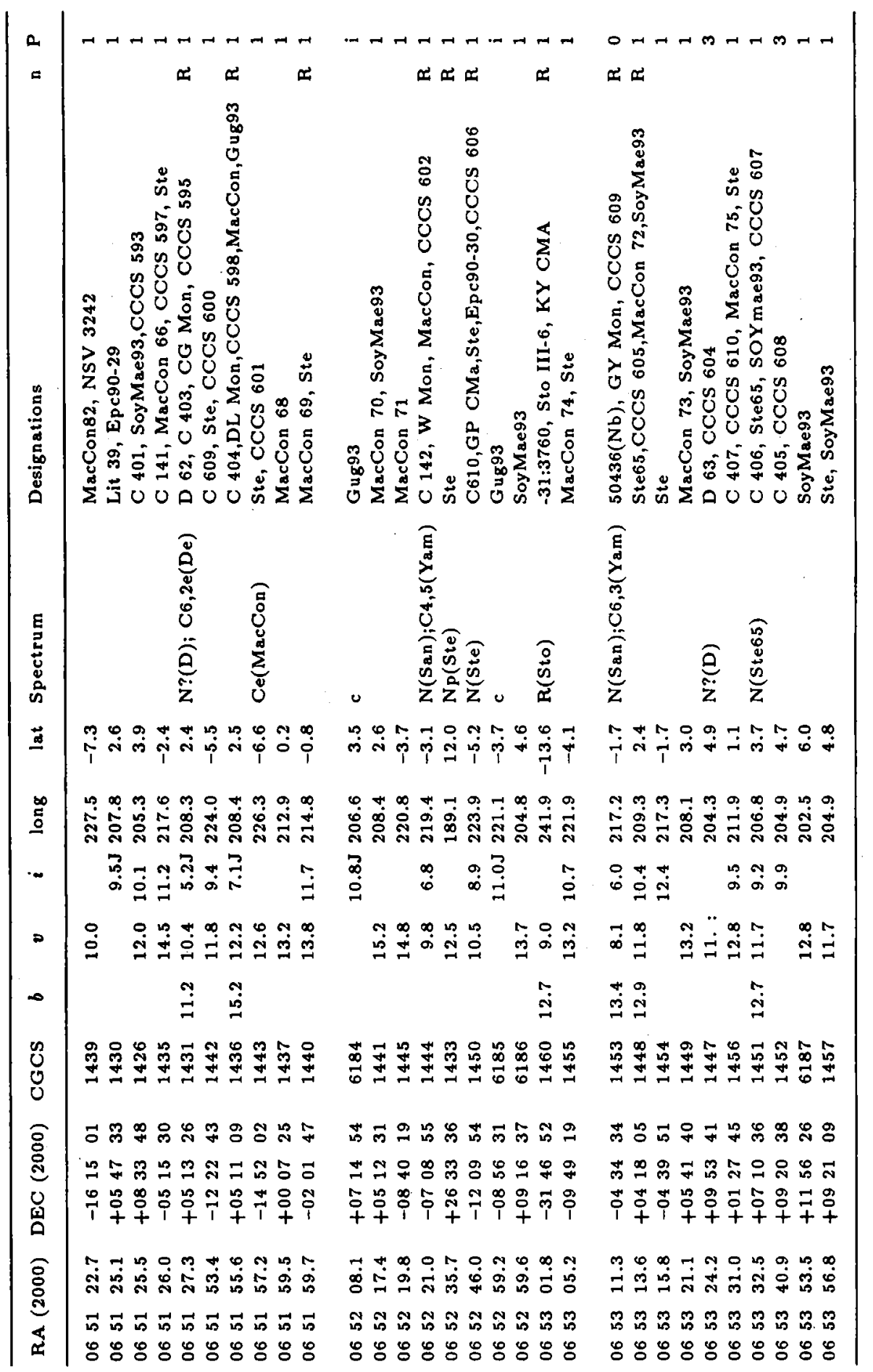




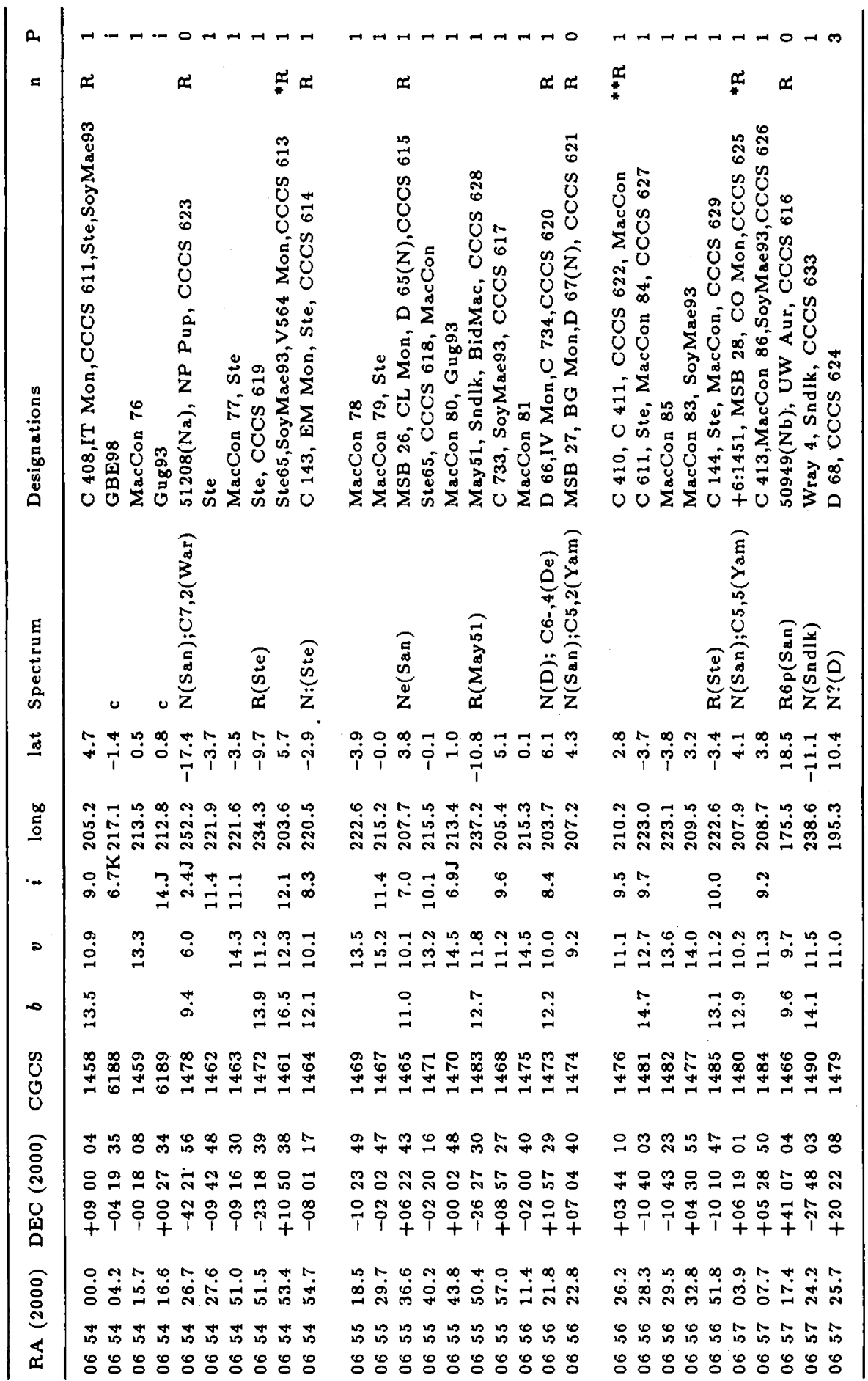




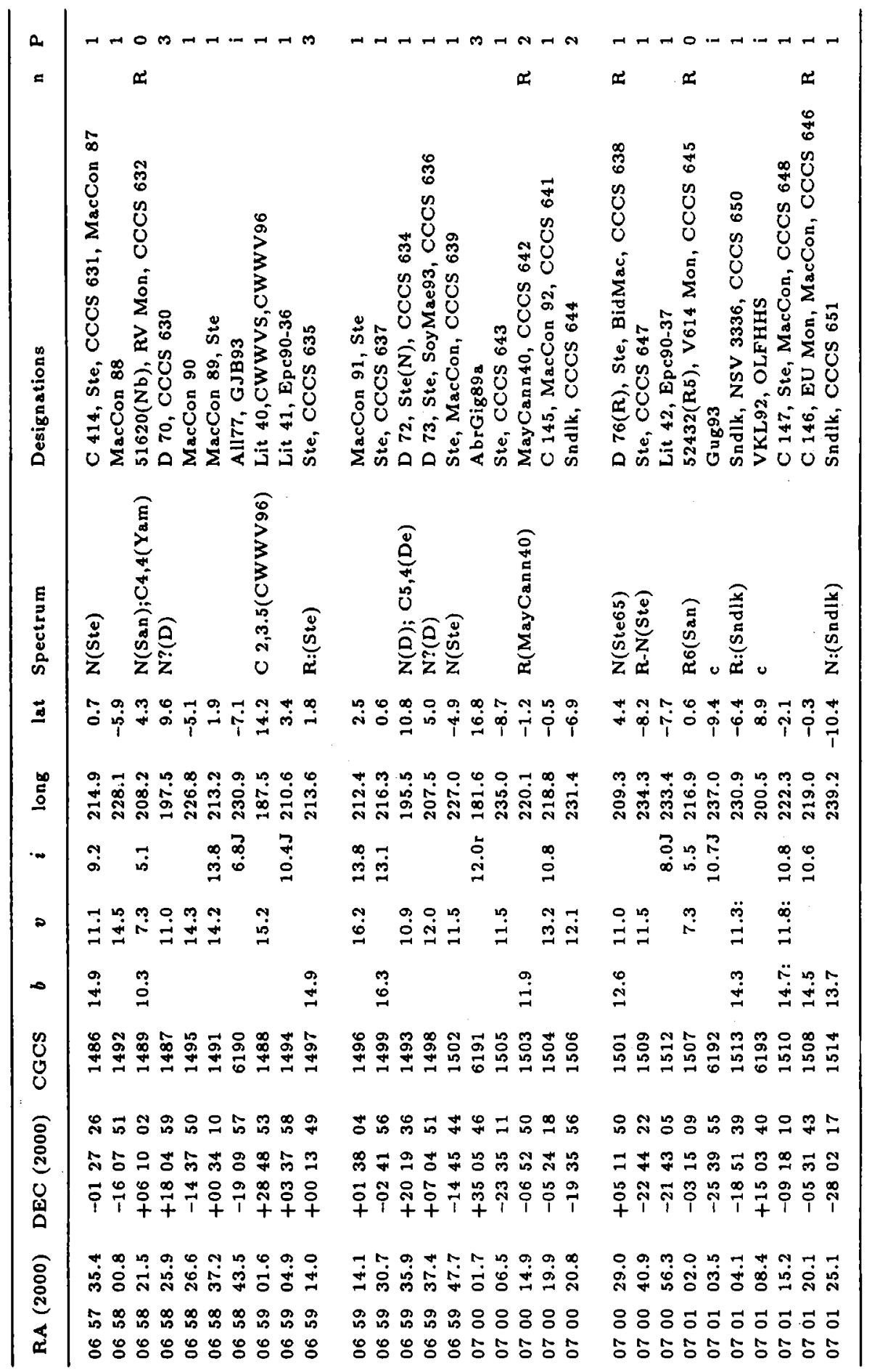




\begin{tabular}{|c|c|c|c|}
\hline a & 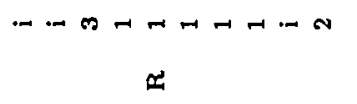 & 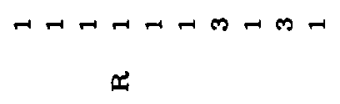 & 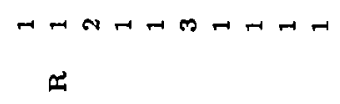 \\
\hline & 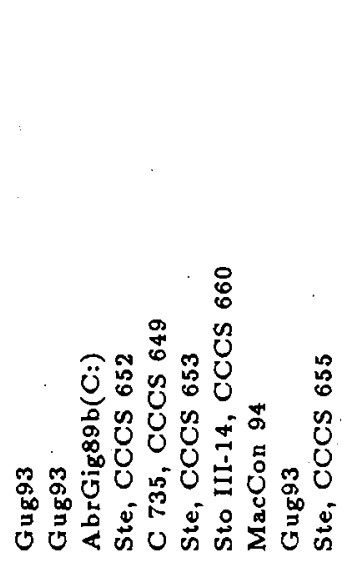 & 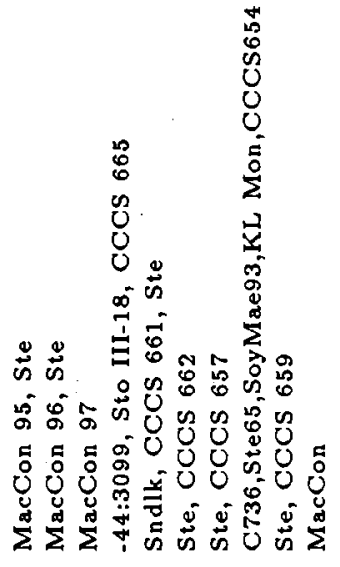 & 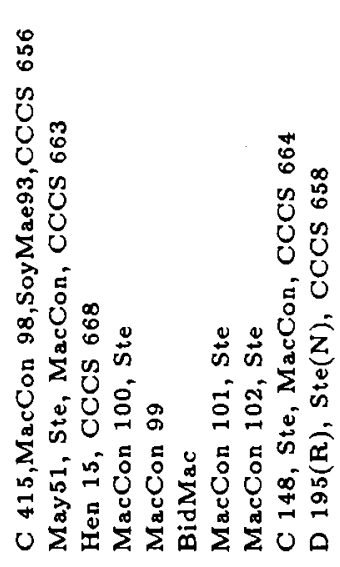 \\
\hline 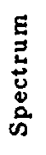 & 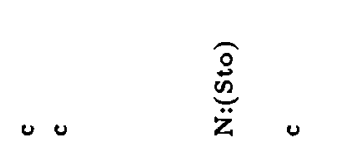 & 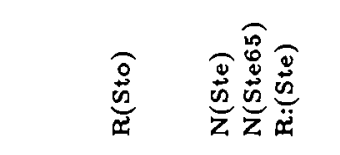 & 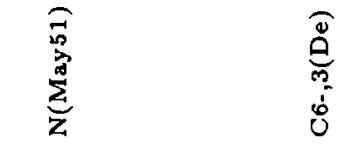 \\
\hline$\underline{\mathrm{d}}$ & 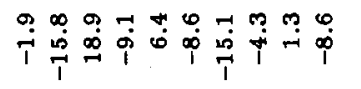 & 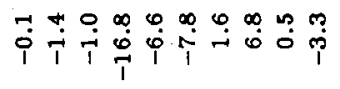 & 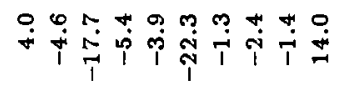 \\
\hline$\stackrel{\infty}{0}$ & 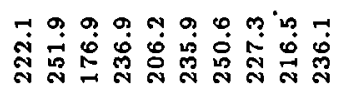 & 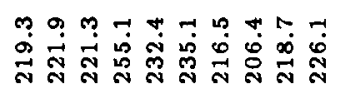 & 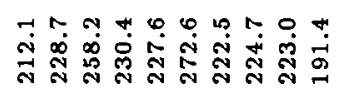 \\
\hline & 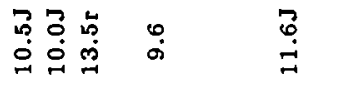 & 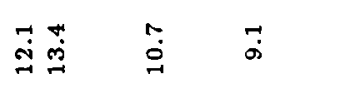 & 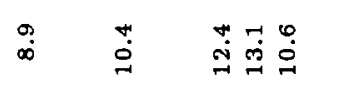 \\
\hline D & 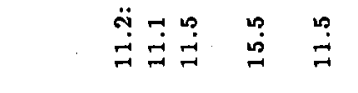 & 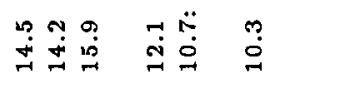 & 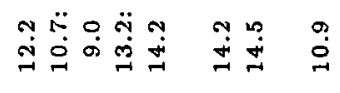 \\
\hline & $\stackrel{\infty}{\dot{m}}$ & 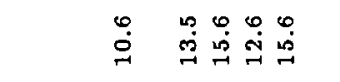 & 苛 \\
\hline$\bigcup_{0}^{8}$ & 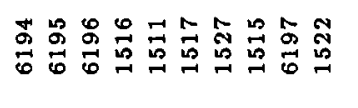 & 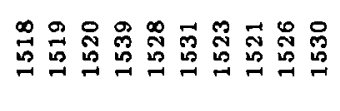 & 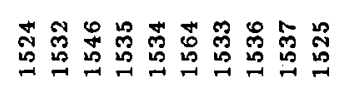 \\
\hline 2 & 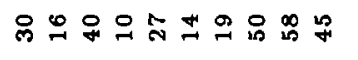 & 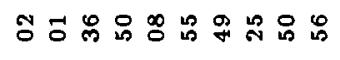 & స \\
\hline U & 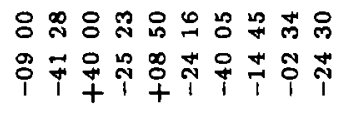 & 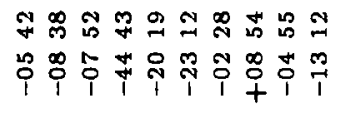 & 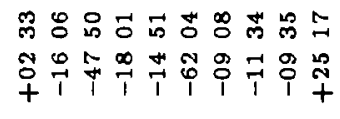 \\
\hline 용 & 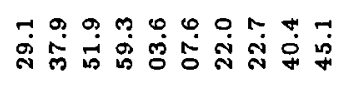 & 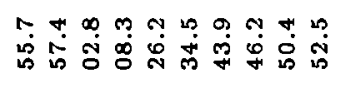 & 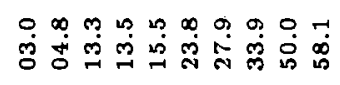 \\
\hline 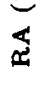 & 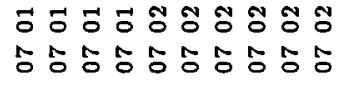 & 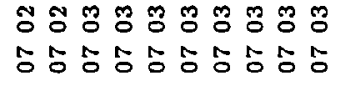 & 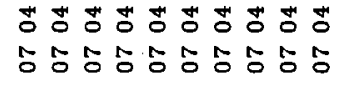 \\
\hline
\end{tabular}




\begin{tabular}{|c|c|c|c|}
\hline$=$ & 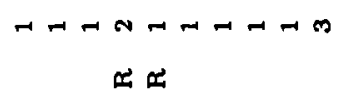 & 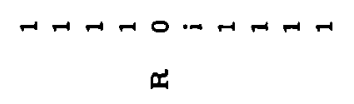 & 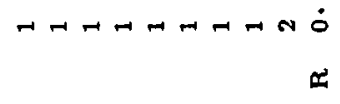 \\
\hline & 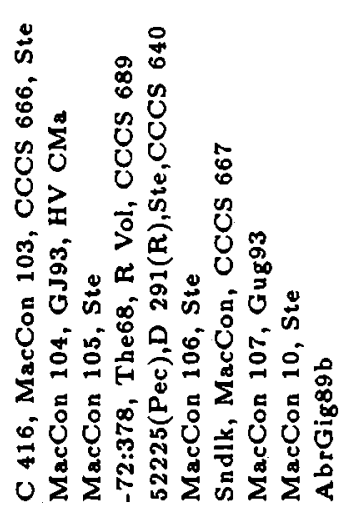 & 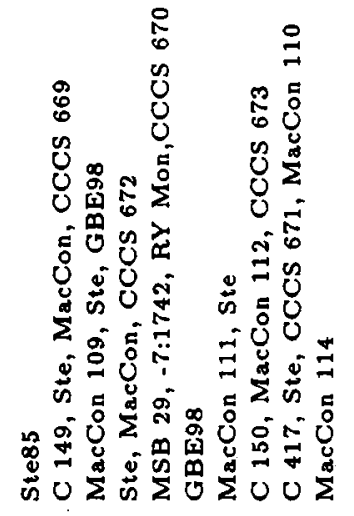 & 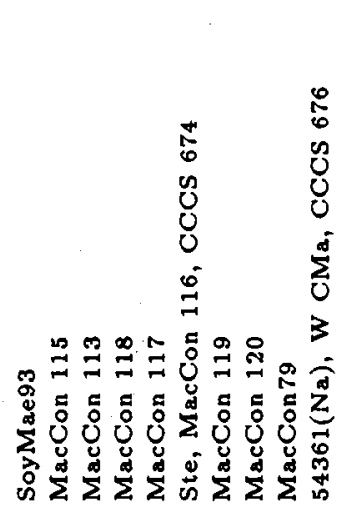 \\
\hline 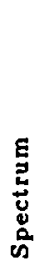 & 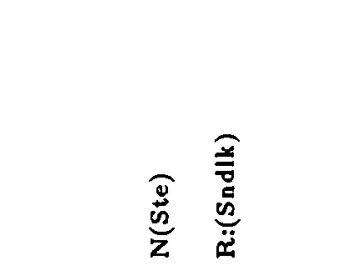 & 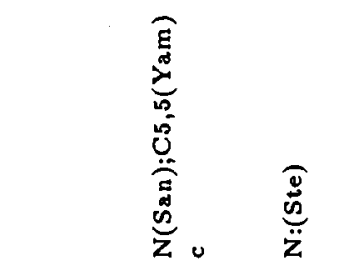 & 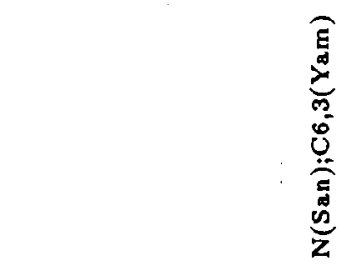 \\
\hline$\underline{\Xi}$ & 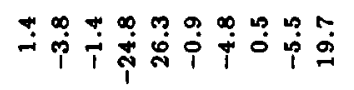 & 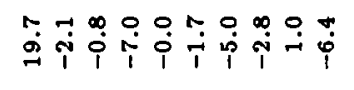 & 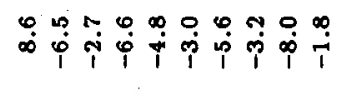 \\
\hline$\stackrel{\infty}{E}$ & 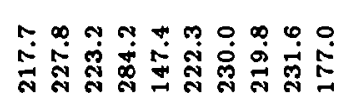 & 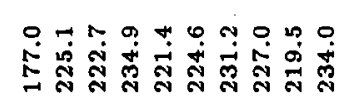 & 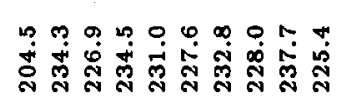 \\
\hline$\cdots$ & 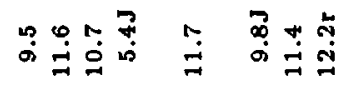 & 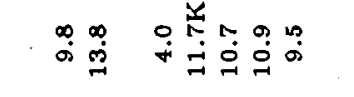 & $\stackrel{2}{+\infty}$ \\
\hline 2 & 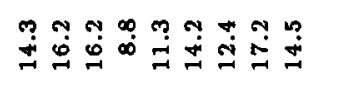 & 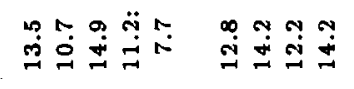 & 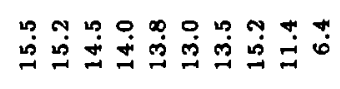 \\
\hline ـ & $\stackrel{\infty}{\stackrel{\infty}{-}}$ & $\stackrel{\circ}{\stackrel{i}{*}}$ & $\stackrel{\ddot{\leftrightarrow}}{\dot{H}}$ \\
\hline 羿 & 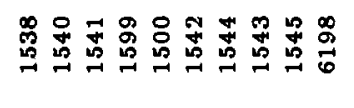 & 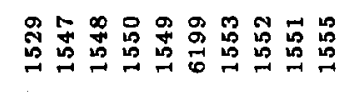 & 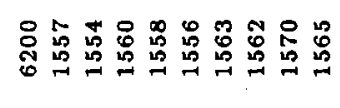 \\
\hline ๑ิ & 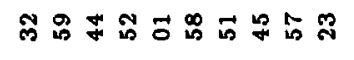 & 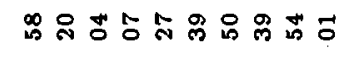 & 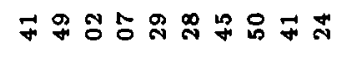 \\
\hline 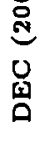 & 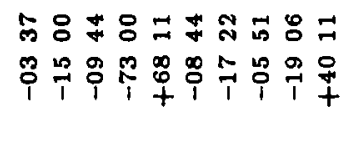 & 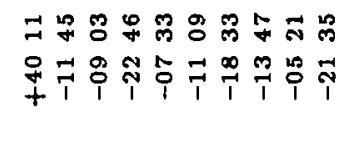 & 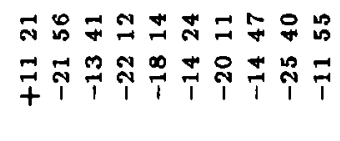 \\
\hline ठั. & 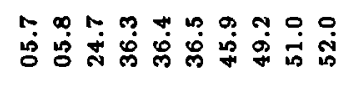 & 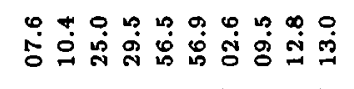 & 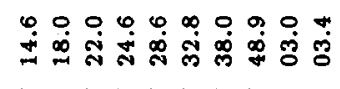 \\
\hline لَّ & 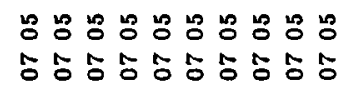 & 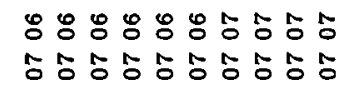 & 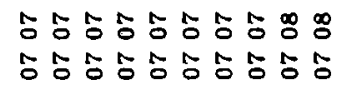 \\
\hline
\end{tabular}




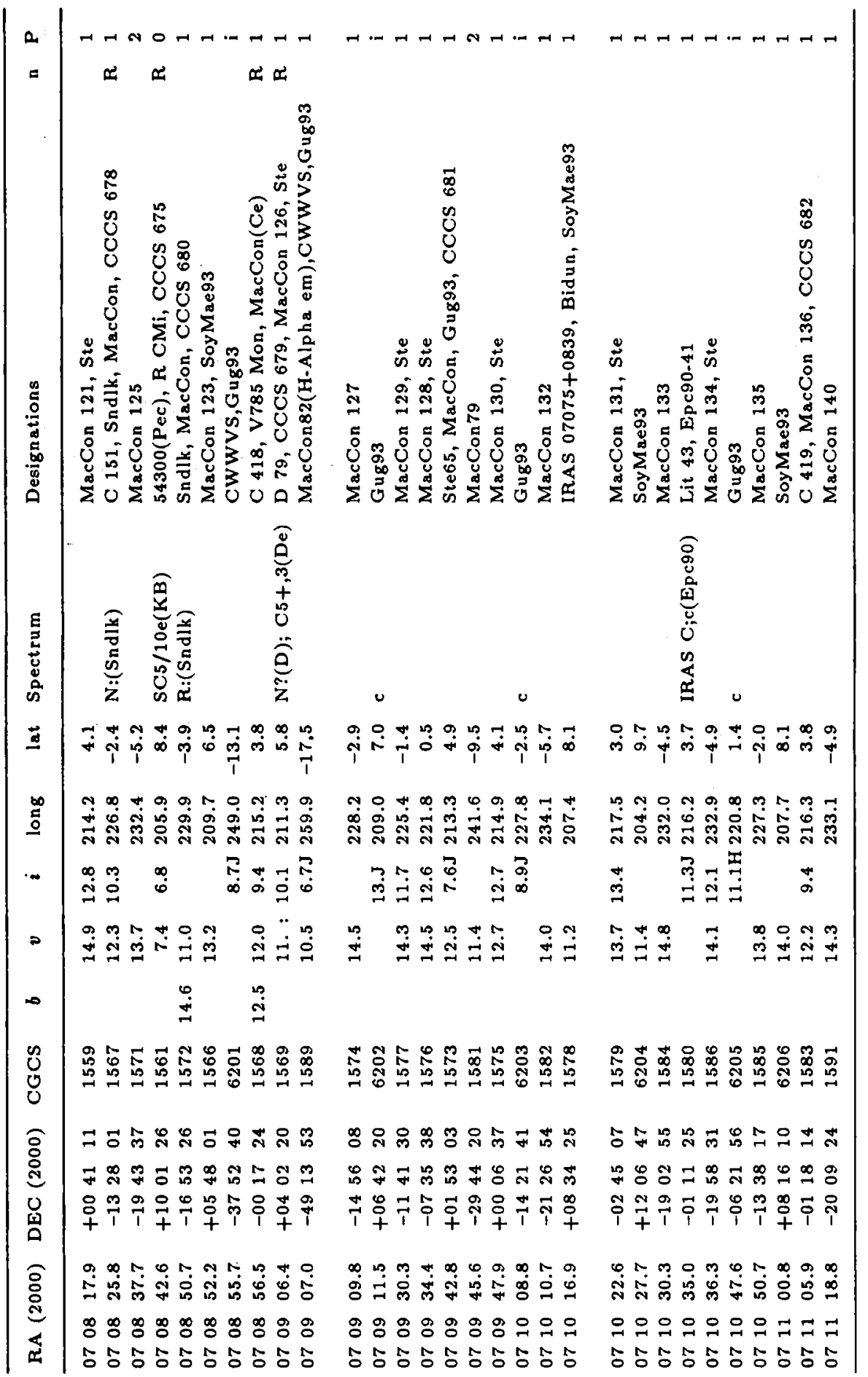




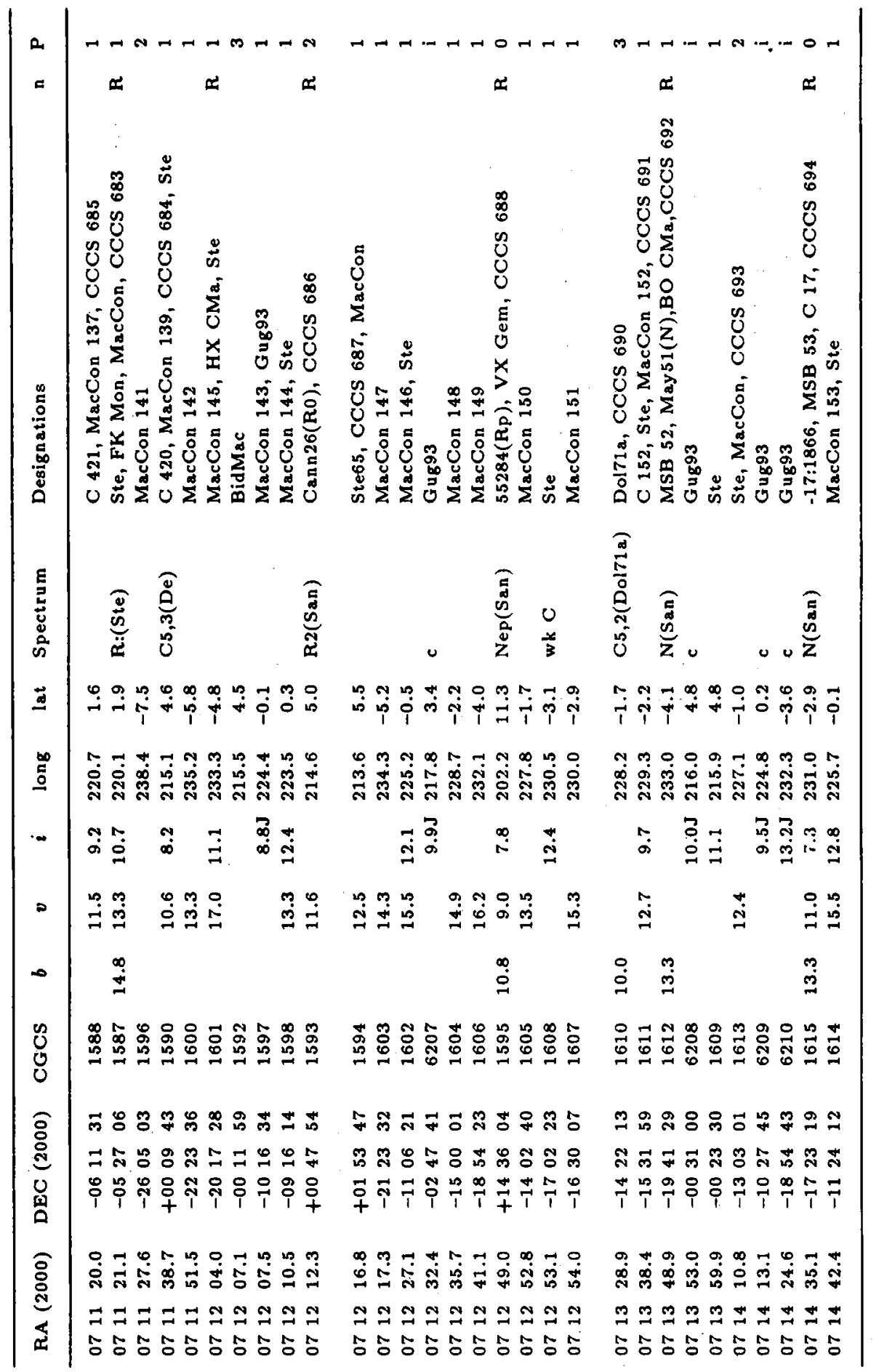




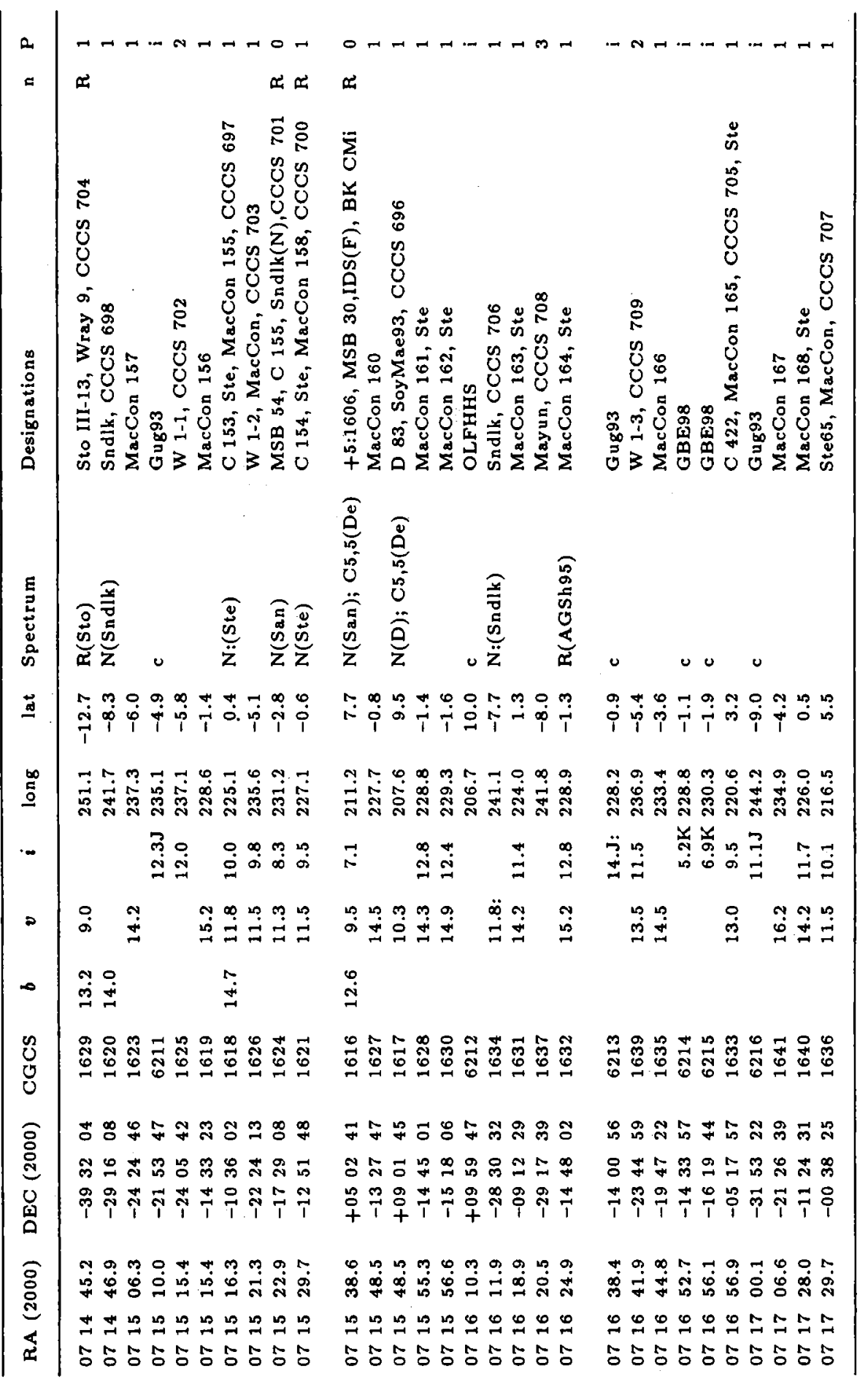




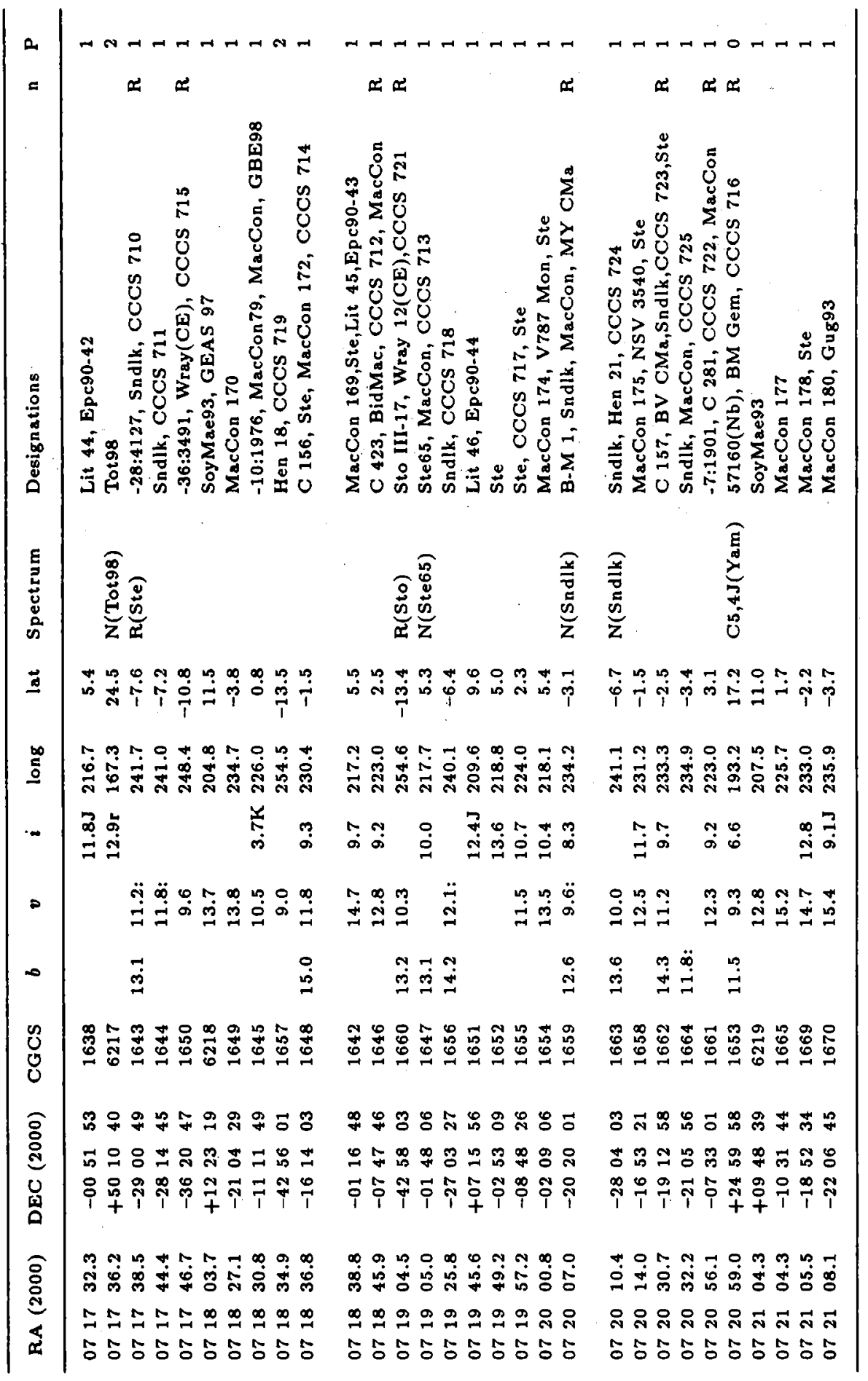




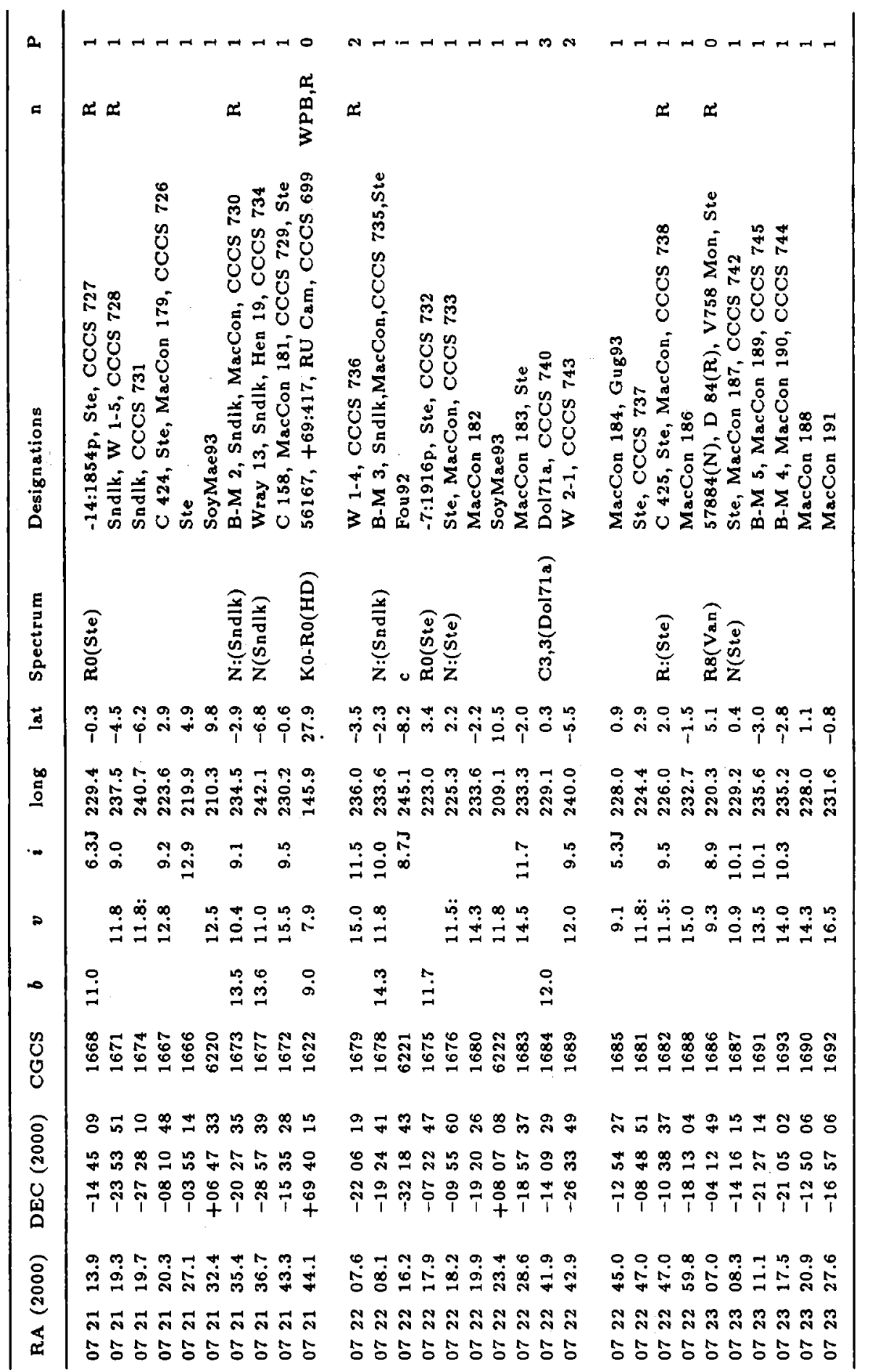




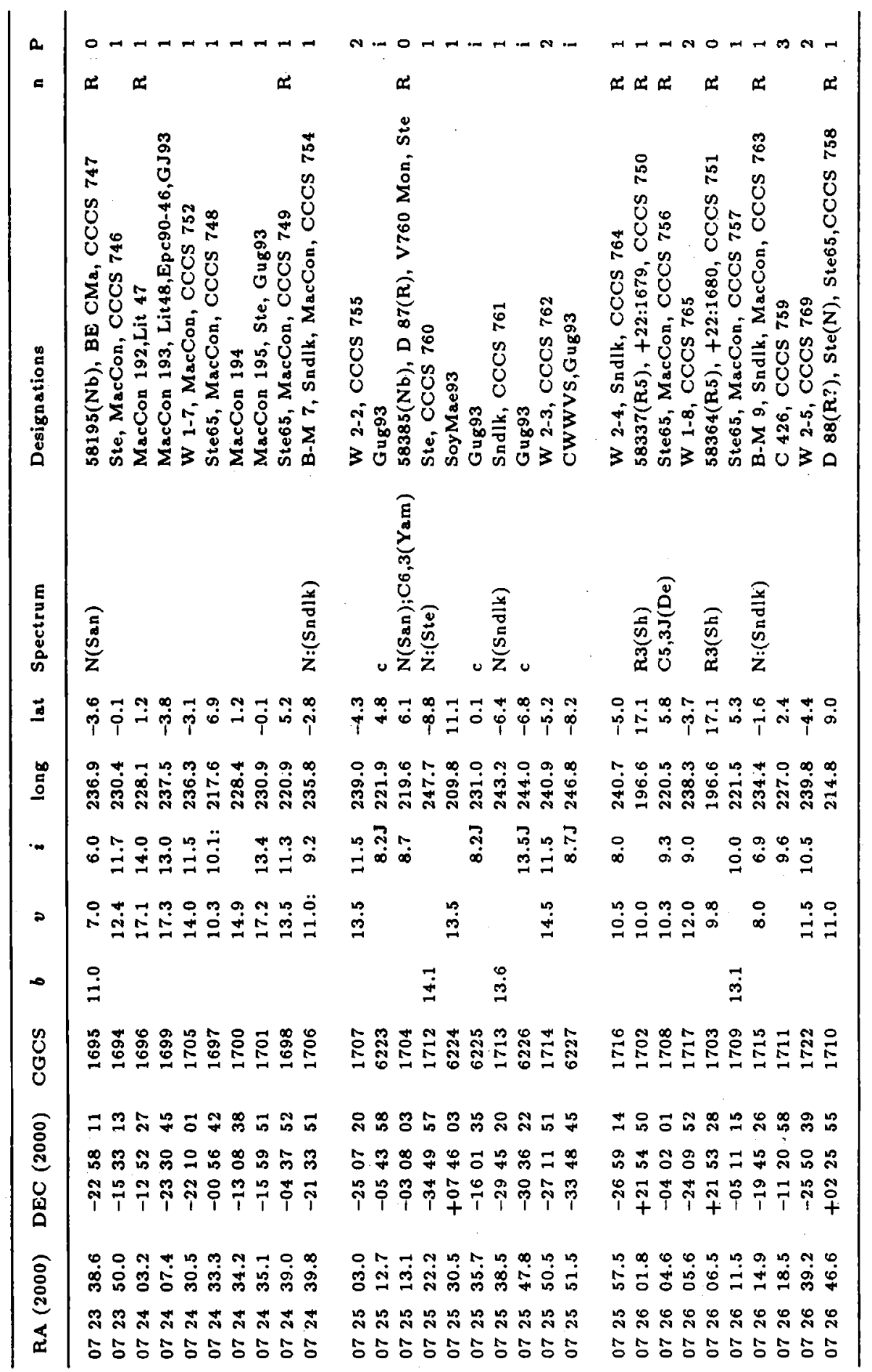




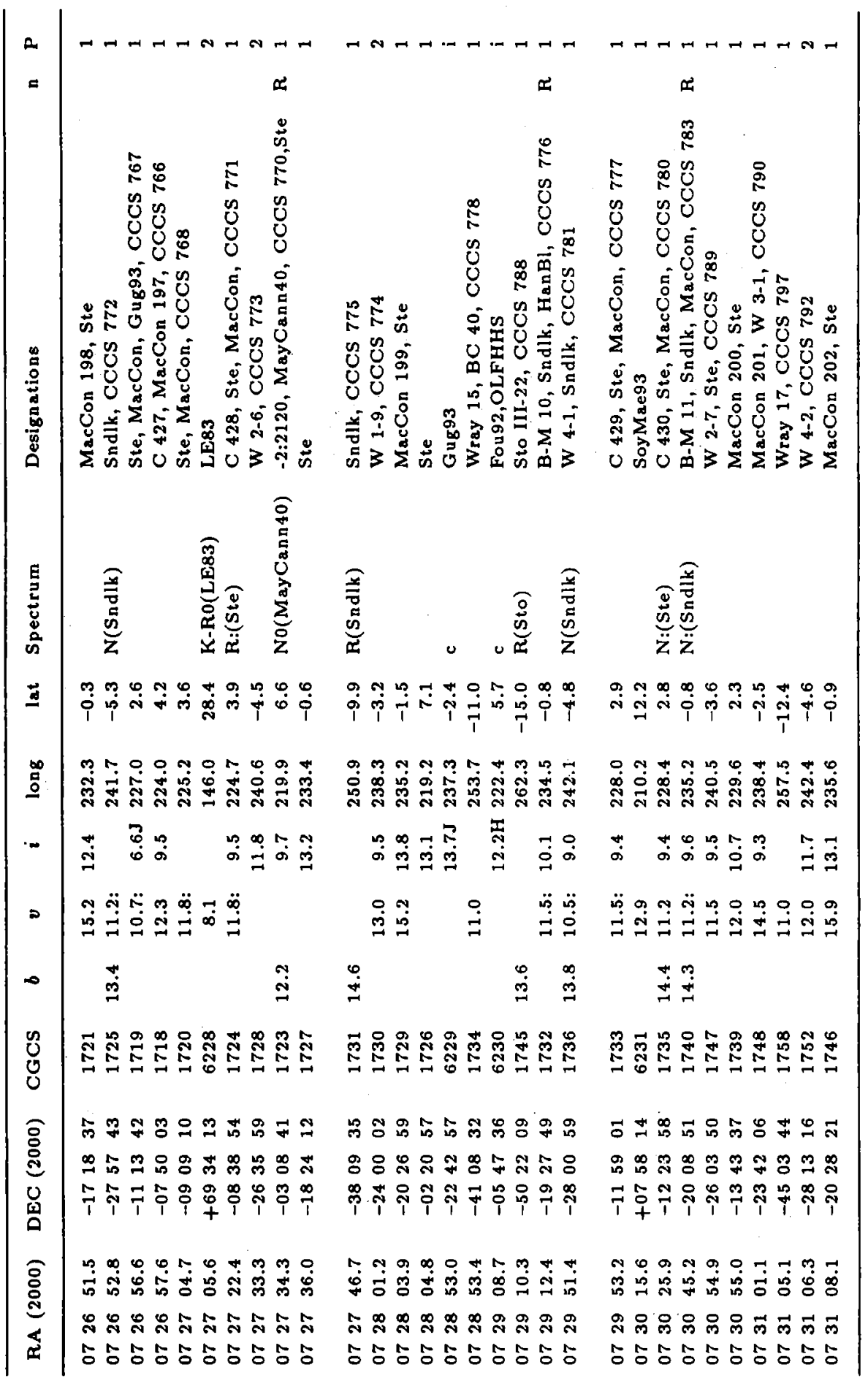




\begin{tabular}{|c|c|c|c|}
\hline$=$ & 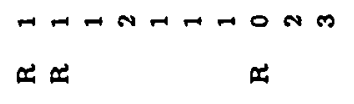 & $\begin{array}{c}x-H A-H-N-N \\
x\end{array}$ & 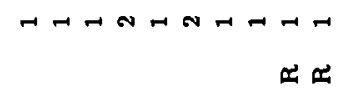 \\
\hline 苾 & 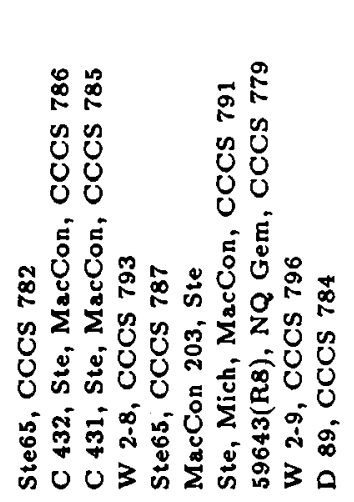 & 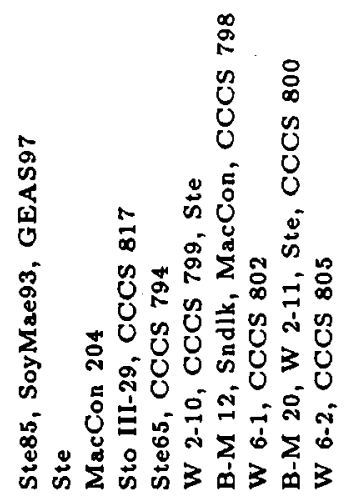 & 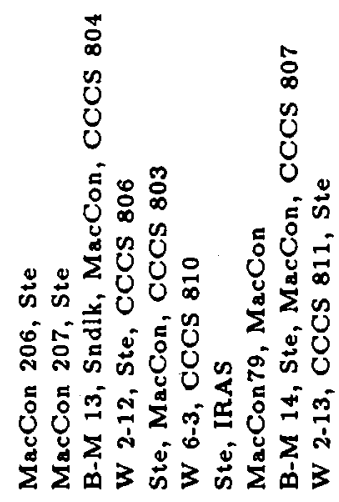 \\
\hline & 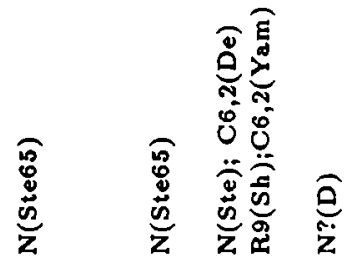 & 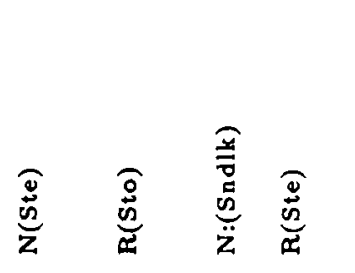 & 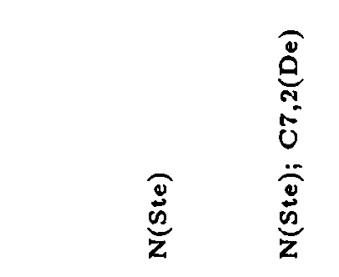 \\
\hline$\underline{\underline{a}}$ & 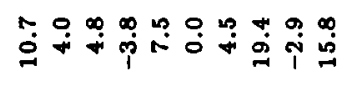 & 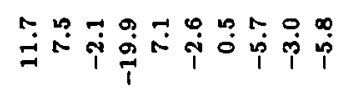 & 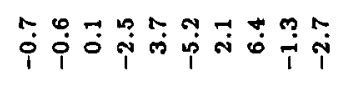 \\
\hline$\stackrel{\infty}{\underline{g}}$ & 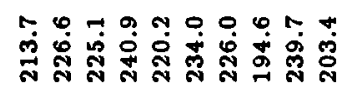 & 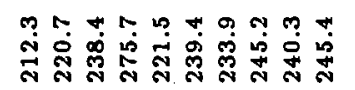 & 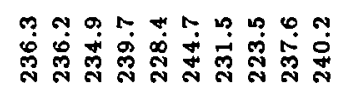 \\
\hline$\cdots$ & 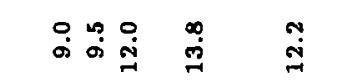 & 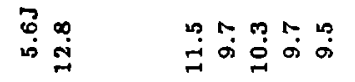 & 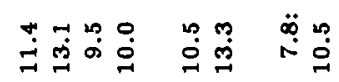 \\
\hline D & 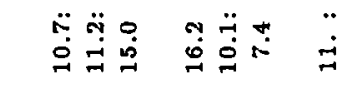 & 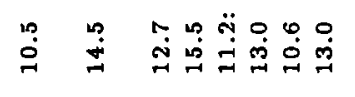 & 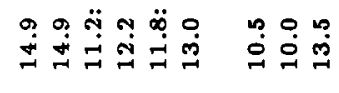 \\
\hline$\infty$ & $\stackrel{\oplus}{\ddot{g}} \stackrel{+}{\ddot{g}}$ & $\stackrel{\sim}{\Psi}$ & $\stackrel{p}{\dot{q}}$ \\
\hline O্ & 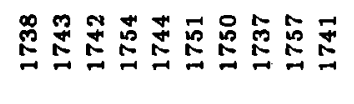 & 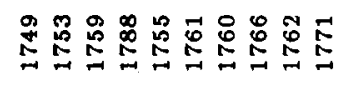 & 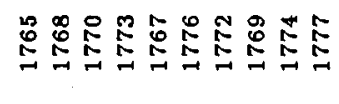 \\
\hline$\widehat{\circ}$ & 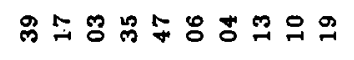 & 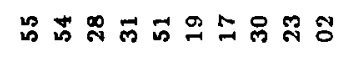 & 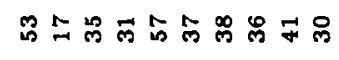 \\
\hline \begin{tabular}{l}
\multirow{3}{*}{} \\
0 \\
0 \\
0 \\
0
\end{tabular} & 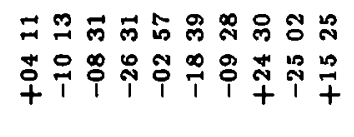 & 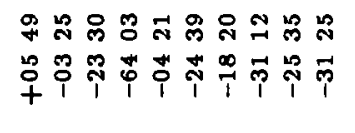 & 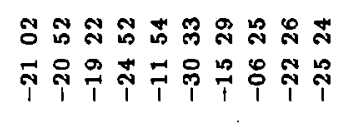 \\
\hline ๕. & 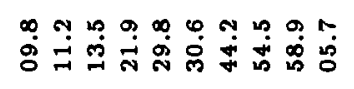 & 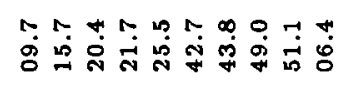 & 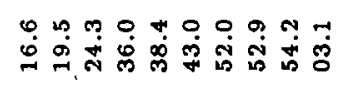 \\
\hline 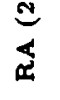 & 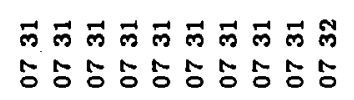 & 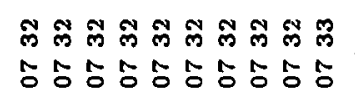 & 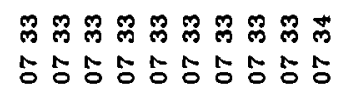 \\
\hline
\end{tabular}




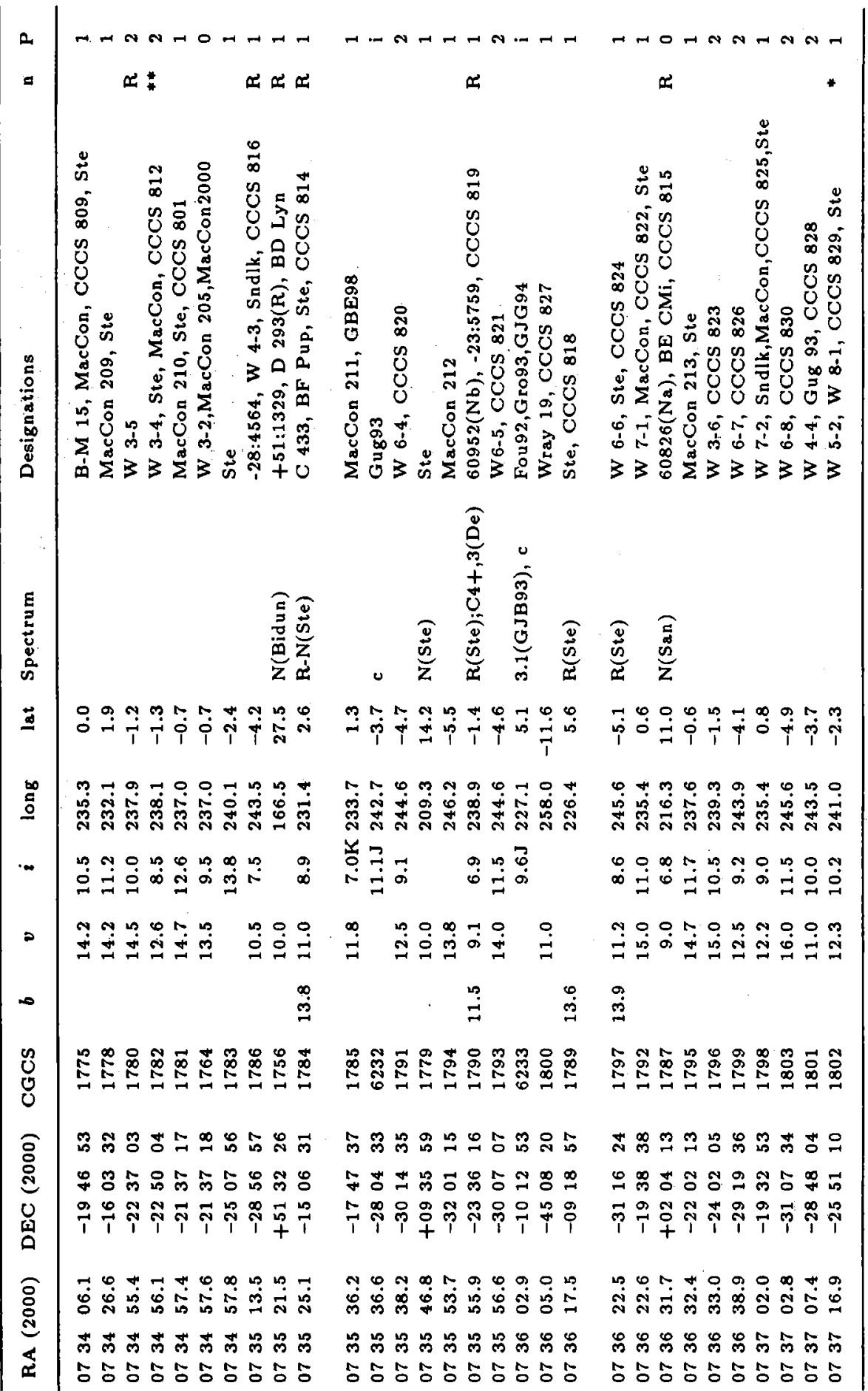




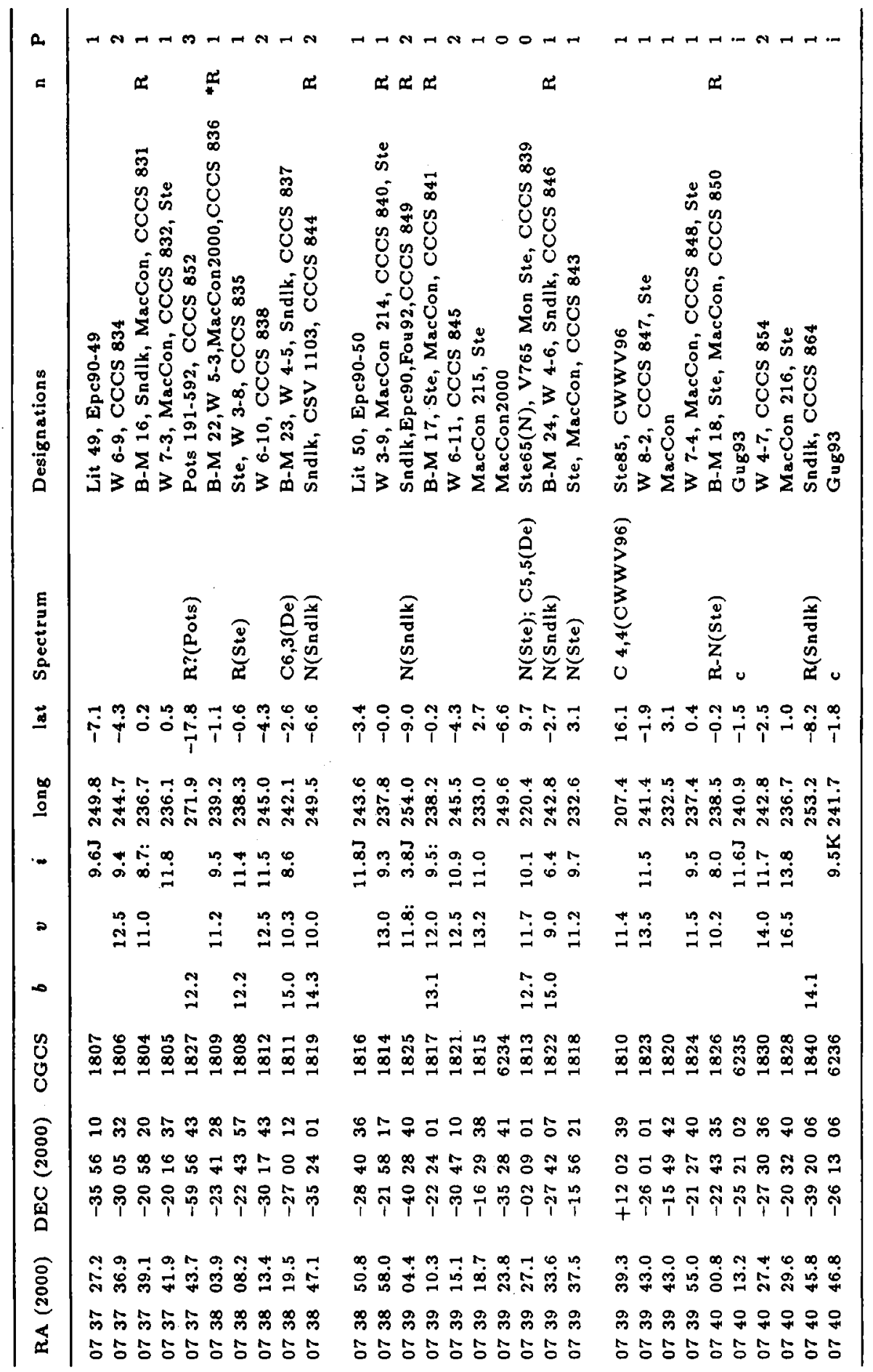




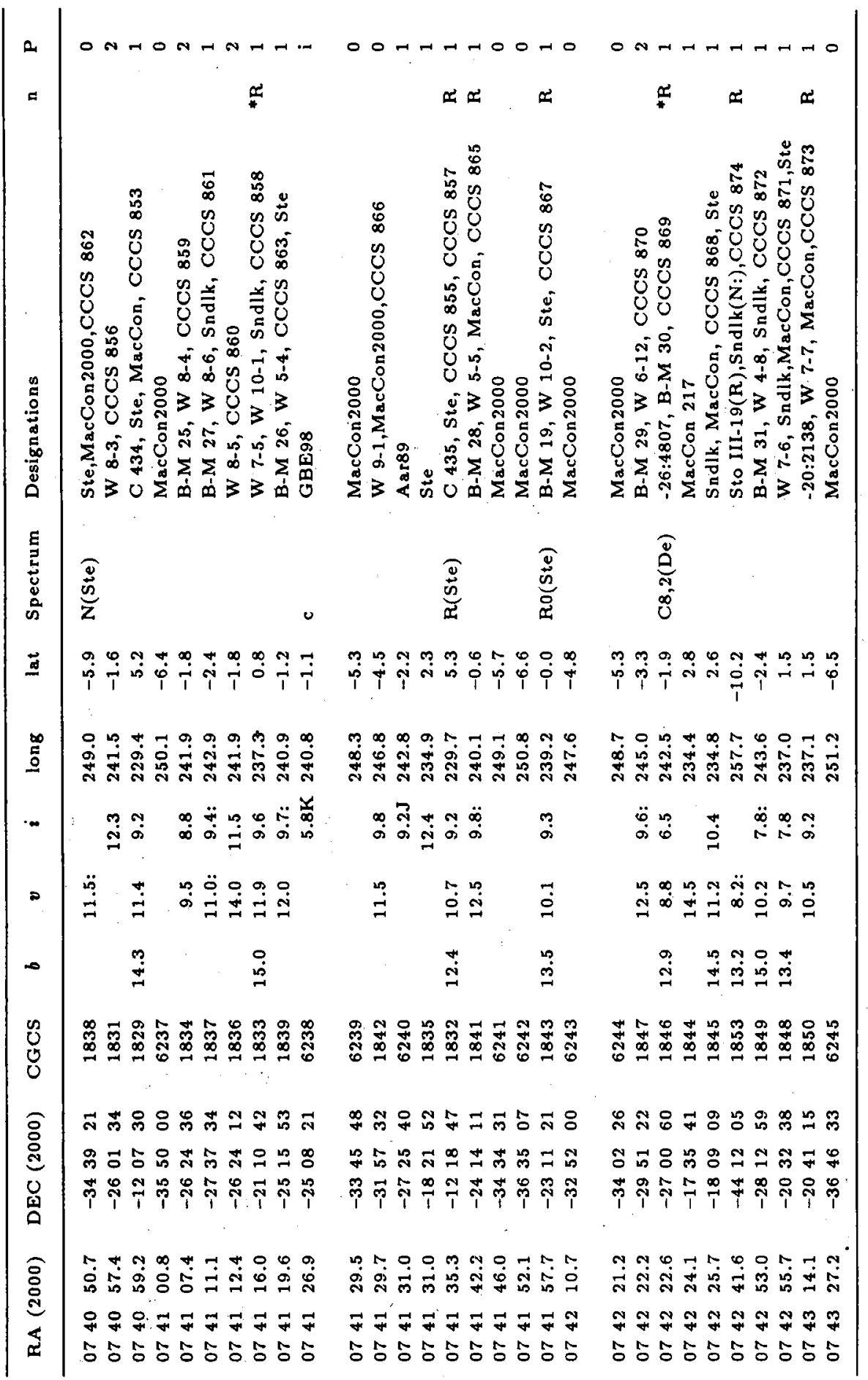




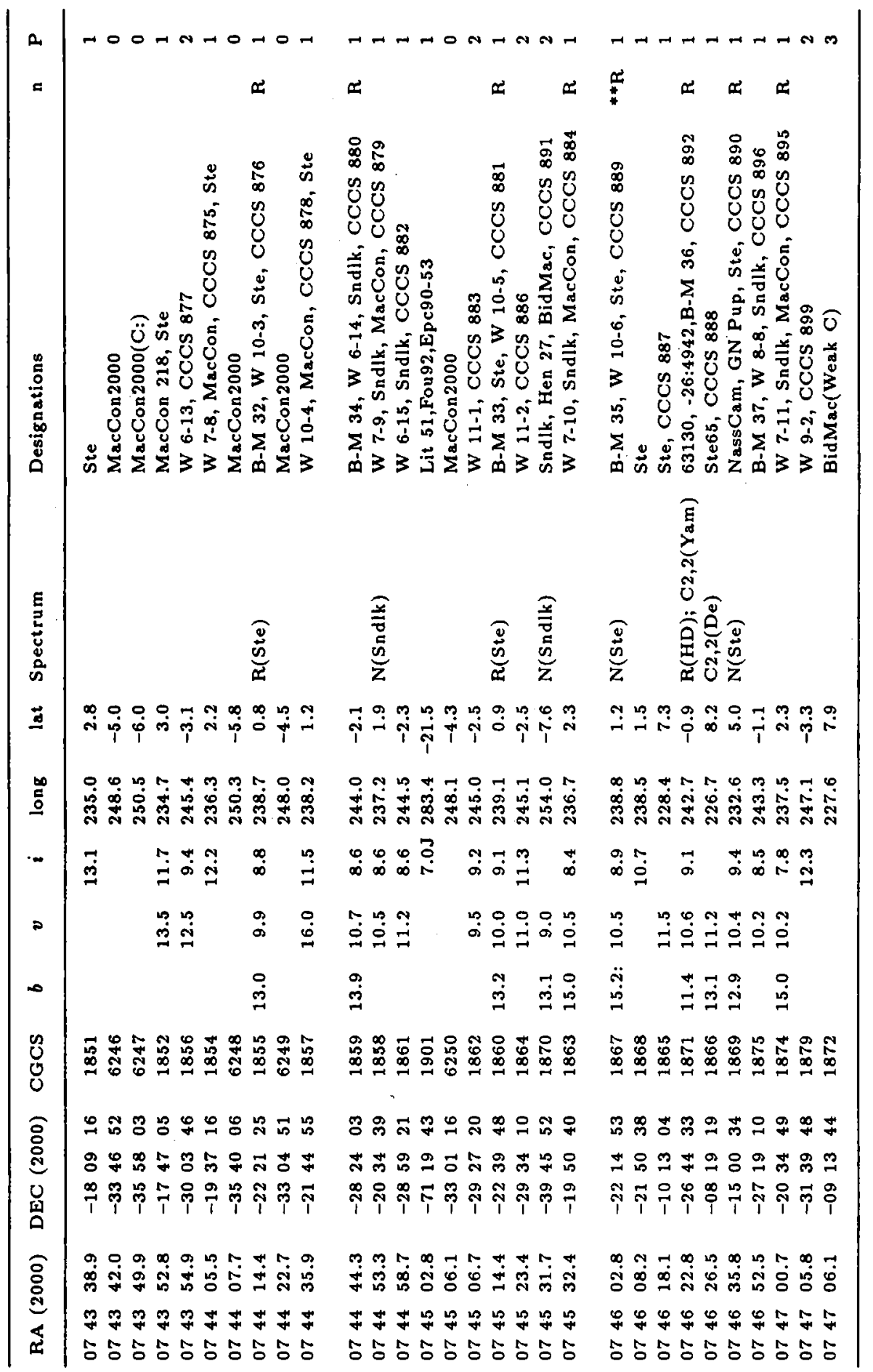




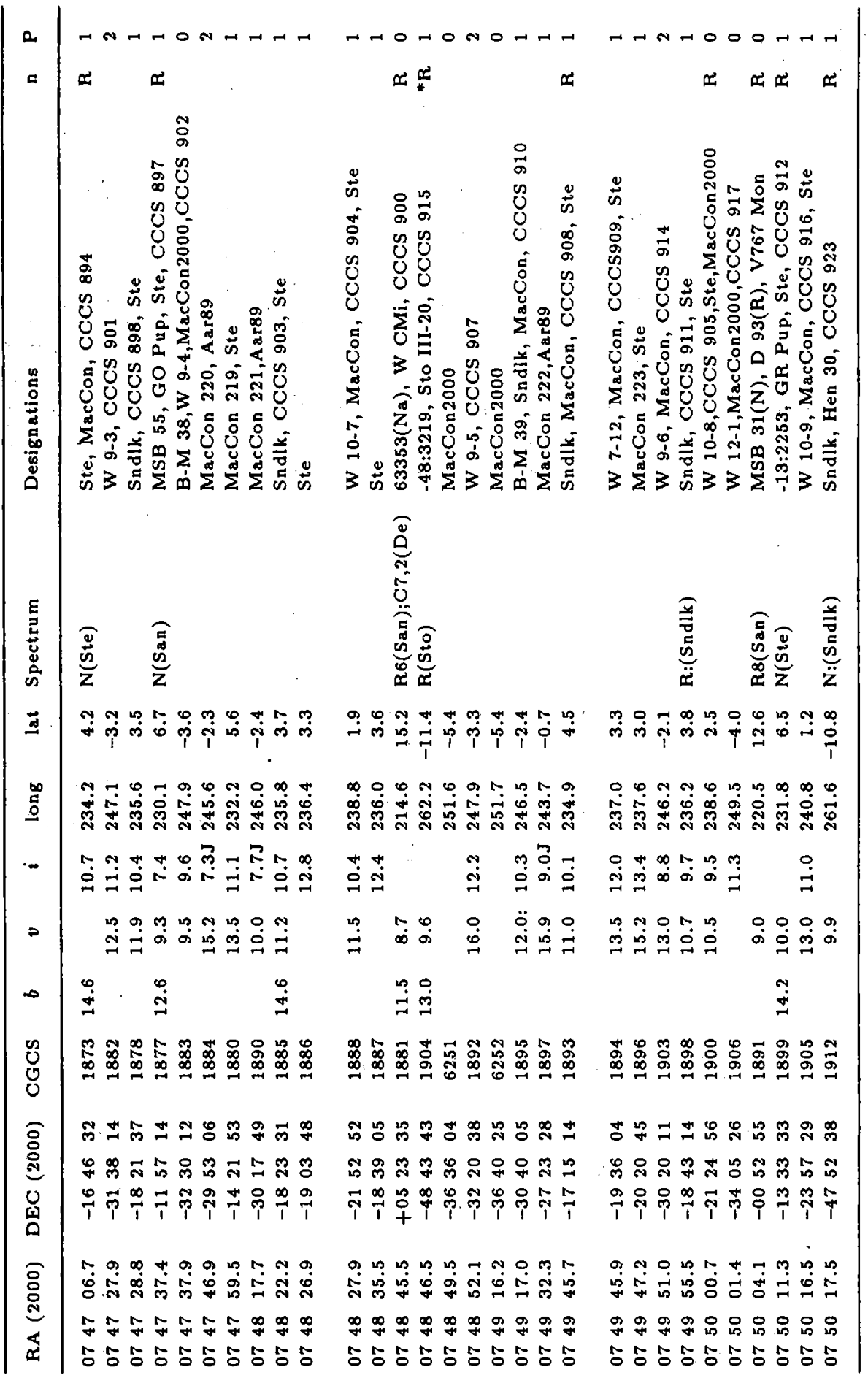




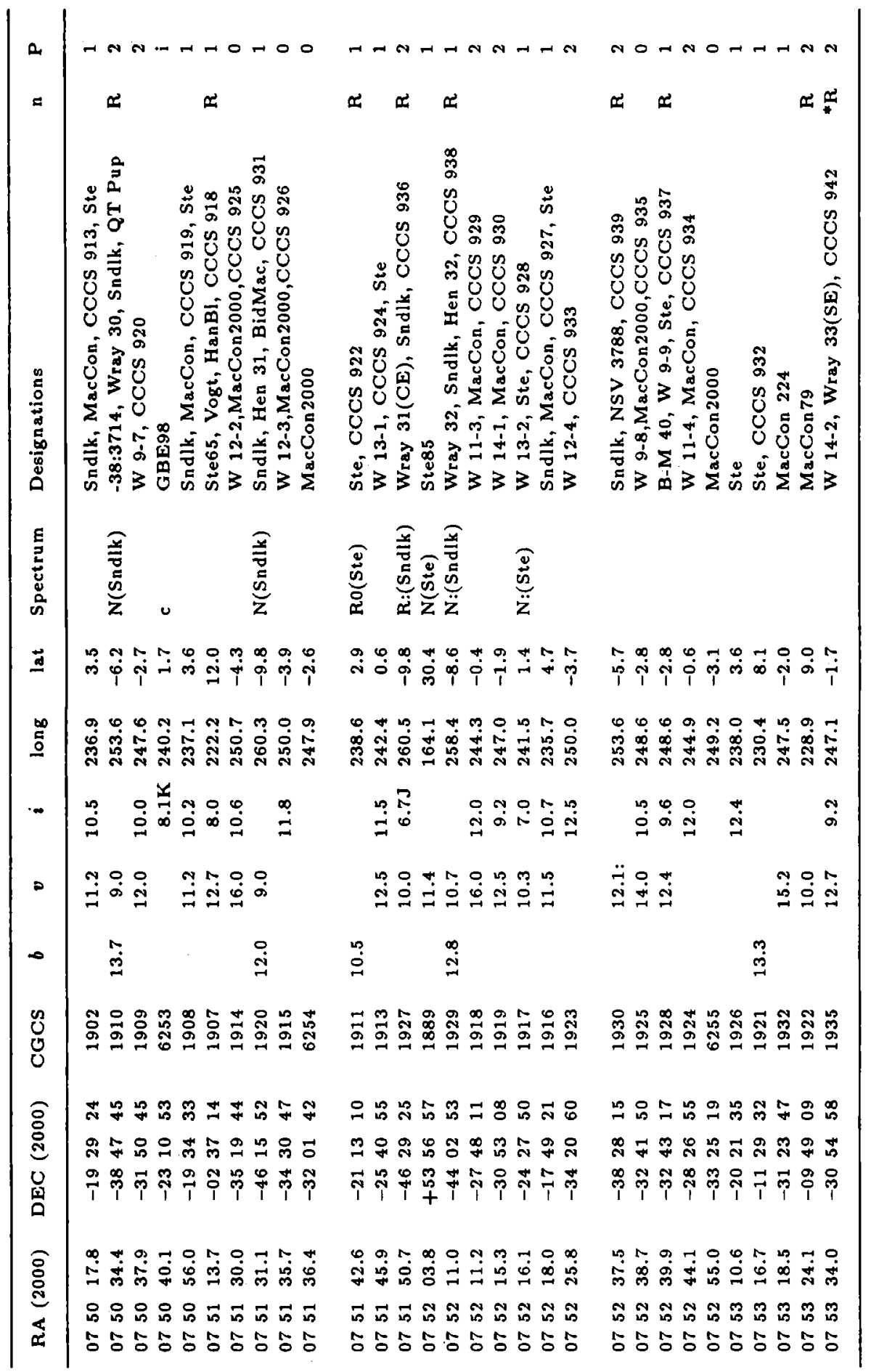




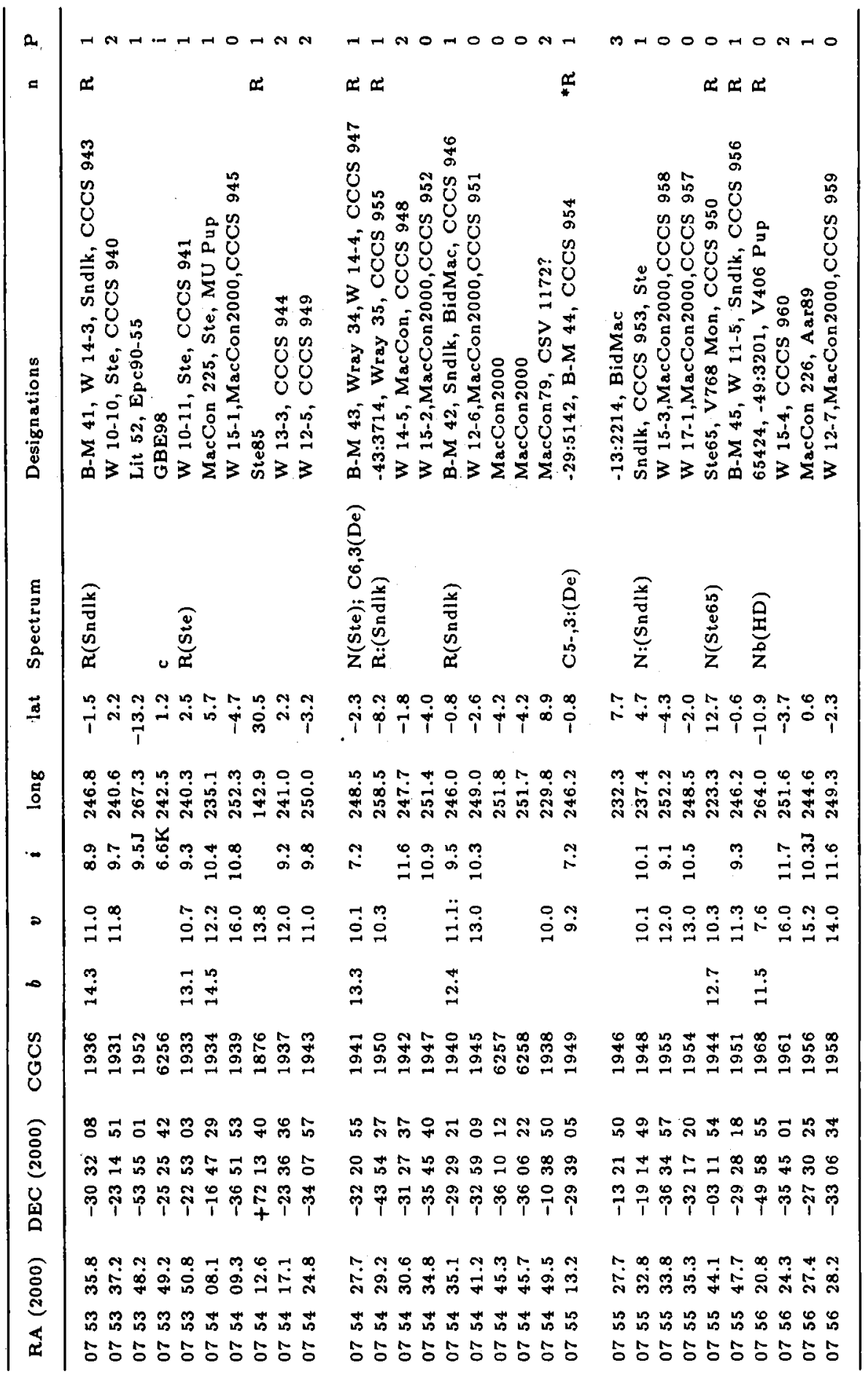




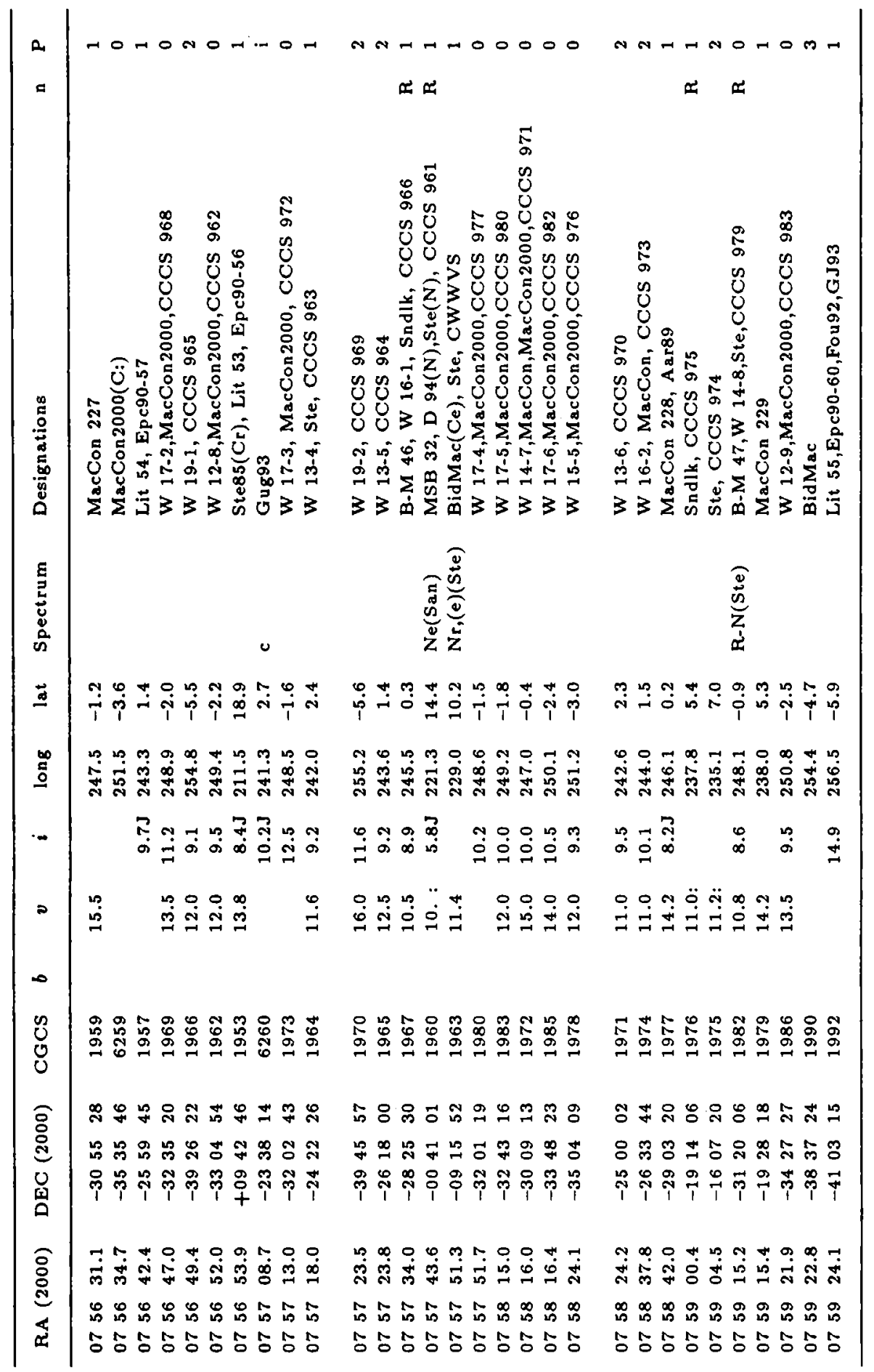




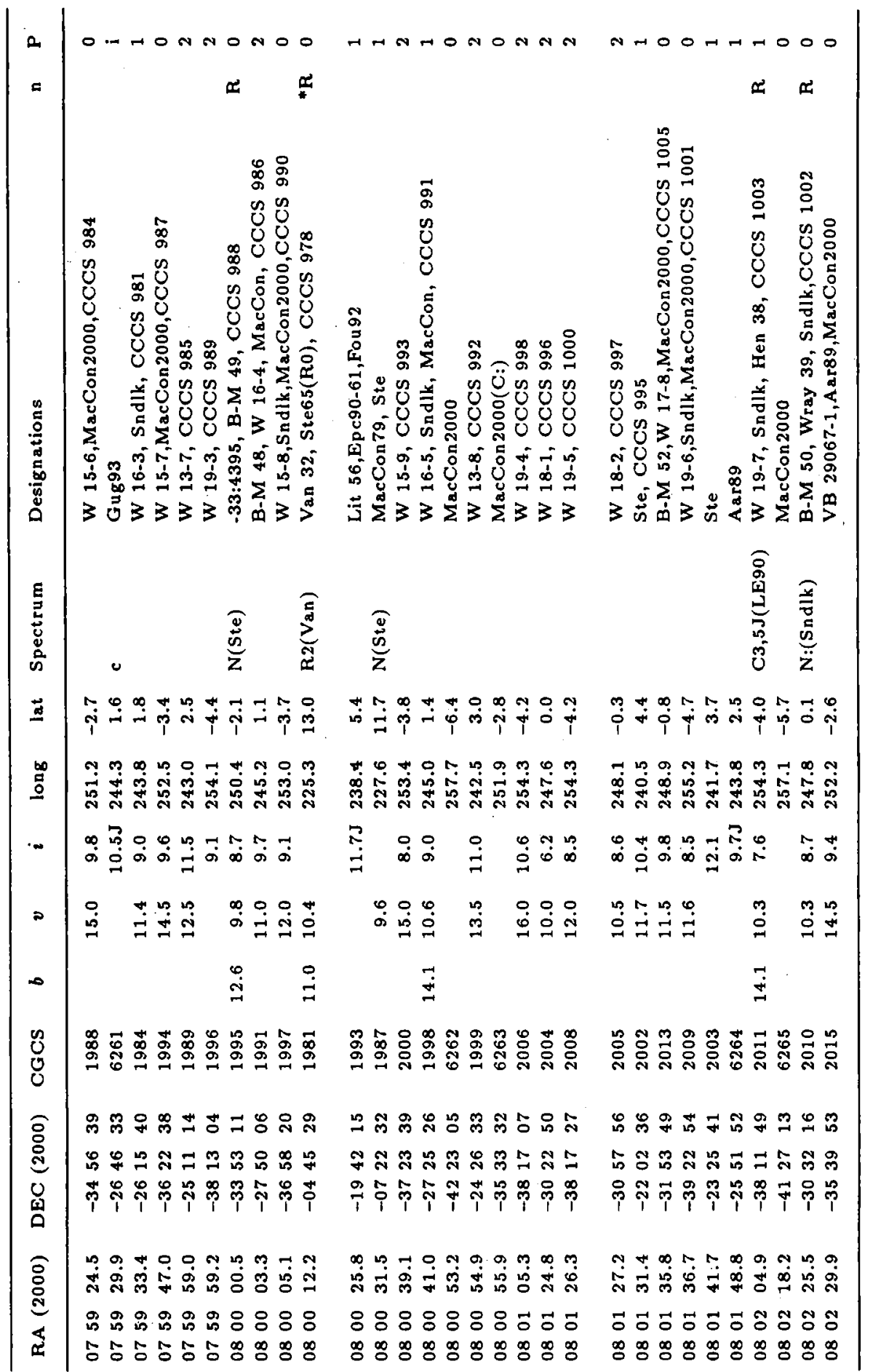




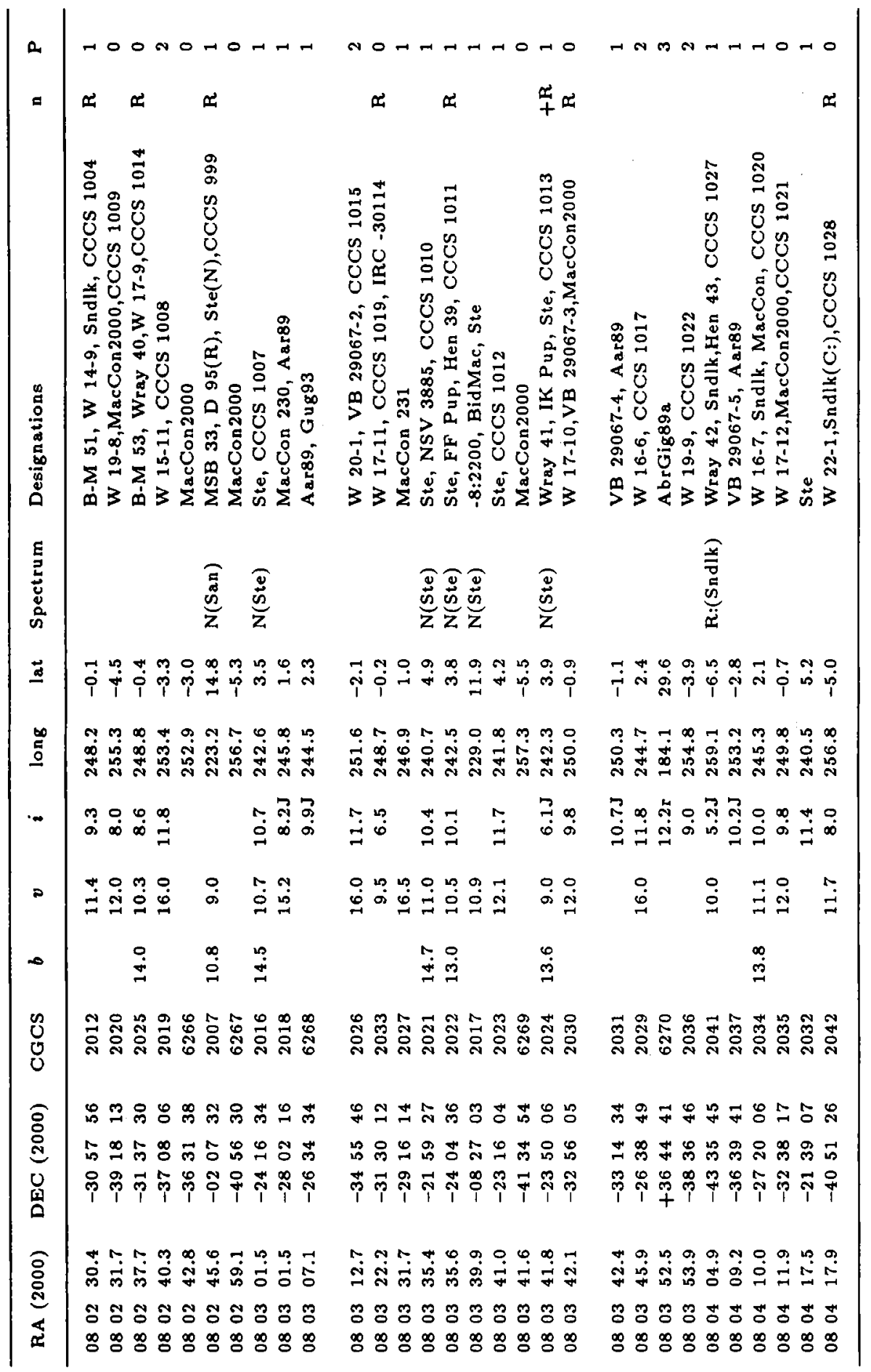




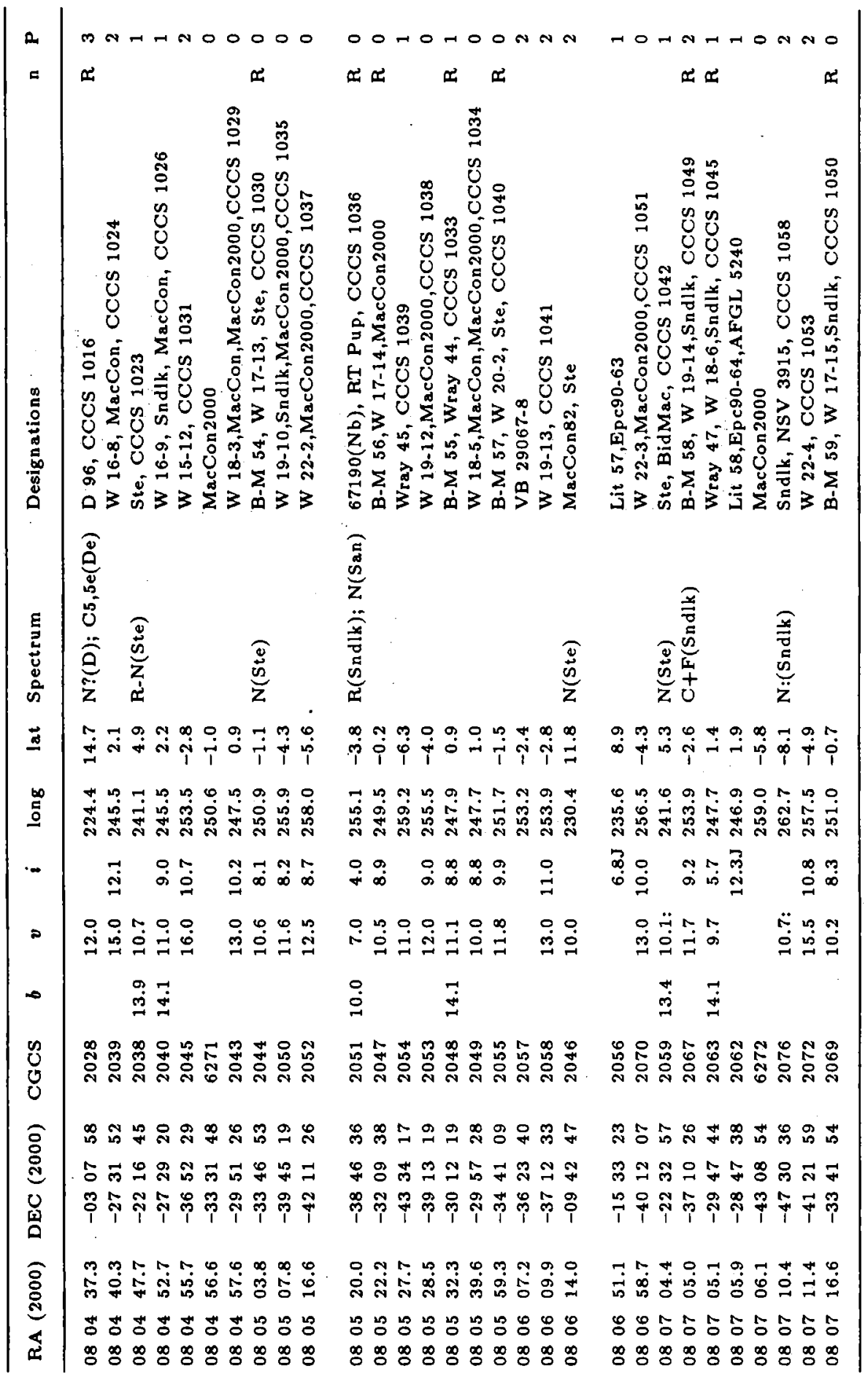




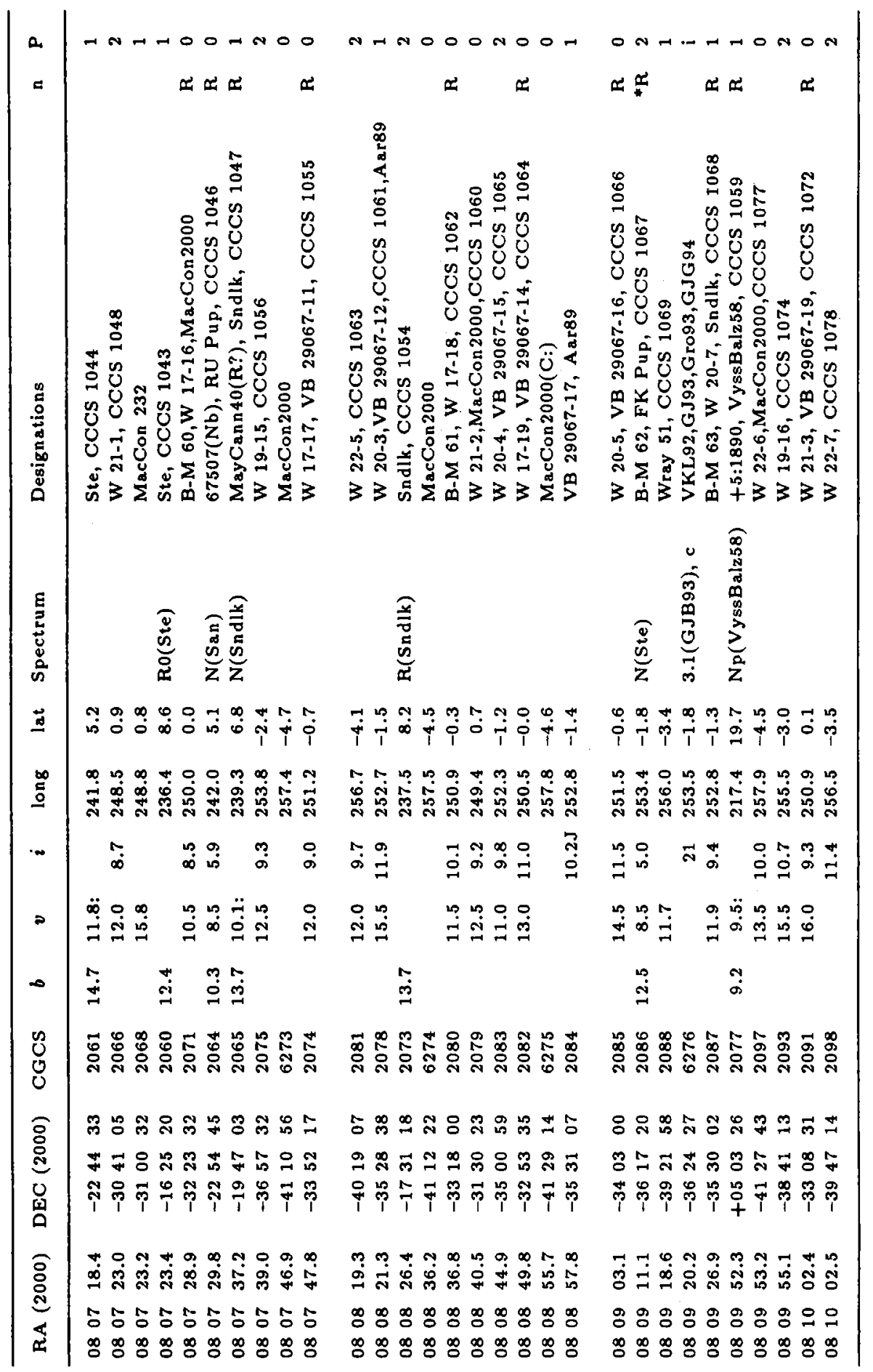




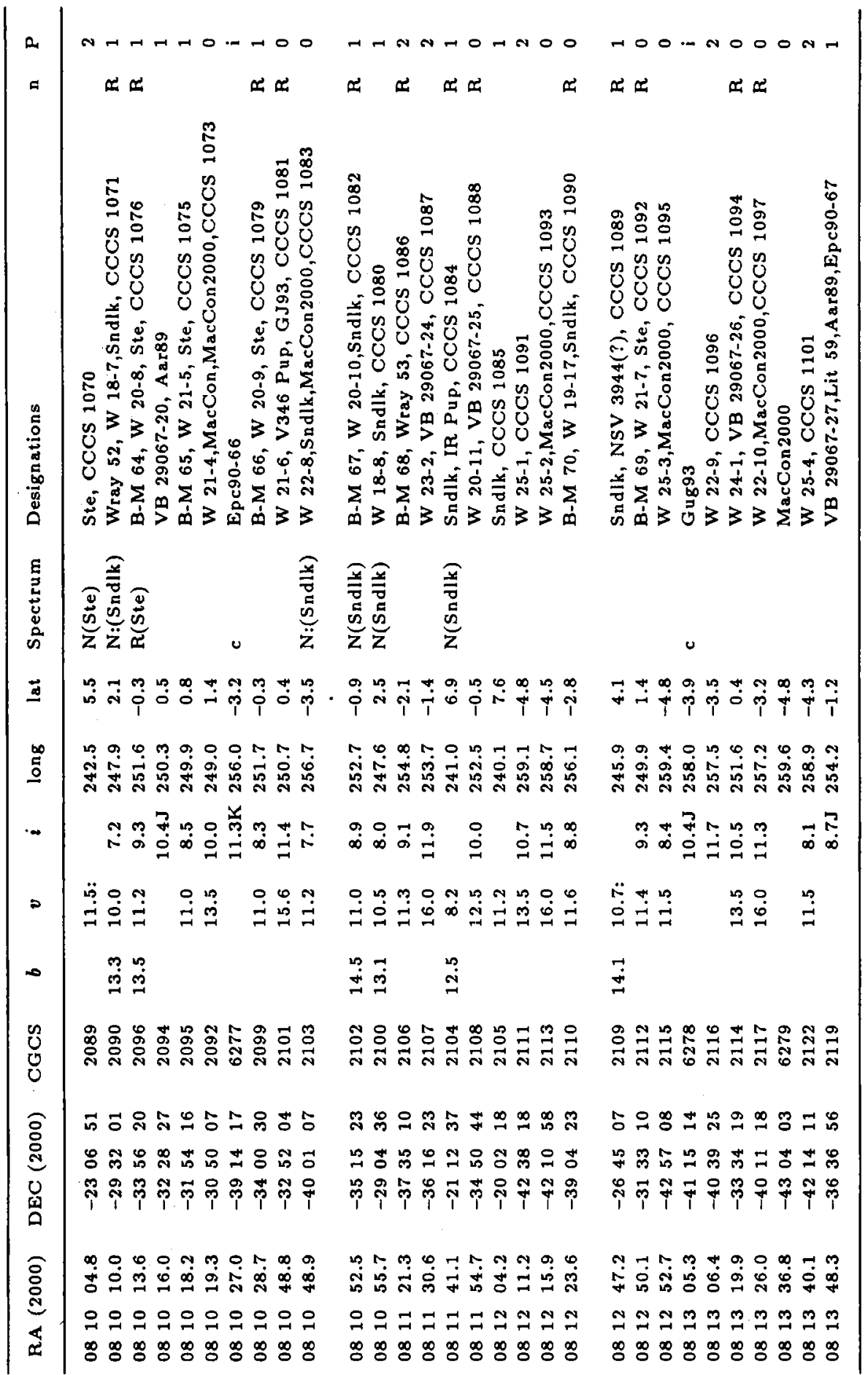




\begin{tabular}{|c|c|c|c|c|c|c|}
\hline$a$ & 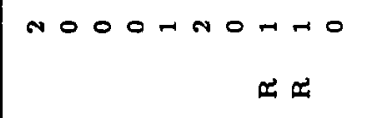 & $n-n O$ & $\begin{array}{c}-00- \\
x\end{array}$ & $x$ & OONHOC & $\begin{array}{c}n \\
\cos \end{array}$ \\
\hline 若 & 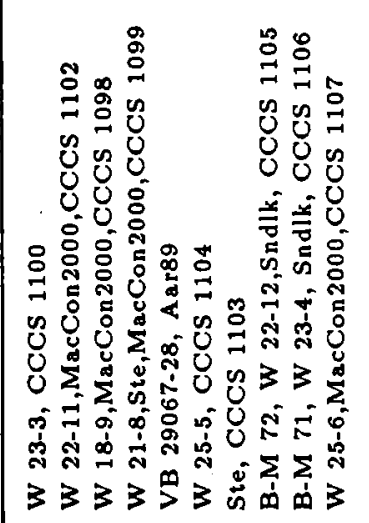 & 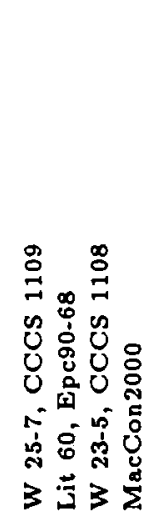 & 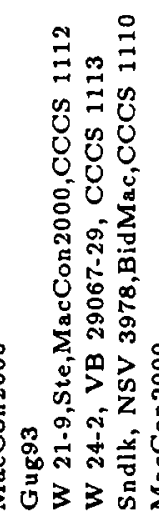 & 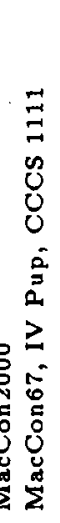 & 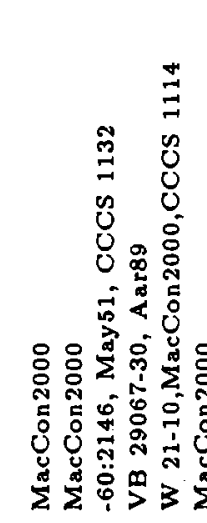 & 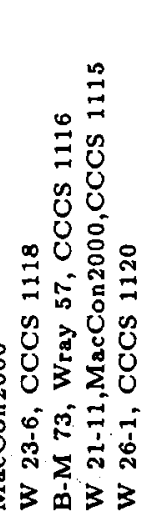 \\
\hline 总 & 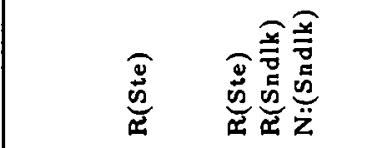 & & 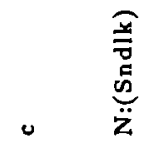 & 离 & 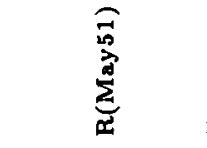 & 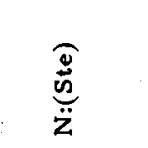 \\
\hline$\underline{\Xi}$ & 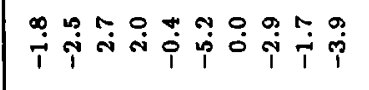 & 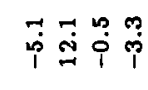 & 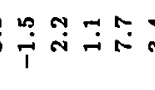 & i⿱ & 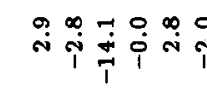 & î \\
\hline$\stackrel{\infty}{\Xi}$ & 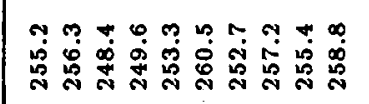 & 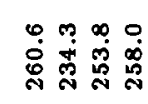 & 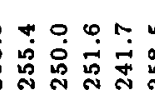 & 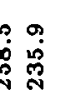 & 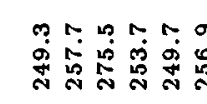 & 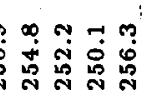 \\
\hline - & 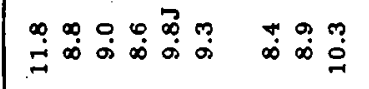 & 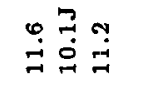 & స̣̃ & & $\begin{array}{l}b \\
\dot{0} \\
\dot{0}\end{array}$ & 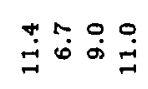 \\
\hline ? & 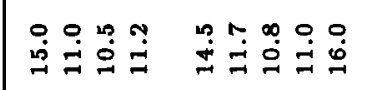 & $\stackrel{\circ}{\text { in }}$ & $\stackrel{\circ}{\stackrel{i}{H}}$ & $\stackrel{?}{\circ}$ & $\stackrel{29}{=}$ & 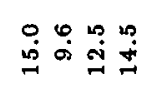 \\
\hline ـ & 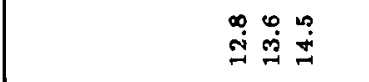 & & $\stackrel{\oplus}{\oplus}$ & $\stackrel{\circ}{\text { ì }}$ & & \\
\hline $\begin{array}{l}0 \\
0 \\
y \\
0\end{array}$ & 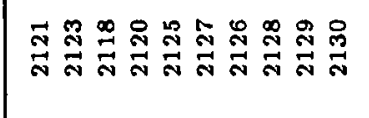 & 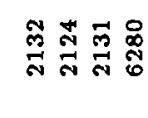 & 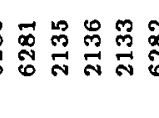 & 药 & 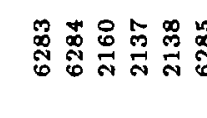 & 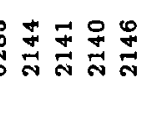 \\
\hline ฮิ & 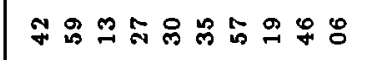 & 品 & 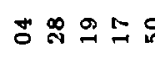 & 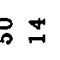 & 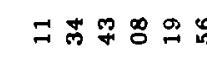 & 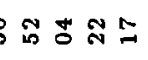 \\
\hline 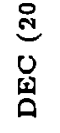 & 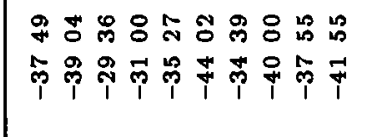 & 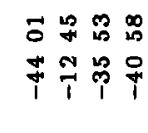 & & & 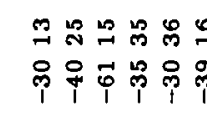 & 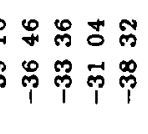 \\
\hline ๖े & 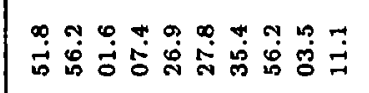 & 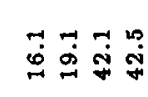 & 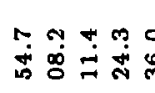 & & 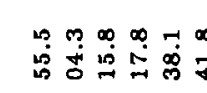 & 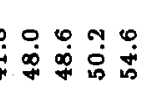 \\
\hline 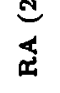 & 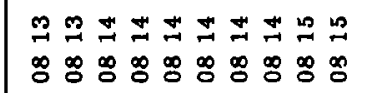 & 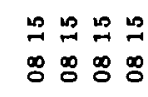 & 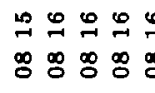 & & 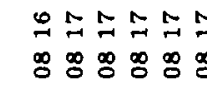 & 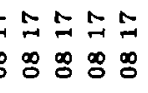 \\
\hline
\end{tabular}




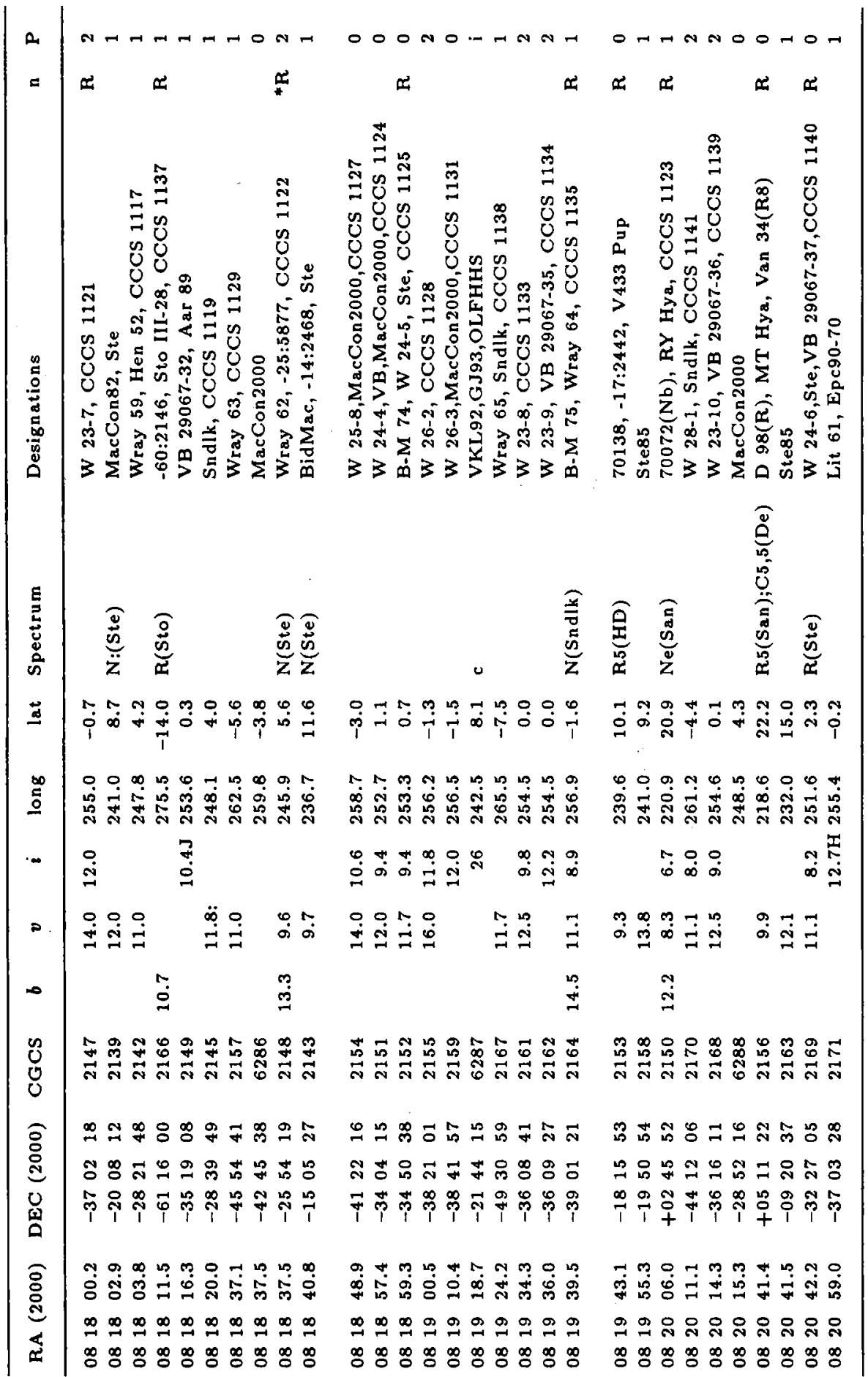




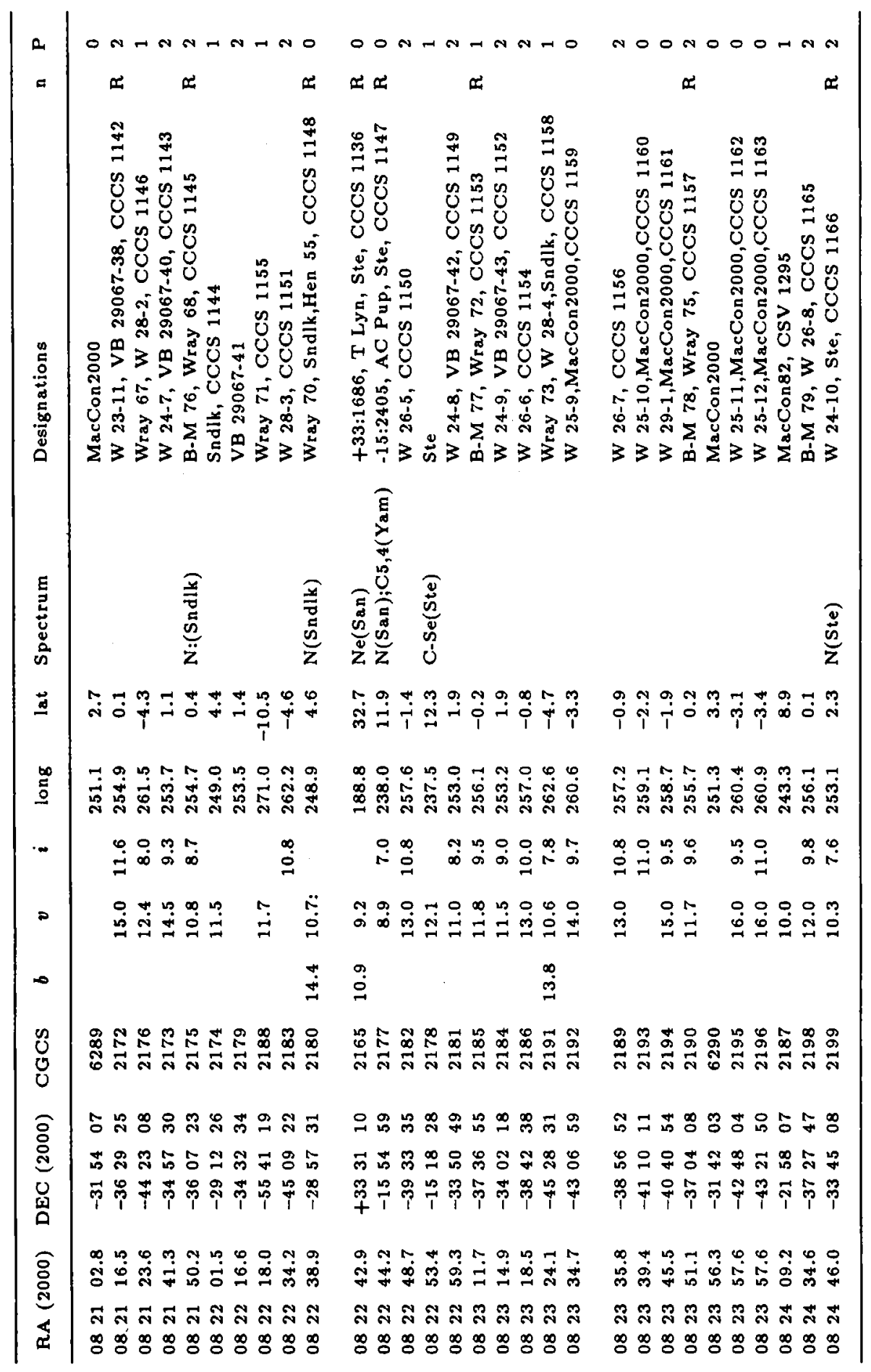




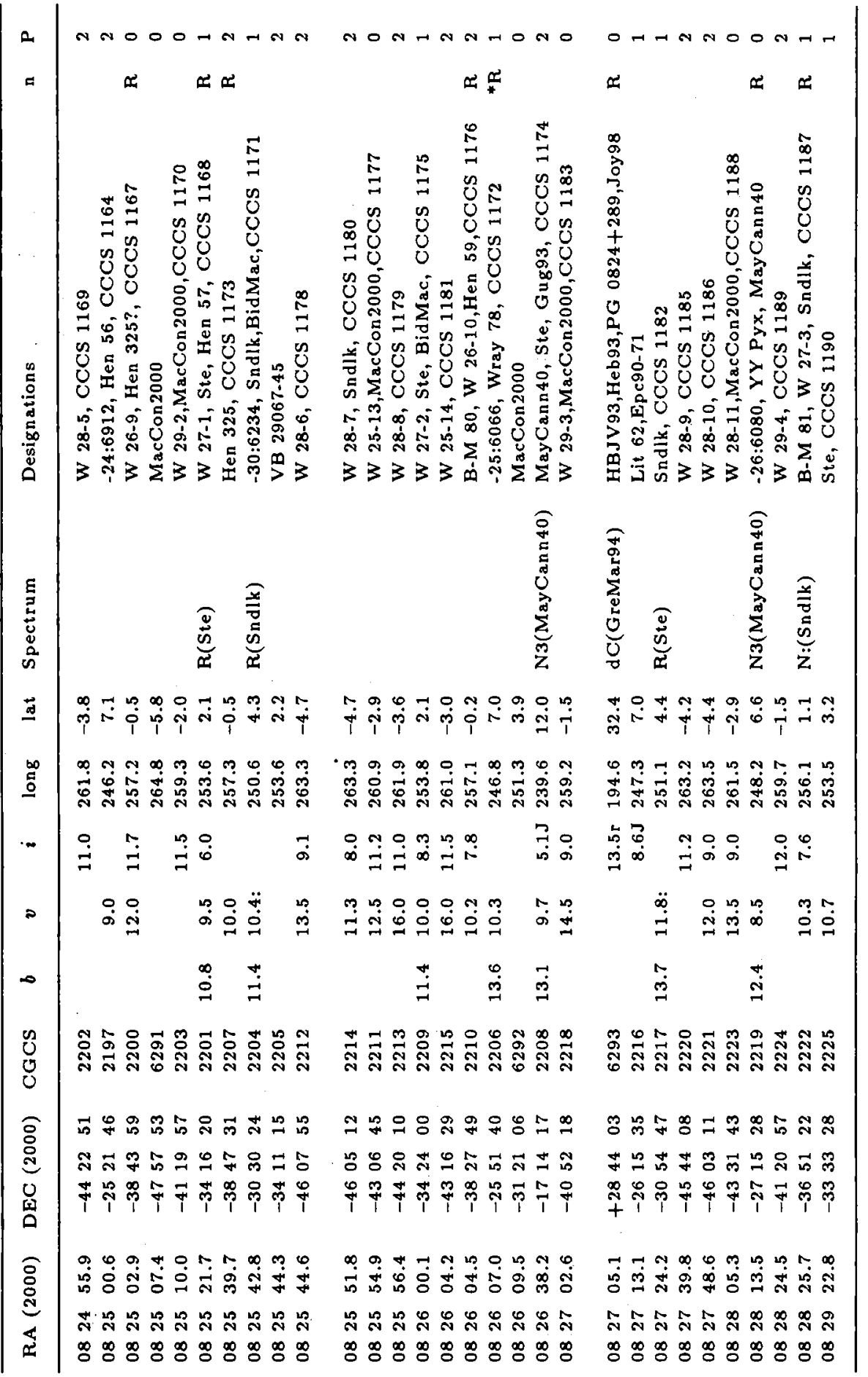




\begin{tabular}{|c|c|c|c|}
\hline a & 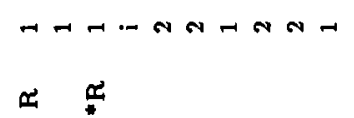 & 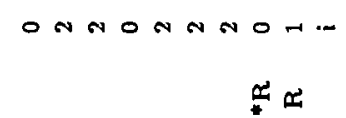 & 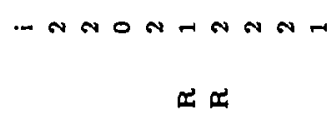 \\
\hline & 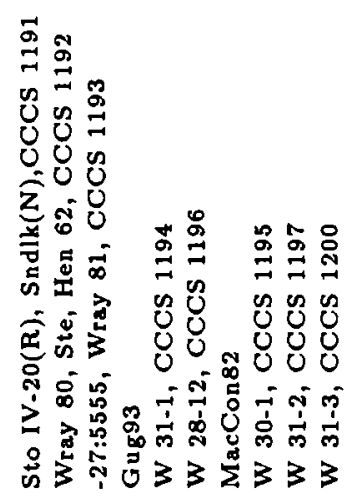 & 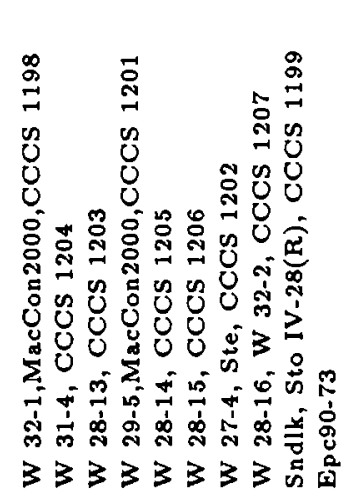 & 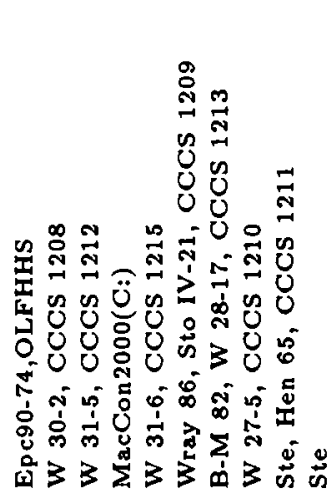 \\
\hline & 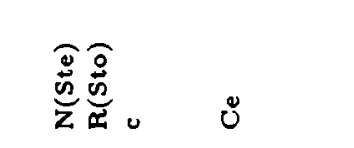 & 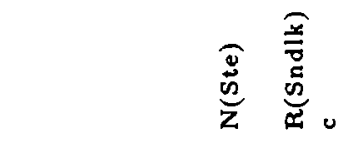 & 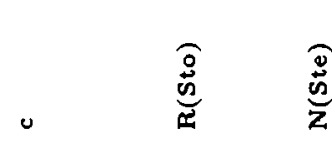 \\
\hline 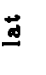 & 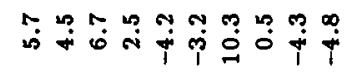 & T̃ & 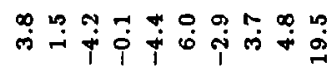 \\
\hline 罯 & 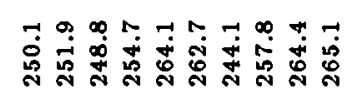 & 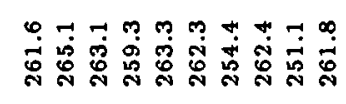 & 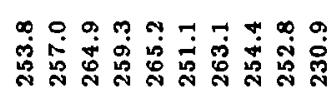 \\
\hline & 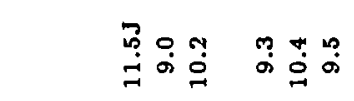 & 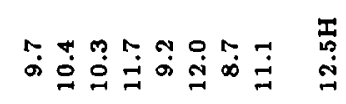 & 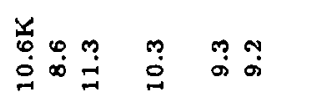 \\
\hline م & 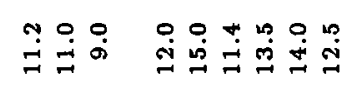 & 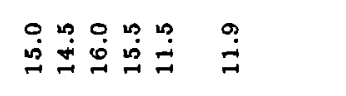 & 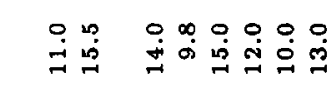 \\
\hline & 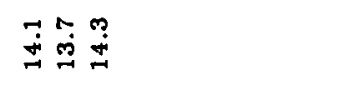 & $\stackrel{\text { มุ }}{\mathbb{N}}$ & $\stackrel{\circ}{\ddot{m}} \quad \stackrel{i}{ت}$ \\
\hline in & 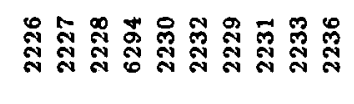 & 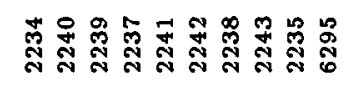 & 品莡 \\
\hline 8 & பั & 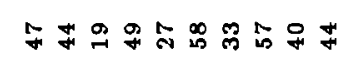 & 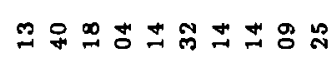 \\
\hline & 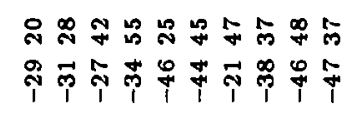 & 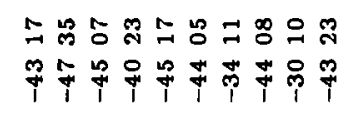 & 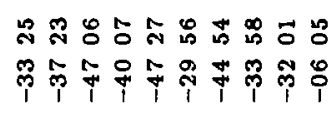 \\
\hline 8 & 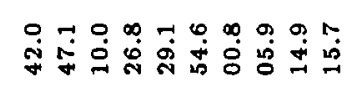 & 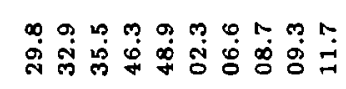 & 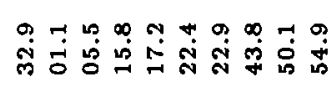 \\
\hline & 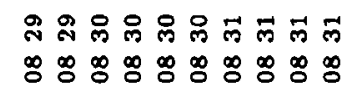 & 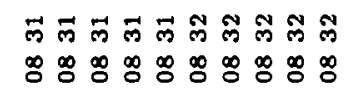 & 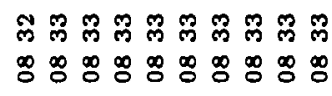 \\
\hline
\end{tabular}




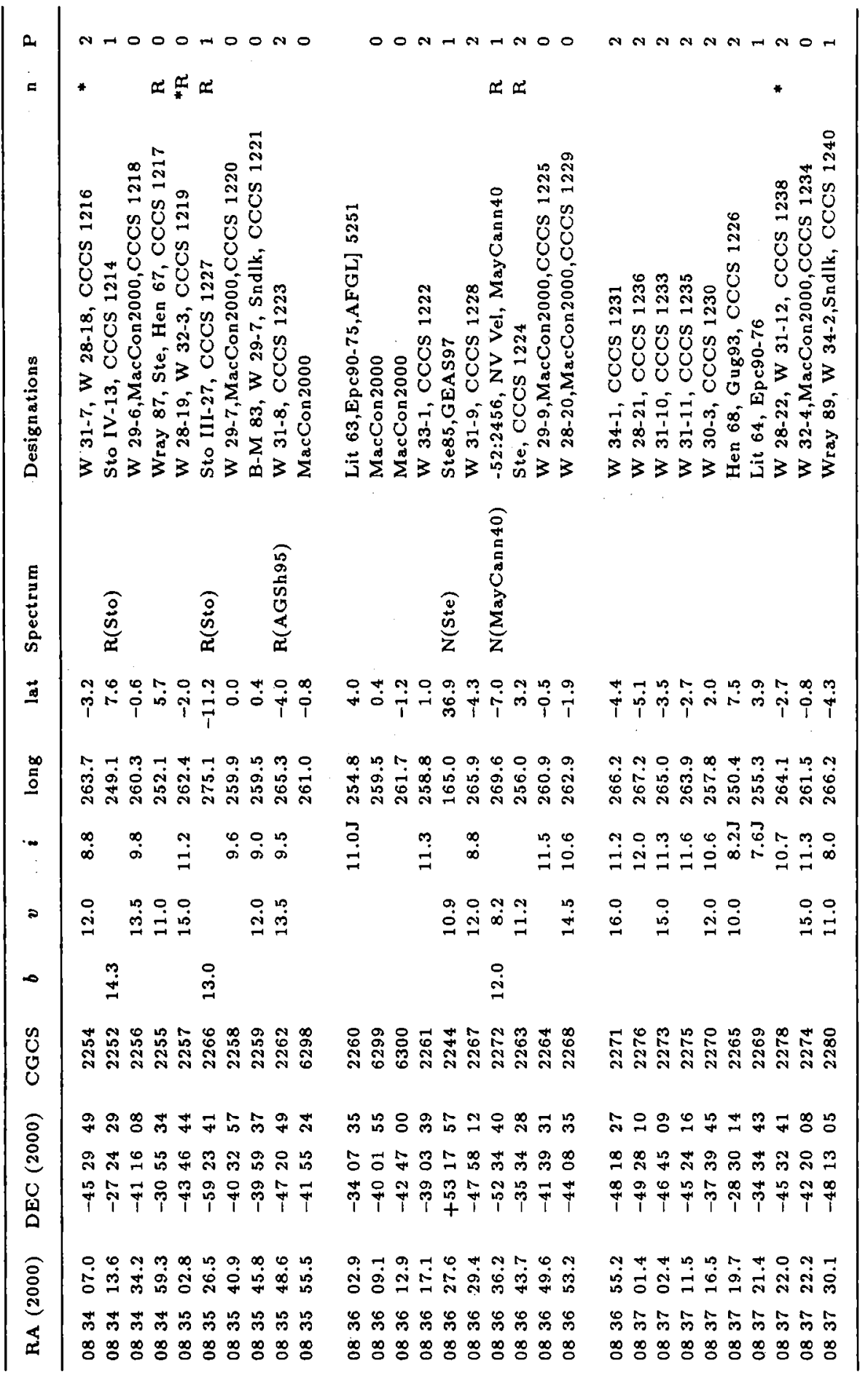




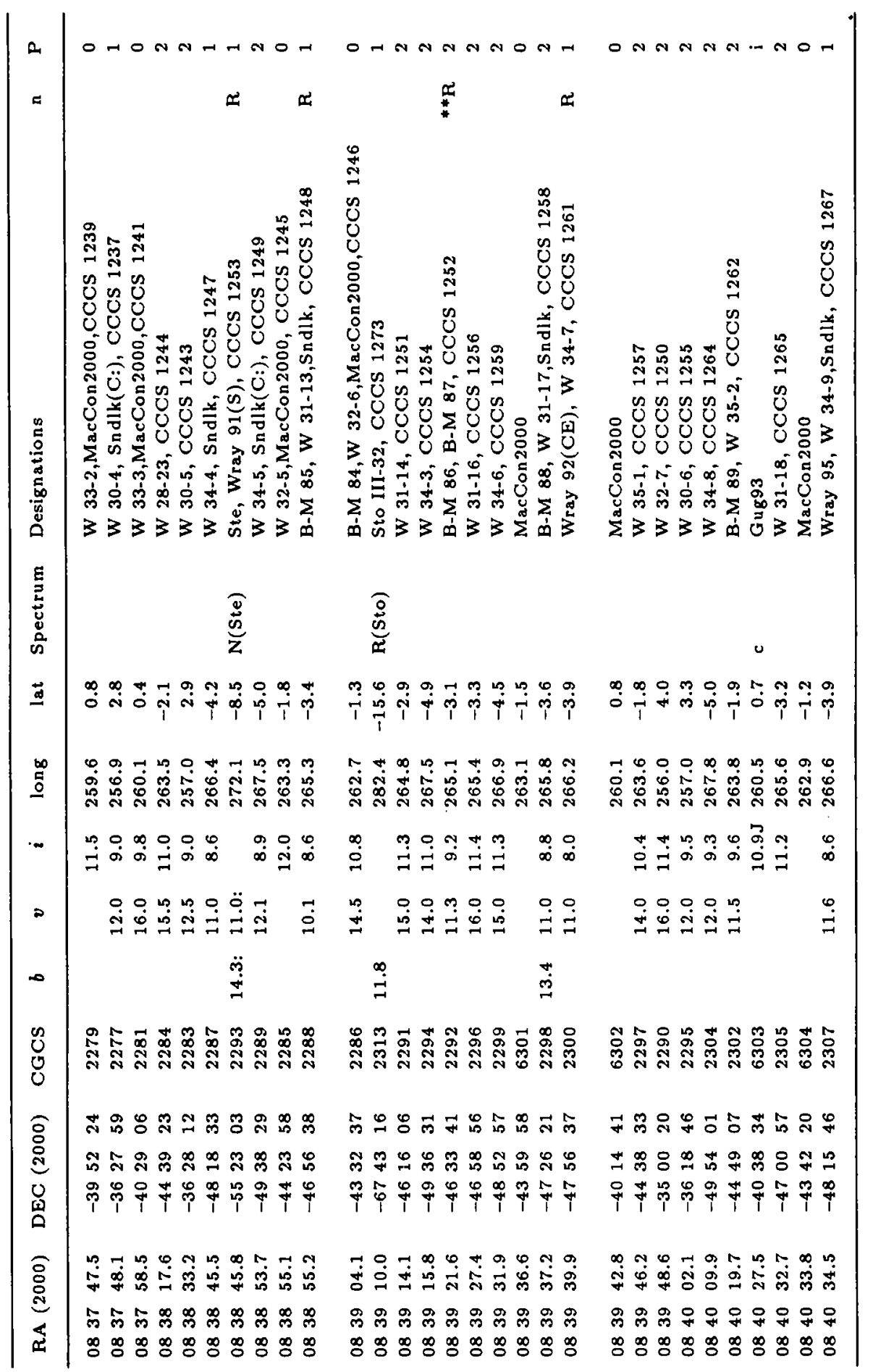




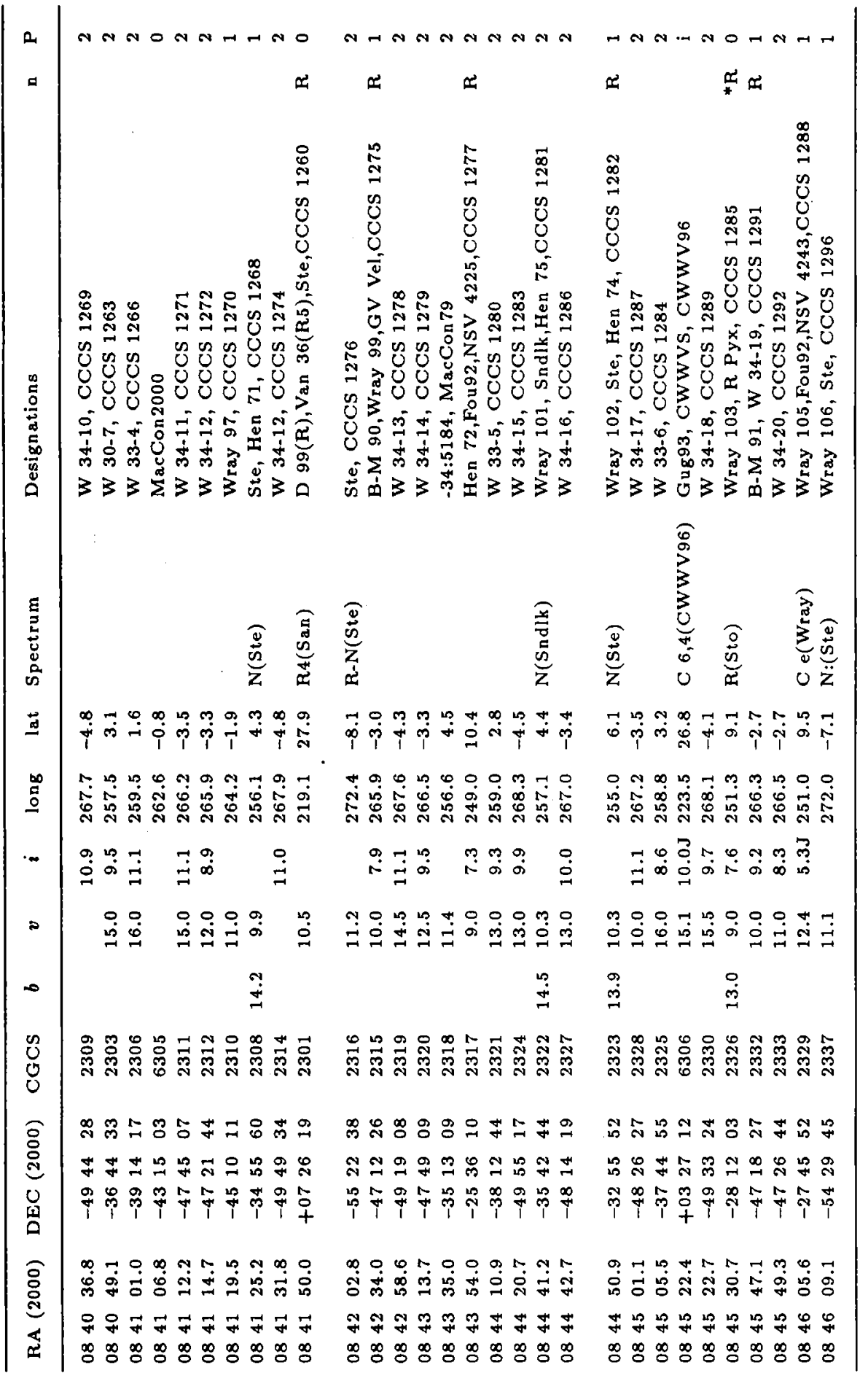




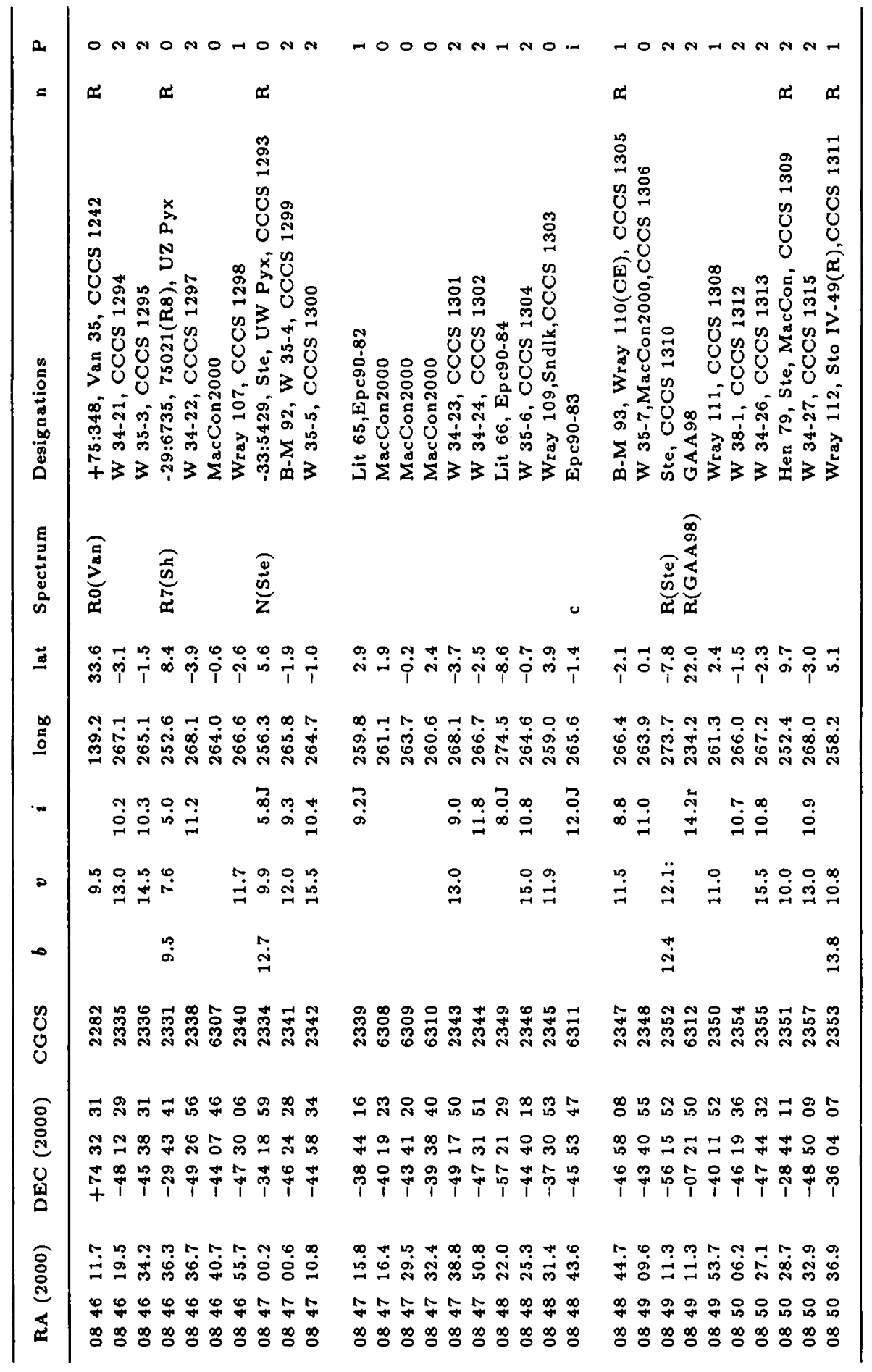




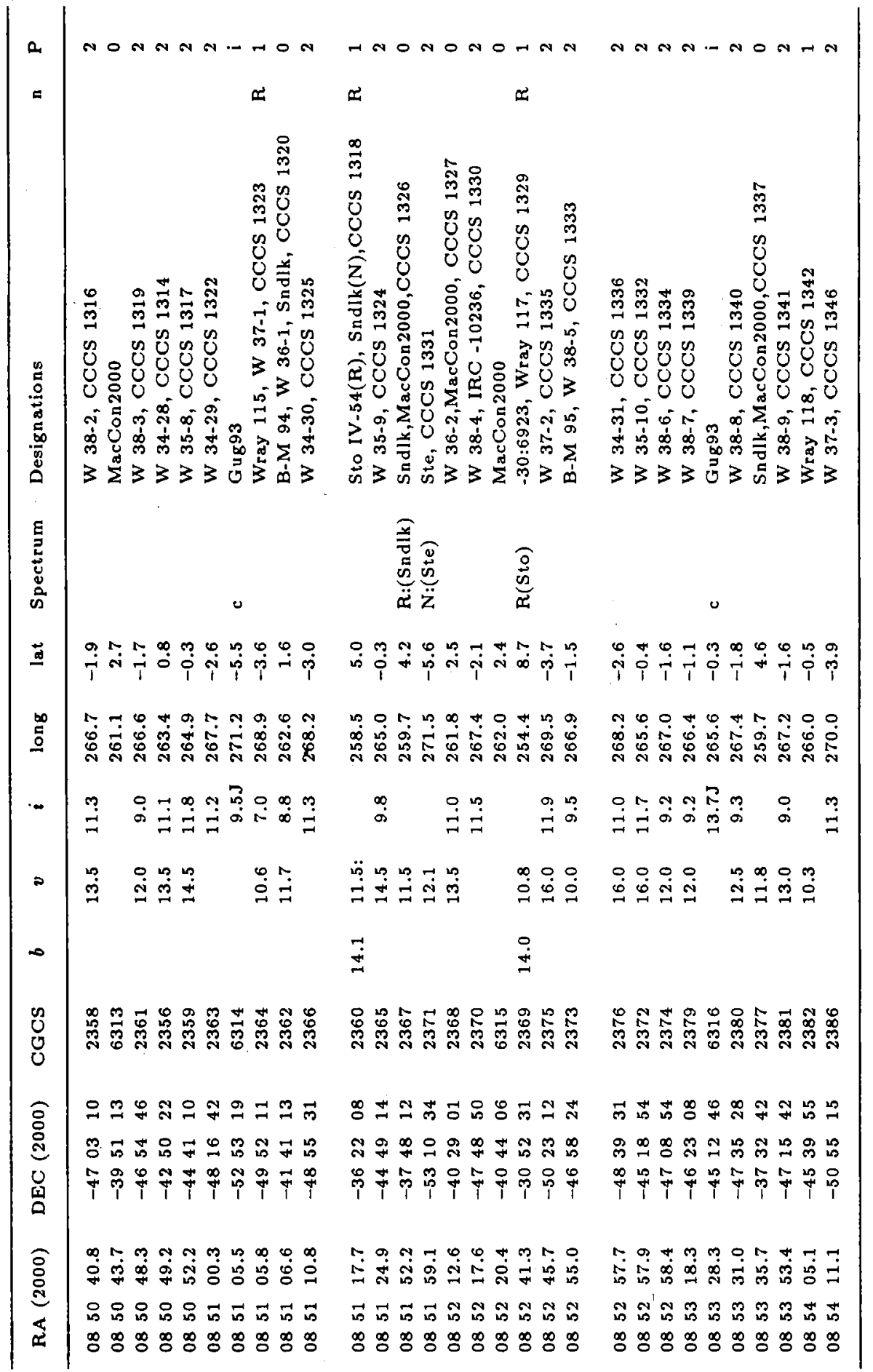




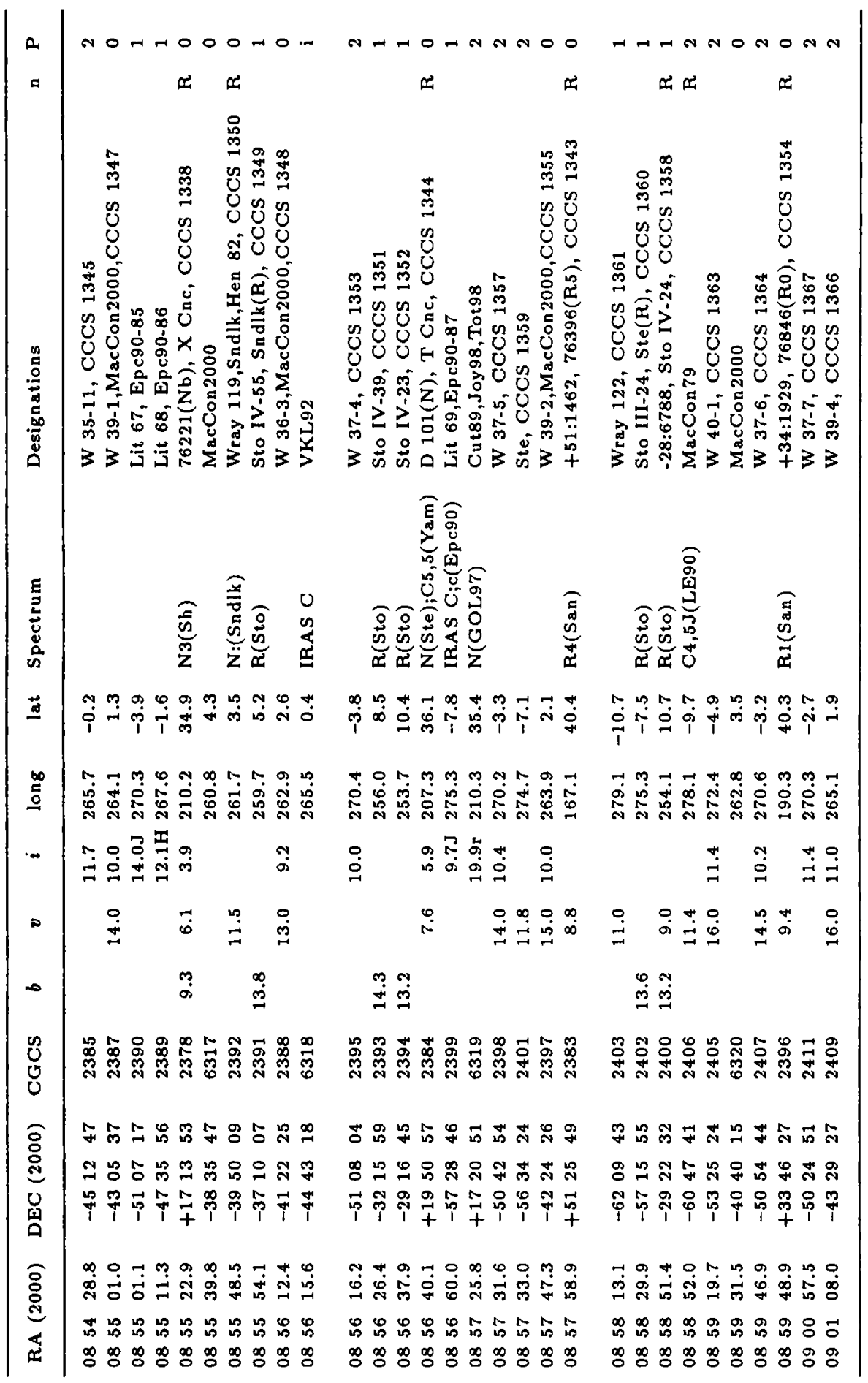




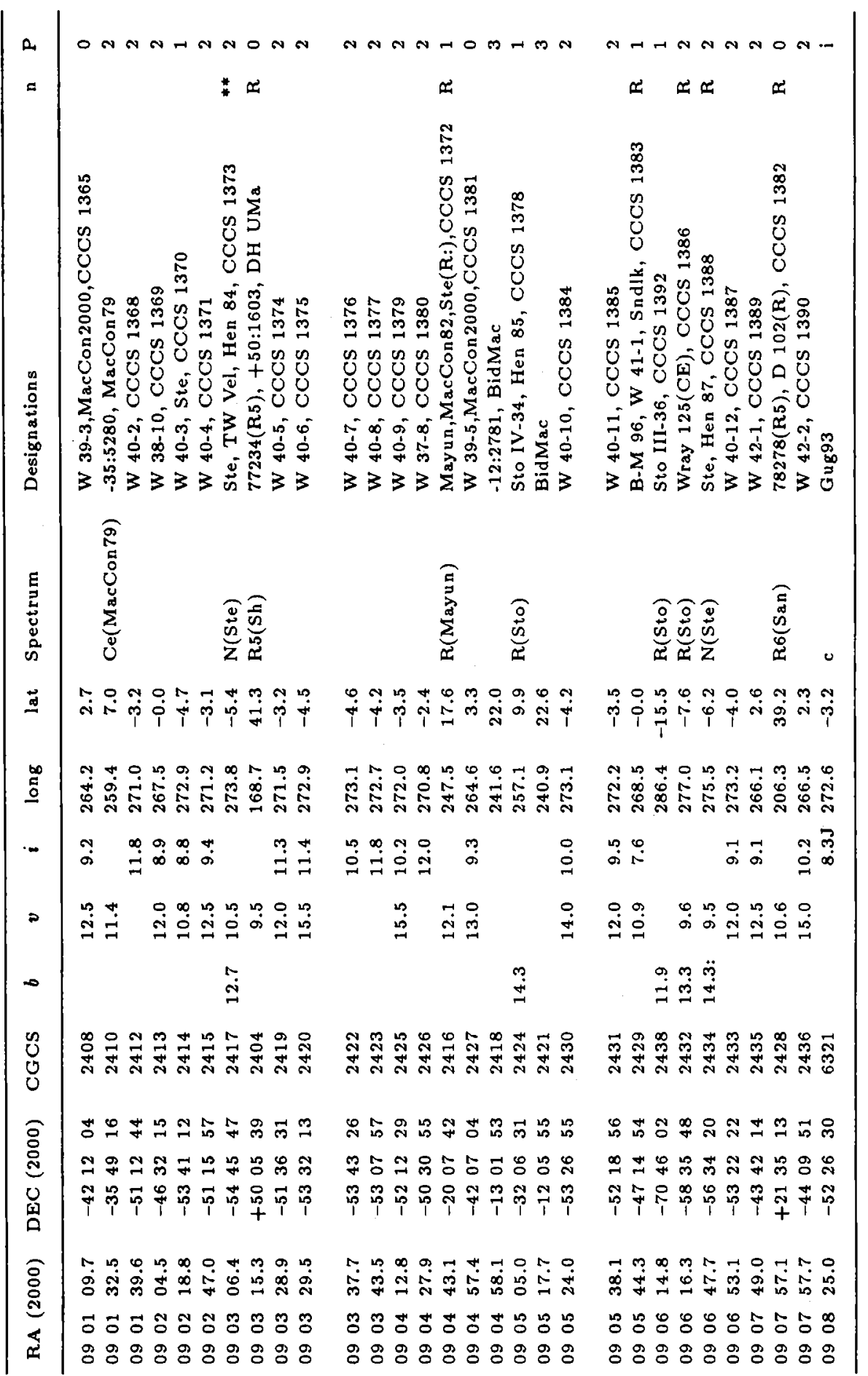




\begin{tabular}{|c|c|c|c|}
\hline$a$ & $n N n-n-O M A n$ & 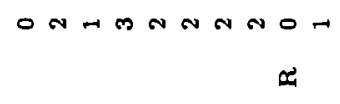 & 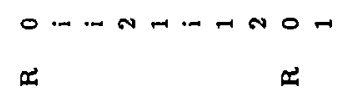 \\
\hline 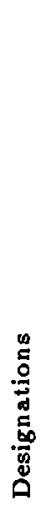 & 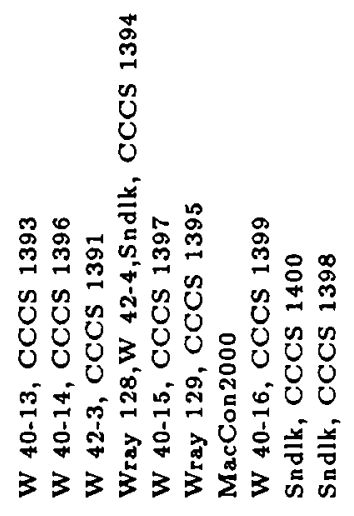 & 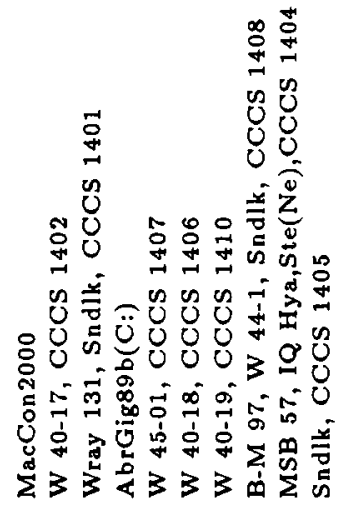 & 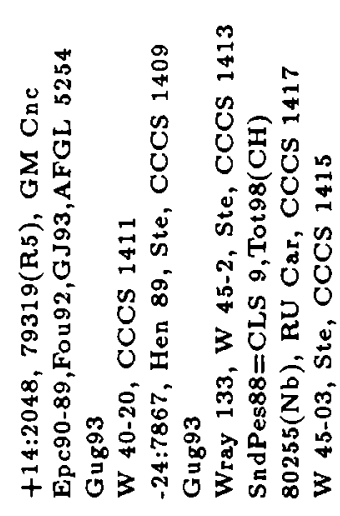 \\
\hline 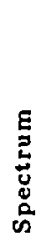 & & 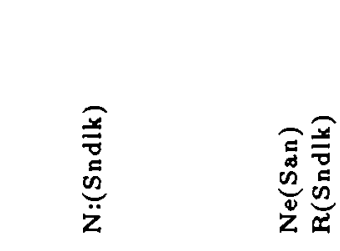 & 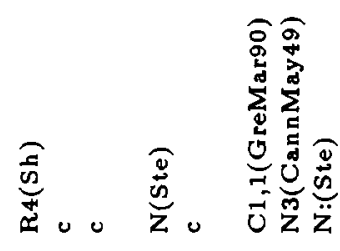 \\
\hline बु & 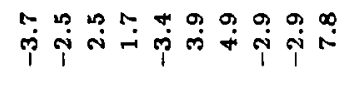 & 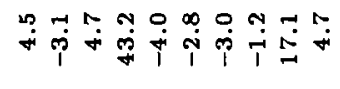 & 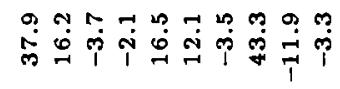 \\
\hline$\stackrel{\infty}{\tilde{g}}$ & 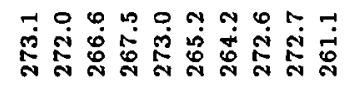 & 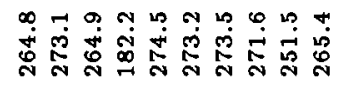 & 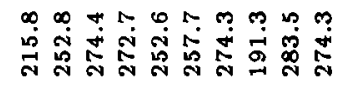 \\
\hline$\cdots$ & 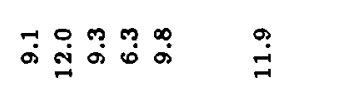 & 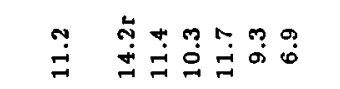 & 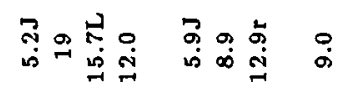 \\
\hline$=$ & 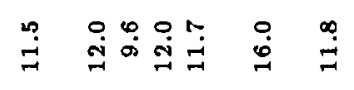 & 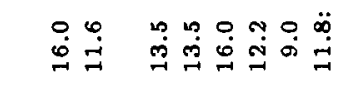 & $\stackrel{\circ}{\circ} \stackrel{0}{=} \stackrel{9}{\circ}$ \\
\hline 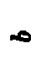 & $\stackrel{\dddot{I}}{ \pm}$ & $\stackrel{\leftrightarrow}{\stackrel{9}{\sim}}$ & $\stackrel{9}{\dot{\theta}} \overline{\dot{H}}$ \\
\hline 号 & 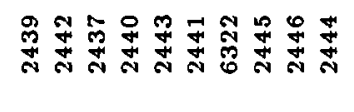 & 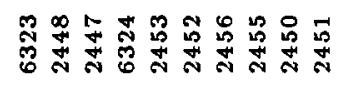 & 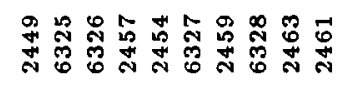 \\
\hline ळ & 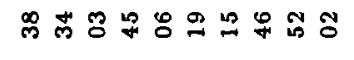 & సี స 용 & 吕需 \\
\hline 造 & 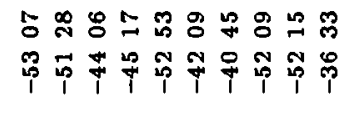 & 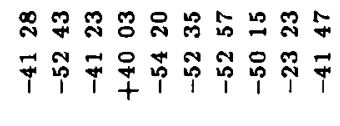 & 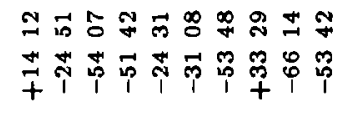 \\
\hline ڤั & 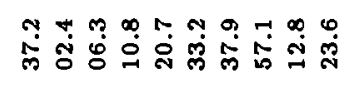 & 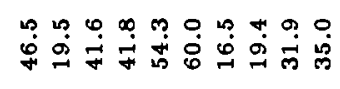 & 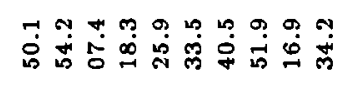 \\
\hline 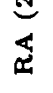 & 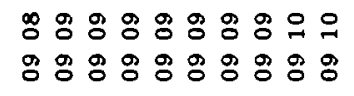 & 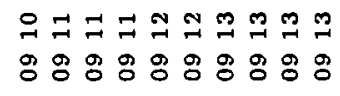 & 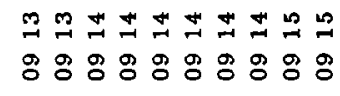 \\
\hline
\end{tabular}




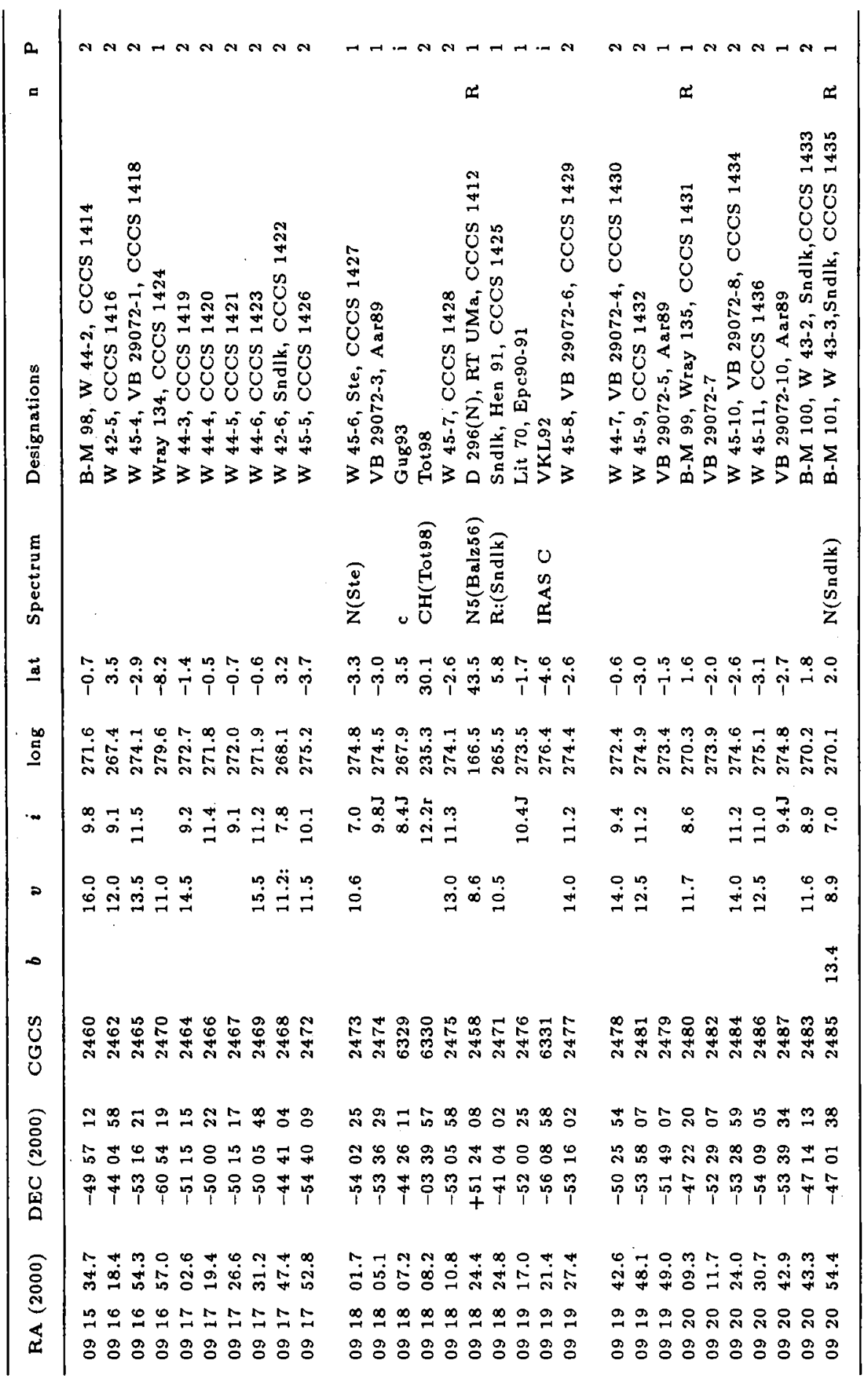




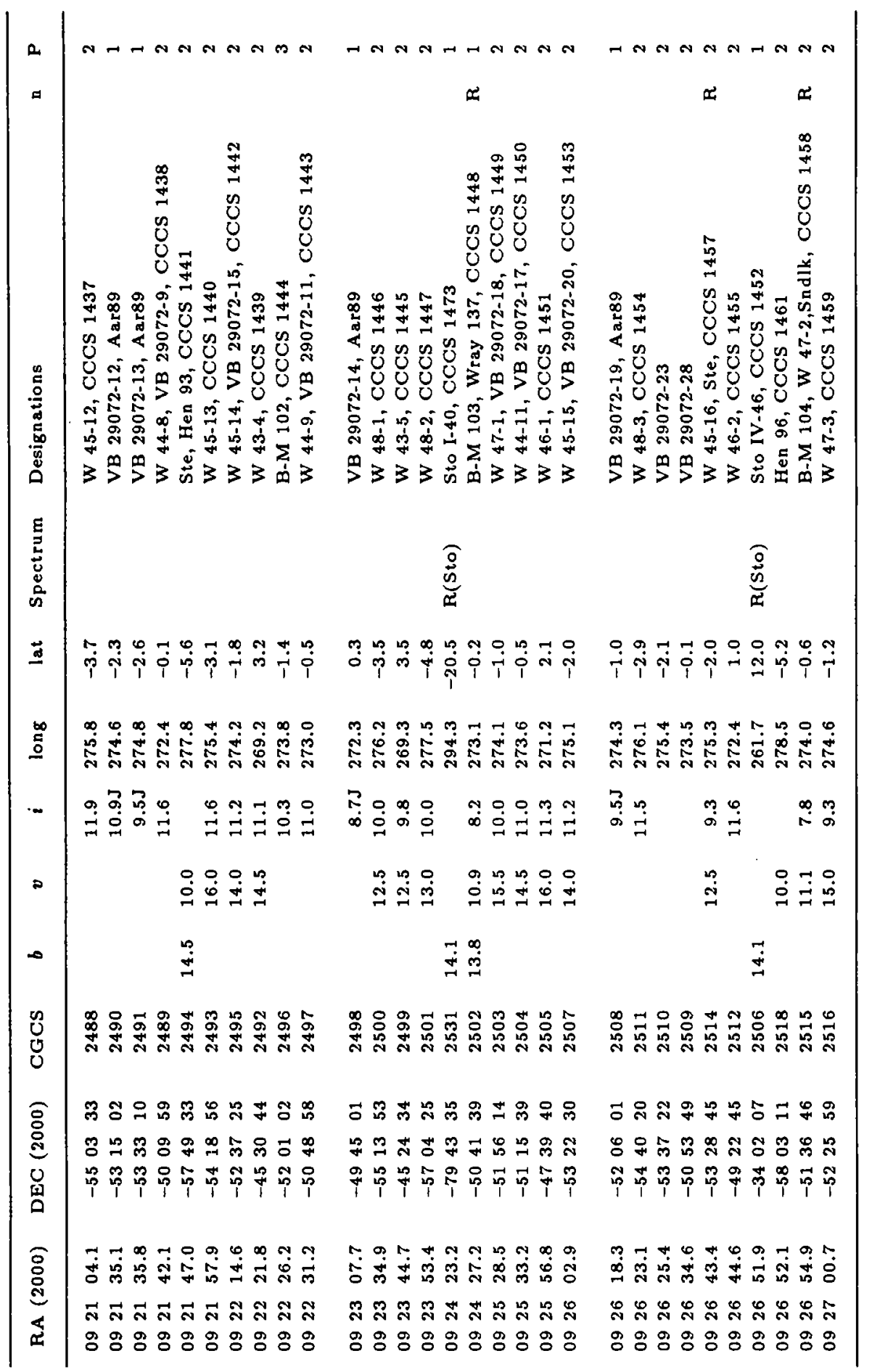




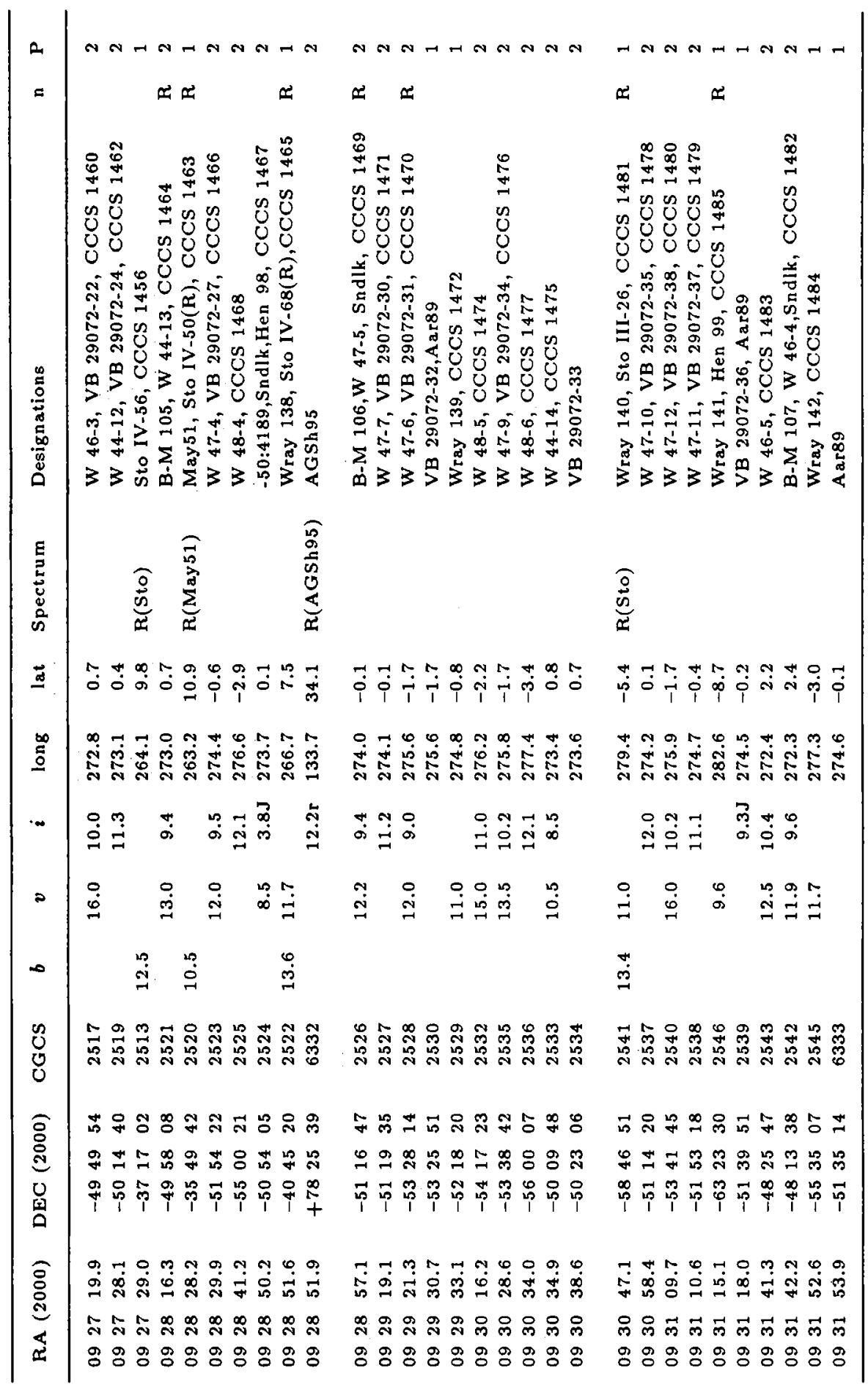




\begin{tabular}{|c|c|c|c|}
\hline a & 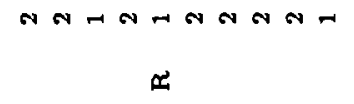 & 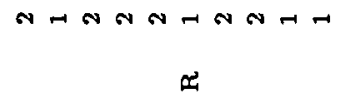 & 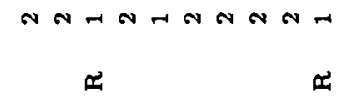 \\
\hline 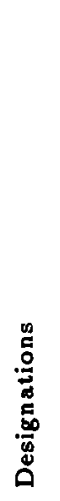 & 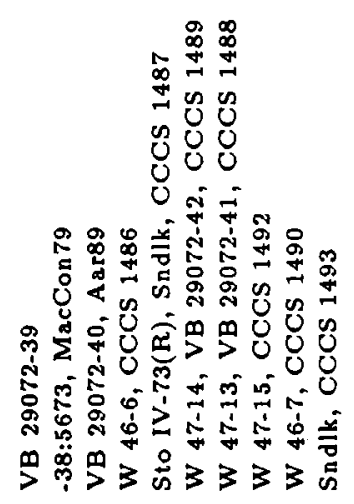 & 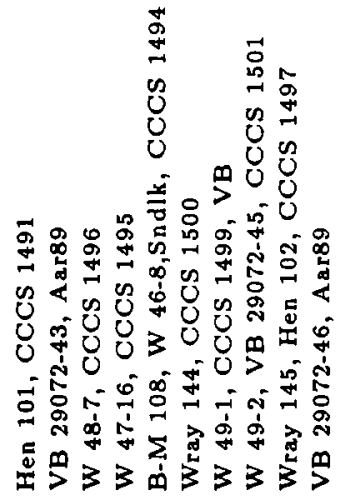 & 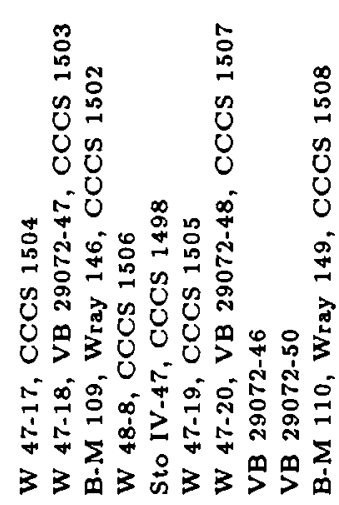 \\
\hline 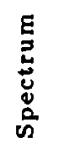 & 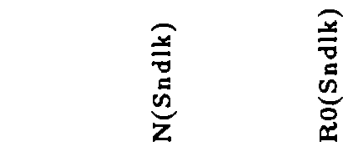 & & 高 \\
\hline$\underline{\Xi}$ & 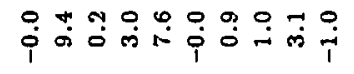 & 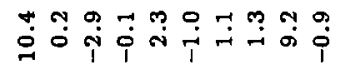 & 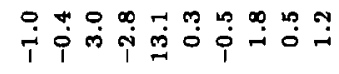 \\
\hline$\stackrel{\infty}{0}$ & 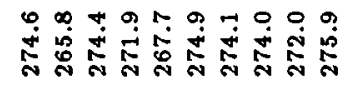 & 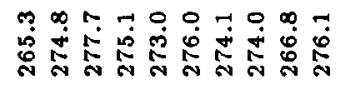 & 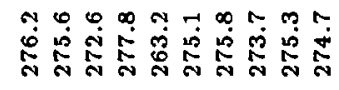 \\
\hline - & 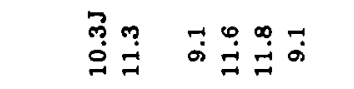 & 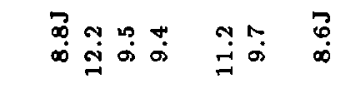 & 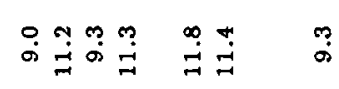 \\
\hline$=$ & 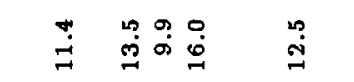 & 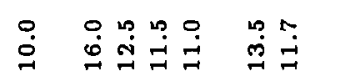 & 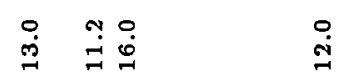 \\
\hline$\infty$ & $\stackrel{\infty}{\stackrel{\infty}{\sim}}$ & & $\underset{\ddot{g}}{\ddot{g}}$ \\
\hline $\begin{array}{l}y \\
y \\
y\end{array}$ & 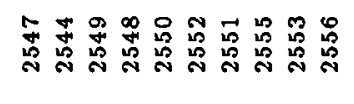 & 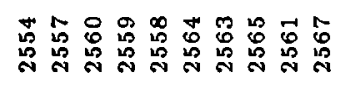 & 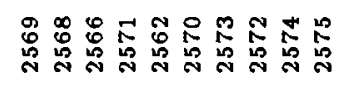 \\
\hline 응 & 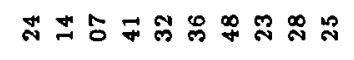 & 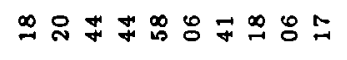 & 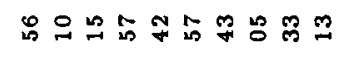 \\
\hline 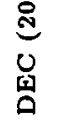 & 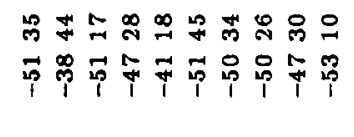 & 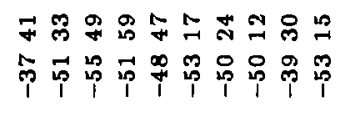 & 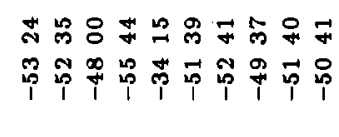 \\
\hline के & 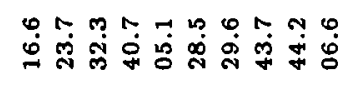 & 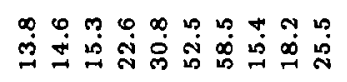 & 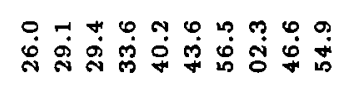 \\
\hline 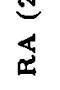 & 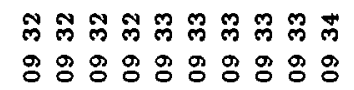 & 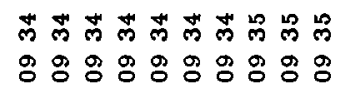 & 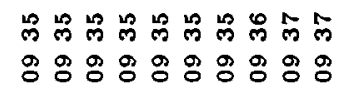 \\
\hline
\end{tabular}




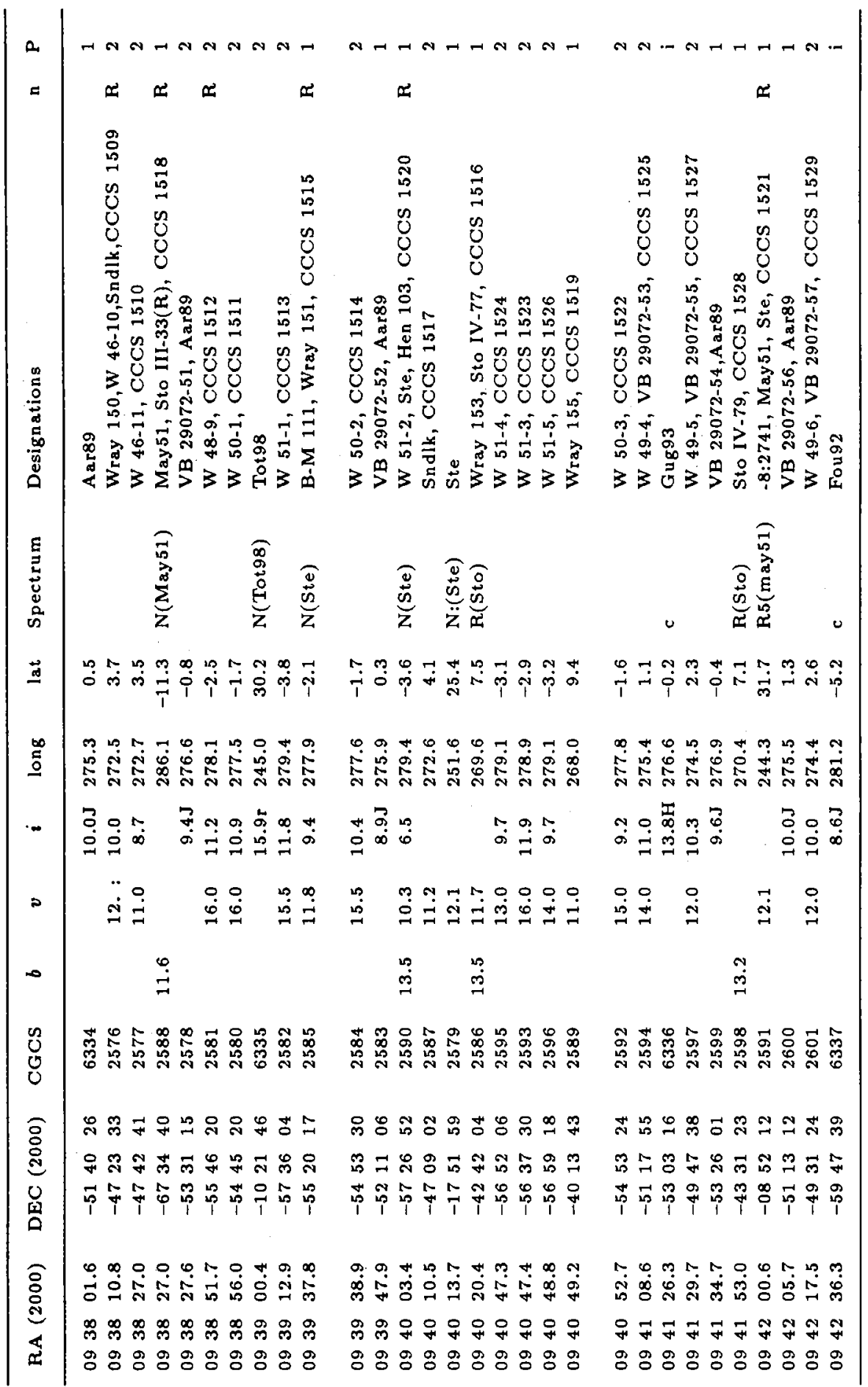




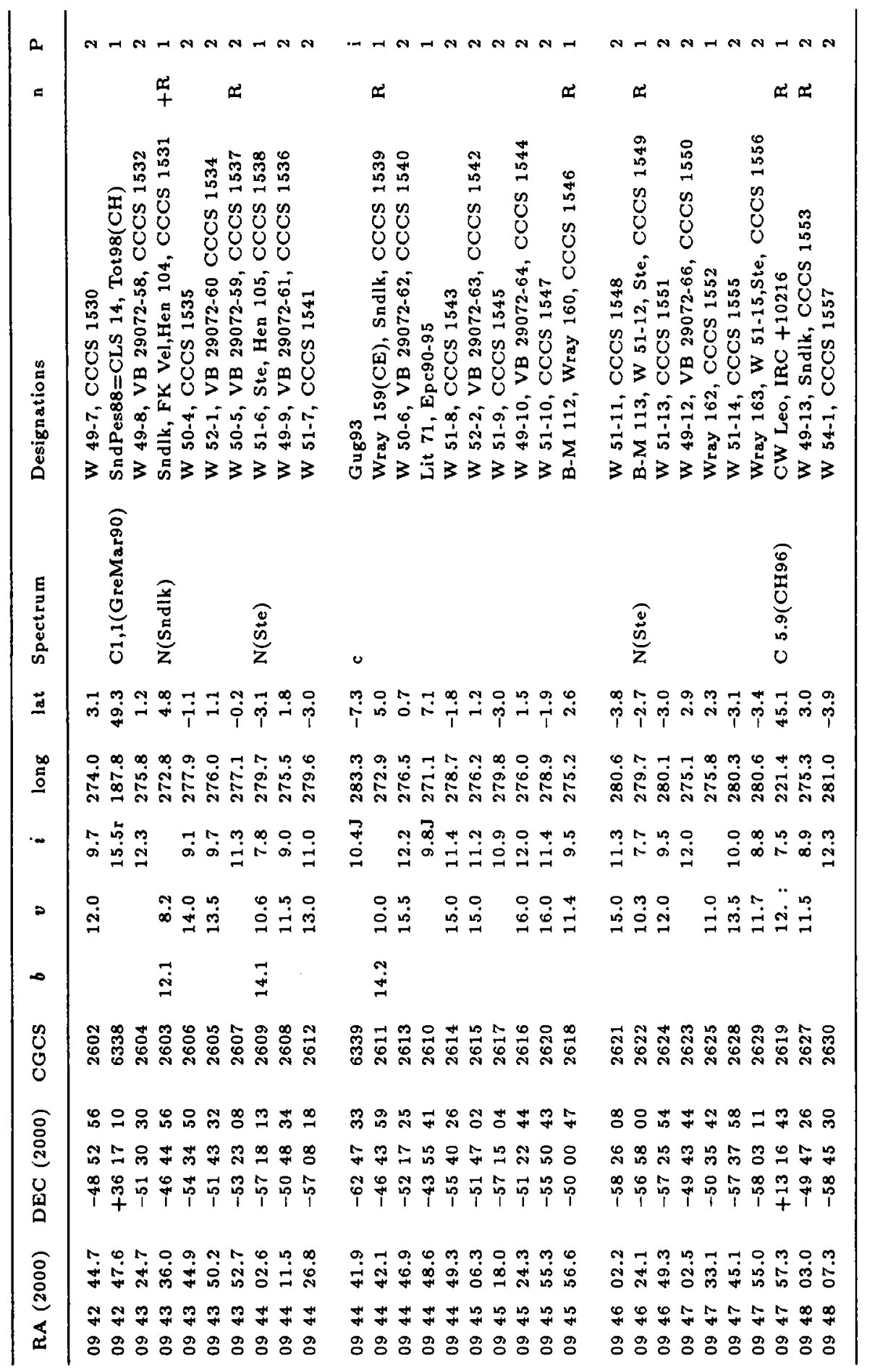




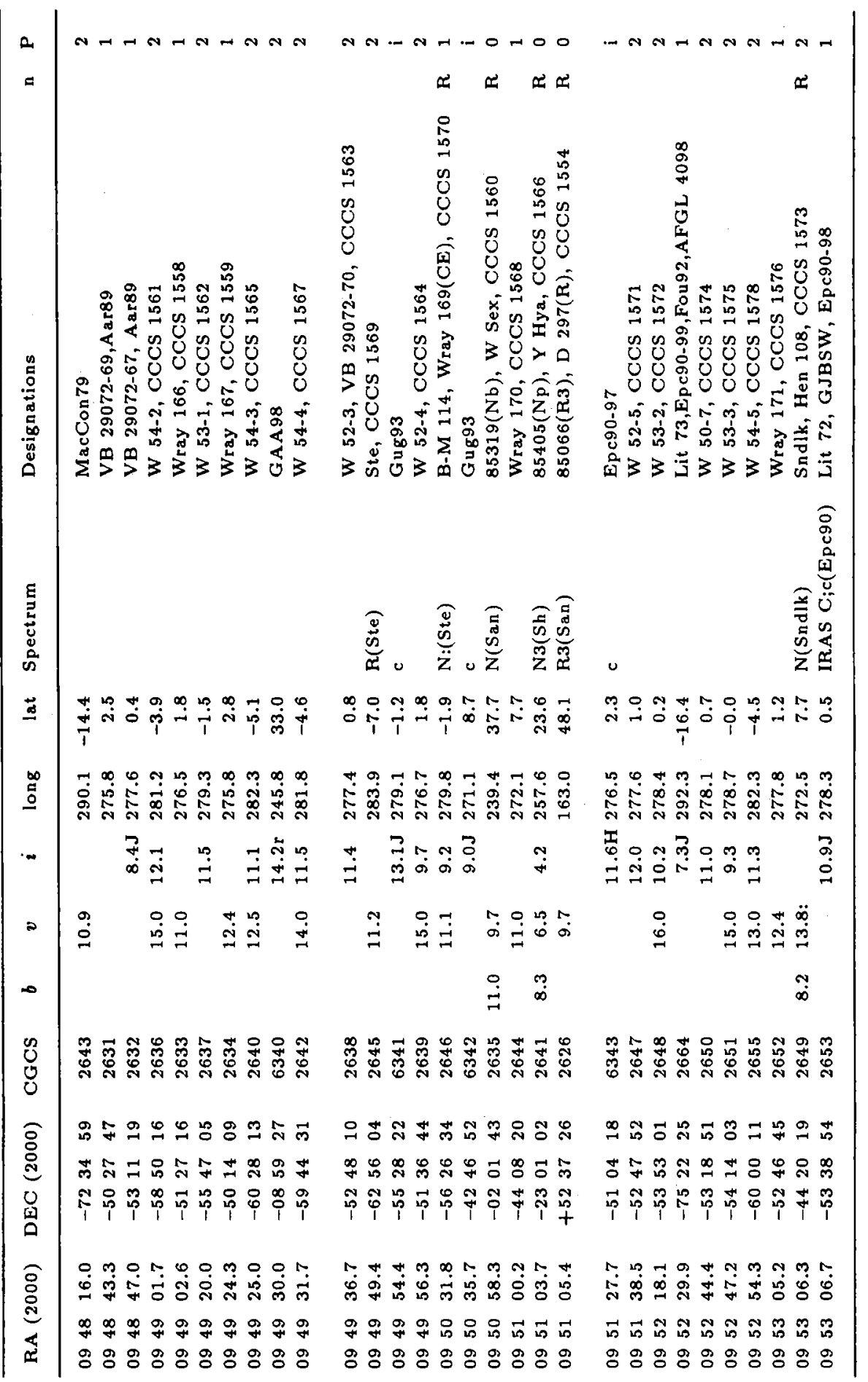




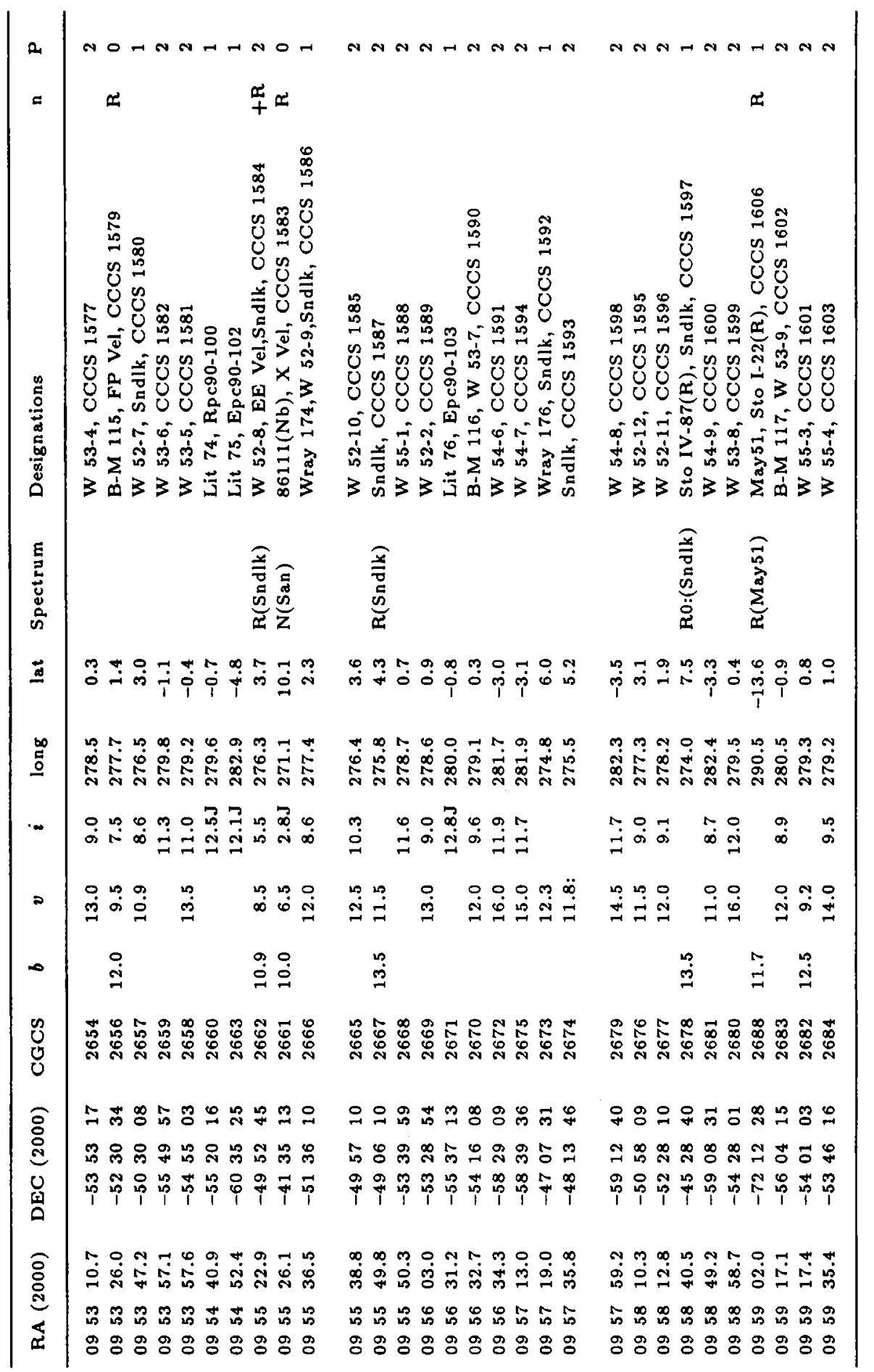




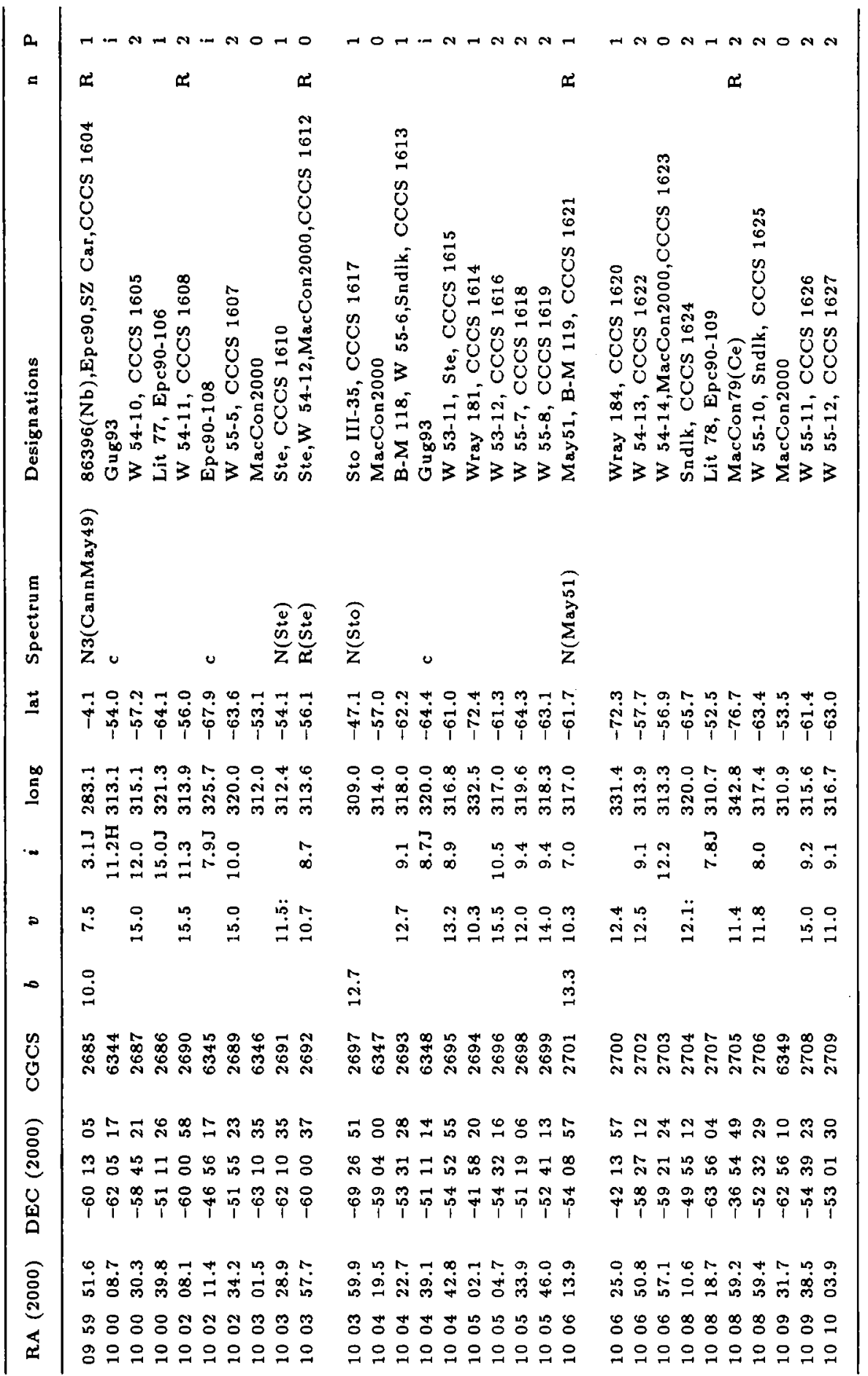




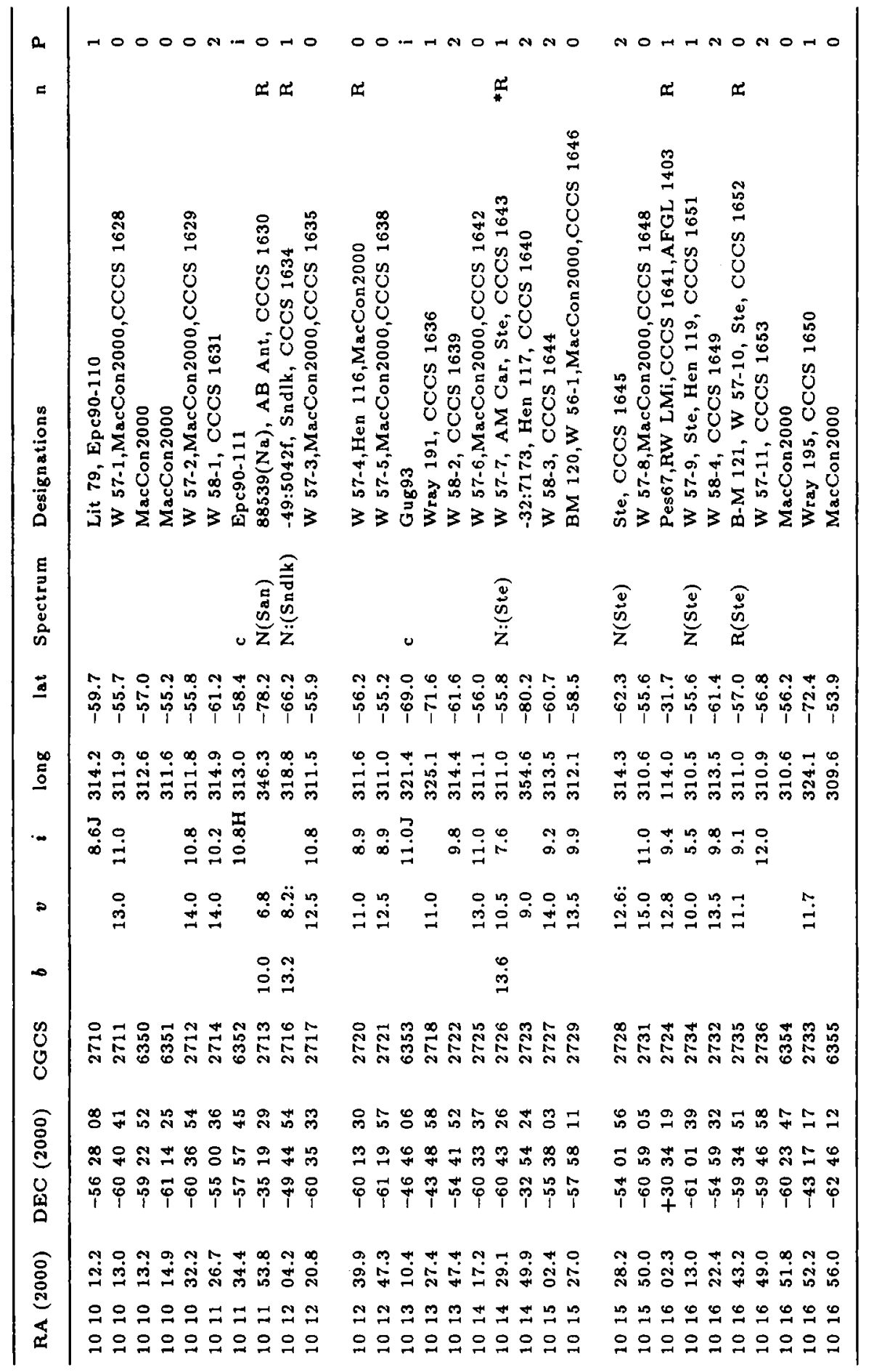




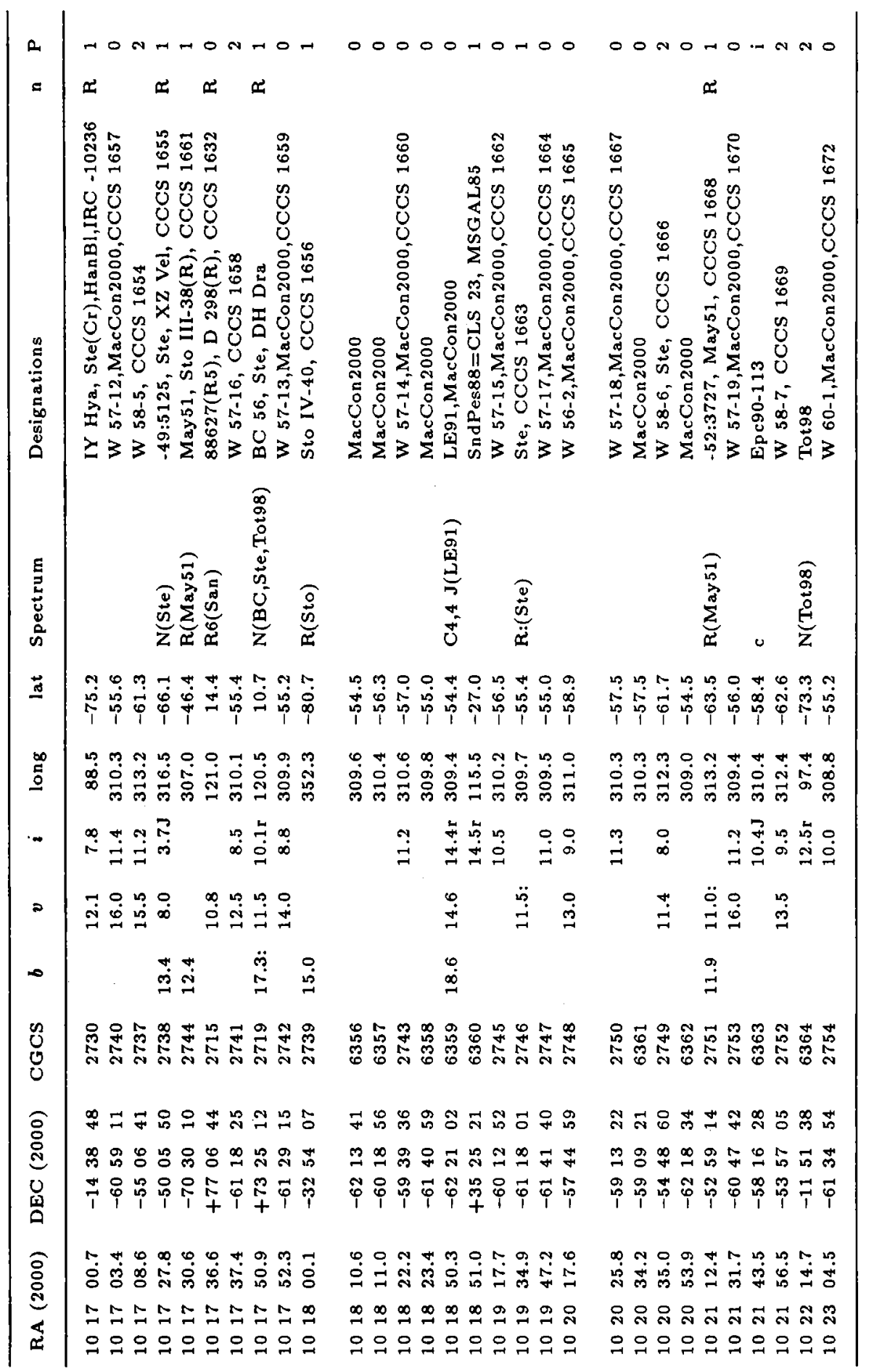




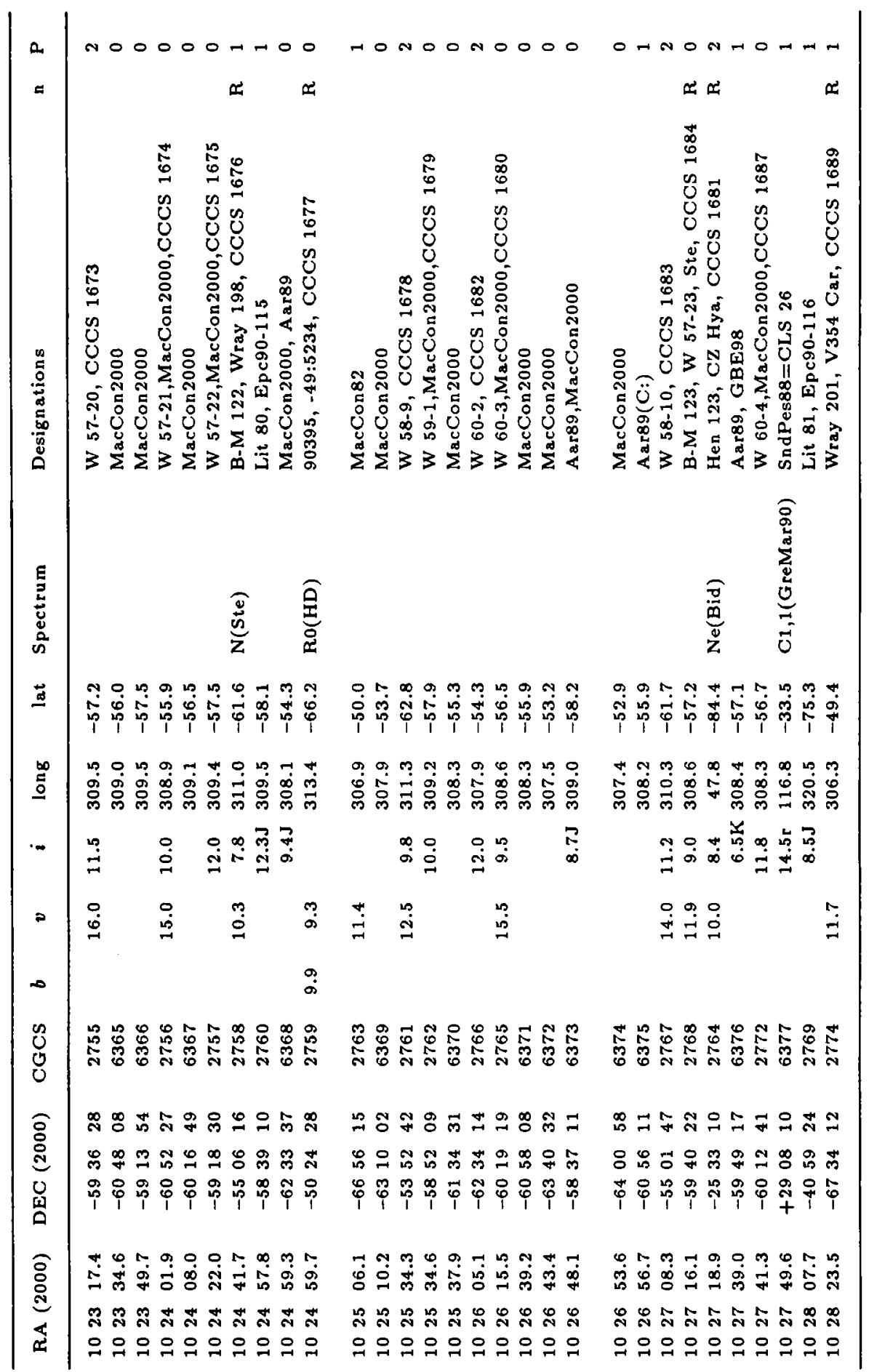




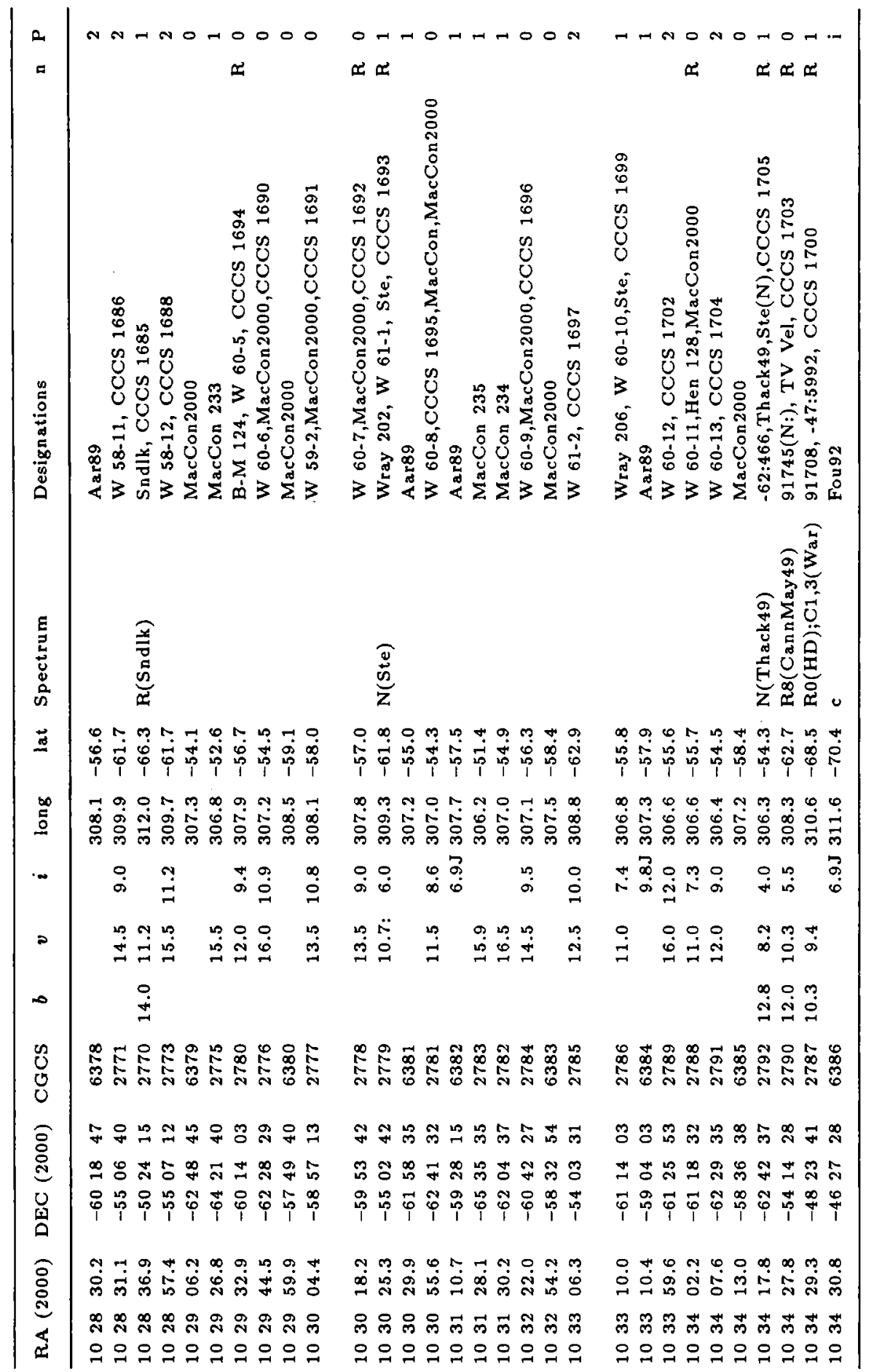




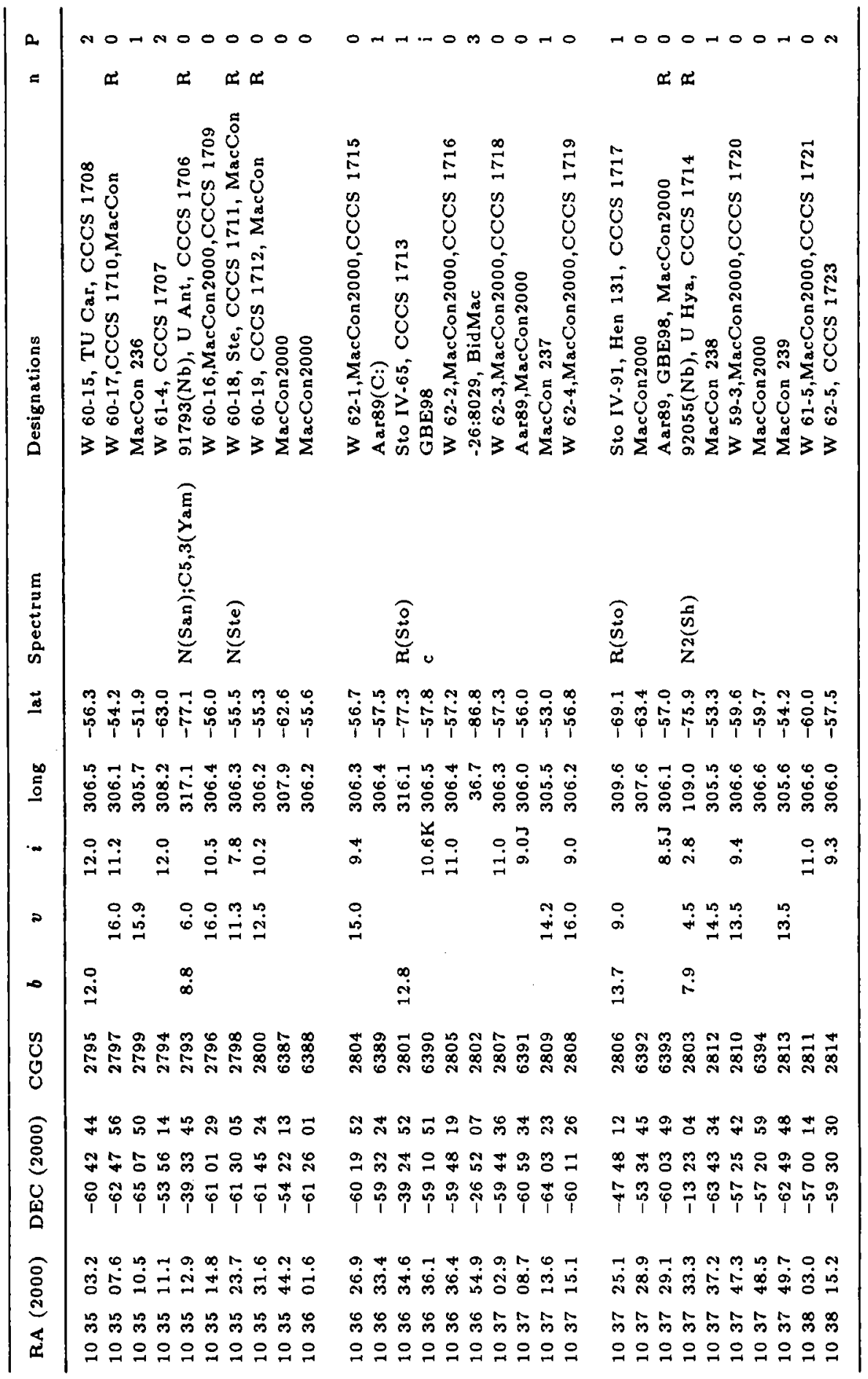




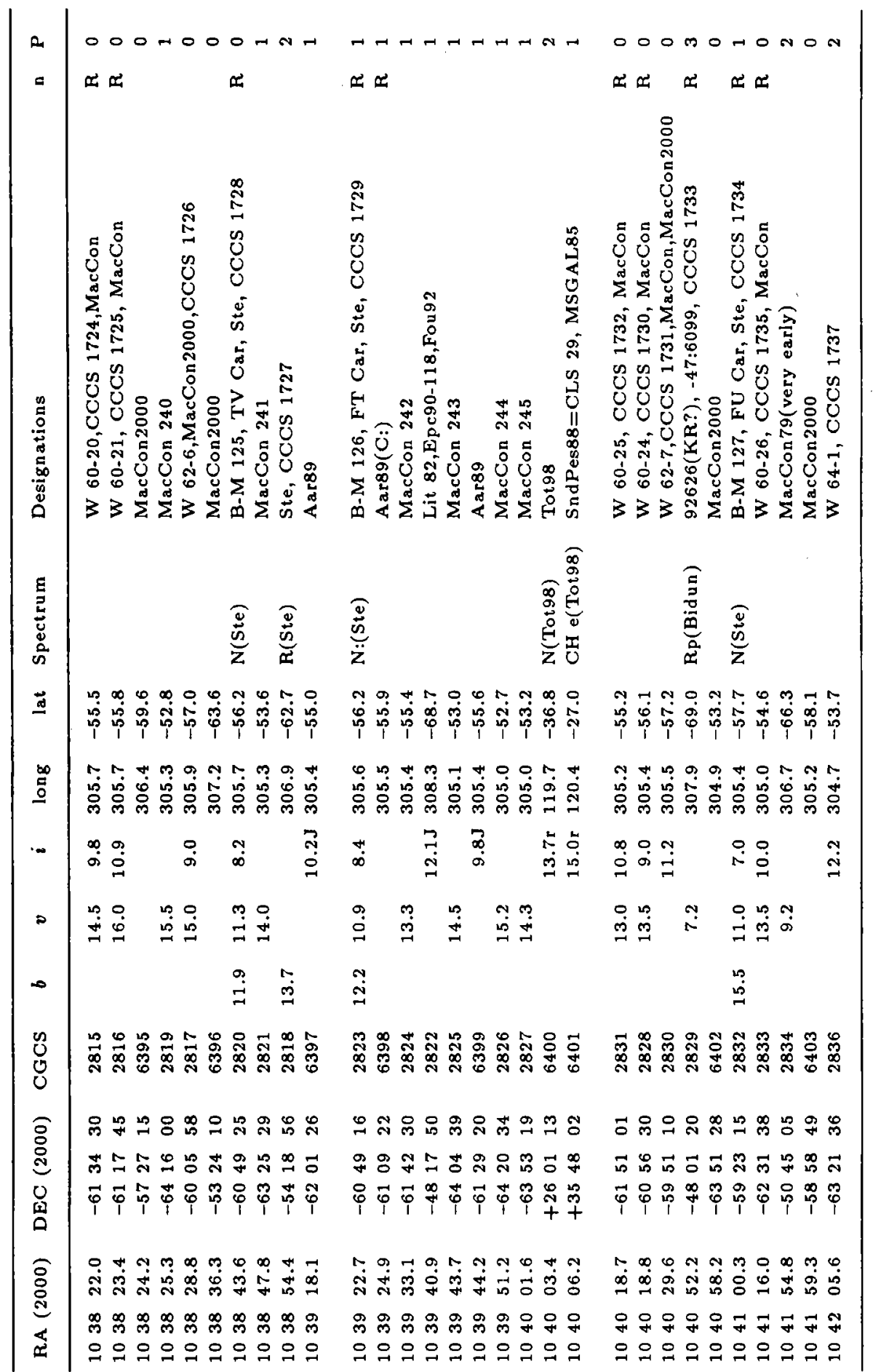




\begin{tabular}{|c|c|c|c|}
\hline a & 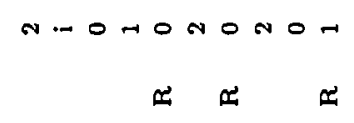 & $\begin{array}{l}0.0 M 0 n O H-h \\
0\end{array}$ & 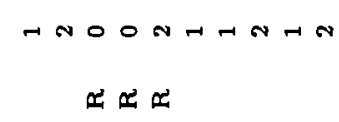 \\
\hline 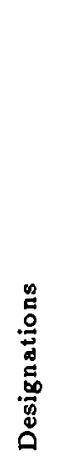 & 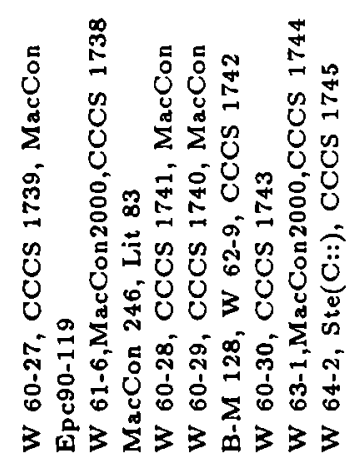 & 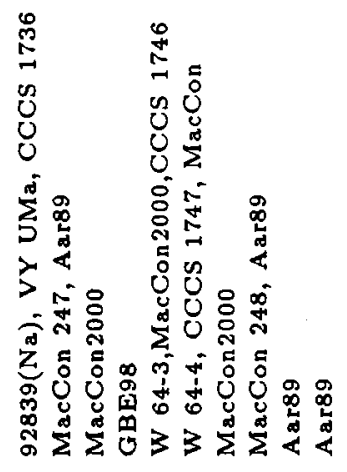 & 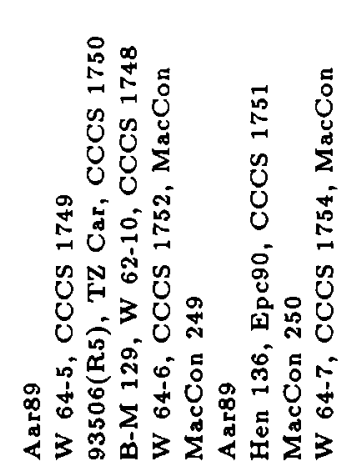 \\
\hline 吾 & 0 & 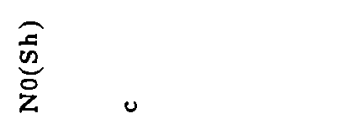 & $\begin{array}{l}\bar{y} \\
\stackrel{5}{Z} \\
z\end{array}$ \\
\hline$\underline{\underline{a}}$ & 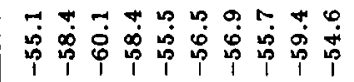 & 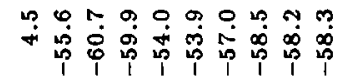 & 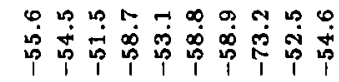 \\
\hline$\stackrel{\infty}{g}$ & 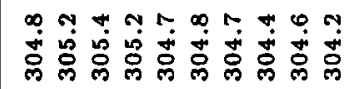 & 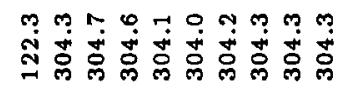 & 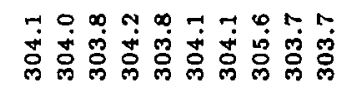 \\
\hline- & 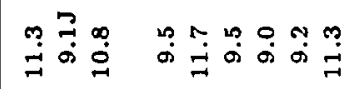 & 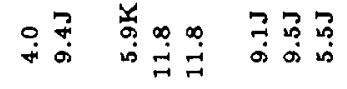 & 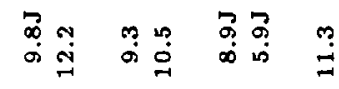 \\
\hline D & 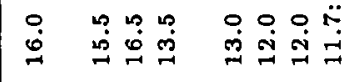 & 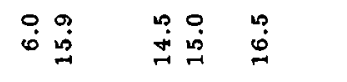 & 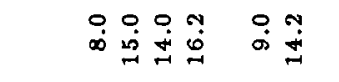 \\
\hline ـ & & $\stackrel{0}{\infty}$ & $\ddot{\infty}$ \\
\hline $\begin{array}{l}n \\
0 \\
0 \\
0\end{array}$ & 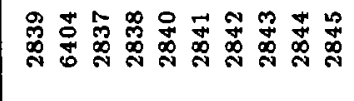 & 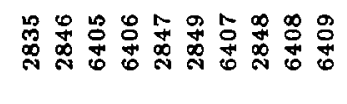 & 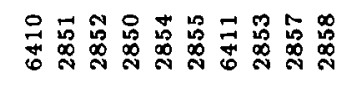 \\
\hline & 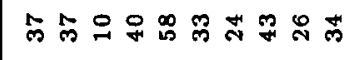 & 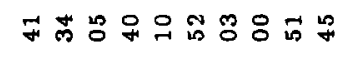 & ณำ \\
\hline $\begin{array}{l}\stackrel{8}{0} \\
\text { 产 } \\
\text { 至 }\end{array}$ & 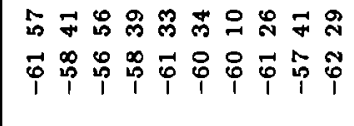 & 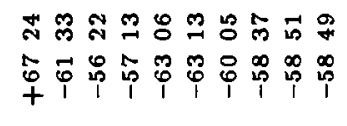 & 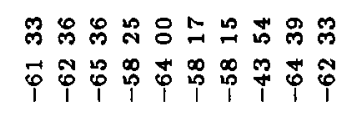 \\
\hline จे & 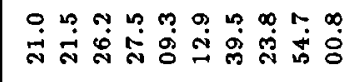 & 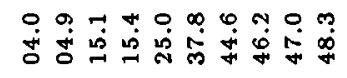 & 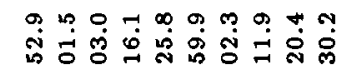 \\
\hline 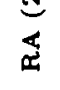 & 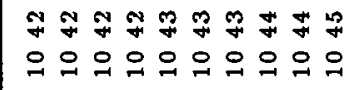 & 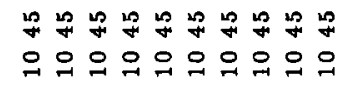 & 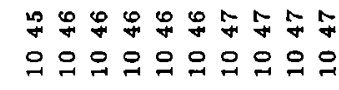 \\
\hline
\end{tabular}




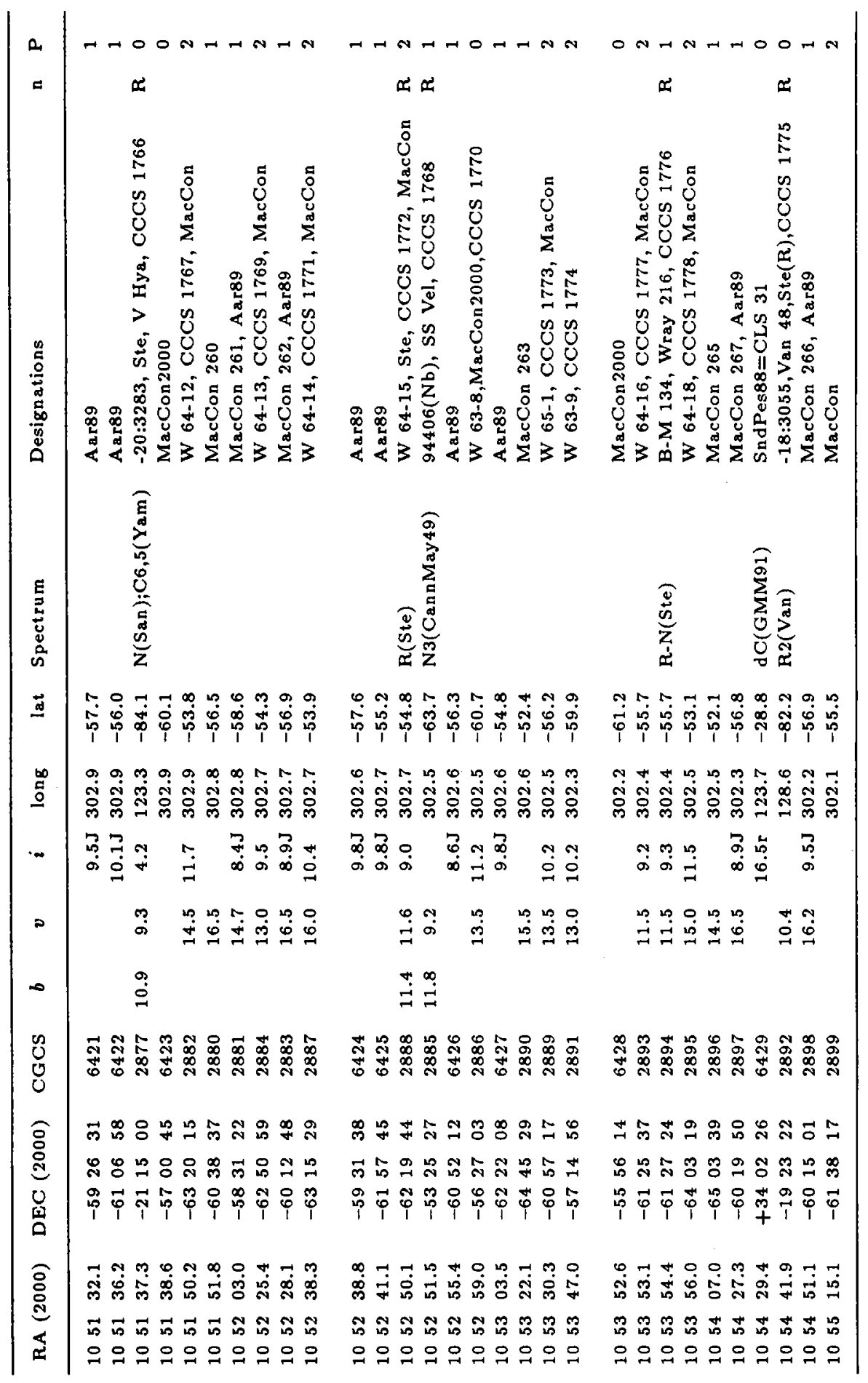




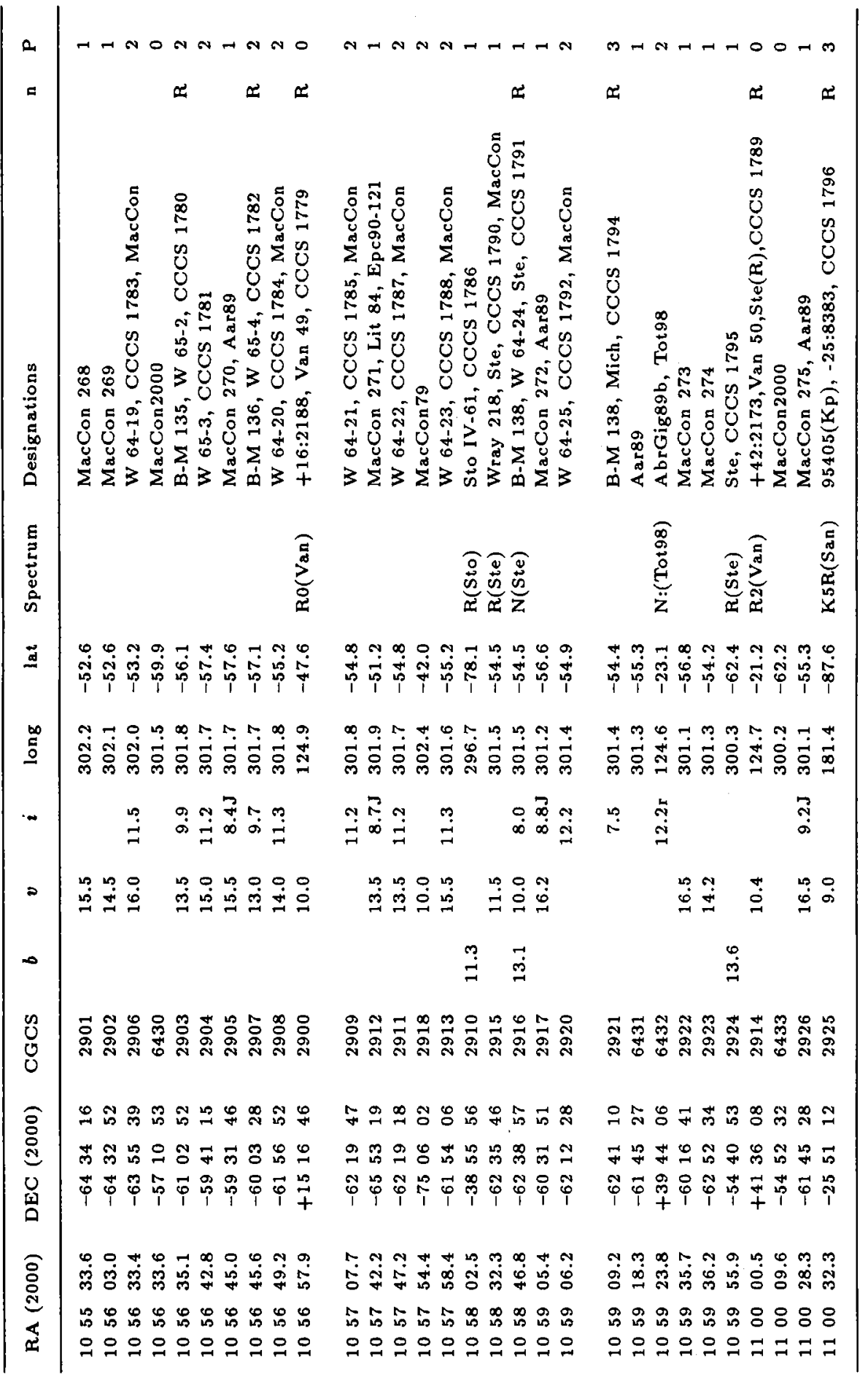




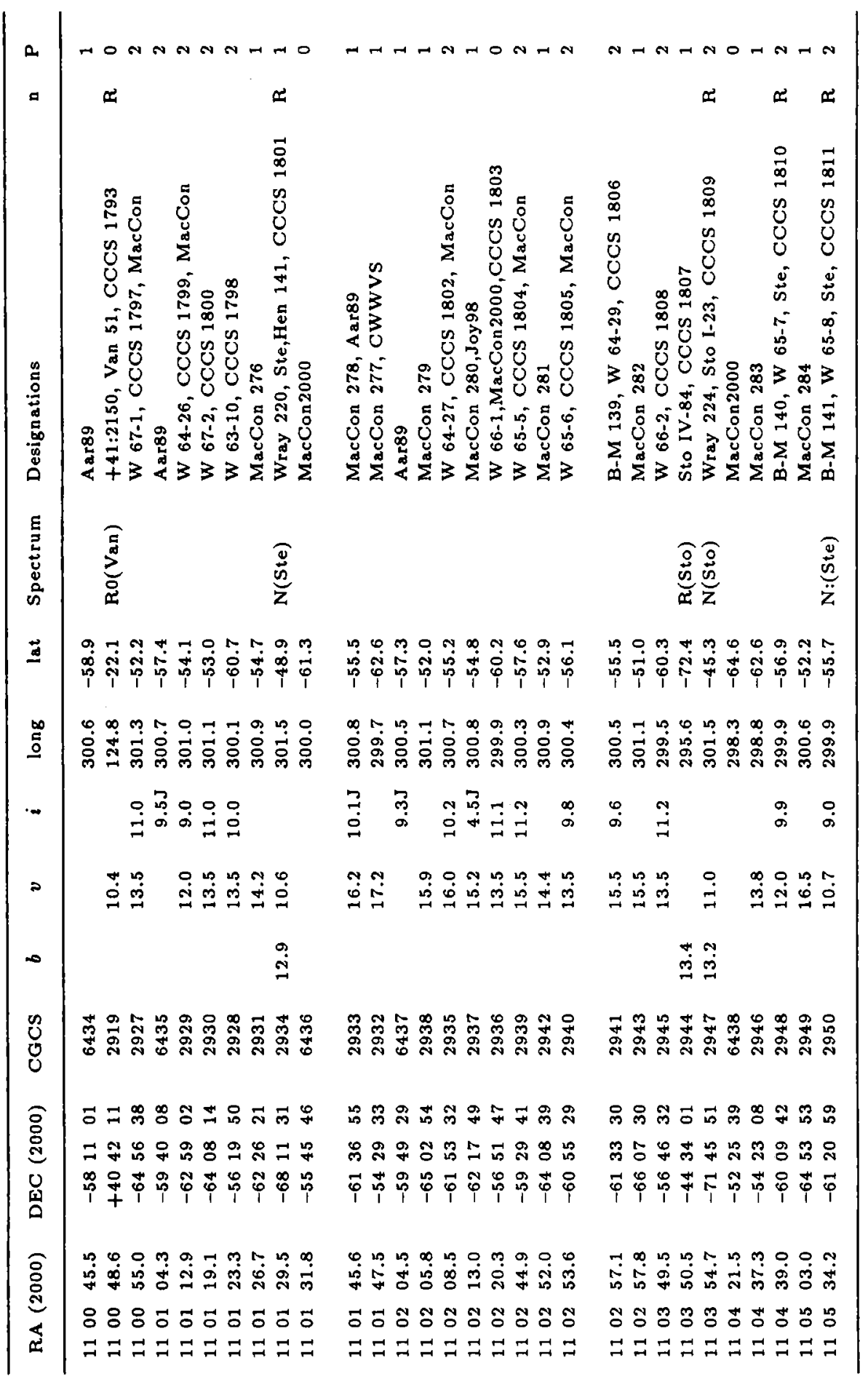




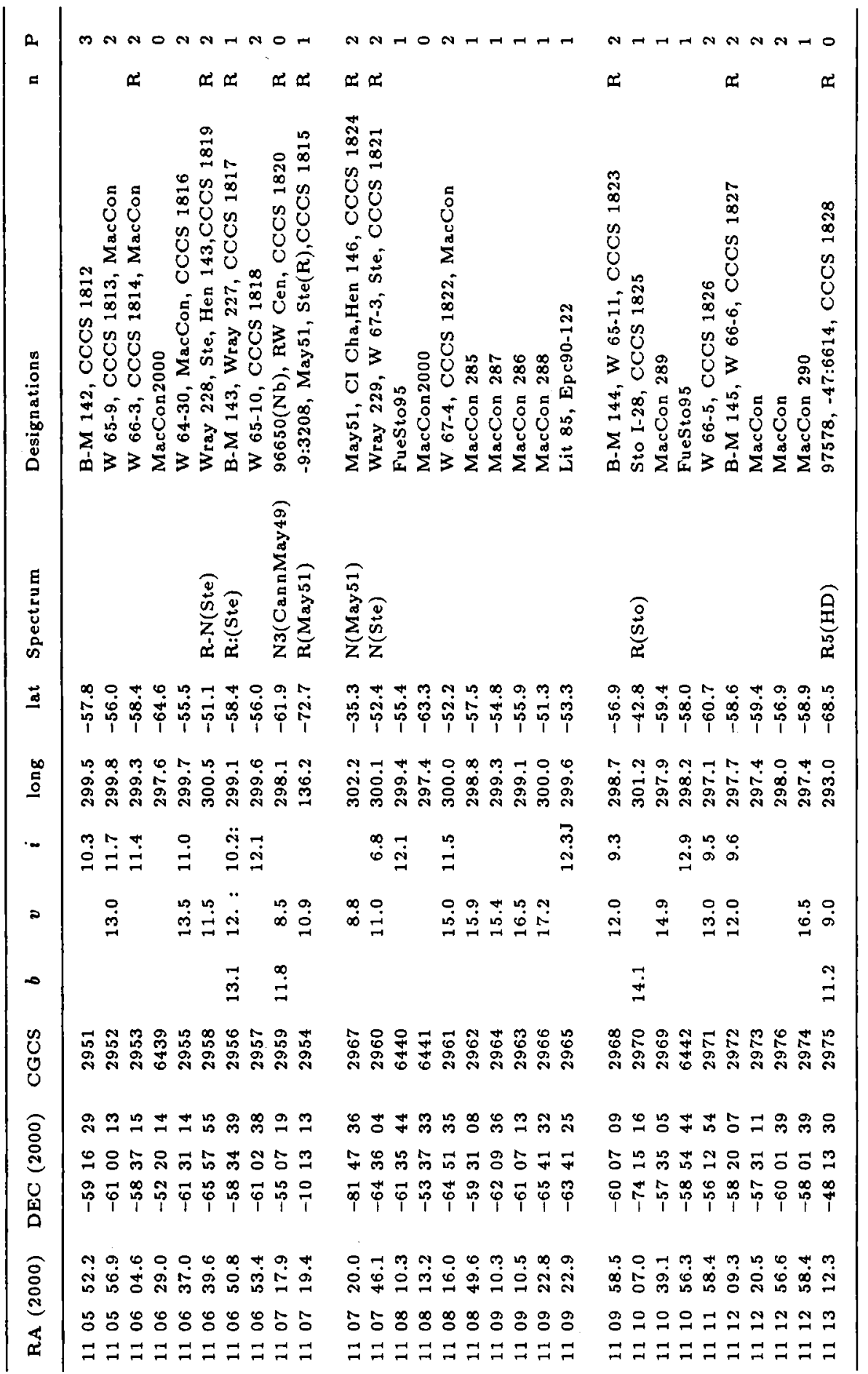




\begin{tabular}{|c|c|c|c|}
\hline a & 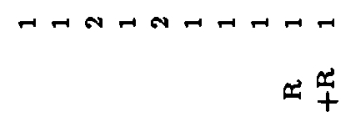 & 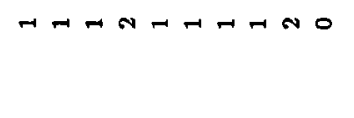 & 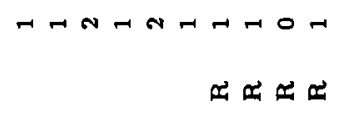 \\
\hline 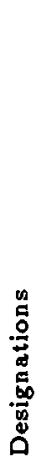 & 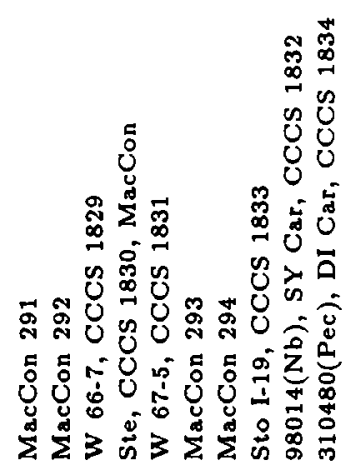 & 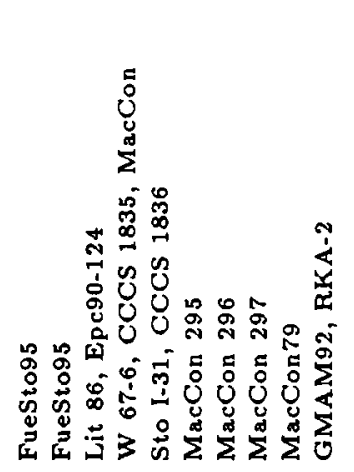 & 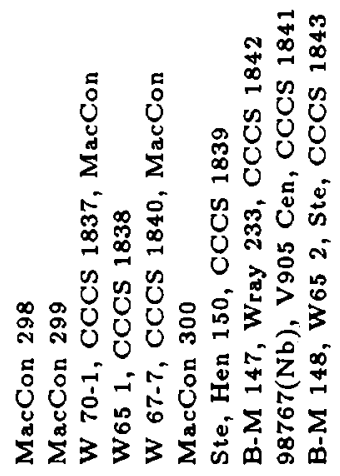 \\
\hline 皇 & 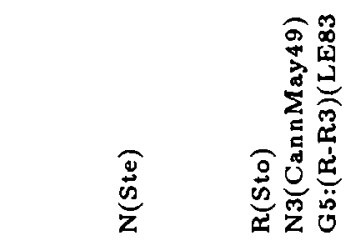 & 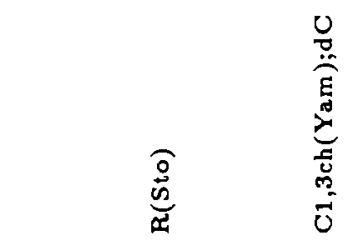 & 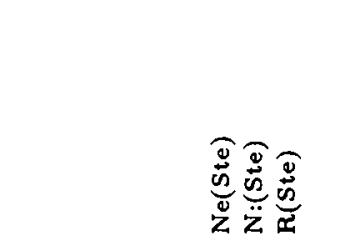 \\
\hline$\Xi$ & 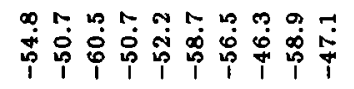 & 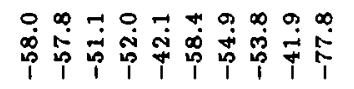 & 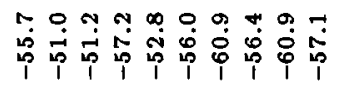 \\
\hline$\stackrel{\infty}{\Xi}$ & 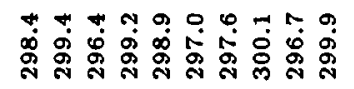 & 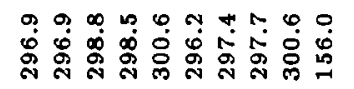 & 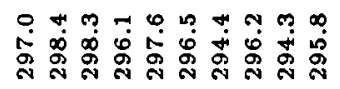 \\
\hline- & $\stackrel{\circ}{\circ} \stackrel{\circ}{=}$ & 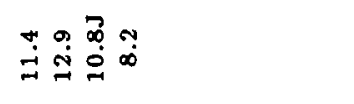 & 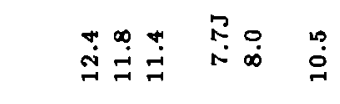 \\
\hline 2 & 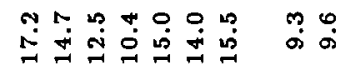 & 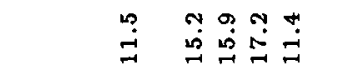 & 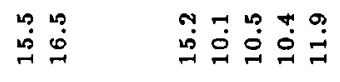 \\
\hline هـ & $\underset{\dot{j}}{\dot{j}} \stackrel{\infty}{=} \stackrel{\infty}{=}$ & $\stackrel{\circ}{\stackrel{i}{i}}$ & $\tilde{\sigma}$ \\
\hline 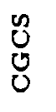 & 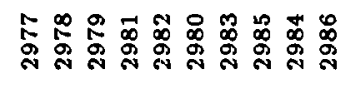 & 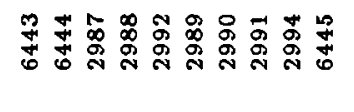 & 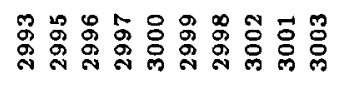 \\
\hline ๖े & 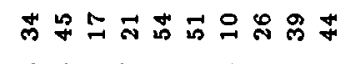 & 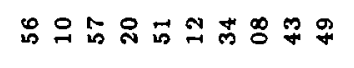 & 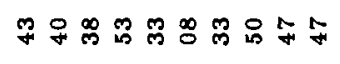 \\
\hline $\begin{array}{l}\text { Si } \\
0 \\
0 \\
0\end{array}$ & 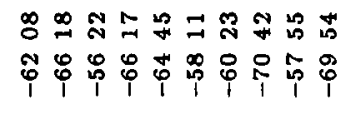 & 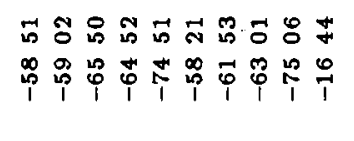 & $\begin{array}{l}\infty \\
0 \\
0\end{array}$ \\
\hline ڤ్రి & 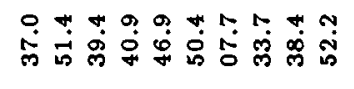 & 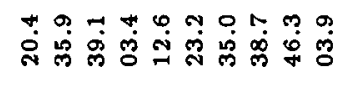 & 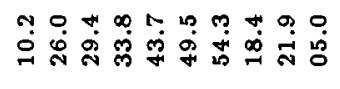 \\
\hline 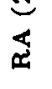 & 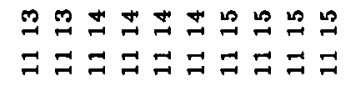 & 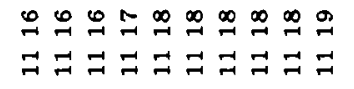 & 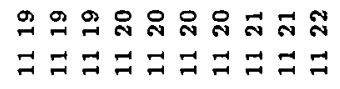 \\
\hline
\end{tabular}




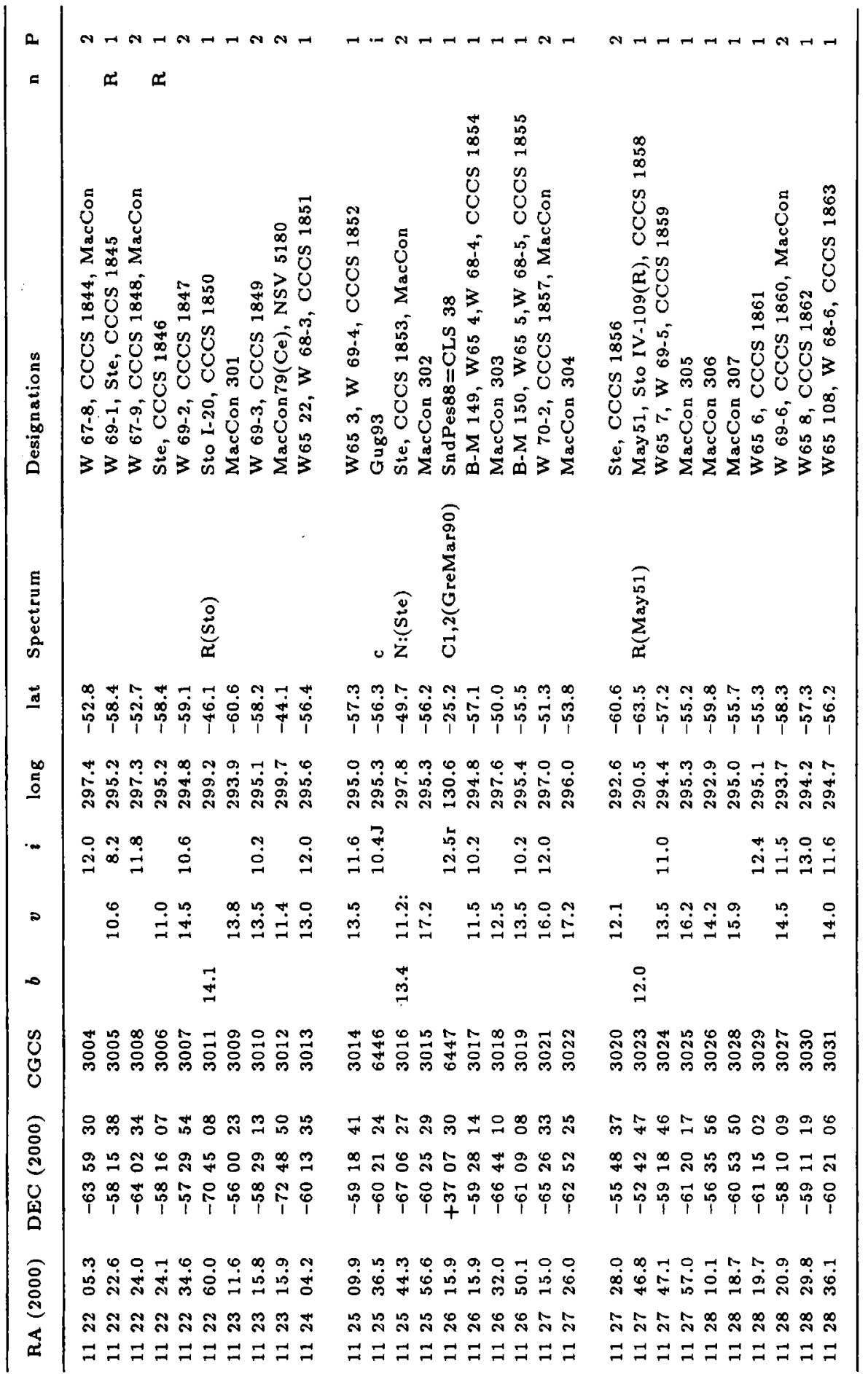




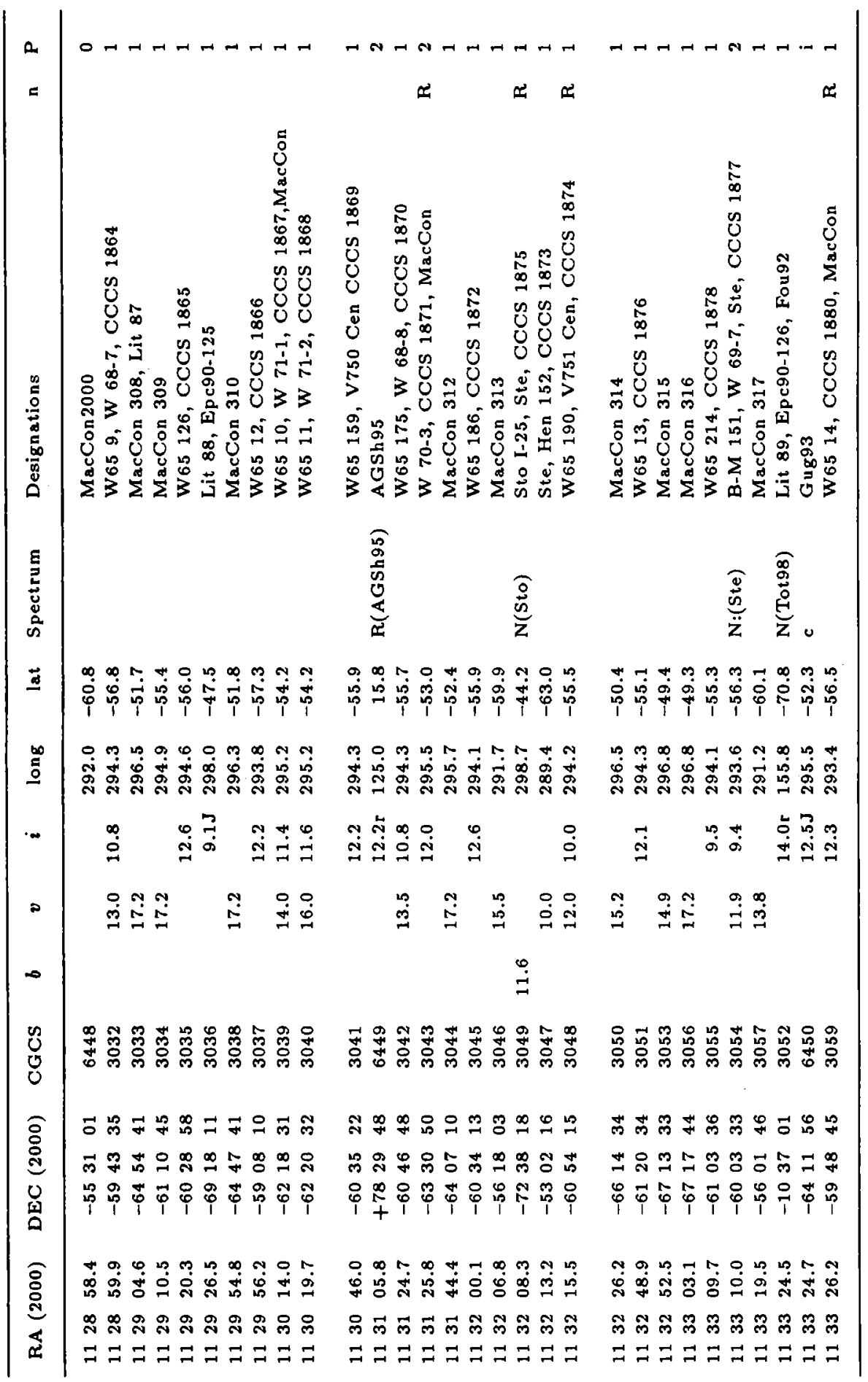




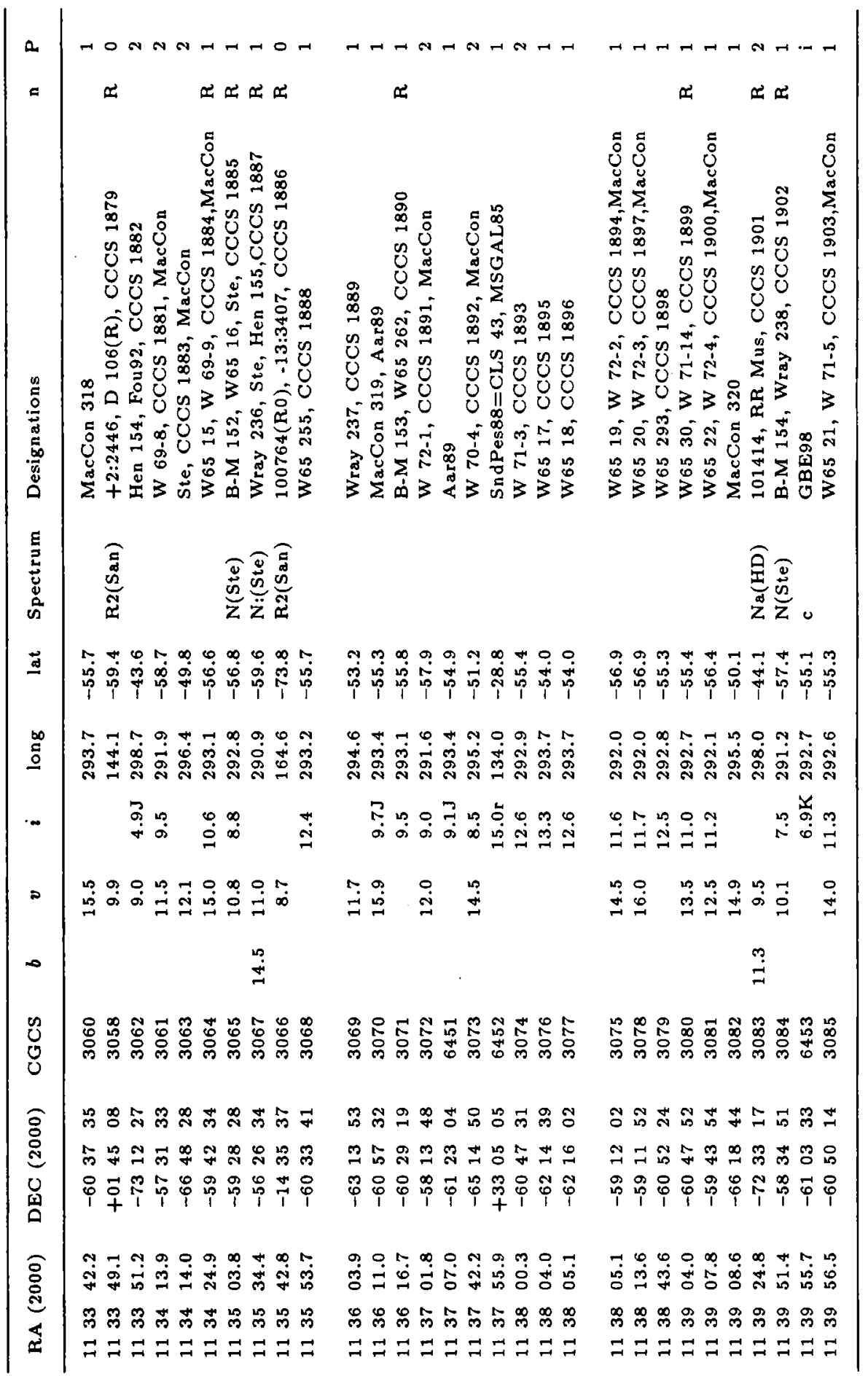




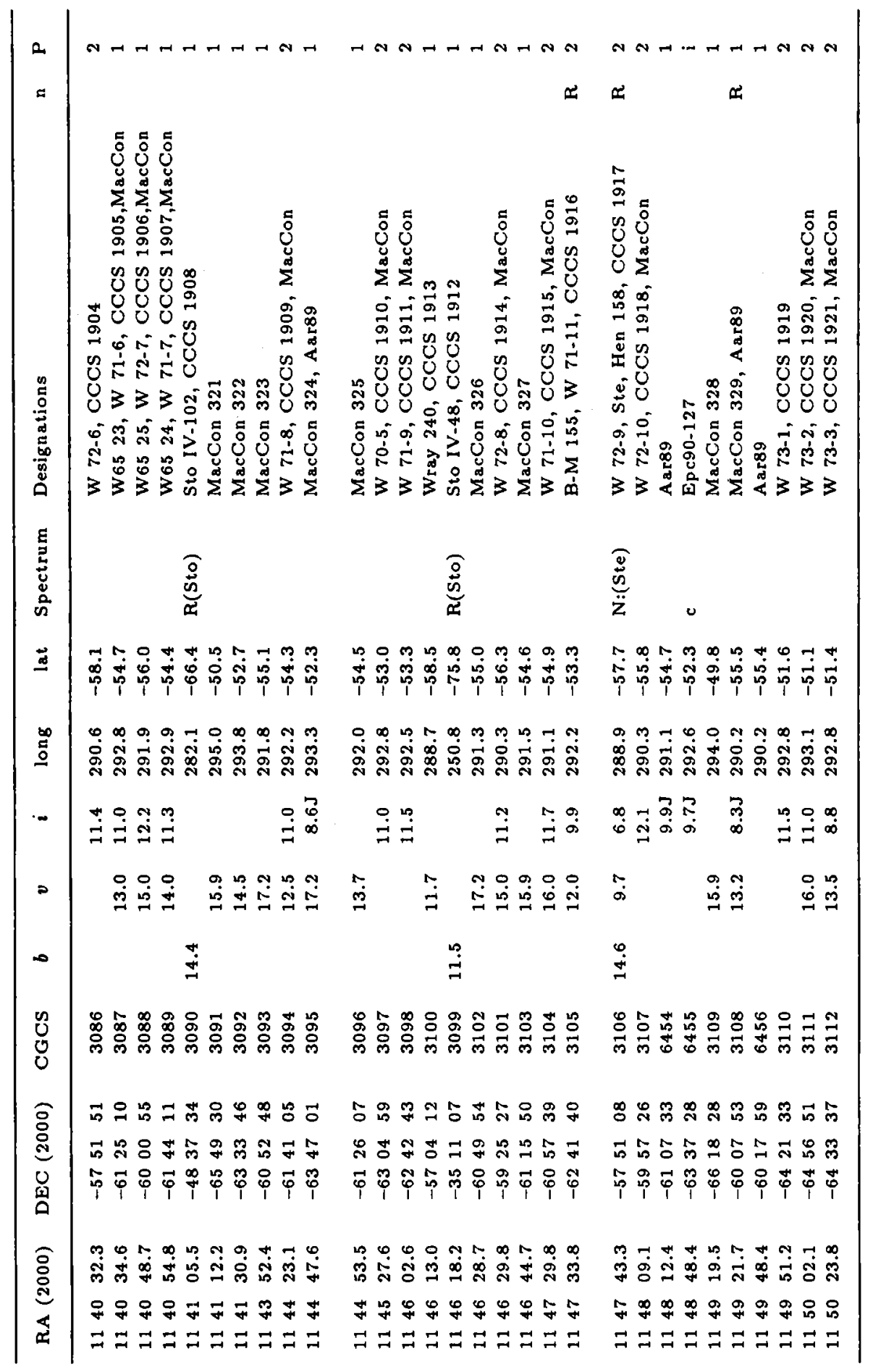




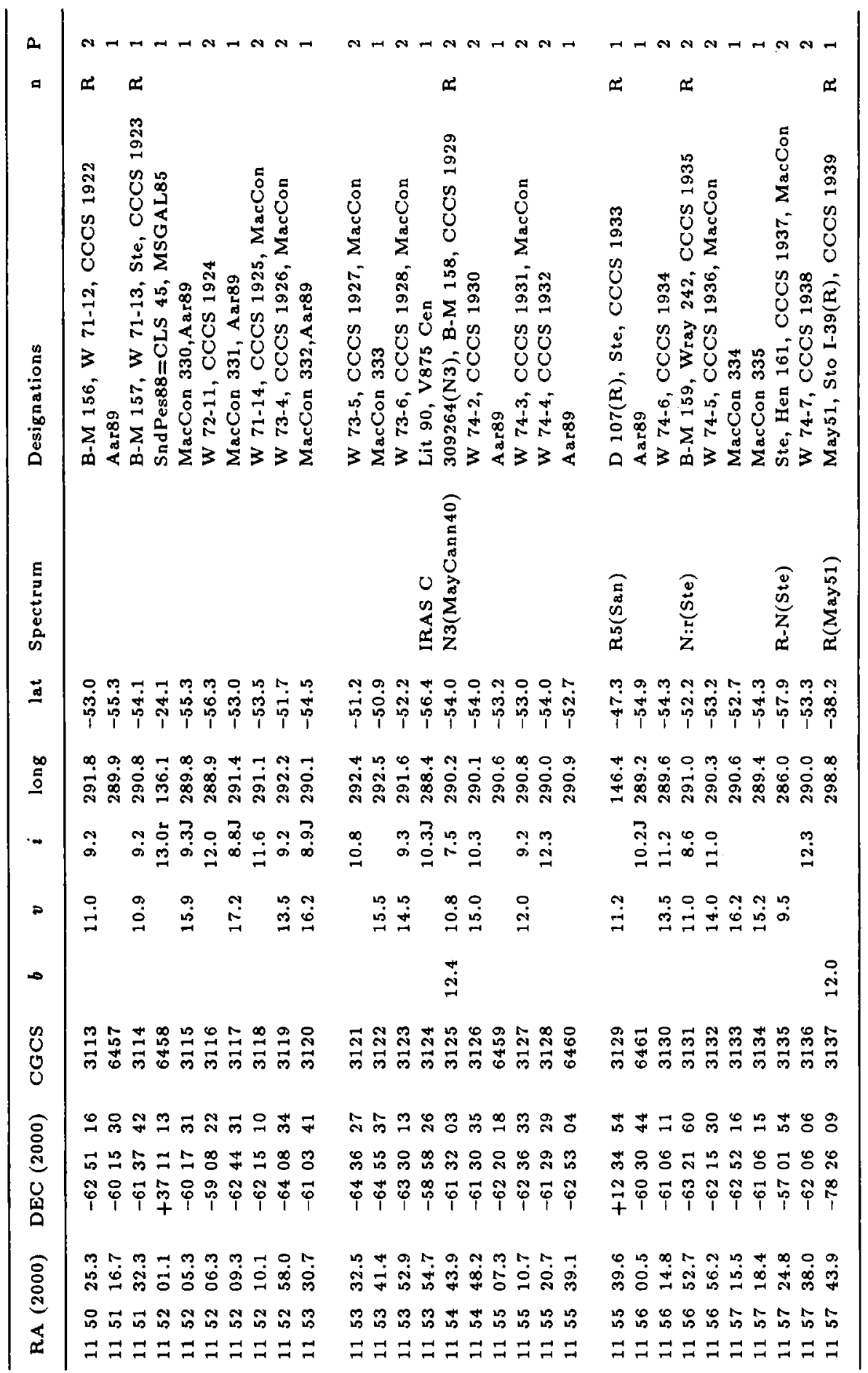




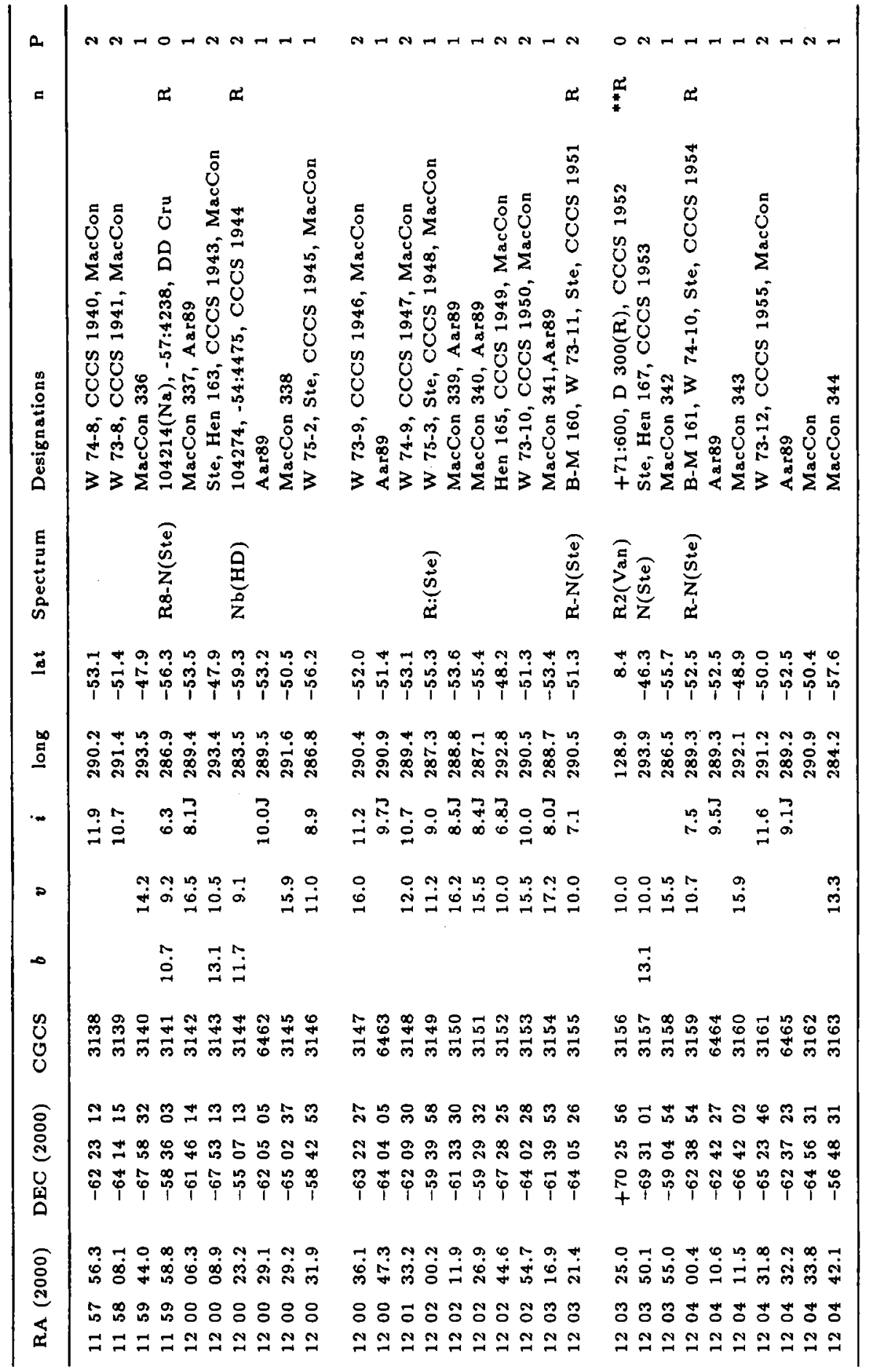


A. Alksnis, A. Balklavs, U. Dzervitis, I. Eglitis et al.

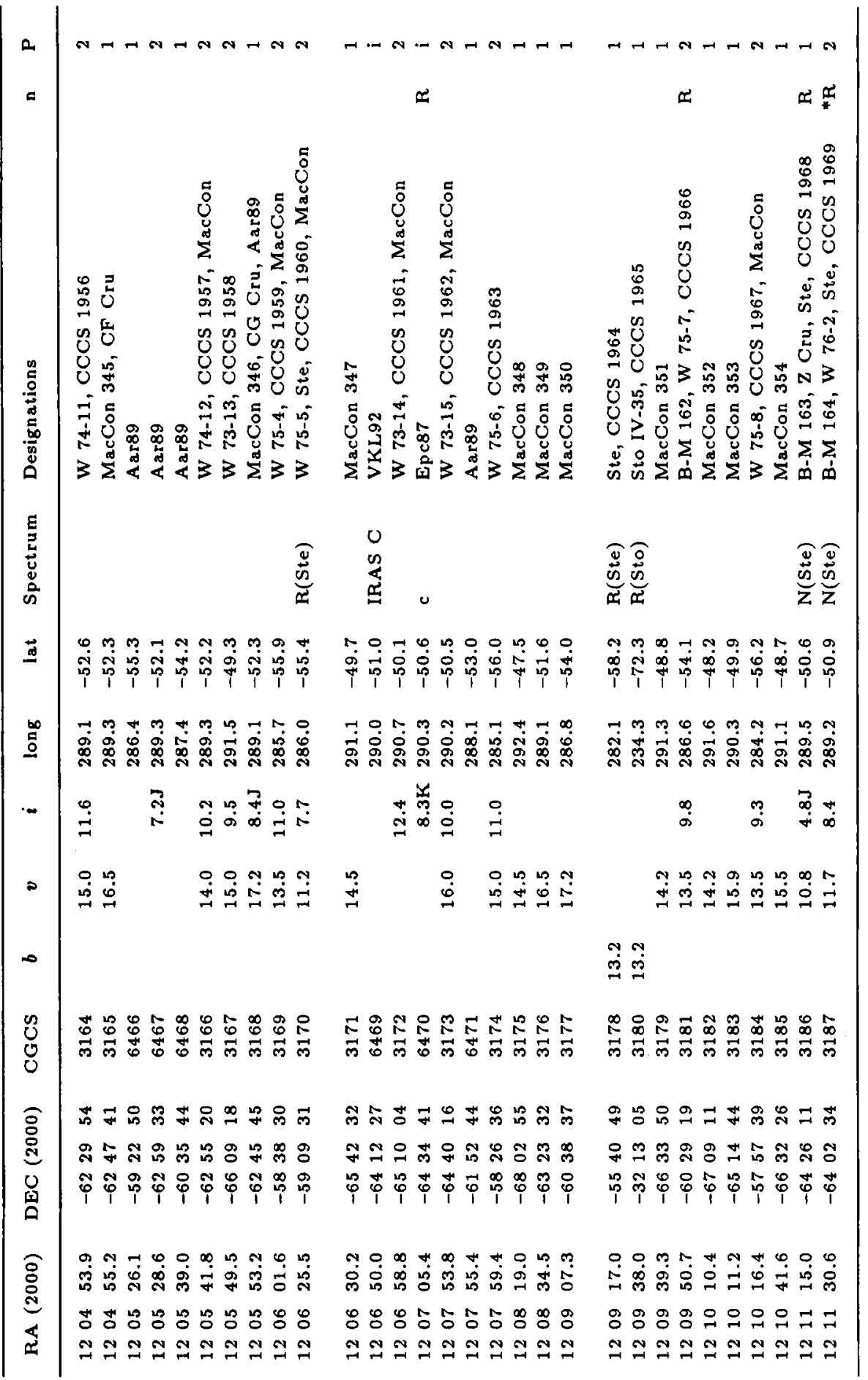




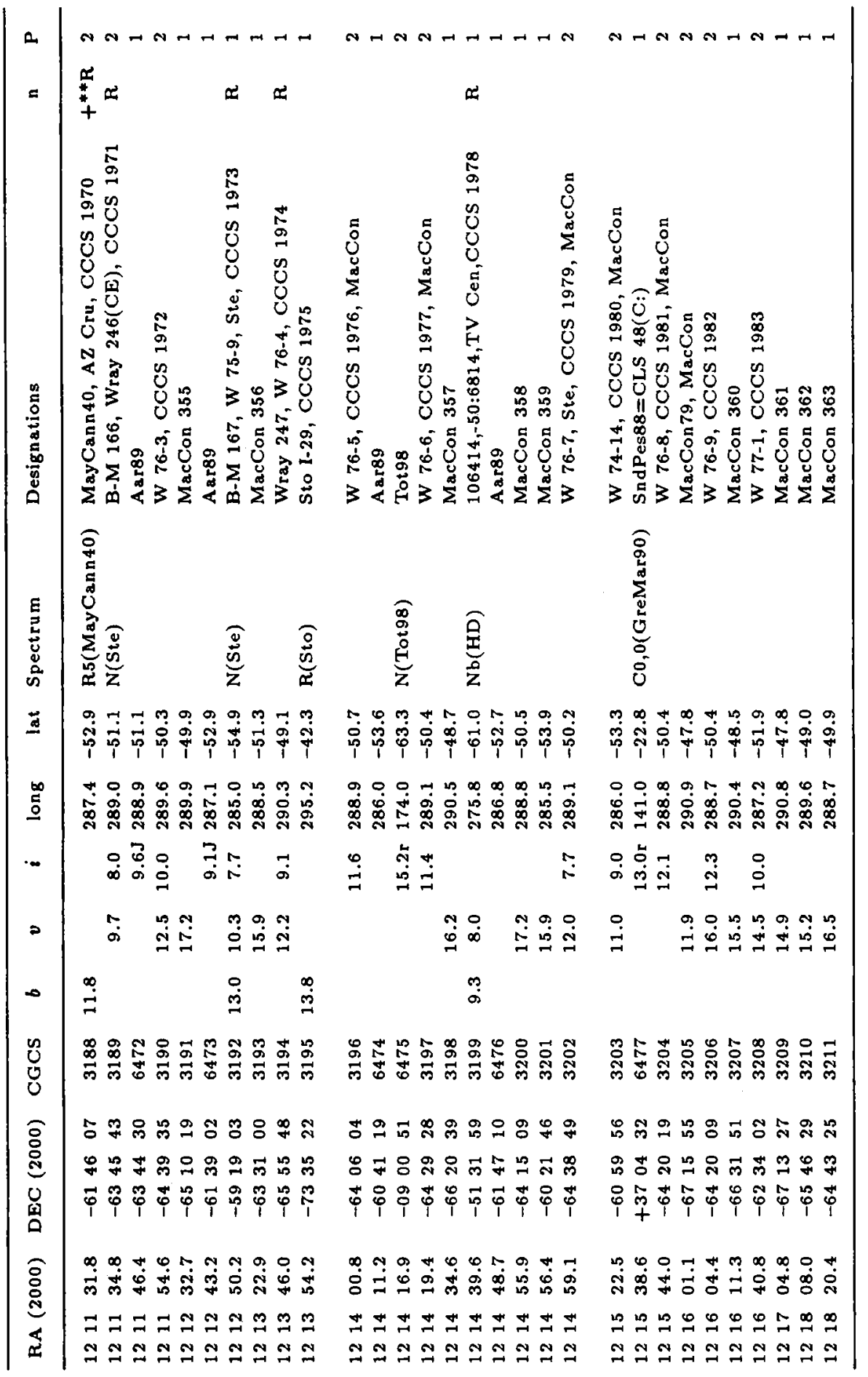




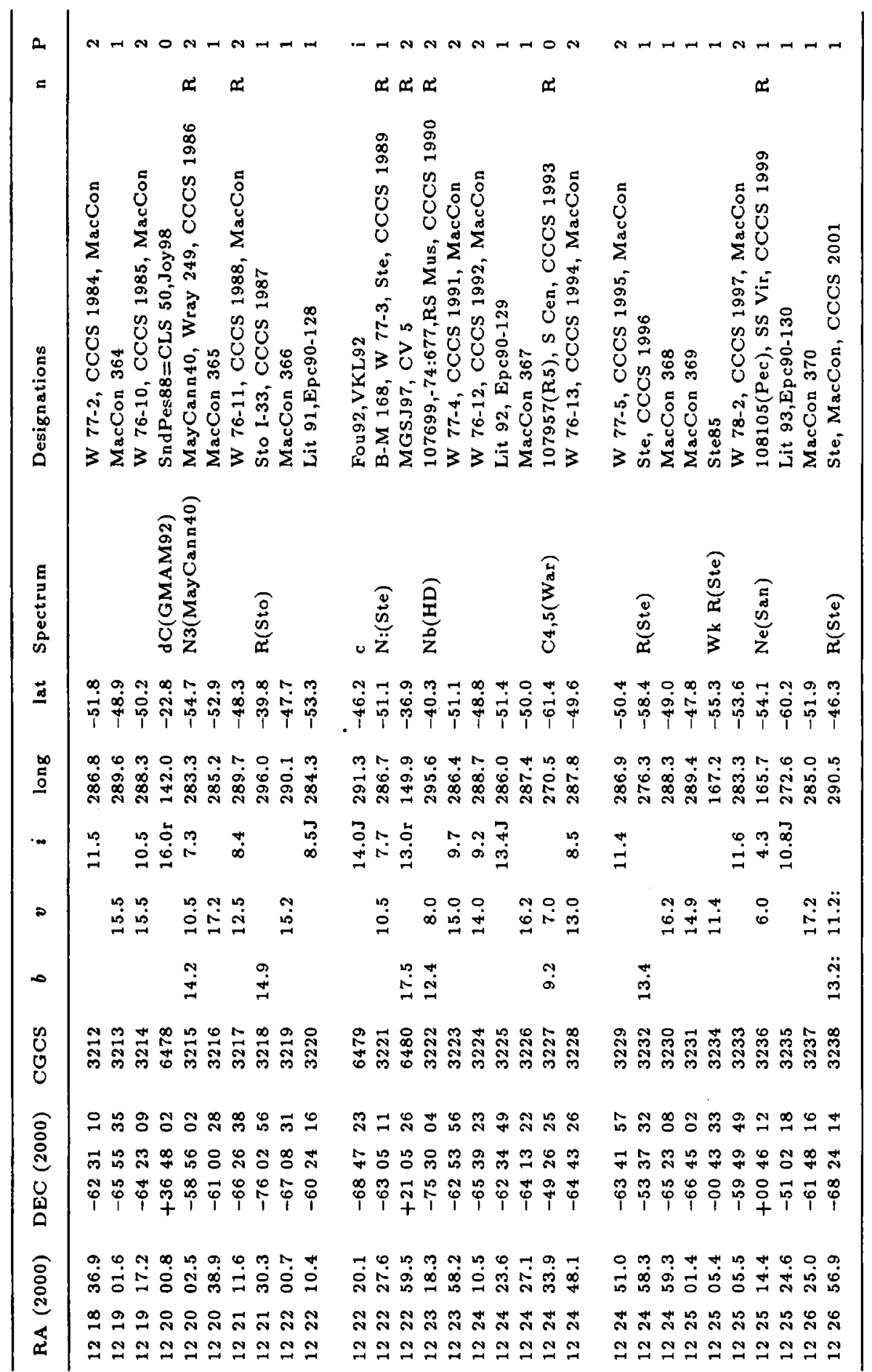




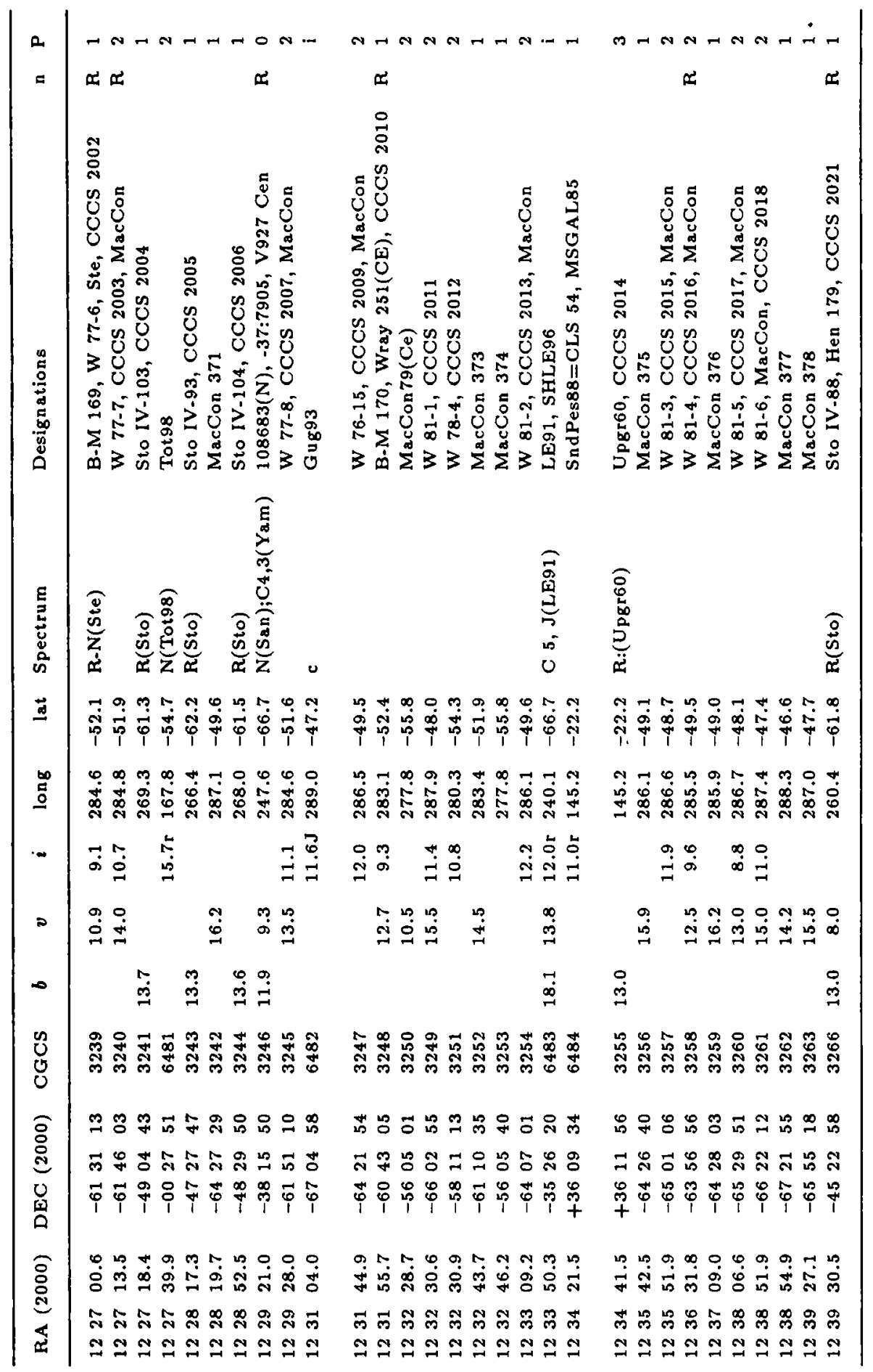




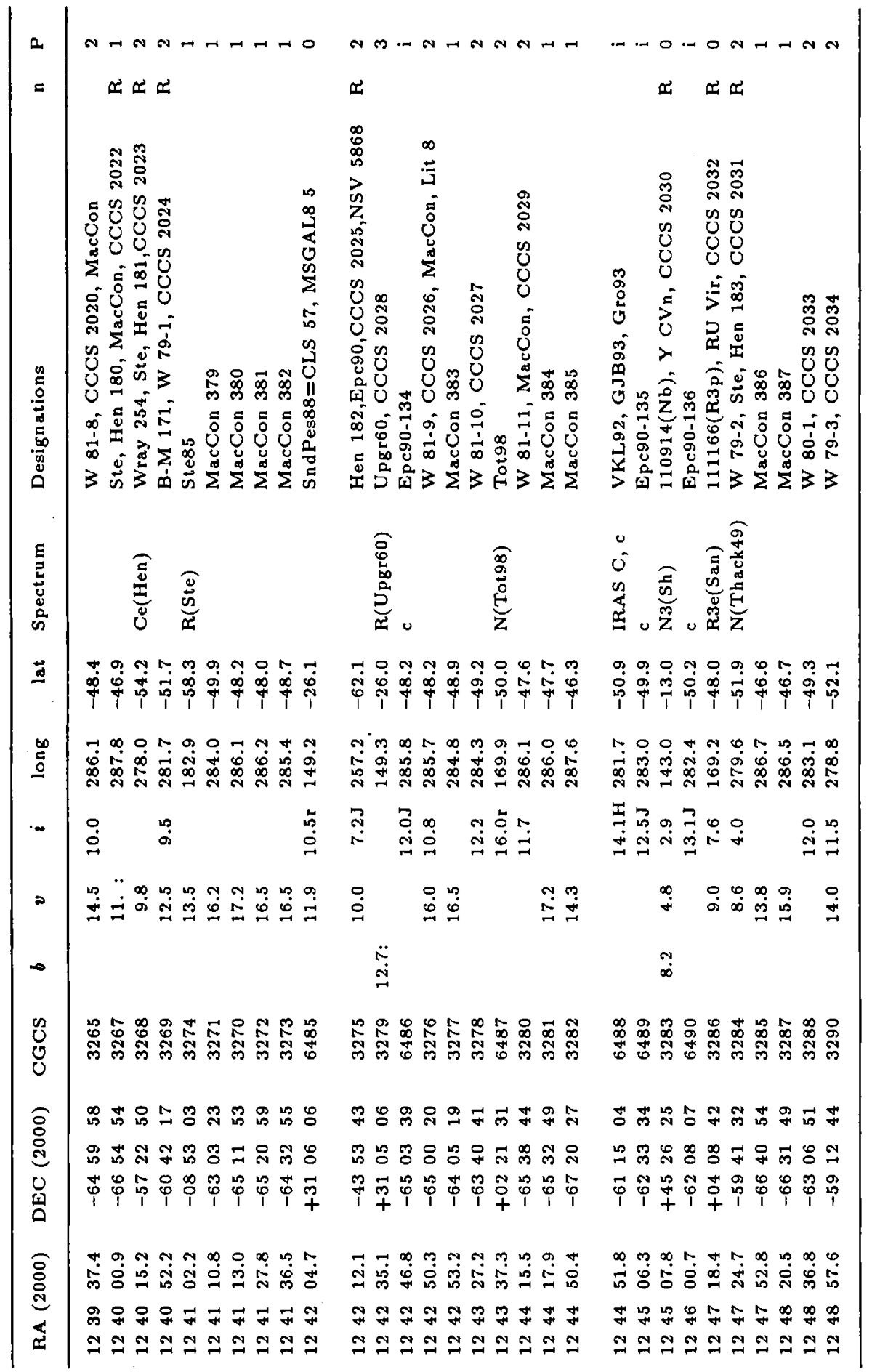




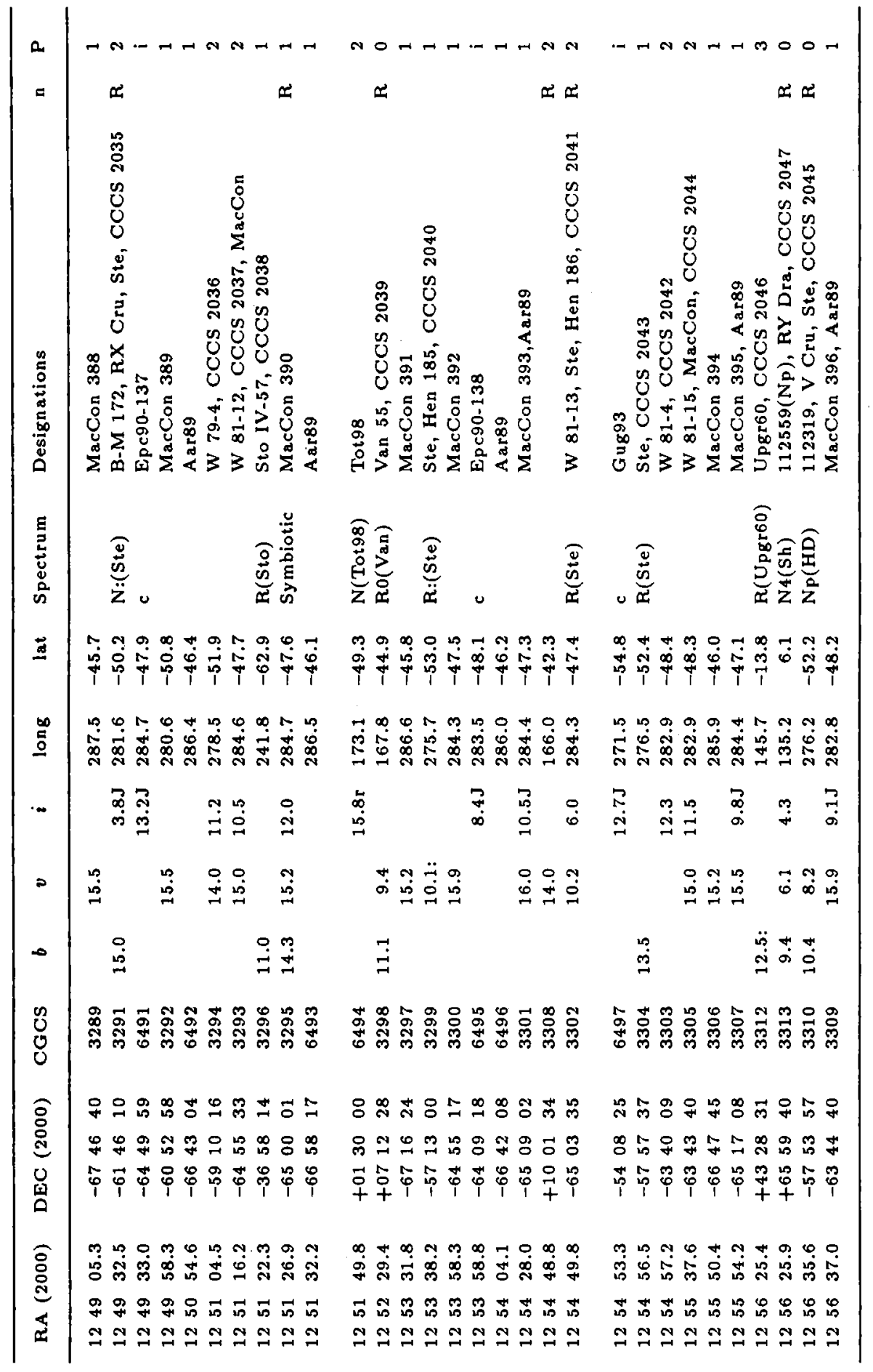




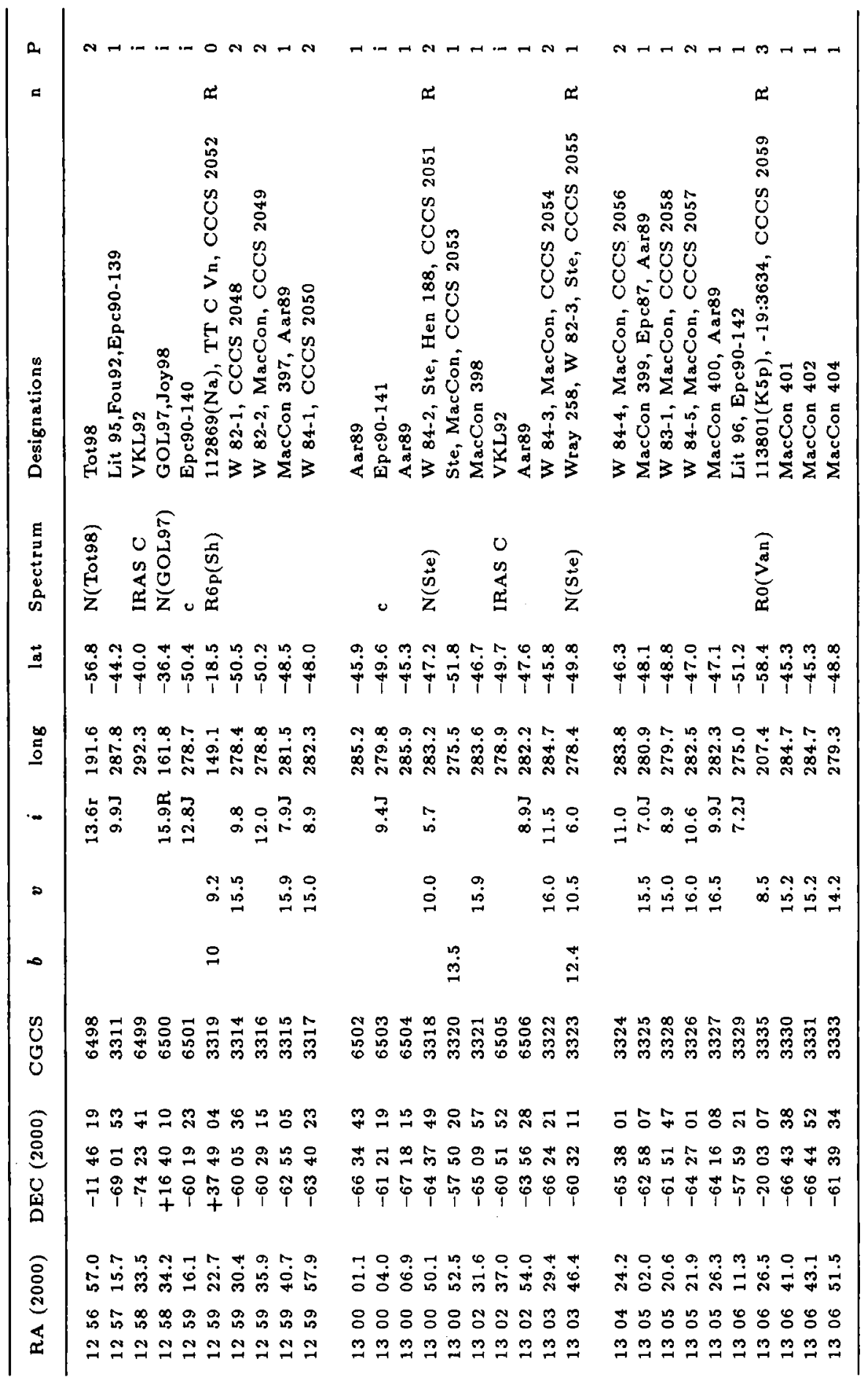




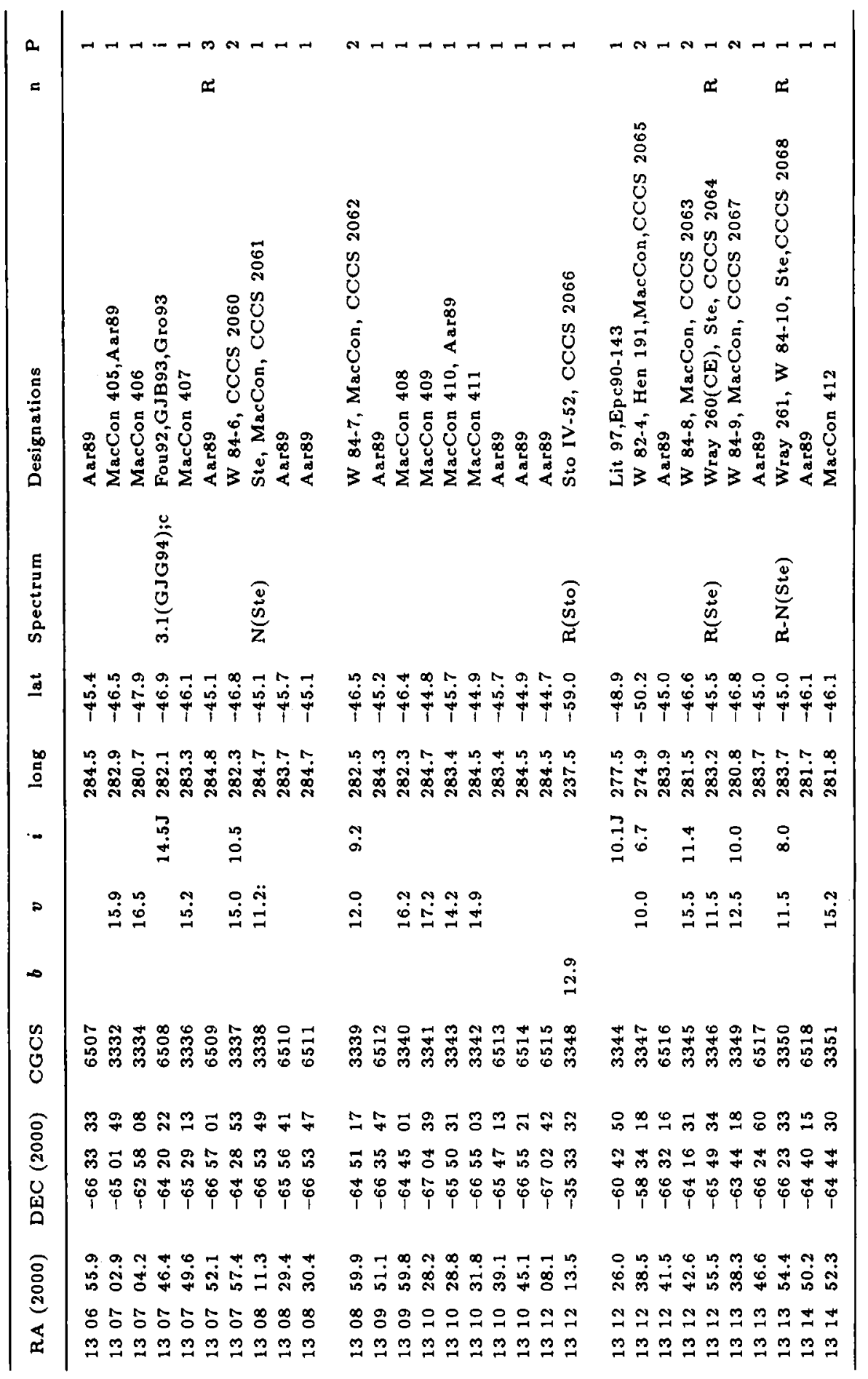




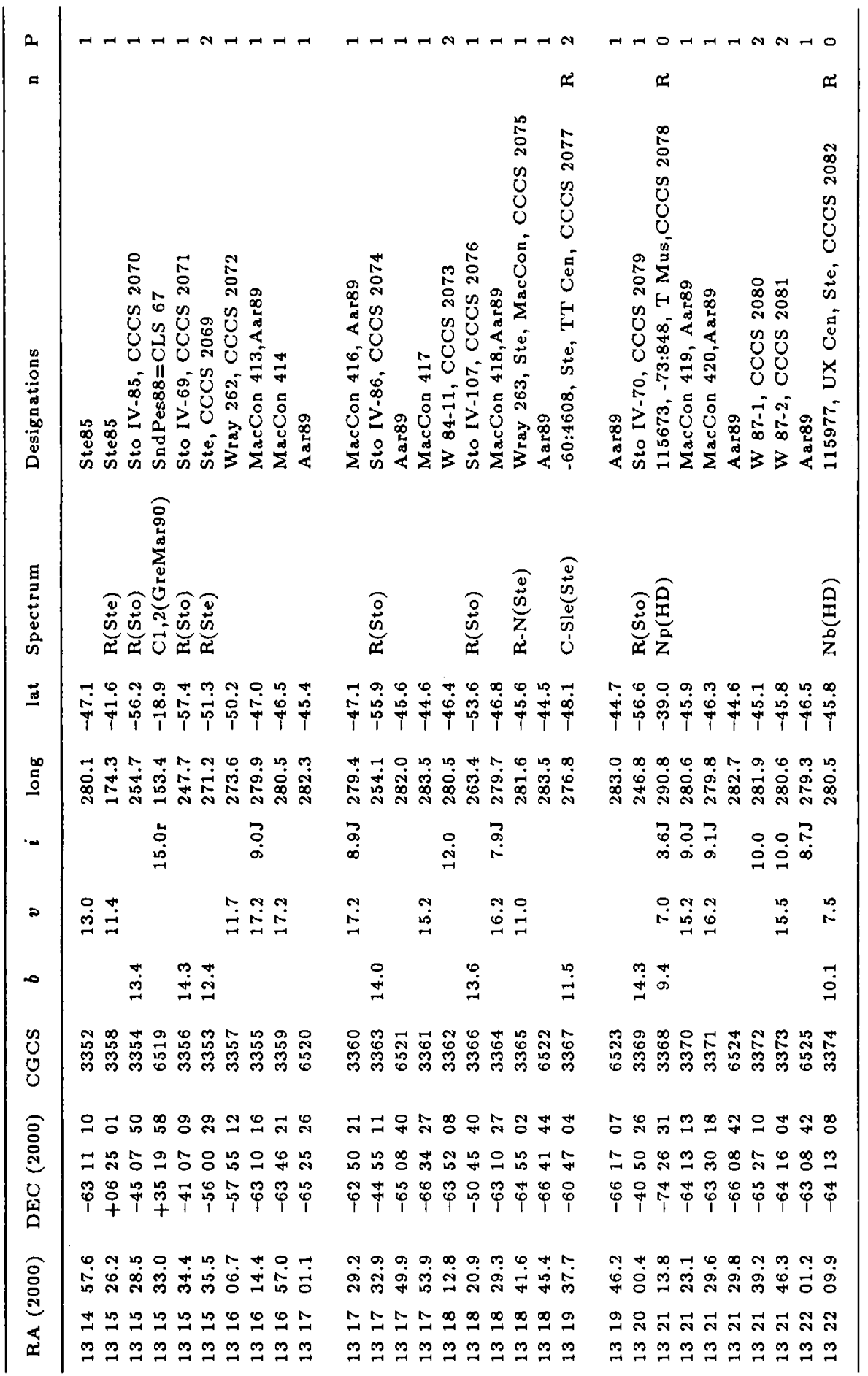




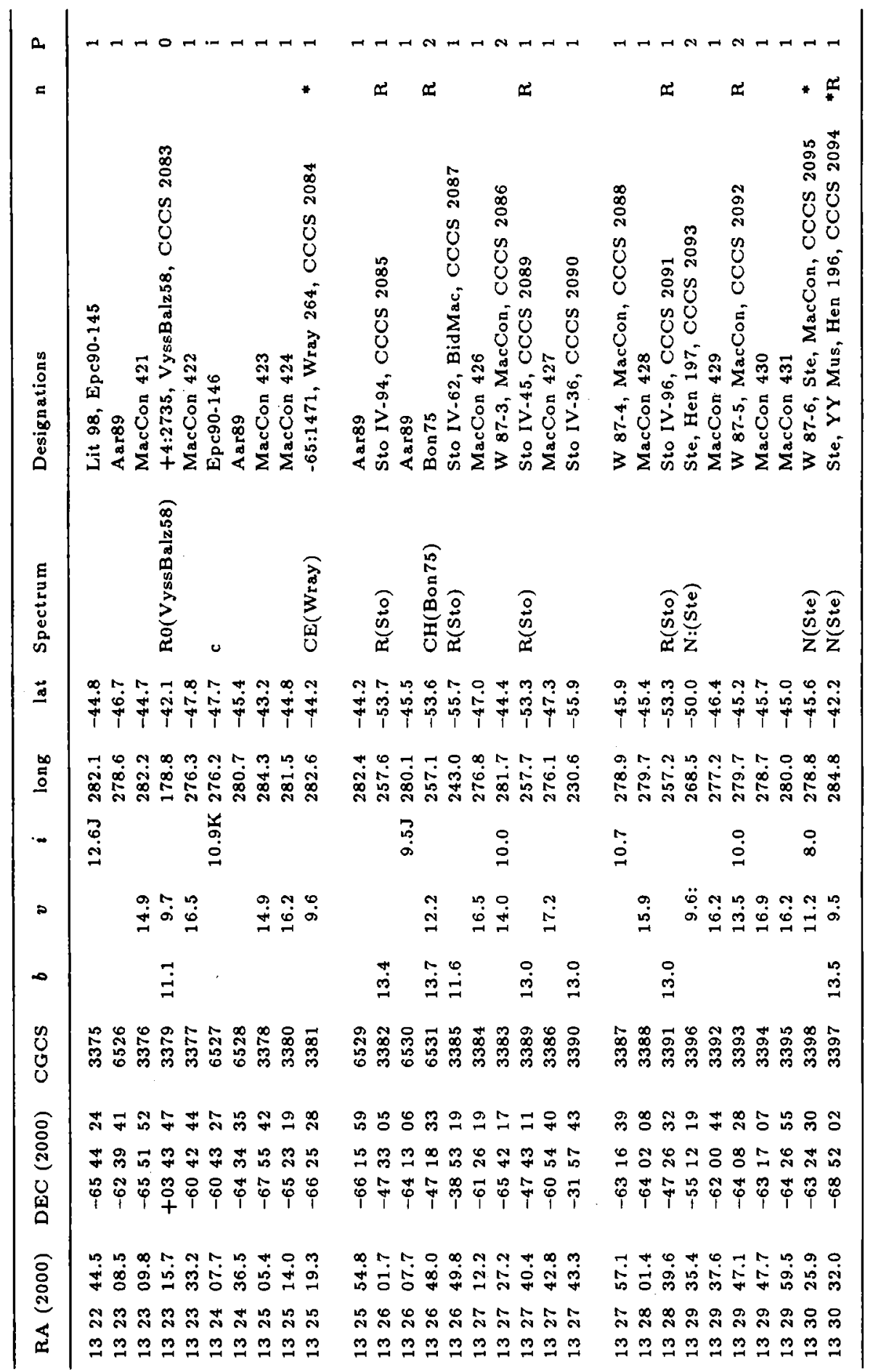




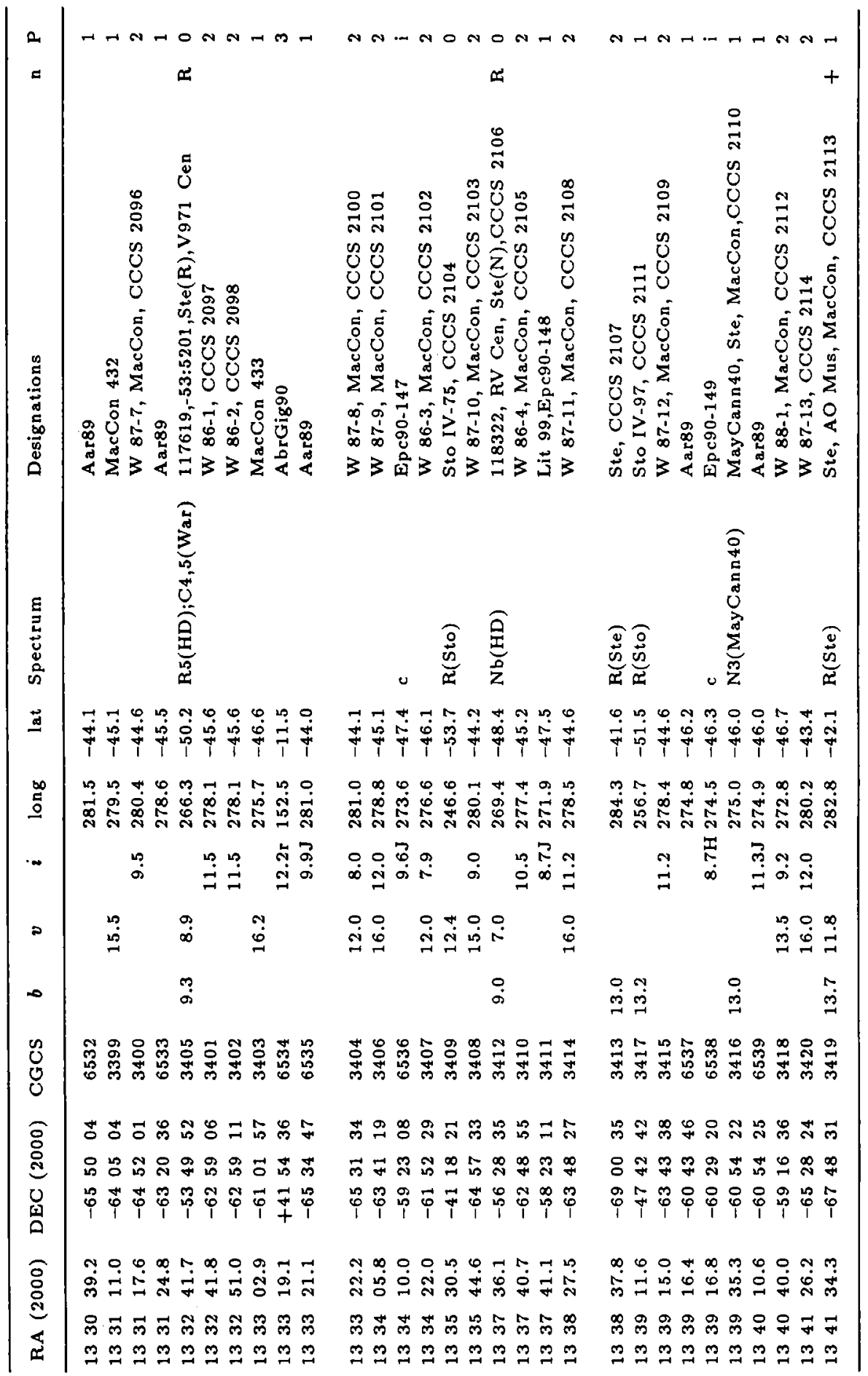




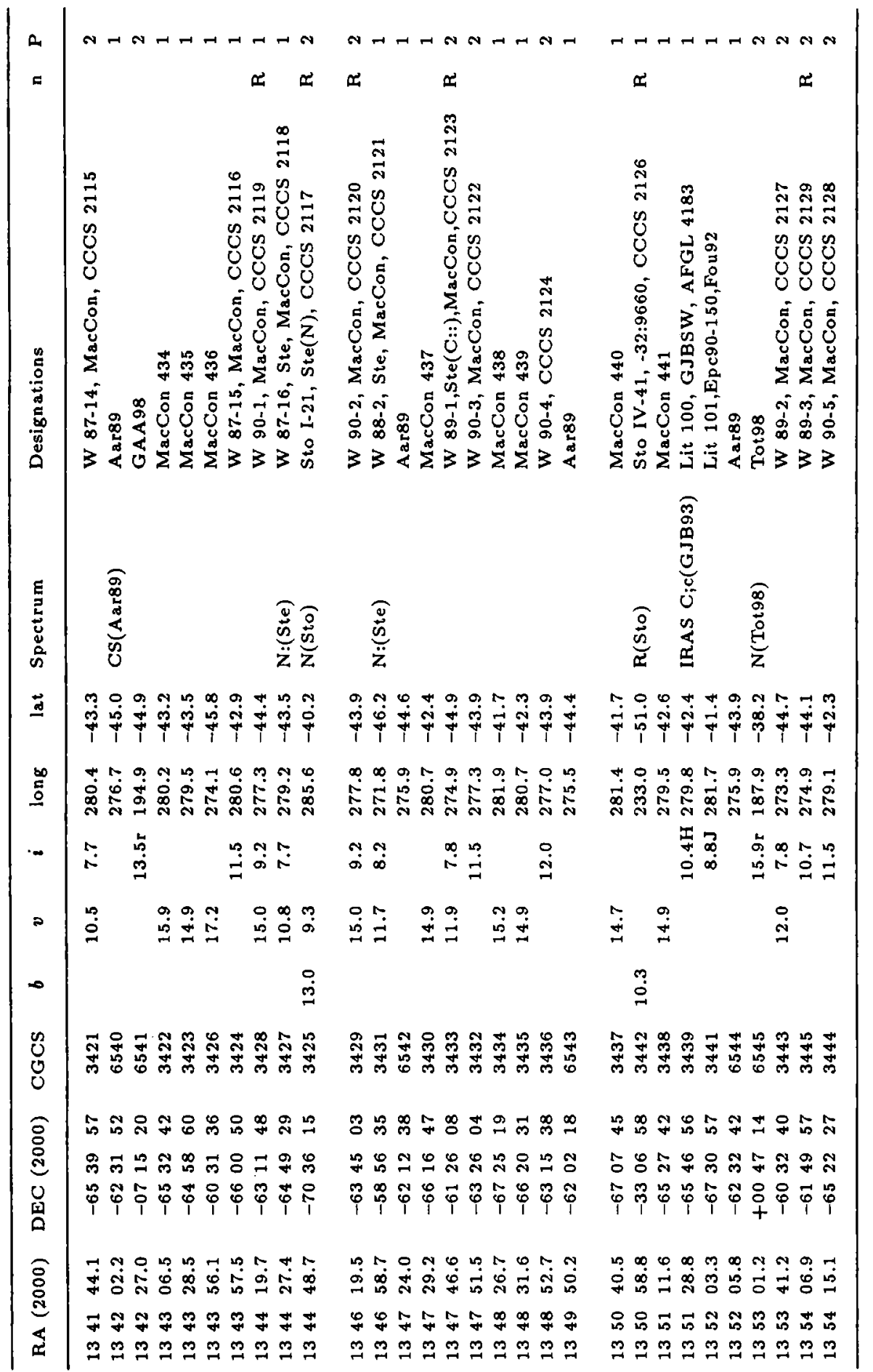




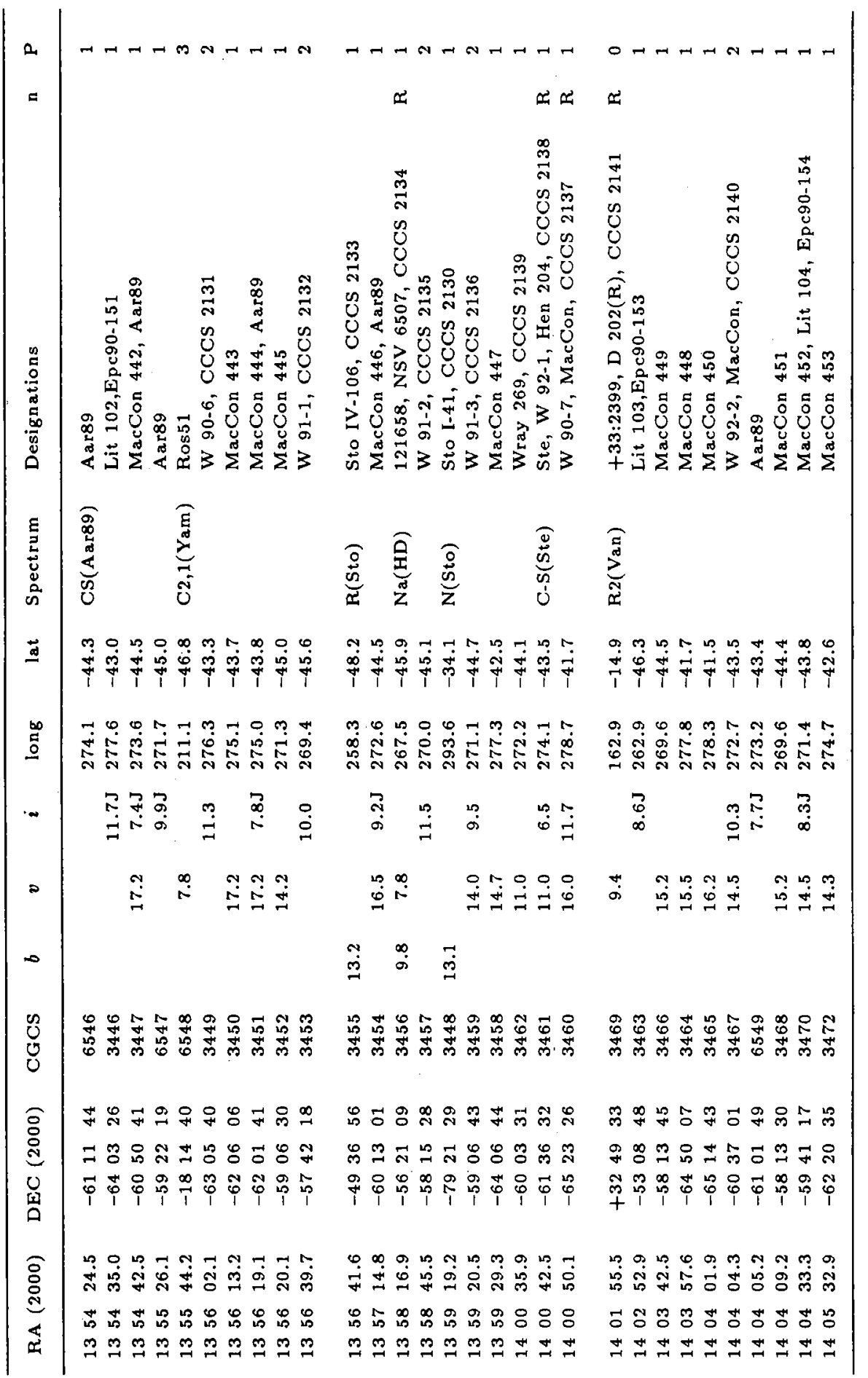




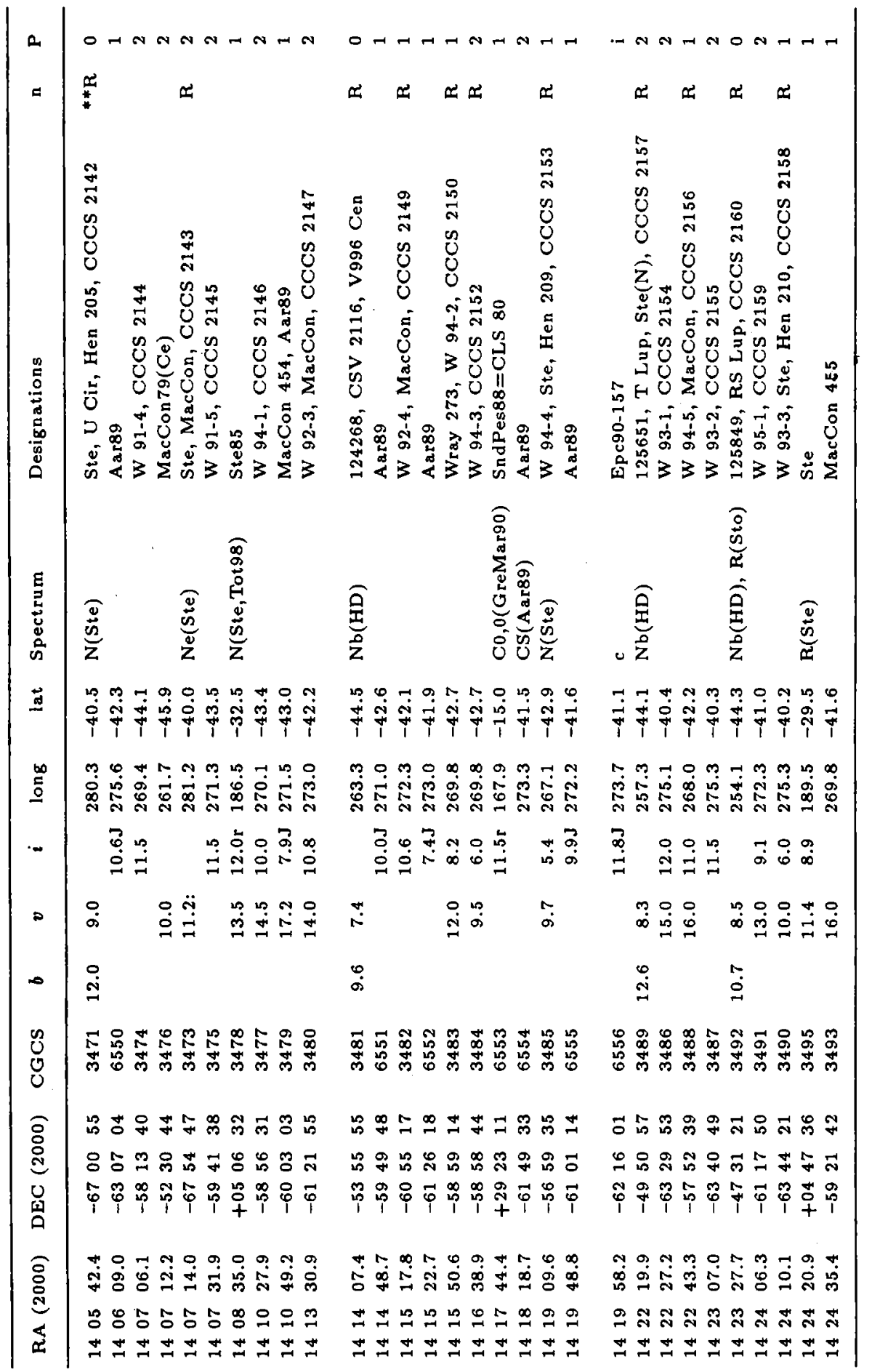




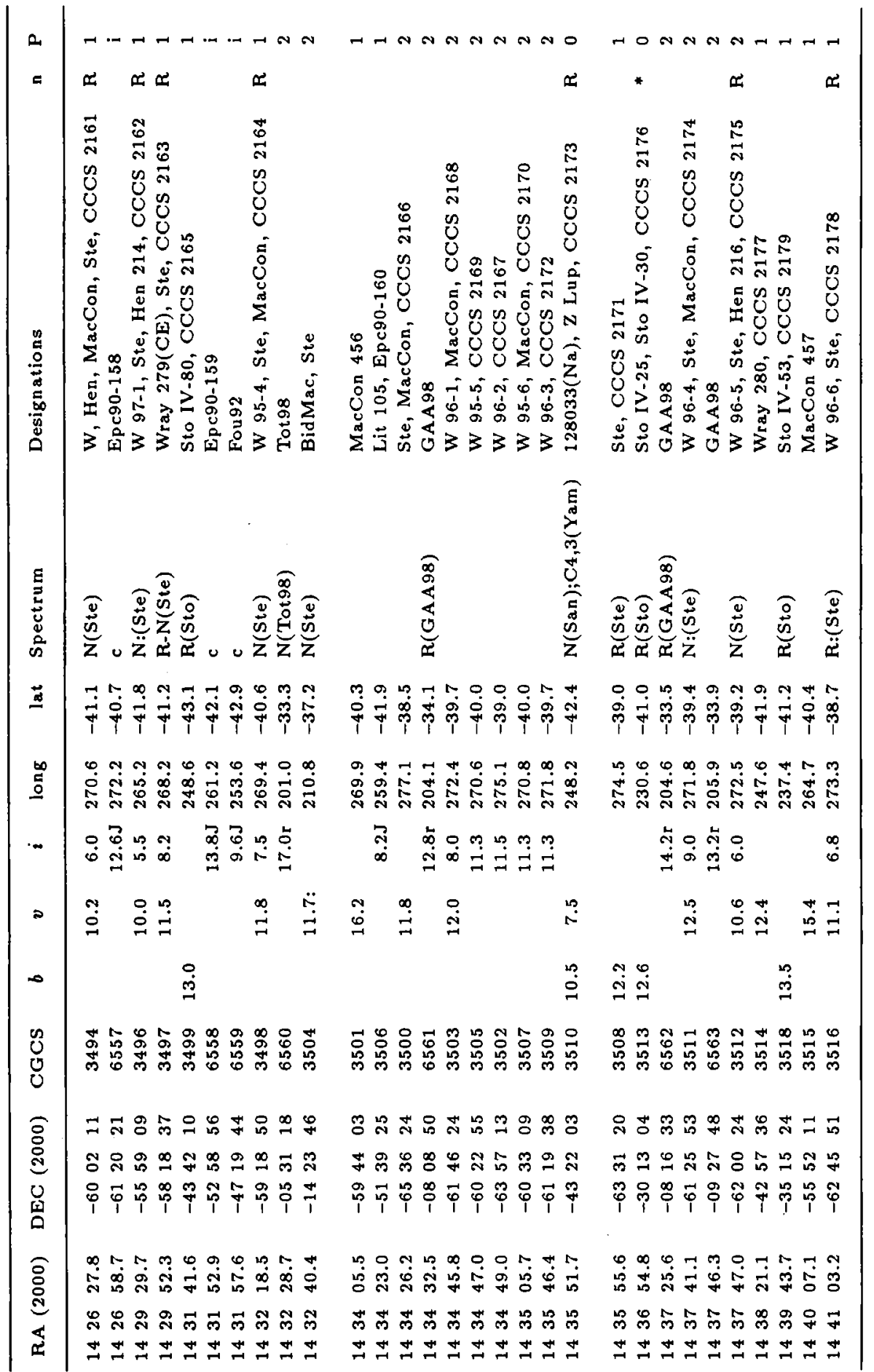




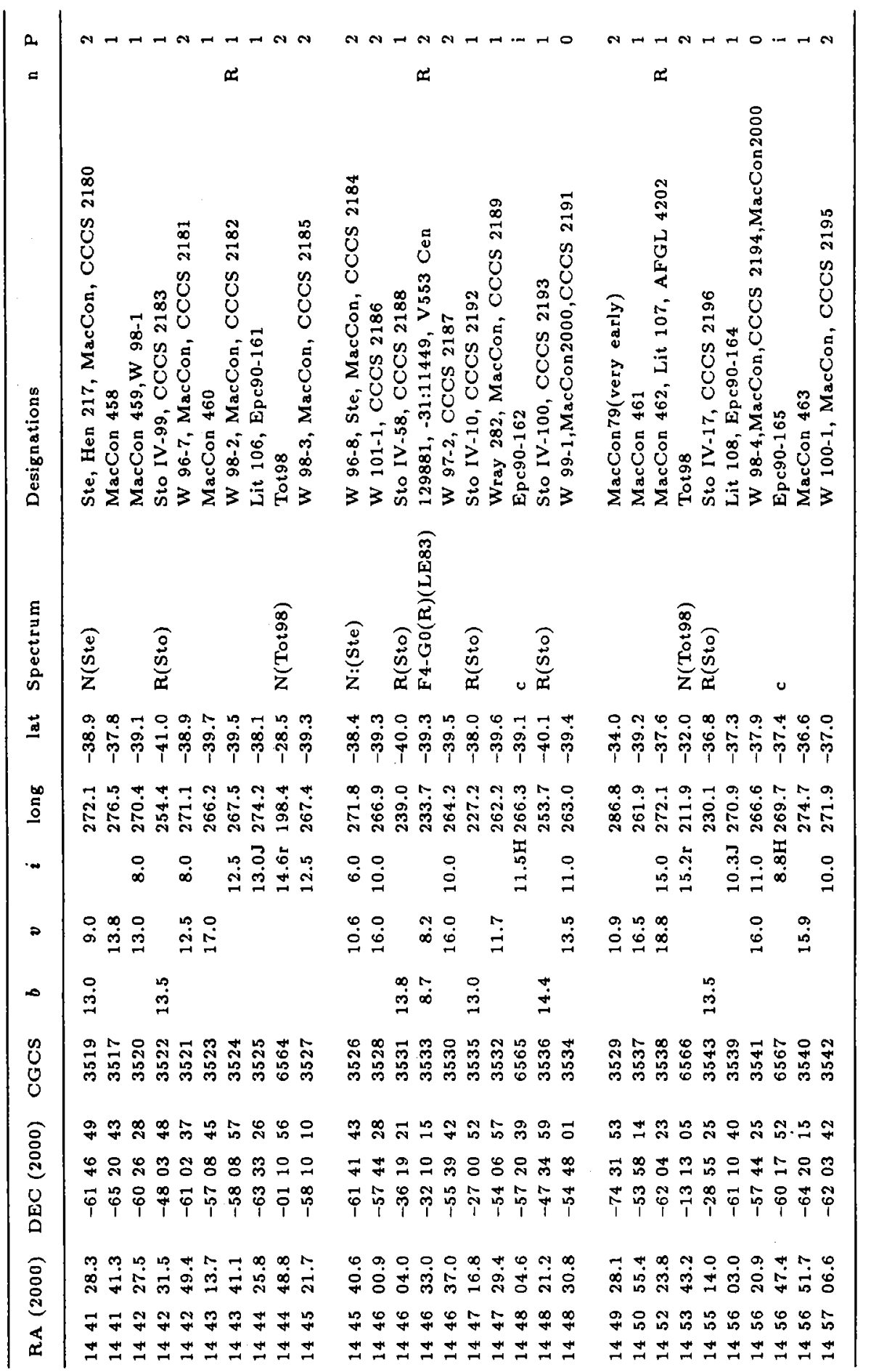




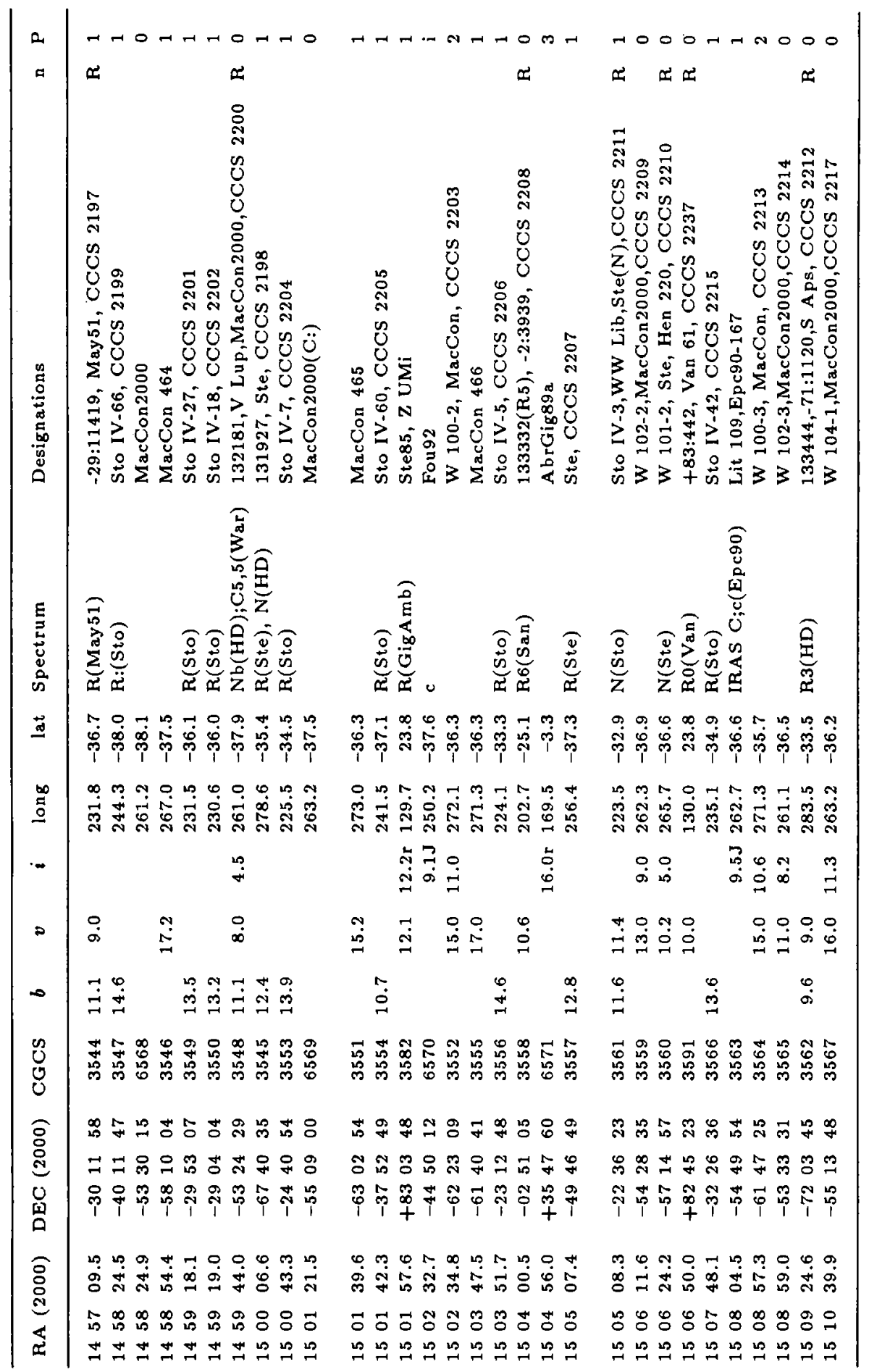




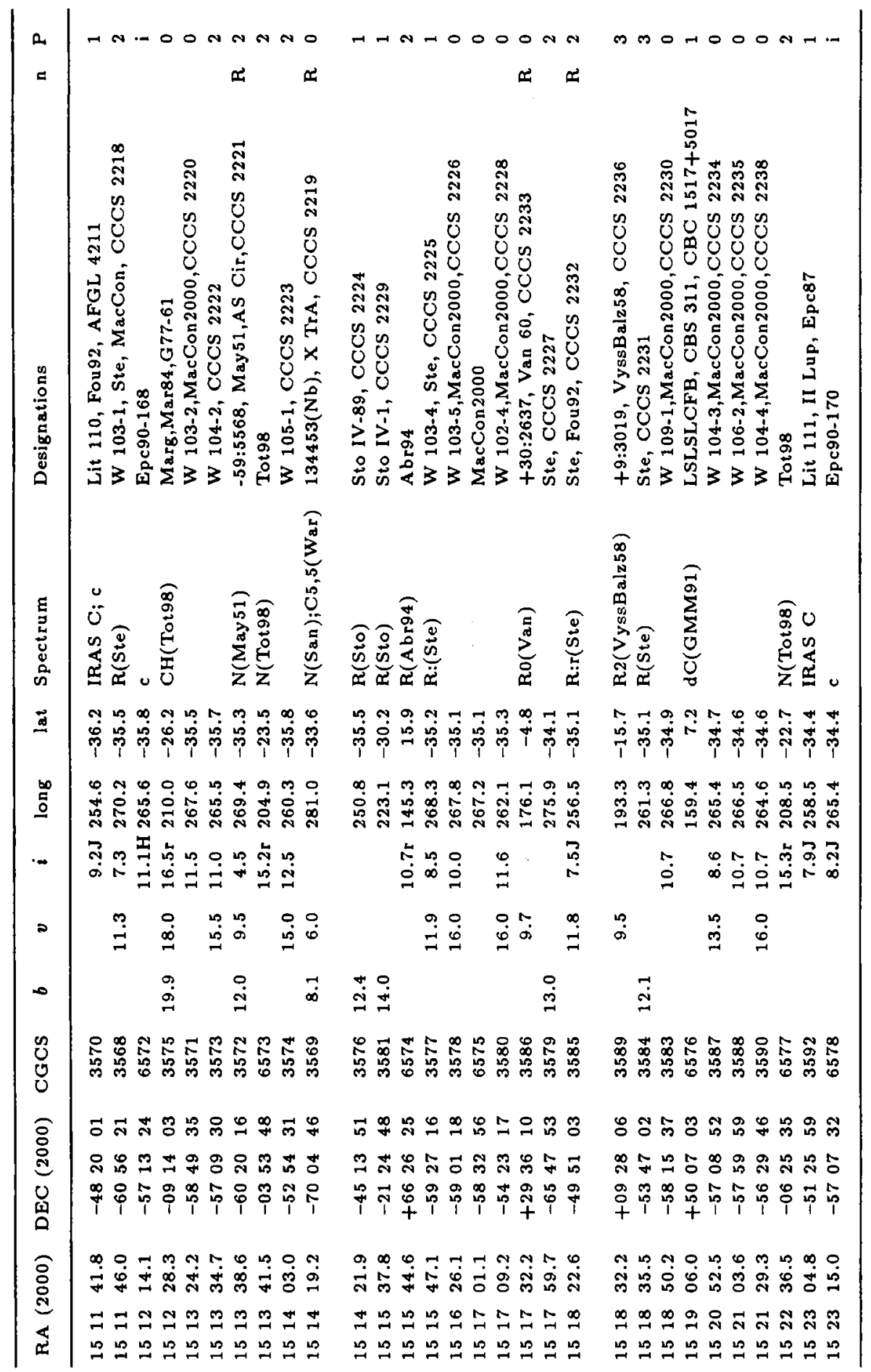




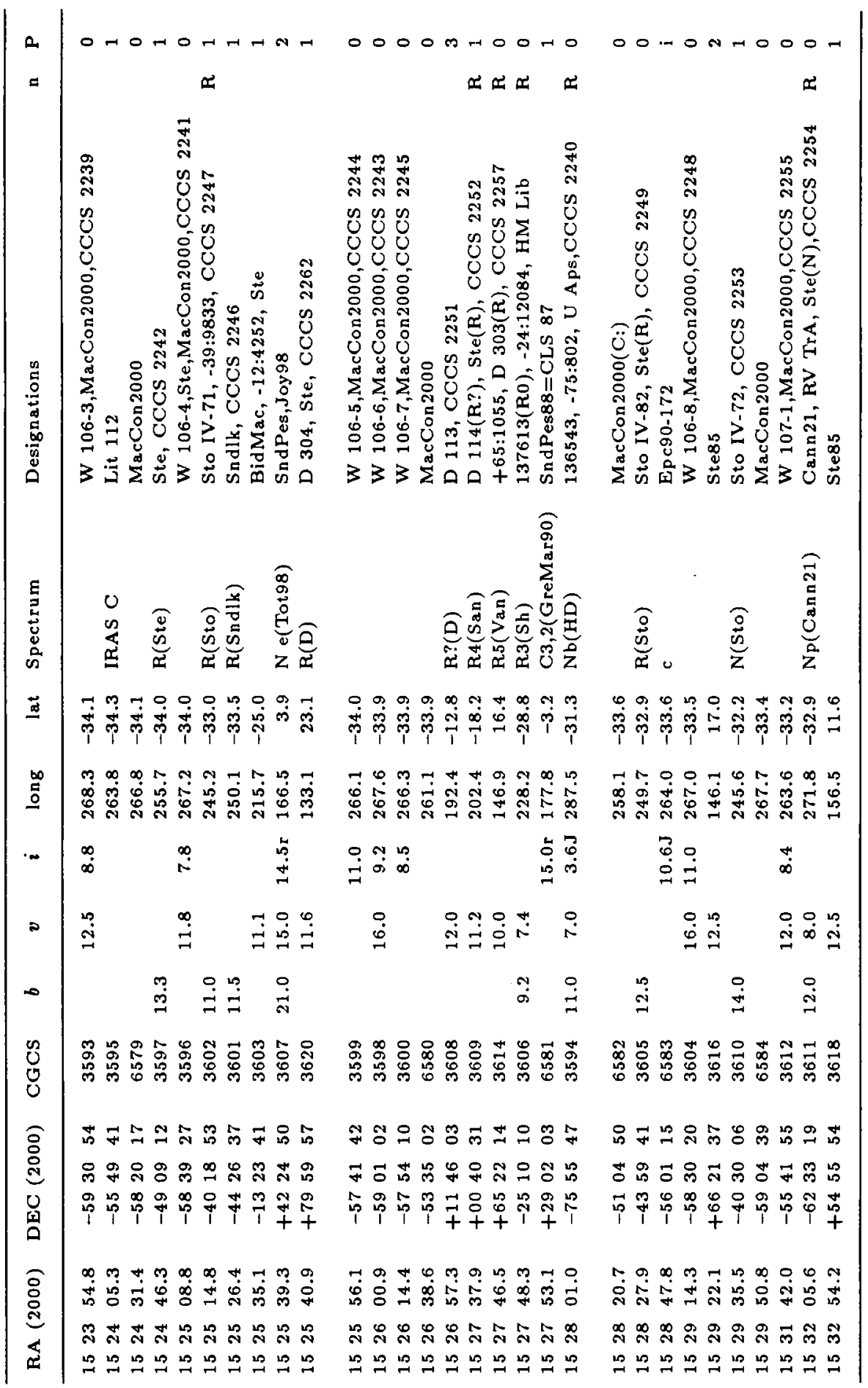




\begin{tabular}{|c|c|c|c|}
\hline 2 & $0-\pi 0 N 00-00$ & $0 h 4 h-4 h-h 0$ & 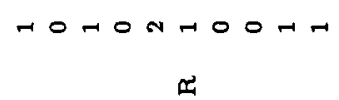 \\
\hline 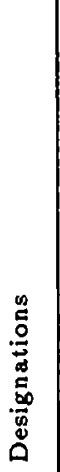 & 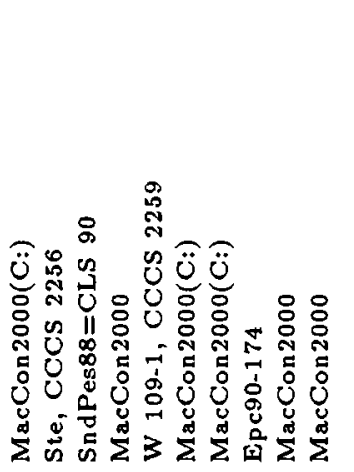 & 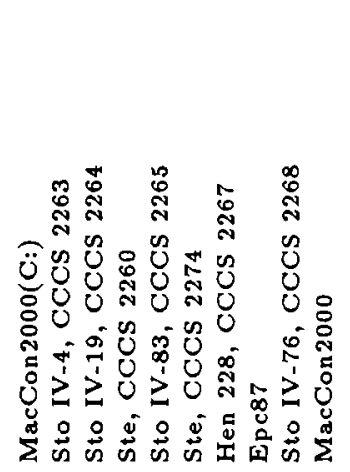 & 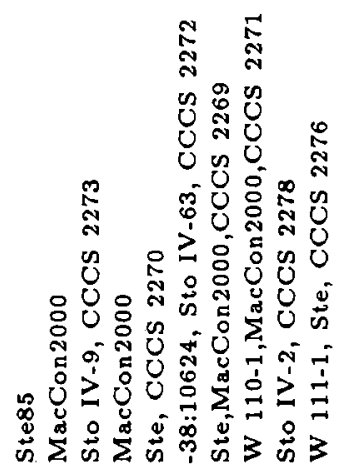 \\
\hline 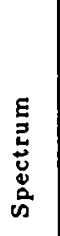 & 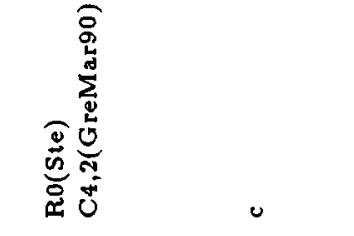 & 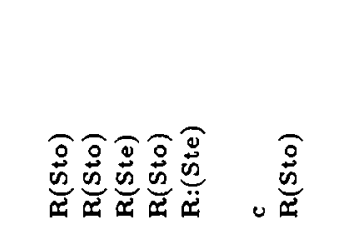 & 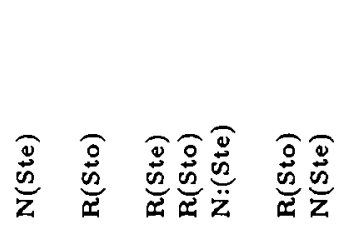 \\
\hline$\underline{g}$ & 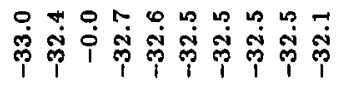 & 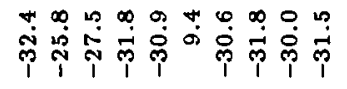 & 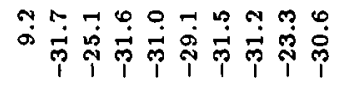 \\
\hline 离 & $\begin{array}{lll}7 & m \\
0 \\
0\end{array}$ & 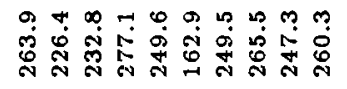 & 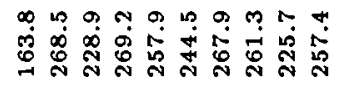 \\
\hline - & 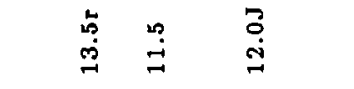 & की & $\stackrel{\mathscr{n}}{\stackrel{\sim}{i}} \stackrel{+}{\infty}$ \\
\hline ? & & $\stackrel{\circ}{\dot{\theta}}$ & $\begin{array}{l}\ddot{ت} \\
\stackrel{\leftrightarrow}{\oplus}\end{array}$ \\
\hline$\infty$ & $\stackrel{m}{\stackrel{M}{*}}$ & 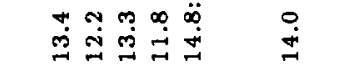 & 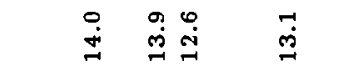 \\
\hline $\begin{array}{l}\text { O } \\
y \\
\text { y }\end{array}$ & 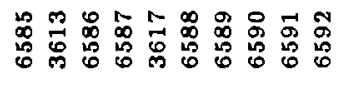 & 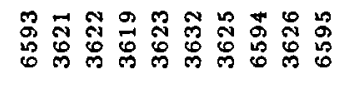 & 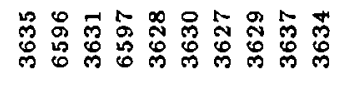 \\
\hline$\widehat{\circ}$ & 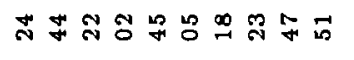 & 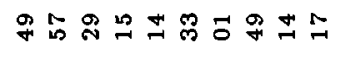 & 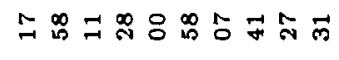 \\
\hline 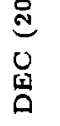 & 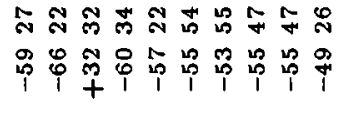 & 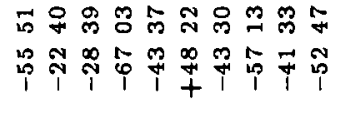 & 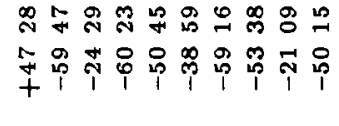 \\
\hline ลे & 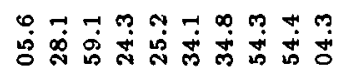 & 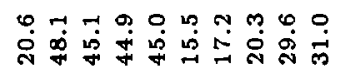 & 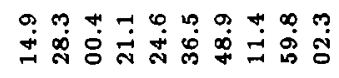 \\
\hline 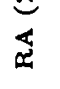 & 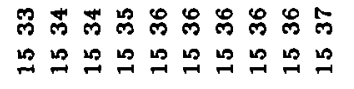 & 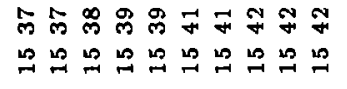 & 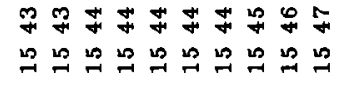 \\
\hline
\end{tabular}




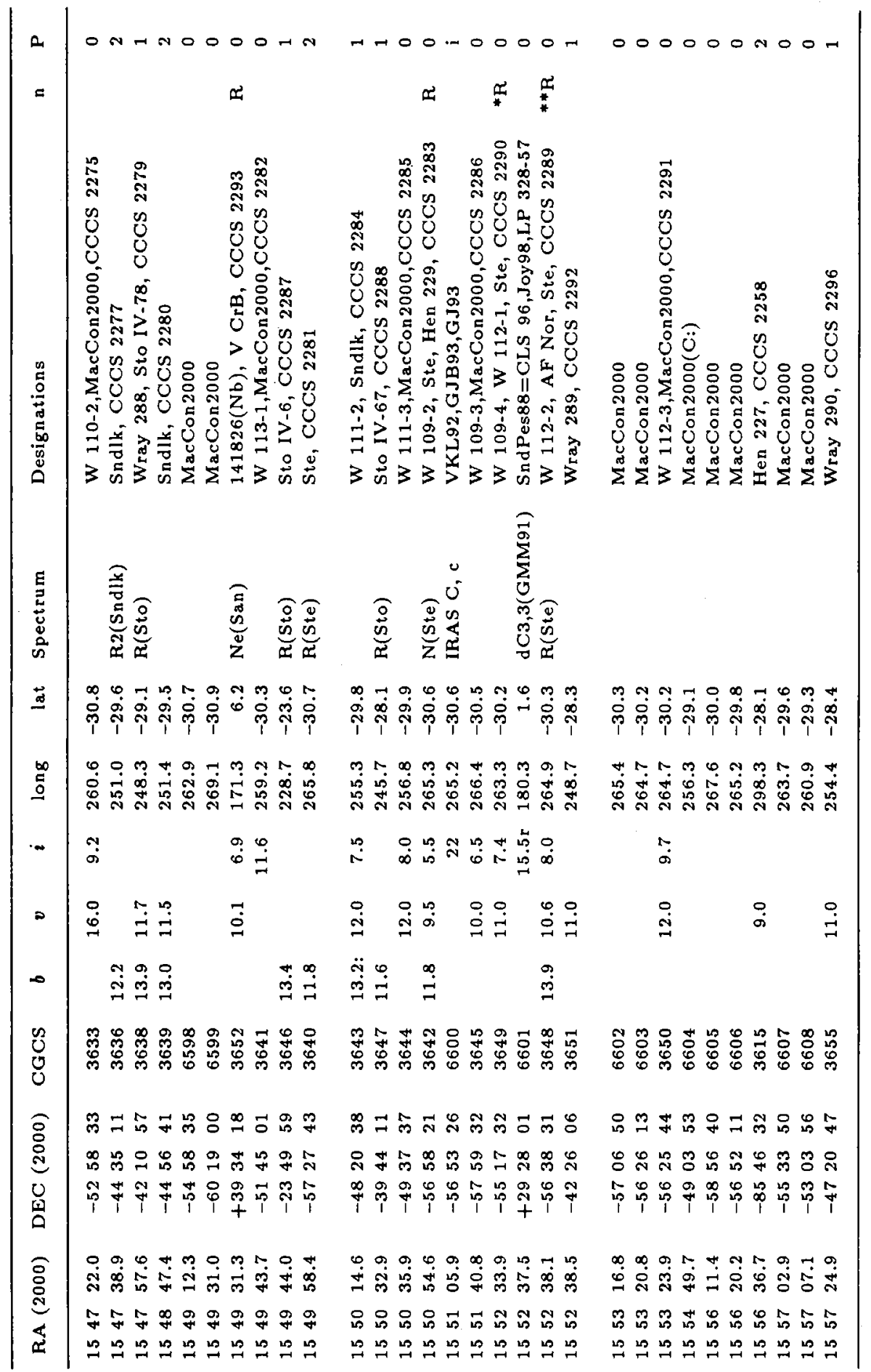




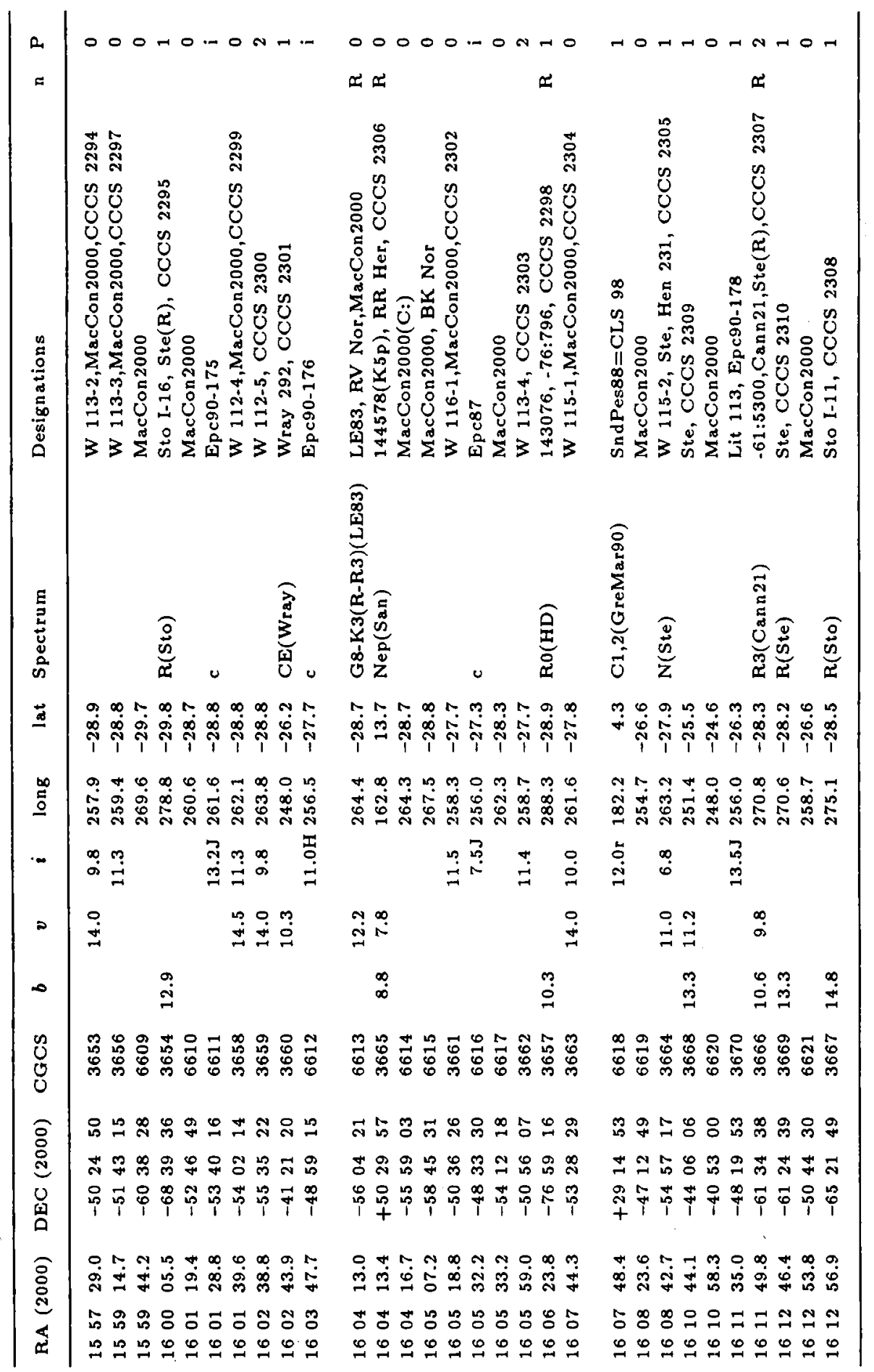




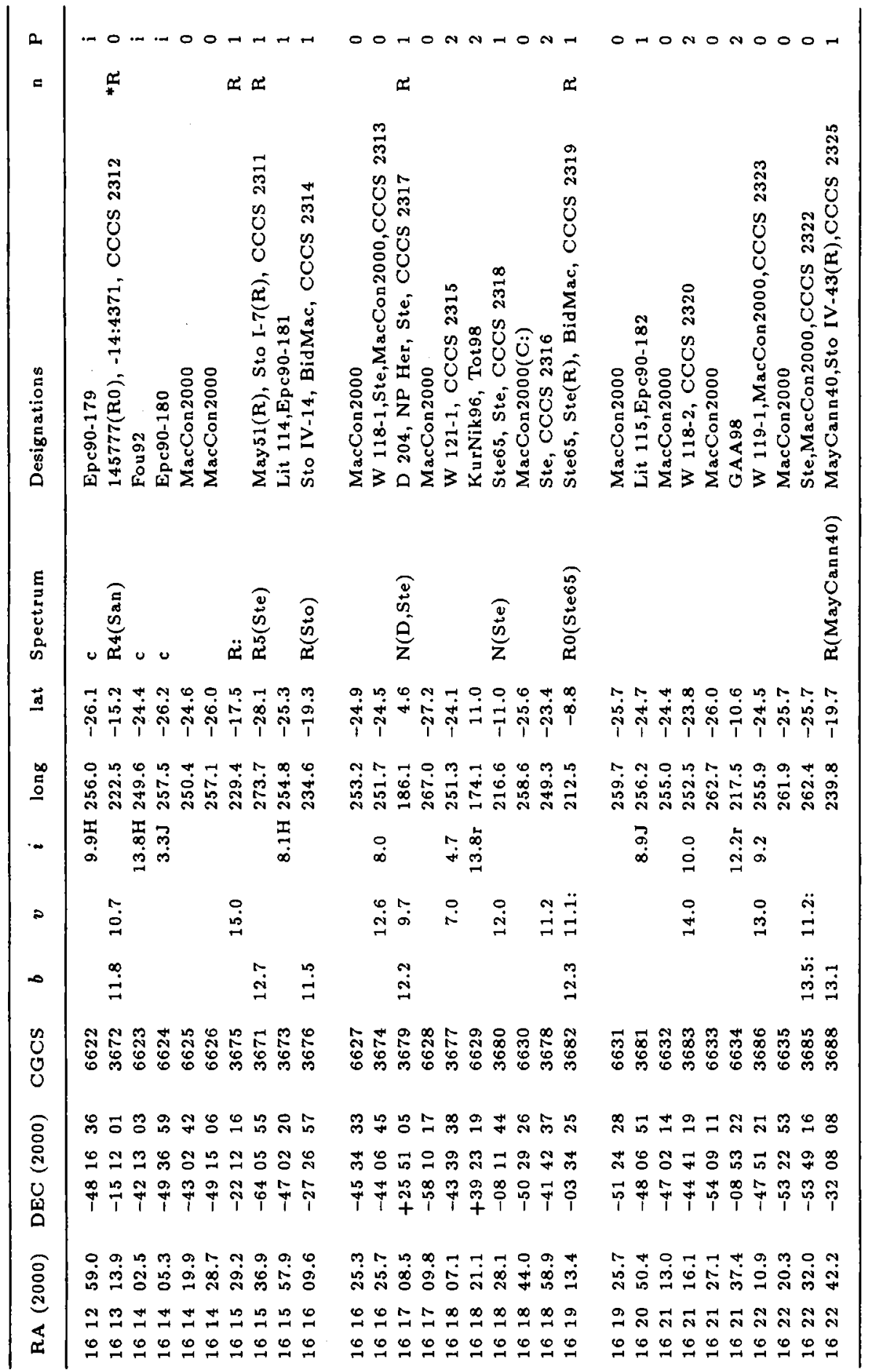




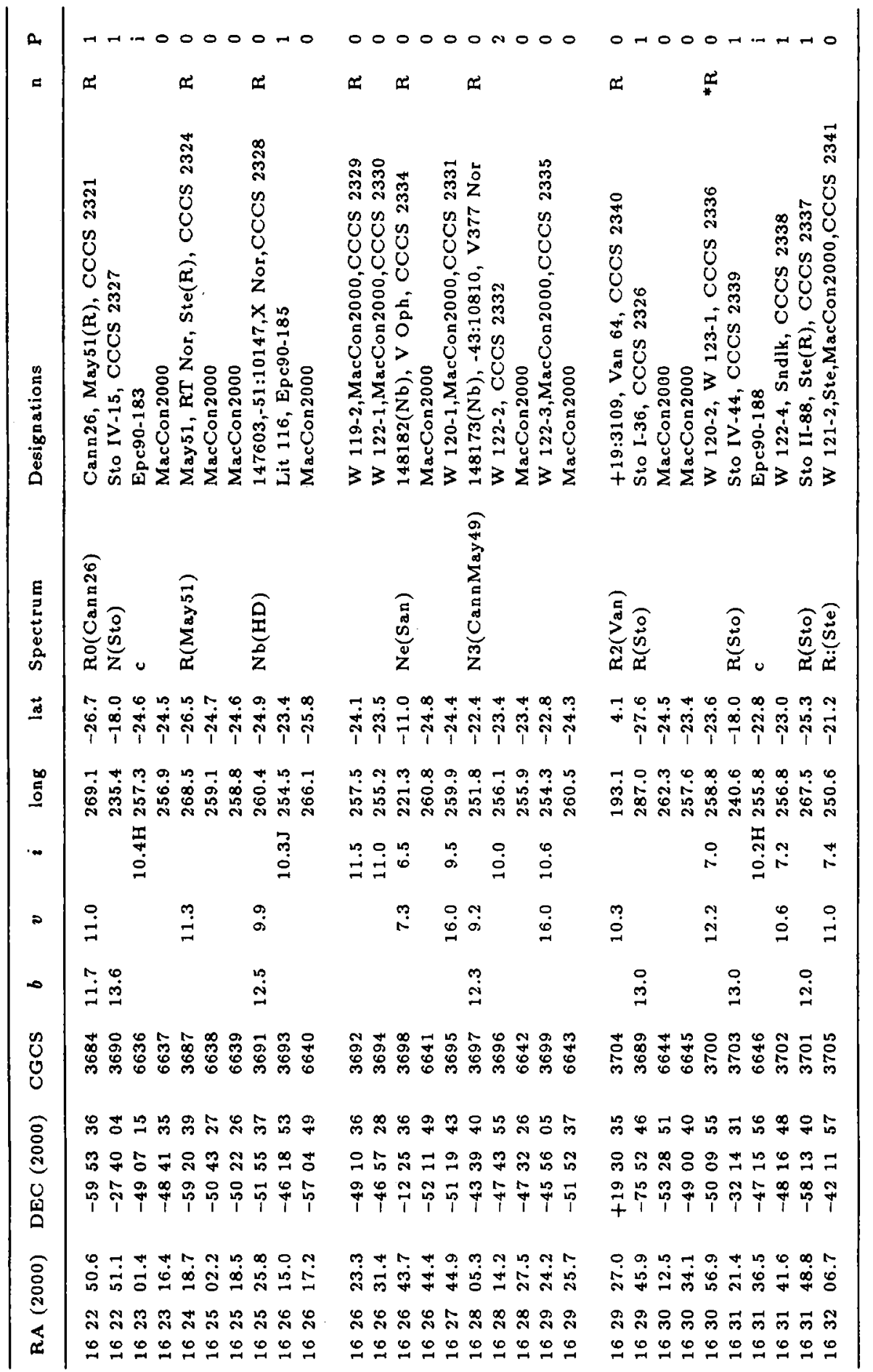




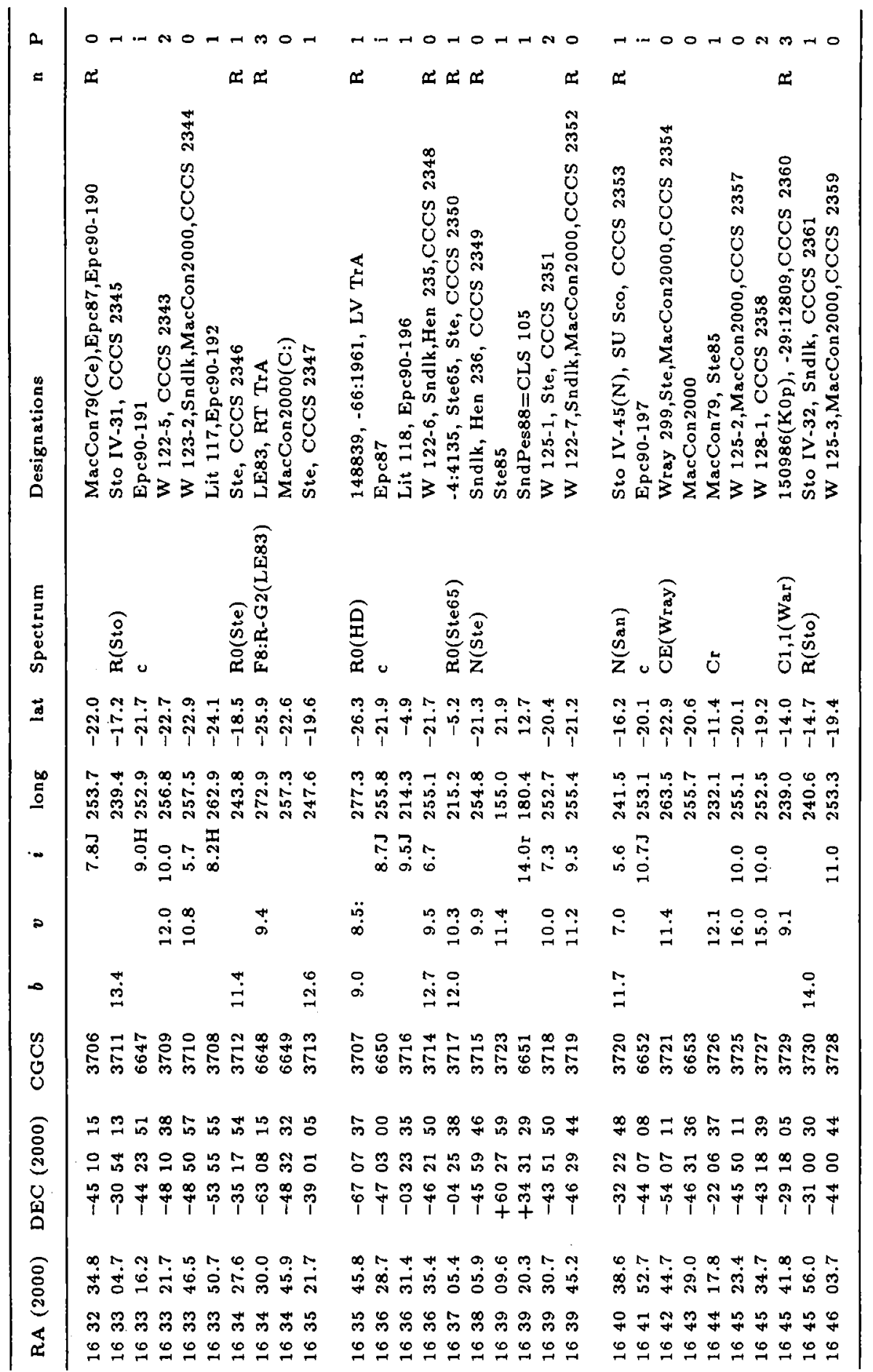




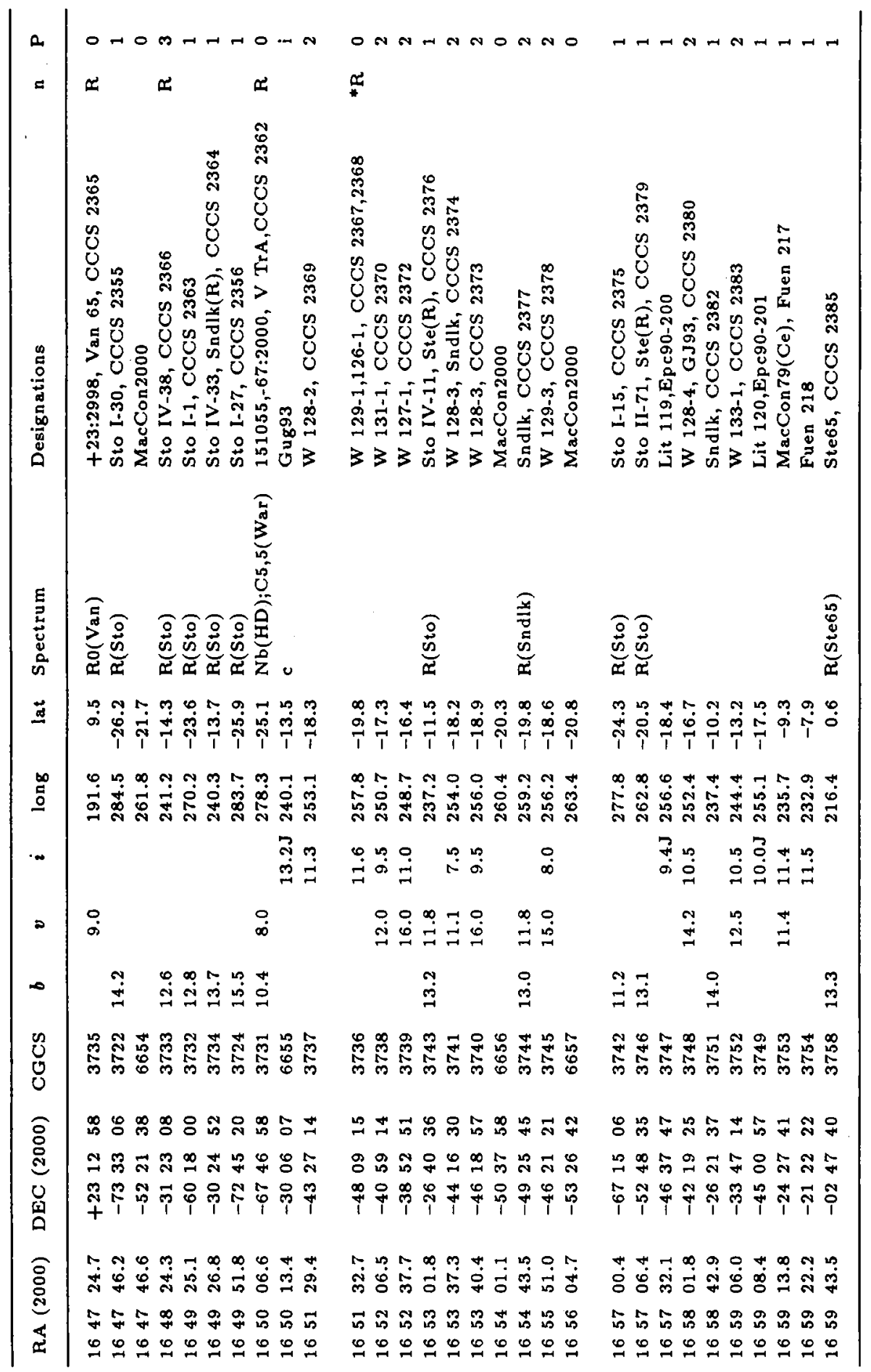




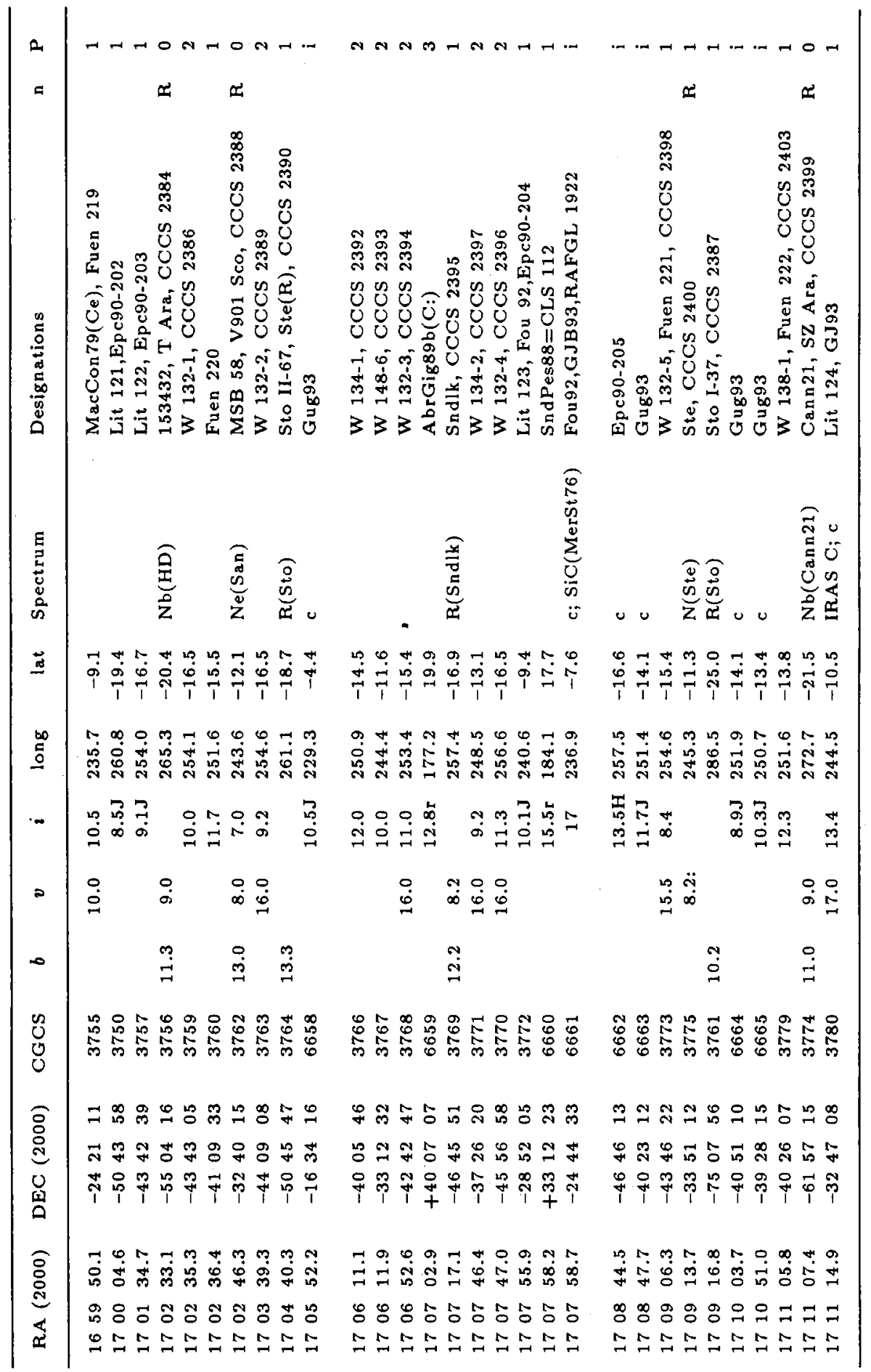




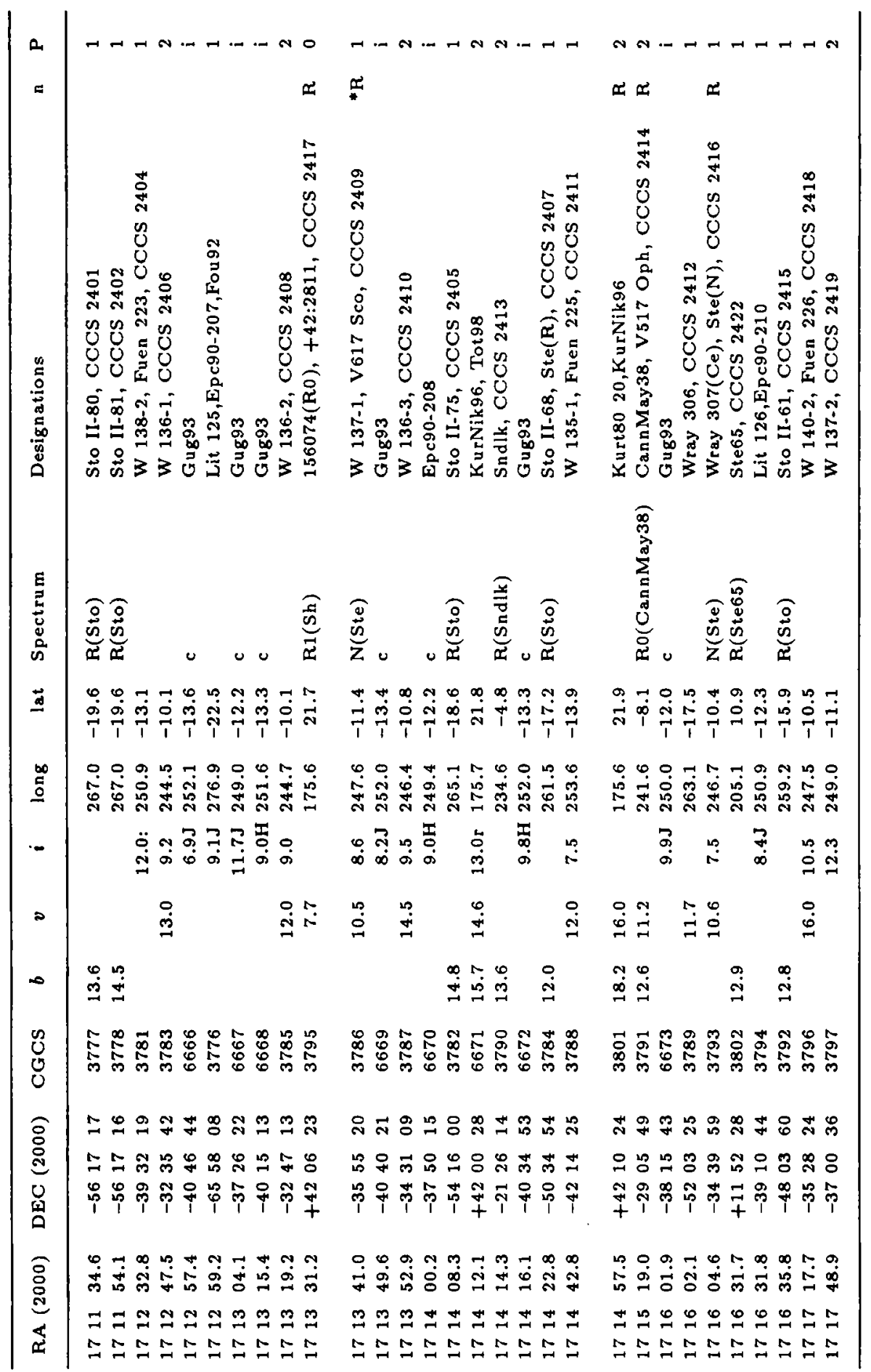




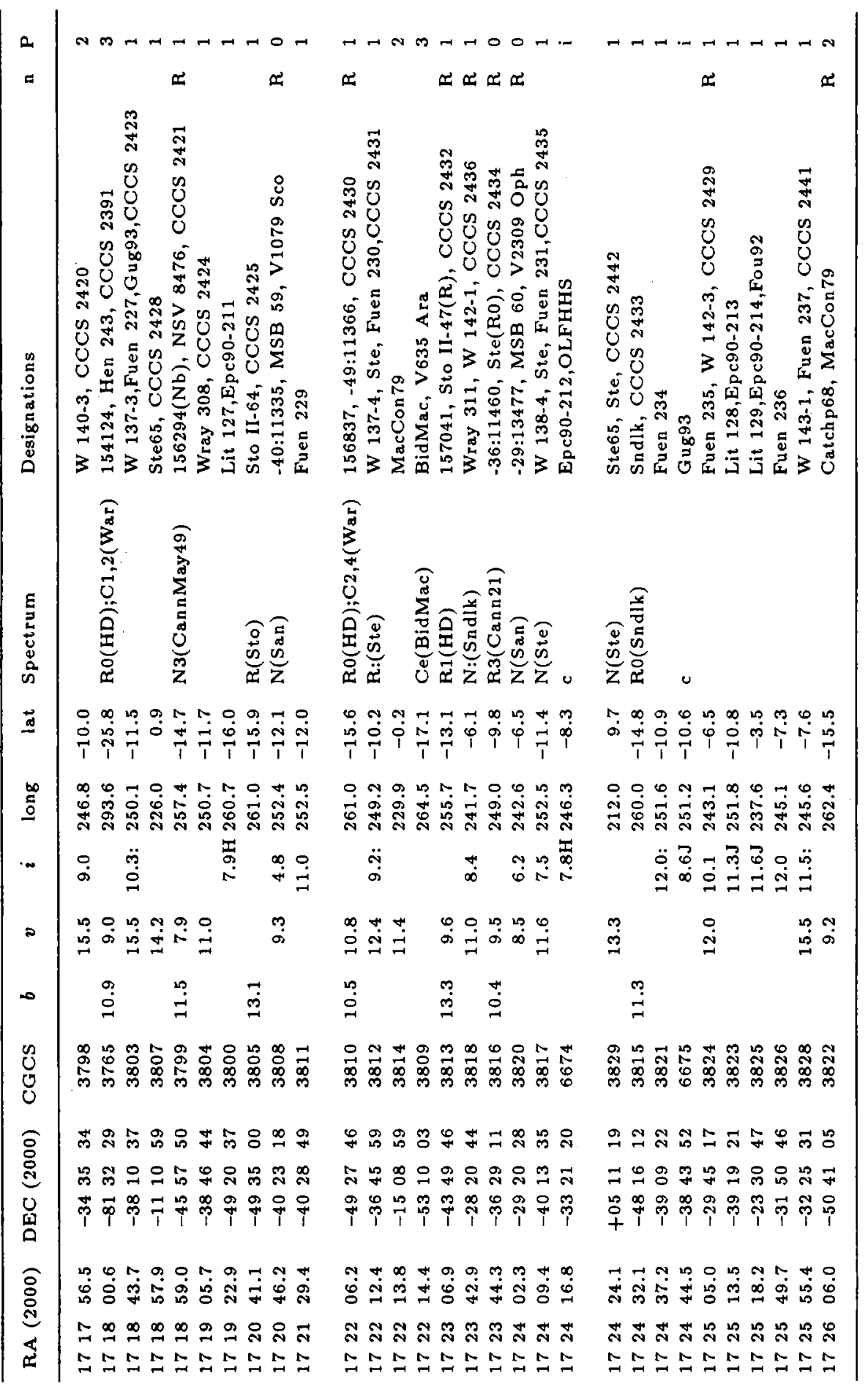




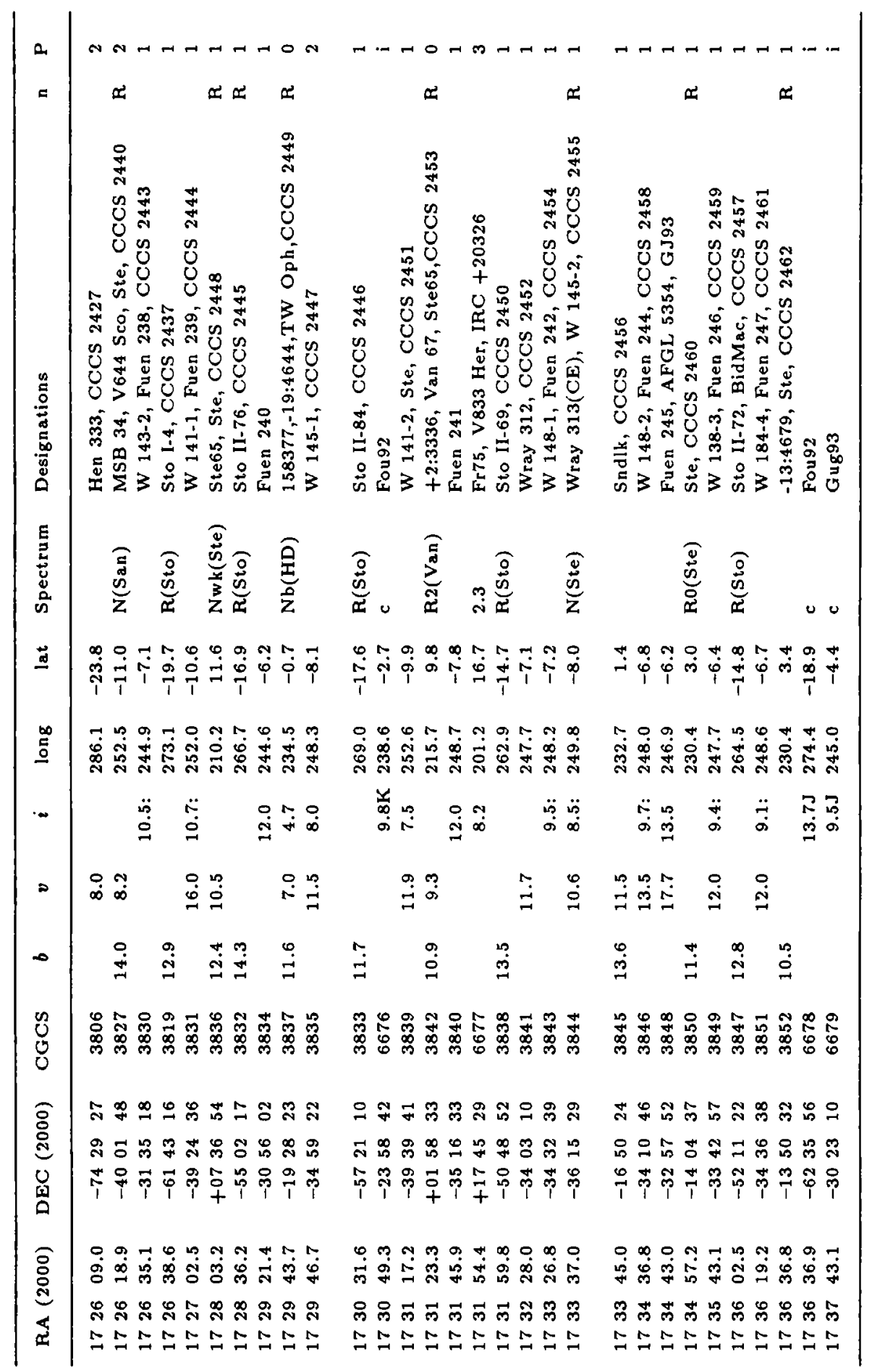




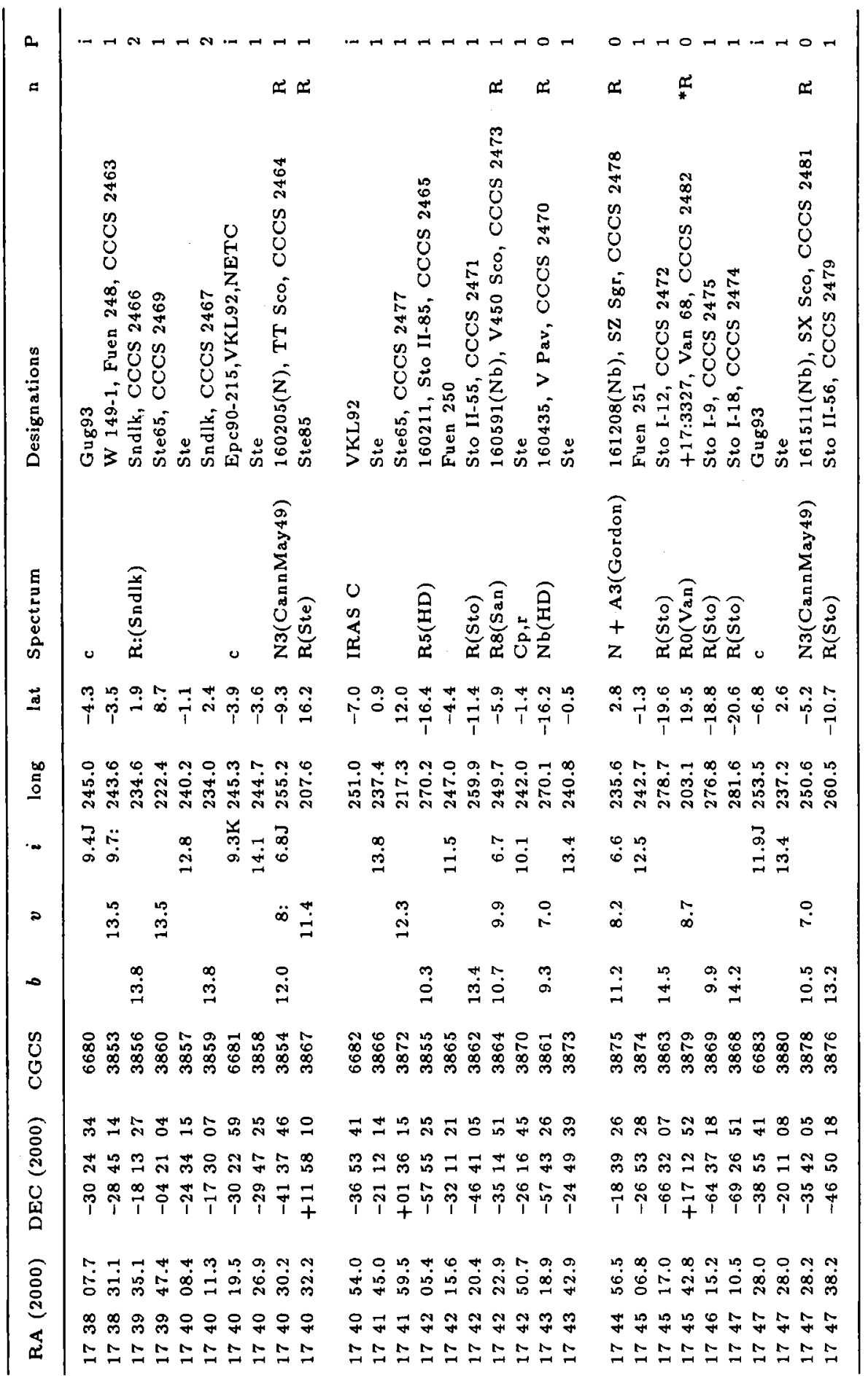




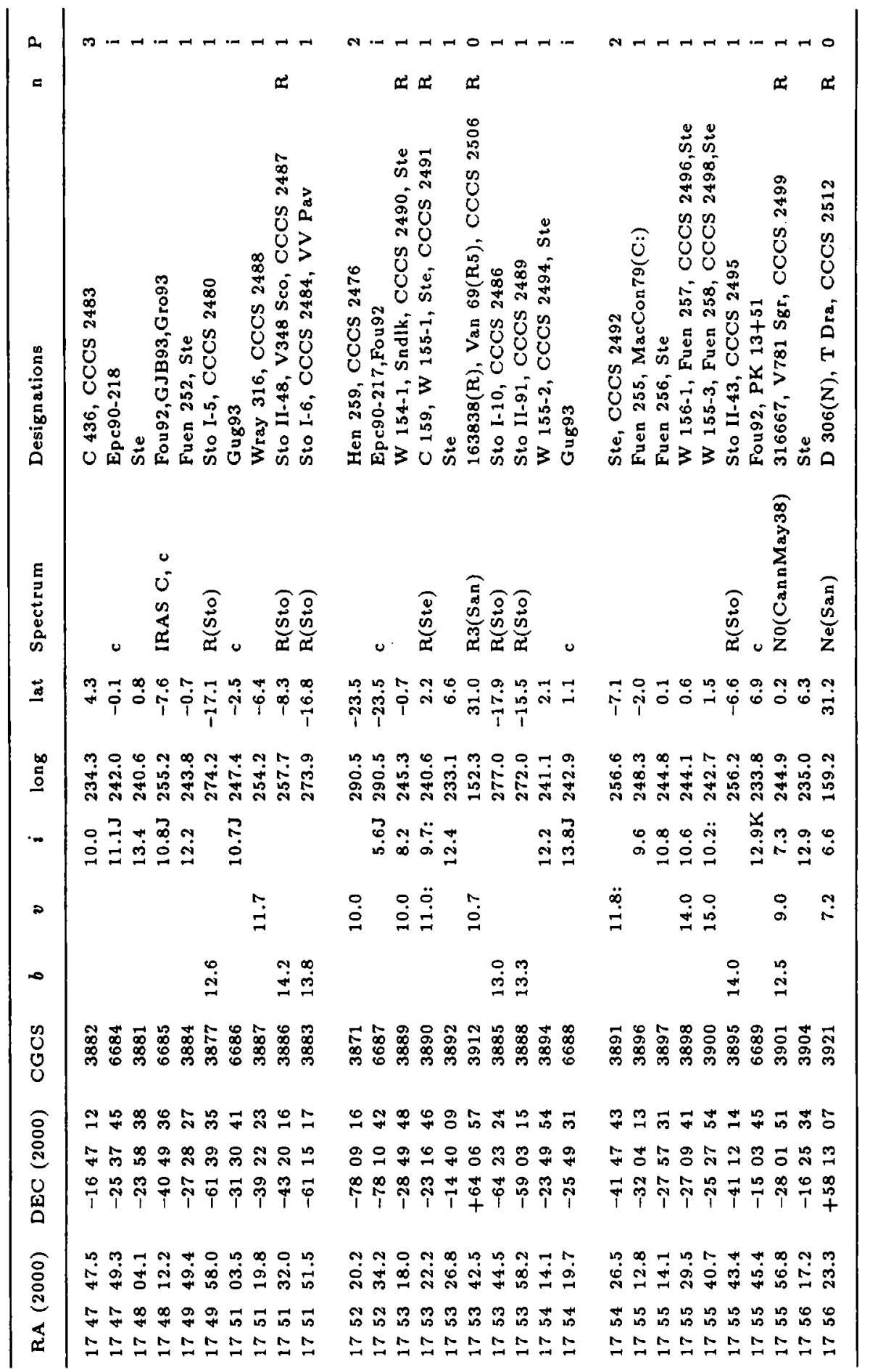




\begin{tabular}{|c|c|c|c|}
\hline a & $\begin{array}{l}x \rightarrow H \rightarrow H \rightarrow H \\
H \rightarrow H\end{array}$ & 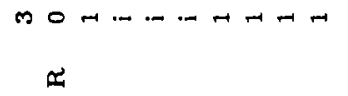 & 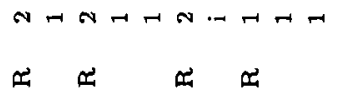 \\
\hline 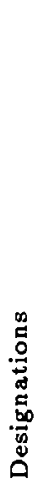 & 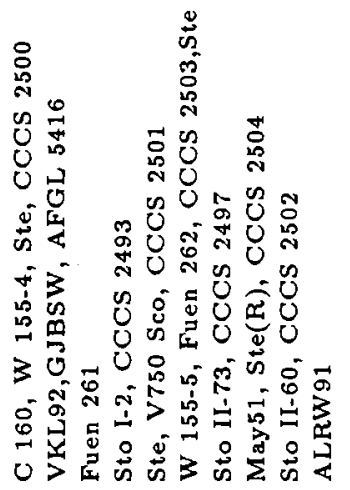 & 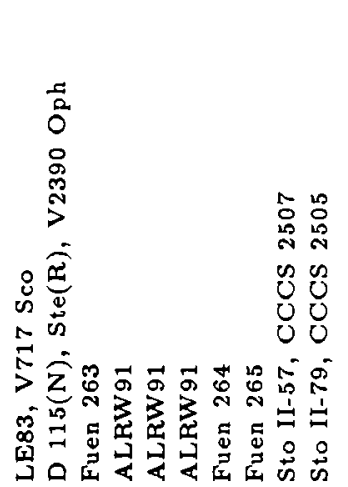 & 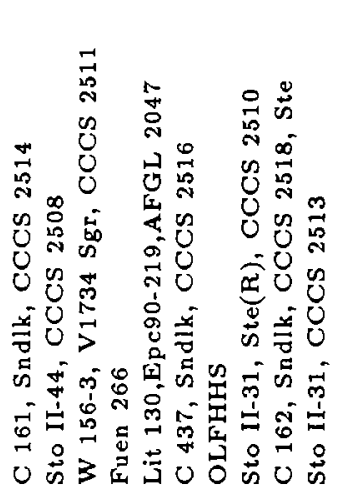 \\
\hline 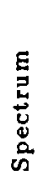 & 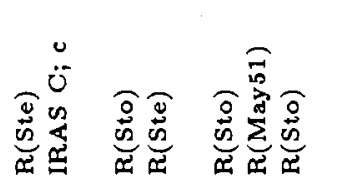 & 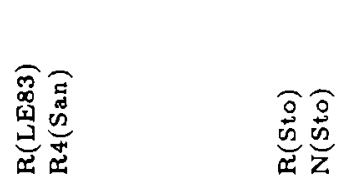 & 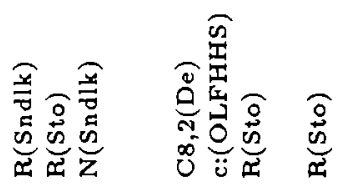 \\
\hline$\stackrel{d}{ }$ & 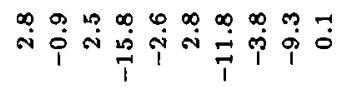 & प̆i & 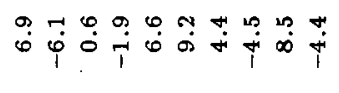 \\
\hline$\stackrel{\text { so }}{0}$ & 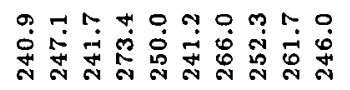 & 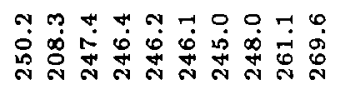 & 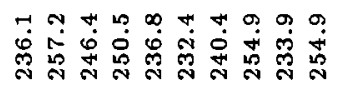 \\
\hline$\rightarrow$ & 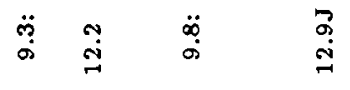 & 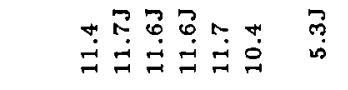 & 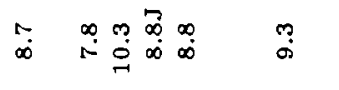 \\
\hline ? & $\stackrel{\leftrightarrow ?}{\dot{H}}$ & $\overrightarrow{\dot{9}} \overrightarrow{9}$ & 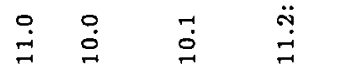 \\
\hline ه & 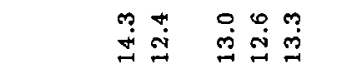 & 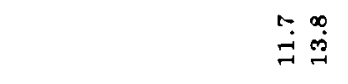 & 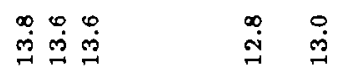 \\
\hline $\begin{array}{l}0 \\
0 \\
0 \\
0\end{array}$ & 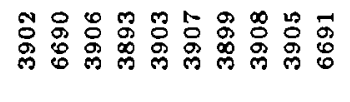 & 조유 & 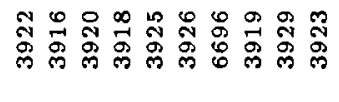 \\
\hline के & 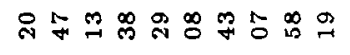 & 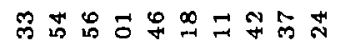 & $\vec{m} \vec{m} 5$ 分 N \\
\hline $\begin{array}{l}\stackrel{\text { D }}{0} \\
\text { 品 } \\
0\end{array}$ & 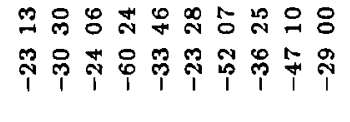 & 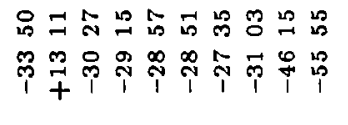 & 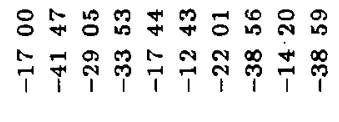 \\
\hline ڤ્ & 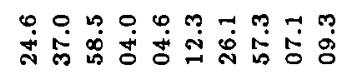 & 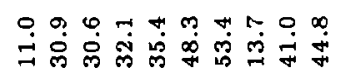 & 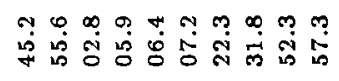 \\
\hline 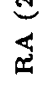 & 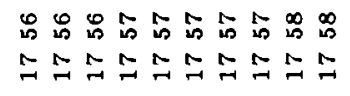 & 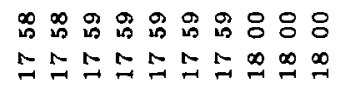 & 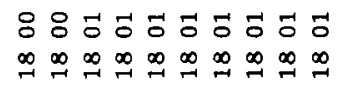 \\
\hline
\end{tabular}




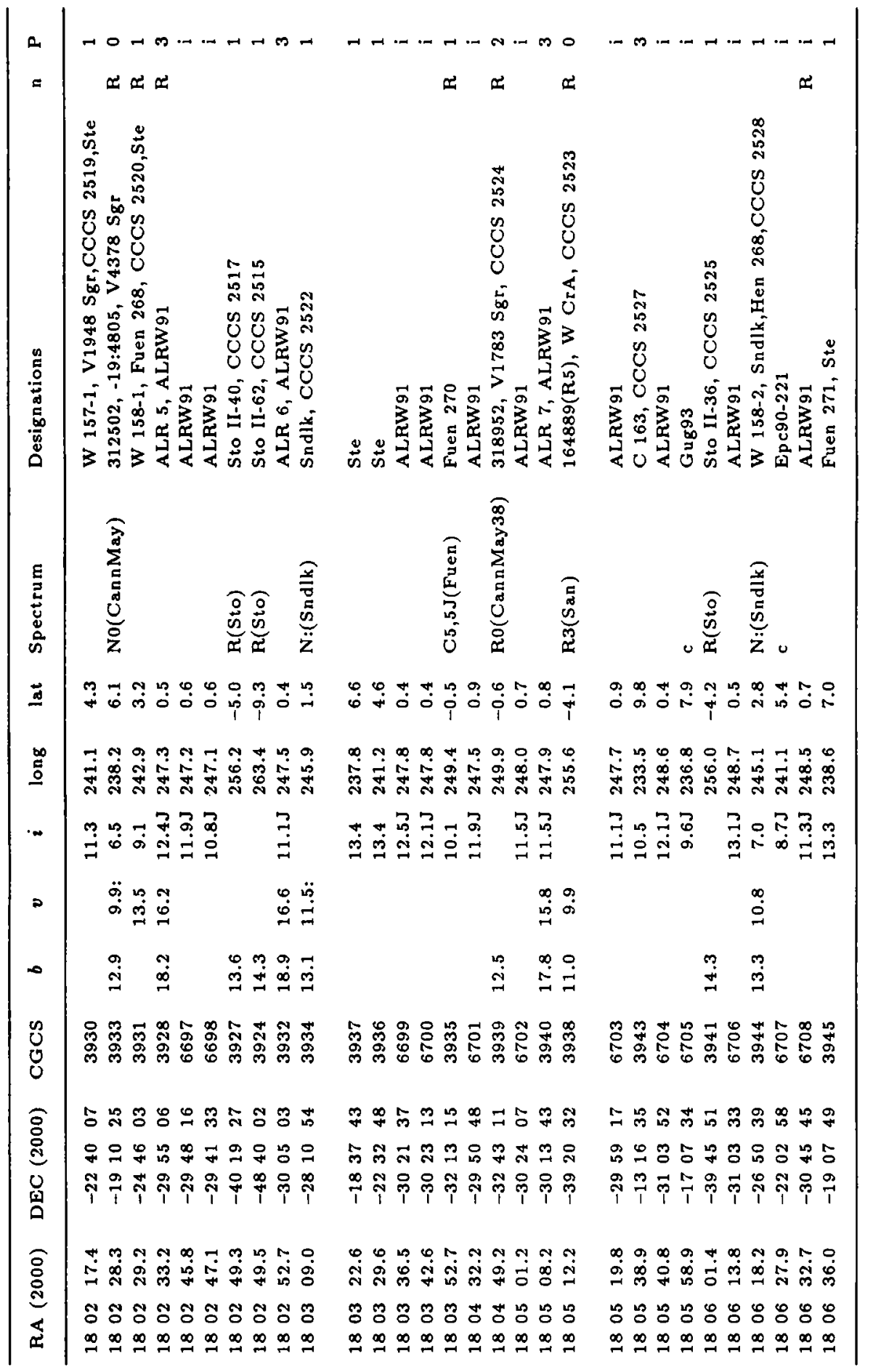




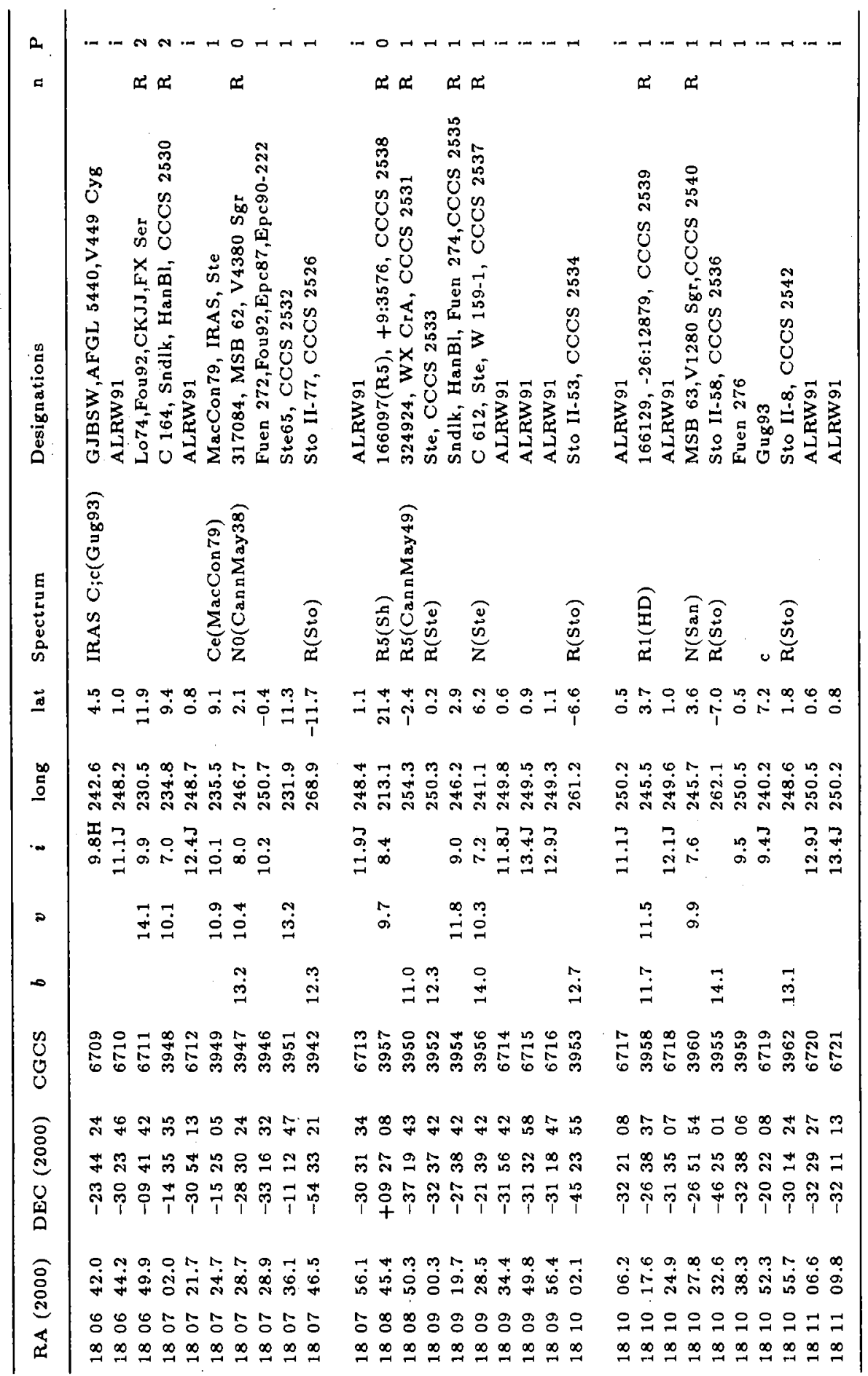




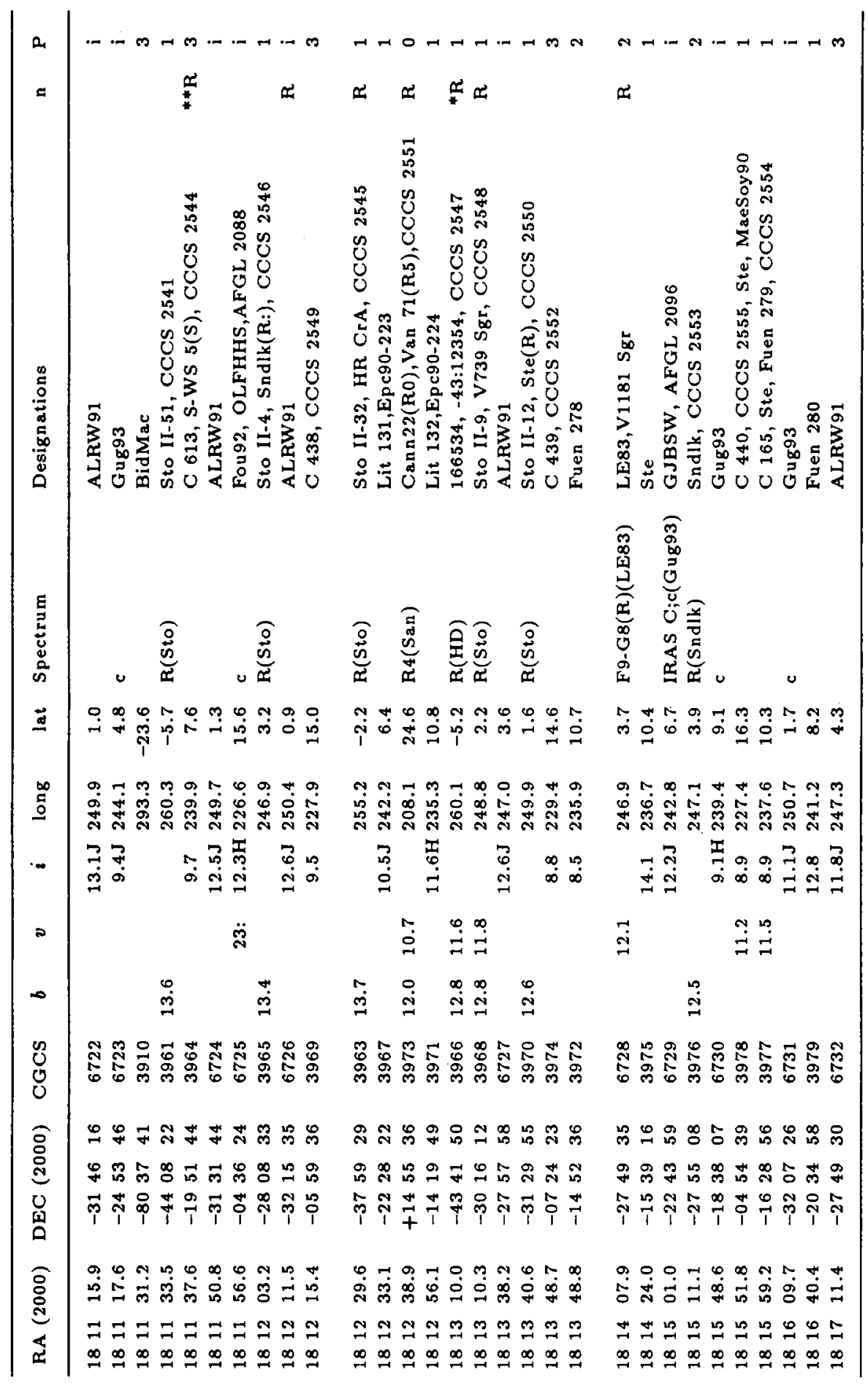




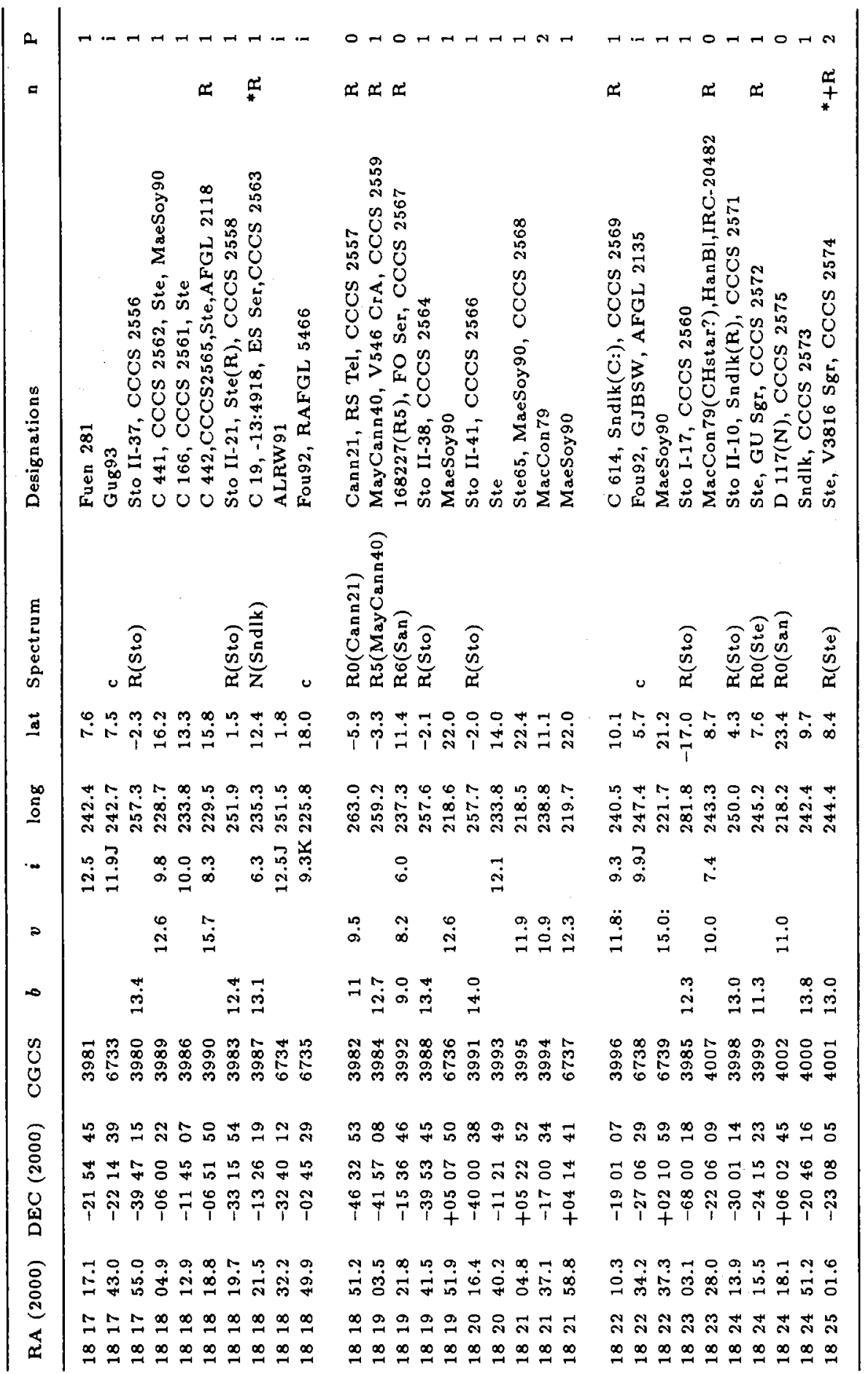




\begin{tabular}{|c|c|c|c|}
\hline a & 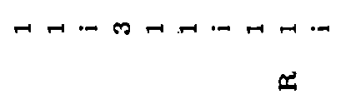 & 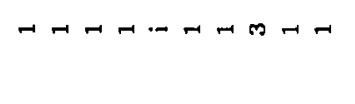 & 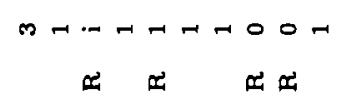 \\
\hline 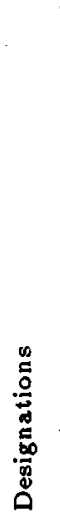 & 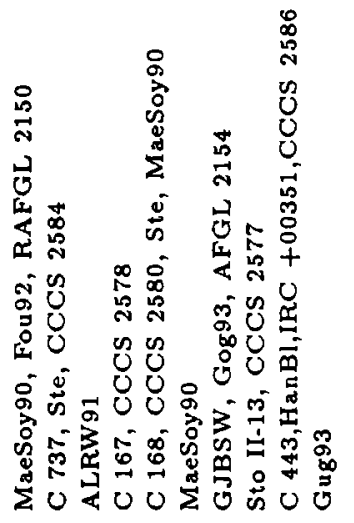 & 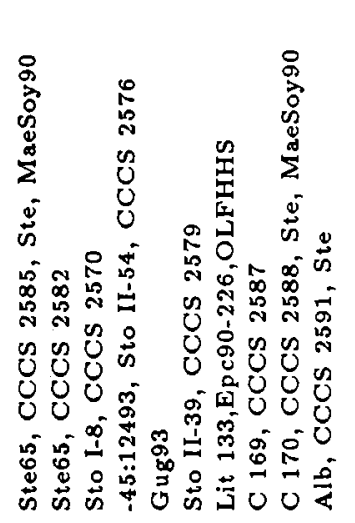 & 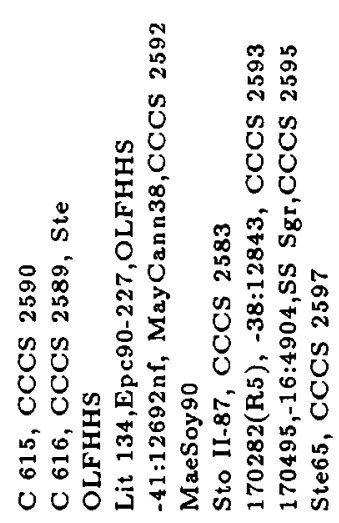 \\
\hline 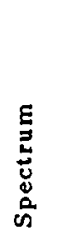 & 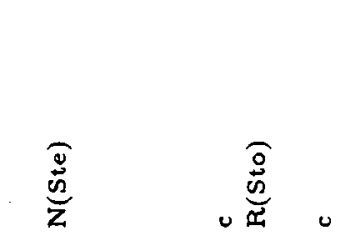 & 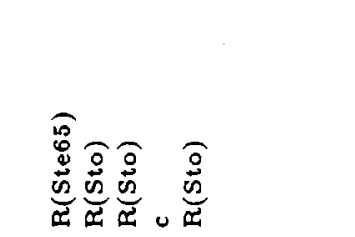 & 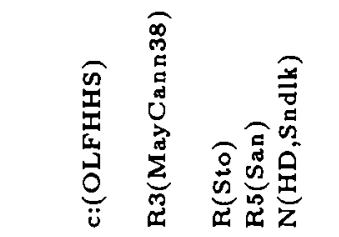 \\
\hline$\underline{d}$ & 禹芦 & 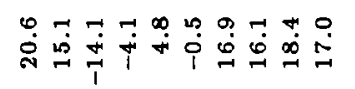 & 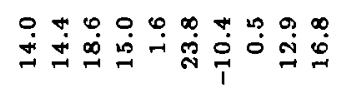 \\
\hline$\stackrel{\infty}{\underline{\Xi}}$ & 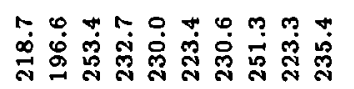 & 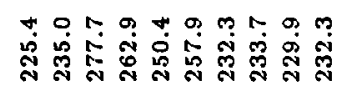 & 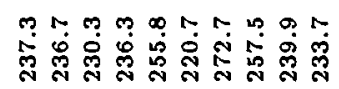 \\
\hline 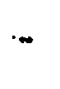 & 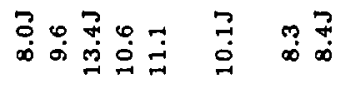 & $\Xi \quad$ 葛 & 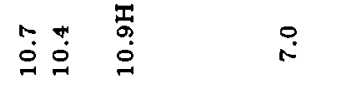 \\
\hline 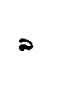 & 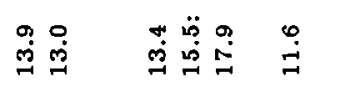 & 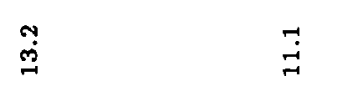 & 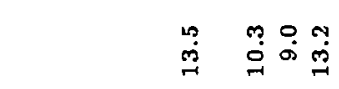 \\
\hline ـ & $\stackrel{9}{g}$ & 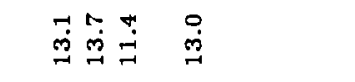 & 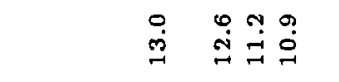 \\
\hline $\begin{array}{l}y \\
0 \\
y\end{array}$ & 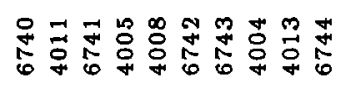 & 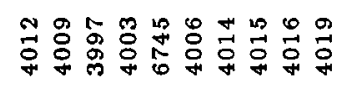 & 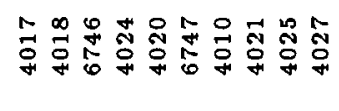 \\
\hline$\widehat{8}$ & 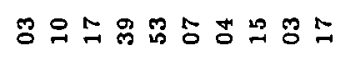 & 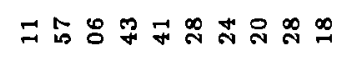 & 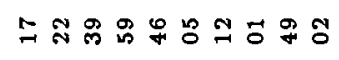 \\
\hline 过 & 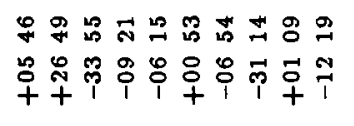 & 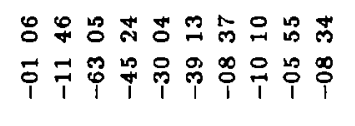 & 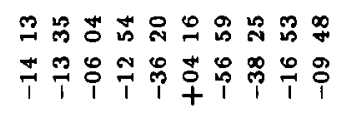 \\
\hline ڤे & 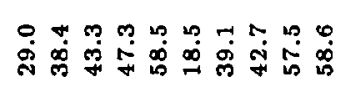 & 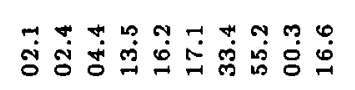 & 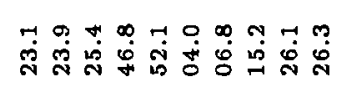 \\
\hline 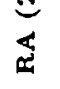 & 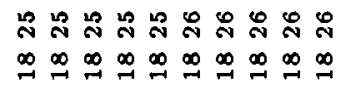 & 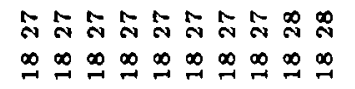 & 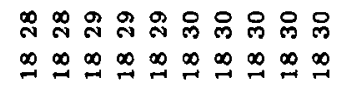 \\
\hline
\end{tabular}




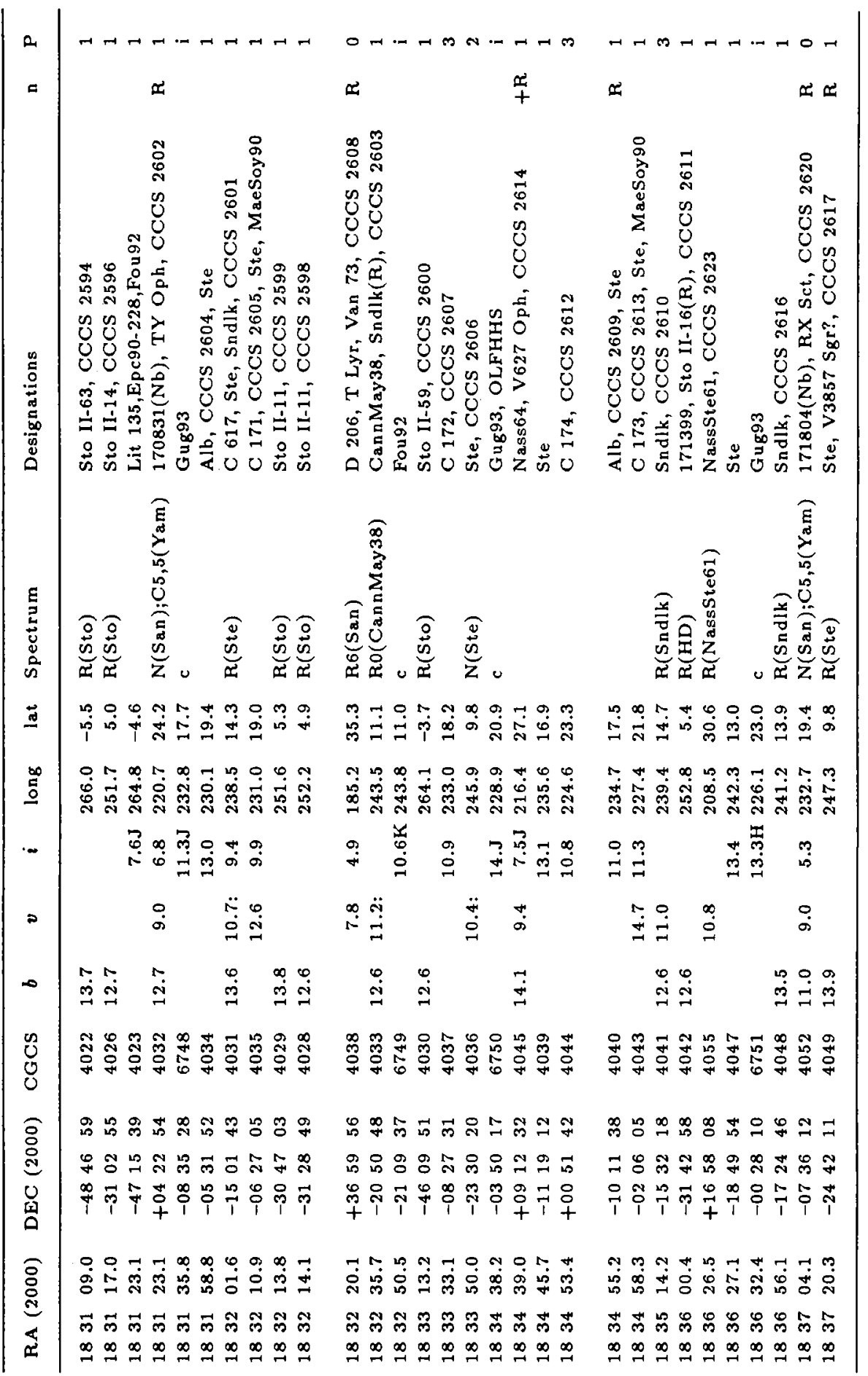




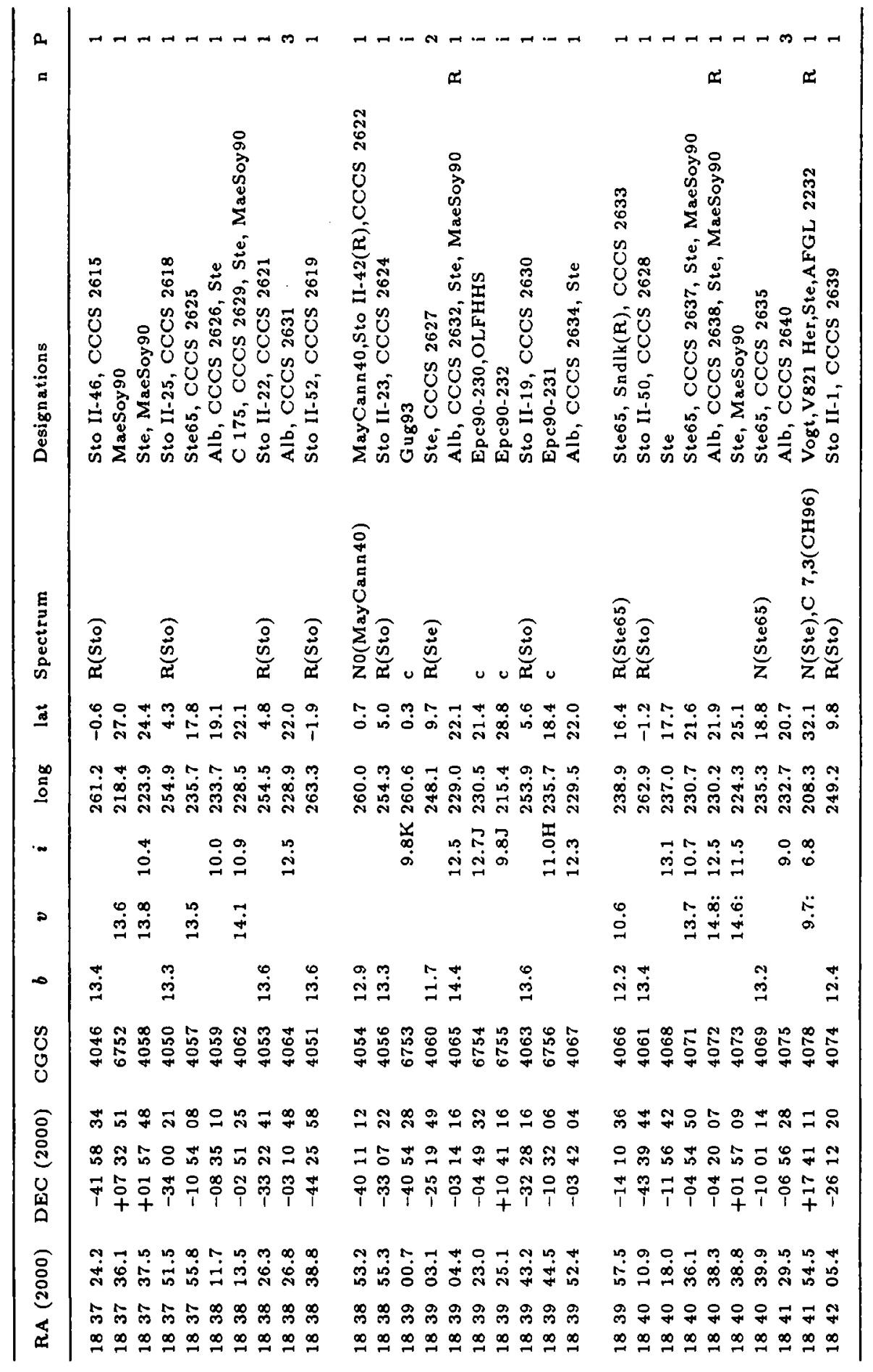




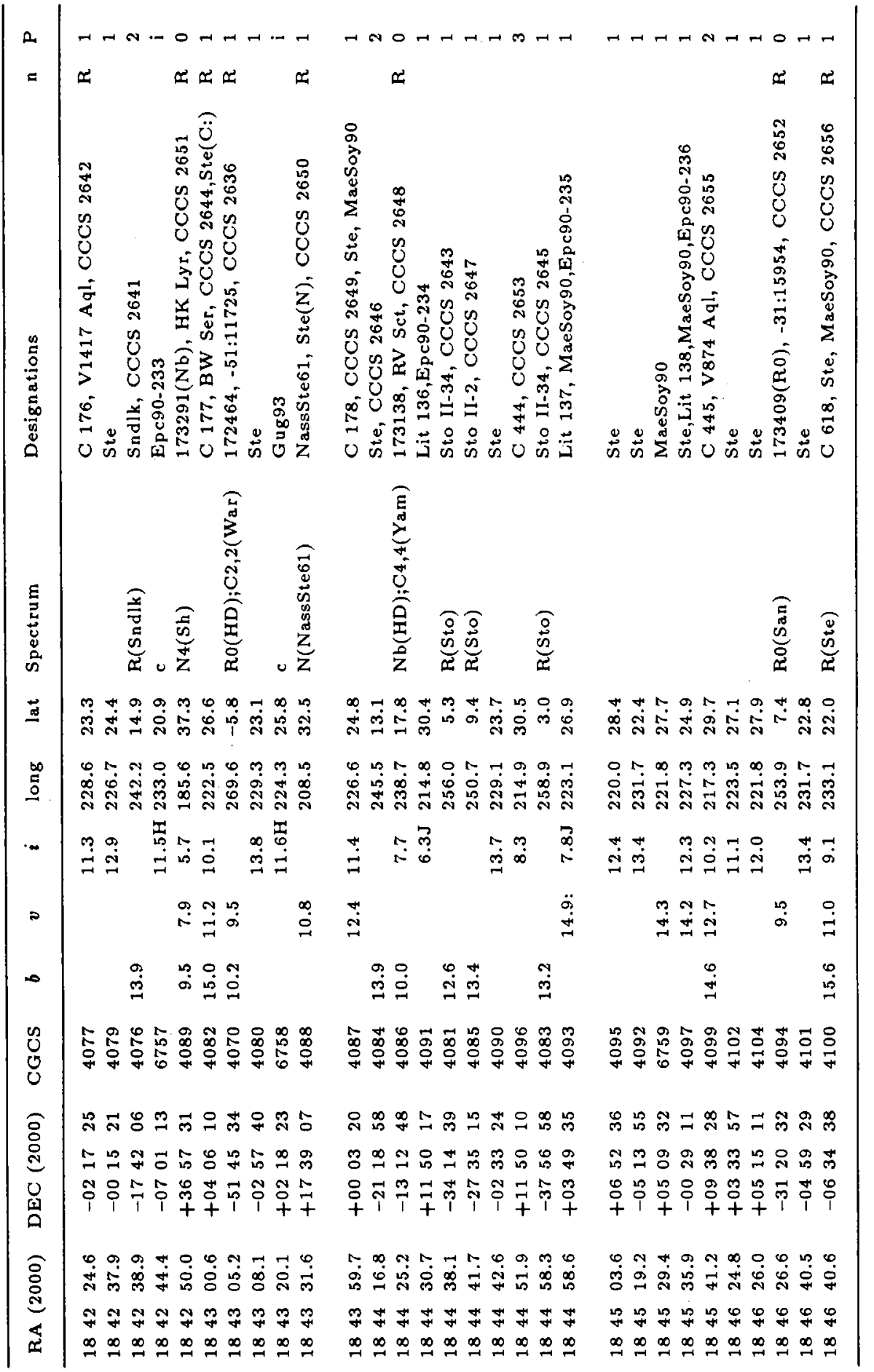




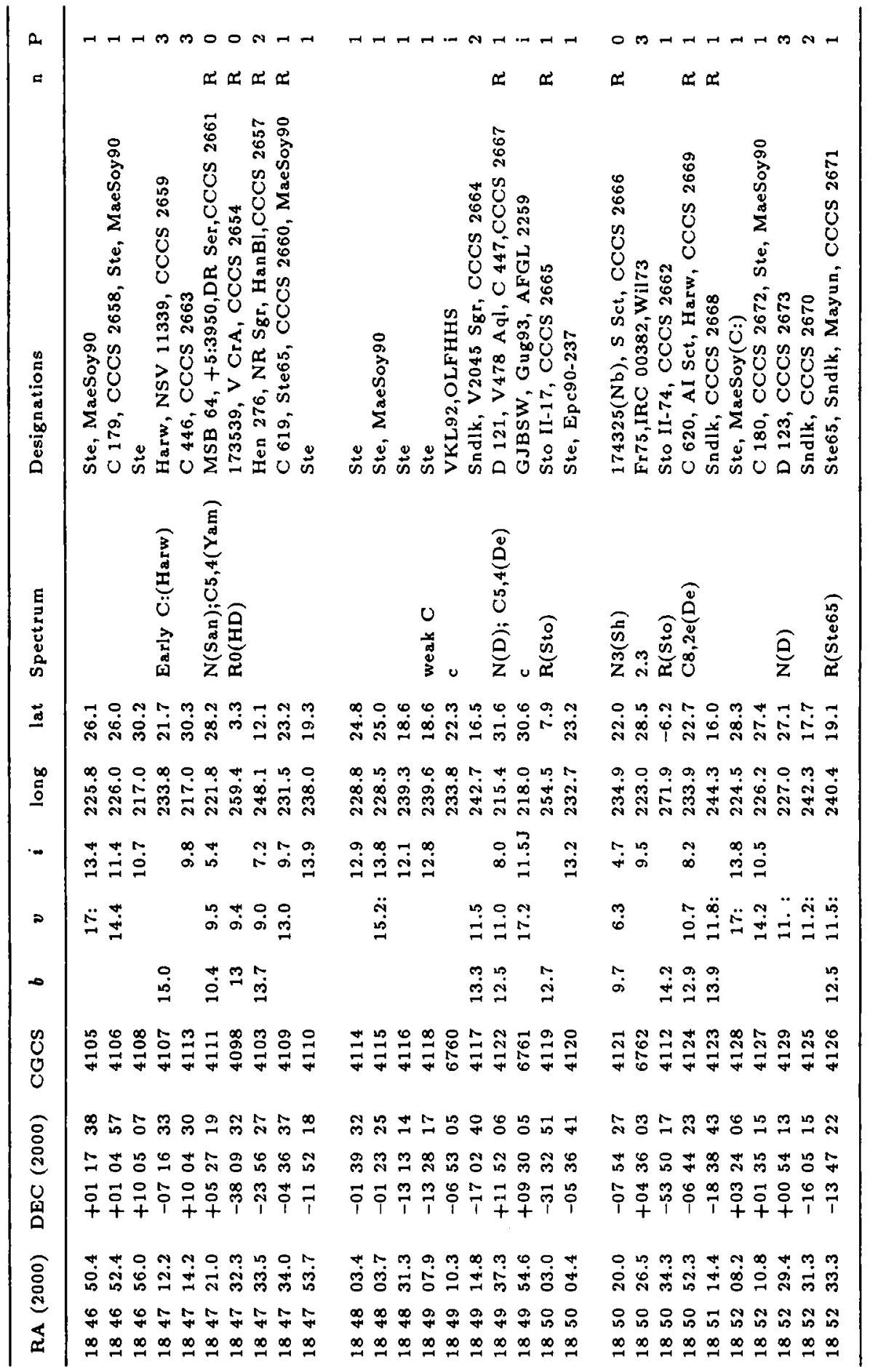




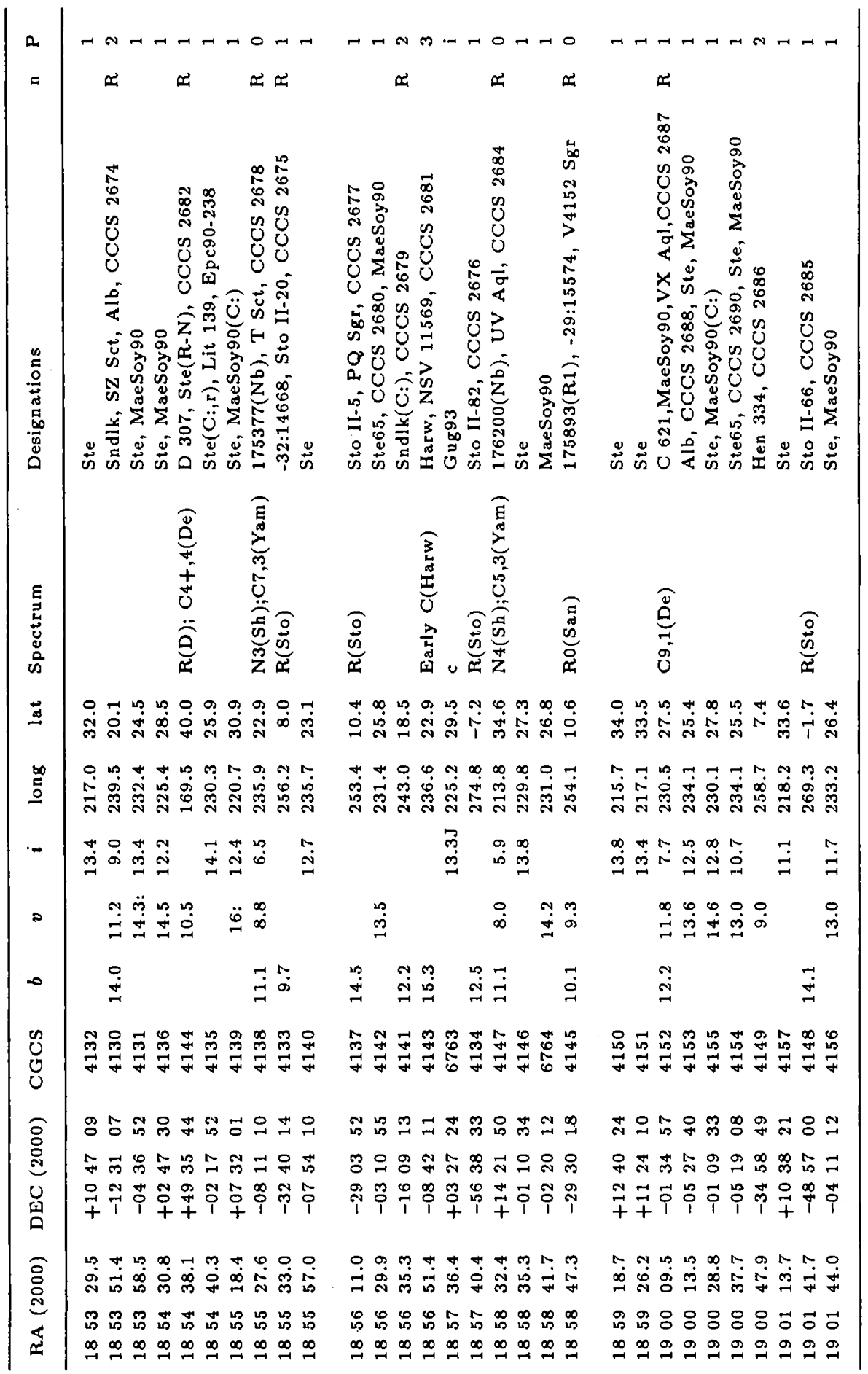




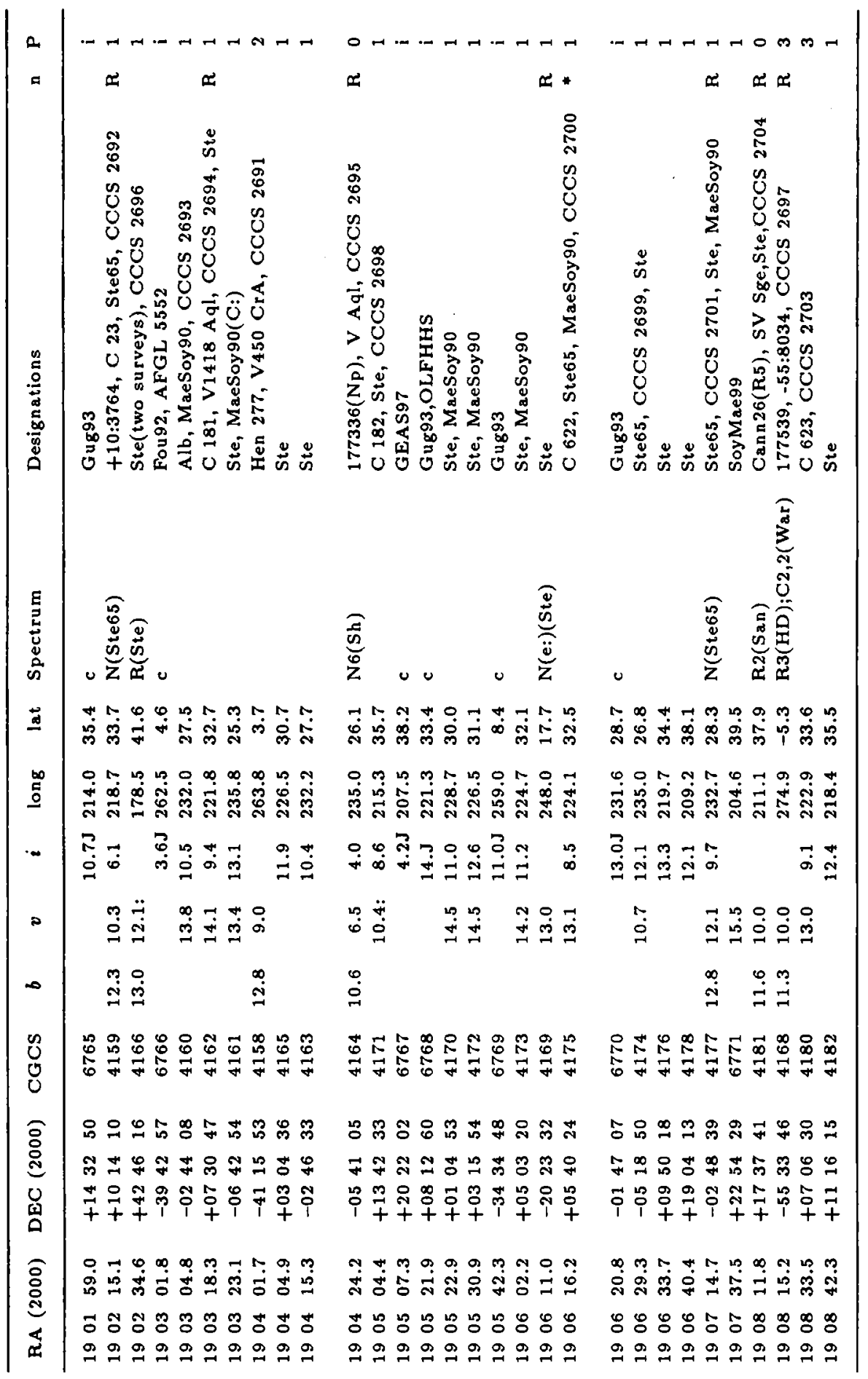




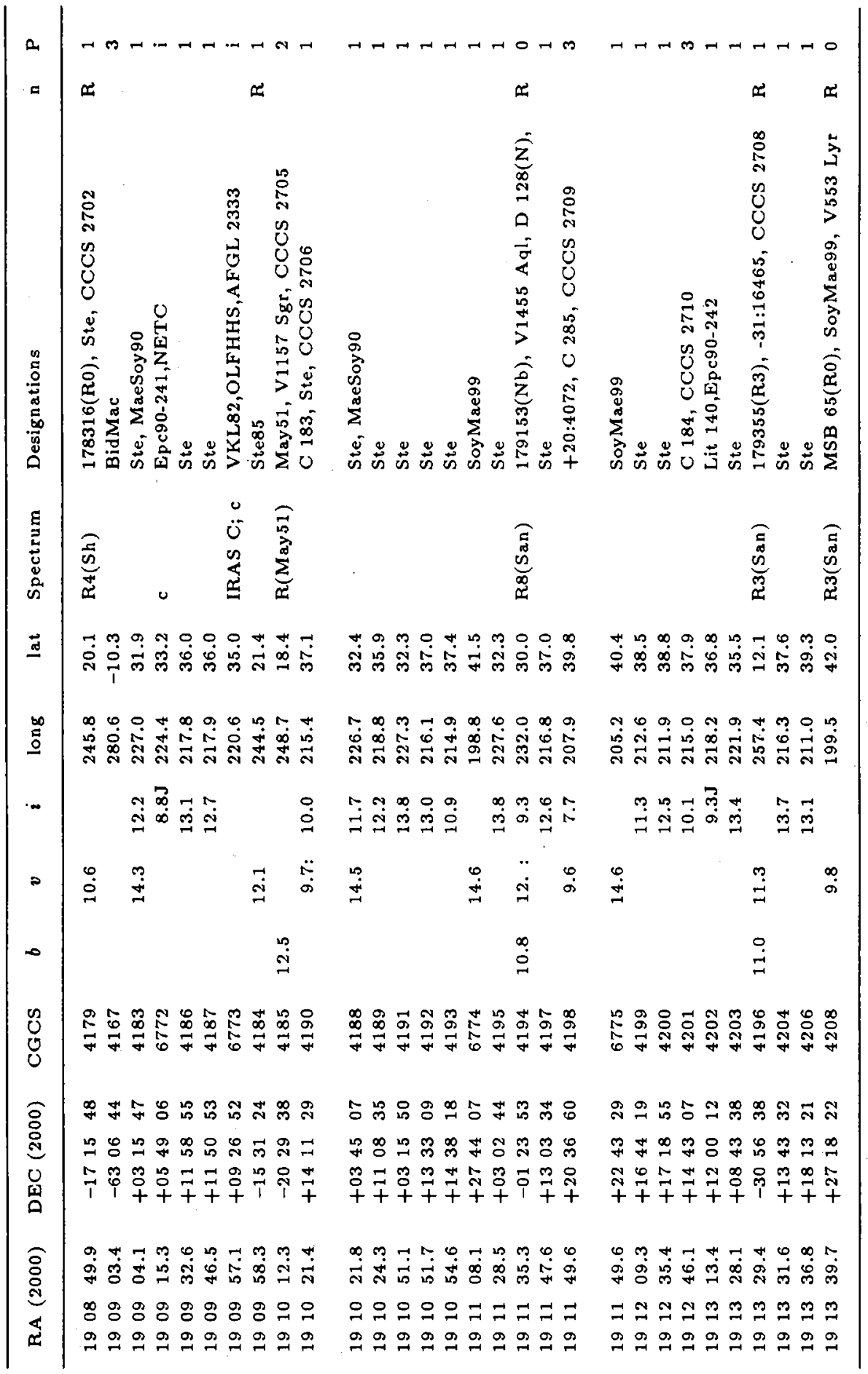




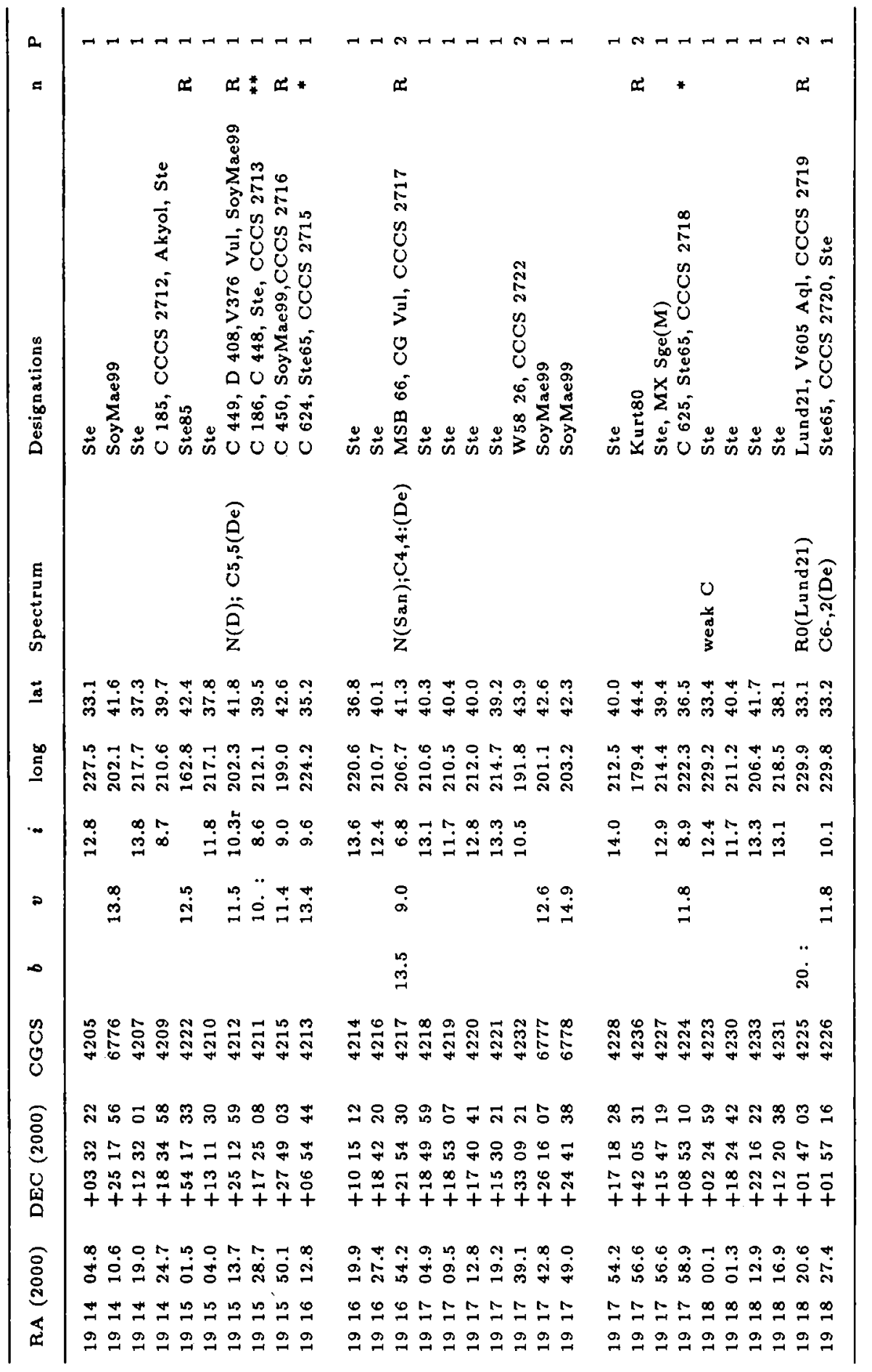




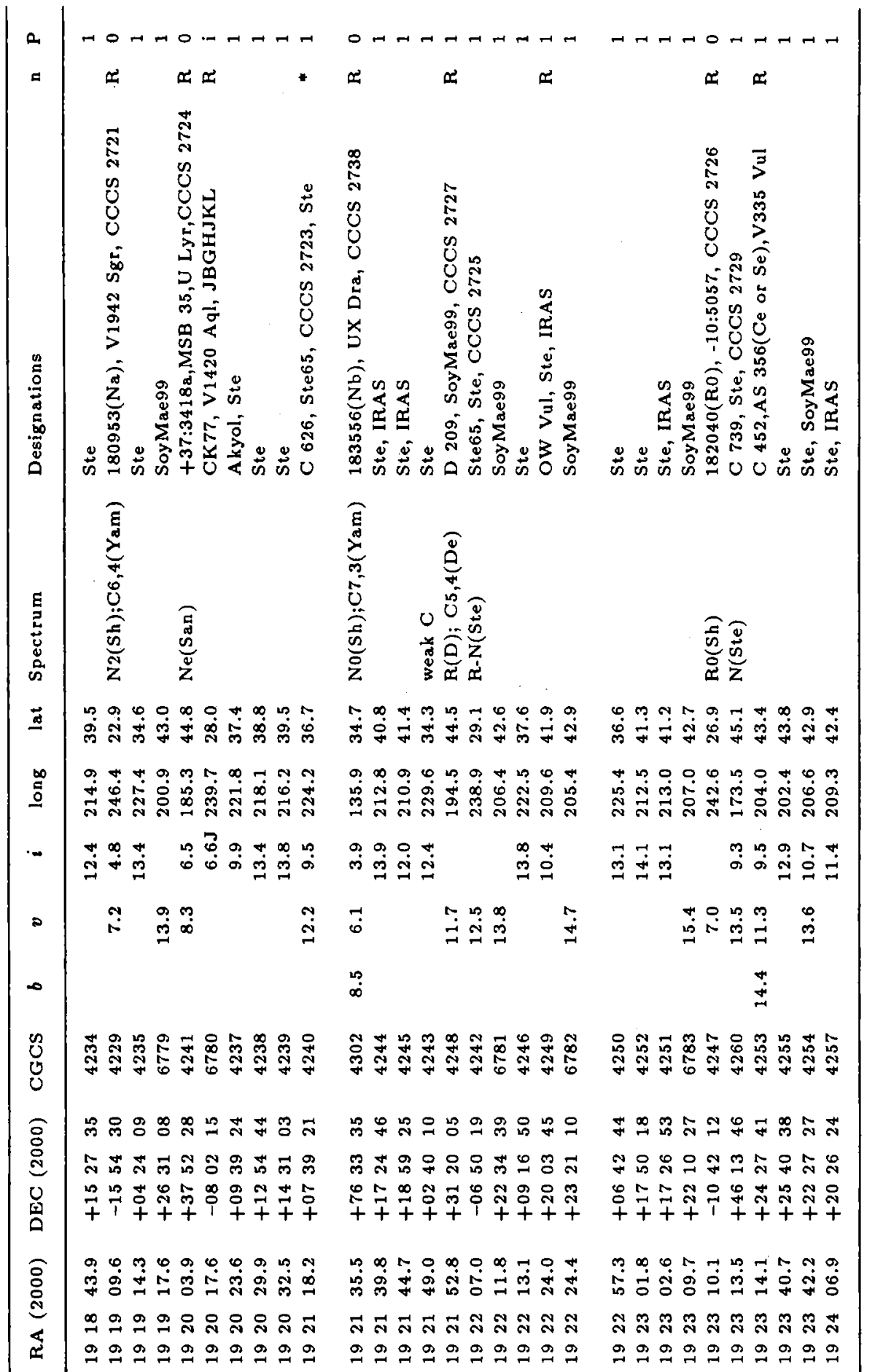




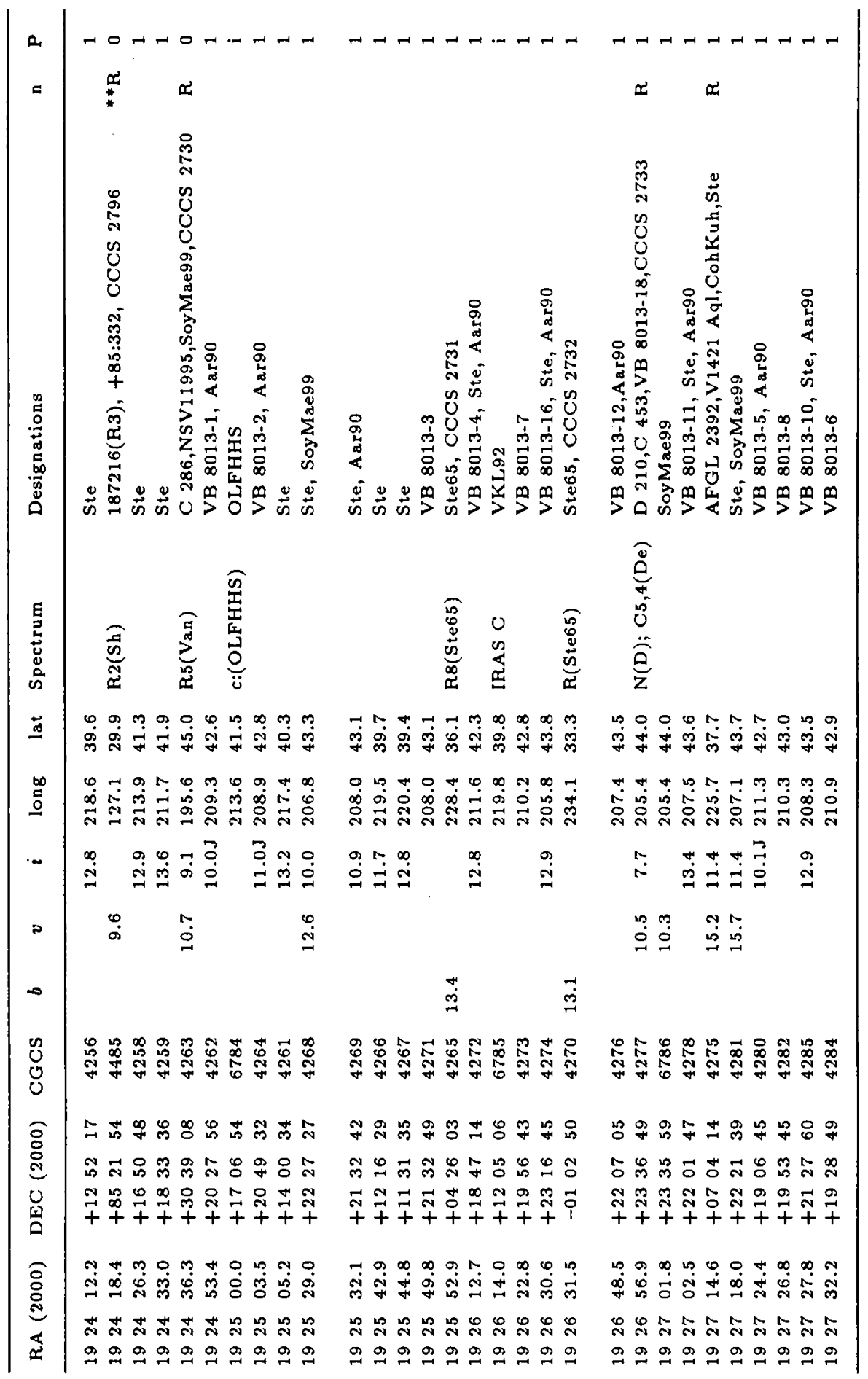




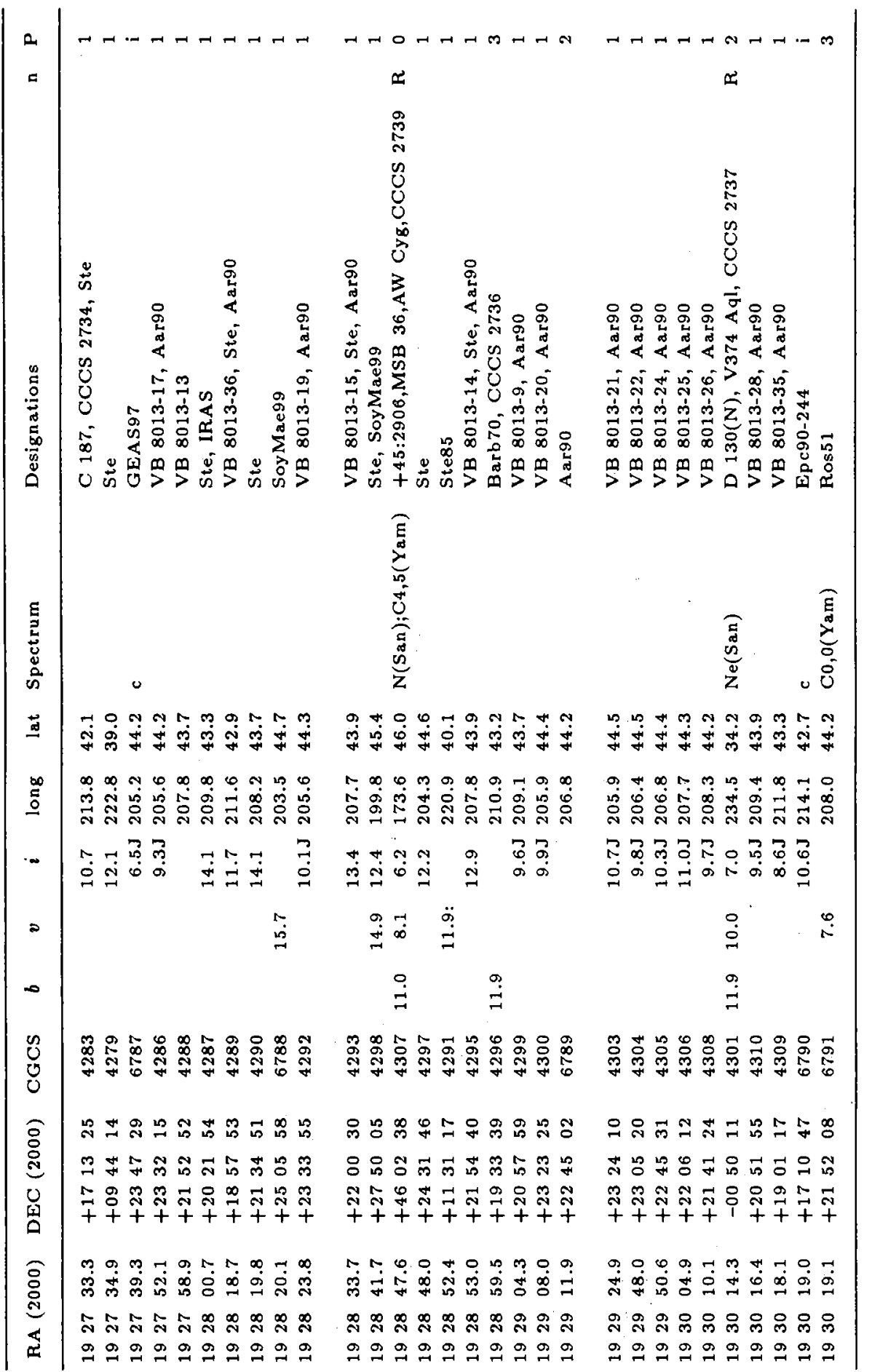




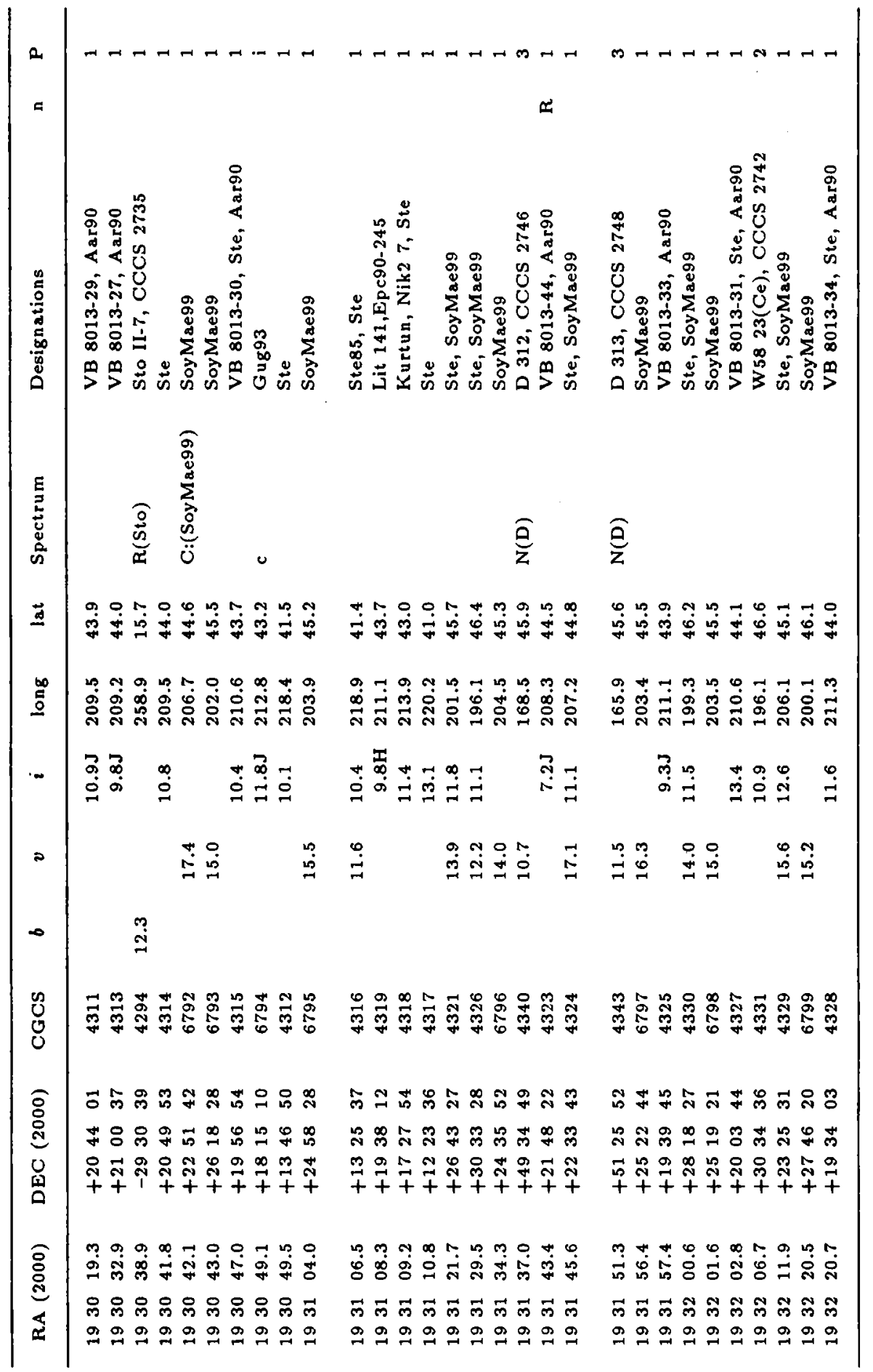




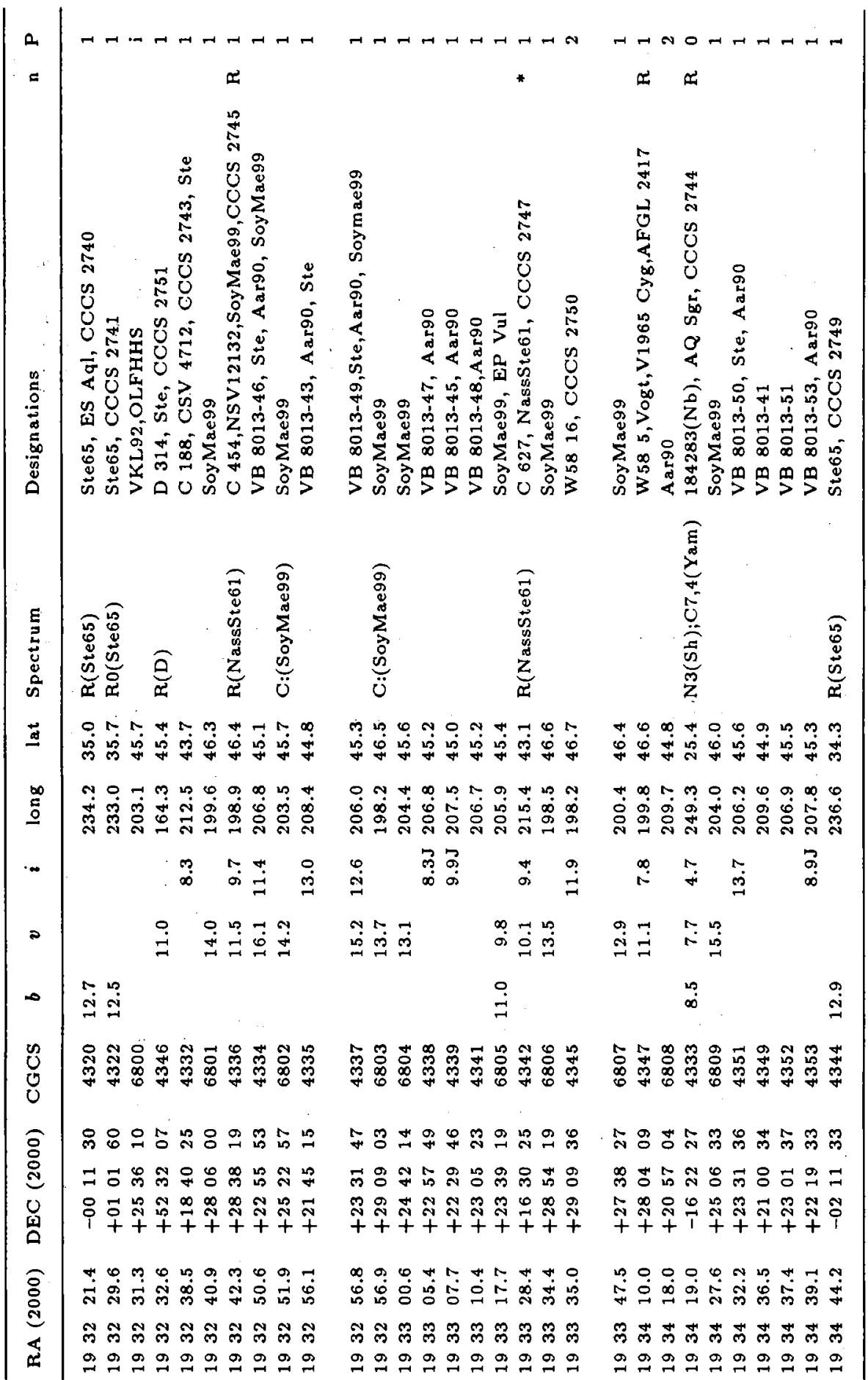




\begin{tabular}{|c|c|c|c|}
\hline 2 & ルコルナルルナルの & $\rightarrow H \rightarrow-n \rightarrow-A \rightarrow H$ & 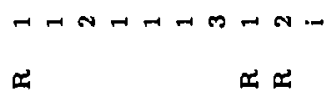 \\
\hline 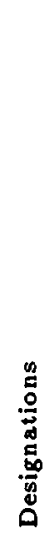 & 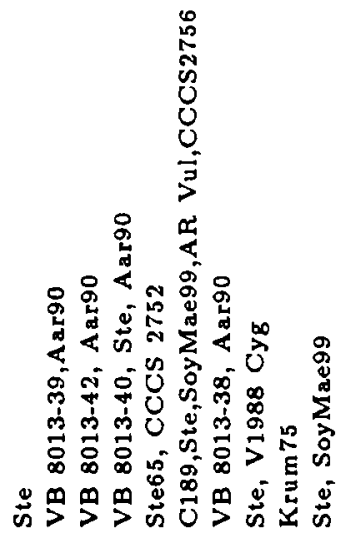 & 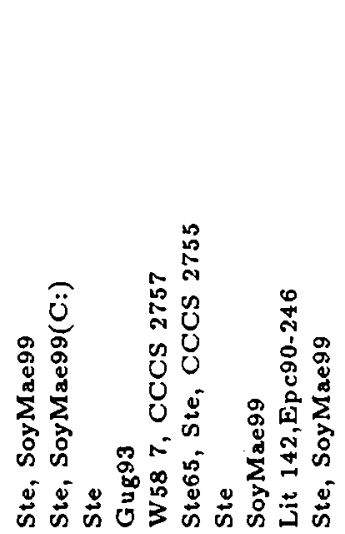 & 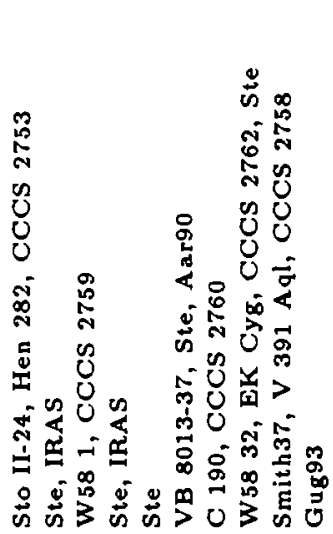 \\
\hline 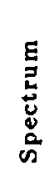 & 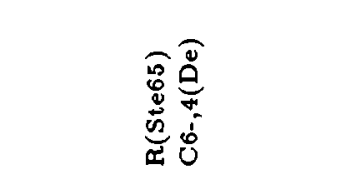 & 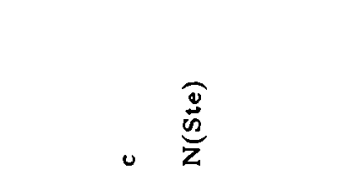 & 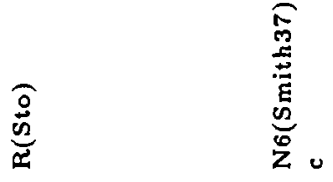 \\
\hline a & 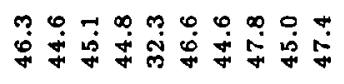 & 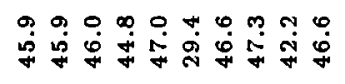 & 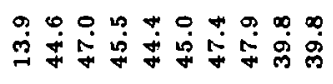 \\
\hline$\stackrel{\infty}{\Xi}$ & 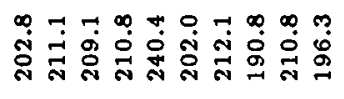 & 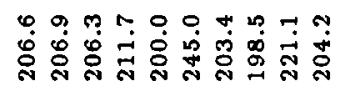 & 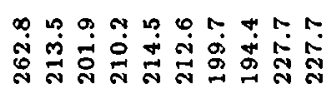 \\
\hline . & 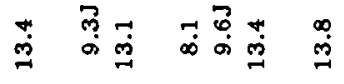 & 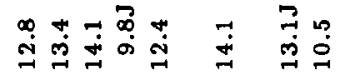 & 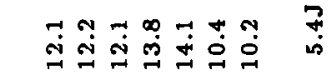 \\
\hline$D$ & $\stackrel{\varphi}{\dot{0}}$ & 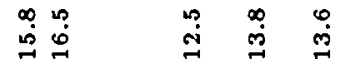 & $\stackrel{\infty}{\leftrightarrows}$ \\
\hline$\infty$ & 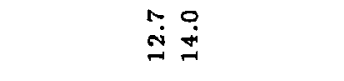 & & 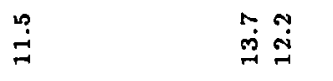 \\
\hline $\begin{array}{l}0 \\
0 \\
0 \\
0\end{array}$ & 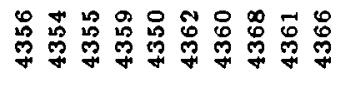 & 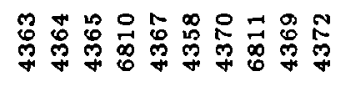 & 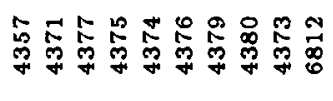 \\
\hline ธิ & 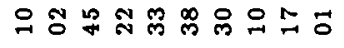 & 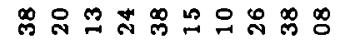 & 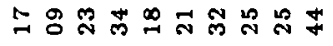 \\
\hline 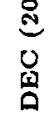 & 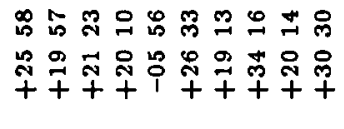 & 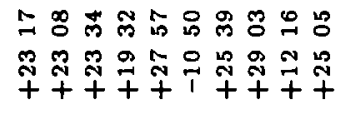 & 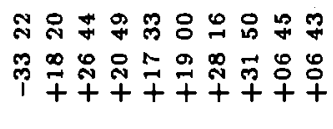 \\
\hline ๕̊․․ & 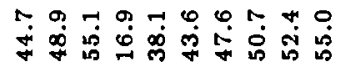 & 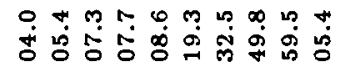 & 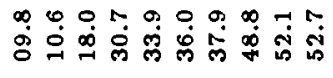 \\
\hline 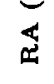 & 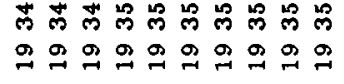 & 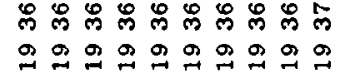 & 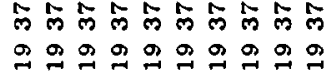 \\
\hline
\end{tabular}




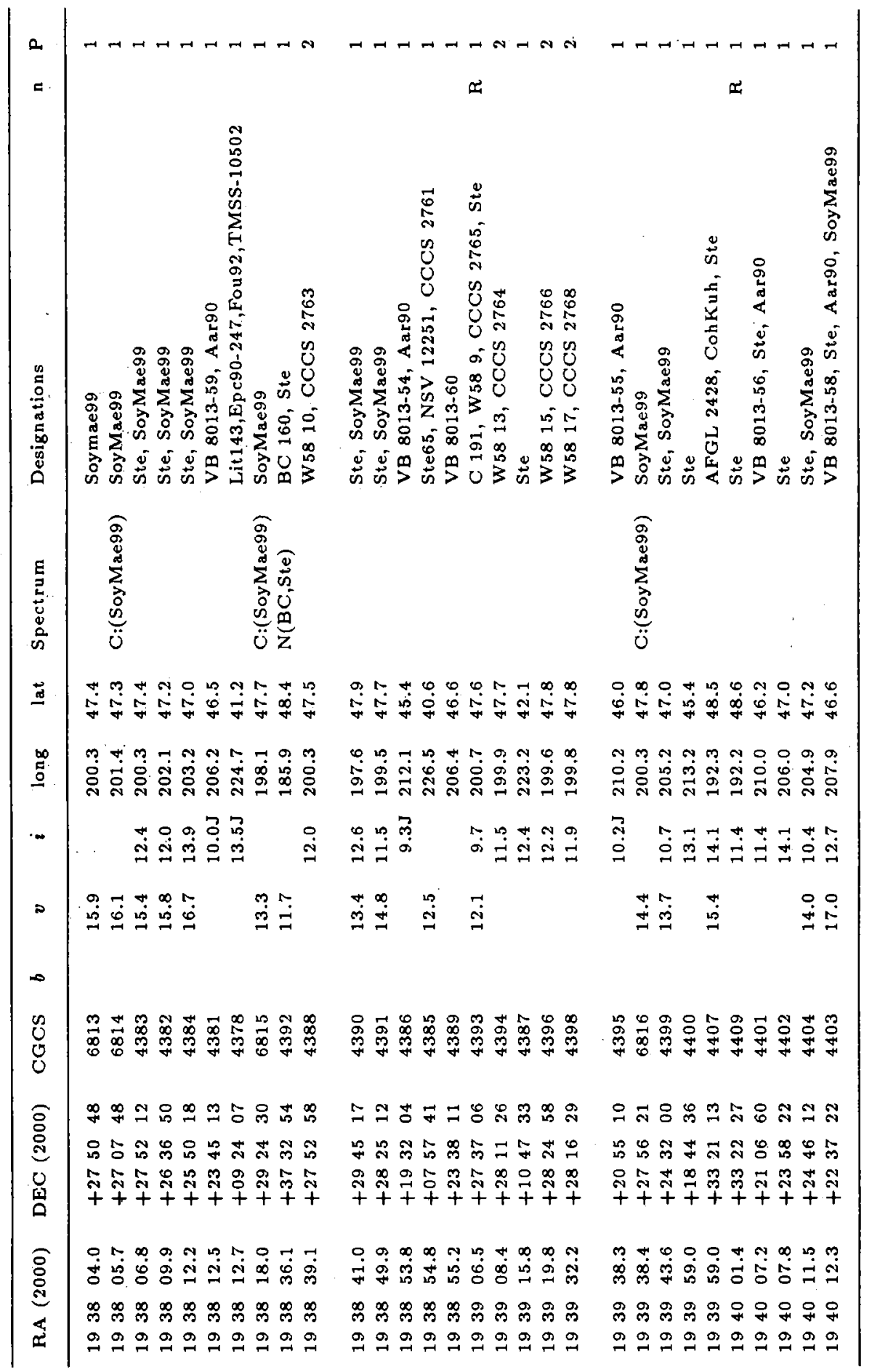




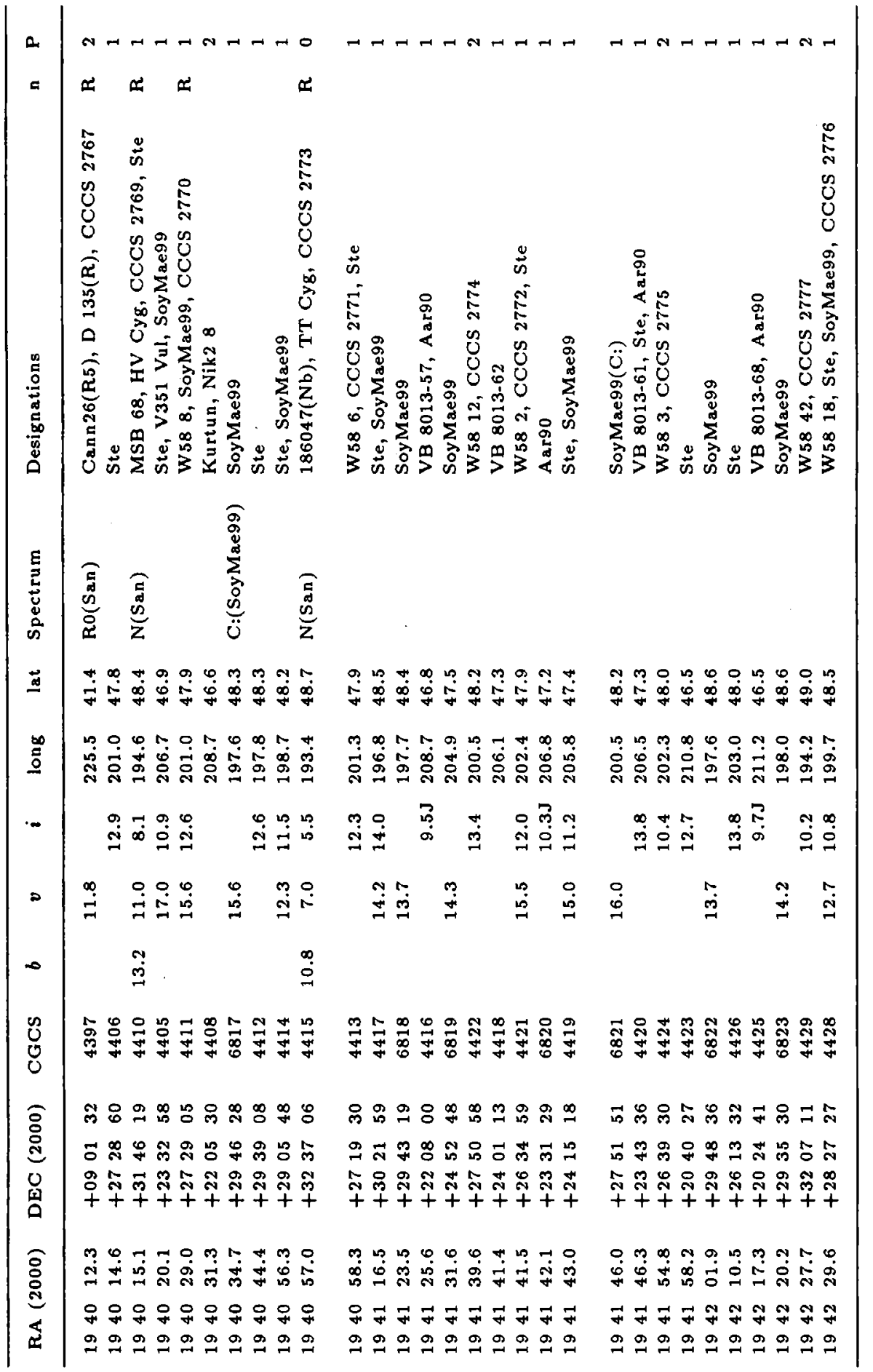




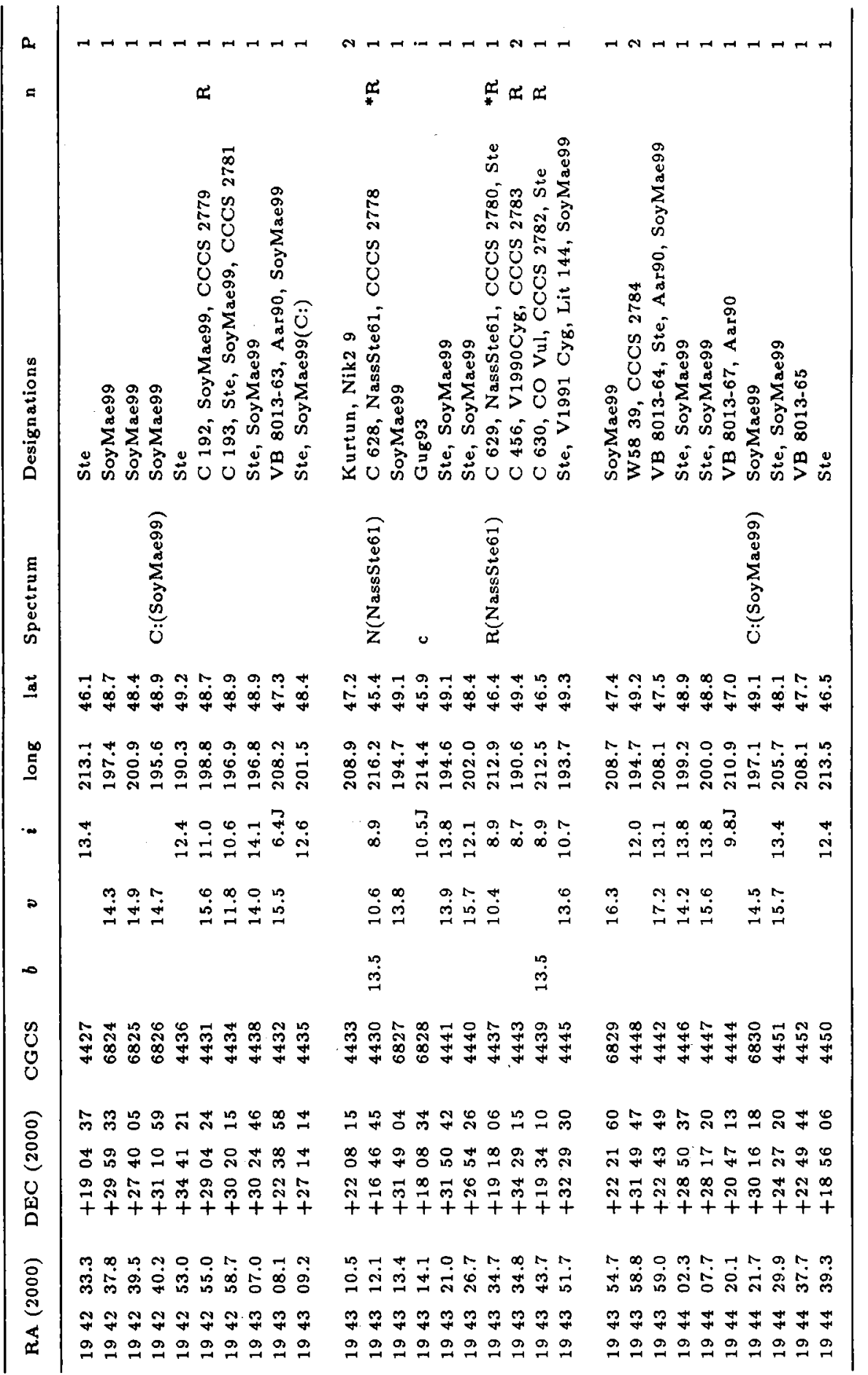




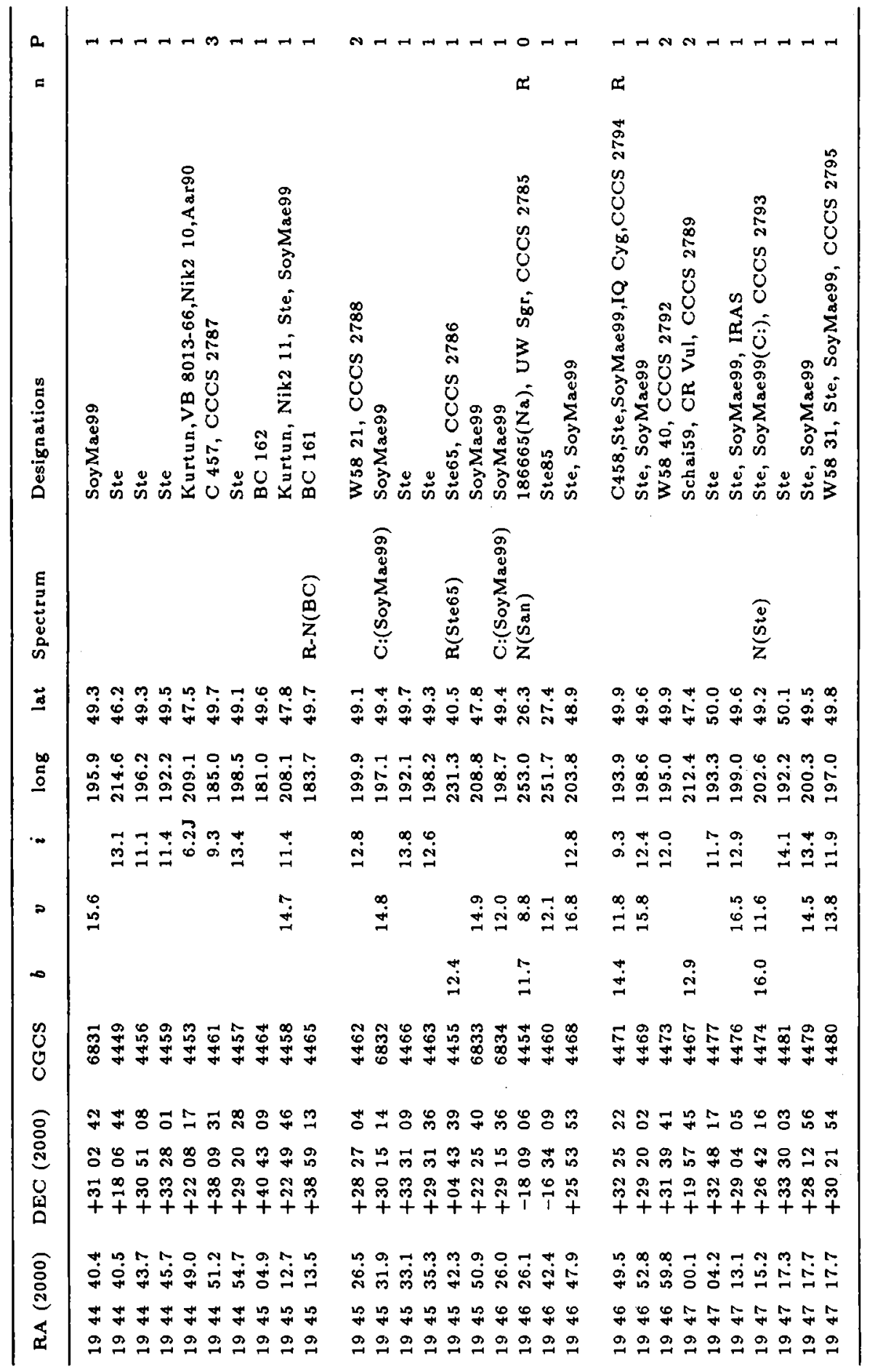




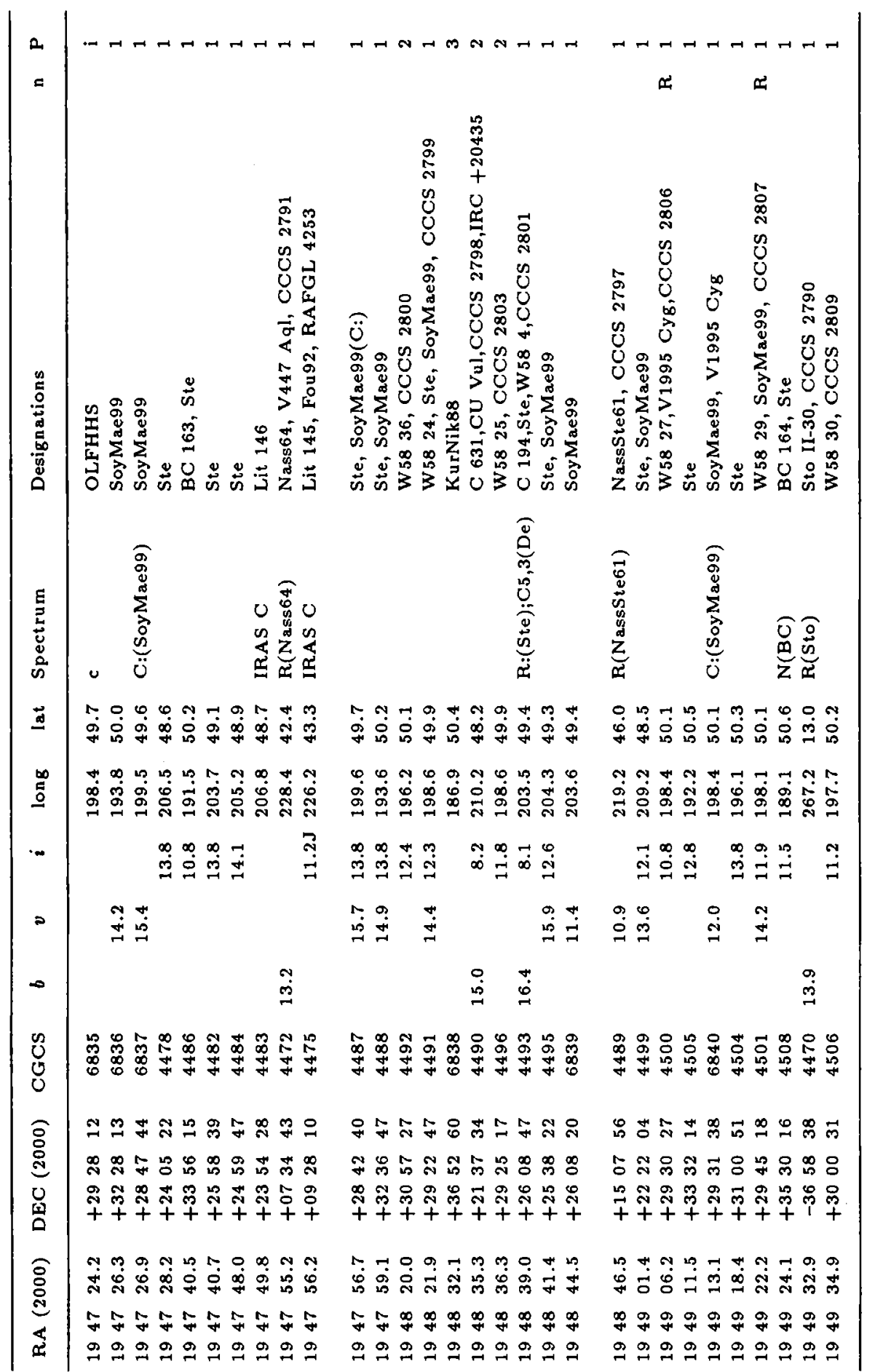




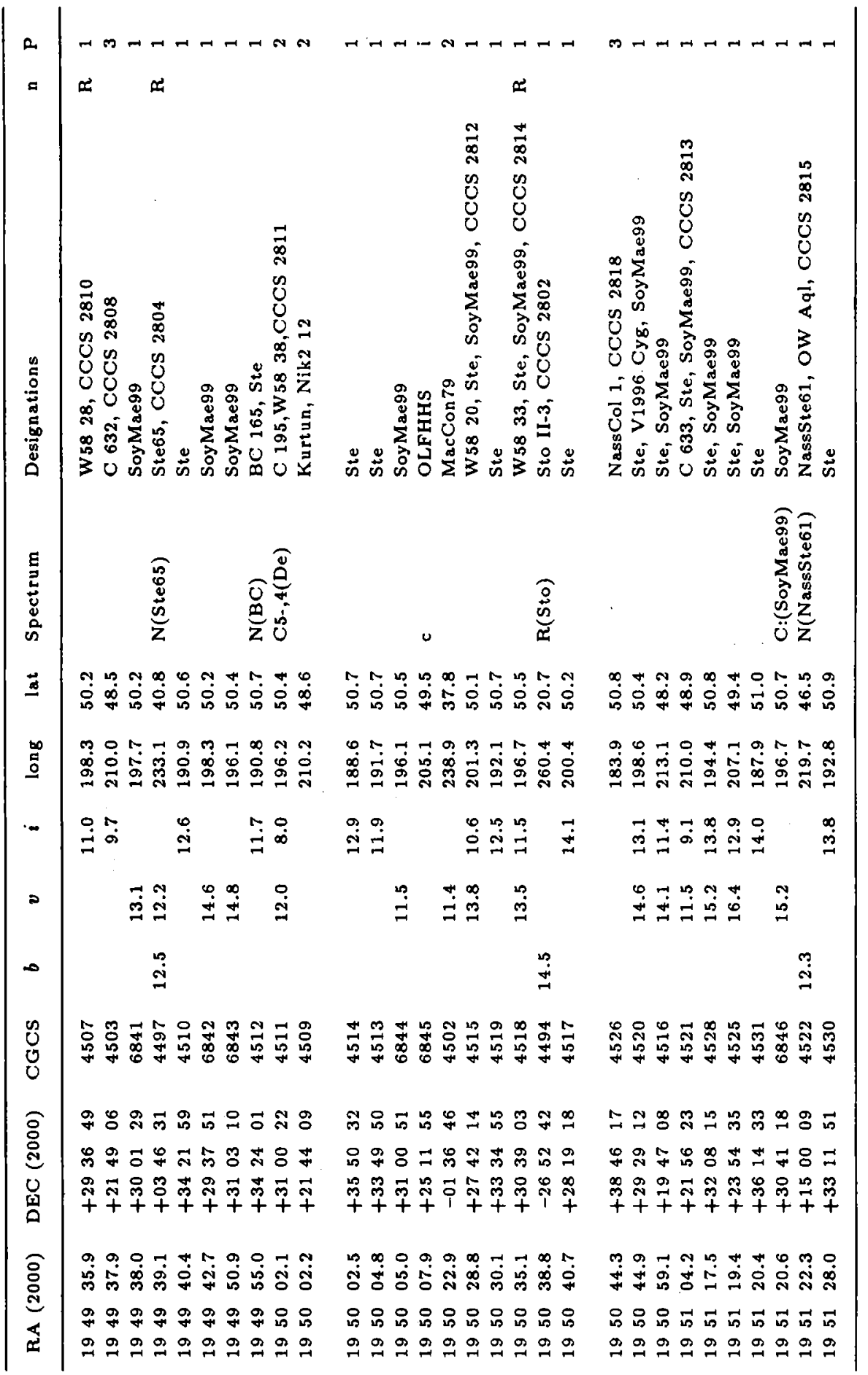




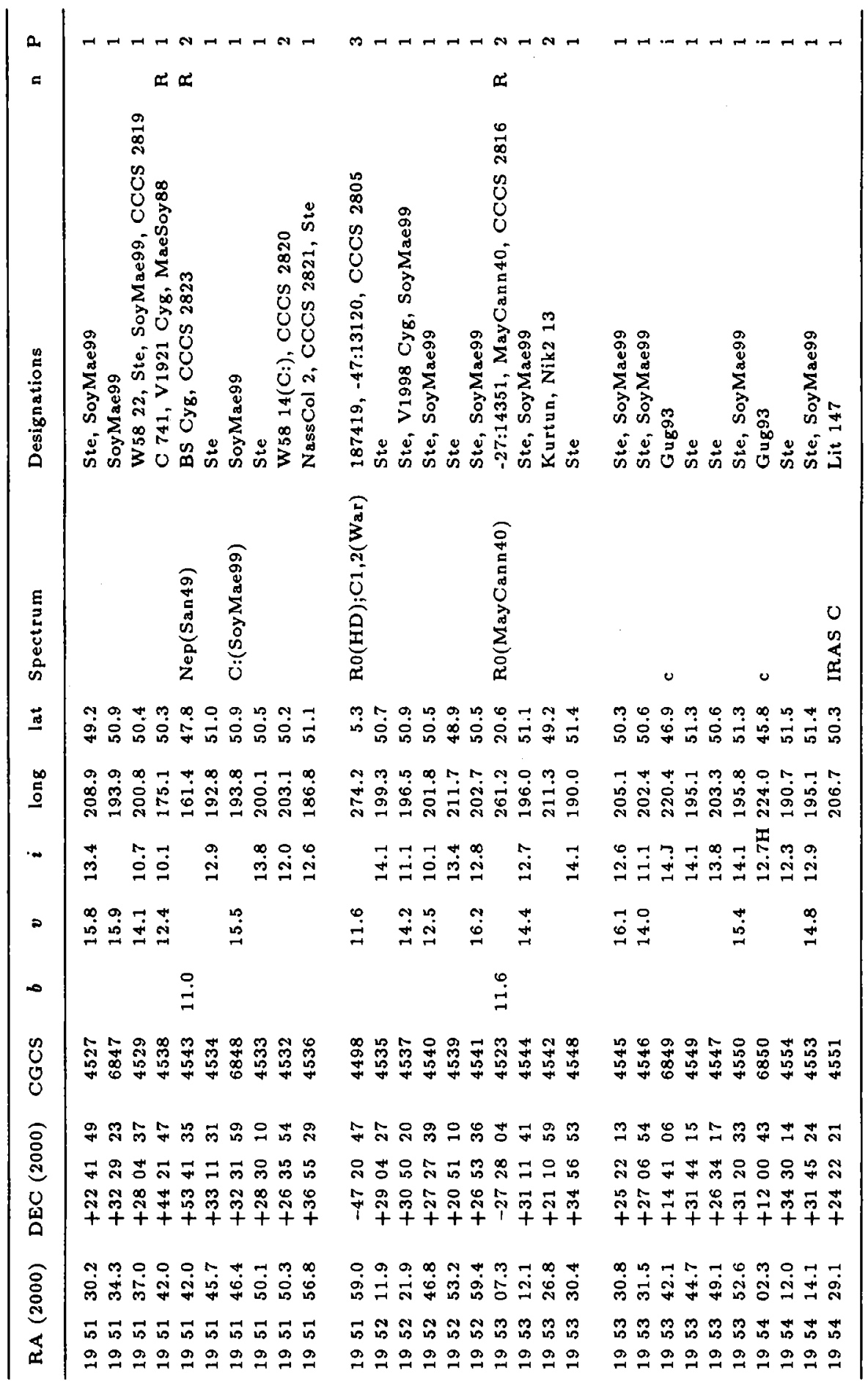




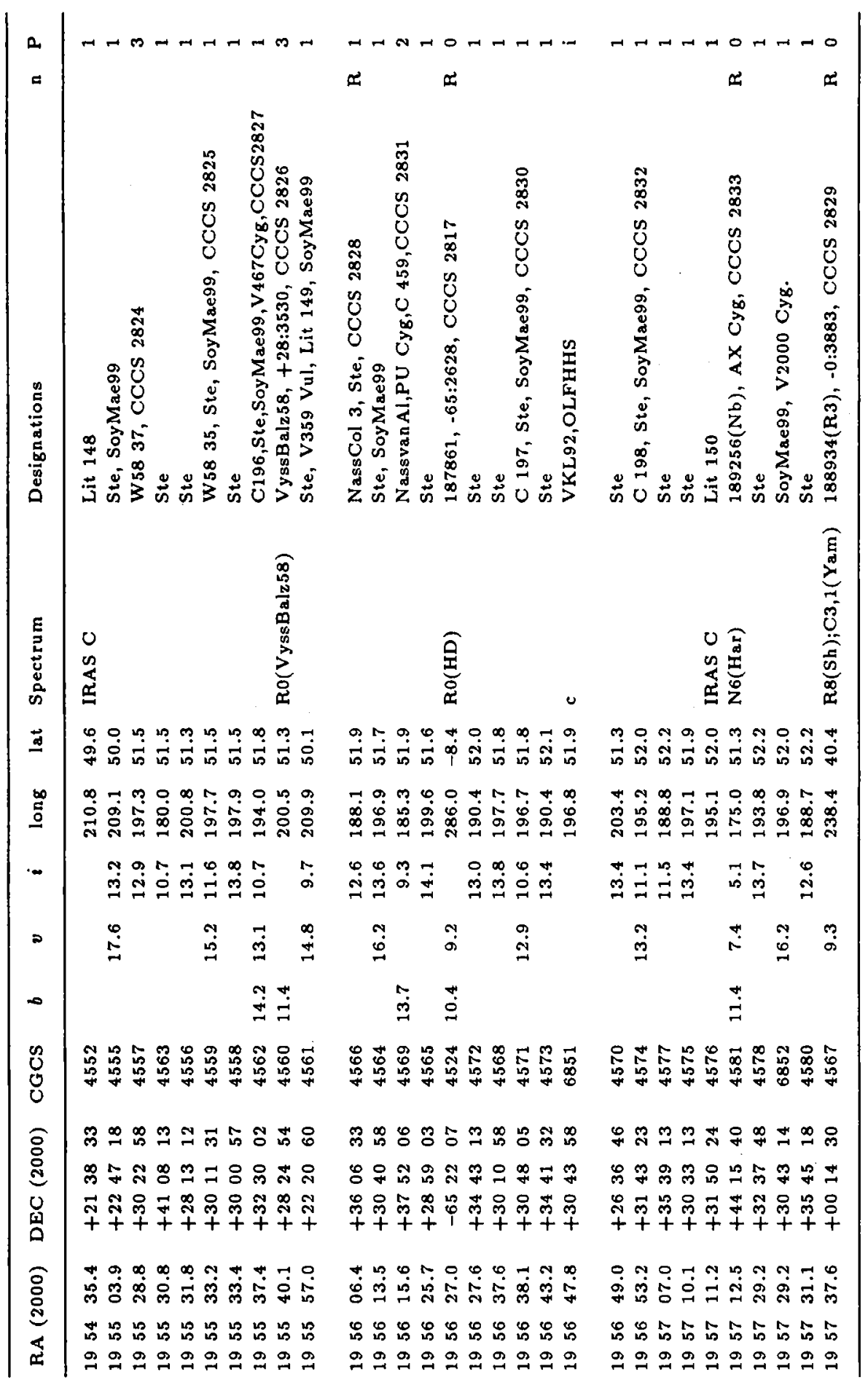




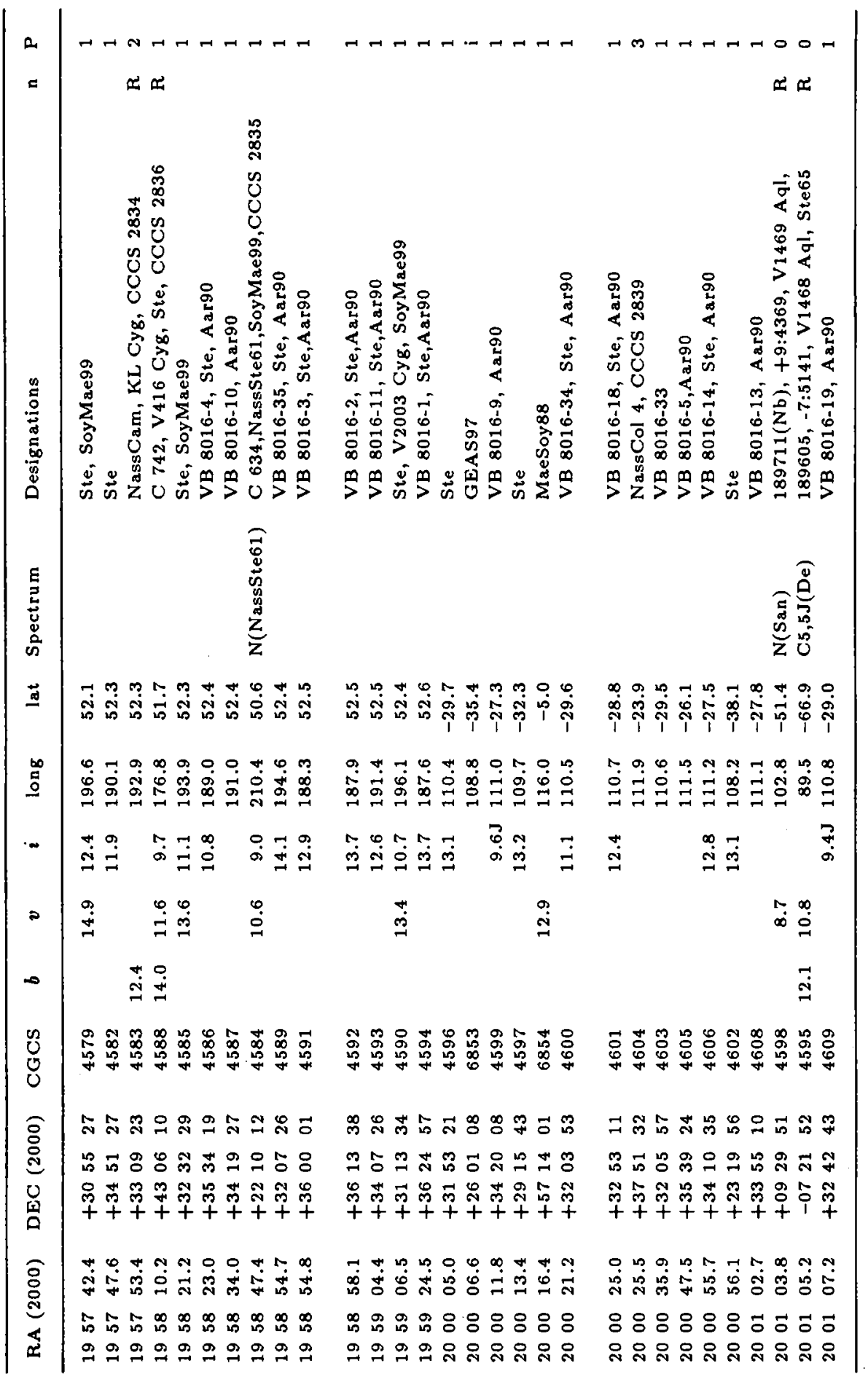




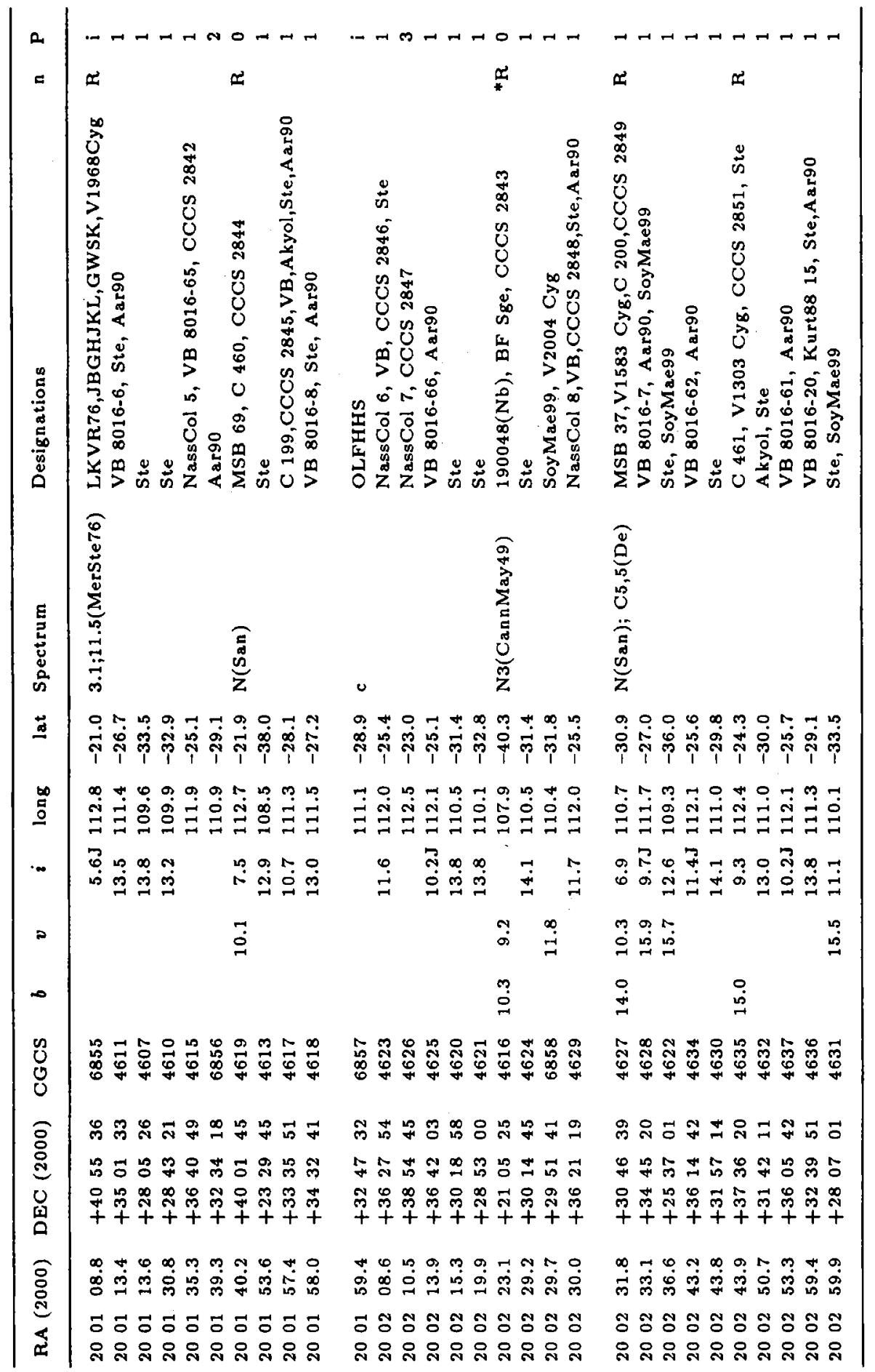




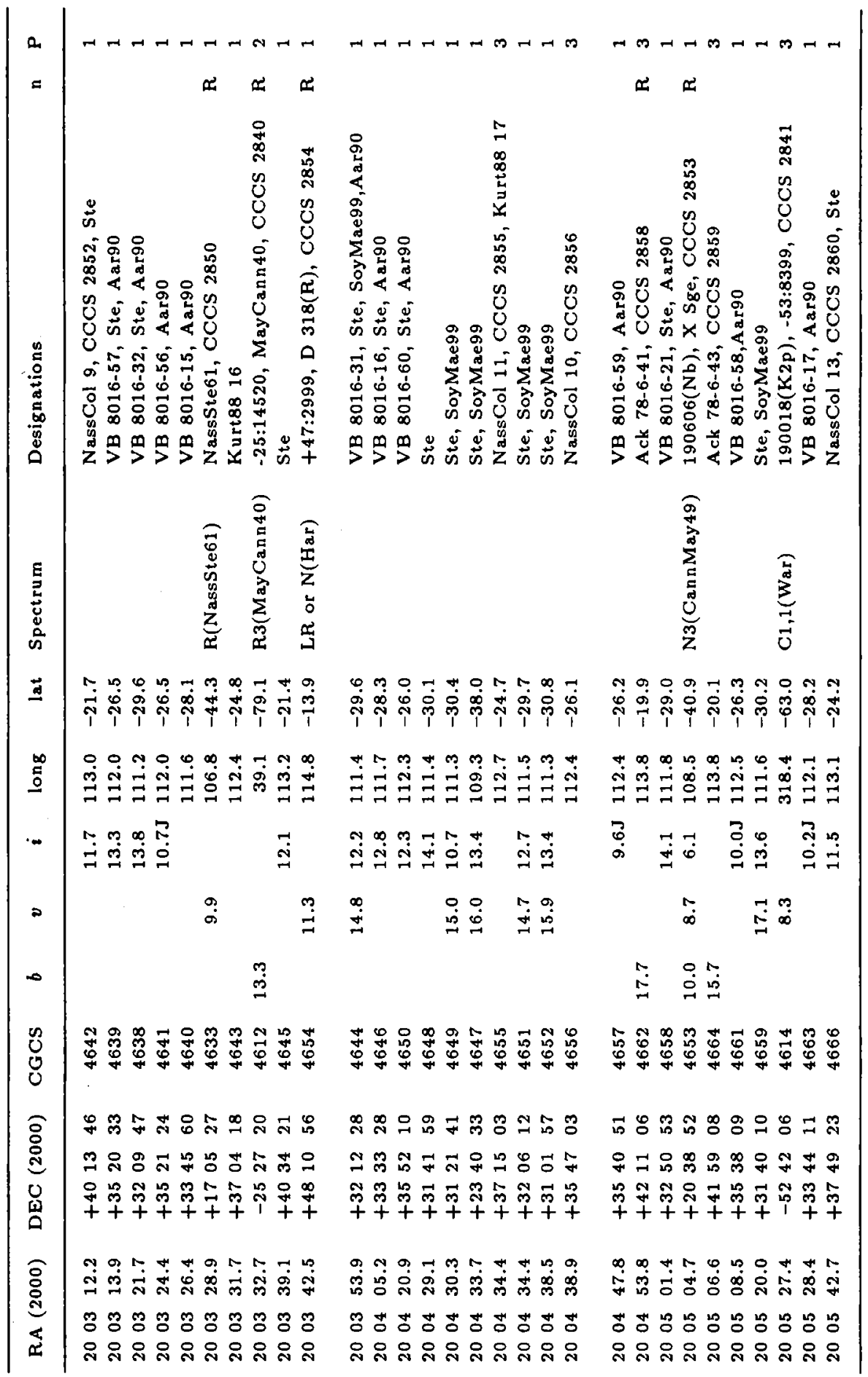




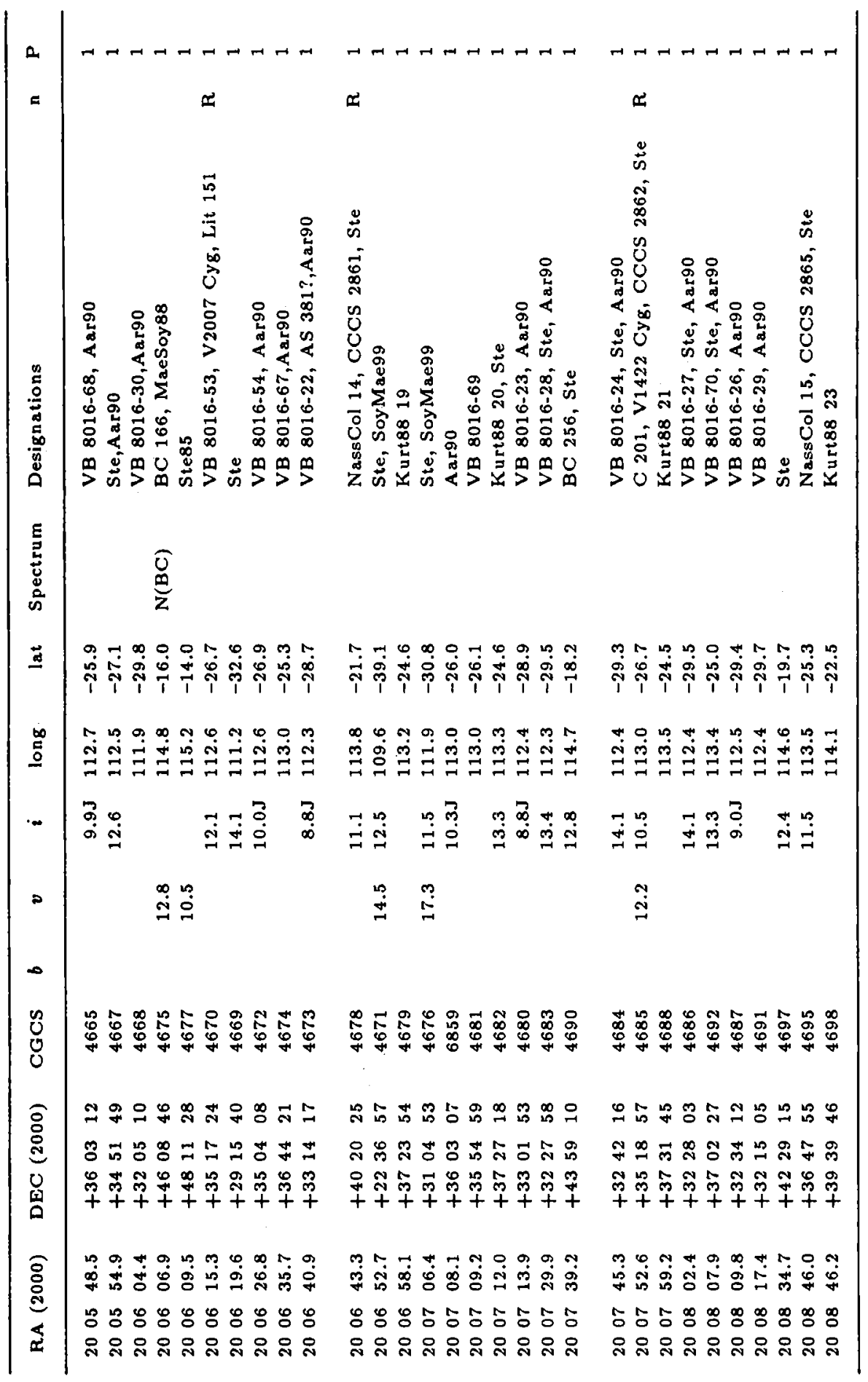




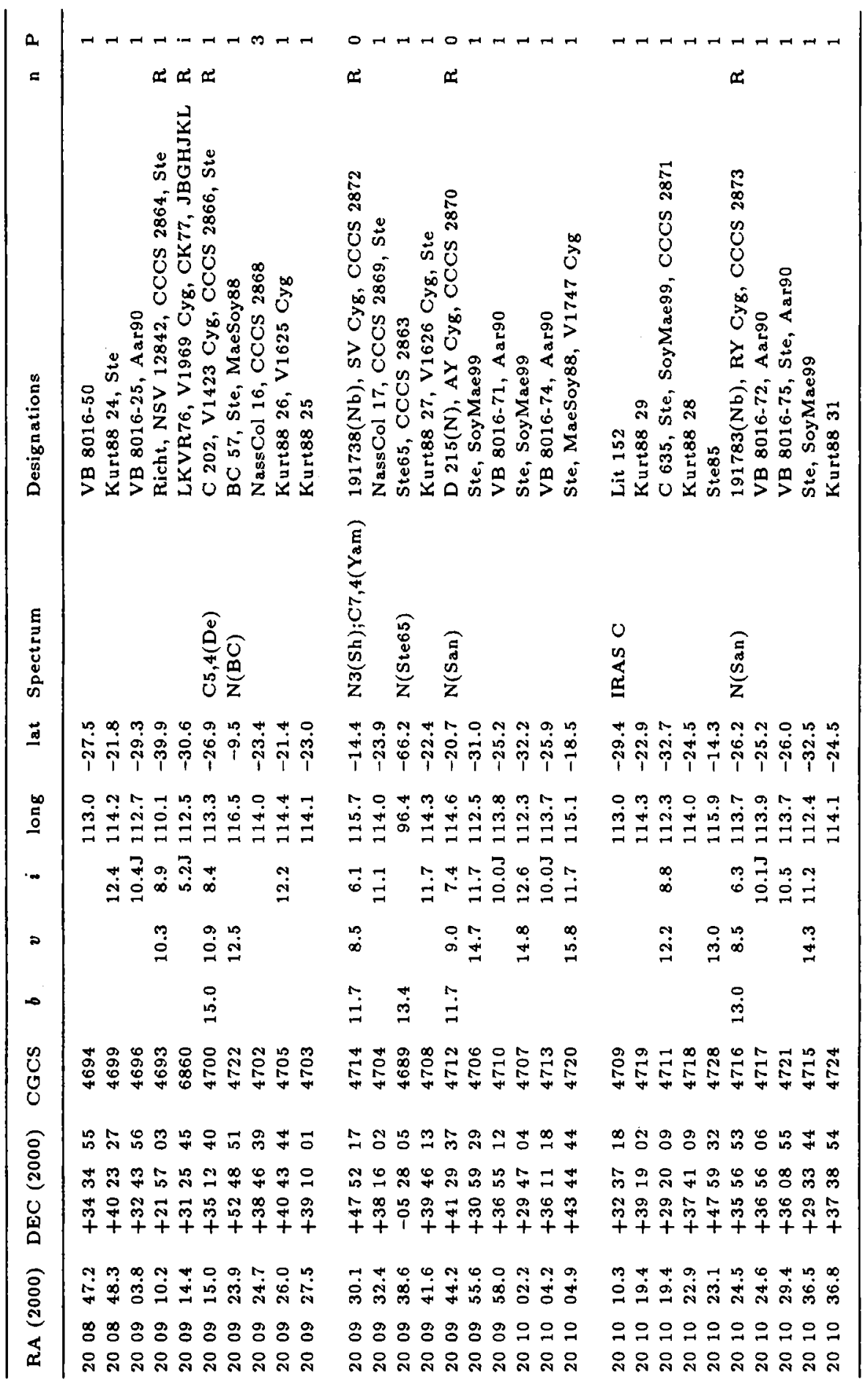




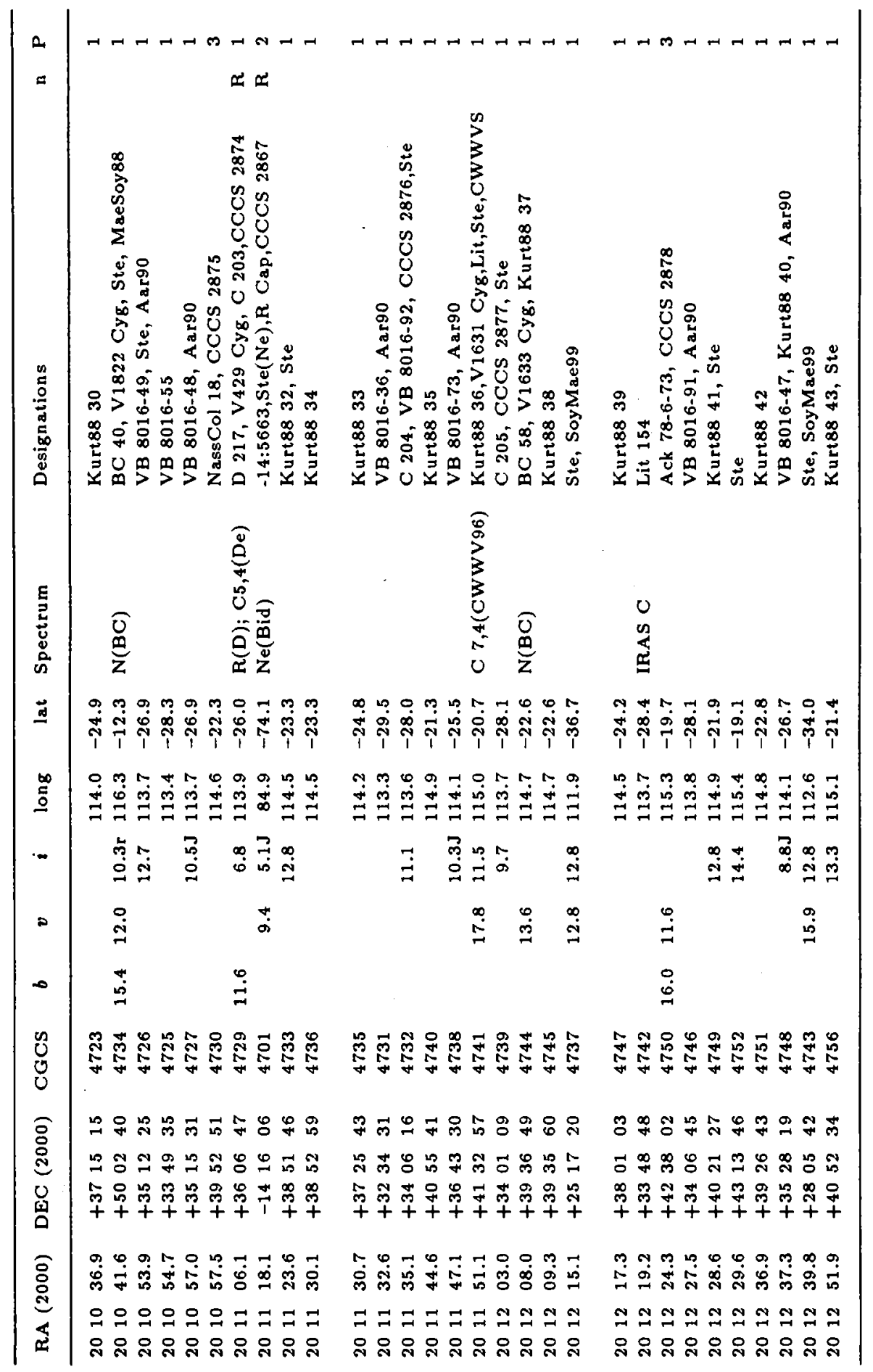




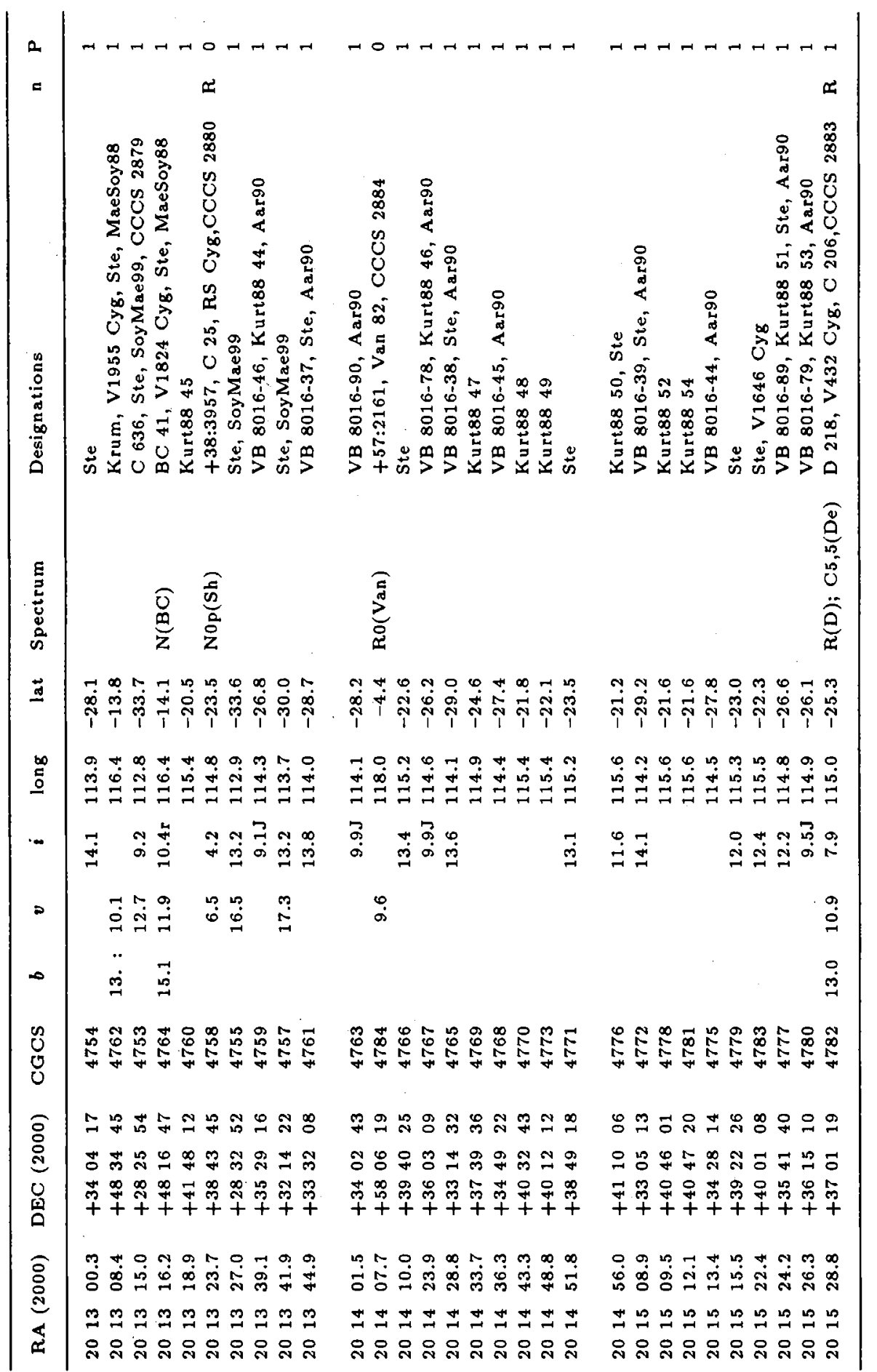




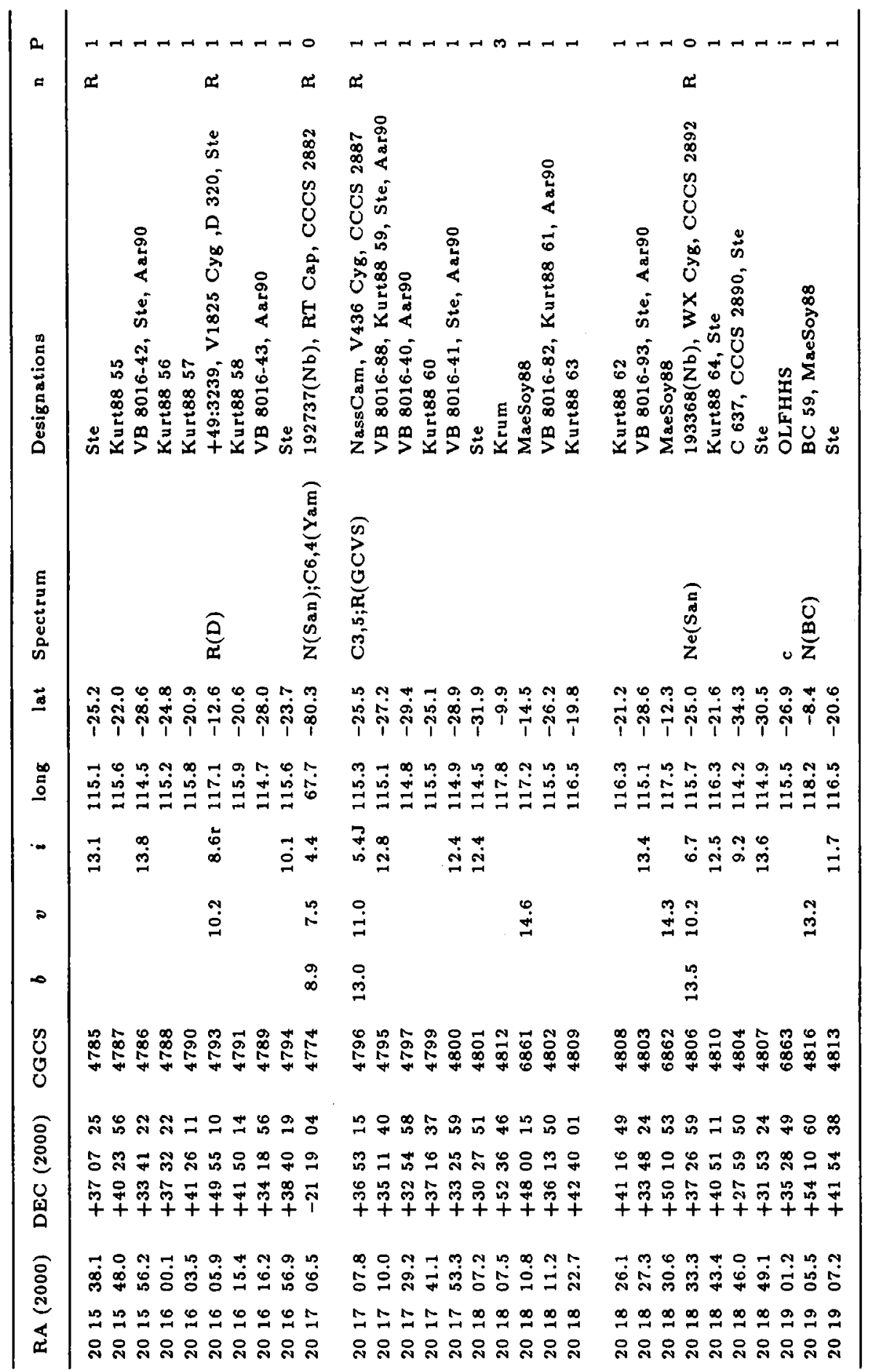




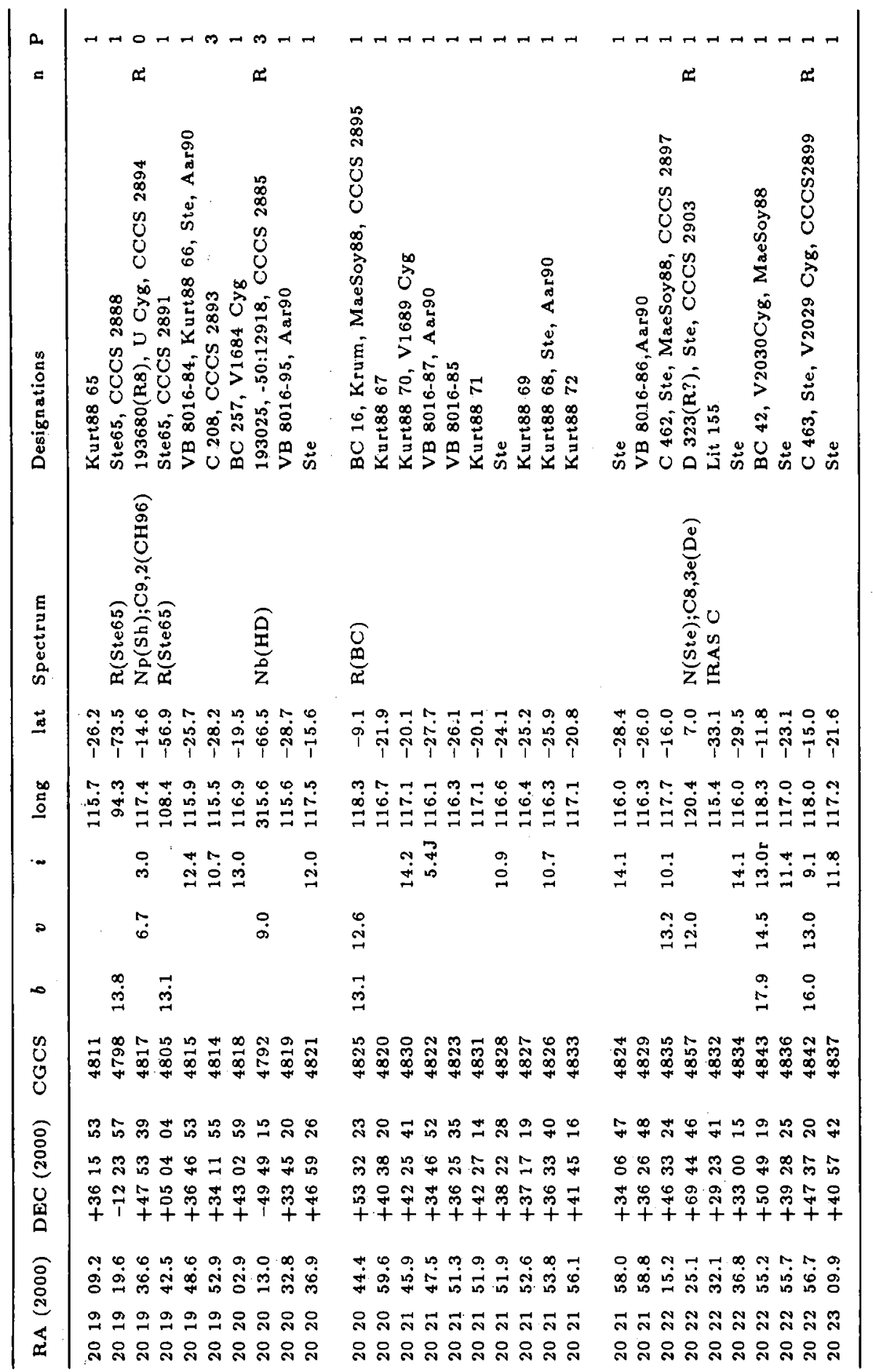




\begin{tabular}{|c|c|c|c|c|}
\hline a & 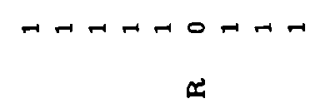 & & 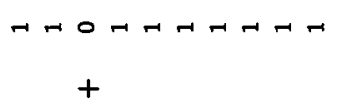 & 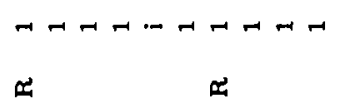 \\
\hline 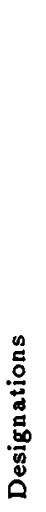 & 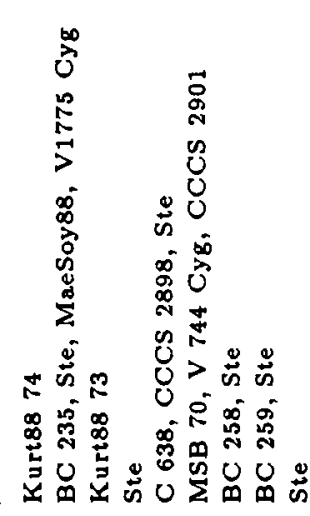 & 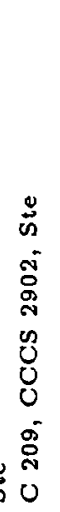 & 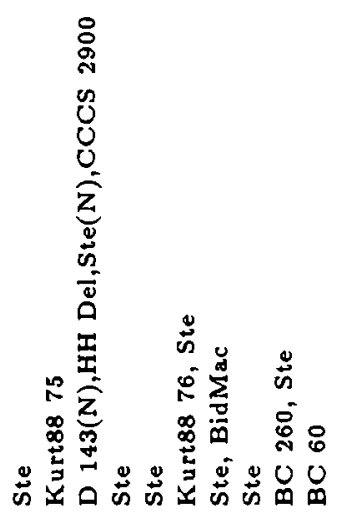 & 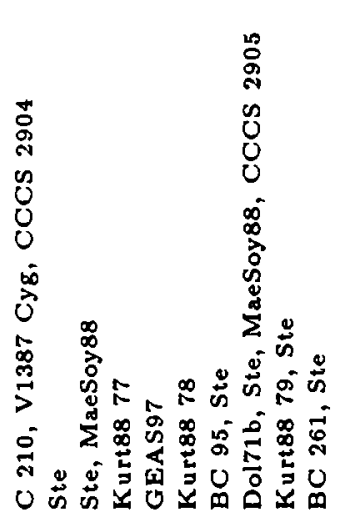 \\
\hline & 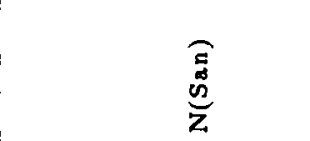 & & $\begin{array}{c}\widehat{c} \\
\substack{\tilde{n} \\
\tilde{z}}\end{array}$ & 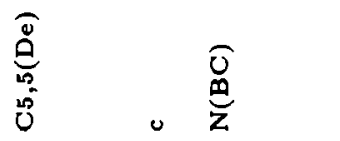 \\
\hline$\underline{\Xi}$ & 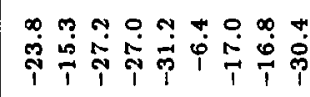 & & 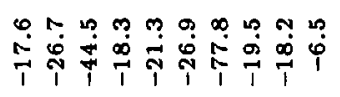 & 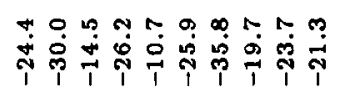 \\
\hline 离 & 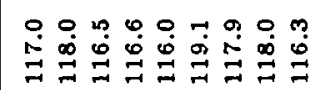 & & 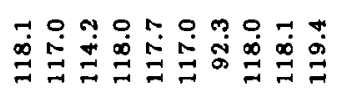 & 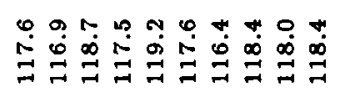 \\
\hline - & 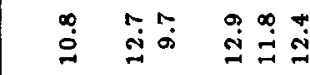 & & 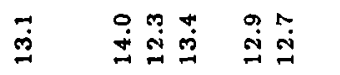 & 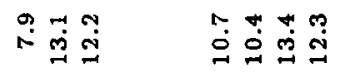 \\
\hline D & $\stackrel{\infty}{\stackrel{0}{0}}$ & & 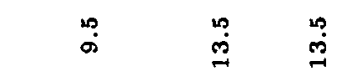 & 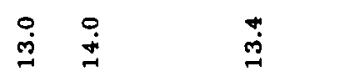 \\
\hline & 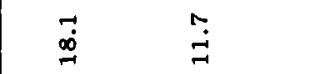 & & $\stackrel{\ddot{m}}{\ddot{\sim}}$ & $\stackrel{\leftrightarrow}{\leftrightarrow}$ \\
\hline $\begin{array}{l}0 \\
0 \\
0 \\
0\end{array}$ & 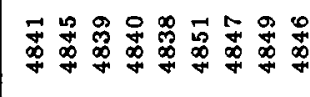 & & 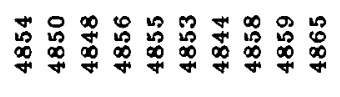 & 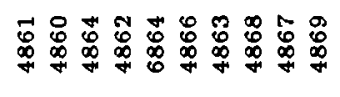 \\
\hline$\widehat{\circ}$ & 吕 & & 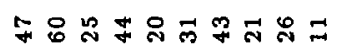 & 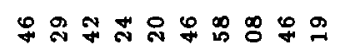 \\
\hline 过 & 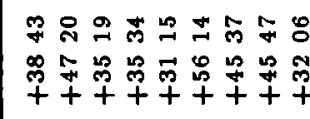 & & 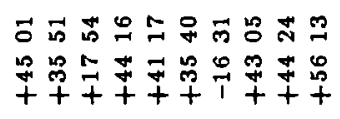 & 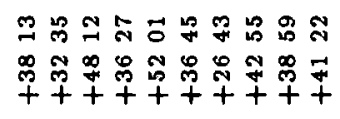 \\
\hline$\widehat{a}$ & 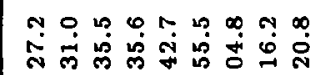 & & 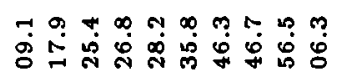 & 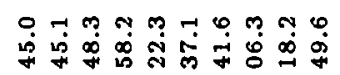 \\
\hline 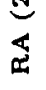 & 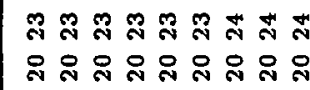 & & 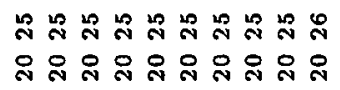 & 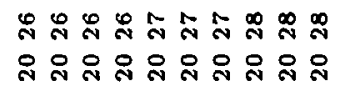 \\
\hline
\end{tabular}




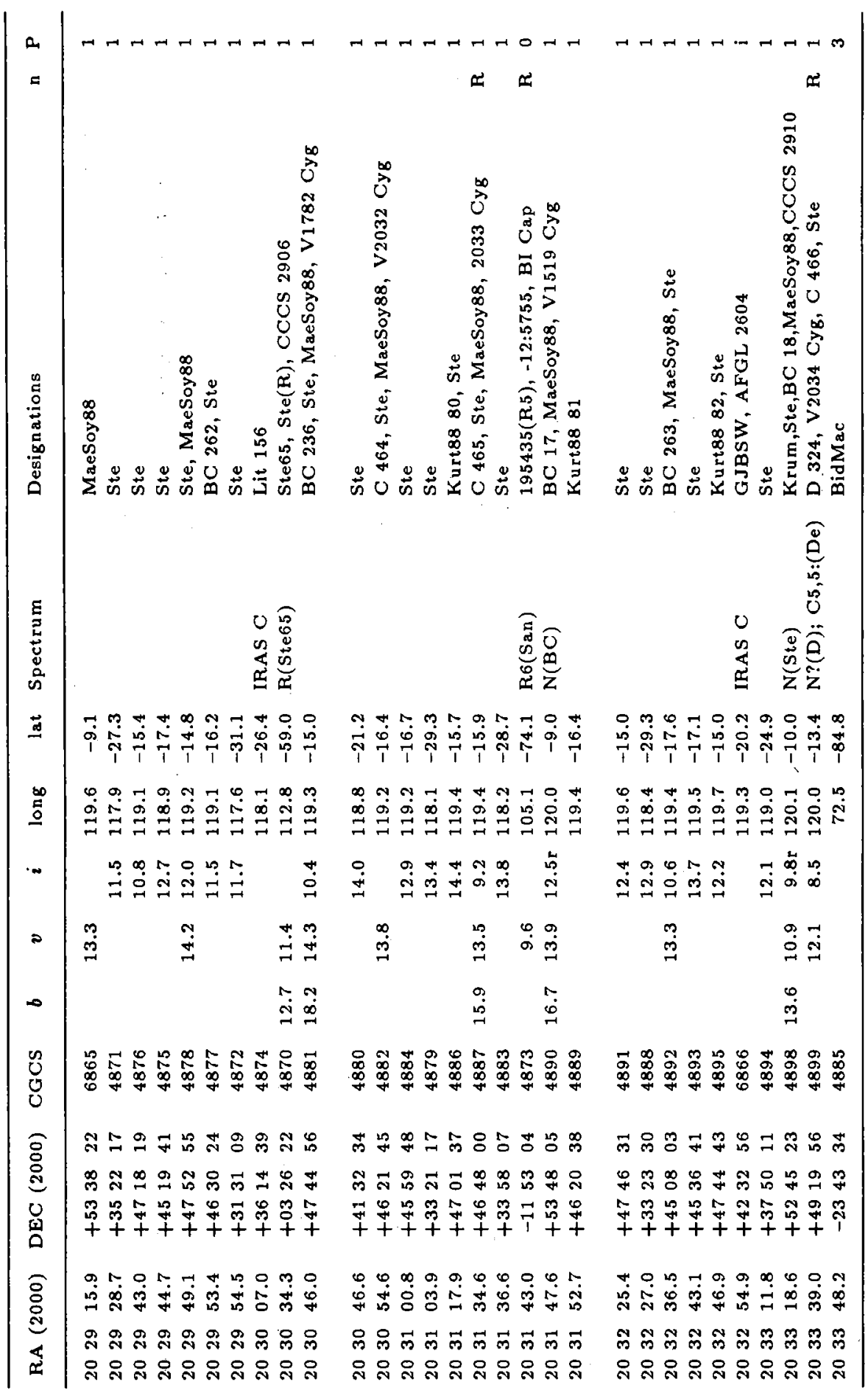




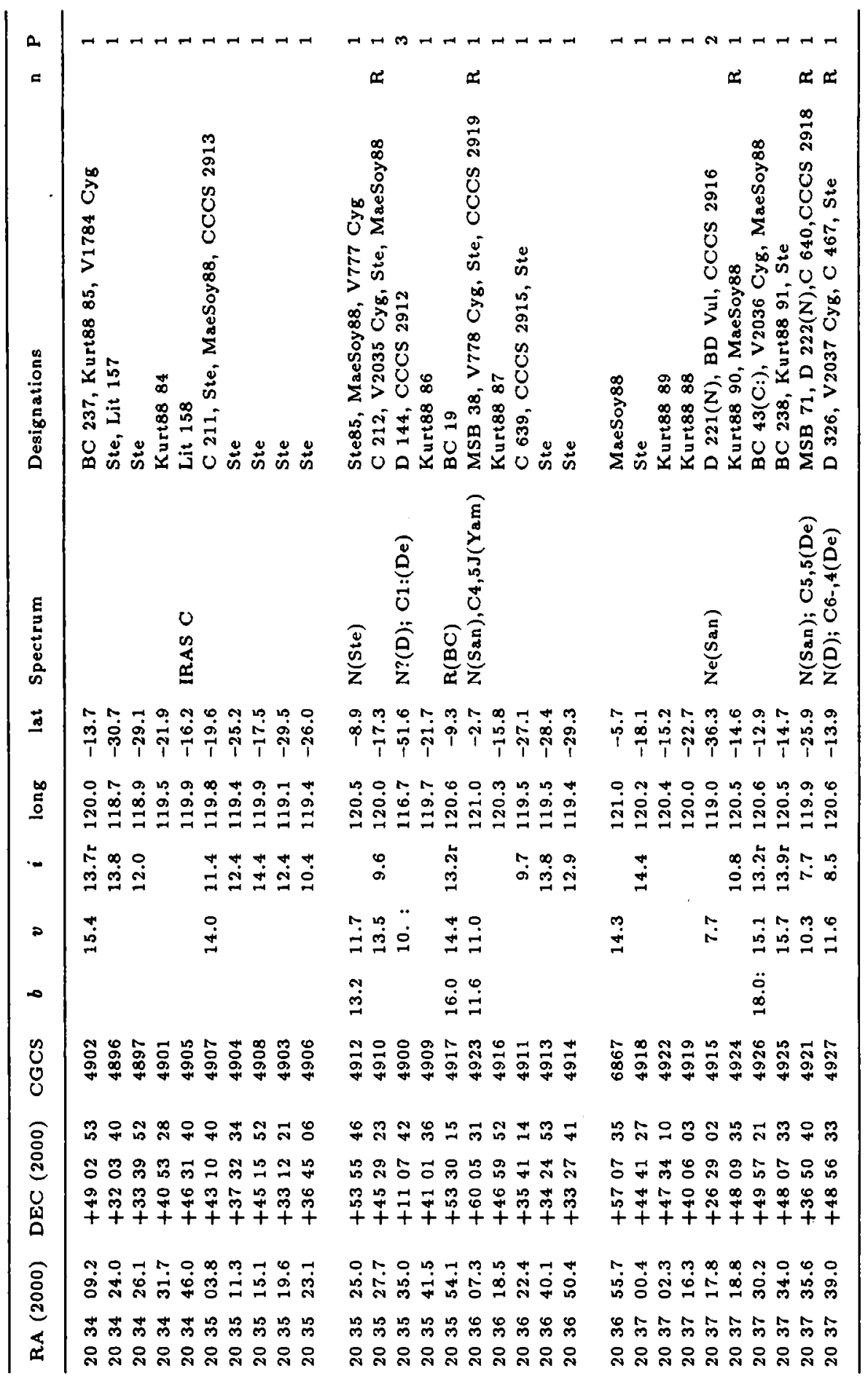




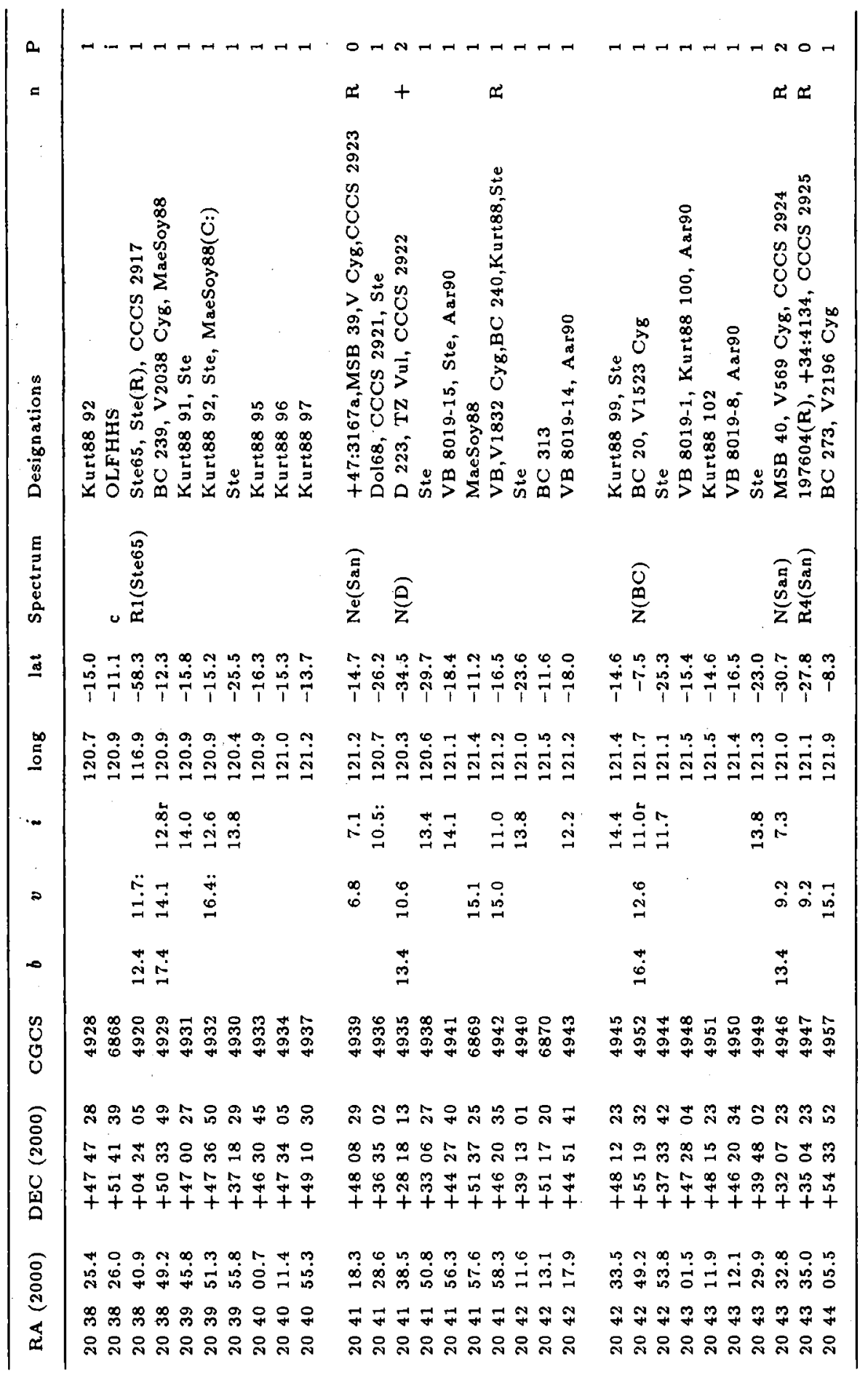




\begin{tabular}{|c|c|c|c|}
\hline a & 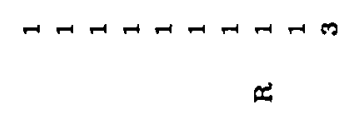 & 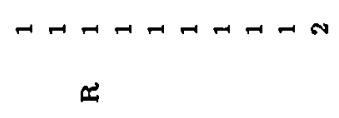 & 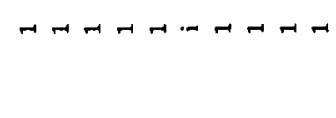 \\
\hline 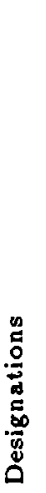 & 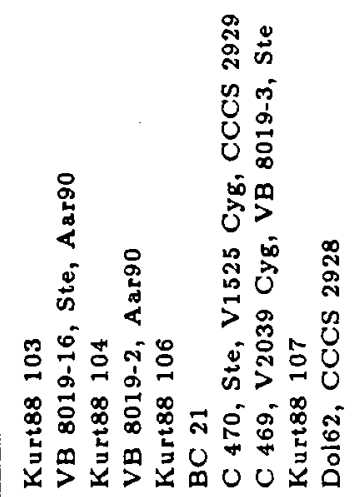 & 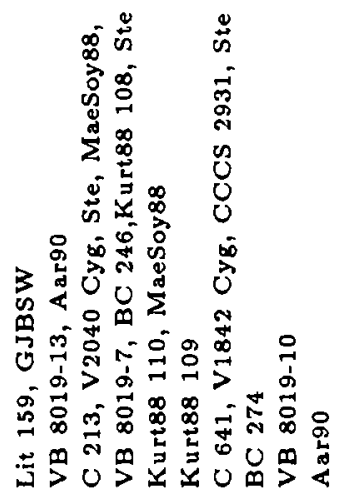 & 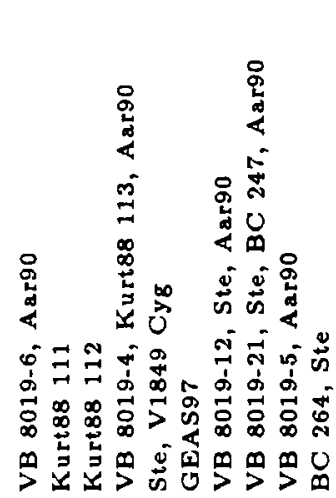 \\
\hline 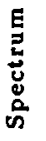 & & 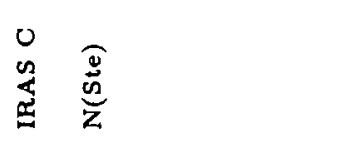 & 0 \\
\hline ప्త & 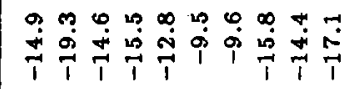 & 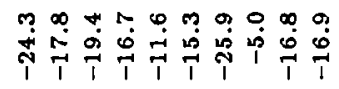 & 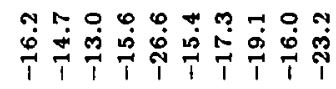 \\
\hline$\stackrel{\infty}{5}$ & 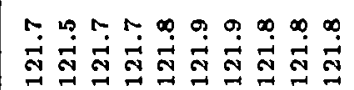 & 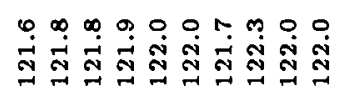 & 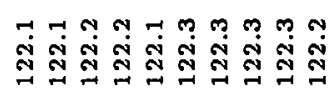 \\
\hline - & 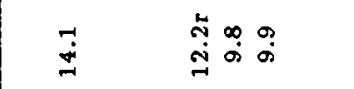 & 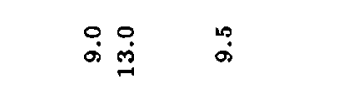 & 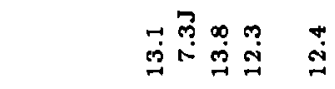 \\
\hline 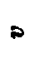 & 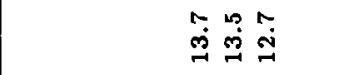 & 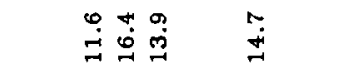 & 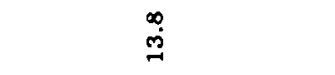 \\
\hline ـ & $\stackrel{+}{\dot{0}} \stackrel{+}{\oplus}$ & $\stackrel{\dot{\rho}}{\dot{\leftrightarrow}}$ & \\
\hline $\begin{array}{l}y \\
\text { O } \\
\text { O }\end{array}$ & 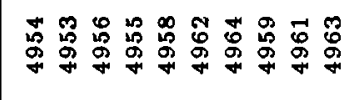 & 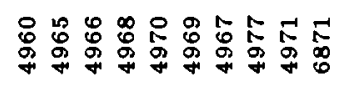 & 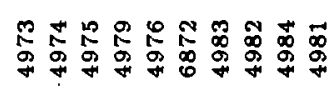 \\
\hline$\widehat{g}$ & 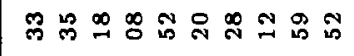 & 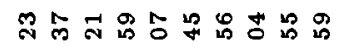 & 里 ส \\
\hline 产 & 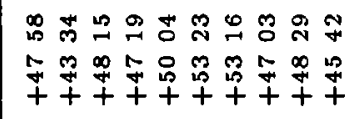 & 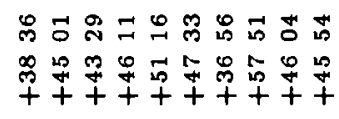 & 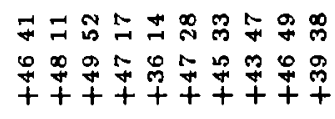 \\
\hline : & 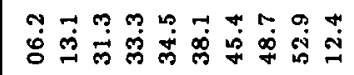 & 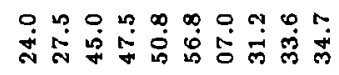 & 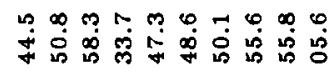 \\
\hline غ & 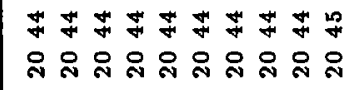 & 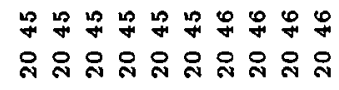 & 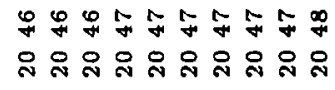 \\
\hline
\end{tabular}




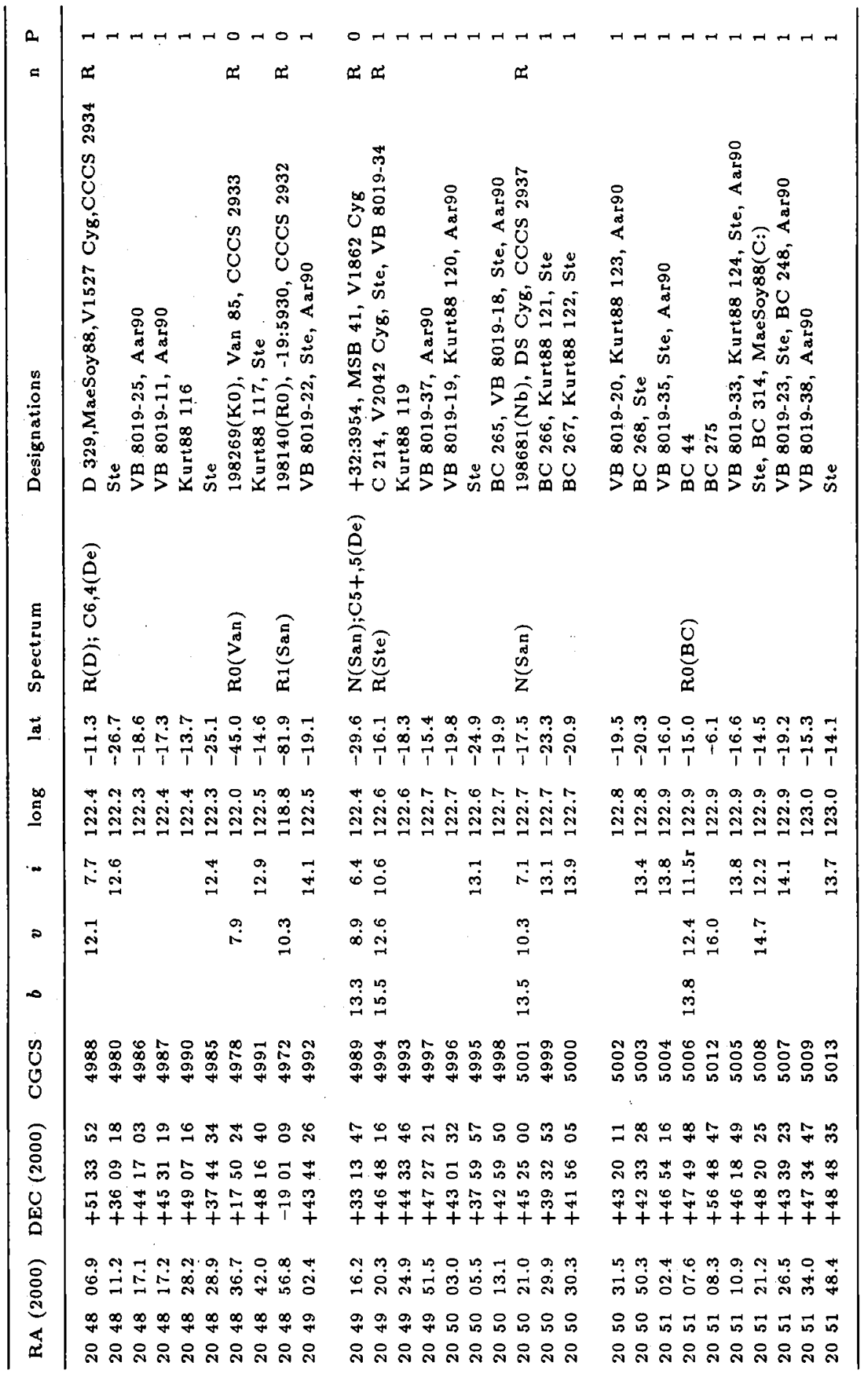




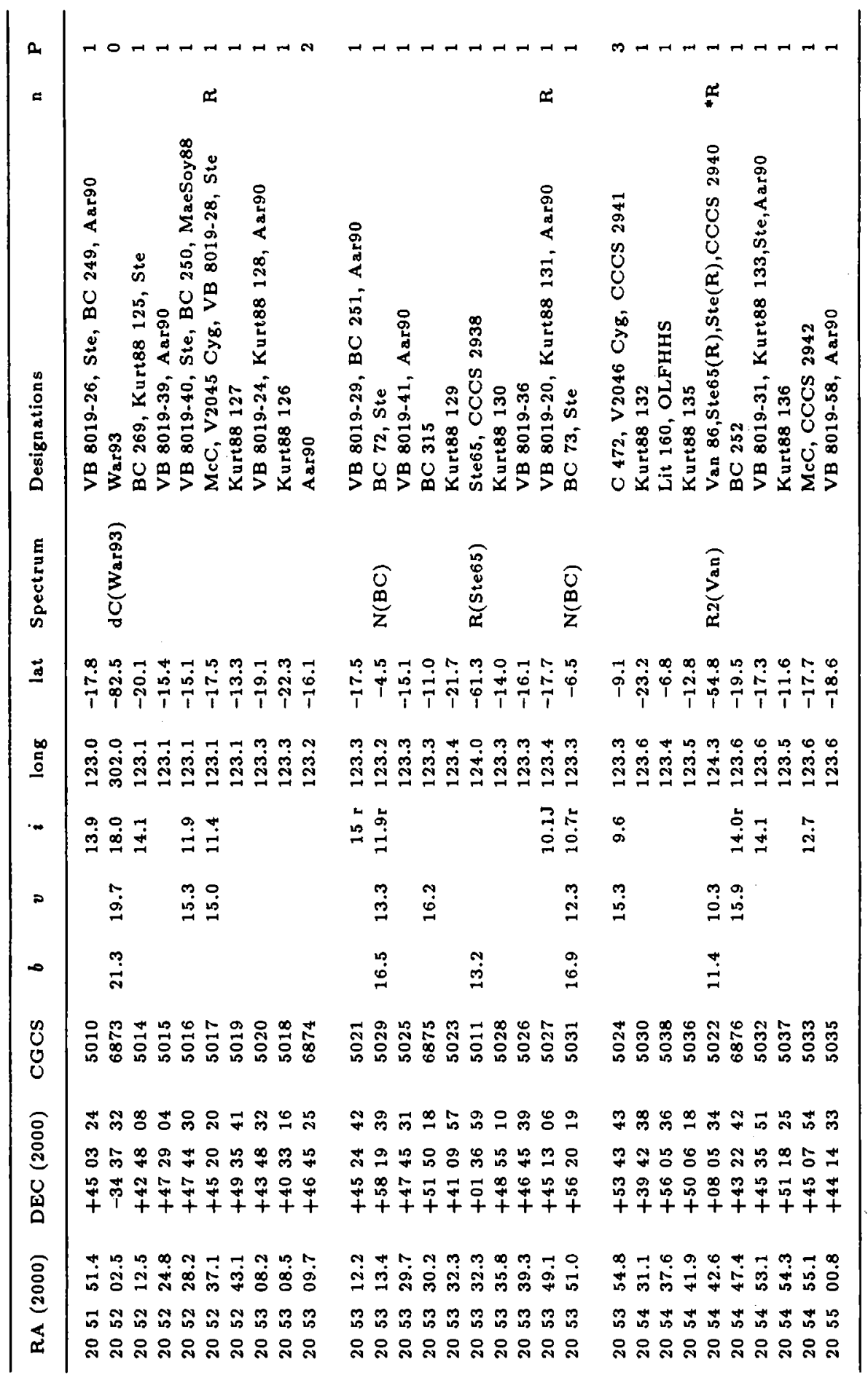




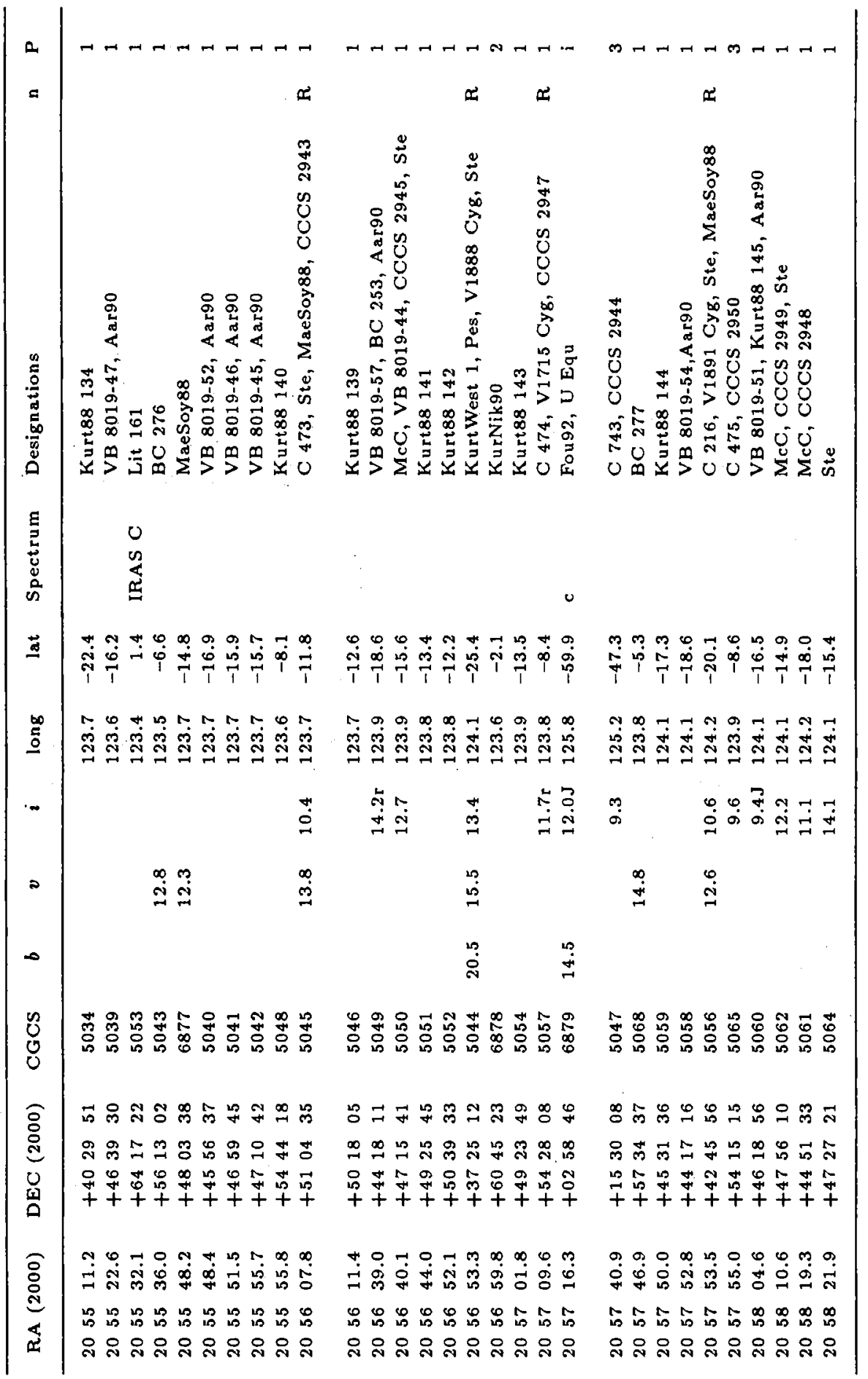




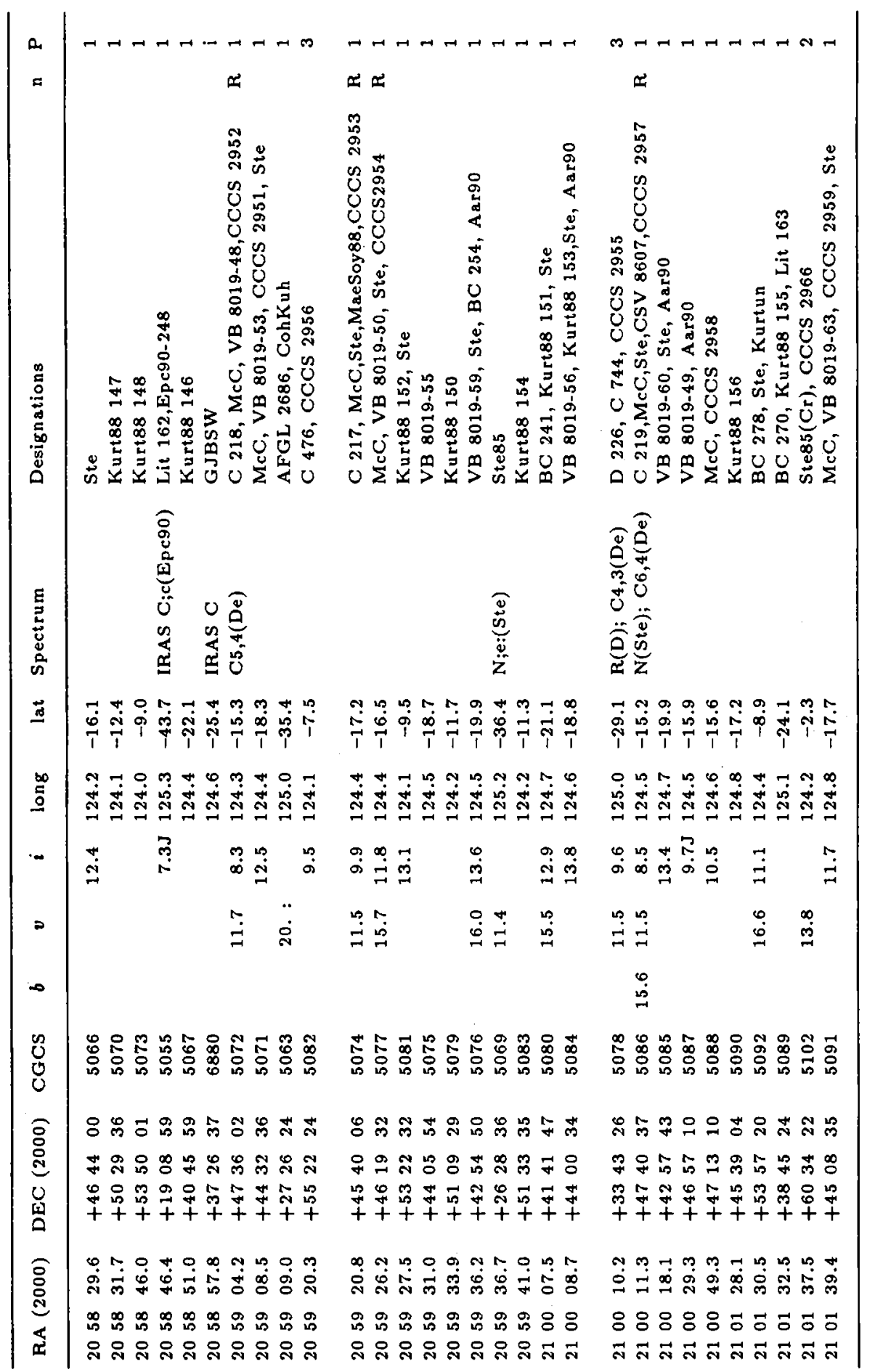




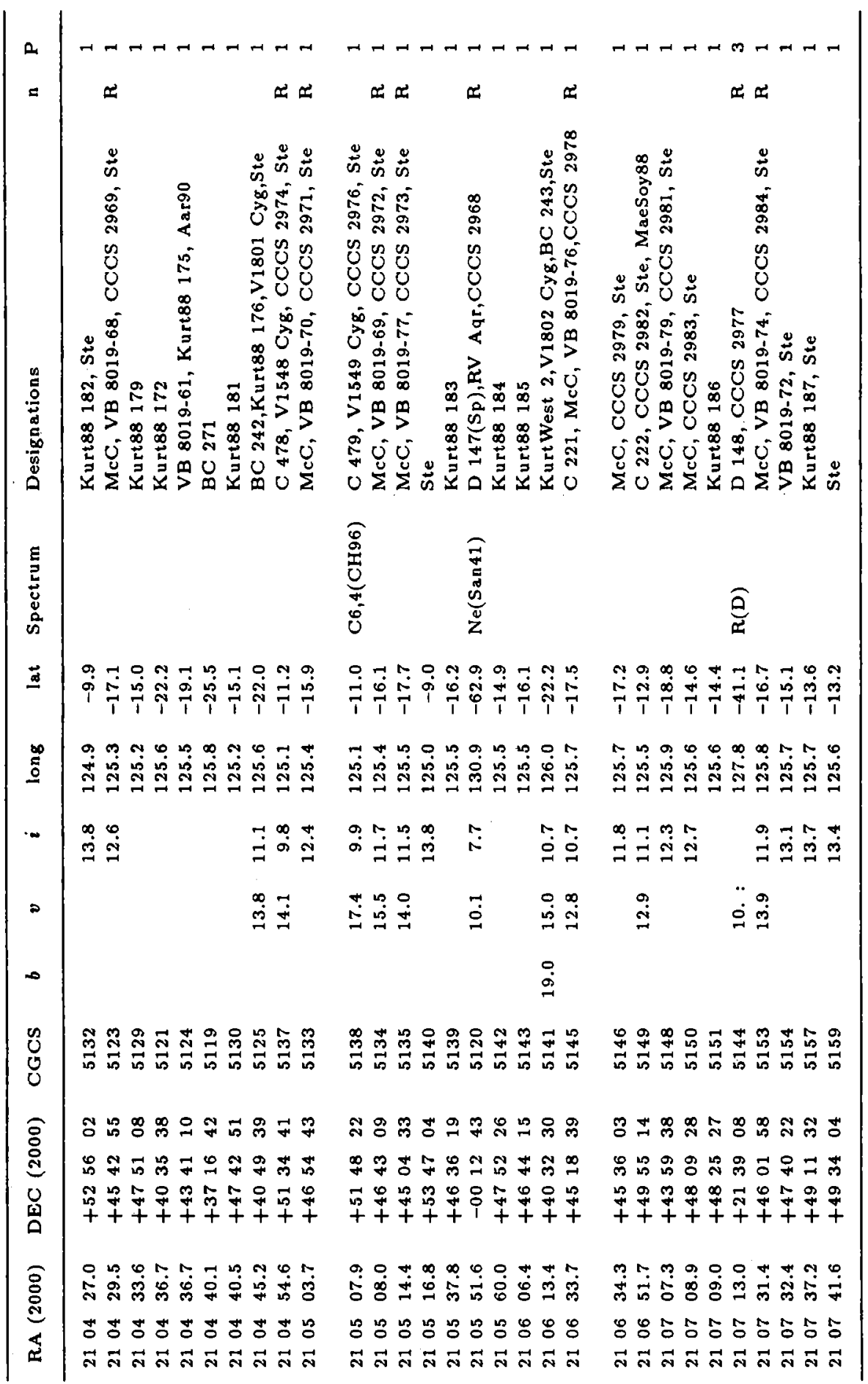




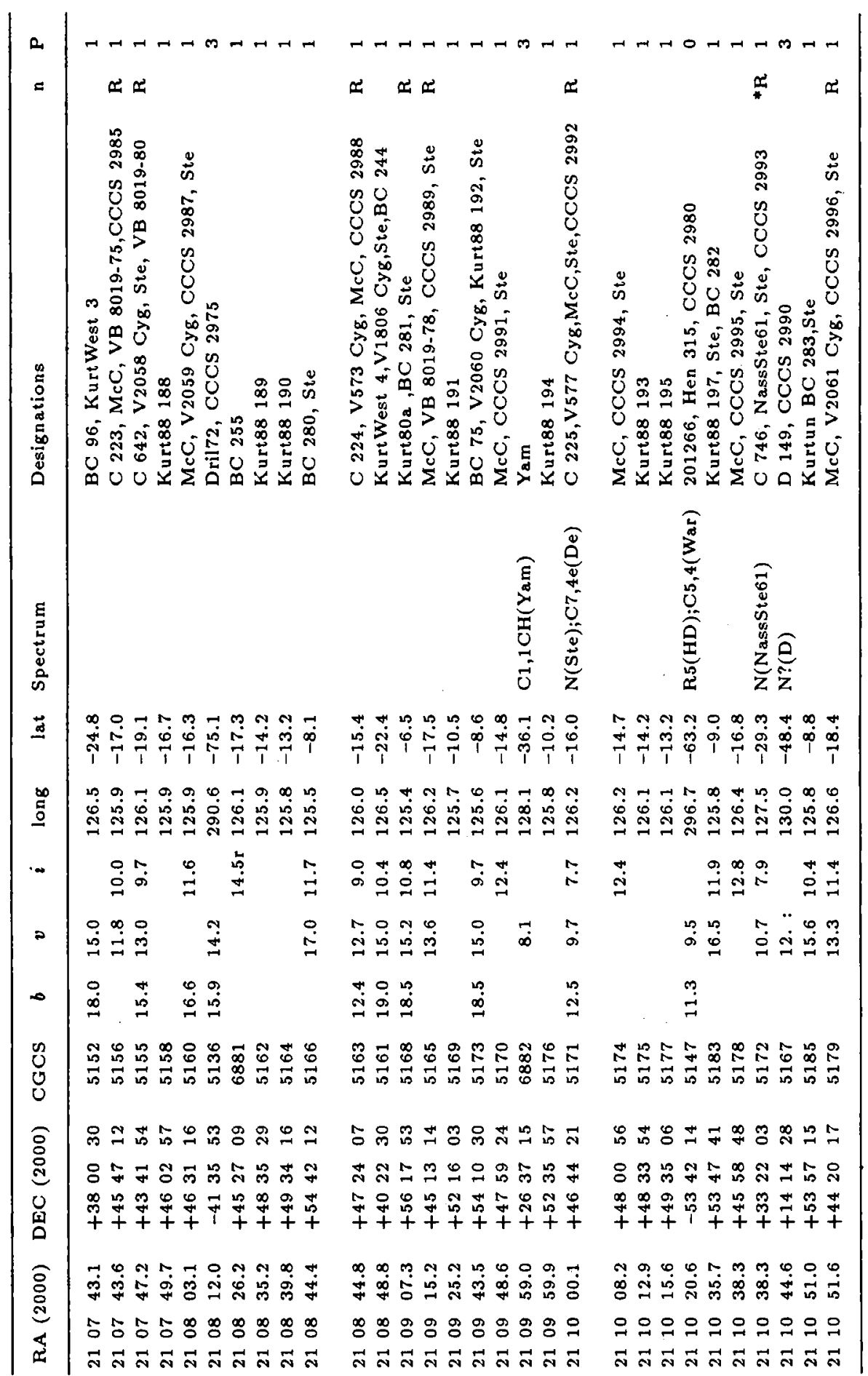




\begin{tabular}{|c|c|c|c|}
\hline a & 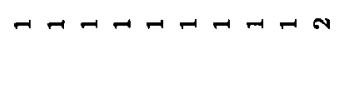 & $\begin{array}{c}0 \\
h a t h y \rightarrow H A t\end{array}$ & 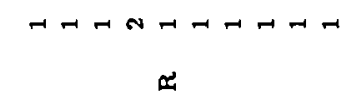 \\
\hline 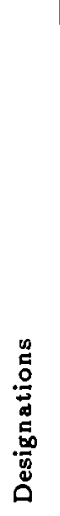 & 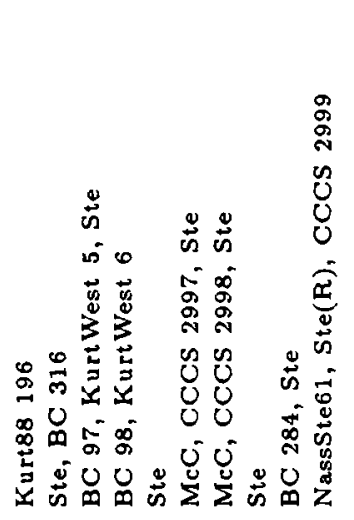 & 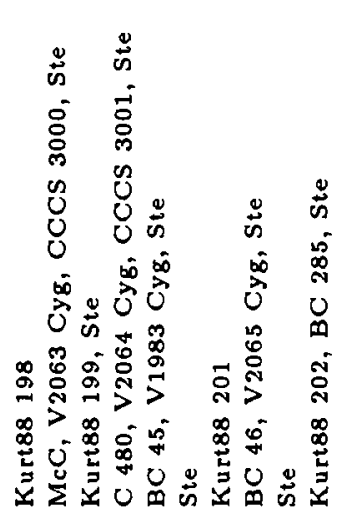 & 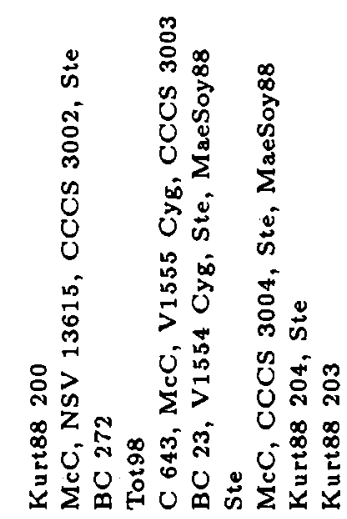 \\
\hline 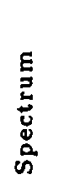 & 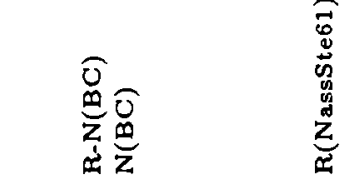 & $\begin{array}{l}\widehat{O} \\
\stackrel{\mathscr{m}}{z}\end{array}$ & $\begin{array}{l}\text { 务 } \\
\stackrel{8}{0} \\
\stackrel{0}{0} \\
\text { 工్ } \\
0\end{array}$ \\
\hline$\underline{\Xi}$ & 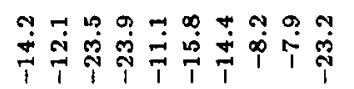 & $\begin{array}{l}0 \\
\dot{0} \\
\end{array}$ & 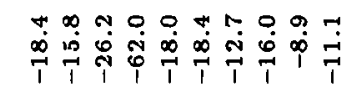 \\
\hline$\stackrel{\infty}{0}$ & 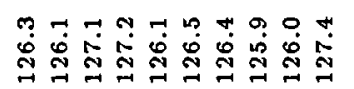 & 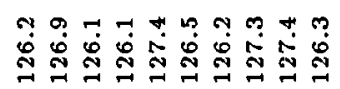 & 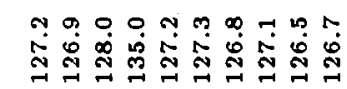 \\
\hline - & 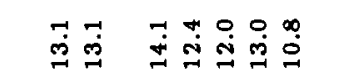 & 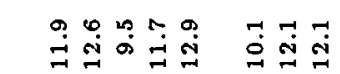 & 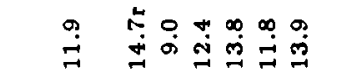 \\
\hline 2 & 苞品虽虽 & $\stackrel{0}{\ddot{m}}$ & 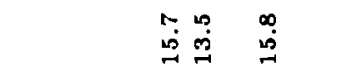 \\
\hline$\infty$ & 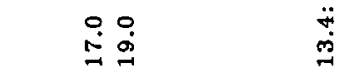 & 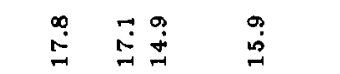 & $\underset{\infty}{\stackrel{0}{\infty}}$ \\
\hline $\begin{array}{l}0 \\
0 \\
0 \\
0\end{array}$ & 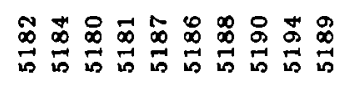 & 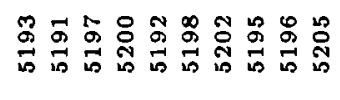 & 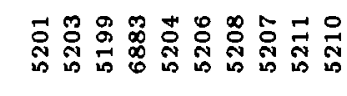 \\
\hline o & 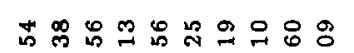 & エ & 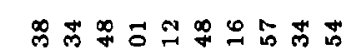 \\
\hline 递 & 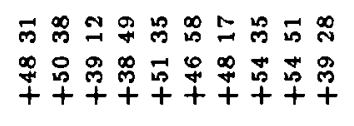 & 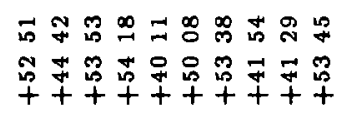 & 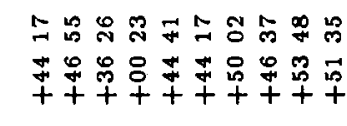 \\
\hline ठั & 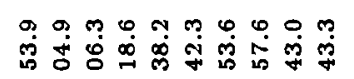 & 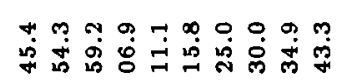 & $\begin{array}{l}\infty \\
\dot{\dot{b}} \\
\dot{n} \\
\dot{v}\end{array}$ \\
\hline (1) & 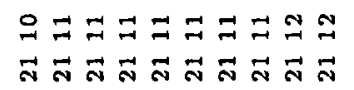 & 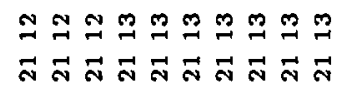 & 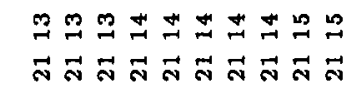 \\
\hline
\end{tabular}




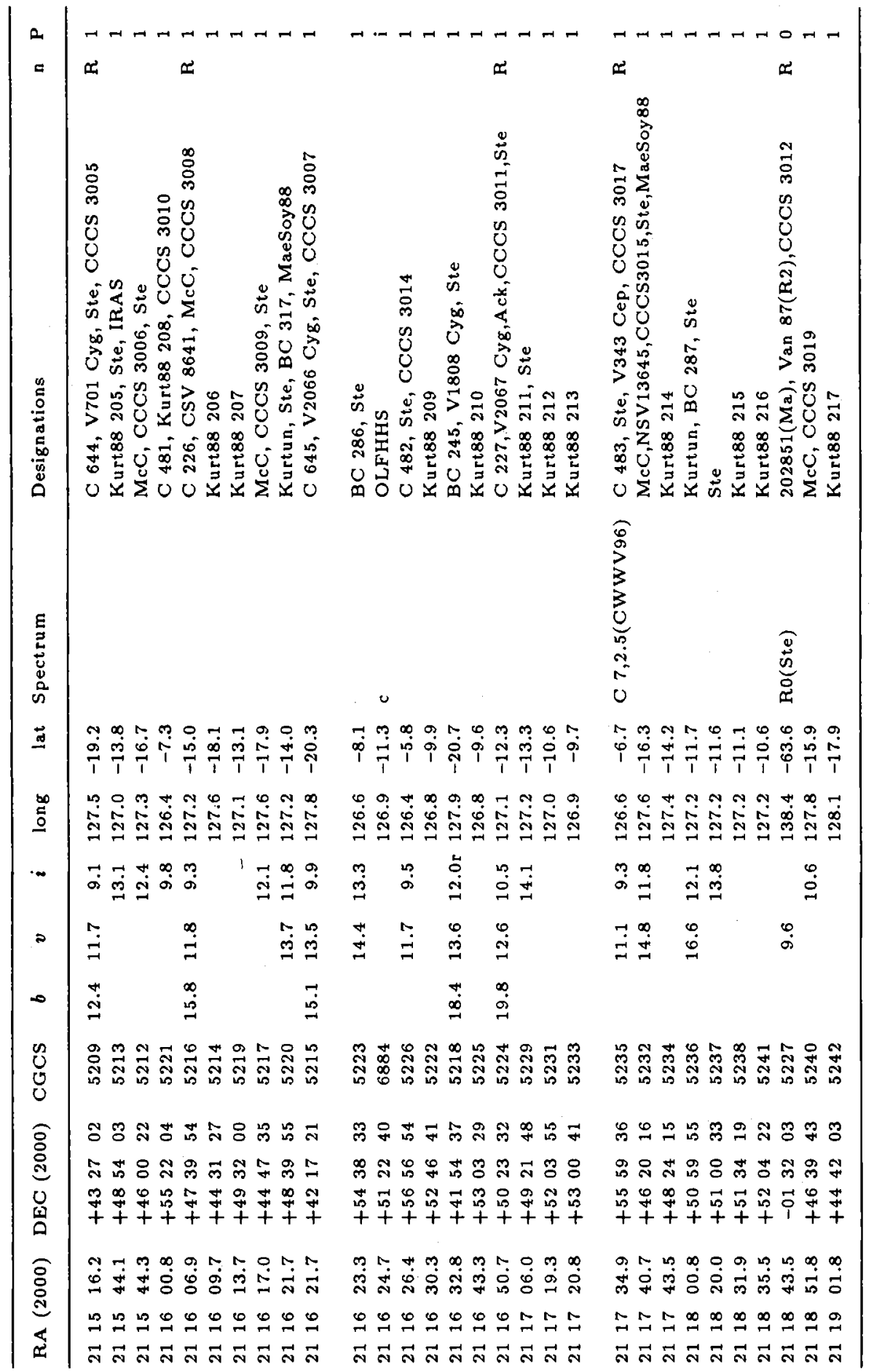




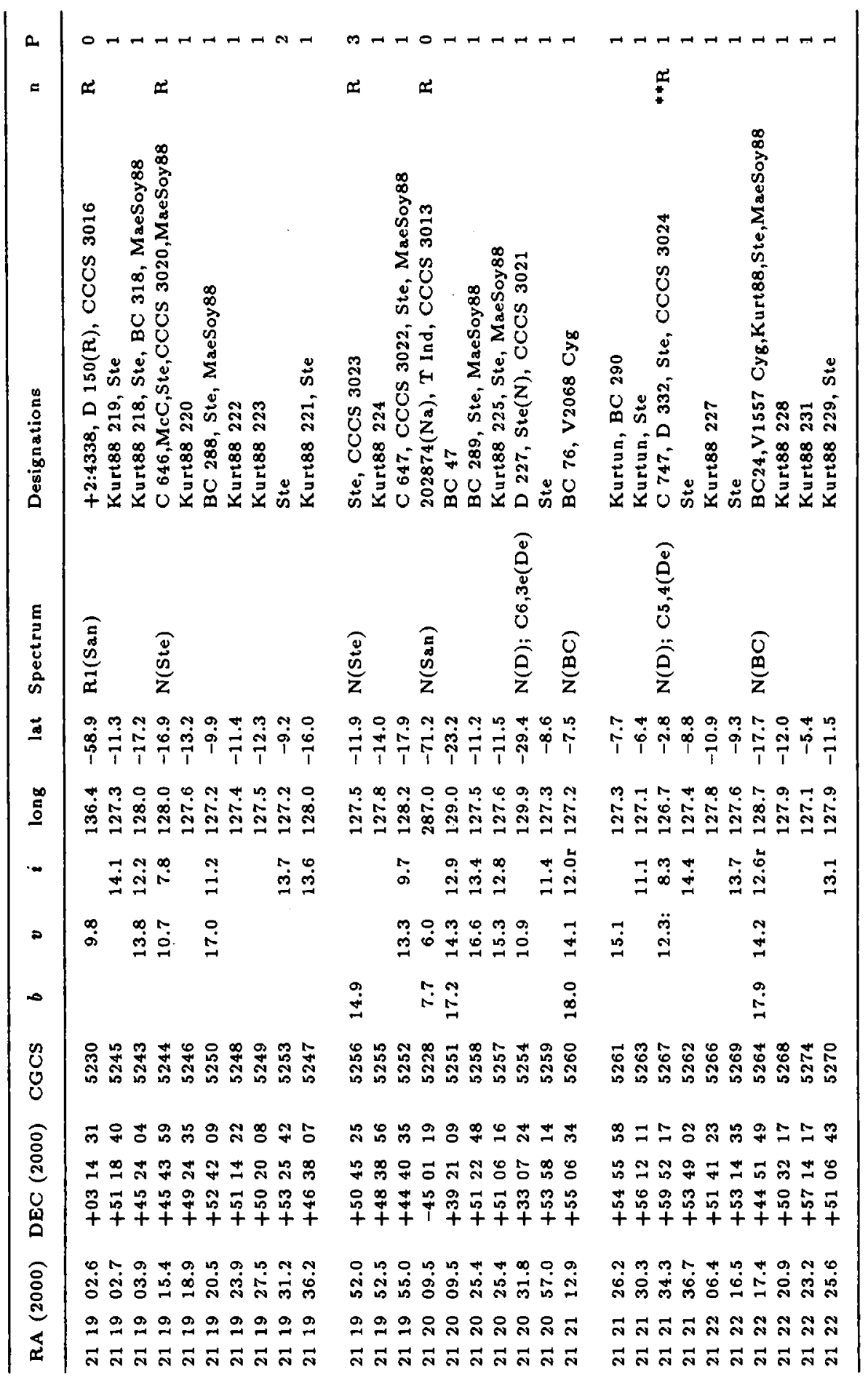




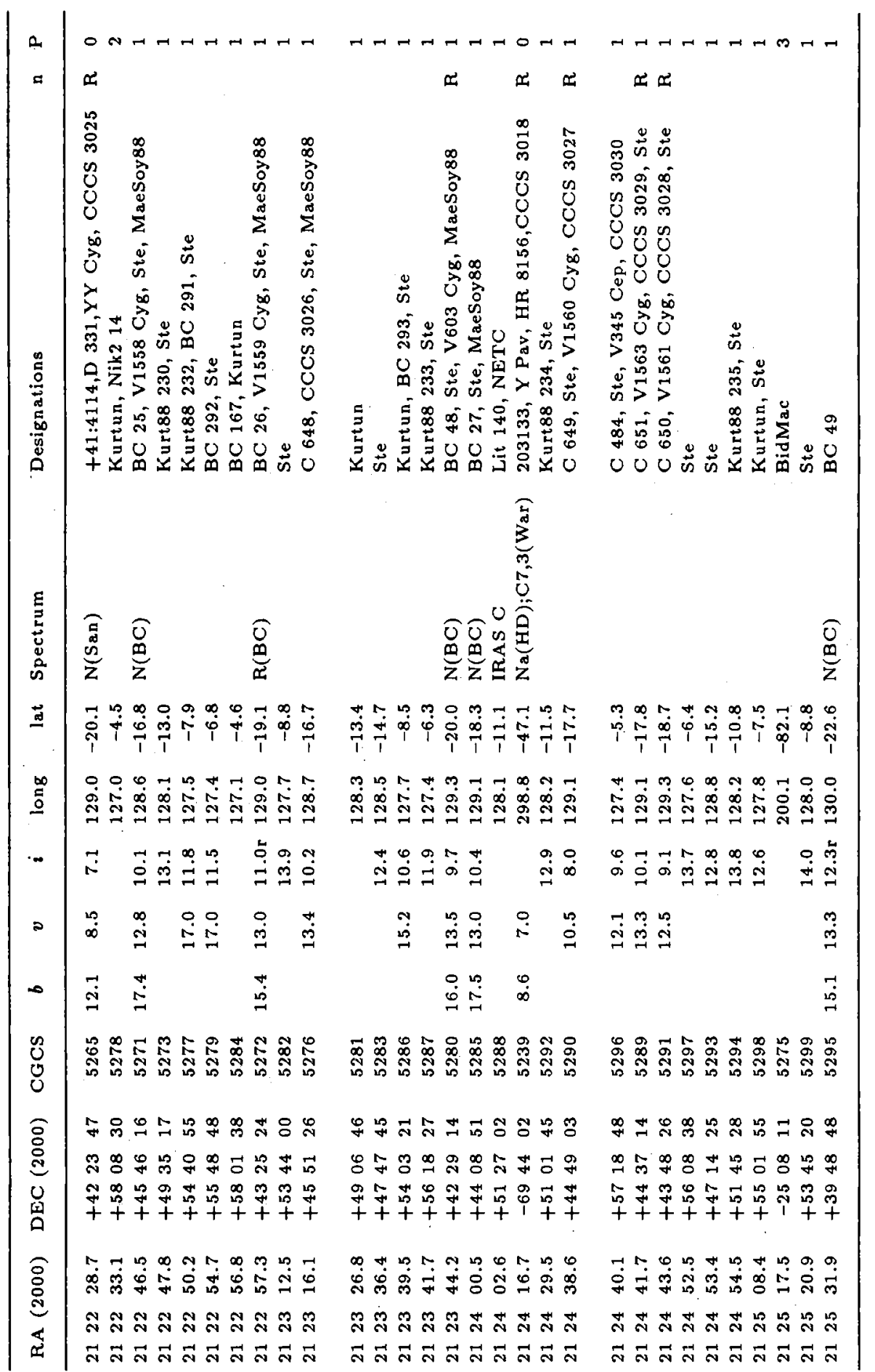




\begin{tabular}{|c|c|c|c|}
\hline a. & 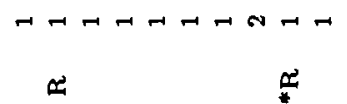 & 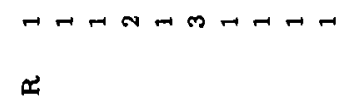 & 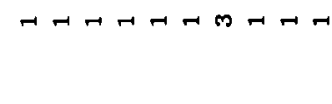 \\
\hline 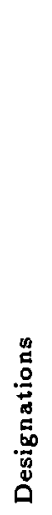 & 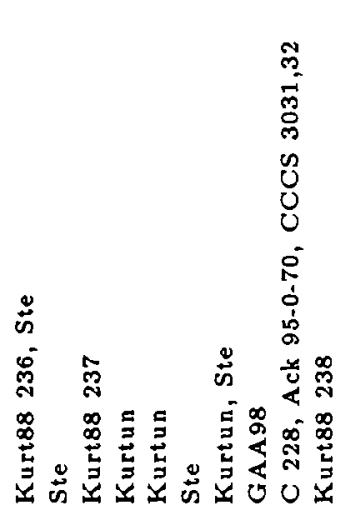 & 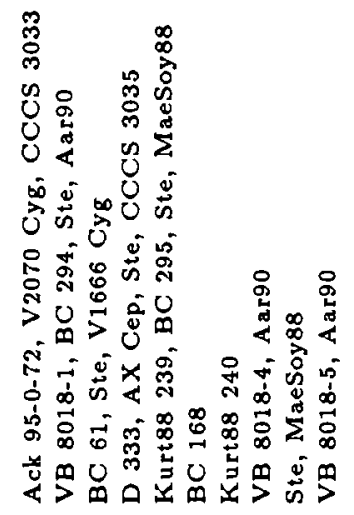 & 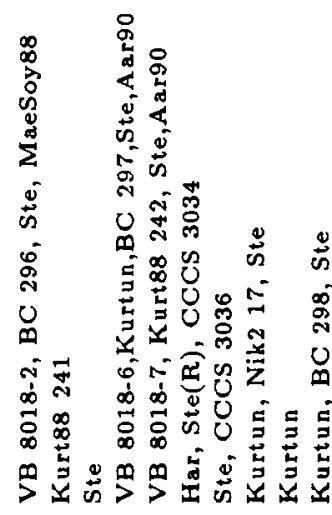 \\
\hline & & 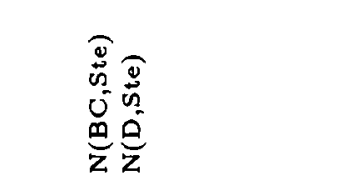 & 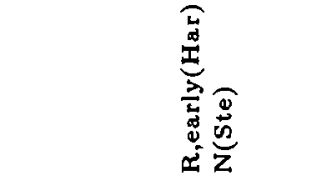 \\
\hline త్ & 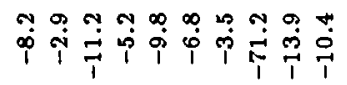 & 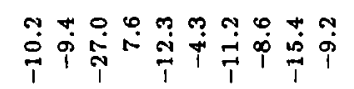 & 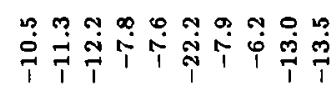 \\
\hline 电 & 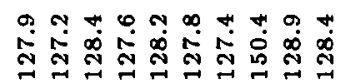 & 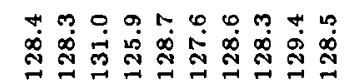 & 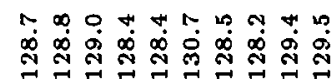 \\
\hline - & 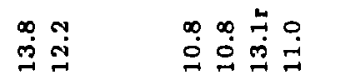 & 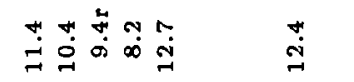 & 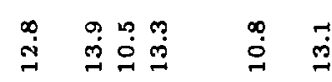 \\
\hline 2 & $\stackrel{\circ}{\stackrel{\rho}{\rightarrow}}$ & 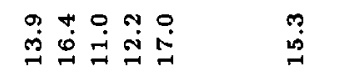 & $\stackrel{\leftrightarrow}{\ddot{D}}$ \\
\hline$\infty$ & $\stackrel{\infty}{\stackrel{\infty}{\sim}}$ & 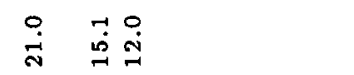 & 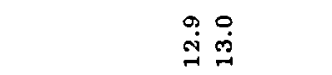 \\
\hline : & 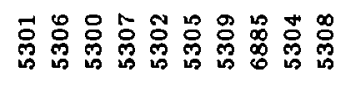 & 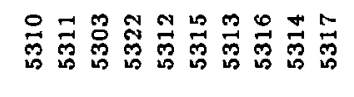 & 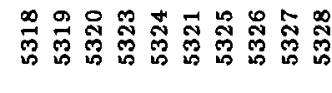 \\
\hline 。 & 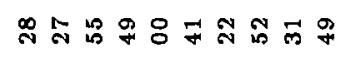 & 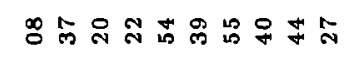 & 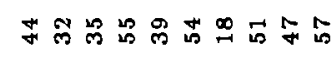 \\
\hline 通 & 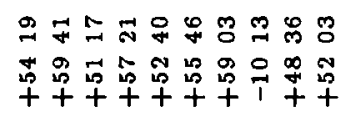 & 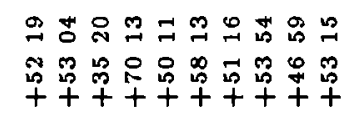 & 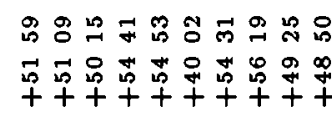 \\
\hline ळे & 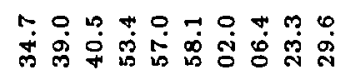 & 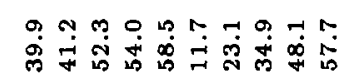 & 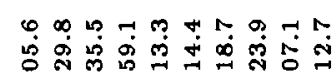 \\
\hline$\sqrt{4}$ & 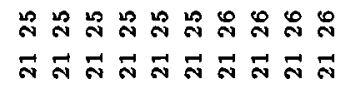 & 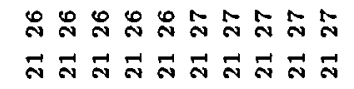 & 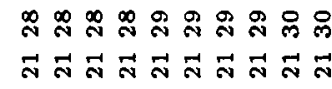 \\
\hline
\end{tabular}




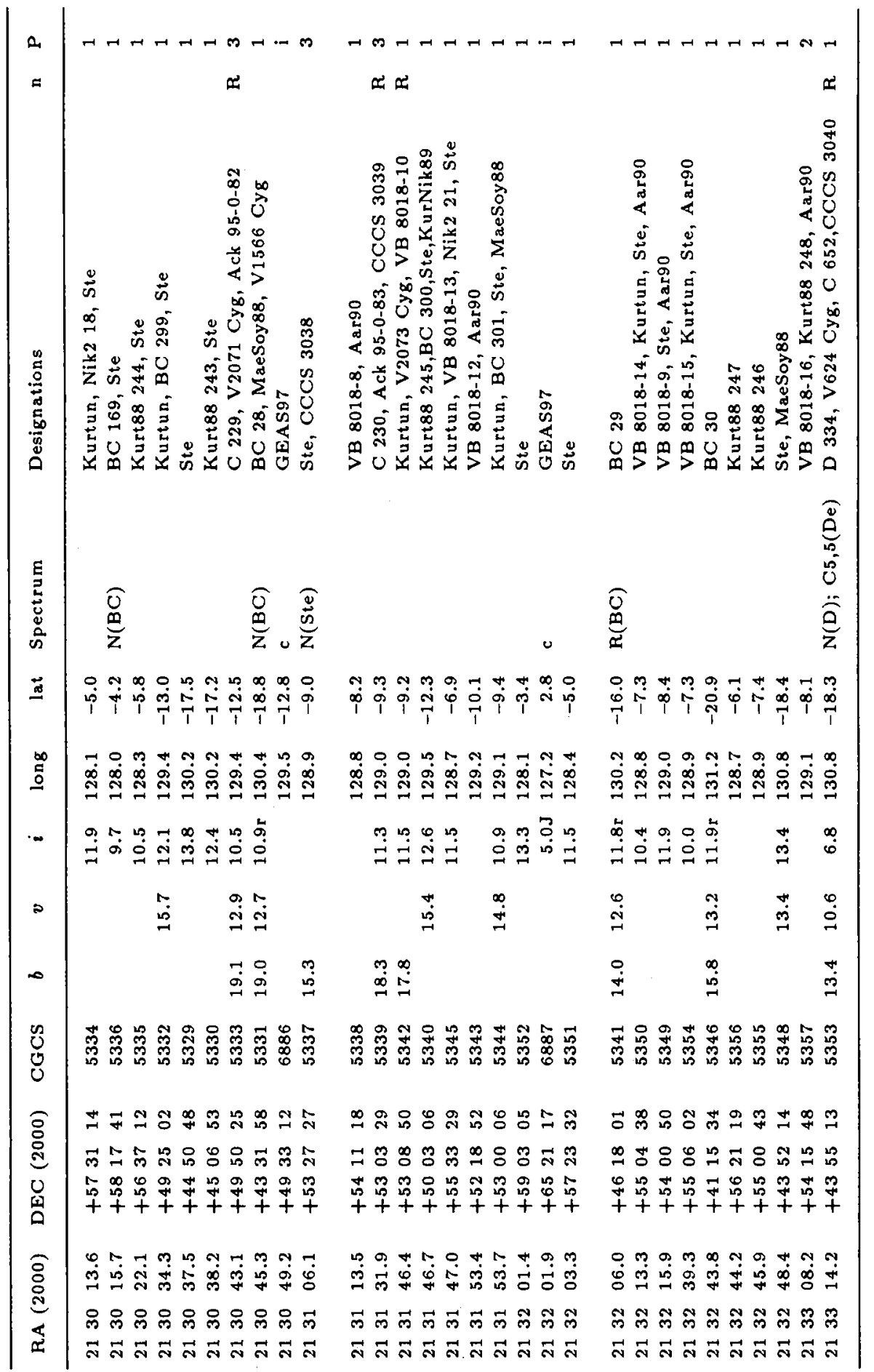




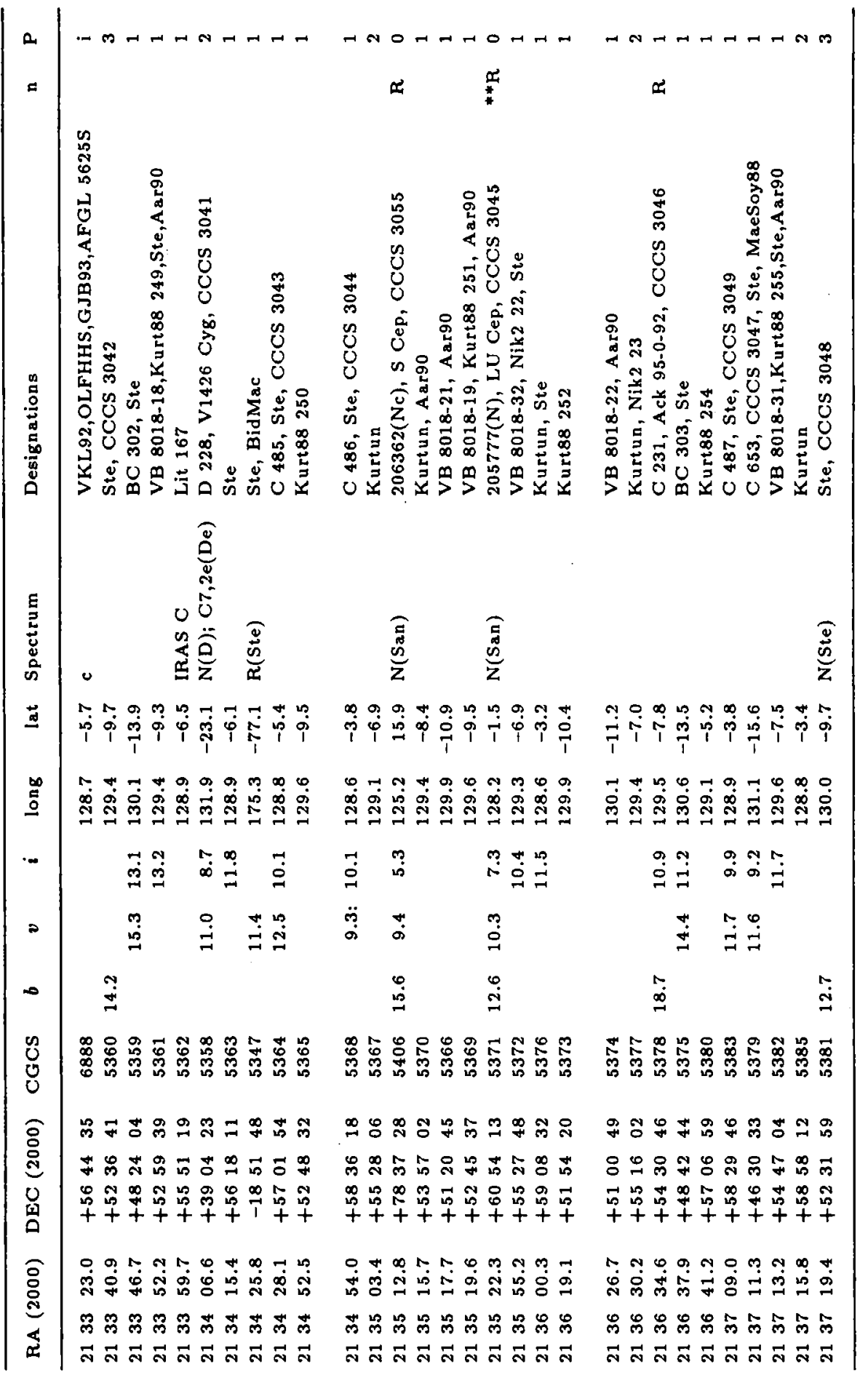




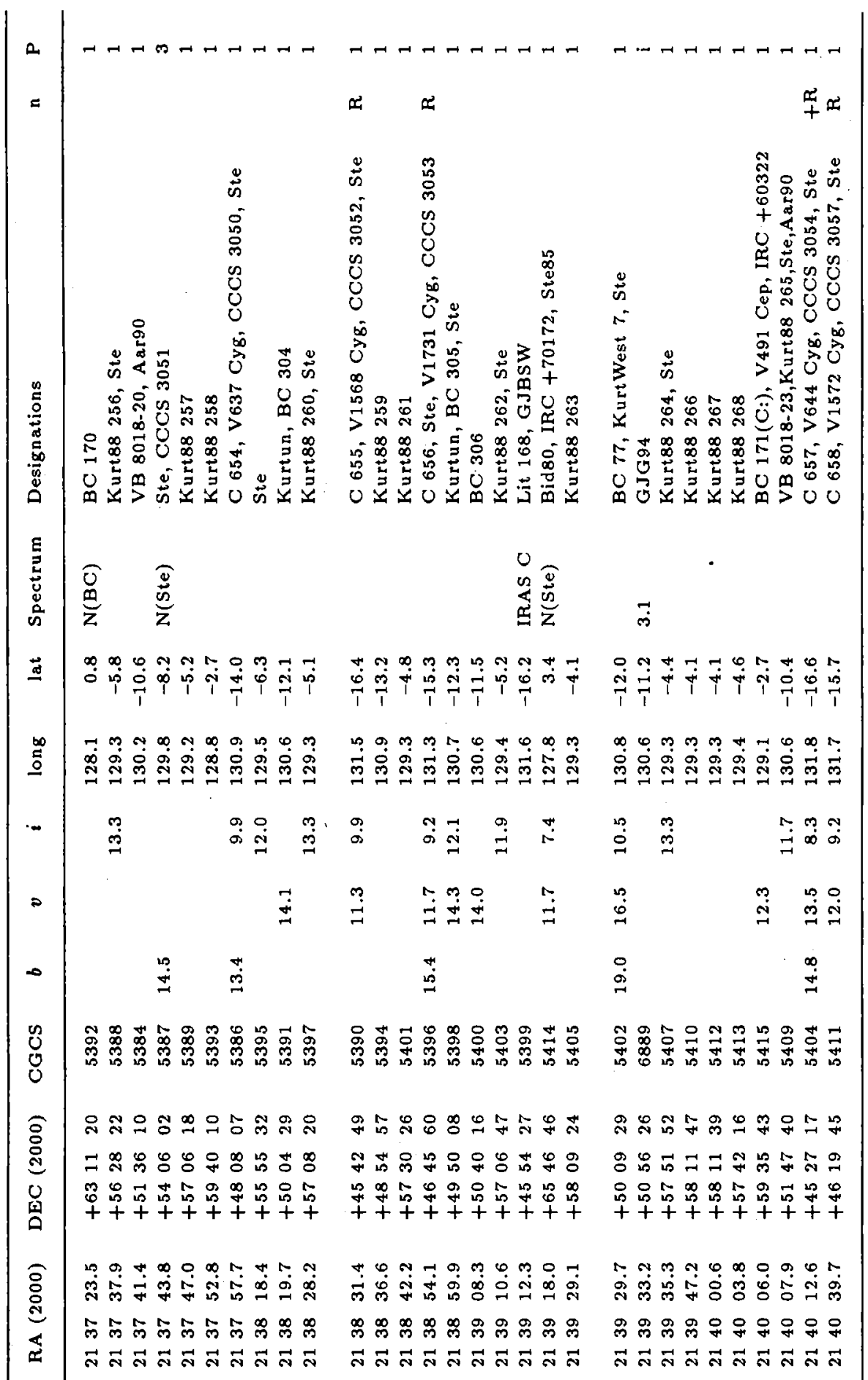




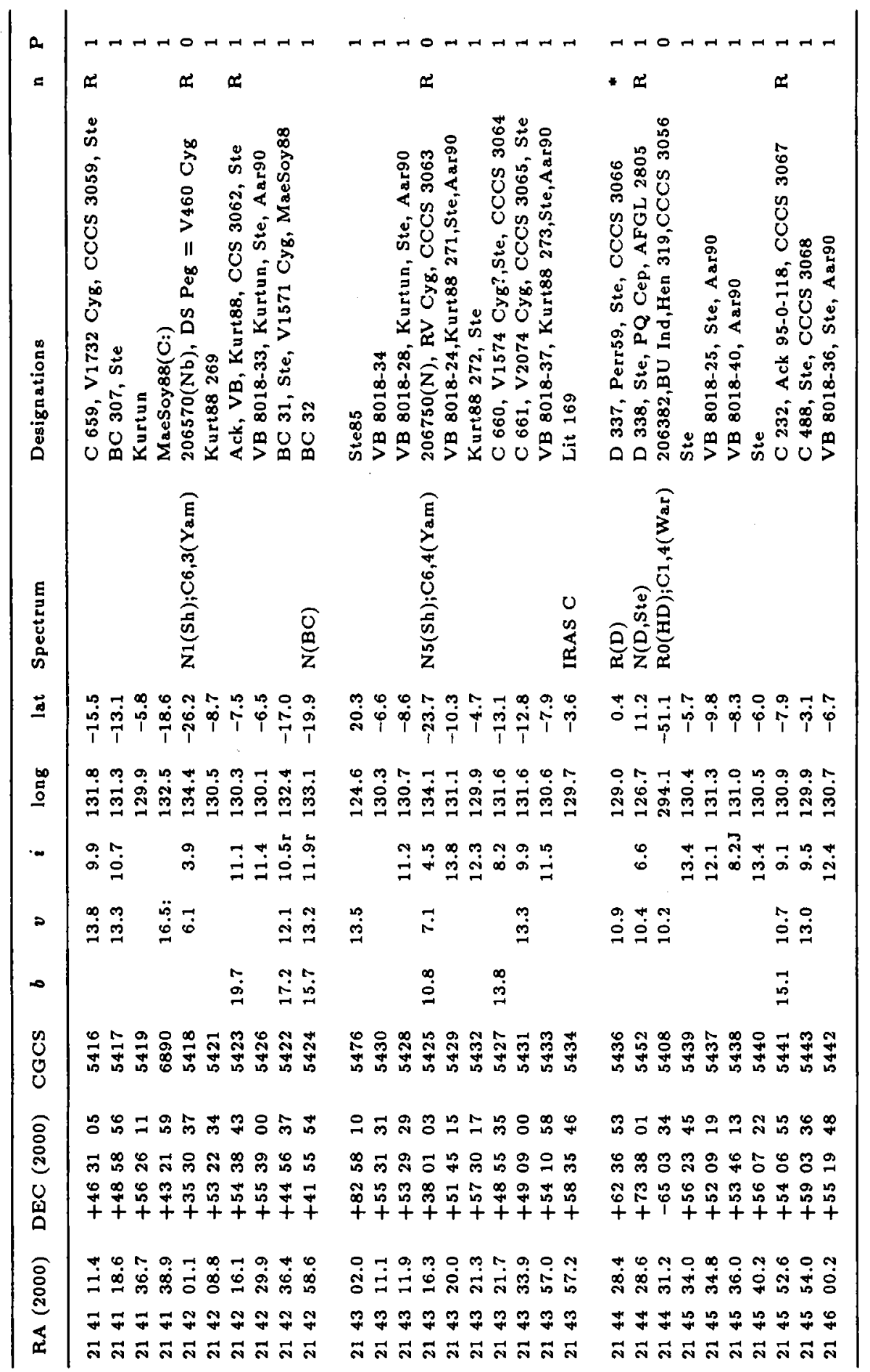




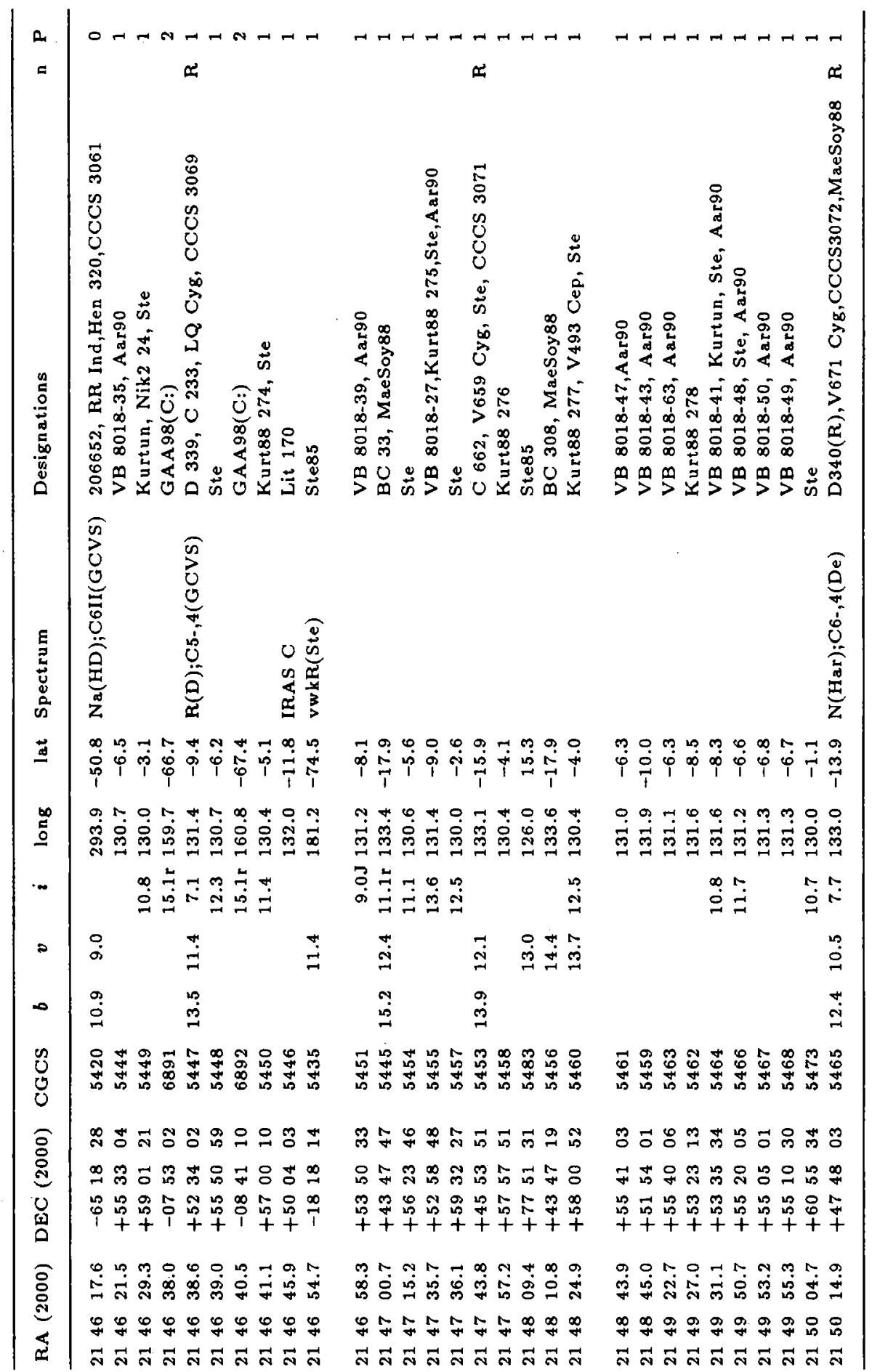




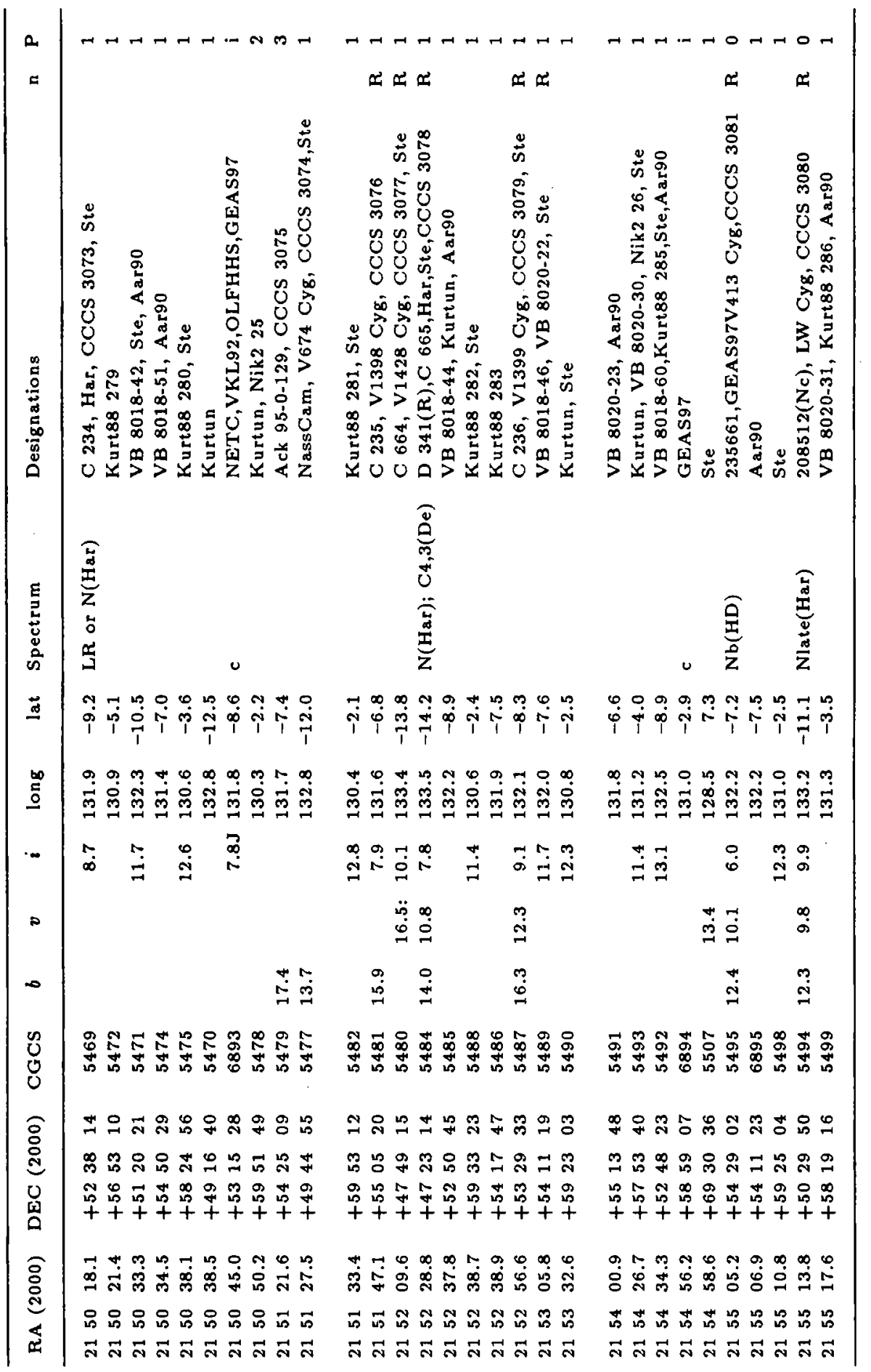




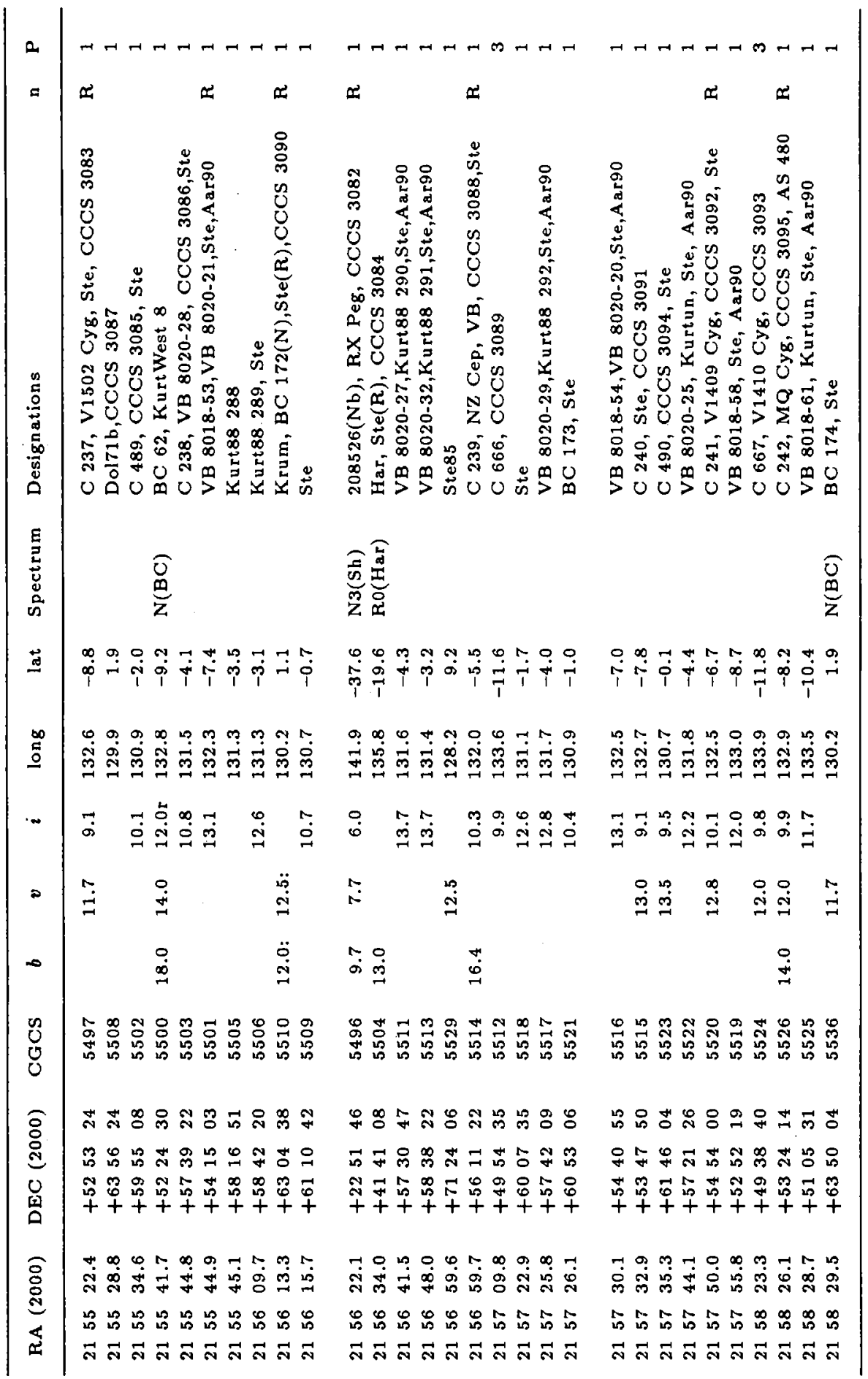




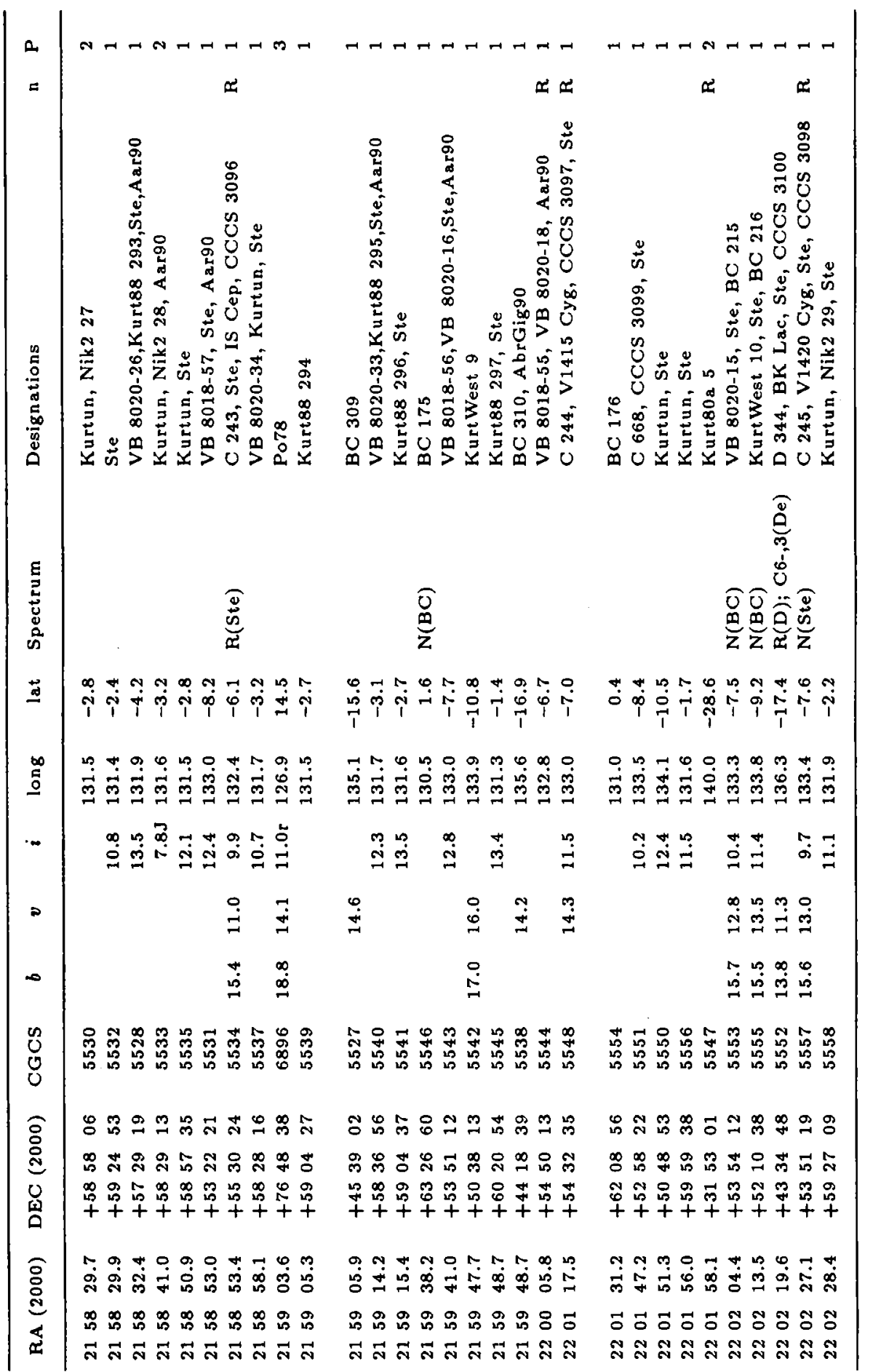




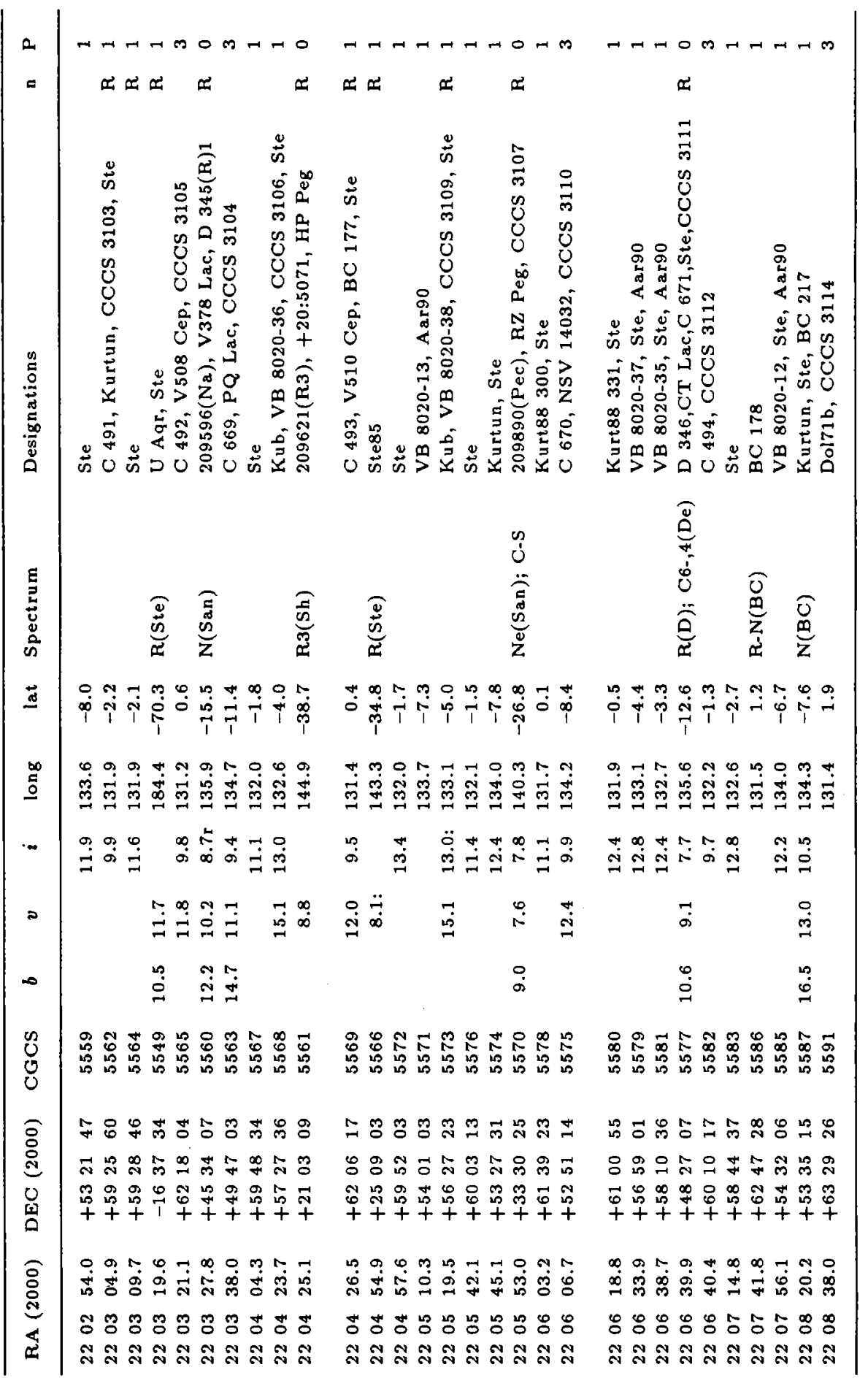




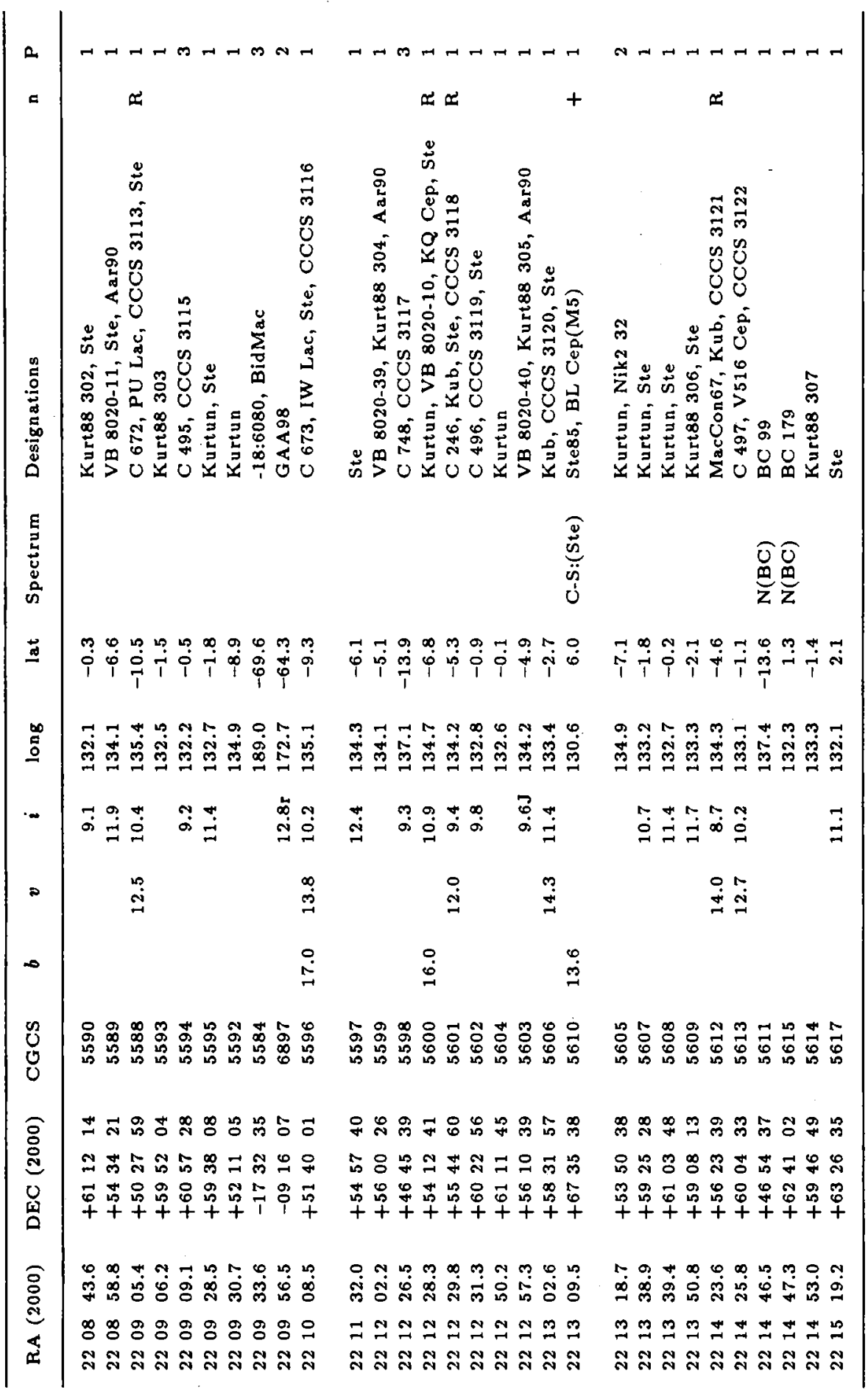




\begin{tabular}{|c|c|c|c|c|}
\hline z & \multicolumn{3}{|c|}{ 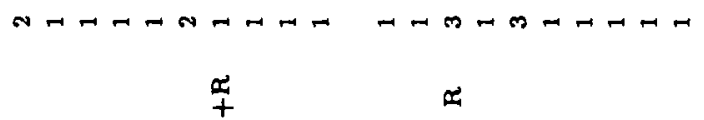 } & $\rightarrow H A \rightarrow H A m-t a$ \\
\hline 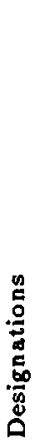 & 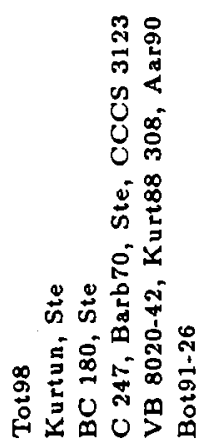 & 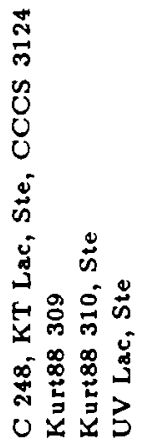 & 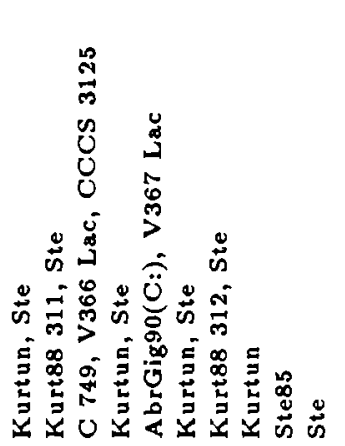 & 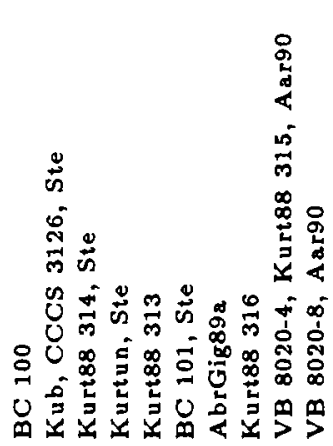 \\
\hline 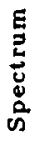 & 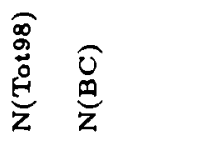 & 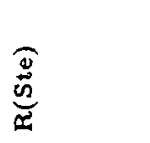 & 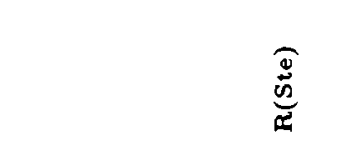 & $\underset{\substack{0 \\
z}}{\substack{n \\
x^{\prime}}}$ \\
\hline 㤩 & 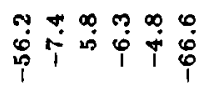 & $\stackrel{\infty}{0} \underset{i}{\dot{1}} \stackrel{0}{0}$ & 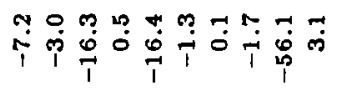 & 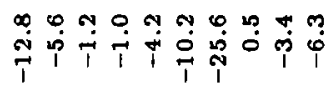 \\
\hline$\underline{E}$ & 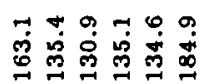 & 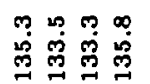 & 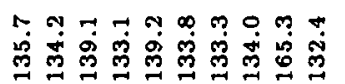 & 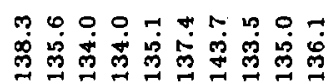 \\
\hline 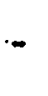 & 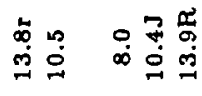 & 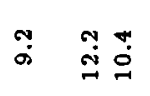 & 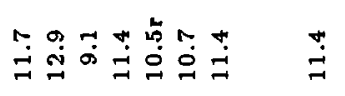 & 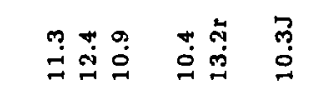 \\
\hline D & $\stackrel{\infty}{\ddot{g}} \stackrel{-}{=}$ & & $\ddot{\theta}$ & $\underset{\infty}{\mathscr{N}}$ \\
\hline ه & $\bar{i}$ & 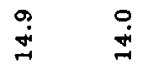 & & \\
\hline$\bigcup_{0}^{\infty}$ & 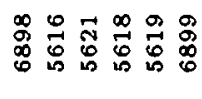 & 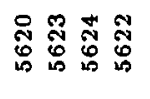 & 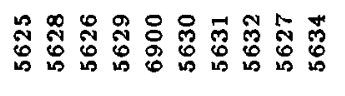 & 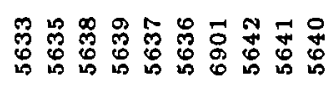 \\
\hline 8 & 吕哭缶品品品 & नิ : : & 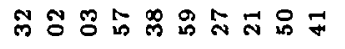 & 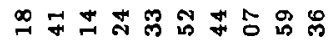 \\
\hline 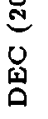 & 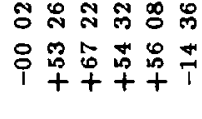 & 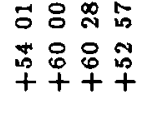 & 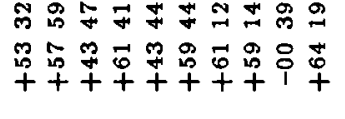 & 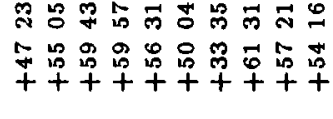 \\
\hline ळे & 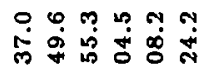 & 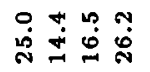 & 品 怘 & 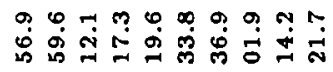 \\
\hline 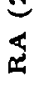 & 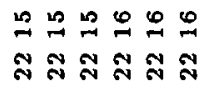 & 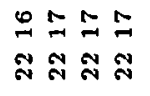 & 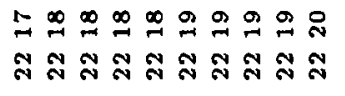 & 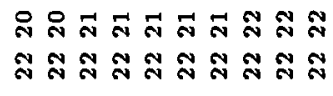 \\
\hline
\end{tabular}




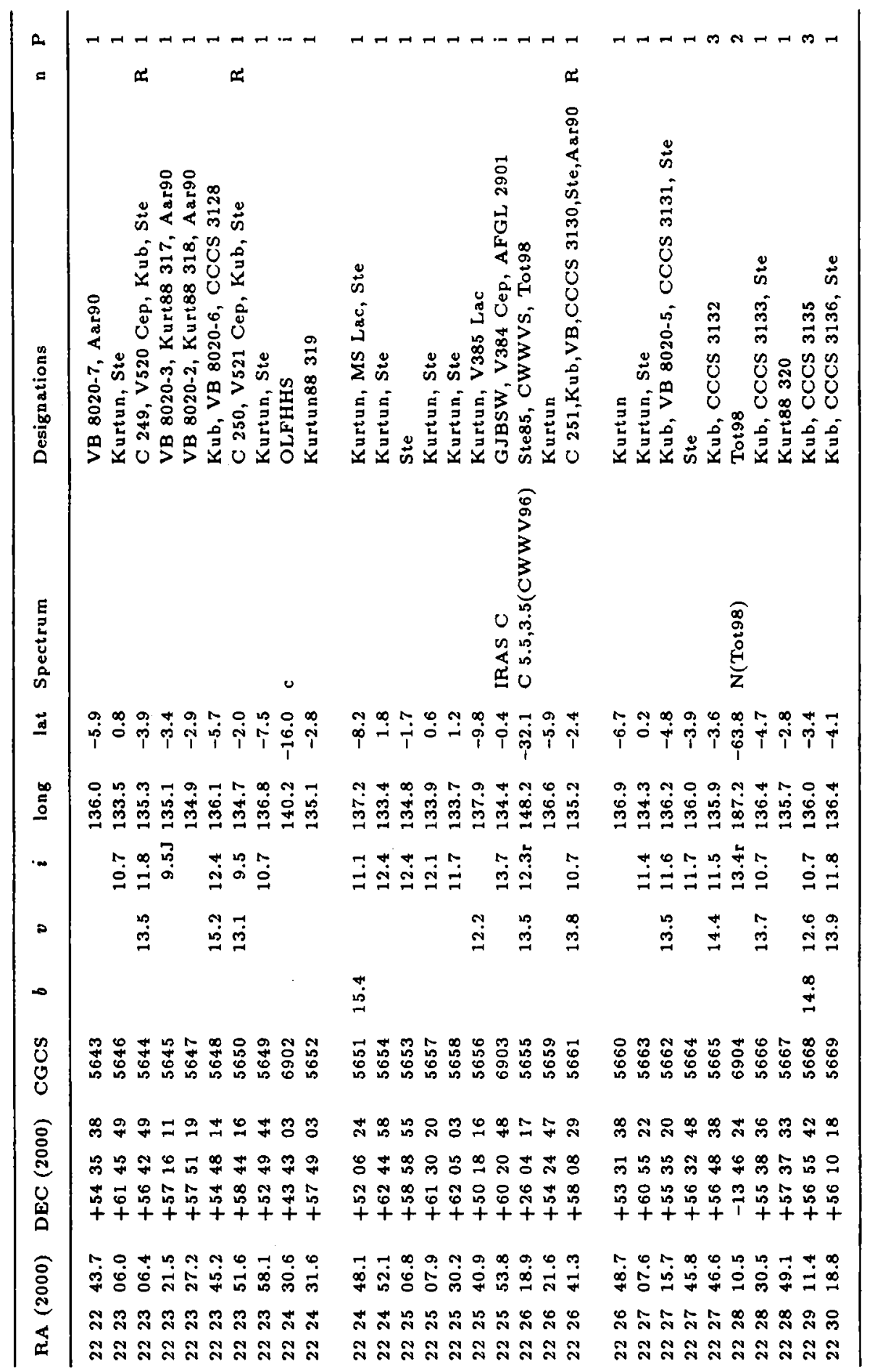




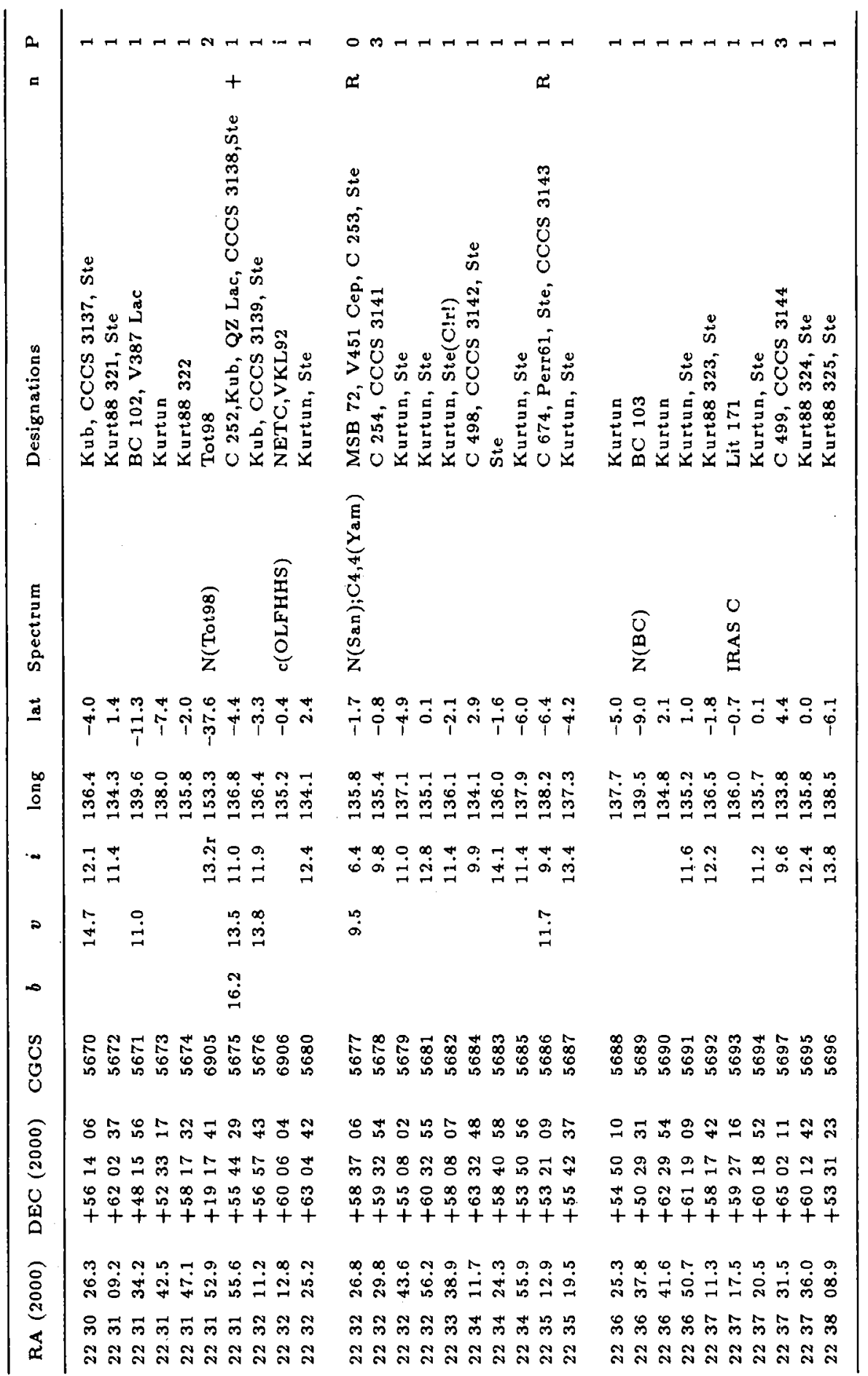




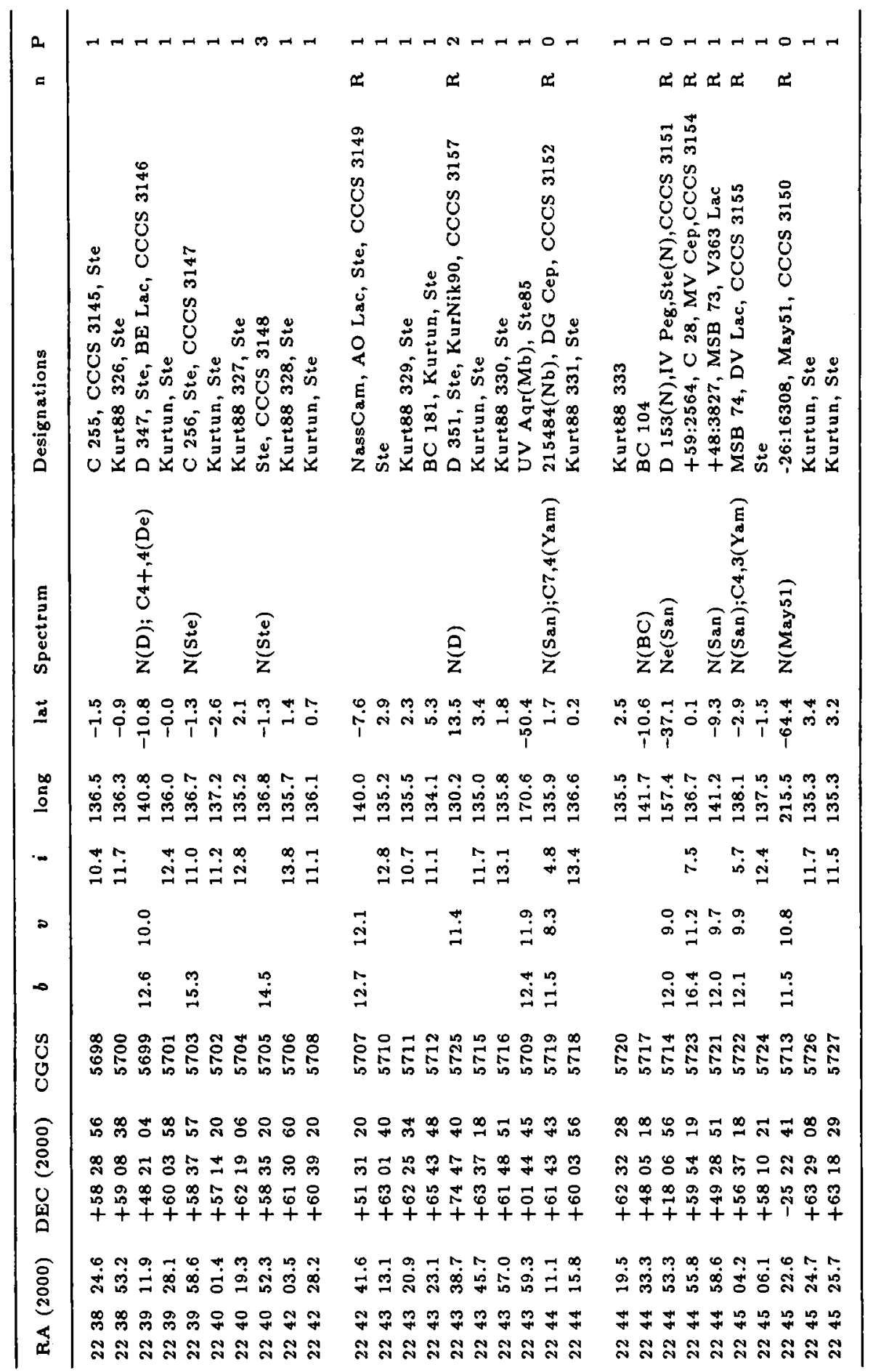




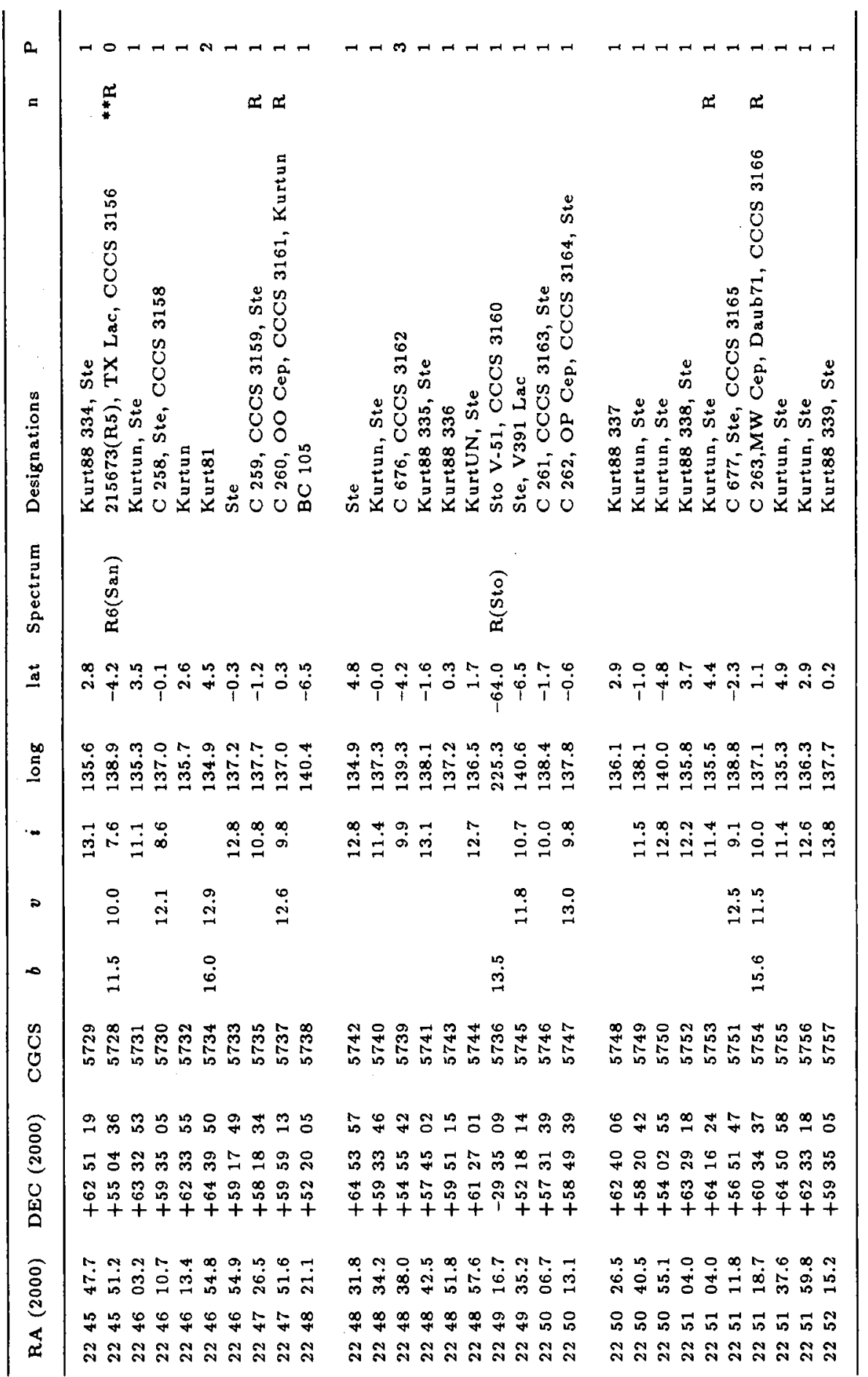




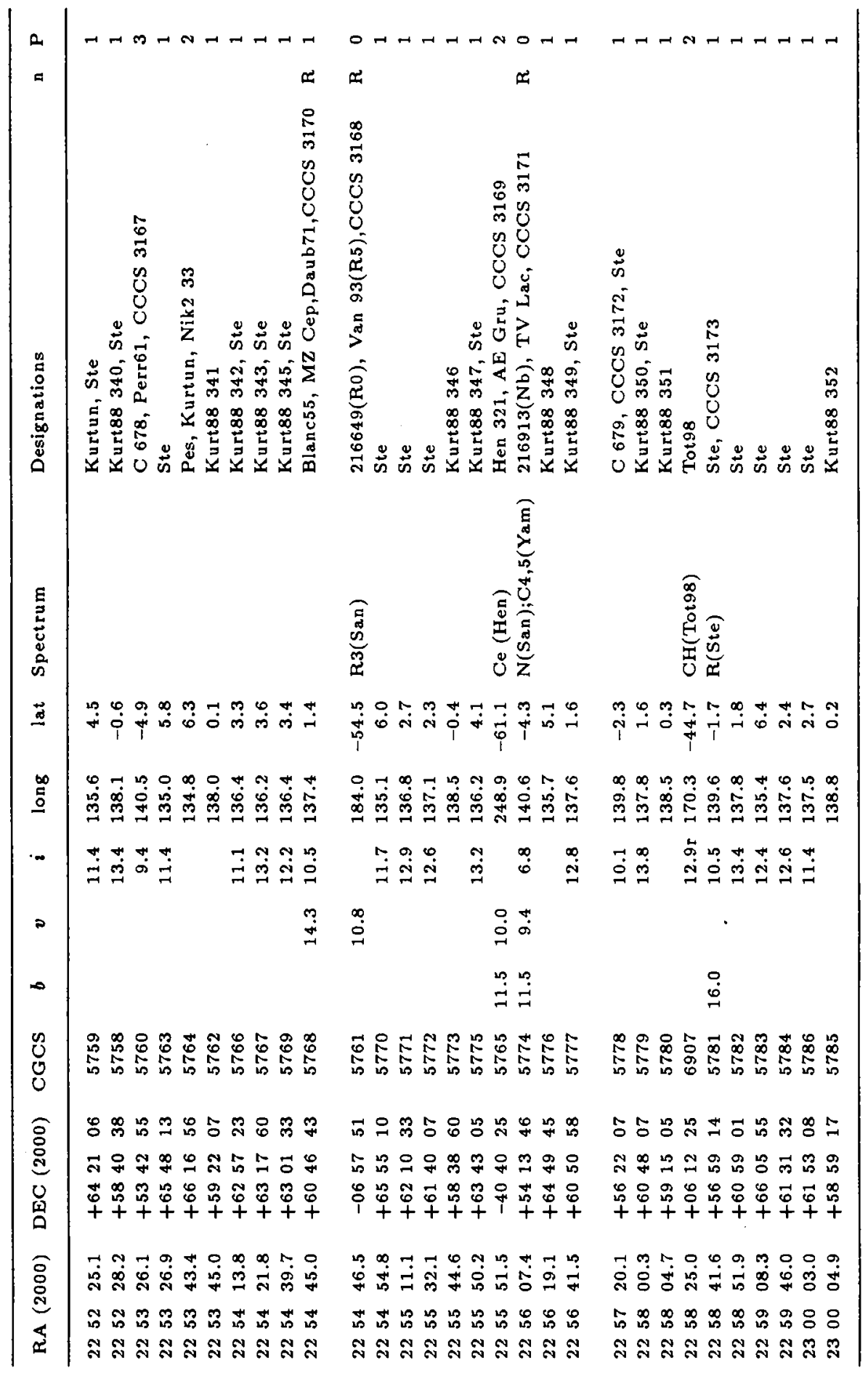




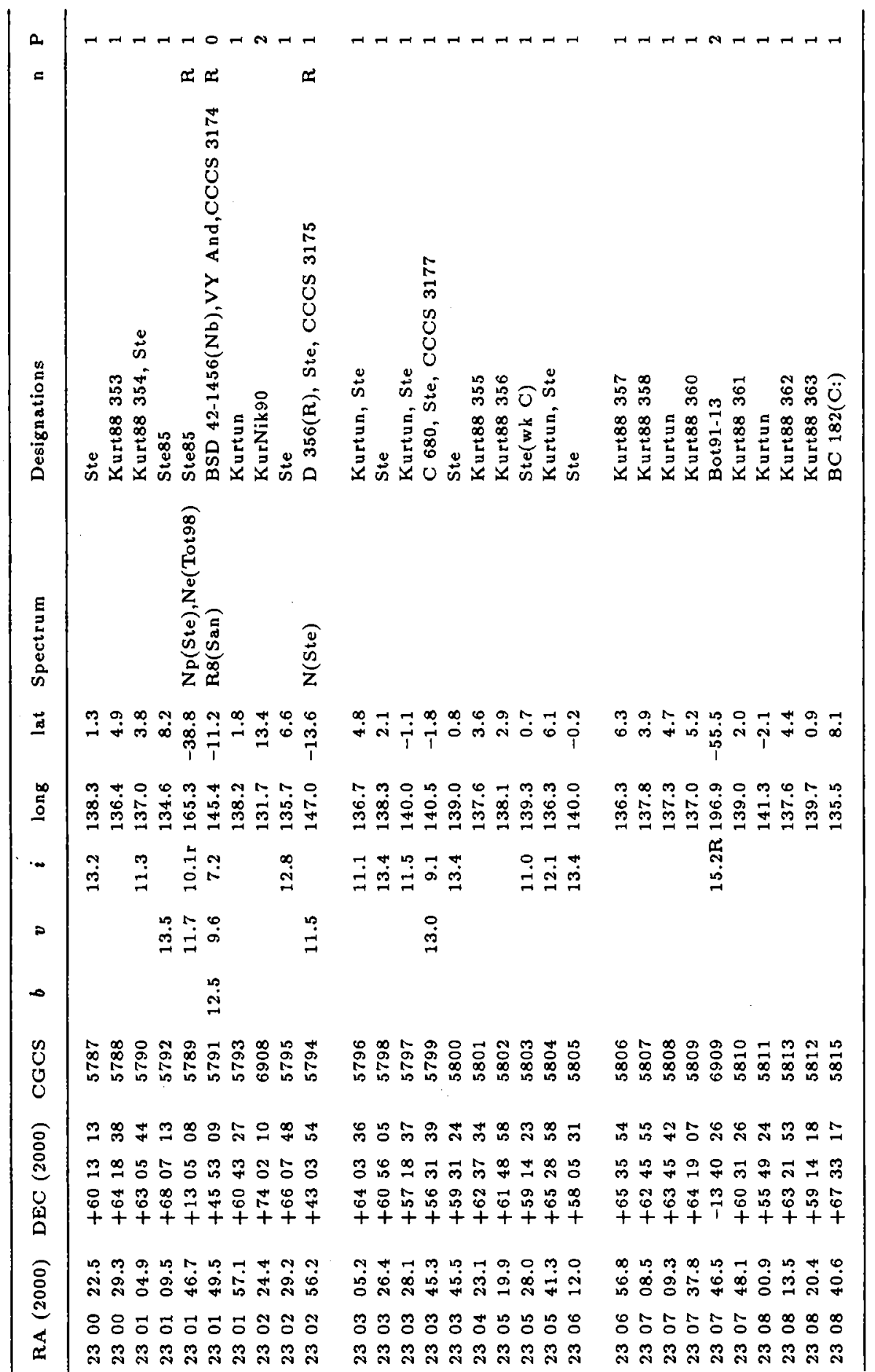




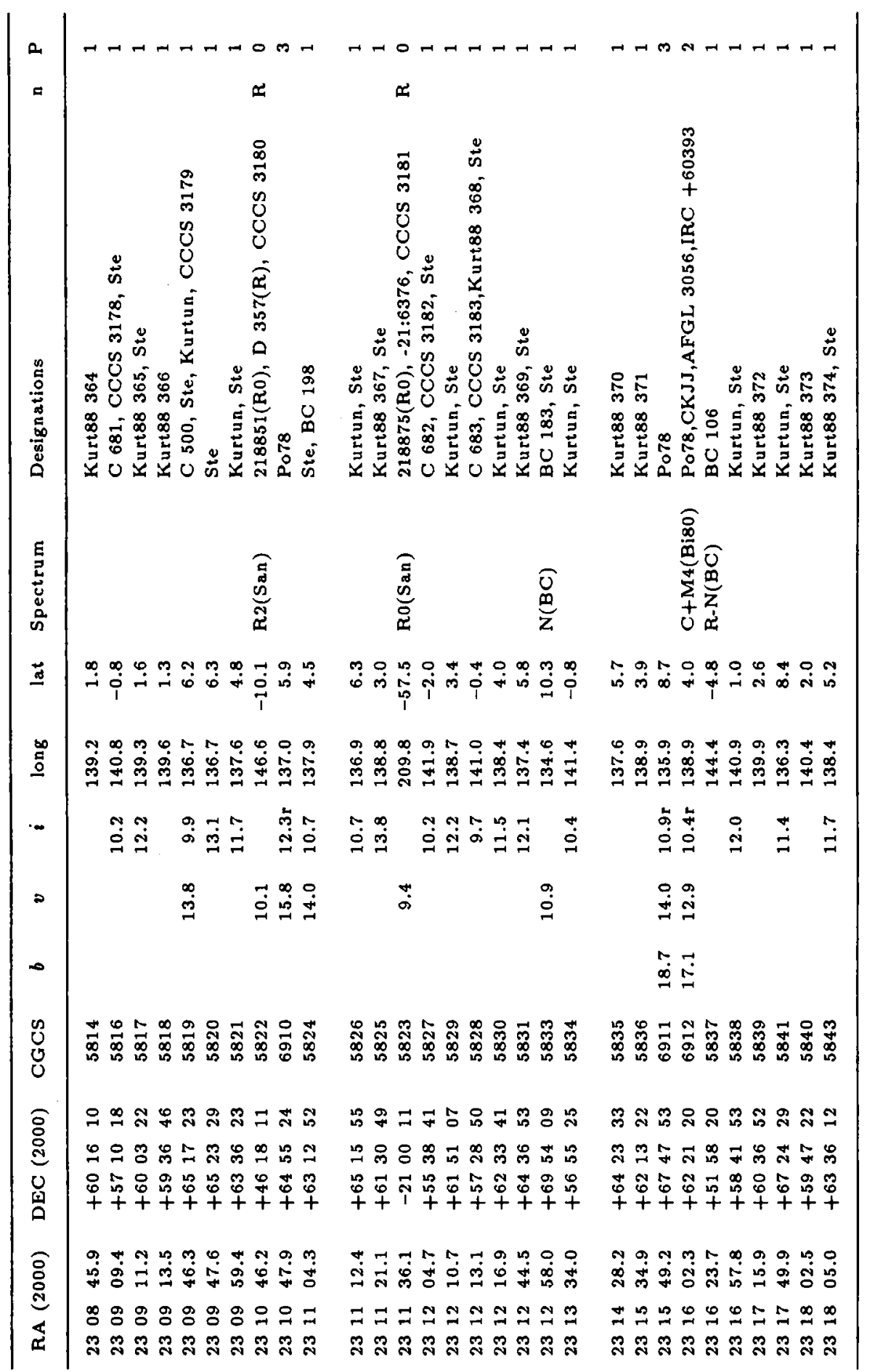




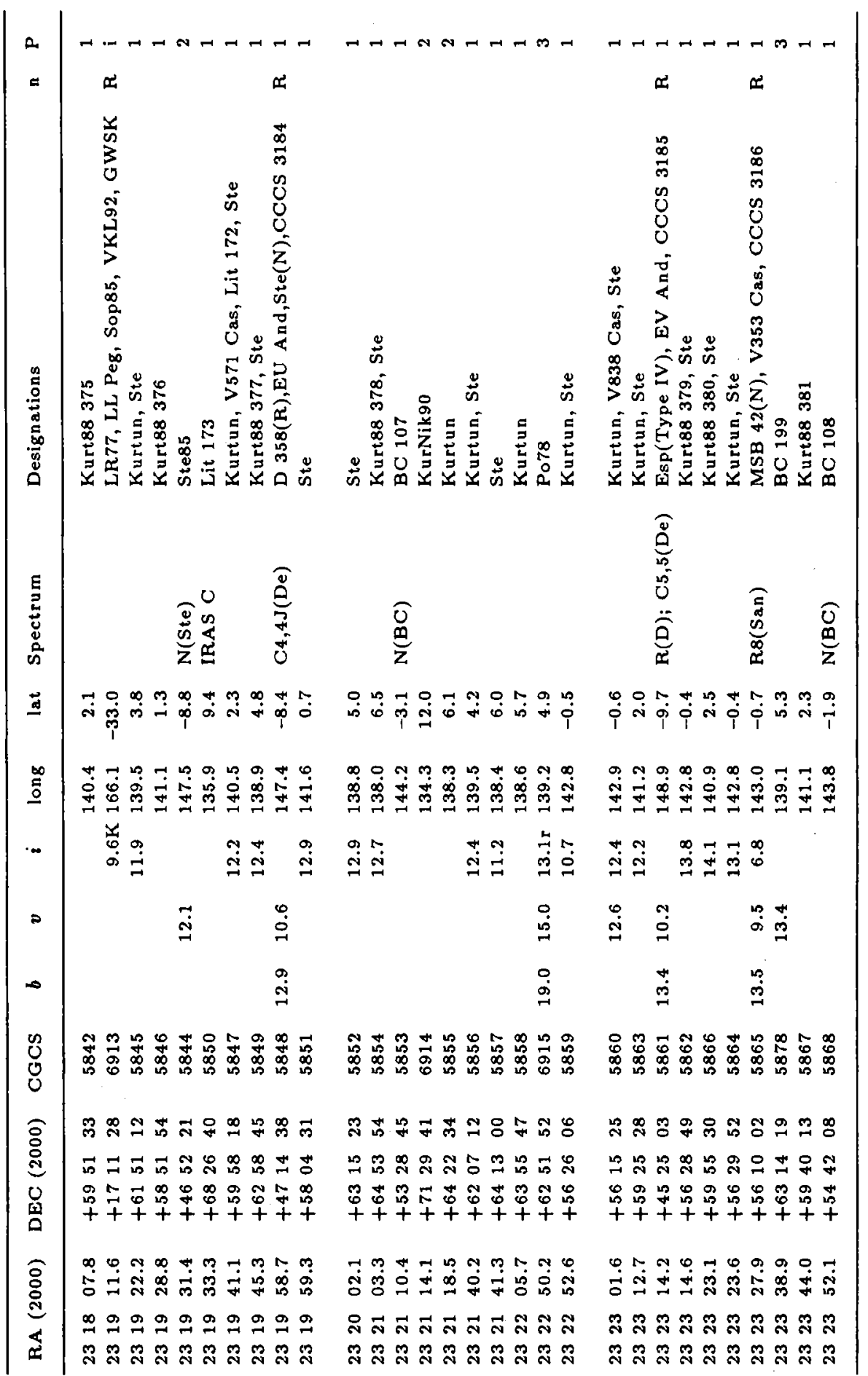




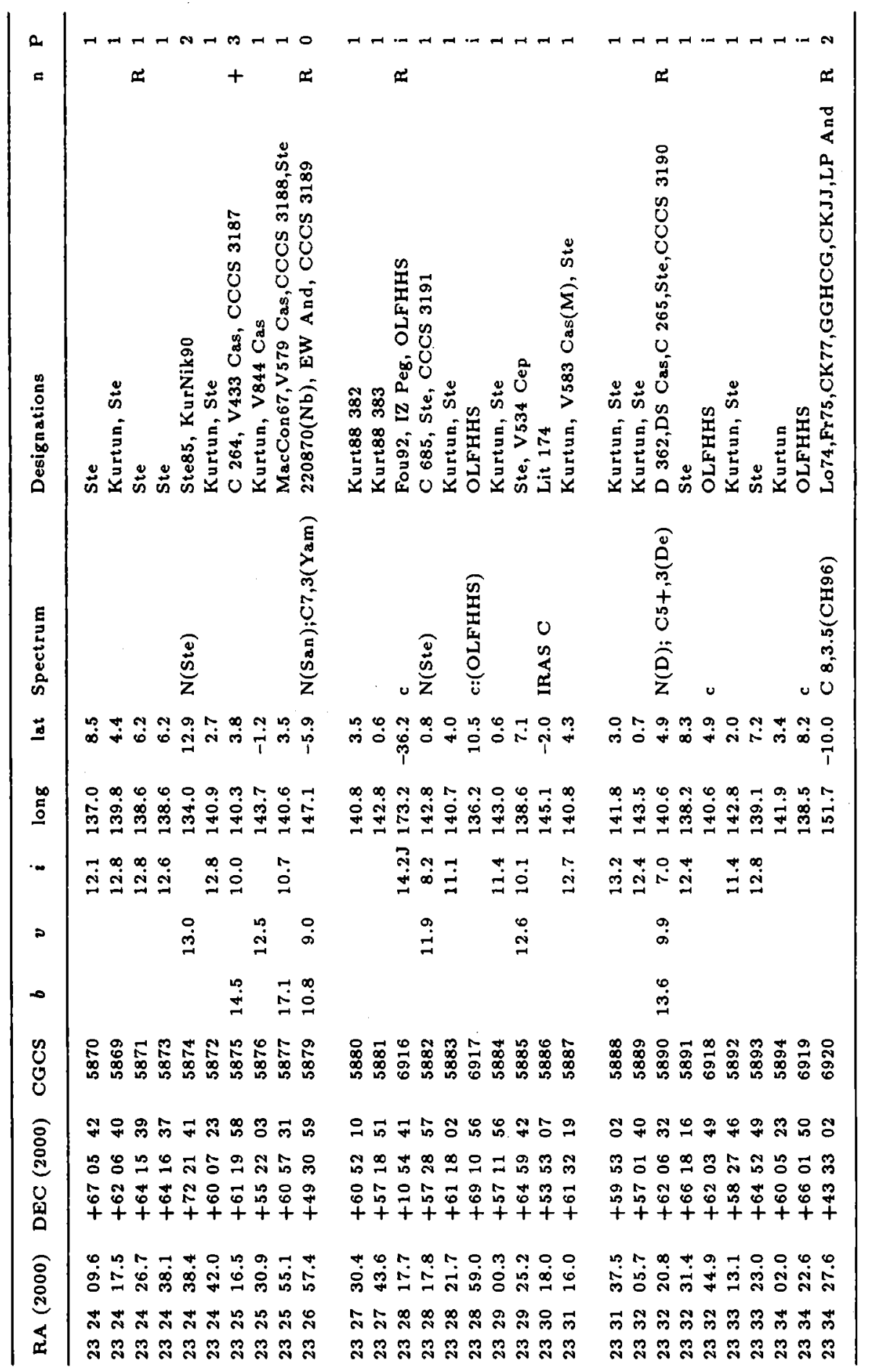




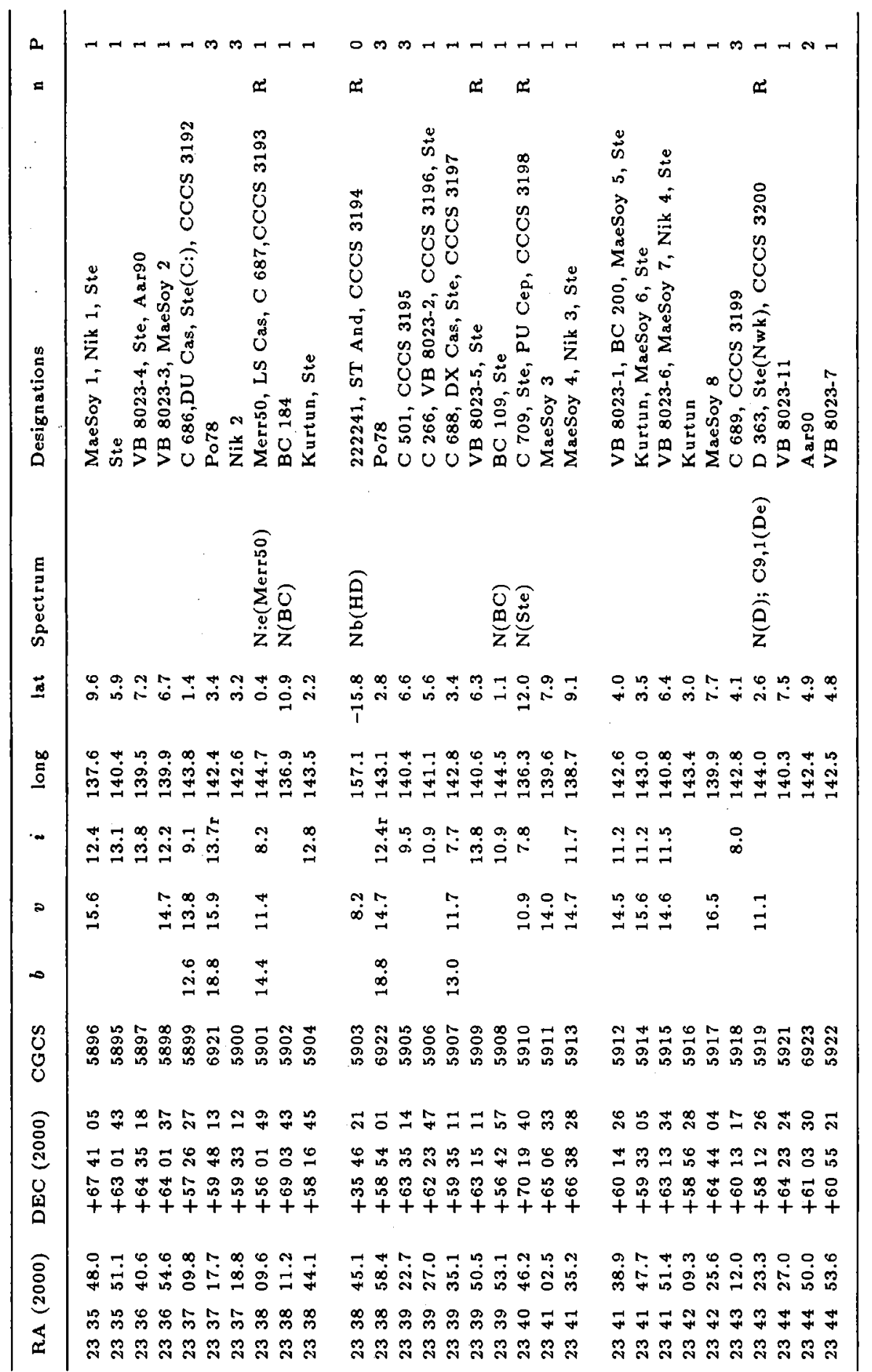




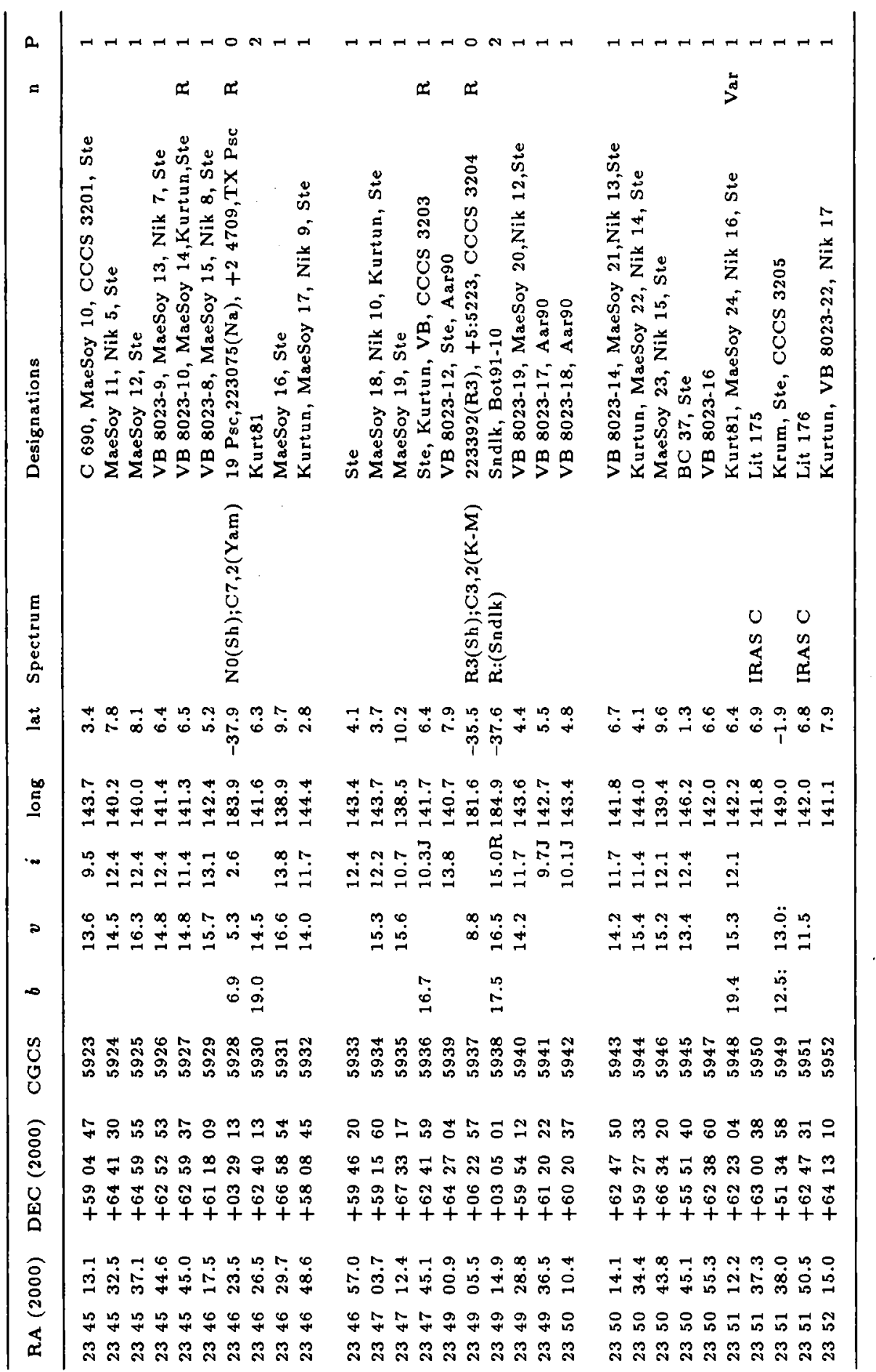




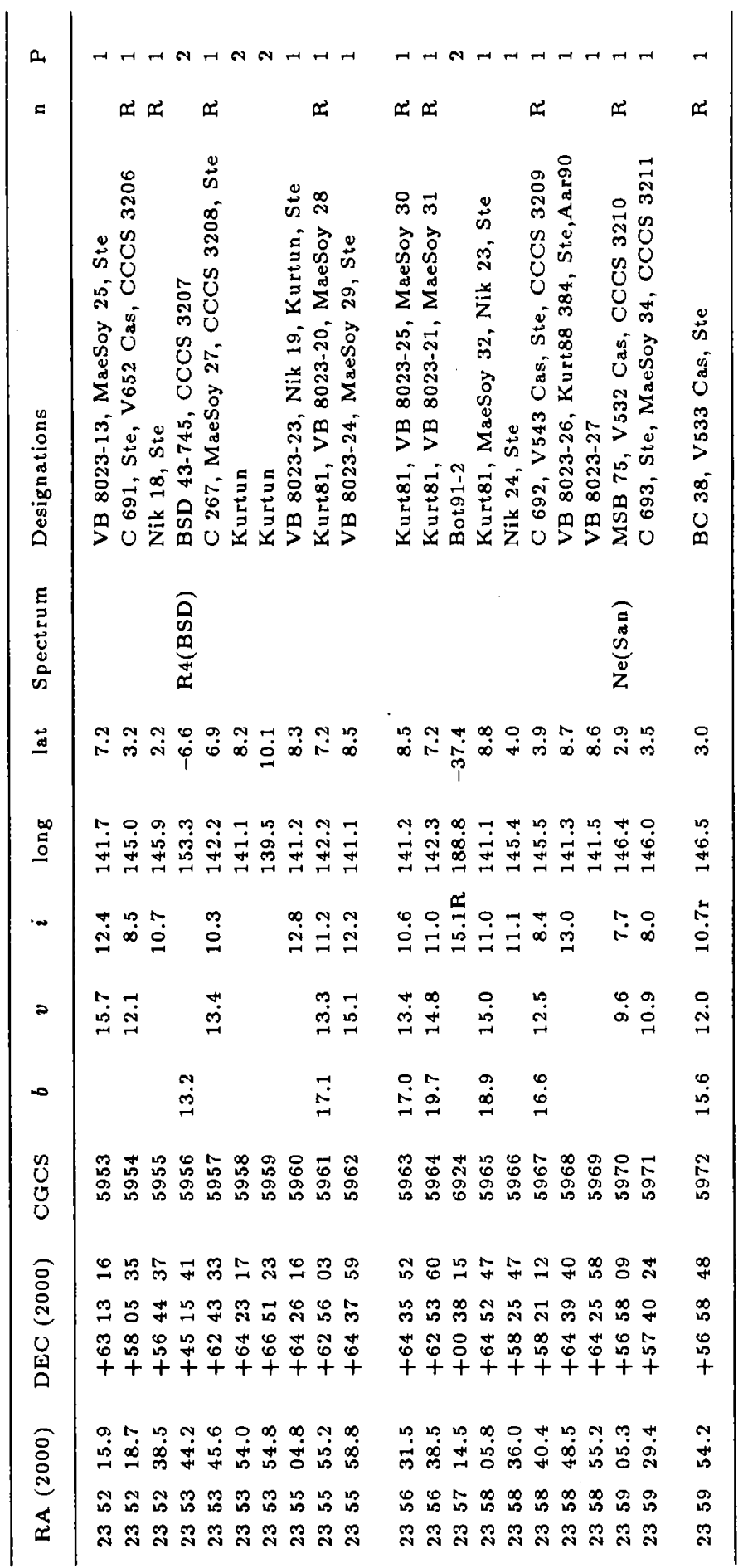


PART II. REMARKS

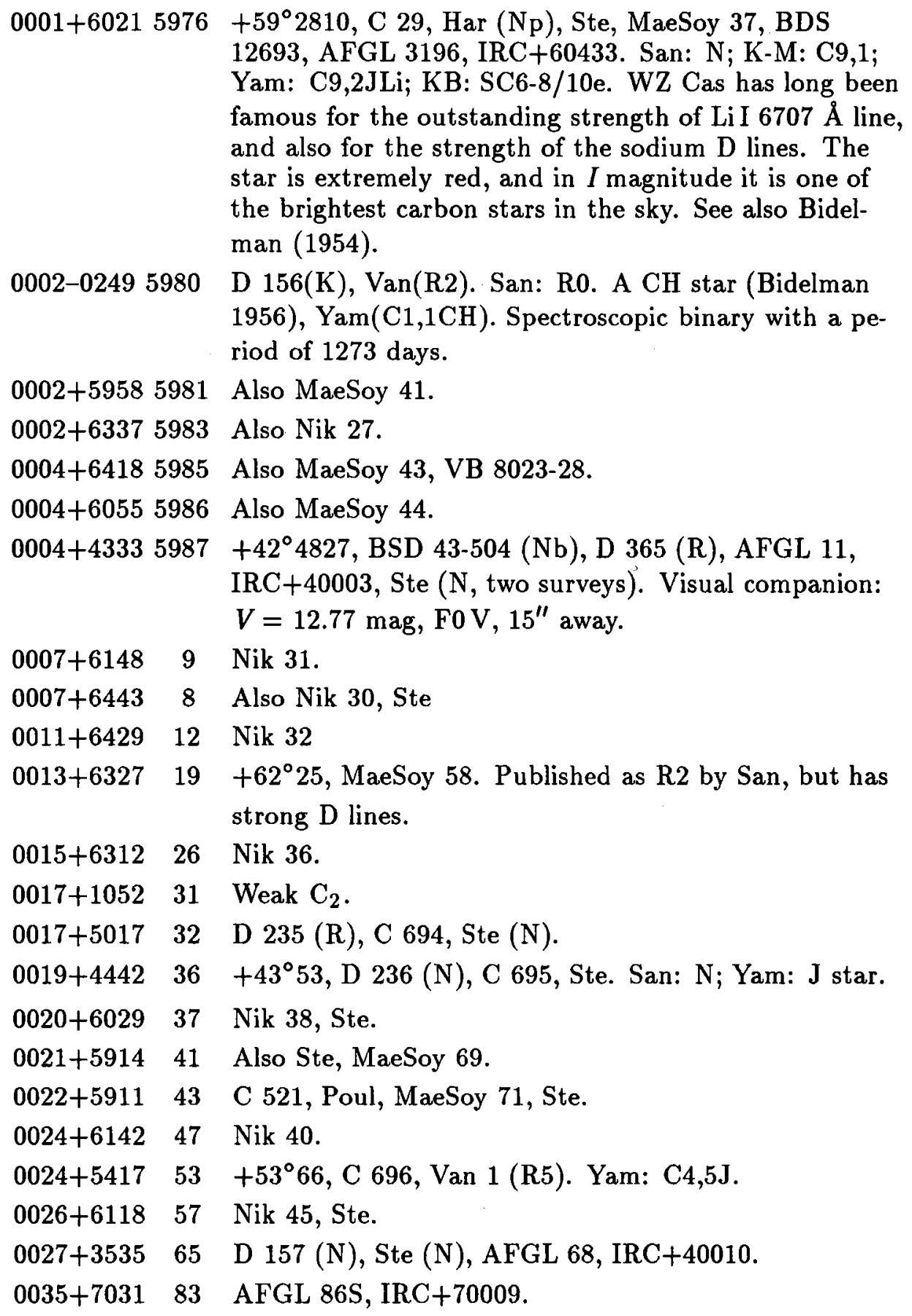


0035+6119 82 MaeSoy 91, CCCS 20.

$0035+580086$ Also MaeSoy 93, Ste.

$0035+603789$ Also Ste.

$0039+604697$ MaeSoy 97, Ste.

$0041+6128100$ Nik 53 .

0041-2619 102 Sanduleak and Philip (1968): probable early R, from color.

$0042+6328101$ Also Ste.

0046+5937 111 Also Ste.

$0050+6317121$ MaeSoy 97.

0050-1904 124 Also BidMac. Weak $\mathrm{C}_{2}$, but fairly strong red CN (Ste).

0052+6019 127 MaeSoy 110, Ste. Case plates suggest variable (Ste).

$0052+6011128$ MaeSoy 111 . Not the nearby $0052+6019$.

0053+6320 131 VB 8024-30, MaeSoy 113.

$0054+2404135+23^{\circ} 123$, Van $98(\mathrm{R})$, Ste (R). A CH star (K-M, Yam $(\mathrm{C} 1,2))$. Spectroscopic binary with a period of 755 days.

$0054+5833136+57^{\circ} 165$, AFGL 4062S, IRC+60026, Ste, MaeSoy 115 .

A CS star (Ste); cf. Keenan (1950). Yam: C7,1e.

$0054+5923134$ Large radial velocity $(-232 \mathrm{~km} / \mathrm{s})$ and proper motion $\left(0.149^{\prime \prime} / \mathrm{yг}\right)$.

$0056+6427143$ MaeSoy 118.

0059+6348 149 MaeSoy 120 .

0059+6044 150 VB 8024-42, MaeSoy 121.

0059+3920154 $\mathrm{C}_{2}$ weak.

$0100+6020157 \quad \mathrm{C}_{2}$ weak.

$0100+6216160$ Nik 67, Ste.

0102+6151 165 Har, Ste, Kurt78 8, MaeSoy 127, AFGL 146S, IRC+60034.

0104+6122 167 MaeSoy 128.

0109-2821 177 Also Slet, CCCS 55.

0110+6154 176 Also Ste.

$0111+5341180$ Yam: C4,3e. 
0112+6211 185 Also Ste, Nik77. 2' position difference with V418 Cas, which is not a carbon star.

$0112+6022187$ MaeSoy 141 .

0113+5951 188 MaeSoy 142, IRC+60041, CCCS 58.

0113+6257 189 MaeSoy 143 .

0116+5738 197 Variable, 13.2-13.8 (BC).

$0116+2546198$ D $159(\mathrm{~N})$, Ste (N), AFGL 188, IRC+30025. Yam:

$\mathrm{C} 7,2$.

0117+67136017 also OLFHHS, VKL92, AFGL 190 .

$0118+6252204$ MaeSoy 151.

$0118+5809205$ CCCS 65. In area of the open cluster NGC 103.

0126-3232 $234-33^{\circ} 525$, AFGL 215, IRC-30015, Slet ( $\left.\mathrm{N}=\mathrm{C} 5,4, \mathrm{SiC}_{2}\right)$,

Hen. San: Np; Yam: C6,4. The star has a detached gas shell.

$0128+6216233$ Also MaeSoy 167.

0129+5853 235 Also MaeSoy 168.

0133+5745 238 D 243(N), C 530, MaeSoy 170, AFGL 6161S, IRC+60056.

Yam: $\mathrm{C} 5,5$.

0134+5743 239 Probably NSV 547 (Bidun).

0140-1856 256 Also BidMac.

0142+6305 253 MaeSoy 179 .

$0142+6336255$ MaeSoy 181.

0145+5357 268 D 244 (R), Van (R5), Har (ER), CCCS 82. Yam:

$\mathrm{C} 3,5 \mathrm{JCH}$.

$0152+5658289$ In area of the open cluster Stock 4.

$0156+5915295+58^{\circ} 334 a$, Ste, MaeSoy 201 AFGL 270S, IRC+60069.

Yam: C5,4e.

$0156+5918296 \quad$ MaeSoy 202.

0158+5512 $297 \mathrm{H} \alpha$ emission star No. 658 in Publ. Vat. Obs. 1, No. 12.

0201+5750 $305 \quad$ MaeSoy 206.

$0201+5818303 \quad$ CCCS 90 .

0202+6106 306 Also Nik3 2 .

$0206+5650310$ Also Ste, two surveys. $\mathrm{H} \alpha$ emission reported by Bidel$\operatorname{man}$ (1965). 

0214+4739 324 May be $S$, not C. Very strong D lines.
0215+1214 327 D 1 (R), Van 5 (R8), Ste. A CH star (K-M, $\operatorname{Yam}(\mathrm{C} 4,4))$.

$0215+6416325$ The quoted RA is by $16 \mathrm{~s}$ greater than Kurtun and DEC is by $1^{\prime}$ smaller (Ste).

0216+6253 326 Also Nik3 13, Ste.

0221+6004 339 Also Bidelman, SoyMae91.

0225-1323 $353 \quad-14^{\circ} 450$ (BidMac).

$0226+5207350$

D $248(\mathrm{~N})$, IRC+50061. Yam: C5,5.

$0227+5859352$

Also SoyMae91.

0228-0721 357 Yam: C4:,0+.

0229-2605 361 Hanbl, IRC-30021. See also Sanford (1944).

$0232+5814365$ CCCS 105. The identification of the carbon star with the variable can be made from the charts published for each.

$0232+5951364$

Also SoyMae91. Identical with CCGCS 362.

$0233+58026034$

$0235+5608372$

Also GWSK, VKL92, AFGL 341.

0235-0926 378

A 6168 star (De). Yam: C4,5.

$0238+5545384$ $-10^{\circ} 513$, Ste (R). San: R4; Yam: C2,3J.

$0238+3910387$

D 250 (R), C 544, Har (LR), Ste. Yam: C4,5J.

$0240+6216388$ $+38^{\circ} 525$, D $160(\mathrm{R})$. Yam: C5,4.

$0244+6018398$

Also SoyMae91.

The quoted position is from an infrared Case plate. (Ste).

$0246+4707403$

BSD 22-3484 (NA), Ste (N:).

$0250+5853409$

SoyMae position is used which differs by $1^{\prime}$ in DEC from Ste.

$0252+3828417$ Probably V448 Per = NSV 966 (Bidun).

$0253+5957415$ SoyMae position is used which differs by about $1.5^{\prime}$ in DEC from Ste.

0311+5754 451 D 255 (R), C 2, Van 8 (R5), Har (ER), Ste, SoyMae91, AFGL 453, IRC+60113, CCCS 131. San: R6; Yam: $\mathrm{C} 4,5 \mathrm{~J}$.

0313+4749461 D 256 (R), Har (EN), Ste, IRC+50088, CCCS 134. Yam: C7,4. 
0312-5719 471 HR $977,-57^{\circ} 626$, Hen.

$0316+5823467$ Also SoyMae91. Dean: C5,3.

0316-2810 $478-28^{\circ} 1082$ (BidMac).

$0318+6128473$ Also SoyMae91.

0324+6035 489 Also SoyMae91.

$0326+4731496$ Also SoyMae91 AFGL 489, IRC+50096.

$0327+4410500+43^{\circ} 726$, D 260(R), Van 9. Yam: C5,3e.

$0328+2040503$ IRAS spectral tracing confirms carbon star.

$0332+5244514$ Also SoyMae91. In area of the open cluster Berk 9.

$0332+4126515$ CCCS 149.

$0339+6141531$ The SoyMae91 position is given.

$0340+5905534$ The SoyMae91 position is given; the Nik3 RA is about $7 \mathrm{~s}$ smaller and DEC is $15^{\prime \prime}$ smaller.

$0340+6105535$ The SoyMae91 position is given.

$0341+6238540+62^{\circ} 596$, D $264(\mathrm{~N}), \mathrm{C} 697, \mathrm{Har}(\mathrm{N} 6)$, Ste $(\mathrm{N})$, IRC+60124. $\mathrm{SiC}_{2}$ bands. Also San: N. The star has two detached gas shells.

$0341+5130541+51^{\circ} 762$, D $265(\mathrm{~N}), \operatorname{MSB} 3(\mathrm{~N})$, Har $(\mathrm{LN})$, Ste (N), SoyMae91.

$0345+5621553$ The SoyMae91 positon is given. NSV 1253 according to the Case paper.

$0345+5355555$ Assumed to be Nik4 13, received with no minutes of declination. The quoted position is from two accordant Ste survey plates.

$0345+4446556$ D $266(\mathrm{~N})$, Ste (N), IRC+40067.

0346+5139559 Also BidMac.

0350-4332 576 Pots 167-194 (R4), Hen 3.

$0352+3951572$ IRC+40070. Revised coordinates. The position of the variable is $2^{\prime}$ away. GJBSW identify it with IRAS $03488+3934$.

$0353+5043574$ The SoyMae91 position is given.

0356+4348 578 C 551, Har (LR or N), Ste (N).

$0358+1154588$ Also Ste (R). NSV 1426. Yam: C4,3.

$0400+5844583$ The SoyMae91 position is given; the Case RA is about $6 \mathrm{~s}$ smaller and DEC is $1^{\prime}$ larger.

0400+2332 594 Yam: C3,3J. 


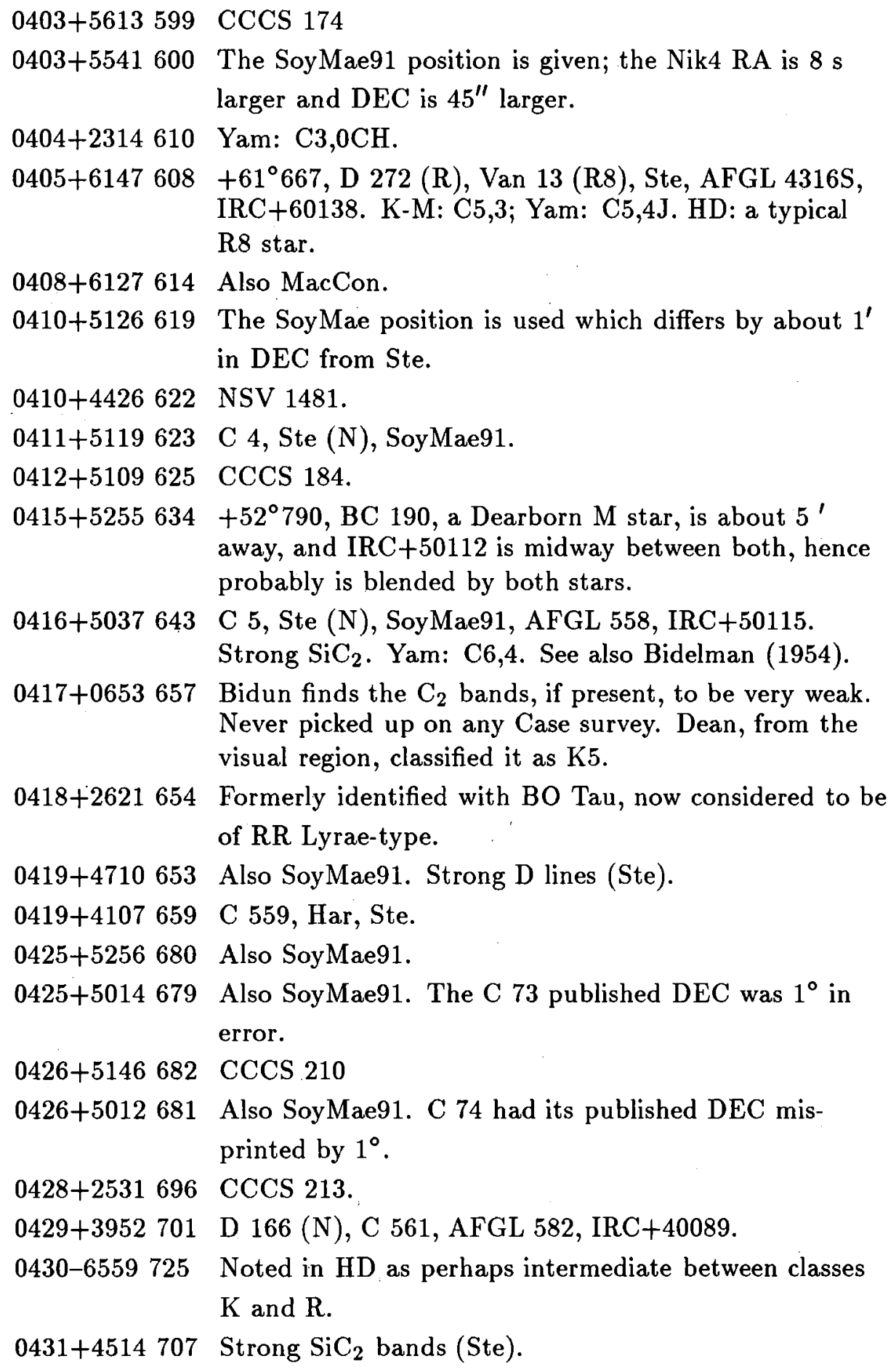

$0418+2621654$ Formerly identified with BO Tau, now considered to be of RR Lyrae-type.

0419+4710 653 Also SoyMae91. Strong D lines (Ste).

$0419+4107659$ C 559, Har, Ste.

$0425+5256680$ Also SoyMae91.

$0425+5014679$ Also SoyMae91. The C 73 published DEC was $1^{\circ}$ in error.

$0426+5146682 \operatorname{CCCS} 210$

0426+5012 681 Also SoyMae91. C 74 had its published DEC misprinted by $1^{\circ}$.

$0428+2531696$ CCCS 213.

0429+3952701 D $166(\mathrm{~N})$, C 561, AFGL 582, IRC+40089.

0430-6559 725 Noted in HD as perhaps intermediate between classes $\mathrm{K}$ and $\mathrm{R}$.

0431+4514 707 Strong $\mathrm{SiC}_{2}$ bands (Ste). 
0431+1739 714 Spectrum Ne quoted by Luyten and Merrill (1954).

0439+4137 737 C 78, Ste(N).

$0442+36066086$ May be protoplanetary nebula in the process of formation (GCVS).

0445-3131 777 Also BidMac.

0447-3612 $788-36^{\circ} 1884$, Hen 5. Yam: C6,3.

$0448+4418771$ Very weak $\mathrm{C}_{2}$.

$0449+3958780$ An LS V OB lies near the same declination and $1.4 \mathrm{~s}$ east of the carbon star. The two stars are of similar brightness on the infrared plate (Ste).

0449+3500 781 D 171 (R), C 504, Van 15 (R8), Har (ER), Ste. Yam: $\mathrm{C} 4,3 \mathrm{CH}$. Spectroscopic binary with a period of 2954 days.

0450+1547 793 D $6(\mathrm{~N})$, C 699, Ste, CCCS 253.

0451+6810 $769+67^{\circ} 350$, D $275(\mathrm{~N}), \mathrm{C} 698$, Ste, AFGL 633, IRC+70055. $\mathrm{HD}$ : a typical star of class Nb; K-M: C5,4.

$0451+4340789$ In area of the cluster NGC 1664, nonmember by proper motion.

$0451+2831794+28^{\circ} 707$, D $172(\mathrm{~N}), \operatorname{Har}(\mathrm{N})$, Ste, MaeSoy2 2, AFGL 639, IRC+30098. GCVS: $\mathrm{C} 4,2-\mathrm{C} 7,4$.

0452+3830 797 Also Ste (CS, probably weak C), AFGL 643, IRC+40101, CCCS 257. Yam: $\mathrm{C} 8,1 \mathrm{~J}$.

0453+4957803 AFGL 4385S, IRC+50130.

$0453+4041805$ The identification chart published by the Gonzaleses (1956) plus a Case plate show that this is their $\mathrm{H} \alpha$ emission star No. 306. The spectrum shows $\mathrm{SiC}_{2}$ bands (Ste). The quoted position is from two accordant plates of the Ste infrared survey.

0453+2246808 MaeSoy2 4.

0454+4954 806 D $276(\mathrm{R})$, C 329, Ste (N).

$0455+4150814$ Not the nearby $0455+4152$.

0457+3207 819 V336 Aur, MaeSoy2 7.

$0458+1654826$ Noted by Luyten (1956) as a very red star.

0459+0807 $828+7^{\circ} 768$, D 9, Ste. KB: SC6/10e; Yam: C8,1e. See also Bidelman (1954). 
0459-1448 833 HD 31996 (Pec), $-15^{\circ} 915$, Ste (N), IRC-10080. K-M: C7,4+; Yam: C7,4e. See also Bidelman (1954). HD: like VX And.

$0500+4548827$ A weak CS star, with no certain $\mathrm{C}_{2}$. Possibly an $\mathrm{S}$ star. Published as a reddened early $\mathrm{M}$ (therefore a presumed supergiant) by Nassau and Blanco (1957), their No. 116.

$0503+5037836+50^{\circ} 1112$, D $277(\mathrm{~N})$, Har $(\mathrm{N})$, AFGL 672, IRC+50135. Yam: C5,4.

$0503+4218839$ Also Fuen 3.

$0505+0110853+0^{\circ} 939, \mathrm{D} 12(\mathrm{~N})$, Ste $(\mathrm{N})$, AFGL 683, IRC +00066 .

San: N; K-M: C5,3. See also Bidelman (1954).

$0509+3900860+38^{\circ} 1035$, D $173(\mathrm{~N}), \mathrm{C} 7, \operatorname{Har}(\mathrm{N})$, Ste, Fuen 7, IRC+40115. Yam: C5,4.

0509+2954 864 MaeSoy2 11.

0509-0530 $875-5^{\circ} 1174$, Ste (N), IRC-10083. San: N.

$0510+3923866$ CCCS 293.

$0510+3949867$ CCCS 292.

$0511+2938874$ MaeSoy2 13.

0511+1450 876 Missing from the longer Dearborn list, but according Ste the Case plates definitely confirm this star in this position. Dean: C6:,9.

$0512+1453879$ Present in the early short list of the Dearborn carbon stars but not in the later longer list of red stars; but the Case plates confirm the carbon star (Ste).

0514+3447 883 Also Fuen 14, Ste.

$0515+1158893+11^{\circ} 755$, Ste (N), Epc90-6, IRC+10081.

0517+2741 896 Also Fuen 21, MaeSoy2 15.

$0517+3212899$ MaeSoy2 16, Ste.

0517-7110931 Very weak $\mathrm{C}_{2}$ (Ste, Sndlk). Within $2^{\circ}$ of a similar star, HD 36598. In the Large Magellanic Cloud field; Fehrenbach reports high velocity.

0518+3515 900 Also Fuen 23, Ste.

0519+3547 904 D 178 (N), C 8, Har (EN), Upgr68, Ste, Fuen 25, AFGL 4401S, IRC+40120. San: N. Visual companion $V=13.43 \mathrm{mag}, 24^{\prime \prime}$ away.

$0521+3723910$ Also Fuen 28 . 


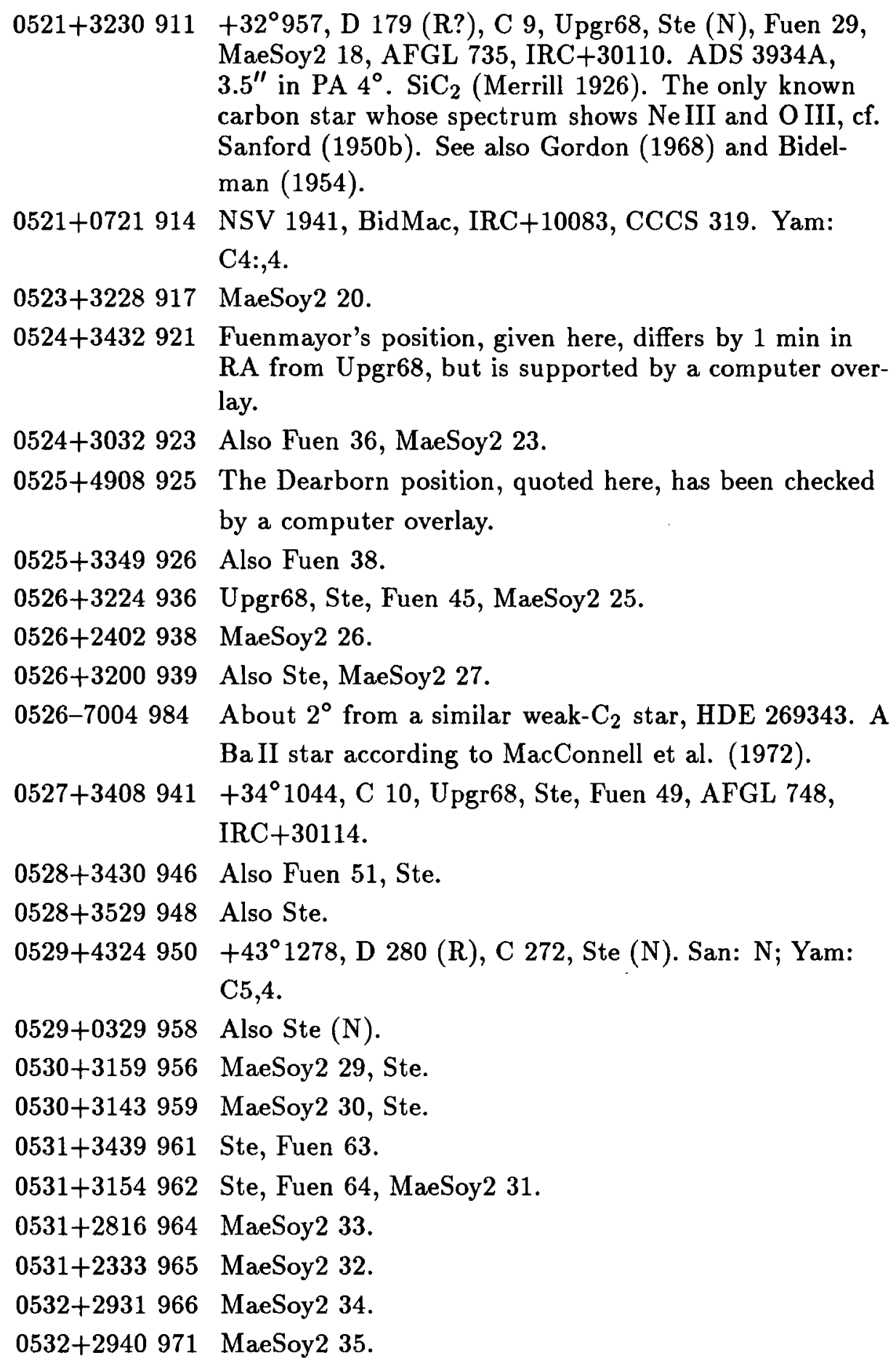




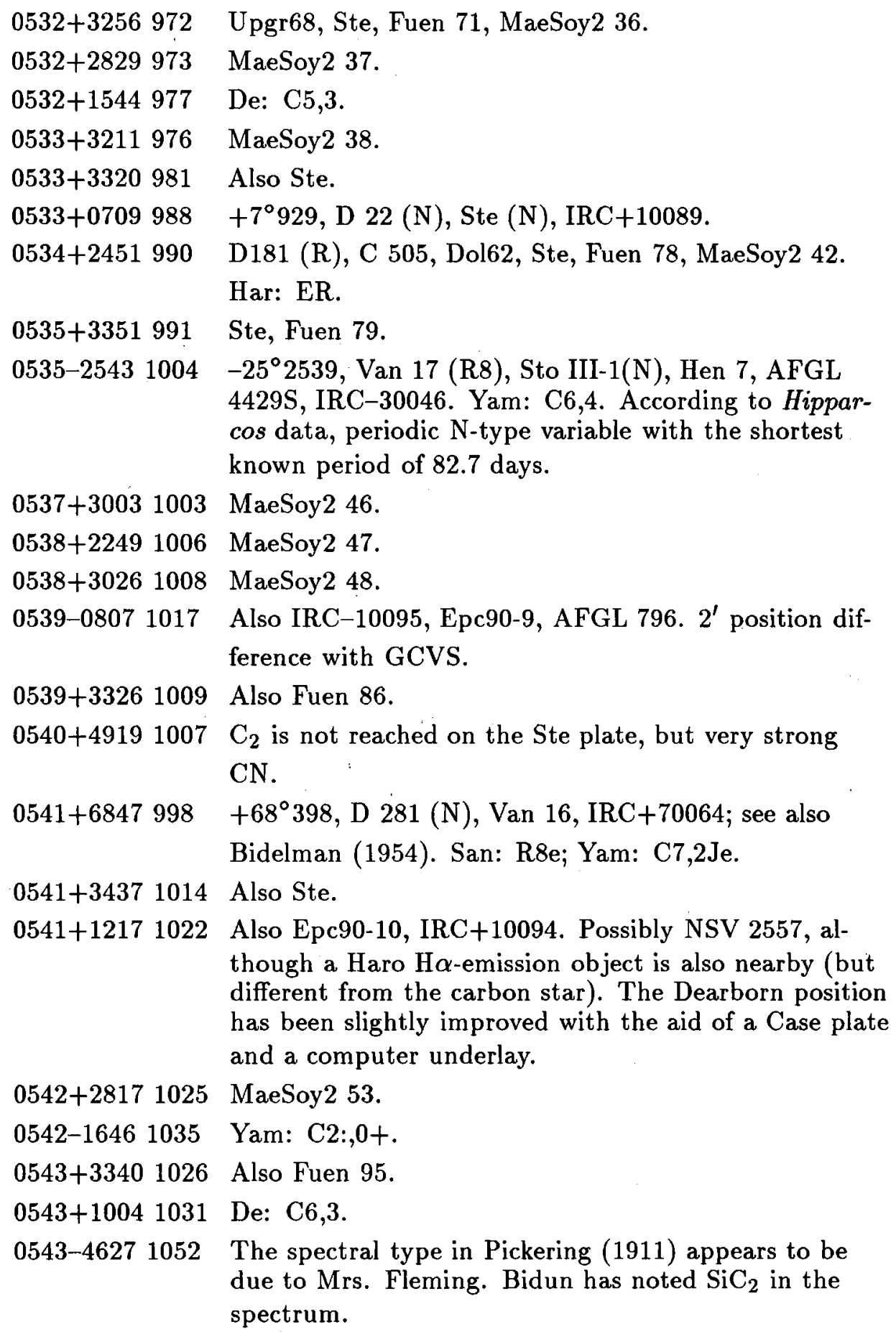
$\mathrm{CN}$.

$0541+6847998+68^{\circ} 398$, D $281(\mathrm{~N}), \operatorname{Van} 16, \mathrm{IRC}+70064$; see also Bidelman (1954). San: R8e; Yam: C7,2Je.

$0541+34371014$ Also Ste.

0541+1217 1022 Also Epc90-10, IRC+10094. Possibly NSV 2557, although a Haro $\mathrm{H} \alpha$-emission object is also nearby (but different from the carbon star). The Dearborn position has been slightly improved with the aid of a Case plate and a computer underlay.

0542+2817 1025 MaeSoy2 53.

0542-1646 1035 Yam: C2:,0+.

0543+3340 1026 Also Fuen 95.

0543+1004 1031 De: C6,3.

0543-4627 1052 The spectral type in Pickering (1911) appears to be due to Mrs. Fleming. Bidun has noted $\mathrm{SiC}_{2}$ in the spectrum. 
$0545+24251038+24^{\circ} 943$, D $183(\mathrm{~N})$, C 11 , Van (C:), Ste (N:), Har (N), Fuen 100, MaeSoy2 57, AFGL 390, IRC+20120. Spectrum composite, see Gordon (1968). Sanford: N. Spectroscopic binary.

$0545+25291041$ MaeSoy2 58.

$0545+20411042+20^{\circ} 1083$, D $30(N), C 507$, Van (N), Ste, Har (EN).

San: N; Bid: C7,4e; Yam: C6,4.

$0545+15301043+15^{\circ} 921$, MSB $13(\mathrm{~N})$, D $31(\mathrm{R})$, C 508, Ste (N). Yam:C5,4.

0545+1216 1044 AFGL 393, IRC+20121. Very strong D lines.

$0546+25381049$ MaeSoy2 61.

$0547+28511053$ Also Fuen 105, MaeSoy2 63.

0547+3034 1058 MaeSoy2 65.

$0548+44541057$ MSB 14, D $285(\mathrm{~N})$, IRC+40141.

$0548+30371061+30^{\circ} 1014, \mathrm{D} 184(\mathrm{~N}), \mathrm{C} 273, \mathrm{Har}(\mathrm{LR}$ pec), Ste (R-N), Fuen 110, MaeSoy2 67. San: N.

$0548+26131067$ MaeSoy2 69.

0549+3202 1068 MaeSoy2 70.

0549+1904 6106 Spectral class from GCVS.

$0550+25171078$ MaeSoy2 73.

$0550+22161079$ MaeSoy2 74. In area of the open cluster Berk 72.

0550-0203 1086 Letter from Boeshaar to Bidelman: $V-I=3.7 \mathrm{mag}$.

$0552+32111088$ Ste, Fuen 122, MaeSoy2 76.

0552+2954 1089 NSV 2684, Fuen 123, MaeSoy2 77.

$0552+29501090$ MaeSoy2 78.

$0553+30381092$ MaeSoy2 79.

0554+2838 1096 Also MaeSoy2 84.

$0554+01051101$ Also BidMac.

0554+3011 1098 MaeSoy2 86.

0554-8403 1212 Mayall (1951) identifies with CPD $-84^{\circ} 86$.

$0555+31271099$ Fuen 133, MaeSoy2 87. BN Aur is about $5^{\prime}$ distant from this $\mathrm{C}$ star.

0555+3402 1100 Also Fuen 134 .

$0555+32021103$ Also MaeSoy2 89. CS type in the red spectral region, but enough infrared $\mathrm{CN}$ to be called carbon by Nassau-Blanco, Fuen, MaeSoy, and Ste. AY Aur 
is nearby and seen separately (on the red and infrared objective prism plates) as an $\mathrm{M}$ star.

0556+3352 1104 Har: LR.

0556+3006 1109 NSV 2735, MaeSoy2 93, Ste.

0557+3351 1110 Yam: C2,3.

$0558+28281118$ Fuen 144, MaeSoy2 98.

0559+2447 1126 MaeSoy2 100.

0559-1052 6109 IRC-10104.

0600+3045 1130 Fuen 150, MaeSoy2 103.

0601+3940 1128 D $185(\mathrm{~N})$, Ste, IRC+40151. Bid: C8e; Yam: C7,1e.

$0601+49321129$ Very weak $\mathrm{C}_{2}$.

0601+2105 1133 Also Fuen 153.

0601+2927 1136 Ste, Fuen 156, MaeSoy2 105.

$0601+06381145$ A weak C, like R CMi, according to A. E. Greene's unpublished $\mathrm{Ph} . \mathrm{D}$. thesis (Ohio State).

$0602+29311142$ Ste, Fuen 159, MaeSoy2 107.

0602+2938 1144 Fuen 160, MaeSoy2 108.

0603+2559 1149 Also MaeSoy2 110.

0603+0725 6113 Also Fou92, Gug93, GJBSW, AFGL 865, OLFHHS, VKL82.

0604+4717 1148 Also Ste (N).

0604+2652 1151 Fuen 164, MaeSoy2 111.

0604+1010 1158 Silicate CSE. Water maser emission at $22 \mathrm{GHz}$ (Engels 1994).

0607-0435 1168 Weak $\mathrm{C}_{2}$.

0608+2242 1169 Fuen 170, MaeSoy2 116.

0609+4054 1167 De: C6,2.

0609+3001 1172 MaeSoy2 117.

$0610+32031173$ MaeSoy2 118.

$0610+30371177$ MaeSoy2 119.

$0610+26001179+26^{\circ} 1117$, D $189(\mathrm{~N})$, C 276, Har (N), Ste, Fuen 183, MaeSoy2 120, IRC+30143. San: N.

$0610+08081183$ Very red.

0611+2414 1185 MaeSoy2 122. 
$0612+27391189$ MaeSoy2 123.

0617+0831 1222 Also C 12, SoyMae93, Epc90-16, AFGL 910, IRC+10113.

0617-0015 $1226-0^{\circ} 1246$, D $39(\mathrm{~N})$, MSB 16, May51 (N), BidMac, CCCS 482.

0618+2008 1227 Also Fuen 201.

0618-0107 1230 CCCS 486.

0619+0519 1236 De: C6,4e.

$0621+07201246$ D 41 (N), C 131, Epc-18, AFGL 920, IRC+10118. San: N; Yam: C4,3e.

$0623+08291254$ IRC+10119.

0623-0727 1256 Also BidMac. CCCS 504.

0623-1029 1258 The quoted position is from a single Tololo plate, which differs from Mayall's position by $0.4 \mathrm{~min}$ in RA and $3^{\prime}$ in DEC (Ste).

0623-2703 1263 Sto III-2 (N), Hen 10, BidMac ( $\left.\mathrm{SiC}_{2}\right)$, HanBl, IRC30060, CCCS 507. N + A5 V, according to Richer (1972). Spectroscopic binary.

$0624+47421244+47^{\circ} 1291 \mathrm{a}, \mathrm{D} 289(\mathrm{R})$, Ste (N). Yam: C6,2e. There is no doubt, the $\mathrm{N}$ star was incorrectly identified by the IDS catalog with ADS $4988\left(+47^{\circ} 1291\right)$, which is actually half a degree distant from $+47^{\circ} 1291 \mathrm{a}$. See also Bidelman (1954).

$0624+25011251+25^{\circ} 1250$, D $190(\mathrm{~N}), \operatorname{Har}(\mathrm{N})$, Ste, IRC+30150. Yam: $\mathrm{C} 5,3 \mathrm{e}$.

0624+0826 1257 Also SoyMae93.

$0625+14431264+14^{\circ} 1283$, BL Ori, D 46, C 277, BSD 74-1087(NA), Fuen 209, AFGL 934, IRC+10121. San: N.

$0625+20571268$ NSV 2957. The Case list mentions an emission-like feature at $850 \mathrm{~nm}$.

0625-0932 6149 Also GJ93, GWSK.

0626+1904 1269 MSB 21, D 47 (N), C 387, AFGL 937, IRC+20145, $\operatorname{Ste}\left(\mathrm{N}, \mathrm{SiC}_{2}\right)$. Yam: $\mathrm{C} 5,4$.

0627+2236 1272 Perr59 misidentify it with HDE 257290 (K0). The quoted position is from Ste red survey plate.

0630+1228 1290 D $48(\mathrm{R})$, C 278, Ste (N), SoyMae93. Yam: C4,5J. 
$0631+16061292$ Ste (N:), Fuen 215.

0631-0531 1293 A CS star, showing no $\mathrm{C}_{2}$ on a $600 \AA / \mathrm{mm}$ objective prism plate of the blue region (Ste).

$0634+42301300+42^{\circ} 1571$, D $290(\mathrm{R})$, Ste (N), IRC+40157. Yam: $\mathrm{C} 4,5$.

$0634+23231305$ Also Ste (N).

0634+1604 1309 D $51(\mathrm{~N})$, C 279, Ste (N), AFGL 959, IRC+201521. K-M: C8,3; Yam: C8,3e. Strong D lines:

0636+3826 1316 HR 2405, $+38^{\circ} 1539$, D 192(N), Ste(N), AFGL 966, IRC+40158. Yam: C6,4. Sanford (1926) noted variable strength of the (much later identified) $\mathrm{SiC}_{2}$ bands.

$0636+06041319$ Very red.

0636+0325 6167 AFGL 971.

0637-1205 $1332 \quad \mathrm{SiC}_{2}$ bands.

$0638+0859616914^{\prime \prime}$ from RZ Mon (M6).

0638-0425 1336 Near, but different from, V397 Mon.

$0639+22361337$ D 52, Ste.

0639+0519 1346 Also SoyMae93.

0640+1846 1349 Also Ste (N:). Yam: C3:,3.

0640+1234 1352 Also SoyMae93.

0641-2009 1356 Also BidMac.

0641-2216 1357 HanBl (C), AFGL 4521S, IRC-20101.

$0642+31271355+31^{\circ} 1388, \mathrm{D} 193(\mathrm{R}), \mathrm{C} 700, \operatorname{Van} 21(\mathrm{R} 8)$, Ste(N), IRC+30163.

0643-0845 1373 Also MacCon. CCCS 568.

0643-0532 1375 MacCon 33, Ste.

0644+0319 $1378+3^{\circ} 1381$, D $56(\mathrm{~N})$, C 13, MacCon, HanBl, SoyMae93, IRC+00 125. San: N. Very red.

0644-7239 1425 CPD $-72^{\circ} 523=\mathrm{CD}-72^{\circ} 353$.

$0645+09401383$ Not C 396, though near. Also Ste, SoyMae93.

0646-1252 1392 Strong D lines.

$0647+07121398$ SoyMae93 position indicated, the Case RA is by $7 \mathrm{~s}$ too large.

0647+0013 1400 MacCon 43. 
0647+0040 1401 D $60(\mathrm{~N}), \mathrm{C}$ 140, MacCon 44, HanBl, AFGL 4531S, IRC+00125. GCVS: C4-5,4-5.

0649+0404 1415 MacCon 54, AFGL 1017, IRC+00131. Near, but different from $+4^{\circ} 1462$.

$0649+02581417$ MacCon 55, SoyMae93, IRC+00131.

0650-4153 1446 Hen 12, BidMac.

$0651+05131431$ MacCon 64, SoyMae93.

0651+0511 1436 MacCon 67, Gug93, SoyMae93.

0651-0201 1440 Also SoyMae93.

$0652+26331433 \quad \mathrm{C}_{2}$ not clearly visible.

0652-0708 1444 Also Ste.

0652-1209 1450 Ste, MacCon, Epc90-58, AFGL 4538S.

0653+0418 1448 Weak $\mathrm{C}_{2}$.

0653-0434 $1453-4^{\circ} 1708$, D $64(\mathrm{R})$, C 14, Van 22 (R8), Ste (N:), MacCon, IRC+00136.

0653-3146 1460 Hen 13, BidMac, CCCS 612.

0654+1050 1461 Fairly strong D lines.

0654+0900 1458 Also SoyMae93.

0654-0801 1464 C 409, MacCon; see also Bidelman (1954). Yam: $\mathrm{C} 6,3 \mathrm{e}$.

0654-4221 1478 HR 2591, $-42^{\circ} 2818$, Sto III-16(N), Hen 14. Yam: C7,2.

0655+0622 1465 Also C 409, MacCon, AFGL 1038, IRC+10144. Yam: C6,3e.

0656+1057 1473 Also SoyMae93.

0656+0704 1474 Also SoyMae93.

0656+0344 1476 MacCon 82, Ste.

$0657+41071466+41^{\circ} 1556$, D 194 (R), Ste (R). Yam: C4,5J.

$0657+06191480$ D $69(\mathrm{~N})$, C 412, MacCon, SoyMae93. Coord. from the last source are given, the position indicated by Ste is about $2.5^{\prime}$ in error.

0658+0610 $1489+6^{\circ} 1462$, D 71(R), C 280, MacCon, SoyMae93, AFGL 1045, IRC+10146. $\mathrm{SiC}_{2}$ noted by Sanford (1926).

$\mathrm{K}-\mathrm{M}: \mathrm{C} 5,4$.

0700+0511 1501 Also MacCon, SoyMae93. De: C5,4. 
0700-0652 1503 Never found on any Case plate, despite special searching for it (Ste).

0701-0315 $1507 \quad-3^{\circ} 1685$, D 77 (R), Van 23 (R5), Ste, MacCon 93, AFGL 1053, IRC+00141. K-M: C4,4; Yam: C4,5J.

0701-0531 1508 Also Ste.

$0702+08501511$ SoyMae93 position is indicated.

0703-4443 1539 Also BidMac. A brighter star is SW (Sto).

0704-1606 1532 NSV 3361, Ste.

0705+6811 1500 HD: Resembles VX And. Yam: C4:,4J.

0705-7300 1599 Flem12 (Md?), Hen 16. Called a carbon star by Bidelman (1954) purely on the basis of its color index; confirmed by The (1968).

0706-0733 1549 C 15, Ste, HanBl, AFGL 1070, IRC-10149, MacCon. $\mathrm{K}-\mathrm{M}$ : C6,5. $\mathrm{SiC}_{2}$ bands noted by Sanford (1926).

They are very strong, comparable to V.Aql.

0708+1001 $1561+10^{\circ} 1428$, D 78 (Sp), C 701, IRC+10154. Yam:

C7,1J. A CS star. See also Bidelman (1954) and Stephenson (1965).

0708-0017 1568 CCCS 677, Ste.

0708-1155 $1565-11^{\circ} 1805$, C 16, Van 24 (R8), Ste (N), MacCon, AFGL 1075, IRC-10152. HD 54306, a B star $V=8.76 \mathrm{mag}$, $158^{\prime \prime}$ away; see Gordon (1968).

0708-1328 1567 MacCon 122.

0709+0402 1569 Also SoyMae93.

0711-0527 1587 MacCon 138.

0712-2017 1601 AFGL 1055.

0712+0047 1593 Yam: C2,3J.

0712+1436 $1595+14^{\circ} 1598$, BSD 75-459, D $81(\mathrm{~N}), \mathrm{C} 702$, AFGL 4574S, IRC+10156, Ste (close to CS). Bid: C8e; De: C9,1e; Yam: C7,2e. Outstanding D lines on a Case plate.

0713-1941 1612 Sndlk (N), MacCon, Ste.

0714-1723 1615 NSV 3482, Sndlk (N:), MacCon 154, Ste. Yam: C5:,3:. 0714-3932 1629 Also Hen 17. 
0715+0502 1616 D $82(\mathrm{~N})$, Ste65 (N, strong $\left.\mathrm{SiC}_{2}\right)$, SoyMae93, AFGL 1092, IRC+10158, CCCS 695. Yam: C5,5. IDS F star $60^{\prime \prime}$ is in PA $200^{\circ}$ from the carbon star. In outskirts of the open cluster NGC 2353.

0715-1251 1621 Very strong D lines noted on a Case plate by Ste.

0715-1729 1624 Also MacCon 159, Ste.

0717-2900 1643 Has M-type visual companion, see ApJ, 172, L115, 1972.

0717-3620 1650 In area of the open cluster Col. 135.

0718-0747 1646 MacCon 171, Ste.

0719-4258 1660 Hen 18, BidMac, NSV 3537.

$0720+24591653+25^{\circ} 1643$, D $196(R)$, Ste (R-N), IRC+30182. Yam:

C5,4J. Silicate CSE.

0720-20201659 CCCS 720. SiC 2 (Sandlk). De: C4+,3.

0720-0733 1661 MacCon 176, Ste.

0720-1912 1662 Also MacCon.

0721+6940 1622 D 292 (R). K-M: C0,1-C3,1; Yam: C0,1. See note in GCVS.

0721-1445 1668 Weak $\mathrm{C}_{2}$, but very strong $\mathrm{CH}$ and $\mathrm{CN}$ in the blue spectral region.

0721-2353 1671 Westerlund's position was in error by 1 min in RA, according to MacConnell.

0721-2027 1673 De: C5,4. Also Ste.

0722-1038 1682 Silicate CSE. Water maser emission at $22 \mathrm{GHz}$ (Engels 1994).

0722-2206 1679 Probably, this is MacCon 180 which has RA by $1 \mathrm{~min}$ smaller.

0723-0412 $1686-3^{\circ} 1873$, Van 26, MacCon, CCCS 739. San: N; Yam: C5,4; De: $\mathrm{C} 4+, 3$.

0723-2258 $1695-22^{\circ} 1850$, B-M 6, W 1-6, Ste (R8), Hen 20, MacCon. De: C4,3J; Yam: C5,5J.

0724-0437 1698 Silicate CSE. Water maser emission at $22 \mathrm{GHz}$ (Engels 1994).

0724-1252 1696 Also GJ93.

0725-0308 $1704-2^{\circ} 2101$, MacCon 196, CCCS 753. De: C6,2.

0725-2659 1716 Very strong D lines. 
0726+2154 1702 D 85 (R), Van 27 (R5), Ste (R). San: R4; De:

C3,2; Yam: C3,3J. HD likens this star to HD 58364

$(0726+2153$ of the present catalog), which according to Bidelman has the same proper motion.

0726+2153 1703 D 86(R), Van 28(R5), Ste(R). Yam: C3,3J De: C4,3p.

Common proper motion with $0726+2154$.

0726-0402 1708 Very strong D lines.

$0726+02251710$ Published as $+2^{\circ} 1677$ in the Dearborn lists, but the Case plates show that the BD star is of early type. (Ste).

0726-1945 1715 Also Ste. Extremely red, see IAU Circ. 2118, 1968. Very strong red CN. De: C5,4.

0727-0308 1723 Also Ste65(N:). De: C4,2.

0729-1927 1732 AFGL 1131, IRC-20131, MacCon, NSV 3610, Ste.

0730-2008 1740 Also Ste.

$0731+24301737+24^{\circ} 1686$, D $197(\mathrm{~N})$, Van 29 (R8), Ste (N:). San: R6. Greene and Wing (1971) mention hydrogen emission, plus an ultraviolet continuum like $\mathrm{CH}$ Cyg.

0731+0411 1738 Strong $\mathrm{SiC}_{2}$ bands. De: C3,2.

0731-1013 1743 The early Case papers suggest that this star is KQ Mon but the identification chart, as well as the present coordinates, based on accordant plates, indicate that it is not. (Ste).

$0732+05491749$ SoyMae93 position is given, the Ste85 DEC is by $1.3^{\prime}$ larger.

0732-1820 1760 Also Ste.

0733-2226 1774 Also W 3-3. Westerlund's DEC was in error by $1^{\circ}$.

0734-2524 1777 Not the rather nearby CT Pup.

0734-2237 1780 W 3-4 was originally published with its DEC $1^{\circ}$ in error. Ste confirmed the position given here via two independent surveys, neither of which finds anything in the position of the nearby W 3-5.

$0735+51321756$ Also Ste(R-N). CCCS 795. Bidun has noted strong $\mathrm{SiC}_{2}$ bands, hence the star is of class N. De: C5,4; Yam: C4,5J.

0735-1506 1784 Also MacCon.

0735-2856 1786 Also Hen 22. 
0735-2336 1790 B-M 21, Ste, W 5-1, MacCon. San: N. Quite red, but a Tololo plate shows strong violet $\mathrm{CN}$, which makes it class $R$ in Shane's system. (Ste).

$0736+02041787+2^{\circ} 1715$, D $90(\mathrm{R})$, Van 30 (R8), Ste (N), HanBl, IRC+00158. K-M: C5,5; De: C5,4; Yam: C5,5.

0737-2058 1804 Also W 3-7, Ste. W 3-7 was published with a $1^{\circ}$ declination error.

0738-2341 1809 Also MacCon.

0738-2158 1814 Westerlund's position is $1^{\circ}$ declination error. There is no star in the original W 3-9 position in the Ste infrared survey, which confirms MacConnell's position.

0738-3524 1819 Wray 21, Hen 23.

0739-2224 1817 Also W 3-10. The W DEC is $1^{\circ}$ in error.

0739-2742 1822 Hen 24, HanBl, AFGL 4621S, IRC-30077. De: C5,3.

0739-4028 1825 Strong D lines.

0740-2243 1826 Also W 3-11, where the published DEC is $1^{\circ}$ in error.

0741-1218 1832 BidMac, MacCon.

0741-2110 1833 Also MacCon, Ste. In area of the open cluster Pis 3.

0741-2414 1841 Also Ste.

0741-2311 1843 Also MacCon.

0742-2700 1846 Strong D lines.

0742-4412 1853 Also Hen 26. Strong D lines.

0743-2041 1850 Also Ste.

0744-2221 1855 Also MacCon.

0744-2824 1859 In area of the open cluster Haffner 14, $2^{\prime}$ away from its center.

0745-2239 1860 BidMac, MacCon.

0745-1950 1863 Also Ste.

0746-2214 1867 Also MacCon. The published B-M 35 position was apparently precessed from the wrong equinox.

0746-1500 1869 Also MacCon.

0746-2644 1871 Also Ste(R).

0747-1646 $1873 \quad \mathrm{SiC}_{2}$ bands.

0747-2034 1874 Also Ste.

0747-1157 1877 HanBl, IRC-10177, MacCon. 
$0748+05231881+5^{\circ} 1797$, D $91(\mathrm{~N})$, Van $31(\mathrm{R} 8)$, Ste (N). Yam: C7,2.

0748-4843 1904 Wray 29, Han 28, BidMac.

0749-1715 1893 In area of the open cluster Berk 40, $2^{\prime}$ away from its center.

0750-0052 1891 Also Ste(R-N), CCCS 906. De: C5,5J; Yam: C5,5J. Has an A6 III-V: visual companion, see Gordon (1968), $4^{\prime \prime}$ away.

0750-1333 1899 Also MacCon.

0750-2124 1900 Westerlund's published RA is too small by $1 \mathrm{~min}$ (MacCon).

0750-3847 1910 Also Hen 29, CCCS 921. Strong D lines. In area of the open cluster NGC 2477.

0750-4752 1912 Extremely red.

0751-2113 1911 In the blue region the $\mathrm{C}_{2}$ is nearly as weak as in the stars called $\mathrm{K} 5 \mathrm{R}$ in the HD catalog.

0751-0237 1907 Also Lo74, AFGL 1189, IRC+00162, V633 Mon. Hanson and Blanco classify it as $\mathrm{S}+\mathrm{C}$ ?

0751-4629 1927 NSV 3779. Strong D. lines.

0752-3243 1928 Quite red.

0752-4402 1929 In area of the open cluster NGC 2477.

0752-3828 1930 NSV 3788. Lies within the apparent boundaries of NGC 2477, a large open cluster.

0753-0949 1922 MacCon: may be CH-like star.

0753-3054 1935 Also MacCon.

0753-3032 1936 Also MacCon.

$0754+72131876$ Called carbon from the redness and CN strength on a red-region Case plate, underexposed in the region of the $\mathrm{C}_{2}$ band (Ste).

0754-3220 1941 Ste, Hen 33.

0754-4354 1950 Sndlk, Hen 35.

0755-0311 1944 De: C6-,4.

0755-2939 1949 Wray 36, Sndlk, W 14-6, Hen 34, MacCon, CCCS 956.

0755-2928 1951 Also MacCon, IRC-30106.

0756-4958 1968 Sto III-21 (R), Hen 36. CCCS 967. 


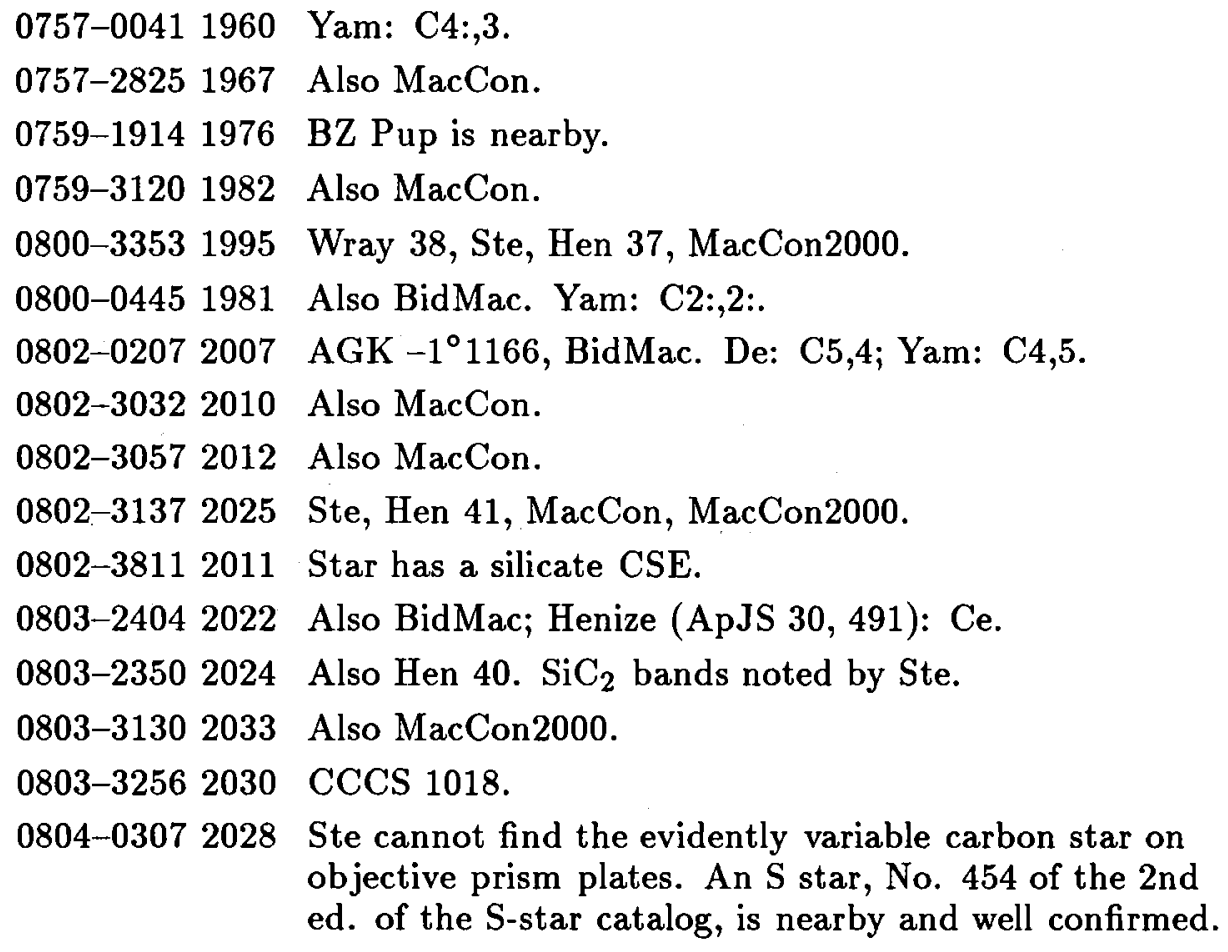
objective prism plates. An S star, No. 454 of the 2nd ed. of the S-star catalog, is nearby and well confirmed.

0804-4051 2042 Also MacCon2000.

0805-3346 2044 Also VB 29067-6, MacCon2000.

0805-3209 2047 CCCS 1033. B-M note an M2 star 25" SE of the carbon star.

0805-3012 2048 W 18-4, Sndlk, MacCon.

0805-3846 $2051-38^{\circ} 4049$, W 19-11, Sndlk, Hen 44. Strong D lines noted on a Tololo plate, but independently classified $\mathrm{R}$ on three blue-region Tololo plates (Ste). War, Yam: C6,2.

0805-3441 2055 Also VB 29067-7.

0807-2947 2063 Hen 45, MacCon. Near the open cluster NGC 2533. 0807-2254 $2064-22^{\circ} 2160$, Wray 48, Ste(N), Hen 46, AFGL 4666S, IRC-20156. MacCon2000. Yam: C5,4.

0807-1947 2065 Also BidMac, NSV 3910.

0807-3710 2067 The F component completely dominates the blue spectral region (Ste).

0807-3341 2069 Also VB 29067-10. 


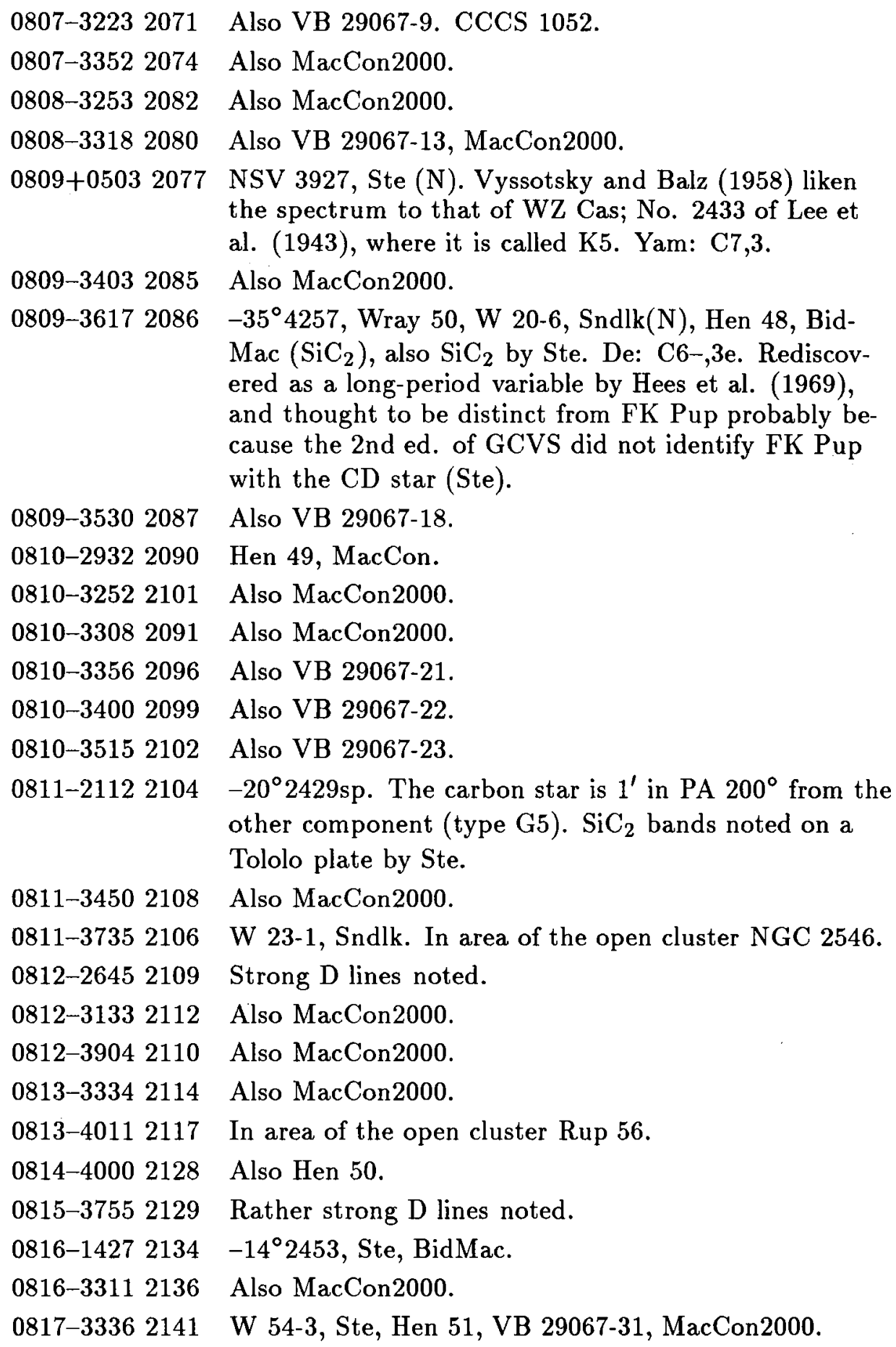
the spectrum to that of WZ Cas; No. 2433 of Lee et al. (1943), where it is called K5. Yam: C7,3.

0809-3403 2085 Also MacCon2000.

0809-3617 $2086-35^{\circ} 4257$, Wray 50, W 20-6, Sndlk(N), Hen 48, Bid$\mathrm{Mac}\left(\mathrm{SiC}_{2}\right)$, also $\mathrm{SiC}_{2}$ by Ste. De: C6-,3e. Rediscovered as a long-period variable by Hees et al. (1969), and thought to be distinct from FK Pup probably because the 2nd ed. of GCVS did not identify FK Pup with the CD star (Ste).

0809-3530 2087 Also VB 29067-18.

0810-2932 2090 Hen 49, MacCon.

0810-3252 2101 Also MacCon2000.

0810-3308 2091 Also MacCon2000.

0810-3356 2096 Also VB 29067-21.

0810-3400 2099 Also VB 29067-22.

0810-3515 2102 Also VB 29067-23.

0811-2112 $2104-20^{\circ} 2429 \mathrm{sp}$. The carbon star is $1^{\prime}$ in PA $200^{\circ}$ from the other component (type G5). $\mathrm{SiC}_{2}$ bands noted on a Tololo plate by Ste.

0811-3450 2108 Also MacCon2000.

0811-3735 2106 W 23-1, Sndlk. In area of the open cluster NGC 2546.

0812-2645 2109 Strong D lines noted.

0812-3133 2112 Also MacCon2000.

0812-3904 2110 Also MacCon2000.

0813-3334 2114 Also MacCon2000.

0813-4011 2117 In area of the open cluster Rup 56.

0814-4000 2128 Also Hen 50.

0815-3755 2129 Rather strong D lines noted.

0816-1427 $2134-14^{\circ} 2453$, Ste, BidMac.

0816-3311 2136 Also MacCon2000.

0817-3336 2141 W 54-3, Ste, Hen 51, VB 29067-31, MacCon2000. 
0818-3702 2147 In outskirts of the open cluster Pis 1.

0818-2554 2148 Ste, Hen 53.

0818-3450 2152 Also VB 29067-33, MacCon2000.

0818-6116 2166 Also BidMac.

0819-1815 2153 Van 33 (R5), Ste (Re). CCCS 1126. San: R7; Yam: C5,5J.

0819-3901 2164 W 26-4, Sndlk.

$0820+02452150+3^{\circ} 1958$, D (N), Ste (N), HanBl, AFGL 1243, IRC+00172. De: C5,4; Yam: C6,4e. See also Bidelman (1954).

0820+05112156 Also Ste (R-N). CCCS 1130. Yam: C3,5.

0820-3227 2169 Also MacCon2000.

0821-3629 2172 Blanco's position is given. Westerlund's position is 1 $\min \mathrm{W}$ and $2.6^{\prime} \mathrm{S}$.

0821-3607 2175 W 23-12, Sndlk, Hen 54, VB 29067-39. In outskirts of the open cluster NGC 2579.

0822+3331 2165 K-M: C6,3; Yam: C7,1e. See also Bidelman (1954). Ref 191 in that bibliography for this star should be Ref 185.

0822-1554 $2177 \mathrm{HanBl,} \mathrm{IRC-20165.}$

0822-2857 2180 Also MacCon2000. Strong $\mathrm{SiC}_{2}$.

0823-3736 2185 W 23-13, Sndlk. The published declinations for this star range through nearly $5^{\prime}$, rather uniformily. The one given here, from a single Tololo plate, is supported by a computer underlay. (Ste).

0823-3704 2190 W 23-14, Sndlk.

0824-3345 2199 Also VB 29067-44.

0825-3416 2201 Also BidMac.

0825-3843 2200 Also MacCon2000.

0825-3847 2207 Not found on a deep red Tololo plate, perhaps identical with No. 0825-3843 (Ste).

0826-2551 2206 Sndlk, Hen 58, BidMac.

0826-3827 2210 Very red.

$0827+28446293$ Also Tot98. Double-line spectroscopic binary: dwarf carbon star + hot white dwarf (Heber et al., A\&A, 267, L31, 1993). 
0828-2715 2219 Wray 79, Sndlk (N), Hen 60, CCCS 1184.

0828-3651 2222 Also Hen 61. De: C6-,4.

0829-2920 2226 Also BidMac.

0830-2742 2228 Sto IV-12, Sndlk, Hen 63.

0832-3010 2235 Also BidMac.

0832-4408 2243 Also MacCon2000.

0833-2956 2247 Sndlk, Hen 64.

0833-4454 2251 Also Hen 66.

0834-3055 2255 Also MacCon2000.

0835-4346 2257 Also MacCon2000.

0835-5923 2266 Stock and Wroblewski note a brighter star SW.

0836-3534 2263 Henize (1976): Se or Ce. The spectrum does look Slike on a Tololo red-region plate, but shows the 5635 $\mathrm{C}_{2}$ band head. (Ste).

0836-52342272 Sto III-23, Sndlk (N:), Hen 69, BidMac, CCCS 1232. 0838-4656 2288 Also Hen 70.

0839-4633 2292 W 31-15, Sandlk. The published coordinates of B-M 86 , here identified with B-M 87 , are $8^{h} 35.9^{m},-46^{\circ} 12^{\prime}$. If it is not B-M 87 then either its coordinates are in error or it is a variable, since Westerlund did not find it. It is also not visible on a Tololo red plate on which B-M 87, published as 0.4 infrared magnitude brighter, is well exposed (Ste).

0838-5523 2293 The Ste classification as carbon is from one blue and one red Tololo plate, independently.

0839-4756 2300 Also Sndlk.

0841+0726 2301 De: C4,3; Yam: C3,4J.

0842-4712 2315 W 31-20, Sndlk, Hen 73.

0843-2536 2317 Also IRC-30132, HanBl, AFGL 5232.

0844-3255 2323 Strong $D$ lines and weak $4737 \AA \mathrm{C}_{2}$ (Ste).

0845-2812 $2326-27^{\circ} 5879$, Sto IV-16, Hen 76, IRC-30133.

0845-4718 2332 Also Sndlk, NSV 4246. Strong D lines noted on a Tololo plate by Ste, as well as $\mathrm{H} \alpha$ emission, the latter also by Henize (1976). 
0846-2943 2331 Van 37, Sto IV-22(R), Hen 77, IRC-30134, CCCS 1290. San: R8; War: C6,3; Yam C5,5J. Common proper motion with HD 75022, K0 III (Eggen 1971, see also Gordon 1968).

0846+7432 2282 Yam: C3,0.

0847-3418 2334 Hen 78, BidMac. Bright $\mathrm{H} \beta$ and $\mathrm{H} \gamma$.

0848-4658 2347 W 34-25, Sndlk.

0850-2844 2351 Also Sto IV-12 (S).

0850-3604 2353 Sndlk(N), Hen 80.

0851-3622 2360 NSV 4283.

0851-4952 2364 Sndlk, Hen 81.

0852-3052 2369 Sto IV-29, Ste (R).

$0855+17132378$ HR 3541, +17 ${ }^{\circ} 1973$, D $100(\mathrm{~N})$, AFGL 1298, IRC+20206.

Weak $\mathrm{SiC}_{2}$ noted by Shane and by Sanford. K-M:

C5,4; Yam: C5,4.

0855-3950 2392 Also MacCon2000.

$0856+19502384+20^{\circ} 2243$, Van 40, Ste, AFGL 1301, IRC+20207. San:

R6. The IDS gives a companion star.

$0857+51252383$ D 294 (R), Van 39 (R2). K-M: C1p2 (CH star).

0858-2922 2400 Also Hen 83.

0858-6047 2406 Star has a silicate CSE.

0859+3346 2396 D 199(R), Van 41(R2), Ste(R). Yam: C3,2.

$0903+50052404$ D 295(R), CCCS 1362, Van 42(R5), Ste(R). San: R6;

De: C4,3; Yam: C3,5J.

0904-2007 2416 Mayun provided a considerably different declination, it looks like a sign error in the precession.

0905-4714 2429 B-M note strong infrared CN.

0906-5835 2432 Sto III-25, Hen 86.

0906-5634 $2434 \quad \mathrm{SiC}_{2}$ bands.

$0907+21352428$ Van 43 (R5), Ste (R-N). De: C4,2; Yam: C3,4J.

0913+1412 2449 D 103 (R), Van 44 (R5), Ste, CCCS 1403. San: R6;

K-M: C4,4; Yam: C4,4J.

0913-2323 2450 Hen 88, HanBl, IRC-20184.

0915-6614 $2463-65^{\circ} 754$, Sto III-31(N), Hen 90.

$0918+51242458+52^{\circ} 1378$, Ste(N). Yam: C4,4; De: C4+,4.

0920-4722 2480 W 43-1, Sndlk. 
0920-47012485 Also Hen 92 .

0924-5041 2502 W 44-10, Sndlk, Hen 94, VB 29072-16.

0926-5328 2514 Also VB 29072-25. Noted as very red on a Tololo plate (Ste).

0926-5136 2515 Hen 95, VB 29072-21.

0928-3549 2520 Also BidMac. CPD -35 3524 (May51), CD-35 5709 (Sto).

0928-4958 2521 Also VB 29072-26.

0928-4045 2522 Sndlk (N:), Hen 97.

0928-5116 2526 Also VB 29072-29.

0929-5328 2528 Blanco's position is given. Westerlund's position is 1.9' west.

0930-5846 2541 Also Ste (N:).

0931-6323 2546 NSV 4524?

0933-4118 2550 Also Hen 100.

0934-5317 2564 In outskirts of the open cluster NGC 2925.

0935-4800 2566 Also W 46-9.

0937-5041 2575 W 49-3, Sndlk, VB 29072-49.

0938-4723 2576 Westerlund's visual mag. of 16 is far too faint to appear on Wray's plates or Ste Tololo plates, so the star possibly is variable.

0938-5546 2581 An S star is close by, independently found by Ste and MacCon79.

0938-6734 2588 Also BidMac.

0939-5520 2585 W 48-10, Ste.

0940-5726 2590 Very strong D lines on Tololo plates, on which the $\mathrm{C}_{2}$ bands are weak. Rather like some $S$ stars in the $580-$ $680 \mathrm{~nm}$ region. Definite $\mathrm{C}_{2}$ is present on a Tololo slit spectrogram (Ste).

0942-0852 2591 Also Ste. Yam: C3,4.

0943-4644 2603 Also BidMac.

0943-5323 2607 Blanco's position is given; Westerlund's is $1.9^{\prime} \mathrm{E}$.

0944-46432611 Also Hen 329.

0945-5000 2618 W 49-11, VB 29072-65.

0946-5658 2622 Also Hen 106. 
0947+1316 2619 Also Wil73. Carbon classification by Herbig and Zappala, ApJ 162, L15.

0948-4947 2627 Also VB 29072-68.

0950-0201 $2635-1^{\circ} 2312$, D $104(\mathrm{~N})$, BSD 101-10 (Nb), Ste (R-N). De: C6,3; Yam: C4,4. See also Bidelman (1954).

0950-5626 2646 Also Ste.

0951+5237 2626 Van 45 (R5), Ste (R). De: C3,2; Yam: C2,3. Spectroscopic binary with a period of 2902 days.

0951-2301 $2641-22^{\circ} 2739$, Hen 107, Ste(N), IRC-20199. San: N; K-M: $\mathrm{C} 5,4$; Yam: $\mathrm{C} 4,5 . \mathrm{SiC}_{2}$ noted by Shane and $\mathrm{HD}$.

0953-4420 2649 NSV 4667. Strong $\mathrm{SiC}_{2}$ bands. A slit spectrogram by Catchpole, PASP $80,744,1968$, confirms it as a carbon star.

0953-5230 2656 Wray 172, W 52-6, Sndlk, Hen 109. In outskirts of the open cluster Col. 213.

0955-4135 $2661-40^{\circ} 5616$, Sto IV-74, Hen 111.

0955-4952 2662 Hen 110, BidMac.

0959-6013 $2685-59^{\circ} 2676$, CannMay49, Ste (N), Hen 112. Near NGC 3114 .

0959-7212 2688 Also BidMac.

1002-6000 2690 In area of the open cluster NGC 3114.

1003-6000 2692 In area of the open cluster NGC 3114.

1006-5408 2701 Wray 185, W 55-9, Sndlk (N), Hen 113.

1008-3654 2705 Definitely variable between two plates, according to MacCon.

1011-3519 $2713-34^{\circ} 6528$, Sto IV-51, Hen 114. Yam: C6,3.

1012-4944 2716 Also Hen 115. The carbon star is $90^{\prime \prime}$ in PA $160^{\circ}$ from a CD star.

1012-6013 2720 CCCS 1637.

1014-6043 2726 Also Hen 118. The position given here is the mean of two accordant Tololo plates (coordinates spread $0.2 \mathrm{~s}$ in RA, 4" in DEC); the GCVS position for AM Car (3rd ed.) agrees with this to its indicated precision of $1^{\prime}$. The next nearest candidate for this SR variable is a middle-M star $3.6 \mathrm{~s} \mathrm{~W}$ of the $\mathrm{C}$ star and $2.3^{\prime} \mathrm{N}$ of it. (Ste). 
1016+3034 2724 CIT 6, Wil73, Lo74, IRC+30129. Position by Clausen et al., 1987, ApJS 65, 385.

1016-5934 2735 Also MacCon2000.

$1017+77062715$ Van 46 (R5), Ste. Yam: C3,5.

$1017+73252719$ Originally noted by Kowal in IAUC 2394 as a very red star at rather high galactic latitude.

1017-1438 2730 AFGL 1406, IRC-10236.

1017-5005 2738 Also Hen $120 . \mathrm{SiC}_{2}$ bands noted by Ste.

1021-5259 2751 Sndlk (R), BidMac.

1024-5506 2758 W 58-8, Ste, Hen 122.

1024-5024 2759 Also Ste (R0).

1027-2533 $2764-24^{\circ} 9072$, HanBl, AFGL 4781S, IRC-30165.

1027-5940 2768 Also MacCon2000.

1028-6734 2774 Also Hen 124.

1029-6014 2780 Also MacCon2000.

1030-5953 2778 In area of the open cluster Col. 223.

1030-5502 2779 Also Hen 125.

1034-4823 2787 Sto IV-101 (R), Sndlk (R0), Hen 127.

1034-5414 2790 Wray 207, Ste, W 61-3, Hen 330, BidMac (Ce), Mac-

Con2000.

1034-6118 2788 CCCS 1701.

1034-6242 2792 W60-14, Hen 130, BidMac.

1035-3933 2793 HR $4153,-38^{\circ} 6579$, Sto IV-64 (N), Hen 129. The star has two detached dust shells.

1035-6130 2798 Also MacCon2000.

1035-6145 2800 Also MacCon2000.

1035-6247 2797 Also MacCon2000.

1037-6003 6393 GBE98 identifies it with IRAS 10356-5947 with difference from the Aar 89 coordinates by $2 \mathrm{~s}$ in RA and $65^{\prime \prime}$ in DEC.

1037-1323 2803 HR 4163, $-12^{\circ} 3218$, Ste (N), AFGL 1427, IRC-10242. K-M: C7,3; Yam: C6,3. Weak $\mathrm{SiC}_{2}$ noted by Shane and by Sanford (1926).

1038-6049 2820 W 60-22, Hen 132, MacCon, MacCon2000. Strong D lines. 
1038-6117 2816 Also MacCon2000.

1038-6134 2815 Also MacCon2000.

1039-6049 2823 W 60-23, Hen 133, MacCon.

1039-6109 6398 Given position is $36^{\prime \prime}$ from HW Car, a Cepheid variable.

1040-4801 2829 Bidun's source is a $98 \AA / \mathrm{mm}$ objective prism plate.

1040-6056 2828 Also MacCon2000.

1040-6151 2831 Also MacCon2000.

1041-5923 2832 Wray 21 (Ce), W 62-8, Hen 134, MacCon.

1041-6231 2833 Also MacCon2000.

1043-6010 2842 Also MacCon, MacCon2000. In area of the open cluster Col. 228.

1043-6133 2840 Also MacCon2000.

$1045+67242835$ HR 4195, +68 617 , D $299(\mathrm{~N})$, Van $47(\mathrm{R} 8)$, Ste (N), AFGL 1433, IRC+70100. K-M: C6,3; Yam: C6,3.

1045-6229 2845 Also MacCon.

1046-5825 2850 Also MacCon, MacCon2000. B-M noted strong infrared $\mathrm{CN}$.

1046-6536 $2852-64^{\circ} 496$, Ste, Hen 135 , MacCon. Independently classified as $\mathrm{N}$ by Ste on three Tololo plates.

1046-6400 2854 In area of the open cluster IC 2602.

1048-5709 2865 Also MacCon2000.

1048-5837 2863 Also MacCon2000.

1048-6011 2862 Also MacCon, MacCon2000.

1049-5525 2868 Ste, Hen 137, MacCon2000.

1049-6035 6415 Two stars very close.

1050-6104 2875 Hen 138, MacCon.

1051-2115 2877 AFGL 1439, IRC-20218. K-M: C7,5. The IDS lists a companion, whose spectral type, according to Gordon (1968), is K0 III:, $V=11.58 \mathrm{mag}, 46^{\prime \prime}$ away. See also Bidelman (1954). CO mm emission shows fast bipolar molecular outflow. According to Knapp et al. (A\&A, $351,97,1999)$ it has orbiting dust cloud $3.5 \cdot 10^{14} \mathrm{~cm}$ away from the star and circumstellar envelope with radius $8 \cdot 10^{16} \mathrm{~cm}$.

1052-5325 $2885 \quad-52^{\circ} 4032$, Sndlk (N), Hen 139. 
1052-6219 2888 In area of the open cluster Rup 162.

1053-6127 2894 W 64-17, Ste, MacCon. Wray's RA is $\sim 15 \mathrm{~s}$ in error. The eclipsing variable FL Car has a published position only $1^{\prime} \mathrm{N}$ of this.

1054-1923 2892 Yam: C2,1.

1056+1516 2900 Yam: C3,1.

1056-6102 2903 Also MacCon. In area of the open cluster Col. 236.

1056-6003 2907 Also MacCon.

1058-6238 2916 Hen 140, MacCon. Note proximity to Wray 218, No. 1058-6235. These are definitely two different carbon stars (Ste, MacCon).

1059-6241 2921 Peculiar spectrum.

1100+4136 2914 Yam: C3,2CH. Spectroscopic binary with a period of 328 days.

1100+4042 2919 Yam: C3,1.

1100-2551 2925 Also BidMac. War: C2,1; Yam: C2,0 (very early; wide absorption at $4226 \AA$ ).

1101-6811 2934 Also BidMac.

1103-7145 2947 Also Hen 142.

1104-6009 2948 Also MacCon.

1105-6120 2950 Also MacCon.

1106-5837 2953 In area of the open cluster NGC 3532.

1106-5834 2956 W 66-4, Ste, MacCon. In area of the open cluster NGC 3532.

1106-6557 2958 Also MacCon.

1107-1013 2954 Yam: C1,3CH.

1107-5507 $2959-54^{\circ} 3975$, Ste (N), Hen 144, MacCon2000.

1107-6436 2960 Hen 145, MacCon.

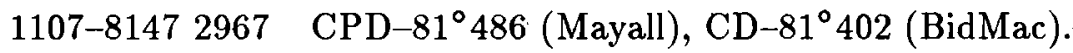

1109-6007 2968 Also MacCon. In area of the open cluster Col. 240.

1112-5820 2972 Also MacCon.

1113-4813 2975 Sto IV-92(R), Sndlk(R0), Hen 147. According to the Hipparcos data, the nearest known carbon star with trigonometric parallax 31.18 mas.

1115-5755 $2984-57^{\circ} 3809, \mathrm{~B}-\mathrm{M} 146, \mathrm{~W} 66-8$, Ste(N), Hen 148. In area of the open cluster Rup 164. 
1115-6954 2986 CannMay 49 (Pec), Ste (R), BidMac. Carbon cepheid with a period of 29.2 days.

1120-5544 $2998 \mathrm{H} \alpha$ emission; strong D lines. Not the nearby carbon star HD 98767 (1121-5545).

1121-5545 $3001-55^{\circ} 3979$, CannMay 49(N3), Ste, Mich, Hen 149.

1121-6017 3002 W 68-1, Ste, Hen 151, Mich.

1122-5938 3003 Also W 68-2.

1122-5815 3005 Double with 1122-5816.

1122-5816 3006 Double with $1122-5815$ and is included in the cata$\log$ for this reason, though it is definitely not a carbon star.

1131-6330 3043 In area of the open cluster Rup 94.

1132-6054 3048 Also W 68-9.

1132-7238 $3049 \quad 2^{\prime} \mathrm{N}$ of CD-71 770.

$1133+01453058$ May51 (R), Van 52 (R2), Ste (R). Yam: C3,3.

1133-5948 3059 The nearby IW Cen, according to the identification chart, is of $B$ type - at least in the blue spectral region.

1134-5942 3064 Blanco's position is given. Westerlund's is $1.8^{\prime} \mathrm{S}$.

1135-5928 3065 W 69-10, Hen 153, MacCon.

1135-1435 3066 Van $53(\mathrm{R} 2)$, Ste $\left(\mathrm{R}\right.$, weak $\left.\mathrm{C}_{2}\right)$. K-M: C0,2; Yam: C1,1.

1135-5626 3067 Also MacCon.

1136-6029 3071 Also MacCon.

1139-6047 3080 Also MacCon.

1139-7233 $3083-71^{\circ} 778$, Sto I-26 (N), Ste (N), Hen 156.

1139-5834 3084 W 72-5, Ste, Hen 157, Mich, MacCon.

1147-6241 3105 Also MacCon.

1147-5751 3106 Also MacCon.

1149-6007 3108 Difference between MacCon 329 and Aar89 coordinates is $0.6 \mathrm{~s}$ in RA and $50^{\prime \prime}$ in DEC.

1150-6251 3113 Also MacCon.

1151-6137 3114 Also MacCon.

1154-6132 3125 MayCann40, W 74-1, Ste (N:), Henize 160, MacCon.

$1155+12343129$ A CH star (Bidelman 1956). Yam: C4:,3CH. 
1156-6321 3131 W 73-7. Ste (red), MacCon.

1157-7826 3137 CPD-77²61 (May51), Hen 162, BidMac.

1159-5836 3141 W 75-1, Ste, Hen 331, MacCon, CCCS 1942. War:

C3,2.

1200-5507 3144 Ste (N), Hen 164.

1203-6405 3155 Hen 166, MacCon.

$1203+70253156$ Van 54. Yam: C2,3CH.

1204-6238 3159 Hen 168, MacCon. Not WX Cru.

1207-6434 6470 May be Mira-type variable CL Cru 36" away.

1209-6029 3181 Also MacCon.

1211-6426 3186 W 76-1, Hen 169, MacCon. The quoted position is a weighted mean of many sources.

1211-6402 3187 Also MacCon. Westerlund identifies it with B-M 166.

1211-6146 3188 B-M 165, Wray 245, W 74-13, Ste, Hen 170, MacCon. There is only one carbon star here, though the Harvard papers imply two. The May51 coordinates are in error (Ste).

1211-6345 3189 Ste, Hen 171, MacCon. Near, but distinct from, B-M 154. Strong D lines.

1212-5919 3192 Hen 172, Mich, MacCon.

1213-6555 3194 Also MacCon.

1214-5131 3199 Sto IV-108 (R), Hen 173.

1220-5856 3215 W 78-1, Ste (N), Hen 174, Mich, MacCon.

1221-6626 3217 Not BE Mus (Bidun).

$1222+21056480$ Also Fea97.

1222-6305 3221 Mich, MacCon.

1223-7530 3222 Sto I-34 (N), Ste (N), Hen 175.

1224-4926 $3227-48^{\circ} 7401$, Sto IV-105(R), Hen 176. San: Np.

$1225+00463236+1^{\circ} 2694$, D 108(N), Ste(N), HanBl, IRC+00 217.

K-M: C6,3; Yam C5,3e. $\mathrm{SiC}_{2}$ bands. See also Bidelman (1954). Shane (Np) found the spectrum closely similar to $\mathrm{U}$ Cyg at minimum. HD likens it to $\mathrm{VX}$ And.

1227-6131 3239 Mich, MacCon.

1227-6146 3240 In outskirts of the open cluster NGC 4349. 
1229-3815 3246 Sto IV-59(N), Hen 178. CCCS 2008.

1231-6043 3248 W 78-3, MacCon.

1236-6356 3258 The $I$ mag is from Westerlund, Uppsala Medd. No. 130 , and differs almost by 2 mag from $W$.

1239-4522 3266 Also BidMac.

1240-6654 3267 Strong D lines.

1240-5722 3268 Balmer emission also noted by Bidun; strong D lines.

1240-6042 3269 Also MacCon. In outskirts of the open cluster Tr 20.

1242-4353 3275 Fou92 identifies it with IRAS 12394-4338 which is about $2^{\prime}$ away.

$1245+45263283$ HR 4846, D 301 (R), Upgr60 (N), Ste, AFGL 1576, IRC+50219. San: N, $\mathrm{SiC}_{2}$; K-M: C5,4; Yam: C5,5J.

1247-5941 3284 About $2^{\prime}$ from $\beta$ Crucis.

$1247+04083286+4^{\circ} 2651 \mathrm{a}, \mathrm{D} 109(\mathrm{~N})$, Ste (Np,r), HanBl, AFGL 1579, IRC+00224. See also Bidelman (1954). On a Case plate, strong $\mathrm{D}$ lines and $5635 \AA \mathrm{C}_{2}$, but weak $\mathrm{CN}$ (Ste).

1249-6146 3291 W 80-2, Hen 184, MacCon ( $\mathrm{H} \alpha$ emission).

1251-6500 3295 A somewhat confusing case. Published, on the basis of meagre data, as weakly symbiotic by Sndlk and Ste (ApJ, 185, 899); called symbiotic by Weaver (PASP, $84,854)$, who saw no bands in the photographic infrared; confirmed symbiotic by David Allen (MNRAS 184, 601), who reported very weak TiO. However, MacCon finds infrared $\mathrm{CN}$ and on that basis calls it a carbon star (Ste).

$1252+07123298$ Component $\mathrm{C}$ of $\mathrm{BD}+8^{\circ} 2654$. Vyssotsky and Balz (1958) gives type G5 for component $\mathrm{A}$ and type G0 for component $\mathrm{B}$. In the $\mathrm{AG}$ catalog, $\mathrm{A}$ is $\mathrm{AG}+7^{\circ} 1683$, $\mathrm{B}$ is $+7^{\circ} 1685$ and $\mathrm{C}$ is $+7^{\circ} 1684$. Included in Burnham's double star catalog as BDS 6293. Yam: C2,0ch. A CH-type star. Spectroscopic binary with a period of 571 days.

1254-6503 3302 Also MacCon.

1254+1001 3308 Reported by Phillips and Terlevich (PASP 95, 43, 1983) as a possible $\mathrm{CH}$ star.

1256-5753 3310 Hen 187, MacCon. Hydrogen emission; see also Bidelman (1954). Also has strong D lines. 
$1256+65593313+66^{\circ} 780$, D $302(\mathrm{~N})$, Ste (N!), AFGL 1588, IRC+70116. $\mathrm{K}-\mathrm{M}$ : $\mathrm{C} 4,4$ : Yam: $\mathrm{C} 4,5 \mathrm{~J}$. $\mathrm{SiC}_{2}$ is quite strong and noted in HD (though not yet identified as such).

$1259+37493319+38^{\circ} 2389$, D 200 (R), Van 56 (R5), Upgr60 (R), Ste (R). K-M: Cp,5; Yam: C3,5CH. Long known as a $\mathrm{CH}$ star (Keenan and Morgan 1941, Bidelman 1956).

1300-6437 3318 Also MacCon.

1303-6032 3323 Mich, Hen 189, MacCon.

1306-2003 3335 Also Van 57. K-M: C1,1; Yam: C2,1.

1307-6657 $6509 \quad 3^{\prime}$ uncertainty in position.

1312-6549 3346 Hen 192, MacCon.

1313-6623 3350 Also MacCon.

1319-60473367 Hen 193 (C), MacCon (C). A remarkable spectrum variable. Sometimes shows $\mathrm{C}_{2}$ and $\mathrm{ZrO}$ bands. Evidently belongs to CS type (Ste).

1321-7426 3368 Sto I-32 (N), Hen 194.

1322-6413 $3374-63^{\circ} 839$, Hen 195, MacCon.

1326-4718 6531 In the center of the globular cluster Omega Cen.

1326-4733 3382 In the globular cluster Omega Cen.

1327-4743 3389 In the globular cluster Omega Cen.

1328-4726 3391 In the globular cluster Omega Cen.

1329-6408 3393 In area of the open cluster Col. 271.

1330-6852 3397 Also MacCon. Ste identifies with the variable because, except of similarity in coordinates, the discovery paper says the variable is quite red.

1332-5349 3405 Also Hen 198. CCCS 2099.

1337-5628 $3412-55^{\circ} 5254$, Hen 199, MacCon.

1344-7036 3425 Also Hen 200. $\mathrm{SiC}_{2}$ noted.

$1344-63113428$ MacConnell's position is given. Westerlund's is $1.4^{\prime} \mathrm{N}$.

1346-6345 3429 MacConnell's position is given; Westerlund's is $1.6^{\prime} \mathrm{N}$.

1347-6126 3433 The star has a silicate CSE.

1350-3306 3442 Also BidMac, with the same BD number but DEC is discordant by $15^{\prime}$.

1354-61493445 $2^{\prime}$ away from the center of the open cluster NGC 5316.

1358-5621 $3456-55^{\circ} 5435$, Ste (N), Hen 203, MacCon. 
1400-6523 3460 MacConnell's position is given; Westerlund's is $1.4^{\prime} \mathrm{W}$.

1400-6136 3461 Also MacCon. Although called a CS star by Ste, this star is not the nearby S or MS star No. 838 in the $S$ star catalog (2nd ed.).

1401+3249 3469 Van 58, Ste. Yam: C3,3. De: C4,2. HD gives for it spectral class $\mathrm{K} 0$, but this type probably refers to the nearby $+33^{\circ} 2400$.

1405-6700 3471 Also MacCon.

1407-6754 3473 $\mathrm{H} \alpha$ emission noted on a Tololo plate.

1414-5355 $3481-53^{\circ} 5490$, Ste (N), Hen 207, CCCS 2148.

1415-6055 3482 MacConnell's position is given; Westerlund's is $1.5^{\prime} \mathrm{S}$.

1415-5859 3483 Hen 208, MacCon. In outskirts of the open cluster Rup 167.

1416-5858 3484 In area of the open cluster Rup 167.

1419-5659 3485 NSV 6614, MacCon. Strong red CN, but weak $\mathrm{C}_{2}$.

1422-5752 3488 MacConnell's position is given; Westerlund's is $1.1^{\prime} \mathrm{N}$.

1422-4950 $3489-49^{\circ} 8685$, Wray 274, Hen 211.

1423-4731 $3492-46^{\circ} 9289$, Wray 275, Sto IV-98, Hen 212.

1424-6344 3490 Strong D lines.

1426-6002 3494 Also MacCon. Strong D lines.

1429-5559 3496 Also MacCon.

1429-5818 3497 W 94-6, MacCon.

1432-5918 3498 Strong D lines.

1435-4322 $3510-42^{\circ} 9465$, Sto IV-81(N), Hen 215.

1437-6200 3512 Also MacCon.

1441-6245 3516 Quite red.

1443-5808 3524 MacConnell's position is given; Westerlund's is $3.2^{\prime} \mathrm{W}$ and $1^{\prime} \mathrm{S}$.

1446-3210 3533 CCCS 2190. Herbig (Her52) classifies it as Gp, but notes that $\mathrm{C}_{2}$ is visible at low dispersion. Carbon cepheid with a period of 2.1 days.

1452-6204 3538 Also GJ93, GJBSW.

1457-3011 3544 Sto IV-26 (R), Hen 218, BidMac.

1459-5324 $3548-52^{\circ} 6392$, Ste (N:), W 102-1, Hen 219.

1504-02513558 D 111 (R), Van 59 (R5), Ste (R-N). Yam: C4,4J; De:

$\mathrm{C} 4,3$. 


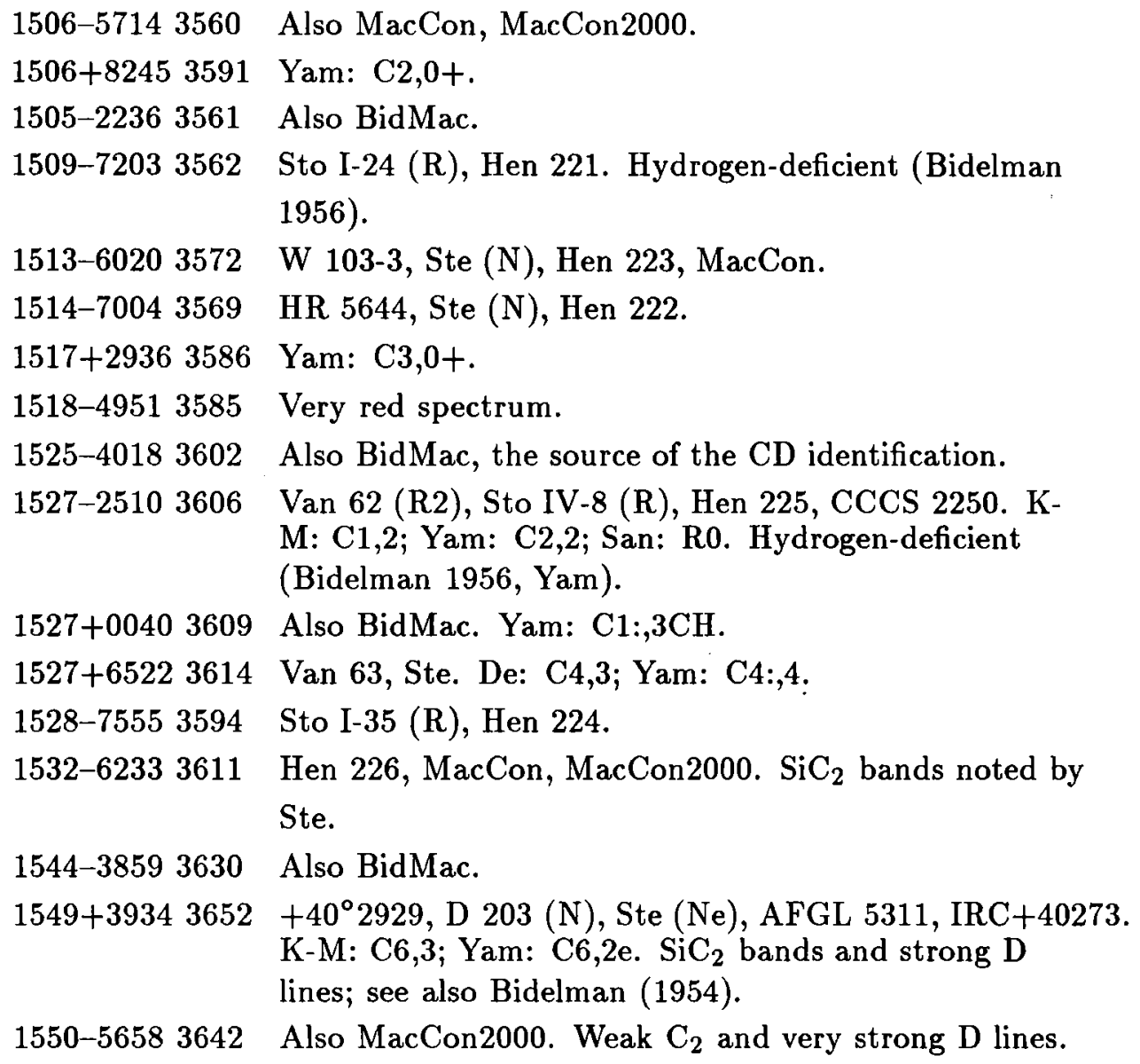

1527+0040 3609 Also BidMac. Yam: C1:,3CH.

1527+6522 3614 Van 63, Ste. De: C4,3; Yam: C4:,4.

1528-7555 3594 Sto I-35 (R), Hen 224.

1532-6233 3611 Hen 226, MacCon, MacCon2000. $\mathrm{SiC}_{2}$ bands noted by Ste.

1544-3859 3630 Also BidMac.

$1549+39343652+40^{\circ} 2929$, D $203(\mathrm{~N})$, Ste (Ne), AFGL 5311, IRC+40273.

$\mathrm{K}-\mathrm{M}: \mathrm{C} 6,3$; Yam: $\mathrm{C} 6,2 \mathrm{e} . \mathrm{SiC}_{2}$ bands and strong $\mathrm{D}$ lines; see also Bidelman (1954).

1550-5658 3642 Also MacCon2000. Weak $\mathrm{C}_{2}$ and very strong $\mathrm{D}$ lines.

1552-5517 3649 Also MacCon2000.

1552-5638 3648 Also MacCon2000.

$1604+50293665+50^{\circ} 2251$, Ste (Np, nearly CS). K-M: C7,2(at min); Yam: C8,1e; KB: SC 5/10e=C2,2. See also Bidelman (1954).

1604-5604 6613 Carbon cepheid with a period of 32.3 days.

1606-7659 3657 Pots 204-304, Sto I-38 (R), Mich (CH star).

1611-6134 3666 Also BidMac. War: C1,1. Blue plate shows weak $\mathrm{C}_{2}$ and strong CN (Ste).

1613-1512 3672 MayCann40 (R3), Ste (R). Yam: C1:1CH; also CH by Bidelman (1956). 
1615-6405 3671 Ste, BidMac.

1615-2212 3675 Discovery by Longmore and Allen, ApJ, 18, L159. Said to be symbiotic.

$1617+25513679$ Also Mich, Bidun. De: C6,3e.

1619-0334 3682 De: C3,2.

1622-5953 3684 Sto II-89 (R), Ste (R5). Mayall (1951) indicated two carbon stars in this vicinity, but there seems to be only one $\left(\mathrm{CPD}-59^{\circ} 6710=\mathrm{CoD}-59^{\circ} 6131\right)$ (Ste).

1624-5920 3687 Also Sto II-90(R). Hydrogen-deficient (Bidelman 1956).

1625-5155 3691 Sndlk (N:), Hen 232, MacCon2000. Strong D lines.

1626-4910 3692 Near the open cluster NGC 6314.

1626-1225 3698 Also $-12^{\circ} 4510$, AFGL 1859, IRC-10339. K-M: C7,4;

Yam: C7,3e; Bid: C7,3e. See also Bidelman (1954).

1628-4339 3697 CannMay49, Ste(N), Hen 233, CCCS 2333; San: N.

$1629+19303704$ Also NSV 7765. Yam: $\mathrm{C} 1,1 \mathrm{CH}$.

1630-5009 3700 Also MacCon2000.

1632-4510 3706 Also MacCon2000. MacCon find it definitely variable between two plates.

1634-3517 3712 Weak $\mathrm{C}_{2}$ and strong violet $\mathrm{CN}$.

1634-6308 6648 Carbon cepheid with a period of 1.95 days.

1636-4621 3714 Also MacCon2000.

1635-6707 3707 Also Sto I-13 (R), CCCS 2342. War: C1p,1; hydrogendeficient.

1637-0425 3717 Also BidMac.

1638-4559 3715 Also MacCon2000. Strong D lines.

1639-4629 3719 Very red.

1640-3222 $3720-32^{\circ} 11900$, Ste (N), Hen 323, AFGL 1878, IRC-30268. Yam: C5,5. Strong $\mathrm{SiC}_{2}$.

1645-2918 3729 HD likens its spectrum to K5R.

$1647+23123735$ NSV 7969. Yam: C3,0+.

1648-3123 3733 Position in Sto is essentially for LSS 3773. The present position is by Sndlk.

1650-6746 3731 Sto I-14 (N), Hen 237.

1651-4809 3736 Also MacCon2000. 
1702-5504 $3756 \mathrm{CD}-54^{\circ} 7100=\mathrm{CPD}-54^{\circ} 7975$. In the IDS double with CPD-54 $4^{\circ} 7976$. Sto II-78 (R), Hen 240.

1702-3240 3762 Ste (N), Hen 241, HanBl, IRC-30277.

1709-3351 3775 Red. Very strong D lines.

1711-6157 3774 -61 5736 , Pots 196.1037(Nb), Sto I-3 (R), Hen 244.

1713-3555 3786 Ste, Fuen 224. Very red, with strong CN and D lines. A color index of +4.0 is mentioned in the variability discovery paper (Swope, Ann. Harvard Obs. 109, No. 9 ).

1713+4206 3795 D 305 (R), Van 66 (R0), KurNik96. San: N; K-M: C1,2; Yam: C3,1. Included in Sanford's (1950a) atlas of carbon star spectra.

$1714+42103801$ Unpublished revised coordinates by Ste.

1715-2905 3791 Also Sndlk(R), IRAS17121-2902. According to Kilkenny et al. (Observatory, 112, 158, 1992), it is probable $\mathrm{R}$ CrB-type variable.

1716-3439 3793 W 140-1, Hen 247. Strong D lines.

1718-4557 $3799-45^{\circ} 11383$, Sndlk (R:), Hen 248.

1720-4023 3808 Ste (R:), W 138-3, Hen 250, Fuen 228, CCCS 2429.

1722-4927 3810 Sto II-65 (R), BidMac.

1723-4349 3813 Also Ste.

1723-3629 3816 San: R3.

1723-2820 3818 Sndlk, Fuen 232. The position given by Wray appears to be for V521 Oph, an M-type long-period variable.

1724-2920 3820 W 142-2, Sndlk (N:r), HanBl, AFGL 1961, IRC-30293, Fuen 233. Yam: $\mathrm{C} 7:, 3$.

1725-2945 3824 The reputed planetary nebula PK $356+3^{\circ} 1$ has this position, within the published precision of $1^{\prime}$. The planetary nebula is defined by $\mathrm{H} \alpha$ emission seen by P.S. The on an objective prism plate, without any continuum. Ste have seen the same at the plate limit of a red plate with no clear-cut carbon star candidate. The N1, N2 nebular lines may be visible superimposed on another spectrum. The quoted position, which is that of the $\mathrm{H} \alpha$ emission to a small fraction of an arcminute, is from Fuen; the W DEC is $4^{\prime}$ further north (Ste).

1726-5041 3822 MacCon: J-star. 
1726-4001 $3827-39^{\circ} 11452$, Hen 253. GCVS: C4-5,5. The star has detached gas shell. In the discovery paper announcing the variability, Swope (1943) quoted a color index of +5.0 .

1728-5502 3832 Sto: a star of similar brightness is nearby (to W).

1728+0736 3836 De: $\mathrm{C} 6,2$. Weak $\mathrm{C}_{2}$, strong infrared $\mathrm{CN}$.

1729-1928 3837 Sndlk (N), Hen 254, Ste (N), HanBl, AFGL 1971, IRC-20364. San: N; K-M: C6,5; Yam: C5,5. Strong $\mathrm{SiC}_{2}$ bands.

1731+0158 3842 NSV 9082. De: C4,1; Yam: C3,1CH.

1733-3615 3844 Ste, Hen 255, Fuen 243.

1734-1404 3850 Weak $\mathrm{C}_{2}$ and strong violet $\mathrm{CN}$.

1736-1350 3852 Weak $\mathrm{C}_{2}$ and very strong violet $\mathrm{CN}$.

1740-4137 $3854-41^{\circ} 11893$, Ste $(\mathrm{N})$, Hen 256. San: N; Yam: C4,4. $\mathrm{SiC}_{2}$ bands noted by Ste.

$1740+11583867$ Strong $\mathrm{C}_{2}$.

1742-3514 $3864-35^{\circ} 11829$, CannMay49(N3), W 148-5, Ste (R), Hen 258, Fuen 249.

1743-5743 $3861-57^{\circ} 8687$; in IDS. Sto II-86 (N), Hen 257. War: C6,4.

1744-1839 $3875-18^{\circ} 4634$, ADS 10751A, C 282, Sndlk (N+A5), Hen 260, AFGL 5134S, IRC-20382. Yam: C7,3. The IDS misprints the spectral type as M. Gordon (1968) gives A3 for the companion $\left(2^{\prime \prime}\right.$ in PA $\left.215^{\circ}\right)$.

$1745+17123879$ Yam: C2,0+.

1747-3542 $3878-35^{\circ} 11923$, Ste $(\mathrm{N})$, Hen 261. War: C5,4; Yam: C4,4.

1751-4320 3886 The identification with the variable star is by Sto.

1753-2849 3889 Hen 262, Fuen 253. Strong D lines.

1753-2316 3890 Also Fuen 254.

1753+6406 3912 Yam: C2,2.

1755-2801 3901 W 156-2 Sndlk (N), Hen 263, HanBl, AFGL 5156S, IRC-30333, Fuen 259. Very strong red CN.

1756-2313 3902 Also Fuen 260.

1756+5813 $3921+58$ 1772a, Ste (N) AFGL 2040, IRC+60255. Bid: C8e; Yam: C6,2e. Sanford's radial velocities (PASP, 53, 291, 1941) show the $14^{\prime \prime}$ distant companion UY Dra to be optical. 
1758+1311 3917 CCCS 2509. Yam: C3,3CH.

1800-1700 3922 Silicate CSE. Water maser emission at $22 \mathrm{GHz}$ (Engels 1994).

1801-3856 3919 The position given by Sto is about $3^{\prime}$ in error.

1801-2905 3920 Sndlk, Fuen 267. Strong D lines.

1801-1243 3926 Strong D lines.

1802-2446 3931 In outskirts of the open cluster NGC 6523.

1802-1910 3933 MSB $61(\mathrm{~N})$, Ste (N), Sndlk (N:), BidMac, HAnBl, AFGL 5181S, IRC-20420, Fuen 269, CCCS 2521. San: N.

1802-2955 3928 V3896 Sgr (M6:) is $25^{\prime \prime}$ away.

1803-3213 3935 The star has a silicate dust envelope.

1804-3243 3939 Hydrogen-deficient according to Lloyd Evans, IAU Colloq. No. 106.

1805-3920 $3938-39^{\circ} 12196$, Sto II-35 (R), Ste (R), Hen 266.

1806-0941 6711 AFGL 2067, IRC-10396.

1806-3045 6708 Very strong D lines.

1807-2830 $3947-28^{\circ} 14163$, Wray 320, Sndlk (N), Hen 269, Fuen 273, San: N.

1807-1435 3948

AFGL 5197S, IRC-10397.

1808-3719 3950

Sto II-29 (R), Ste (R). Hydrogen-deficient (Bidelman 1956).

1808+0927 3957 D 116 (R), C 703, Van 70 (R5), Ste (R). San: R4;

Yam: C3,5J.

1809-2738 3954 AFGL 2076, IRC-30364.

1809-2139 3956 Also Fuen 275.

1810-2638 3958 Also Sndlk (R).

1810-2651 3960 Sndlk (N:), Hen 270, HanBl, AFGL 2082, IRC-30365, Fuen 277. GCVS: C6-7,3. Strong D lines.

1811-1951 3964 An extremely red star which in different papers is considered either as a carbon or S-type star. May be an $\mathrm{SC}$ star or a weak carbon star (Ste).

1812-3215 6726 Very strong D lines.

1812-3759 $3963 \quad \mathrm{H} \alpha$ emission (MacCon).

1812+1455 3973 BSD 86-707 (R8), Ste (R). Yamashita (PASJ, 26, 159) consideres there must be two carbon stars. 
1813-4341 3966 Sto II-49, BidMac. BidMac omit the HD identification. Sto notes a star of comparable brightness $W$ of the carbon star.

1813-3016 3968 Also Sndlk (R).

1814-2749 6728 Carbon cepheid with a period of 21.3 days.

1818-4632 $3982-46^{\circ} 12279$. Hydrogen-deficient (Bidelman 1956).

1818-1326 3987 AFGL 2114, IRC-10409. The variable was not identified with the BD star by Maffei in the variability announcement (Frascati Contr. No. 2). A large color index is noted. Ste, Sndlk and Bidun find strong $\mathrm{SiC}_{2}$ bands.

1818-0651 3990 The position quoted is from the Ste infrared survey, supported by a computer underlay. It differs in RA from $\mathrm{C} 442$ by $0.3 \mathrm{~min}$. Comparison of the old and new plates shows they are the same star, and that the 5 mag difference in estimated $I$ magnitude is real; the star is (presumably a long-period) variable. $\mathrm{H} \alpha$ emission noted by Cohen and Kuhi (PASP, 89, 829) (Ste).

1819-4157 $3984-41^{\circ} 12644$, Sto II-45 (R), BidMac.

1819-1536 $3992-15^{\circ} 4923$, C 20, Pots 134-1901 (R), Van 72 (R5), Sndlk (R), Fuen 282, IRC-20464. Yam: C4,5J.

1822-1901 3996 Also Fuen 283.

1823-2206 4007 Also MacCon2000.

1824-2415 3999 Classified as an RCB variable and hydrogen-deficient star according to Bidelman (1956). Weak but definite $\mathrm{C}_{2}$, plus fairly strong $\mathrm{CN}$.

1825-2308 4001 M spectral type has been published for the variable, but it refers to a nearby star.

$1826+01094013$ Also MaeSoy90, AFGL 2158.

1829-0604 $6746 \quad 6^{\prime \prime}$ away from the $\alpha$ Cyg-type variable V431 Sct.

1829-3620 4020 Sto II-87 (R).

1830-3825 4021 Sto II-33 (R), Hen 274. War: C4,4.

1830-1653 4025 C 511, Sndlk, HanBl, IRC-20488. San: R3; Yam:

C3,4. Classification as $\mathrm{N}$ was independently done on four plates by Ste.

$1831+04224032+4^{\circ} 3779$, C 283, D $118(\mathrm{~N})$, HanBl, MaeSoy90, AFGL 2180, IRC +00353 . 
$1832+36594038+36^{\circ} 3168$, Ste, AFGL 2187, IRC+40321. K-M: C6,5; Yam: C6,5J. Npec by D.

1834-1011 4040 The position given here is the mean of three accordant plates of the Ste infrared survey. The Albers position is $18^{h} 29.1^{m},-10^{\circ} 11.8^{\prime}$.

$1834+09124045$ The variable was previously identified with the carbon star from the coordinates alone by Ste.

1837-2442 4049 An MS star lies within $2^{\prime}$ of the C star, and it is unclear which is the variable.

1837-0736 4052 $-7^{\circ} 4633$, C 21, Van, HanBl, MaeSoy90, AFGL 2203, IRC-10441.

1839-0314 4065 The position given here, from infrared survey, is confirmed by a computer underlay; it differs from Albers by about $9^{\prime}$ in DEC (Ste).

1840-0420 4072 In area of the open cluster Dol 33.

$1841+17414078$ IRC+20370.

1842-0217 4077 AFGL 2233, IRC+00365.

$1842+36574089+36^{\circ} 3243$, D $207(\mathrm{~N})$, Ste (N), AFGL 2240, IRC +40325 . San $\mathrm{N}$, with weak $\mathrm{SiC}_{2}$; K-M: C6,4; Yam: C7,4.

1843-5145 4070 Also Sto II-70(R).

$1843+17394088$ Very weak $\mathrm{C}_{2}$, strong $\mathrm{D}$ lines.

$1843+04064082$ Also MaeSoy90.

1844-1312 $4086-13^{\circ} 5083$, C 512, Sndlk. San: R3.

1846-3120 4094 Also Van 74. Hydrogen-deficient (Bidelman 1956).

1846-0634 4100 The MaeSoy90 position is used.

1847-3809 $4098-38^{\circ} 13089$.

1847-2356 4103 Also IRC-20518.

1847-0436 4109 The DEC published in the Case discovery list is by $2^{\circ}$ in error.

$1847+05274111$ D 120(N), C 22, MaeSoy90, AFGL 5295S, IRC+10379. Visual companion $V=11.8 \mathrm{mag}, 28^{\prime \prime}$ away.

$1849+11524122$ Also Ste.

1850-3132 4119 V963 Sgr?

1850-0754 4121 HR 7089, C 513. San: N; K-M: C5,4; Yam: C6,4. The star has two detached gas-dust shells. 
1850-0644 4124 HanBl, MaeSoy90, IRC-10 469. Harwood noted quite red color.

1851-1838 4123 Not the nearby V2054 Sgr.

1853-1231 4130 The GCVS position is quoted here.

$1854+49354144$ Very strong $\mathrm{C}_{2}$.

1855-3240 4133 Also BidMac.

1855-0811 $4138-8^{\circ} 4764, \mathrm{C} 514, \mathrm{HanBl}$, IRC-10475. San: N.

1856-1609 4141 Very weak $\mathrm{C}_{2}$.

1858-2930 4145 Van 75, Sto II-6(R), CCCS 2683. Hydrogen-deficient (Bidelman 1956).

$1858+14214147+14^{\circ} 3729$. D $126(\mathrm{~N})$, C 284 . San: N. Visual companion $V=12.05 \mathrm{mag}, 20^{\prime \prime}$ away.

1900-0134 $4152 \mathrm{HanBl}$, IRC+00404. The MaeSoy90 positon is given; the Ste RA and DEC are smaller by $4.5 \mathrm{~s}$ and $1.25^{\prime}$, respectively. A CS or SC star, with further entries in the S-star catalog.

1902+1014 4159 Yam: C8,1; KB: SC 6:/9e = C6:,2+.

1903+0730 4162 Also MaeSoy90. Nassau and Blanco noted this star to be very red.

1904-0541 4164 HR 7220. San: N; K-M: C6,4; Yam: C5,4. Very strong $\mathrm{SiC}_{2}$ bands.

1906-2023 4169 The position is only about $3^{\prime}$ from that of V1076 Sgr, an RR Lyrae-type star.

1907-0248 4177 De: C4:,4:. Quite red.

1908-5533 4168 Also Hen 335.

1908-1715 $4179-17^{\circ} 5492$. San: R2.

1908+1737 4181 Also D 127(R). Hydrogen-deficient (Bidelman 1956).

1909-1531 4184 Spectrum resembles CS star.

1911-0123 4194 C 738, MaeSoy90, CCCS 2707.

1913-3056 4196 Also Sto II-18 (R).

1913+2718 4208 CCCS 2711. Yam: C8,2J. SoyMae99 position used here differs by $4.4 \mathrm{~s}$ from Ste.

$1915+54174222$ Silicate CSE.

$1915+25124212$ CCCS 2714. SoyMae99 position used here differs by 6 $\mathrm{s}$ and $0.5^{\prime}$ from Ste. 
$1915+27494215$ SoyMae99 position used here differs by $10.6 \mathrm{~s}$ and $0.5^{\prime}$ from Ste.

$1916+21544217$ D $129(\mathrm{~N}), \mathrm{C} 451$.

$1917+42054236$ Unpublished corrected coordinates by Ste.

$1918+01474225$ A unique, nova-like variable star. Bidelman (1971) has summarised its history and suggested that it should have been supernova, whose spectra superficially resemble R stars. Van den Bergh (1971) has pointed out that a weak planetary nebula of low surface brightness, which does not seem to be expanding, surrounds this position. This weakens Bidelman's original hypothesis of an extragalactic supernova, but leaves the true nature obscure; Van de Bergh's suggestion that it is a slow nova is not reconcilable with Lundmark's description of the spectrum. Infrared objective prism plates of the region by Stephenson showed no carbon star in the region in 1971. One of Lundmark's original spectrograms was recently recovered by Lynga at Lund and sent to Bidelman at the Warner and Swasey Observatory. Lundmark's classification of the spectrum as type $R$ is undoubtedly correct. The only peculiarity is that the G-band is weak or absent; Bidelman (unpublished) now thinks the star may be an exceptional type of $R$ CrB star. (Ste).

1919-1554 4229 $-16^{\circ} 5272$, Pots $135-671(\mathrm{~N})$, Van 76 (R8), Ste (N, 3 plates). San: N.

1920+3752 4241 Also Ste (N). Yam: C4,5e. See also Bidelman (1954). In area of the open cluster NGC 6791.

1920-0802 6780 Also Fou92, AFGL 2368, NSV 11912, LeB92, GJB93. $1921+76334302+76^{\circ} 734$, D $309(\mathrm{~N})$, Ste (N). San: N; K-M: C7,3. $1921+31204248$ Near, but different from, V378 Lyr.

$1922+20034249$ Rosino et al. in A\&AS 31, 314, give a type of M6 for the Mira variable since named OW Vul. However, their identification chart leaves no doubt that this star is a carbon star and variable. (Ste).

1923+2427 4253 Also SoyMae99. CCCS 2728. Its position used here differs by $8.5 \mathrm{~s}$ from Ste. Carbon symbiotic binary (Munari et al. 1999, IAUC 7329).

1923-1042 4247 Also Van 77 (R2). San: R2; K-M: C1,2; Yam: C2,2Hd. Although hydrogen-deficient, cited in HD as a typical R0 star. 
$1924+30394263$ Van 78, W58 19, NassSte61 (R). Erroneously identified with a BD star by Nassau and Blanco (1957).

$1924+85214485$ D 315, D 316 (R), Van 79 (R5), Ste. San: R3; Yam: $\mathrm{C} 3,3 \mathrm{CH}$. Also an early-listed CH star by Bidelman (1956).

$1926+23364277$ Also SoyMae99.

1927+0704 4275 C5-9e by Allen et al., ApJ, 217, 109, 1977.

$1928+46024307$ D $310(N)$, C 706, Ack 78-12-7, Ste (N).

1930-0050 4301 C 740, HanBl, IRC+00438.

$1931+21484323$ Hydrogen-deficient.

$1932+28384336$ NassSte61, W58 11.

1934-1622 $4333-16^{\circ} 5360$, Ste (N). San: N.

$1934+28044347$ IRC +30374 .

1937-3322 4357 Also BidMac.

1937+0645 4373 Also D $133(\mathrm{~N})$. San: N; Yam: C4,4.

$1937+31504380$ Also W 32, SoyMae99.

1939+2737 4393 Also SoyMae99.

$1940+09014397$ Also Perr59. The published Dearborn DEC is $13^{\prime}$ in error.

$1940+33224409$ This and the nearby AFGL carbon star are separately seen on the same infrared survey plate by Ste.

$1940+31464410$ D 212 (N?), C 455, W58 34, SoyMae99.

$1940+32374415+32^{\circ} 3522$, D 213 (R), W58 41, SoyMae99. CannMay49: N3; Bid: C6,4e; Yam: C5,4. The star has two detached gas shells.

$1940+27294411$ SoyMae99 position used here differs by $3.6^{\prime}$ from Ste.

$1942+29044431$ SoyMae99 position given here differs by $6 \mathrm{~s}$ and $1^{\prime}$ from Ste.

$1943+16464430$ CCCS 2778 .

1943+1918 4437 Also VB 8013-70.

$1943+19344439$ Also VB 8013-69, whose DEC is $2^{\prime} \mathrm{N}$ of the quoted GCVS position.

$1943+34294443$ The originally published Case DEC was slightly in error. Vogt (AJ, 78, 389) reobserved the carbon star and considered it to be $\mathrm{BD}+34^{\circ} 3680$.

1946-1809 $4454-18^{\circ} 5480$, Ste (N). Yam: C7,1J. 
$1946+32254471$ The quoted position, from one plate of Ste infrared survey, differs by $18 \mathrm{~s}$ in RA from the GCVS. Ste position has been confirmed by a computer underlay. The original identification of the variable with the carbon star was by Nassau and Cameron.

$1949+29454501$ SoyMae99 position used here differs by $11 \mathrm{~s}$ and $1.2^{\prime}$ from Ste.

1949+2936 4507 SoyMae99 position differs by 1' in DEC.

$1949+29304500$ Also SoyMae99. The GCVS position is about $2^{\prime}$ different.

1949+0346 4497 De: C3:,2. Red.

1950+3039 4518 Exceptionally strong infrared CN.

1951+5341 4543 De: CS; GCVS: C6-7,-e; KB: SC8/8, with strong $\mathrm{CaCl}$. See also Bidelman (1954).

$1951+44214538$ CCCS 2822.

1953-2728 4523 Also BidMac, who classifies the star as a $\mathrm{CH}$ star.

1956-6522 4524 War: C1p, CH star.

$1956+36064566$ Not the nearby KK Cyg.

$1957+00144567$ D 137 (R), Van 80 (R8), Ste (R). San: R4.

$1957+44154581+43^{\circ} 3425$, D $317(\mathrm{~N})$, BSD 39-1464 (NA), C 707, Har, MaeSoy88, Ste. San: N, strong $\mathrm{SiC}_{2}$; Yam: C4,5. Listed in IDS.

1957+3309 4583 Strong $\mathrm{H} \alpha$ emission (MacConnell 1967).

$1958+43064588$ Also MaeSoy88.

2001-0721 4595 Also Ste (N). CCCS 2837. San R4; De: C5,5. Silicate CSE.

$2001+09294598$ D $138(R), \operatorname{Van} 81(R 8)$, Ste (N), CCCS 2838. Yam: $\mathrm{C} 4,3 \mathrm{CH}$.

2001+4001 4619 De: C6,3; Yam: C5,3.

2001+4055 6855 Also AFGL 2494, GJBSW, VKL92, GJG94, GEAS97, MS76b, GJB93, OLFHHS.

$2002+21054616+20^{\circ} 4390 f$, D $139(\mathrm{~N})$, SoyMae99. San: N; Yam: C4,3.

$2002+30464627$ Also SoyMae99. The position of the last source is used which differs by $1^{\prime}$ from Ste.

$2002+37364635$ In outskirts of the open cluster Dolidze 37 . 
2003-2527 4612 Also BidMac.

$2003+17054633$ Not the nearby RZ Sge.

$2003+48104654$ Har, Ste (N), MaeSoy88.

$2004+42114662$ In area of the open cluster Dolidze 38.

$2005+20384653+20^{\circ} 4417$, D 140(N), SoyMae99. San: N; De: C6-,5;

Yam: C6,4. Visual companion $V=13.2 \mathrm{mag}, 6^{\prime \prime}$ away.

$2006+35174670$ Also Ste.

$2006+40204678$ Also Kurt88 18.

$2007+35184685$ Also VB 8016-52.

$2009+21574693$ Also SoyMae99. The Richt source makes this the N preceding component of a close binary.

2009+3125 6860 Also MS76b, GJBSW, GEAS97, AFGL 2513, GJB93, GWSK.

2009+3512 4700 Also VB 8016-51.

$2009+41294712+41^{\circ} 3632, \mathrm{C} 288$, Har (N), Ste, Ack 78-6-61. De:

C6-,3; Yam: C6,3e. In outskirts of the open cluster Dolidze 2.

$2009+47524714+47^{\circ} 3031$, D $319(\mathrm{~N})$, Van $(\mathrm{N})$, Har $(\mathrm{N})$, Ste, MaeSoy88. IDS object, visual companion $45^{\prime \prime}$ away.

$2010+35564716+35^{\circ} 4002$, D $216(\mathrm{~N})$, C 24, Ste (N:), VB 8016-77. De: C5,4; Yam: C6,4. Near the open cluster NGC 6883.

$2011+36064729$ Also VB 8016-76. An M7 star is about 70" in PA $30^{\circ}$ from the carbon star, mentioned by Schaifers (1959) and confirmed by Ste.

2011-1416 4701 Strong D lines.

2013+3843 4758 San: Ne; K-M: C8,2; Yam: C8,2e. Listed in IDS; see also Bidelman (1954). Shane noted unusually large violet opacity for this spectral type.

2015+3701 4782 Ack 78-0-6, VB 8016-80.

$2015+37074785$ Also NSV 12948. Yam: C4,1CH.

2016+4955 4793 NSV 12973, Har (LR or N), MaeSoy88.

2017-2119 4774 -21 ${ }^{\circ} 5672$, Ste (N), HanBl, IRC-20 585. ADS 13616A.

2017+3653 4796 Ack 78-0-6, VB 8016-81.

$2018+37264806+37^{\circ} 3876$, D 219 (N), C 207, Ack 78-0-19. Bid: C8e;

Yam: C9,2JLi. San noted that the Li I $6707 \AA$ line 
is nearly as strong in this spectrum as in that of the lithium star WZ Cas.

$2019+47534817+47^{\circ} 3077$, D $321(\mathrm{~N})$, C 289, Ste. San: Ne(SiC2); KM: C7,2-C9,2 Yam: C8,2e. Numerous observers have discussed the hydrogen line emission, cf. Bidelman (1954). The star is a prototype of carbon stars exhibiting $\mathrm{CaCl}$ bands, cf. Sanford, PASP, 54, 158, 1942.

2020-4949 4792 NSV 12993, Hen 305.

$2022+69444857$ NSV 13068. The Ste $\mathrm{N}$ type is based on two plates. $2022+47374842$ Also MaeSoy88.

2023+5614 4851 D $322(\mathrm{R})$, Ste (N). Yam: C4,4.

$2026+38134861$ Ack 78-0-52.

$2027+26434863$ The published DEC is from the Ste infrared survey. $2031+46484887$ CCCS 2909

2031-1153 4873 NSV 13120, Van 83 (R5), Ste (very strong red $\mathrm{C}_{2}$ ). Yam: C3,4J.

2033+49194899 CCCS 2911

$2035+45294910$ CCCS 2914

2036+6005 4923 D 325 (R). De: C5-,5; Yam: C4,5J. Silicate CSE. Water maser emission at $22 \mathrm{GHz}$ and weak $\mathrm{OH}$ maser at $1667 \mathrm{MHz}$ (Engels 1994).

$2037+36504921$ Also Hen 312 .

$2037+48564927$ CCCS 2920

$2037+48094924$ The MaeSoy88 position is given; the Kurt88-90 position is by $15 \mathrm{~s}$ smaller in RA and by $2^{\prime}$ larger in DEC.

2041+4808 4939 D $327(\mathrm{~N}), \mathrm{C} 468$. Yam: C5,3e. See also Bidelaman (1954).

$2041+46204942$ Also Aar90.

$2043+32074946$ D $224(\mathrm{~N})$, Hen 313. Yam: C5,4; De: C5,4. The carbon star is $2.5^{\prime} \mathrm{E}$ of the $\mathrm{B} 8-\mathrm{A} 0$ star $\mathrm{BD}+31^{\circ} 4201$.

$2043+35044947$ Van 84 (R2). Yam: C3,2.

$2044+47034959$ Also MaeSoy88.

$2045+43294966$ Also VB 8019-17.

2048-1901 4972 Also Ste (R). 
$2048+17504978+17^{\circ} 4421$. Spectroscopic binary with a period of 1295 days. A CH star according to Yamashita $(\mathrm{C} 1, \mathrm{OCH})$ and Bond (PASP, 82, 321).

$2048+51334988$ C 471, Ste.

2049+3313 4989 D 225 (N), C 708, Hen 314, Ste, CCCS 2935. Yam: C6,4. ADS $14338 \mathrm{~A}$. The probably physical companion is G6 III (Gordon 1968), $V=11.0 \mathrm{mag}, 10^{\prime \prime}$ away.

2049+4648 4994 MaeSoy88, CCCS 2936.

$2050+45255001$ D $330(R)$, C 215, Ste (N), Ste, VB 8019-27, MaeSoy88. GCVS: $\mathrm{C} 3,2-\mathrm{C} 5,4$.

$2052+45205017$ MaeSoy88, CCCS 2939.

$2053+45135027$ Aar90 identifies it with IRAS $22239+2548$ which has a position differing from Ste by $3 \mathrm{~s}$ in RA and by $50^{\prime \prime}$ in DEC.

2054+0805 5022 De: C2,3; Yam: C3,2.

2056+3725 5044 AFGL 2679, CohKuh.

$2056+51045045$ In area of the open cluster Berk 53 .

$2057+54285057$ Also Kurtun.

$2057+42455056$ CCCS 2946.

2059+4736 5072 V1541 Cyg. MaeSoy88.

2059+4540 5074 Also Kurt88 149.

2059+4619 5077 Also MaeSoy88.

$2100+47405086$ Also MaeSoy88.

$2101+51075093$ Also MaeSoy88.

$2102+47325098$ Kurt88's DEC differs by $10^{\prime}$ from Lit.

2102+4607 5097 CCCS 2961.

2102+45395101 Also Kurt88 159.

2103+44095105 MaeSoy88, CCCS 2964.

2103+4507 5110 MaeSoy88, CCCS 2967.

2104+4542 5123 Also Kurt88 180.

$2104+53205131$ The $\mathrm{CN}$ absorption is unusually wide on an infrared Case plate. Also Kurt88 180.

$2104+51345137$ Also MaeSoy88.

2105-0012 5120 Ste(Ne), HanBl, IRC+00499. Yam: C6,3e. The Dearborn observers described the red-region objective-prism spectrum in much the same terms as that of the SC 
star GP Ori, but strong $\mathrm{C}_{2}$ bands have been seen on slit spectrograms. The star is extremely red, as noted at Dearborn.

$2105+46435134$ Also MaeSoy88.

2105+46545133 NSV 13524 (Ste).

2105+4504 5135 Also MaeSoy88.

2105-0012 5120 GCVS: C6-7,2-4.

2106+4518 5145 Also MaeSoy88.

$2107+21395144$ CS-like on a $600 \AA / \mathrm{mm}$ blue-region Case plate; $\mathrm{C}_{2}$ not seen (Ste).

$2107+46015153$ Also MaeSoy88.

$2107+45475156$ Also Ste, MaeSoy88.

$2107+43415155$ MaeSoy88, CCCS 2986.

$2108+47245163$ Also VB 8019-73, MaeSoy88.

$2109+56175168$ Kurt has corrected the Kurt80a position to $21^{h} 06^{m} 06^{s}$, $+55^{\circ} 53.6^{\prime}$. The quoted position is for $\mathrm{BC} 281$, which

Ste infrared survey confirms to $0.1 \mathrm{~s}$ and $1^{\prime \prime}$.

$2109+45135165$ Also MaeSoy88.

2110+4644 5171 Also MaeSoy88. Strong $\mathrm{SiC}_{2}$ bands.

2110+4420 5179 Also MaeSoy 88.

$2110+33225172$ NSV 13579. Also Hen 316, with a $20^{\prime}$ DEC error.

2113+4011 5192 V529 Cyg only 3.6' away.

$2114+44415204$ Also Ste, MaeSoy88.

$2115+43275209$ MaeSoy88 position is used. The Case and Ste RA is 1 $\mathrm{s}$ smaller and DEC is about $2^{\prime}$ larger.

$2116+50235224$ Also MaeSoy 88.

2116+4739 5216 Also Ack 95-0-22, Ste, MaeSoy88.

$2117+55595235$ Presumably AS 462, although Ste sees any $\mathrm{H} \alpha$ emission on objective prism plate.

2118-0132 $5227-2^{\circ} 5503$, Ste (wkR). Yam: C3,1CH. A Case plate confirms that the BD star is a carbon star. There is no nearby $\mathrm{M}$ star to account for the HD classification (Ste).

$2119+03145230$ Van 88 (R2), Ste (R). Yam: C3,2.

$2119+45435244$ Strong D lines.

$2119+50455256 \quad \mathrm{SiC}_{2}$ bands. 
2120-4501 5228 HR 8145, Hen 317. War: C7,3; Yam: C7,2.

$2121+59525267$ The published Dearborn position is evidently a misprint. Case plates show only one carbon star, approximately in the position given here. (Ste).

$2122+42235265$ C 516, Har(N), Ste(N), MaeSoy88. Yam: C6,3e.

$2123+42295280$ The GCVS gives the spectral type M4, but Ste has not found any M-type candidate nearer than a dozen minutes on the red and infrared plates. The position given here is the mean of Ste and BC.

2124-69445239 $-70^{\circ} 1827$, Hen 318.

$2124+44495290$ Also MaeSoy88.

$2124+44375289$ Also MaeSoy88.

$2124+43485291$ Also MaeSoy 88 .

$2125+59415306$ There is a nearby S star, No. 1273 in the 2nd ed. of the Ste $\mathrm{S}$ star catalog, $2.5^{\prime} \mathrm{N}$ and $1^{\prime} \mathrm{W}$ of this carbon star. GCVS: C6,0-C7,3e.

$2126+52195310$ Also Ste, MaeSoy 88.

2126+4836 5304 V1564 Cyg, Ste, MaeSoy88. Two Case infrared plates, one quite deep, show only one carbon star here, in the quoted position (Ste).

$2130+49505333$ CCCS 3037.

2131+5303 5339 Also Kurtun, Nik2 20, VB 8018-11.

$2131+53085342$ Also Nik2 19, Ste. Different from nearby $2131+5303$.

2133+4355 5353 Also Ste, MaeSoy88.

$2135+60545371+60^{\circ} 2267$, D 335 (R), Ste. Yam: C5:,4; GCVS: C4$5,4-5$.

$2135+78375406+77^{\circ} 827$, D $336(\mathrm{~N})$, Ste (N). K-M: C7,4; Yam: C7,3e. See also Bidelman (1954). HD: a typical star of class Nc.

$2136+54305378$ Also VB 8018-30, Kurt88 253, Ste.

$2138+45425390$ Also MaeSoy 88 .

$2138+46455396$ Also MaeSoy88.

$2140+45275404$ Also MaeSoy88. Nassau and Cameron (1956) made the original identification of the carbon star with the variable. With the published identification chart plus further Case plates, Ste confirms the identification.

$2140+46195411$ Also MaeSoy88. 
$2141+46315416$ Also MaeSoy88.

2142+3530 5418 HR 8297, D 229 (R), Ste (N), CCCS 3060. K-M: C6,3; San N. Note that two variable star names have been assigned to this star.

$2142+54385423$ Also VB 8018-29, Kurt88 270.

$2143+38015425+37^{\circ} 4407$, D $230(\mathrm{~N})$, Ste (N). Strong $\mathrm{SiC}_{2}$ bands noted by Sanford (N) and Shane. K-M: C6,4. In IDS. See also Bidelman (1954).

$2144+73385452$ CCCS 3070. De: C6-,3e. $\mathrm{SiC}_{2}$ bands.

$2145+54065441$ NSV 13876, VB 8018-38, Ste.

2146+5234 5447 Har (LR or N), Ste, VB 8018-26.

$2147+45535453$ Also MaeSoy88.

$2150+47485465$ C 663, Har, Ste.

2151+5505 5481 Ack 95-0-131, VB 8018-52.

$2152+53295487$ Ack 95-0-132, VB 8018-45.

$2152+47495480$ Also MaeSoy88.

$2152+47235484$ Also MaeSoy88.

$2153+54115489$ Also Kurt88 284.

$2155+50295494+49^{\circ} 3673$, D 343(N), C 517, Van 89, Har, Ste. San: R3; Yam: C5,4. HD likens its spectrum to VX And. In IDS.

$2155+54295495$ D $342(\mathrm{~N})$, C 26, Ste (N), Har (N). De: C5+,4; Yam: $\mathrm{C} 5,4$.

2155+5253 5497 Also VB 8018-59.

$2155+54155501$ Also Kurt88 287.

$2156+22515496+22^{\circ} 4508$, D 151(N), Ste(N). San: N; K-M: C5,5;

Yam: C4,4J.

$2156+56115514$ The position is from two plates of the Ste infrared survey. The identification with NZ Cep is by Ste with reference to published identification chart though the difference in position is $3^{\prime}$.

$2156+63045510$ The positions by Ste and BC agree very well and are given here. Krum's coordinates for the same star are almost half a degree in error.

$2157+54545520$ Also VB 8020-19.

$2158+53245526$ Also Ste.

$2158+55305534$ Also VB 8018-62. 
$2200+54505544$ Also Kurt88 298.

$2201+31535547$ Unpublished corrected coordinates by Ste.

2201+5432 5548 Also VB 8020-17. Silicate CSE. Water maser emission at $22 \mathrm{GHz}$ (Engels 1994).

2202+53515557 Kurtun, VB 8020-14.

2203-1637 5549 Hydrogen-deficient (Bidelman 1956). See also ApJ, $233,205,1979$. Ste classification was assigned without knowledge of the identity of the star.

$2203+45345560$ BSD 41-2321 (R), CCCS 3101, Har (N), Ste. Yam: $\mathrm{C} 4:, 3$. In IDS. The probable physical companion is F8 III-V: (Gordon 1968).

$2203+59255562$ Also Nik2 30. The position given here, from one plate of the Ste infrared survey, is $4^{\prime} \mathrm{S}$ of Nik2 and Kurtun, and $1^{\prime}$ from Case; it is supported by a computer underlay.

$2203+59285564$ Not the nearby star $2203+5925$.

$2204+62065569$ CCCS 3108 .

2204+21035561 D $152(\mathrm{R})$, Van 90 (R), Ste (R), CCCS 3102. K-M: C3p,2 (CH star); Yam: C2,2CH; San: R3. GCVS: $\mathrm{C} 1,2 \mathrm{CH}(\mathrm{R} 6)$. Visual companion $V=12.96 \mathrm{mag}, 9^{\prime \prime}$ away. Spectroscopic binary with the period 407 days. Outstandingly high radial velocity: $-\mathbf{3 8 1} \mathrm{km} / \mathrm{s}$.

$2204+25095566 \quad \mathrm{C}_{2}$ very weak. $2205+33305570+32^{\circ} 4335$, Ste (wkC to CS). Bid: C9e; Yam: C9,1e; KB: SC5-9/9-e. RY Peg, now considered nonvariable, is about $20^{\prime \prime}$ away.

$2205+56275573$ Also Kurt88 299.

$2206+48275577$ Dean notes strong Li I.

$2209+50275588$ Also Kurtun.

$2212+54125600$ Also Nik2 31. VB's position is given; Kurtun's is $2.2^{\prime}$ S. Nik2 gives $53^{\circ} 43^{\prime}$.

$2212+55445601$ Also VB 8020-43.

$2214+56235612$ Also VB 8020-41.

$2216+54015620$ Also VB 8020-9. Weak $\mathrm{C}_{2} 4737 \AA$ band. $2^{\prime}$ away from the center of the open cluster IC 1442 .

$2218+43475626$ AFGL 2881.

$2223+58445650$ CCCS 3129 . 
$2223+56425644$ CCCS 3127.

$2226+58085661$ The VB position is $2.5^{\prime} \mathrm{S}$ of the quoted one.

$2232+58375677$ CCCS 3140 .

$2235+53215686$ Probably NSV 14234 .

$2242+51315707$ Bright $\mathrm{H} \beta$ and $\mathrm{H} \gamma$ (Mich).

$2243+74475725$ The IRAS position is given.

$2244+18065714$ Strong D lines.

2244+59545723 Daub71 (CSV 1742), Ste. Near the open cluster NGC 7419 .

$2244+61435719+60^{\circ} 2432$, D $348(\mathrm{~N})$, C 27 , Van $91(\mathrm{R} 8)$, Ste (N), Har (LR or N), Kurt88 332, AFGL 2948, IRC+60365. K$\mathrm{M}: \mathrm{C} 6,4$.

2244+4928 5721 D 349 (R), Har (LR), Ste, CCCS 3153. Yam: C3:,4.

In IDS; companion F6 III-V: (Gordon 1968), 6.3" away.

$2245+56375722$ D 350 (N?), C 257, Ste (N:).

2245-2522 5713 . Sto V-1 (N), BidMac.

2245+5504 5728 D 352 (R), C 675, Van 92 (R5), Har (ER), Ste. Yam:

C3:,4J. The published Dearborn position is in error.

$2247+58185735$ Near the open cluster NGC 7654 .

$2247+59595737$ Also Ste.

$2251+64165753$ The Kurtun position is $6.5^{\prime} \mathrm{S}$ of the one given here, which is from two accordant positions from the Ste infrared survey.

$2251+60345754$ Also Ste. Near the open cluster NGC 7419.

2254-0657 5761 Also Ste(R).

$2254+60465768$ Near the open cluster NGC 7419. Kurt88 344, Ste.

$2256+54135774+53^{\circ} 3033$, D 354 (R), C 518, Har (LR), Ste.

$2301+13055789$ Close to CS in type.

$2301+45535791+45^{\circ} 4121$, D $355(\mathrm{~N})$, Ste, AFGL 3015, IRC+50455.

Yam: C3,5J; GCVS: C3,5-C4,4-5.

$2302+43035794$ De: C6-,4e. The Ste $\mathrm{N}$ classification is obtained independently from two plates.

2310+4618 5822 Van 94 (R2), Ste (R). Yam: C2,2.

2311-2100 5823 Van 95 (R2), Ste (R). War: C1,3; Yam: C3:,3.

$2319+47145848$ Also Dol62. Silicate CSE. 
2319+1711 6913 Also Jon78, Fou92, GJB93, JBGHJKL, GJG94, OLFHHS, AFGL 3068, GJB93.

$2323+45255861$ D 359, Ste.

2323+5610 5865 D 360(N), C 684, Ste, AFGL 5764S, IRC+60403. Yam: $\mathrm{C} 4,5 \mathrm{~J}$.

$2324+64155871$ The nearby carbon star No. $2324+6416$ is separately seen on two survey plates.

$2326+49305879+48^{\circ} 4051$, D $361(\mathrm{R} ?)$, Ste (N).

$2328+10546916$ AFGL 3099.

$2332+62065890$ IRC+60412.

2334+43336920 Also JVGHJKL, GJBSW, MS76a, GJB93, CKJJ, IRC+40540, AFGL3116, NSV 14623.

$2338+56015901$ Also Ste; IRAS.

$2338+35465903+34^{\circ} 4974$, D $233(\mathrm{R})$, Ste (N). San: R3e; De: C6,4e; Yam: C4,3e. The classifications of this variable star are unusually discordant, reflecting at least some spectrum variation. See also Bidelman (1954).

$2339+63155909$ Blanco's position is that of V441 Cas, called as S on the basis of its redness. There is, however, a carbon star only $7^{\prime}$ away (found and measured in deep infrared survey), and its position is given here. (Ste).

$2340+70195910$ Also $+69^{\circ} 1349$.

2343+5812 5919 MaeSoy 9. Close to type CS on red plates, which is also reflected by Dean's classification.

$2345+62595927$ Also Nik 6 .

2346+0329 5928 D $154(\mathrm{~N})$, Ste (N), HR 9004, AFGL 3147, IRC+00532, CCCS 3202. HD: a typical star of class Na. San: N; $\mathrm{K}-\mathrm{M}: \mathrm{C} 6,2$.

$2347+62415936$ Nik 11 .

$2349+06225937$ D 155 (R), Van 96 (R2), Ste. HD: a typical star of class R3. San: R3; Yam: C3,2.

$2352+58055954$ MaeSoy 26.

$2352+5644595540^{\prime \prime}$ away from M-type variable DH Cas.

$2353+62435957$ Also Kurtun.

$2355+62565961$ Also Nik 20, Ste.

$2356+64355963$ Also Nik 22, Ste. 
$2356+62535964$ Also Nik 21, Ste.

2358+5821 5967 Also MaeSoy 33.

$2359+56585970$ D 364 (R), C 519, Ste (N). Yam: C6,3e. The C 519 list identifies with $\mathrm{BD}+56^{\circ} 3126$, which is $\mathrm{HDE} 240465$, type F8. The Case identification is in error; see Gordon (1968). See also Bidelman (1954). V533 Cas, a fainter carbon star, is nearby; member of the open cluster NGC 7789.

$2359+565859727^{\prime}$ away from V532 Cas. In outskirts of the open cluster NGC 7789. 